Federal Reserve Bank of Dallas

Globalization and Monetary Policy Institute

Working Paper No. 74

http://www.dallasfed.org/assets/documents/institute/wpapers/2011/0074.pdf

\title{
A Redux of the Workhorse NOEM Model with Capital Accumulation and Incomplete Asset Markets*
}

\author{
Enrique Martínez-García \\ Federal Reserve Bank of Dallas
}

February 2011

\begin{abstract}
I build a symmetric two-country model that incorporates nominal rigidities, local-currency pricing and monopolistic competition distorting the goods markets. The model is similar to the framework developed in Martínez-García and Søndergaard (2008a, 2008b), but it also introduces frictions in the assets markets by restricting the financial assets available to two uncontingent nominal bonds in zero-net supply and by adding quadratic costs on international borrowing (see, e.g., Benigno and Thoenissen (2008) and Benigno (2009). The technical part of the paper contains three basic calculations. First, I derive the equilibrium conditions of the open economy model under local-currency pricing and incomplete asset markets. Second, I compute the zero-inflation (deterministic) steady state and discuss what happens with a non-zero net foreign asset position. Third, I derive the log-linearization of the equilibrium conditions around the deterministic steady state. The quantitative part of the paper aims to give a broad overview of the role that incomplete international asset markets can play in accounting for the persistence and volatility of the real exchange rate. I find that the simulation of the incomplete and complete asset markets models is almost indistinguishable whenever the business cycle is driven primarily by either non-persistent monetary or persistent productivity (but not permanent) shocks. In turn, asset market incompleteness has more sizeable wealth effects whenever the cycle is driven by persistent (but not permanent) investment-specific technology shocks, resulting in significantly lower real exchange rate volatility.
\end{abstract}

JEL codes: F31, F37, F41

\footnotetext{
* Enrique Martínez-García, Research Department, Federal Reserve Bank of Dallas, 2200 N. Pearl Street, Dallas, TX 75201. enrique.martinez-garcia@dal.frb.org. 214-922-5262. I would like to thank Janet Koech, María Teresa Martínez-García, Jens Søndergaard, Jian Wang, Mark Wynne, and many others for helpful discussions. I also gratefully acknowledge the support of the Federal Reserve Bank of Dallas. All remaining errors are mine alone. The views in this paper are those of the author and do not necessarily reflect the views of the Federal Reserve Bank of Dallas, or the Federal Reserve System.
} 


\section{Introduction}

Finding a macroeconomic model that can simultaneously replicate the volatility and persistence of the real exchange rate (RER) has been a challenge. One strand of the literature in particular has focused on imperfections in the goods markets (nominal rigidities) as a possible source of RER fluctuations. Not surprisingly, many so-called New Open Economy Macro (NOEM) models have been written for the purpose of investigating the stylized facts of the RER by looking more closely at the pricing decisions of firms. Most of those models, however, isolate the frictions in the goods markets and take for granted that international asset markets ought to be complete.

The functioning of international asset markets determines the extent to which households can efficiently insure amongst themselves to smooth their consumption in the presence of country-specific shocks. Asset markets are viewed as crucial for the propagation and transmission of business cycle fluctuations across countries. Hence, a natural question becomes how sensitive the results in the NOEM literature are to the assumption of complete international asset markets. In this paper, I extend the full-fledge NOEM model with capital accumulation of Martínez-García and Søndergaard (2008a, 2008b) by abandoning the assumption of complete asset markets in order to provide a tractable framework that would easily lend itself to quantitative evaluation of the role of the complete markets assumption.

I adopt a standard incomplete international asset markets structure that restricts the financial assets available to just two uncontingent nominal bonds in zero-net supply (issued in two countries) adding a quadratic cost on international borrowing tied to the real net foreign asset position of the home country (see, e.g., Benigno and Thoenissen (2008) and Benigno (2009)). This set-up represents a clear departure from the complete international asset market assumption, but it also ensures that the solution of the model would be stationary up to a first-order approximation (see also the discussion on closing small open economy models in Schmitt-Grohé and Uribe (2003)). A more in-depth exploration of the complex role of asset markets may reveal important insights that cannot be easily mapped into this reduce form characterization of international asset markets, but that goes beyond the scope of this paper and is left for future research.

The emphasis of the paper is clearly on the technical side and the draft is predominantly focused on the characterization of the first principles of this open-economy setting, and on the many issues surrounding the determination of a steady state and a first-order approximation of the equilibrium conditions - specially whenever the trade balance might be different than zero in steady state. Nonetheless, I extensively document the simulated moments of the model under complete and incomplete asset markets and provide some basic insights about the implications of these experiments that complement (and refine) the work in MartínezGarcía and Søndergaard (2008a, 2008b).

The intuition in Martínez-García and Søndergaard (2008a, 2008b) is rather straightforward. Adding capital gives households in both countries a margin of intertemporal adjustment, thereby making the consumption and RER paths smoother. Capital accumulation contributes to significantly lower the consumption and RER volatility in the NOEM model-irrespective of the shocks driving the cycle. Adjustment costs slow the response of investment to shocks, making it costlier for households to adjust intertemporally and pushing the volatility of consumption and the RER up. Without severely constraining the use of capital accumulation, only in response to monetary shocks we can expect sufficiently volatile RERs.

However, the persistence of the RER falls short if monetary shocks are the primary driver of the business

cycle (see also Chari et al. (2002)). High RER persistence tends to occur in response to persistent productiv- 
ity shocks if the Taylor (1993) monetary policy rule also has a very inertial component. With non-persistent monetary shocks, the persistence is often less but it is also tied to the specification of the Taylor (1993) monetary policy and the adjustment cost function. Still, deviations from the law of one price are larger and more important to account for RER fluctuations when the model is primarily driven by monetary shocks (see, e.g., Betts and Devereux (2000)).

When I depart from the assumption of complete international asset markets, which imposes perfect international risk-sharing and a tight link between the RER and relative consumption, I find that a bond economy subject to international borrowing costs and the workhorse NOEM model with complete asset markets generate very similar international business cycle patterns in response to productivity and monetary shocks (see also Baxter and Crucini (1995), Heathcote and Perri (2002) and Chari et al. (2002)). Asset market incompleteness, however, tends to result in significantly lower RER volatility whenever the business cycles are primarily driven by (persistent but not permanent) investment-specific technology (IST) shocks.

Investment-specific technology (IST) shocks, however, also induce excessive investment volatility and countercyclical consumption that are inconsistent with the data. Interestingly, the optimal decision to postpone consumption to invest more in response to a positive IST shock leads the RER to appreciate on impact while domestic output increases, but the opposite occurs with either productivity or monetary shocks.

The remainder of the paper is structured as follows: section 2 describes my two-country NOEM model with capital accumulation and incomplete asset markets, while section 3 outlines the optimality conditions, section 4 characterizes the zero-inflation steady state, and section 5 derives in detail the log-linearization of

the equilibrium conditions of the model. Section 6 covers a further refinement of the model to incorporate variable capital utilization, and section 7 summarizes the parameterization strategy used for the simulations. Section 8 highlights the quantitative findings, and section 9 concludes. There is also an Appendix that describes the set of log-linear equilibrium conditions and includes all the Tables and Figures in the paper.

\section{The Monetary Open Economy with Incomplete Markets}

Here, I briefly describe the structure of the monetary open economy model with incomplete international asset markets.

\subsection{The Intertemporal Consumption and Savings Problem}

I specify a stochastic, two-country general equilibrium model. Each country is populated by a continuum of infinitely lived (and identical) households in the interval $[0,1]$. In each period, the domestic households' utility function is additively separable in consumption, $C_{t}$, and labor, $L_{t}$. Domestic households maximize,

$$
\sum_{\tau=0}^{+\infty} \beta^{\tau} \mathbb{E}_{t}\left[\frac{1}{1-\sigma^{-1}}\left(C_{t+\tau}\right)^{1-\sigma^{-1}}-\frac{1}{1+\varphi}\left(L_{t+\tau}\right)^{1+\varphi}\right]
$$

where $0<\beta<1$ is the subjective intertemporal discount factor. The elasticity of intertemporal substitution satisfies that $\sigma>0(\sigma \neq 1)$ while the inverse of the Frisch elasticity of labor supply satisfies that $\varphi>0$.

I assume that domestic households are able to trade two nominal risk-less (uncontigent) bonds denominated in domestic and foreign currency, respectively. Hence, the domestic household maximizes its lifetime 
utility in (1) subject to the sequence of budget constraints described by,

$$
P_{t}\left(C_{t}+X_{t}\right)+T_{t}+\frac{1}{I_{t}} B_{t+1}+\frac{1}{I_{t}^{*}} S_{t} B_{t+1}^{F *}+\frac{\mu}{2} \frac{P_{t}}{I_{t}^{*}}\left(\frac{S_{t} B_{t+1}^{F *}}{P_{t}}-\bar{a}\right)^{2} \leq B_{t}+S_{t} B_{t}^{F *}+W_{t} L_{t}+Z_{t} K_{t}+P r_{t}
$$

and the law of motion for capital given by,

$$
K_{t+1} \leq(1-\delta) K_{t}+V_{t} \Phi\left(X_{t}, X_{t-1}, K_{t}\right) X_{t}
$$

where $W_{t}$ is the domestic nominal wage, $P_{t}$ is the domestic consumption price index (CPI), $P r_{t}$ are the nominal profits generated by the domestic firms, and and $T_{t}$ is a lump-sum nominal tax levied on the domestic households. ${ }^{1}$ Moreover, $X_{t}$ is domestic real investment, $K_{t}$ stands for domestic real capital, $Z_{t}$ defines the nominal rental rate of capital, and $V_{t}$ is an exogenous, investment-specific technology (IST) shock.

$B_{t+1}$ is the payoff in period $t+1$ of the (uncontingent) risk-free bond denominated in units of the domestic currency, acquired by the domestic household at the end of period $t$. The implicit nominal gross interest rate on this bond is $I_{t} . B_{t+1}^{F *}$ is the payoff in period $t+1$ of the (uncontingent) risk-free bond denominated in foreign currency and acquired by the domestic household at the end of period $t$. The implicit nominal gross interest rate on this bond is $I_{t}^{*}$, while $S_{t}$ denotes the nominal exchange rate. As in Benigno (2009), I also assume that there is a quadratic cost function (e.g. an international borrowing cost) that penalizes changes in the real foreign asset position of the domestic economy, $\frac{S_{t} B_{t+1}^{F *}}{P_{t}}$, whenever it deviates from constant real reference level of $\bar{a}$. The quadratic borrowing cost is then re-scaled by $\frac{P_{t}}{I_{t}^{*}}$ (for analytical convenience) and multiplied by the parameter $\mu>0$ to fix its size.

The foreign households maximize their lifetime utility (analogous to (1)) subject to a law of motion for capital similar to the one described in (3). I follow Benigno and Thoenissen (2008) in assuming that foreign households are able to trade only on (uncontigent) risk-free bonds denominated in foreign currency. Hence, the foreign households' budget constraint is described by,

$$
P_{t}^{*}\left(C_{t}^{*}+X_{t}^{*}\right)+T_{t}^{*}+\frac{1}{I_{t}^{*}} B_{t+1}^{*} \leq B_{t}^{*}+W_{t}^{*} L_{t}^{*}+Z_{t}^{*} K_{t}^{*}+P r_{t}^{*}+T r_{t}^{*}
$$

$B_{t+1}^{*}$ is the payoff in period $t+1$ of the (uncontingent) risk-free bond denominated in units of the foreign currency, acquired by the foreign household at the end of period $t$. As before, the implicit nominal gross interest rate on this bond is $I_{t}^{*}$. I assume that the foreign households receive the profits from the foreign firms in equal proportion, $\mathrm{Pr}_{t}^{*}$. Foreign households also count as revenue the international borrowing costs paid by the domestic households in trading foreign bonds, i.e.,

$$
\operatorname{Tr}_{t}^{*}=\frac{\mu}{2} \frac{P_{t}}{S_{t} I_{t}^{*}}\left(\frac{S_{t} B_{t+1}^{F *}}{P_{t}}-\bar{a}\right)^{2}
$$

where $T r_{t}^{*}$ expresses the international borrowing costs paid by the domestic households in units of the foreign currency. This asymmetry in the financial market structure between domestic and foreign households is made

\footnotetext{
${ }^{1}$ Fiscal policy is not fully incorporated into the model. However, I include a balanced budget rule (no government borrowing) and lump-sum taxes to finance a subsidy to firms meant to neutralize the mark-up distortion associated with the assumption of monopolistic competition. In case no subsidy was introduced, then the lump-sum tax would simply be equal to zero, i.e. $T_{t}=0$.
} 
for simplicity. For an extension of this set-up in which domestic and foreign households can trade in bonds denominated in both currencies, see Benigno (2009). I can re-interpret the model presented here as a polar case of Benigno (2009) in which the costs of international borrowing are prohibitively high for the foreign households, but not for the domestic households. These modelling assumptions introduce a rather standard incomplete asset market structure (see, e.g., Benigno and Thoenissen (2008) and Benigno (2009)) that serve to close the model down while inducing stationarity. ${ }^{2}$

The domestic- and foreign-currency denominated bonds are issued respectively by the domestic and foreign governments in zero-net supply. The bond market clearing conditions can be expressed as,

$$
\begin{aligned}
B_{t} & =0, \\
B_{t}^{*}+B_{t}^{F *} & =0 .
\end{aligned}
$$

I assume that there is no trade in either domestic or foreign shares of firms. Sole ownership of the local firms rests in the hands of the local households. Money is purely a unit of account, but monetary policy has a potential economic impact by regulating short-term nominal interest rates to affect the inflation rate in the presence of nominal rigidities. Embedded in this model is also the assumption that both factor markets (for labor and capital) are homogenous and perfectly competitive within a country, but segregated across countries. In other words, factors can be used for production purposes in any firm within the same country, but they are immobile across borders.

The capital accumulation in (3) may be subject to adjustment costs, $\Phi(\cdot)$. I consider three special cases: the capital adjustment cost (CAC) case, the investment adjustment cost (IAC) case, and the no adjustment costs (NAC) case. The NAC function is simply,

$$
\Phi\left(X_{t}, X_{t-1}, K_{t}\right)=1
$$

The NAC function for the foreign law of motion for capital is the obvious counterpart. This implies that in steady state $\Phi(\bar{X}, \bar{X}, \bar{K})=1, \Phi^{\prime}(\bar{X}, \bar{X}, \bar{K})=0$, and $\Phi^{\prime \prime}(\bar{X}, \bar{X}, \bar{K})=0$. The CAC and IAC adjustment cost functions, however, require a more detailed description.

Capital Adjustment Cost (CAC) Function. The capital adjustment cost (CAC) function (see, e.g., Chari, et al. (2002)) implies that the function $\Phi(\cdot)$ in (3) takes the following form,

$$
\Phi\left(\frac{X_{t}}{K_{t}}\right)=1-\frac{1}{2} \chi \frac{\left(\frac{X_{t}}{K_{t}}-\delta\right)^{2}}{\frac{X_{t}}{K_{t}}},
$$

where $\frac{X_{t}}{K_{t}}$ is the corresponding investment-to-capital ratio, $\delta$ is the depreciation rate appearing in the law of motion for capital, and $\chi \geq 0$ measures the curvature of the cost function. Among the properties of this

\footnotetext{
${ }^{2}$ For more details and other alternatives to close down the model, see Schmitt-Grohé and Uribe (2003).
} 
adjustment cost function that are relevant for my model, I note that,

$$
\begin{aligned}
\Phi^{\prime}\left(\frac{X_{t}}{K_{t}}\right) & =-\frac{1}{2} \chi\left[\frac{2 \frac{X_{t}}{K_{t}}\left(\frac{X_{t}}{K_{t}}-\delta\right)-\left(\frac{X_{t}}{K_{t}}-\delta\right)^{2}}{\left(\frac{X_{t}}{K_{t}}\right)^{2}}\right] \\
\Phi^{\prime \prime}\left(\frac{X_{t}}{K_{t}}\right) & =-\frac{1}{2} \chi\left[\frac{2\left(\frac{X_{t}}{K_{t}}\right)^{3}-2\left(2 \frac{X_{t}}{K_{t}}\left(\frac{X_{t}}{K_{t}}-\delta\right)-\left(\frac{X_{t}}{K_{t}}-\delta\right)^{2}\right) \frac{X_{t}}{K_{t}}}{\left(\frac{X_{t}}{K_{t}}\right)^{4}}\right] .
\end{aligned}
$$

The same adjustment cost formula as in (9) applies to the foreign households' problem.

I assume that in steady state the IST shocks are at their unconditional mean of $\bar{V}=\bar{V}^{*}=1$. Hence, in steady state the adjustment costs dissipate and the investment-to-capital ratio is equal to the depreciation rate (i.e., $\bar{X}=\delta \bar{K}$ and $\bar{X}^{*}=\delta \bar{K}^{*}$ ) as in the NAC case. This implies that $\Phi(\delta)=1, \Phi^{\prime}(\delta)=0$, and $\Phi^{\prime \prime}(\delta)=-\frac{\chi}{\delta}$.

Investment Adjustment Cost (IAC) Function. I also explore the investment adjustment cost function (IAC) used among others by Smets and Wouters (2003) and Christiano, et al. (2005). The IAC specification implies that the function $\Phi(\cdot)$ in (3) takes the following form,

$$
\Phi\left(\frac{X_{t}}{X_{t-1}}\right)=1-\frac{1}{2} \kappa \frac{\left(\frac{X_{t}}{X_{t-1}}-1\right)^{2}}{\frac{X_{t}}{X_{t-1}}},
$$

where $\frac{X_{t}}{X_{t-1}}$ is the corresponding gross rate of investment, and $\kappa \geq 0$ measures the curvature of the cost function. Among the properties of the IAC function that are relevant for my model, I note that,

$$
\begin{aligned}
\Phi^{\prime}\left(\frac{X_{t}}{X_{t-1}}\right) & =-\frac{1}{2} \kappa\left[\frac{2 \frac{X_{t}}{X_{t-1}}\left(\frac{X_{t}}{X_{t-1}}-1\right)-\left(\frac{X_{t}}{X_{t-1}}-1\right)^{2}}{\left(\frac{X_{t}}{X_{t-1}}\right)^{2}}\right] \\
\Phi^{\prime \prime}\left(\frac{X_{t}}{X_{t-1}}\right) & =-\frac{1}{2} \kappa\left[\frac{2\left(\frac{X_{t}}{X_{t-1}}\right)^{3}-2\left(2 \frac{X_{t}}{X_{t-1}}\left(\frac{X_{t}}{X_{t-1}}-1\right)-\left(\frac{X_{t}}{X_{t-1}}-1\right)^{2}\right)\left(\frac{X_{t}}{X_{t-1}}\right)}{\left(\frac{X_{t}}{X_{t-1}}\right)^{4}}\right] .
\end{aligned}
$$

The same adjustment cost formula as in (10) applies to the foreign households' problem.

In steady state, the adjustment costs dissipate again because the net growth of investment is zero. Under this adjustment cost specification it is costly to change the level of investment and the cost increases with the size of the change, but there are no adjustment costs in steady state. Hence, the steady state expression of the law of motion for capital is the same as in the standard NAC case. This also implies that $\Phi(1)=1$, $\Phi^{\prime}(1)=0$, and $\Phi^{\prime \prime}(1)=-\kappa$.

Aggregation Rules and the Price Indexes. I assume that investment, like consumption, is a composite index of domestic and imported foreign varieties. The home and foreign consumption bundles of the domestic household, $C_{t}^{H}$ and $C_{t}^{F}$, as well as the investment bundles, $X_{t}^{H}$ and $X_{t}^{F}$, are aggregated by means of a CES 
index as,

$$
\begin{aligned}
C_{t}^{H} & =\left[\int_{0}^{1} C_{t}(h)^{\frac{\theta-1}{\theta}} d h\right]^{\frac{\theta}{\theta-1}}, C_{t}^{F}=\left[\int_{0}^{1} C_{t}(f)^{\frac{\theta-1}{\theta}} d f\right]^{\frac{\theta}{\theta-1}} \\
X_{t}^{H} & =\left[\int_{0}^{1} X_{t}(h)^{\frac{\theta-1}{\theta}} d h\right]^{\frac{\theta}{\theta-1}}, X_{t}^{F}=\left[\int_{0}^{1} X_{t}(f)^{\frac{\theta-1}{\theta}} d f\right]^{\frac{\theta}{\theta-1}}
\end{aligned}
$$

while aggregate consumption and investment, $C_{t}$ and $X_{t}$, are defined with another CES index as,

$$
\begin{aligned}
C_{t} & =\left[\phi_{H}^{\frac{1}{\eta}}\left(C_{t}^{H}\right)^{\frac{\eta-1}{\eta}}+\phi_{F}^{\frac{1}{\eta}}\left(C_{t}^{F}\right)^{\frac{\eta-1}{\eta}}\right]^{\frac{\eta}{\eta-1}} \\
X_{t} & =\left[\phi_{H}^{\frac{1}{\eta}}\left(X_{t}^{H}\right)^{\frac{\eta-1}{\eta}}+\phi_{F}^{\frac{1}{\eta}}\left(X_{t}^{F}\right)^{\frac{\eta-1}{\eta}}\right]^{\frac{\eta}{\eta-1}} .
\end{aligned}
$$

The elasticity of substitution across varieties produced within a country is $\theta>1$, and the elasticity of intratemporal substitution between the home and foreign bundles of varieties is $\eta>0$. The share of the home goods in the domestic aggregators is $\phi_{H}$, while the share of foreign goods is $\phi_{F}$. I assume the shares are homogeneous, i.e. $\phi_{H}+\phi_{F}=1$. Similarly, I can define the aggregators for the foreign household. The only difference being that the share of the home goods in the foreign aggregators is $\phi_{H}^{*}=\phi_{F}$, while the share of foreign goods in the foreign aggregator is $\phi_{F}^{*}=\phi_{H}$.

The model introduces home-product bias in consumption (Warnock (2003)) as well as in the composition of investment. By assumption, investment goods can only be used for local production after aggregation. This is also the case because of compositional differences across countries. However, all local and foreign varieties can be traded internationally for either consumption or investment purposes. Moreover, the symmetry of the aggregators implies that the corresponding price indexes are identical for investment and consumption bundles. Hence, the relative price between consumption and investment is one as reflected in the budget constraint (that is, in equation (2)).

Under standard results on functional separability, the CPI indexes which correspond to my specification of the domestic aggregators in (13) - (14) and their foreign counterparts are,

$$
\begin{aligned}
P_{t} & =\left[\phi_{H}\left(P_{t}^{H}\right)^{1-\eta}+\phi_{F}\left(P_{t}^{F}\right)^{1-\eta}\right]^{\frac{1}{1-\eta}}, \\
P_{t}^{*} & =\left[\phi_{H}^{*}\left(P_{t}^{H *}\right)^{1-\eta}+\phi_{F}^{*}\left(P_{t}^{F *}\right)^{1-\eta}\right]^{\frac{1}{1-\eta}}=\left[\phi_{F}\left(P_{t}^{H *}\right)^{1-\eta}+\phi_{H}\left(P_{t}^{F *}\right)^{1-\eta}\right]^{\frac{1}{1-\eta}},
\end{aligned}
$$


and the price sub-indexes are,

$$
\begin{aligned}
P_{t}^{H} & =\left[\int_{0}^{1}\left(P_{t}(h)\right)^{1-\theta} d h\right]^{\frac{1}{1-\theta}}, \\
P_{t}^{F} & =\left[\int_{0}^{1}\left(P_{t}(f)\right)^{1-\theta} d f\right]^{\frac{1}{1-\theta}}, \\
P_{t}^{H *} & =\left[\int_{0}^{1}\left(P_{t}^{*}(h)\right)^{1-\theta} d h\right]^{\frac{1}{1-\theta}}, \\
P_{t}^{F *} & =\left[\int_{0}^{1}\left(P_{t}^{*}(f)\right)^{1-\theta} d f\right]^{\frac{1}{1-\theta}}
\end{aligned}
$$

where $P_{t}^{H}$ and $P_{t}^{F}$ are the price sub-indexes for the home- and foreign-produced bundle of goods in units of the home currency. Similarly for $P_{t}^{H *}$ and $P_{t}^{F *}$. I define the real exchange rate as,

$$
R S_{t} \equiv \frac{S_{t} P_{t}^{*}}{P_{t}}
$$

where $S_{t}$ denotes the nominal exchange rate.

\subsection{The Price-Setting Problem}

Each firm supplies the home and foreign market, and sets prices in the local currency (henceforth, localcurrency pricing or LCP pricing). Firms engage in third-degree price discrimination across markets (re-selling is infeasible) and, furthermore, enjoy monopolistic power in their own variety. Frictions in the goods market are modelled with nominal price stickiness à la Calvo (1983). At time $t$ any firm (whether domestic or foreign) is forced to maintain its previous period prices in the domestic and foreign markets with probability $0<\alpha<1$. Instead, with probability $(1-\alpha)$, the firm receives a signal to optimally reset each price.

I assume that production employs a (homogeneous of degree one) Cobb-Douglas technology, i.e.

$$
\begin{aligned}
Y_{t}(h) & =A_{t}\left(K_{t}(h)\right)^{1-\psi}\left(L_{t}(h)\right)^{\psi}, \forall h \in[0,1], \\
Y_{t}^{*}(f) & =A_{t}^{*}\left(K_{t}^{*}(f)\right)^{1-\psi}\left(L_{t}^{*}(f)\right)^{\psi}, \forall f \in[0,1],
\end{aligned}
$$

where $A_{t}$ is the (aggregate) domestic productivity shock and $A_{t}^{*}$ is the (aggregate) foreign productivity shock. The labor share in the production function is represented by $0 \leq \psi \leq 1 .^{3}$ By consistency and market clearing it follows that the aggregate capital accumulated by households in the domestic and foreign country is $K_{t}=\int_{0}^{1} K_{t}(h) d h$ and $K_{t}^{*}=\int_{0}^{1} K_{t}(f) d f$ respectively, while aggregate labor is $L_{t}=\int_{0}^{1} L_{t}(h) d h$ and $L_{t}^{*}=\int_{0}^{1} L_{t}(f) d f$ respectively. Solving the cost-minimization problem of each individual firm yields an efficiency condition linking the capital-to-labor ratios to factor price ratios as follows,

$$
\begin{aligned}
\frac{K_{t}}{L_{t}} & =\frac{K_{t}(h)}{L_{t}(h)}=\frac{1-\psi}{\psi} \frac{W_{t}}{Z_{t}}, \forall h \in[0,1], \\
\frac{K_{t}^{*}}{L_{t}^{*}} & =\frac{K_{t}^{*}(f)}{L_{t}^{*}(f)}=\frac{1-\psi}{\psi} \frac{W_{t}^{*}}{Z_{t}^{*}}, \forall f \in[0,1],
\end{aligned}
$$

\footnotetext{
${ }^{3}$ These expressions reduce to the standard case of linear-in-labor technologies if $\psi=1$.
} 
as well as a characterization for the (pre-subsidy) nominal marginal costs,

$$
\begin{aligned}
M C_{t} & =\frac{1}{A_{t}} \frac{1}{\psi^{\psi}(1-\psi)^{1-\psi}}\left(W_{t}\right)^{\psi}\left(Z_{t}\right)^{1-\psi}, \\
M C_{t}^{*} & =\frac{1}{A_{t}^{*}} \frac{1}{\psi^{\psi}(1-\psi)^{1-\psi}}\left(W_{t}^{*}\right)^{\psi}\left(Z_{t}^{*}\right)^{1-\psi} .
\end{aligned}
$$

The labor force is homogenous within a country and immobile across borders, and the national labor markets are perfectly competitive. Wages equalize in each country (but not necessarily across countries), i.e. $W_{t}(h)=$ $W_{t}$ for all $h \in[0,1]$ and $W_{t}^{*}(f)=W_{t}^{*}$ for all $f \in[0,1]$, and so does the rental rate on capital, i.e. $Z_{t}(h)=Z_{t}$ for all $h \in[0,1]$ and $Z_{t}^{*}(f)=Z_{t}^{*}$ for all $f \in[0,1]$. Then, since the production function is homogeneous of degree one (constant returns-to-scale), this implies that all local firms choose the same capital-to-labor ratio even though they end up producing different amounts. Moreover, the factors of production are compensated according to their marginal product across all firms.

I introduce a government subsidy in each country that is proportional to the local firms' production costs, i.e. $\xi_{t} M C_{t} Y_{t}(h)$ and $\xi_{t}^{*} M C_{t}^{*} Y_{t}^{*}(f)$ respectively in the domestic and foreign countries. The pre-subsidy production costs of a firm are simply a fraction of the (pre-subsidy) nominal marginal costs, $M C_{t}$ and $M C_{t}^{*}$, in (26) - (27) times the output of that firm. Governments only subsidize the production of firms located in their own country, independently of whether the goods are sold locally or exported. Governments set no import tariffs or subsidize the local demand over the export demand, hence not distorting the international relative prices (e.g., the terms of trade and the real exchange rate). Using the pre-subsidy marginal costs described in (26) - (27), I can write the post-subsidy marginal costs that enter into the pricing decisions of firms in both countries as follows,

$$
\begin{aligned}
\left(1-\xi_{t}\right) M C_{t} & =\frac{1}{A_{t}} \frac{1}{\psi^{\psi}(1-\psi)^{1-\psi}}\left(\left(1-\xi_{t}\right) W_{t}\right)^{\psi}\left(\left(1-\xi_{t}\right) Z_{t}\right)^{1-\psi}, \\
\left(1-\xi_{t}^{*}\right) M C_{t}^{*} & =\frac{1}{A_{t}^{*}} \frac{1}{\psi^{\psi}(1-\psi)^{1-\psi}}\left(\left(1-\xi_{t}^{*}\right) W_{t}^{*}\right)^{\psi}\left(\left(1-\xi_{t}^{*}\right) Z_{t}^{*}\right)^{1-\psi} .
\end{aligned}
$$

This characterization of the subsidies suffices for the purpose of either reducing or eliminating the mark-up distortions associated with monopolistic competition in the goods markets.

Remark 1 I must point out that any given subsidy that the government desires to set can be implemented with a combination of wage subsidies, $\xi_{t}^{L}$ and $\xi_{t}^{L *}$, and capital rental subsidies, $\xi_{t}^{K}$ and $\xi_{t}^{K *}$, which satisfies that,

$$
\begin{aligned}
\left(1-\xi_{t}\right) & =\left(1-\xi_{t}^{L}\right)^{\psi}\left(1-\xi_{t}^{K}\right)^{1-\psi} \\
\left(1-\xi_{t}^{*}\right) & =\left(1-\xi_{t}^{L *}\right)^{\psi}\left(1-\xi_{t}^{K *}\right)^{1-\psi}
\end{aligned}
$$

Under these conditions it immediately follows that,

$$
\begin{aligned}
& \frac{1}{A_{t}} \frac{1}{\psi^{\psi}(1-\psi)^{1-\psi}}\left(\left(1-\xi_{t}^{L}\right) W_{t}\right)^{\psi}\left(\left(1-\xi_{t}^{K}\right) Z_{t}\right)^{1-\psi}=\left(1-\xi_{t}\right) M C_{t}, \\
& \frac{1}{A_{t}^{*}} \frac{1}{\psi^{\psi}(1-\psi)^{1-\psi}}\left(\left(1-\xi_{t}^{L *}\right) W_{t}^{*}\right)^{\psi}\left(\left(1-\xi_{t}^{K *}\right) Z_{t}^{*}\right)^{1-\psi}=\left(1-\xi_{t}^{*}\right) M C_{t}^{*} \text {, }
\end{aligned}
$$


which shows that the post-subsidy marginal costs are the same as before. However, the efficiency conditions in $(24)-(25)$ would not be the same with or without subsidies unless the wage and capital rental subsidies are assumed to be the same, i.e. unless $\xi_{t}=\xi_{t}^{L}=\xi_{t}^{K}$ and $\xi_{t}^{*}=\xi_{t}^{L *}=\xi_{t}^{K *}$.

In other words, I impose the assumption that both factors of production are subsidized in the same proportion in order not to distort the allocation of capital and labor while trying to correct for the mark-up distortion with the introduction of these subsidies. Hence, the nominal wage $W_{t}$ and the nominal rental rate of capital $Z_{t}$ denote the amounts perceived by the domestic households in the supply of each factor of production, while the wage $W_{t}^{*}$ and the rental rate of capital $Z_{t}^{*}$ are the amounts perceived by the foreign households. In turn, the cost of a unit of labor and the cost of renting a unit of capital for the domestic firms are $\left(1-\xi_{t}\right) W_{t}$ and $\left(1-\xi_{t}\right) Z_{t}$, and similarly $\left(1-\xi_{t}^{*}\right) W_{t}^{*}$ and $\left(1-\xi_{t}^{*}\right) Z_{t}^{*}$ are the corresponding costs for the foreign firms. Still, the factor price ratios faced by the domestic and foreign firms would be unrelated to the subsidy, and equal to $\frac{W_{t}}{Z_{t}}$ and $\frac{W_{t}^{*}}{Z_{t}^{*}}$ respectively.

The Optimal Pricing Problem. A re-optimizing domestic firm $h$ under LCP pricing chooses a domestic and a foreign price, $\widetilde{P}_{t}(h)$ and $\widetilde{P}_{t}^{*}(h)$, to maximize the expected discounted value of its net profits,

$$
\sum_{\tau=0}^{+\infty} \mathbb{E}_{t}\left\{\alpha^{\tau} M_{t, t+\tau}\left[\begin{array}{l}
\left(\widetilde{C}_{t, t+\tau}(h)+\widetilde{X}_{t, t+\tau}(h)\right)\left(\widetilde{P}_{t}(h)-\left(1-\xi_{t+\tau}\right) M C_{t+\tau}\right)+\ldots \\
\left(\widetilde{C}_{t, t+\tau}^{*}(h)+\widetilde{X}_{t, t+\tau}^{*}(h)\right)\left(S_{t+\tau} \widetilde{P}_{t}^{*}(h)-\left(1-\xi_{t+\tau}\right) M C_{t+\tau}\right)
\end{array}\right]\right\}
$$

where $M_{t, t+\tau} \equiv \beta^{\tau}\left(\frac{C_{t+\tau}}{C_{t}}\right)^{-\sigma^{-1}} \frac{P_{t}}{P_{t+\tau}}$ is the stochastic discount factor (SDF) for $\tau$-periods ahead nominal payoffs (corresponding to the domestic representative household), subject to a pair of demand constraints in each goods market,

$$
\begin{aligned}
& \widetilde{C}_{t, t+\tau}(h)+\widetilde{X}_{t, t+\tau}(h)=\left(\frac{\widetilde{P}_{t}(h)}{P_{t+\tau}^{H}}\right)^{-\theta}\left(C_{t+\tau}^{H}+X_{t+\tau}^{H}\right), \\
& \widetilde{C}_{t, t+\tau}^{*}(h)+\widetilde{X}_{t, t+\tau}^{*}(h)=\left(\frac{\widetilde{P}_{t}^{*}(h)}{P_{t+\tau}^{H *}}\right)^{-\theta}\left(C_{t+\tau}^{H *}+X_{t+\tau}^{H *}\right) .
\end{aligned}
$$

Here, $\widetilde{C}_{t, t+\tau}(h)$ and $\widetilde{C}_{t, t+\tau}^{*}(h)$ indicate the consumption demand for any variety $h$ at home and abroad respectively, given that prices $\widetilde{P}_{t}(h)$ and $\widetilde{P}_{t}^{*}(h)$ remain unchanged between time $t$ and $t+\tau$. Similarly, $\widetilde{X}_{t, t+\tau}(h)$ and $\widetilde{X}_{t, t+\tau}^{*}(h)$ indicate the households' investment demand at those same prices. ${ }^{4}$

Similarly, I characterize the objective of the foreign firm as,

$$
\sum_{\tau=0}^{+\infty} \mathbb{E}_{t}\left\{\alpha^{\tau} M_{t, t+\tau}^{*}\left[\begin{array}{c}
\left(\widetilde{C}_{t, t+\tau}(f)+\widetilde{X}_{t, t+\tau}(f)\right)\left(\frac{\widetilde{P}_{t}(f)}{S_{t+\tau}}-\left(1-\xi_{t+\tau}^{*}\right) M C_{t+\tau}^{*}\right)+\ldots \\
\left(\widetilde{C}_{t, t+\tau}^{*}(f)+\widetilde{X}_{t, t+\tau}^{*}(f)\right)\left(\widetilde{P}_{t}^{*}(f)-\left(1-\xi_{t+\tau}^{*}\right) M C_{t+\tau}^{*}\right)
\end{array}\right]\right\},
$$

\footnotetext{
${ }^{4}$ I derive the demand for variety $h$ in the home and foreign markets by combining the first-order conditions in (46) - (49), section 3 .
} 
where $M_{t, t+\tau}^{*} \equiv \beta\left(\frac{C_{t+\tau}^{*}}{C_{t}^{*}}\right)^{-\sigma^{-1}} \frac{P_{t}^{*}}{P_{t+\tau}^{*}}$ is the foreign SDF. The demand constraints of the foreign firm are,

$$
\begin{aligned}
& \widetilde{C}_{t, t+\tau}(f)+\widetilde{X}_{t, t+\tau}(f)=\left(\frac{\widetilde{P}_{t}(f)}{P_{t+\tau}^{F}}\right)^{-\theta}\left(C_{t+\tau}^{F}+X_{t+\tau}^{F}\right), \\
& \widetilde{C}_{t, t+\tau}^{*}(f)+\widetilde{X}_{t, t+\tau}^{*}(f)=\left(\frac{\widetilde{P}_{t}^{*}(f)}{P_{t+\tau}^{F *}}\right)^{-\theta}\left(C_{t+\tau}^{F *}+X_{t+\tau}^{F *}\right),
\end{aligned}
$$

given that prices $\widetilde{P}_{t}(h)$ and $\widetilde{P}_{t}^{*}(h)$ remain unchanged between time $t$ and $t+\tau .^{5}$

\subsection{The Monetary Policy Rule and Government Budget Constraint}

The Taylor rule is often defined as the trademark of modern monetary policy. In that case the policy instrument of the domestic and foreign monetary authorities are the short-term rates $I_{t}$ and $I_{t}^{*}$ respectively, while $\bar{I}$ and $\bar{I}^{*}$ are their corresponding steady state values. I assume that the monetary authorities of both countries set short-term nominal interest rates according to Taylor (1993) type rules,

$$
\begin{aligned}
I_{t} & =M_{t}\left(I_{t-1}\right)^{\rho_{i}}\left[\bar{I}\left(\frac{\Pi_{t}}{\bar{\Pi}}\right)^{\psi_{\pi}}\left(\frac{Y_{t}}{\bar{Y}}\right)^{\psi_{y}}\right]^{1-\rho_{i}}, \\
I_{t}^{*} & =M_{t}^{*}\left(I_{t-1}^{*}\right)^{\rho_{i}}\left[\bar{I}^{*}\left(\frac{\Pi_{t}^{*}}{\bar{\Pi}^{*}}\right)^{\psi_{\pi}}\left(\frac{Y_{t}^{*}}{\bar{Y}^{*}}\right)^{\psi_{y}}\right]^{1-\rho_{i}},
\end{aligned}
$$

where $M_{t}$ and $M_{t}^{*}$ are the (domestic and foreign) monetary policy shocks or the shocks to the interest rate policy rules, $\Pi_{t} \equiv \frac{P_{t}}{P_{t-1}}$ and $\Pi_{t}^{*} \equiv \frac{P_{t}^{*}}{P_{t-1}^{*}}$ are the (gross) CPI inflation rates, while $Y_{t}$ and $Y_{t}^{*}$ are the respective output levels. Finally, $\bar{\Pi}$ and $\bar{\Pi}^{*}$ are the steady state (gross) CPI inflation rates, and $\bar{Y}$ and $\bar{Y}^{*}$ are the respective steady state output levels. In other words, the monetary policy rules in (38) - (39) respond to local CPI inflation and output deviations from their respective steady state levels. The index captures both a smoothing term and a systematic policy component. This index specification of the Taylor rule takes a more standard form once it is log-linearized.

The fiscal policy in each country is characterized by balanced budgets in every period, and a subsidy to the production costs of the local producers that is fully financed by a lump-sum tax on households. I summarize the government's budget constraints as,

$$
\begin{aligned}
T_{t} & =\xi_{t} M C_{t} \int_{0}^{1} Y_{t}(h) d h=\xi_{t} M C_{t} Y_{t}, \\
T_{t}^{*} & =\xi_{t}^{*} M C_{t}^{*} \int_{0}^{1} Y_{t}^{*}(f) d f=\xi_{t}^{*} M C_{t}^{*} Y_{t}^{*},
\end{aligned}
$$

where $T_{t}$ and $T_{t}^{*}$ are the domestic and foreign lump-sum taxes on households, and $\xi_{t}$ and $\xi_{t}^{*}$ are the domestic and foreign subsidies expressed as a fraction of the production costs. Given the production functions in $(22)$ - (23), the pre-subsidy marginal cost equations in (26) - (27) and the fact that capital-to-labor ratios

\footnotetext{
${ }^{5}$ I derive the demand for variety $f$ in the home and foreign markets by combining the first-order conditions in (46) - (49), section 3 .
} 
in $(24)-(25)$ are equalized across firms within each country, it is possible to write the government budget constraints as follows,

$$
\begin{aligned}
T_{t} & =\xi_{t} M C_{t} A_{t}\left(\frac{K_{t}}{L_{t}}\right)^{1-\psi} \int_{0}^{1} L_{t}(h) d h \\
& =\xi_{t} M C_{t} A_{t}\left(K_{t}\right)^{1-\psi}\left(L_{t}\right)^{\psi} \\
& =\xi_{t} \frac{1}{\psi^{\psi}(1-\psi)^{1-\psi}}\left(W_{t} L_{t}\right)^{\psi}\left(Z_{t} K_{t}\right)^{1-\psi}, \\
T_{t}^{*} & =\xi_{t}^{*} M C_{t}^{*} A_{t}^{*}\left(\frac{K_{t}^{*}}{L_{t}^{*}}\right)^{1-\psi} \int_{0}^{1} L_{t}^{*}(f) d f \\
& =\xi_{t}^{*} M C_{t}^{*} A_{t}^{*}\left(K_{t}^{*}\right)^{1-\psi}\left(L_{t}^{*}\right)^{\psi} \\
& =\xi_{t}^{*} \frac{1}{\psi^{\psi}(1-\psi)^{1-\psi}}\left(W_{t}^{*} L_{t}^{*}\right)^{\psi}\left(Z_{t}^{*} K_{t}^{*}\right)^{1-\psi},
\end{aligned}
$$

where the second equality follows also from the labor market clearing conditions (i.e. $L_{t}=\int_{0}^{1} L_{t}(h) d h$ and $\left.L_{t}^{*}=\int_{0}^{1} L_{t}^{*}(f) d f\right)$. As these government budget constraints illustrate, the value of the subsidy can be expressed as a share of an aggregate function of the labor income, $W_{t} L_{t}$ and $W_{t}^{*} L_{t}^{*}$ respectively, and the capital rental income, $Z_{t} K_{t}$ and $Z_{t}^{*} K_{t}^{*}$ respectively. As expected, in the limit whenever the labor share converges to one, i.e. $\psi \rightarrow 1$, the technology becomes linear-in-labor, and the subsidy becomes equal to a fraction of the labor income for each country.

In Martínez-García and Søndergaard (2008) this tax subsidy is completely ignored, so the implicit assumption is that $\xi_{t}=\xi_{t}^{*}=0$ and $T_{t}=T_{t}^{*}=0$. Often, however, these subsidies are used to neutralize the mark-up distortion introduced by the assumption that firms produce and sell their varieties under monopolistic competition. In order to eliminate this distortion, suffices to set the subsidy in each country to satisfy the following pair of conditions,

$$
\begin{aligned}
& \frac{\theta}{\theta-1}\left(1-\xi_{t}\right)=1 \\
& \frac{\theta}{\theta-1}\left(1-\xi_{t}^{*}\right)=1 .
\end{aligned}
$$

From here it follows that the optimal subsidy for both countries is characterized as,

$$
\xi_{t}=\xi_{t}^{*}=\frac{1}{\theta}<1
$$

which is a function of the elasticity of substitution across varieties produced within a country, i.e. $\theta>1$. The mark-up is also a function solely of the elasticity of substitution across varieties. Since the elasticity is time-invariant, so are the mark-ups and the subsidies (expressed as a share of the production costs) needed.

For simplicity, I treat the subsidies as a secondary policy instrument intended exclusively to deal with the mark-up distortion and, therefore, I assume from now on that the subsidy as a fraction of the production 
costs will be invariant over time and identical across countries, i.e.,

$$
\xi_{t}=\xi_{t}^{*}=\bar{\xi} \in\left[0, \frac{1}{\theta}\right]
$$

where $\bar{\xi}$ also denotes the steady state subsidy in both countries. In turn, the mark-up and the subsidyindependently of whether the subsidy is set to zero or neutralizes partially or totally the mark-up distortiononly affect the dynamics of the model up to a first-order approximation because those terms enter into the computations of the steady state investment share. Rather than choosing a specific value for the subsidy, I view its share over the production costs as another structural parameter of the model.

\section{The Optimality Conditions}

Here, I present the relevant equilibrium conditions of the model. Since the model is built around two mostly symmetric countries, all the first-order conditions reported correspond to the home country unless otherwise noted.

The Optimality Conditions from the Households' Problem. Given the structure described in (11) (12), the solution to the sub-utility maximization problem implies that the home and foreign households' demands for each variety are given by,

$$
\begin{aligned}
C_{t}(h) & =\left(\frac{P_{t}(h)}{P_{t}^{H}}\right)^{-\theta} C_{t}^{H}, X_{t}(h)=\left(\frac{P_{t}(h)}{P_{t}^{H}}\right)^{-\theta} X_{t}^{H}, \forall h \in[0,1], \\
C_{t}(f) & =\left(\frac{P_{t}(f)}{P_{t}^{F}}\right)^{-\theta} C_{t}^{F}, X_{t}(f)=\left(\frac{P_{t}(f)}{P_{t}^{F}}\right)^{-\theta} X_{t}^{F}, \forall f \in[0,1], \\
C_{t}^{*}(h) & =\left(\frac{P_{t}^{*}(h)}{P_{t}^{H *}}\right)^{-\theta} C_{t}^{H *}, X_{t}^{*}(h)=\left(\frac{P_{t}^{*}(h)}{P_{t}^{H *}}\right)^{-\theta} X_{t}^{H *}, \forall h \in[0,1], \\
C_{t}^{*}(f) & =\left(\frac{P_{t}^{*}(f)}{P_{t}^{F *}}\right)^{-\theta} C_{t}^{F *}, X_{t}^{*}(f)=\left(\frac{P_{t}^{*}(f)}{P_{t}^{F *}}\right)^{-\theta} X_{t}^{F *}, \forall f \in[0,1],
\end{aligned}
$$

while the demands for the bundles of home and foreign goods are simply equal to,

$$
\begin{aligned}
C_{t}^{H} & =\phi_{H}\left(\frac{P_{t}^{H}}{P_{t}}\right)^{-\eta} C_{t}, X_{t}^{H}=\phi_{H}\left(\frac{P_{t}^{H}}{P_{t}}\right)^{-\eta} X_{t}, \\
C_{t}^{F} & =\phi_{F}\left(\frac{P_{t}^{F}}{P_{t}}\right)^{-\eta} C_{t}, X_{t}^{F}=\phi_{F}\left(\frac{P_{t}^{F}}{P_{t}}\right)^{-\eta} X_{t}, \\
C_{t}^{H *} & =\phi_{H}^{*}\left(\frac{P_{t}^{H *}}{P_{t}^{*}}\right)^{-\eta} C_{t}^{*}, X_{t}^{H *}=\phi_{H}^{*}\left(\frac{P_{t}^{H *}}{P_{t}^{*}}\right)^{-\eta} X_{t}^{*}, \\
C_{t}^{F *} & =\phi_{F}^{*}\left(\frac{P_{t}^{F *}}{P_{t}^{*}}\right)^{-\eta} C_{t}^{*}, X_{t}^{F *}=\phi_{F}^{*}\left(\frac{P_{t}^{F *}}{P_{t}^{*}}\right)^{-\eta} X_{t}^{*},
\end{aligned}
$$

where the share of the home goods in the foreign aggregator is $\phi_{H}^{*}=\phi_{F}$ and the share of foreign goods in the foreign aggregator is $\phi_{F}^{*}=\phi_{H}$. These equations determine the demand functions in the model.

The equilibrium conditions of the households' problem include a pair of labor supply functions (the 
intratemporal first-order conditions) which can be expressed as,

$$
\begin{aligned}
\frac{W_{t}}{P_{t}} & =\left(C_{t}\right)^{\sigma^{-1}}\left(L_{t}^{s}\right)^{\varphi}, \\
\frac{W_{t}^{*}}{P_{t}^{*}} & =\left(C_{t}^{*}\right)^{\sigma^{-1}}\left(L_{t}^{s *}\right)^{\varphi},
\end{aligned}
$$

plus the appropriate no-Ponzi games, transversality conditions, the budget constraints and the law of motions for capital in both countries. Furthermore, the equilibrium conditions are completed with a set of equations that characterize the bond portfolio allocation of the domestic and foreign households as well as with a pair of equations that account for the capital-investment decisions of each household. The capital-investment conditions, in turn, depend on the choice of the adjustment cost function $\Phi(\cdot)$ in the law of motion for capital in (3), while the bond portfolio allocations depend on the international borrowing costs paid by the domestic households to trade on foreign bonds (in (2) and (4)).

The domestic households' maximization problem in this bond economy (under incomplete international asset markets) can be summarized generically in the following Lagrangian form,

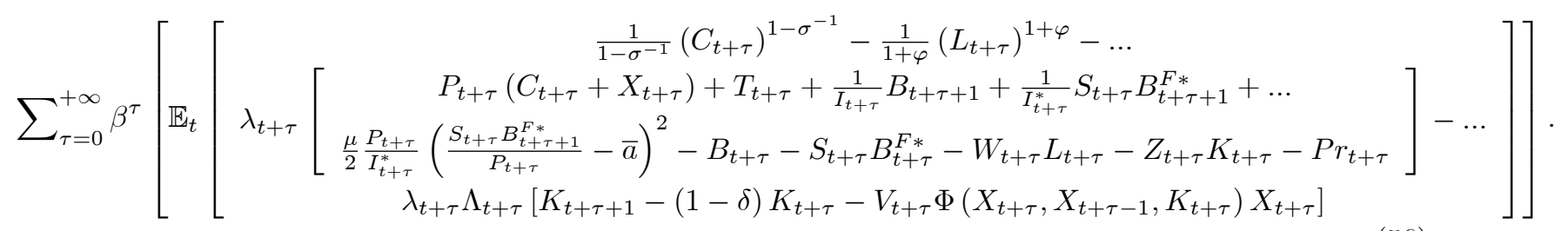

The foreign households' maximization problem in this bond economy (under incomplete international asset markets) can be summarized generically in the following Lagrangian form,

$$
\sum_{\tau=0}^{+\infty} \beta^{\tau}\left[\mathbb{E}_{t}\left[\begin{array}{c}
\frac{1}{1-\sigma^{-1}}\left(C_{t+\tau}^{*}\right)^{1-\sigma^{-1}}-\frac{1}{1+\varphi}\left(L_{t+\tau}^{*}\right)^{1+\varphi}-\ldots \\
-\lambda_{t+\tau}^{*}\left[\begin{array}{c}
P_{t+\tau}^{*}\left(C_{t+\tau}^{*}+X_{t+\tau}^{*}\right)+T_{t+\tau}^{*}+\frac{1}{I_{t+\tau}^{*}} B_{t+\tau+1}^{*}-\ldots \\
B_{t+\tau}^{*}-W_{t+\tau}^{*} L_{t+\tau}^{*}-Z_{t+\tau}^{*} K_{t+\tau}^{*}-P r_{t+\tau}^{*}-T r_{t+\tau}^{*}
\end{array}\right]-\ldots \\
\lambda_{t+\tau}^{*} \Lambda_{t+\tau}^{*}\left[K_{t+\tau+1}^{*}-(1-\delta) K_{t+\tau}^{*}-V_{t+\tau}^{*} \Phi\left(X_{t+\tau}^{*}, X_{t+\tau-1}^{*}, K_{t+\tau}^{*}\right) X_{t+\tau}^{*}\right]
\end{array}\right]\right]
$$

The optimal bond portfolio choices for the domestic and foreign households can be described with the following set of equilibrium conditions,

$$
\begin{array}{rll}
C_{t} & : \quad \lambda_{t} P_{t}=\left(C_{t}\right)^{-\sigma^{-1}} \\
B_{t+1} & : \quad-\lambda_{t} \frac{1}{I_{t}}+\beta \mathbb{E}_{t}\left[\lambda_{t+1}\right]=0 \\
B_{t+1}^{F *} & : \quad-\lambda_{t} \frac{1}{I_{t}^{*}} S_{t}\left[1+\mu\left(\frac{S_{t} B_{t+1}^{F *}}{P_{t}}-\bar{a}\right)\right]+\beta \mathbb{E}_{t}\left[\lambda_{t+1} S_{t+1}\right]=0 \\
C_{t}^{*} & : \quad \lambda_{t}^{*} P_{t}^{*}=\left(C_{t}^{*}\right)^{-\sigma^{-1}}, \\
B_{t+1}^{*} & : \quad-\lambda_{t}^{*} \frac{1}{I_{t}^{*}}+\beta \mathbb{E}_{t}\left[\lambda_{t+1}^{*}\right]=0 .
\end{array}
$$


Then, after some additional algebra, it follows that,

$$
\begin{aligned}
\beta \mathbb{E}_{t}\left[\left(\frac{C_{t+1}}{C_{t}}\right)^{-\sigma^{-1}} \frac{P_{t}}{P_{t+1}} I_{t}\right] & =1, \\
\beta \mathbb{E}_{t}\left[\left(\frac{C_{t+1}}{C_{t}}\right)^{-\sigma^{-1}} \frac{P_{t}}{P_{t+1}} \frac{S_{t+1}}{S_{t}} I_{t}^{*}\right] & =1+\mu\left(N F A_{t+1}-\bar{a}\right), \\
\beta \mathbb{E}_{t}\left[\left(\frac{C_{t+1}^{*}}{C_{t}^{*}}\right)^{-\sigma^{-1}} \frac{P_{t}^{*}}{P_{t+1}^{*}} I_{t}^{*}\right] & =1,
\end{aligned}
$$

where I define the real net foreign asset position (in units of domestic consumption) of the domestic household as $N F A_{t+1} \equiv \frac{S_{t} B_{t+1}^{F *}}{P_{t}}$. Combining the Euler equations of the domestic household in (58) and (59), the following relationship holds true,

$$
\beta \mathbb{E}_{t}\left[\left(\frac{C_{t+1}}{C_{t}}\right)^{-\sigma^{-1}} \frac{P_{t}}{P_{t+1}}\left(\frac{S_{t+1}}{S_{t}} I_{t}^{*}-I_{t}\right)\right]=\mu\left(N F A_{t+1}-\bar{a}\right),
$$

which represents a variant of the uncovered interest rate parity condition where the risk premium appears tied to the real net foreign asset position of the domestic household. This equilibrium condition governs the terms of international risk-sharing in this environment with incomplete asset markets.

Finally, the domestic capital-investment decisions can be described with the following set of additional equilibrium conditions,

$$
\begin{aligned}
C_{t} & : \quad \lambda_{t} P_{t}=\left(C_{t}\right)^{-\sigma^{-1}} \\
K_{t+1} & : \quad \Lambda_{t}=\beta \mathbb{E}_{t}\left[\frac{\lambda_{t+1}}{\lambda_{t}} Z_{t+1}+\frac{\lambda_{t+1}}{\lambda_{t}} \Lambda_{t+1}\left((1-\delta)+V_{t+1} \frac{\partial \Phi\left(X_{t+1}, X_{t}, K_{t+1}\right)}{\partial K_{t+1}} X_{t+1}\right)\right] \\
X_{t} & : \quad 1=\frac{\Lambda_{t}}{P_{t}} V_{t}\left[\Phi\left(X_{t}, X_{t-1}, K_{t}\right)+\frac{\partial \Phi\left(X_{t}, X_{t-1}, K_{t}\right)}{\partial X_{t}} X_{t}\right]+\beta \mathbb{E}_{t}\left[\frac{\lambda_{t+1} \Lambda_{t+1}}{\lambda_{t} P_{t}} V_{t+1} \frac{\partial \Phi\left(X_{t+1}, X_{t}, K_{t+1}\right)}{\partial X_{t}} X_{t+1}\right] .
\end{aligned}
$$

Let me define Tobin's q as $Q_{t} \equiv \frac{\Lambda_{t}}{P_{t}}$. Then, after further manipulation, it is possible to re-write the equilibrium conditions as,

$$
\begin{aligned}
Q_{t} & =\beta \mathbb{E}_{t}\left\{\left(\frac{C_{t+1}}{C_{t}}\right)^{-\sigma^{-1}}\left[\frac{Z_{t+1}}{P_{t+1}}+Q_{t+1}\left((1-\delta)+V_{t+1} \frac{\partial \Phi\left(X_{t+1}, X_{t}, K_{t+1}\right)}{\partial K_{t+1}} X_{t+1}\right)\right]\right\} \\
Q_{t} & =\frac{1-\beta \mathbb{E}_{t}\left\{\left(\frac{C_{t+1}}{C_{t}}\right)^{-\sigma^{-1}}\left[Q_{t+1} V_{t+1} \frac{\partial \Phi\left(X_{t+1}, X_{t}, K_{t+1}\right)}{\partial X_{t}} X_{t+1}\right]\right\}}{V_{t}\left[\Phi\left(X_{t}, X_{t-1}, K_{t}\right)+\frac{\partial \Phi\left(X_{t}, X_{t-1}, K_{t}\right)}{\partial X_{t}} X_{t}\right]}
\end{aligned}
$$

Under no adjustment costs (NAC), the pair of conditions added to account for the capital-investment deci- 
sions of the domestic households are summarized as,

$$
\begin{aligned}
Q_{t} & =\beta \mathbb{E}_{t}\left\{\left(\frac{C_{t+1}}{C_{t}}\right)^{-\sigma^{-1}}\left[\frac{Z_{t+1}}{P_{t+1}}+Q_{t+1}(1-\delta)\right]\right\}, \\
Q_{t} & =\frac{1}{V_{t}}
\end{aligned}
$$

A similar set of derivations allows me to write the following pair of equations for Tobin's $q$ in the foreign country,

$$
\begin{aligned}
& Q_{t}^{*}=\beta \mathbb{E}_{t}\left\{\left(\frac{C_{t+1}^{*}}{C_{t}^{*}}\right)^{-\sigma^{-1}}\left[\frac{Z_{t+1}^{*}}{P_{t+1}^{*}}+Q_{t+1}^{*}(1-\delta)\right]\right\}, \\
& Q_{t}^{*}=\frac{1}{V_{t}^{*}} .
\end{aligned}
$$

The Lagrange multiplier on the law of motion relative to the Lagrange multiplier on the budget constraint expressed in real terms, denoted $Q_{t}$ and $Q_{t}^{*}$ respectively, has the interpretation of being the real shadow price of an additional unit of capital (or Tobin's q). In the neoclassical case without adjustment costs and IST shocks is well-known that Tobin's q is exactly equal to one, as these equations show.

Under capital adjustment costs (CAC), the pair of conditions added to account for the capital-investment decisions of domestic households are,

$$
\begin{aligned}
Q_{t} & =\beta \mathbb{E}_{t}\left\{\left(\frac{C_{t+1}}{C_{t}}\right)^{-\sigma^{-1}}\left[\frac{Z_{t+1}}{P_{t+1}}+Q_{t+1}\left((1-\delta)-V_{t+1} \Phi^{\prime}\left(\frac{X_{t+1}}{K_{t+1}}\right)\left(\frac{X_{t+1}}{K_{t+1}}\right)^{2}\right)\right]\right\} \\
Q_{t} & =\frac{1}{V_{t}\left[\Phi\left(\frac{X_{t}}{K_{t}}\right)+\Phi^{\prime}\left(\frac{X_{t}}{K_{t}}\right) \frac{X_{t}}{K_{t}}\right]}
\end{aligned}
$$

A similar set of derivations allows me to write the following pair of equations for Tobin's $q$ in the foreign country,

$$
\begin{aligned}
Q_{t}^{*} & =\beta \mathbb{E}_{t}\left\{\left(\frac{C_{t+1}^{*}}{C_{t}^{*}}\right)^{-\sigma^{-1}}\left[\frac{Z_{t+1}^{*}}{P_{t+1}^{*}}+Q_{t+1}^{*}\left((1-\delta)-V_{t+1}^{*} \Phi^{\prime}\left(\frac{X_{t+1}^{*}}{K_{t+1}^{*}}\right)\left(\frac{X_{t+1}^{*}}{K_{t+1}^{*}}\right)^{2}\right)\right]\right\}, \\
Q_{t}^{*} & =\frac{1}{V_{t}^{*}\left[\Phi\left(\frac{X_{t}^{*}}{K_{t}^{*}}\right)+\Phi^{\prime}\left(\frac{X_{t}^{*}}{K_{t}^{*}}\right) \frac{X_{t}^{*}}{K_{t}^{*}}\right]} .
\end{aligned}
$$

The Lagrange multiplier on the law of motion relative to the Lagrange multiplier on the budget constraint expressed in real terms, denoted $Q_{t}$ and $Q_{t}^{*}$ respectively, have the interpretation of being the real shadow price of an additional unit of capital (or Tobin's q). Under investment adjustment costs (IAC), the pair of 
conditions added to account for the capital-investment decisions of domestic households are,

$$
\begin{aligned}
Q_{t} & =\beta \mathbb{E}_{t}\left\{\left(\frac{C_{t+1}}{C_{t}}\right)^{-\sigma^{-1}}\left[\frac{Z_{t+1}}{P_{t+1}}+Q_{t+1}(1-\delta)\right]\right\} \\
Q_{t} & =\frac{1+\beta \mathbb{E}_{t}\left\{\left(\frac{C_{t+1}}{C_{t}}\right)^{-\sigma^{-1}}\left[Q_{t+1} V_{t+1} \Phi^{\prime}\left(\frac{X_{t+1}}{X_{t}}\right)\left(\frac{X_{t+1}}{X_{t}}\right)^{2}\right]\right\}}{V_{t}\left[\Phi\left(\frac{X_{t}}{X_{t-1}}\right)+\Phi^{\prime}\left(\frac{X_{t}}{X_{t-1}}\right) \frac{X_{t}}{X_{t-1}}\right]},
\end{aligned}
$$

A similar set of derivations allows me to write the following pair of equations for Tobin's $q$ in the foreign country,

$$
\begin{aligned}
Q_{t}^{*} & =\beta \mathbb{E}_{t}\left\{\left(\frac{C_{t+1}^{*}}{C_{t}^{*}}\right)^{-\sigma^{-1}}\left[\frac{Z_{t+1}^{*}}{P_{t+1}^{*}}+Q_{t+1}^{*}(1-\delta)\right]\right\}, \\
Q_{t}^{*} & =\frac{1+\beta \mathbb{E}_{t}\left\{\left(\frac{C_{t+1}^{*}}{C_{t}^{*}}\right)^{-\sigma^{-1}}\left[Q_{t+1}^{*} V_{t+1}^{*} \Phi^{\prime}\left(\frac{X_{t+1}^{*}}{X_{t}^{*}}\right)\left(\frac{X_{t+1}^{*}}{X_{t}^{*}}\right)^{2}\right]\right\}}{V_{t}^{*}\left[\Phi\left(\frac{X_{t}^{*}}{X_{t-1}^{*}}\right)+\Phi^{\prime}\left(\frac{X_{t}^{*}}{X_{t-1}^{*}}\right) \frac{X_{t}^{*}}{X_{t-1}^{*}}\right]} .
\end{aligned}
$$

Once again, the Lagrange multiplier on the law of motion for capital relative to the Lagrange multiplier on the budget constraint expressed in real terms, denoted $Q_{t}$ and $Q_{t}^{*}$ respectively, have the interpretation of being the real shadow prices of an additional unit of capital (or Tobin's q).

The Optimality Conditions from the Firms' Problem. The necessary and sufficient first-order conditions for the domestic firm producing variety $h$ give me the following pair of price-setting formulas,

$$
\begin{aligned}
\sum_{\tau=0}^{+\infty} \alpha^{\tau} \mathbb{E}_{t}\left[M_{t, t+\tau}\left(\widetilde{C}_{t, t+\tau}(h)+\widetilde{X}_{t, t+\tau}(h)\right)\left(\widetilde{P}_{t}(h)-\frac{\theta}{\theta-1}\left(1-\xi_{t+\tau}\right) M C_{t+\tau}\right)\right] & =0 \\
\sum_{\tau=0}^{+\infty} \alpha^{\tau} \mathbb{E}_{t}\left[M_{t, t+\tau}\left(\widetilde{C}_{t, t+\tau}^{*}(h)+\widetilde{X}_{t, t+\tau}^{*}(h)\right)\left(S_{t+\tau} \widetilde{P}_{t}^{*}(h)-\frac{\theta}{\theta-1}\left(1-\xi_{t+\tau}\right) M C_{t+\tau}\right)\right] & =0 .
\end{aligned}
$$

Similarly, the first-order conditions for the foreign firm producing variety $f$ give me the following price-setting formulas,

$$
\begin{aligned}
& \sum_{\tau=0}^{+\infty} \alpha^{\tau} \mathbb{E}_{t}\left[M_{t, t+\tau}^{*}\left(\widetilde{C}_{t, t+\tau}(f)+\widetilde{X}_{t, t+\tau}(f)\right)\left(\frac{\widetilde{P}_{t}(f)}{S_{t+\tau}}-\frac{\theta}{\theta-1}\left(1-\xi_{t+\tau}^{*}\right) M C_{t+\tau}^{*}\right)\right]=0, \\
& \sum_{\tau=0}^{+\infty} \alpha^{\tau} \mathbb{E}_{t}\left[M_{t, t+\tau}^{*}\left(\widetilde{C}_{t, t+\tau}^{*}(f)+\widetilde{X}_{t, t+\tau}^{*}(f)\right)\left(\widetilde{P}_{t}^{*}(f)-\frac{\theta}{\theta-1}\left(1-\xi_{t+\tau}^{*}\right) M C_{t+\tau}^{*}\right)\right]=0 .
\end{aligned}
$$

Using the law of large numbers and the inherent symmetry of the firms' problem, the price sub-indexes on domestic varieties, $P_{t}^{H}$ and $P_{t}^{H *}$, become,

$$
\begin{aligned}
P_{t}^{H} & =\left[\alpha\left(P_{t-1}^{H}\right)^{1-\theta}+(1-\alpha)\left(\widetilde{P}_{t}(h)\right)^{1-\theta}\right]^{\frac{1}{1-\theta}}, \\
P_{t}^{H *} & =\left[\alpha\left(P_{t-1}^{H *}\right)^{1-\theta}+(1-\alpha)\left(\widetilde{P}_{t}^{*}(h)\right)^{1-\theta}\right]^{\frac{1}{1-\theta}},
\end{aligned}
$$


while the price sub-indexes on foreign varieties, $P_{t}^{F}$ and $P_{t}^{F *}$, are computed as,

$$
\begin{aligned}
P_{t}^{F} & =\left[\alpha\left(P_{t-1}^{F}\right)^{1-\theta}+(1-\alpha)\left(\widetilde{P}_{t}(f)\right)^{1-\theta}\right]^{\frac{1}{1-\theta}}, \\
P_{t}^{F *} & =\left[\alpha\left(P_{t-1}^{F *}\right)^{1-\theta}+(1-\alpha)\left(\widetilde{P}_{t}^{*}(f)\right)^{1-\theta}\right]^{\frac{1}{1-\theta}} .
\end{aligned}
$$

Equations $(78)-(81)$ are a convenient way to reformulate $(17)-(20)$.

Aggregate Output and Relative Price 'Distortions'. The production functions in (22) - (23) can be re-written as,

$$
\begin{aligned}
Y_{t}(h) & =A_{t}\left(\frac{K_{t}}{L_{t}}\right)^{1-\psi} L_{t}(h), \forall h \in[0,1], \\
Y_{t}^{*}(f) & =A_{t}^{*}\left(\frac{K_{t}^{*}}{L_{t}^{*}}\right)^{1-\psi} L_{t}^{*}(f), \forall f \in[0,1],
\end{aligned}
$$

since capital-to-labor ratios are equated across all firms within a country due to factor price equalization (as implied by equations $(24)-(25)$ ). Also because of factor price equalization, all households within a country supply the same amount of labor and capital, that is $L_{t}$ and $K_{t}$ in the domestic country and $L_{t}^{*}$ and $K_{t}^{*}$ in the foreign country. ${ }^{6}$ In turn, factor prices equalize in each country because the factors of production are homogeneous (though immobile across borders) and factor markets are perfectly competitive. However, since the pricing decisions of firms are not synchronized, the amounts of labor and capital allocated to each individual firm will differ in every period.

Across all firms, the capital and labor demands equal their respective supplies. Hence, output can be added up to be expressed as a function of the common (aggregate) productivity shock as well as the aggregate capital and labor supplied by the local households, i.e.

$$
\begin{aligned}
Y_{t} & =\int_{0}^{1} Y_{t}(h) d h=A_{t}\left(K_{t}\right)^{1-\psi}\left(L_{t}\right)^{\psi}, \\
Y_{t}^{*} & =\int_{0}^{1} Y_{t}(f) d f=A_{t}^{*}\left(K_{t}^{*}\right)^{1-\psi}\left(L_{t}^{*}\right)^{1-\psi},
\end{aligned}
$$

where, by labor market clearing, it follows that $L_{t}=\int_{0}^{1} L_{t}(h) d h$ and $L_{t}^{*}=\int_{0}^{1} L_{t}^{*}(f) d f$.

Equations (46) - (53) determine the demand function for each variety produced at home and abroad. Equations (46) - (49) coupled with the market clearing conditions at the variety level allow me to aggregate

\footnotetext{
${ }^{6}$ There is a mass one of identical households in each country all of which follow the same optimal path in their decisions. Then, $L_{t}$ and $K_{t}$ in the domestic country and $L_{t}^{*}$ and $K_{t}^{*}$ in the foreign country represent simultaneously the average and the aggregate supply of labor and capital respectively. Moreover, they also characterize the individual decisions of each household since all households within a country conform to the country average.
} 
output demand as follows,

$$
\begin{aligned}
Y_{t} & =\int_{0}^{1}\left[C_{t}(h)+X_{t}(h)+C_{t}^{*}(h)+X_{t}^{*}(h)\right] d h \\
& =\int_{0}^{1}\left[\left(\frac{P_{t}(h)}{P_{t}^{H}}\right)^{-\theta}\left(C_{t}^{H}+X_{t}^{H}\right)+\left(\frac{P_{t}^{*}(h)}{P_{t}^{H *}}\right)^{-\theta}\left(C_{t}^{H *}+X_{t}^{H *}\right)\right] d h \\
& =\left(\frac{\widetilde{P}_{t}^{H}}{P_{t}^{H}}\right)^{-\theta}\left(C_{t}^{H}+X_{t}^{H}\right)+\left(\frac{\widetilde{P}_{t}^{H *}}{P_{t}^{H *}}\right)^{-\theta}\left(C_{t}^{H *}+X_{t}^{H *}\right), \\
Y_{t}^{*} & =\int_{0}^{1}\left[C_{t}(f)+X_{t}(f)+C_{t}^{*}(f)+X_{t}^{*}(f)\right] d f \\
& =\int_{0}^{1}\left[\left(\frac{P_{t}(f)}{P_{t}^{F}}\right)^{-\theta}\left(C_{t}^{F}+X_{t}^{F}\right)+\left(\frac{P_{t}^{*}(f)}{P_{t}^{F *}}\right)^{-\theta}\left(C_{t}^{F *}+X_{t}^{F *}\right)\right] d f \\
& =\left(\frac{\widetilde{P}_{t}^{F}}{P_{t}^{F}}\right)^{-\theta}\left(C_{t}^{F}+X_{t}^{F}\right)+\left(\frac{\widetilde{P}_{t}^{F *}}{P_{t}^{F *}}\right)^{-\theta}\left(C_{t}^{F *}+X_{t}^{F *}\right),
\end{aligned}
$$

where,

$$
\begin{aligned}
\widetilde{P}_{t}^{H} & \equiv\left[\int_{0}^{1}\left(P_{t}(h)\right)^{-\theta} d h\right]^{-\frac{1}{\theta}}=\left[\alpha\left(\widetilde{P}_{t-1}^{H}\right)^{-\theta}+(1-\alpha)\left(\widetilde{P}_{t}(h)\right)^{-\theta}\right]^{-\frac{1}{\theta}} \\
\widetilde{P}_{t}^{H *} & \equiv\left[\int_{0}^{1}\left(P_{t}^{*}(h)\right)^{-\theta} d h\right]^{-\frac{1}{\theta}}=\left[\alpha\left(\widetilde{P}_{t-1}^{H *}\right)^{-\theta}+(1-\alpha)\left(\widetilde{P}_{t}^{*}(h)\right)^{-\theta}\right]^{-\frac{1}{\theta}} \\
\widetilde{P}_{t}^{F} & \equiv\left[\int_{0}^{1}\left(P_{t}(f)\right)^{-\theta} d f\right]^{-\frac{1}{\theta}}=\left[\alpha\left(\widetilde{P}_{t-1}^{F}\right)^{-\theta}+(1-\alpha)\left(\widetilde{P}_{t}(f)\right)^{-\theta}\right]^{-\frac{1}{\theta}} \\
\widetilde{P}_{t}^{F *} & \equiv\left[\int_{0}^{1}\left(P_{t}^{*}(f)\right)^{-\theta} d f\right]^{-\frac{1}{\theta}}=\left[\alpha\left(\widetilde{P}_{t-1}^{F *}\right)^{-\theta}+(1-\alpha)\left(\widetilde{P}_{t}^{*}(f)\right)^{-\theta}\right]^{-\frac{1}{\theta}}
\end{aligned}
$$

Similarly, equations (46) - (53) coupled with the market clearing conditions at the variety level allow me to aggregate output as follows,

$$
\begin{aligned}
Y_{t} & =\int_{0}^{1}\left[C_{t}(h)+X_{t}(h)+C_{t}^{*}(h)+X_{t}^{*}(h)\right] d h \\
& =\left[\int_{0}^{1}\left(\frac{P_{t}(h)}{P_{t}^{H}}\right)^{-\theta} d h\right] \phi_{H}\left(\frac{P_{t}^{H}}{P_{t}}\right)^{-\eta}\left(C_{t}+X_{t}\right)+\left[\int_{0}^{1}\left(\frac{P_{t}^{*}(h)}{P_{t}^{H *}}\right)^{-\theta} d h\right] \phi_{H}^{*}\left(\frac{P_{t}^{H *}}{P_{t}^{*}}\right)^{-\eta}\left(C_{t}^{*}+X_{t}^{*}\right) \\
& =\phi_{H}\left(\frac{\widetilde{P}_{t}^{H}}{P_{t}^{H}}\right)^{-\theta}\left(\frac{P_{t}^{H}}{P_{t}}\right)^{-\eta}\left(C_{t}+X_{t}\right)+\phi_{F}\left(\frac{\widetilde{P}_{t}^{H *}}{P_{t}^{H *}}\right)^{-\theta}\left(\frac{P_{t}^{H *}}{P_{t}^{*}}\right)^{-\eta}\left(C_{t}^{*}+X_{t}^{*}\right), \\
Y_{t}^{*} & =\int_{0}^{1}\left[C_{t}(f)+X_{t}(f)+C_{t}^{*}(f)+X_{t}^{*}(f)\right] d f \\
& =\left[\int_{0}^{1}\left(\frac{P_{t}(f)}{P_{t}^{F}}\right)^{-\theta} d f\right] \phi_{F}\left(\frac{P_{t}^{F}}{P_{t}}\right)^{-\eta}\left(C_{t}+X_{t}\right)+\left[\int_{0}^{1}\left(\frac{P_{t}^{*}(f)}{P_{t}^{F *}}\right)^{-\theta} d f\right] \phi_{F}^{*}\left(\frac{P_{t}^{F *}}{P_{t}^{*}}\right)^{-\eta}\left(C_{t}^{*}+X_{t}^{*}\right) \\
& =\phi_{F}\left(\frac{\widetilde{P}_{t}^{F}}{P_{t}^{F}}\right)^{-\theta}\left(\frac{P_{t}^{F}}{P_{t}}\right)^{-\eta}\left(C_{t}+X_{t}\right)+\phi_{H}\left(\frac{\widetilde{P}_{t}^{F *}}{P_{t}^{F *}}\right)^{-\theta}\left(\frac{P_{t}^{F *}}{P_{t}^{*}}\right)^{-\eta}\left(C_{t}^{*}+X_{t}^{*}\right),
\end{aligned}
$$


where the share of the home goods in the foreign aggregator is $\phi_{H}^{*}=\phi_{F}$ and the share of foreign goods in the foreign aggregator is $\phi_{F}^{*}=\phi_{H}$. Equations (92) - (93) tie the aggregate output demand in both countries to aggregate consumption, aggregate investment as well as relative prices.

If I define the aggregate output of the bundle of domestic varieties as $Y_{t}^{H}$ and the aggregate output of the bundle of foreign varieties as $Y_{t}^{F *}$, then by market clearing it must hold true that each bundle of goods must be either consumed or invested by the two countries, i.e.

$$
\begin{aligned}
Y_{t}^{H} & =C_{t}^{H}+X_{t}^{H}+C_{t}^{H *}+X_{t}^{H *}, \\
Y_{t}^{F *} & =C_{t}^{F}+X_{t}^{F}+C_{t}^{F *}+X_{t}^{F *} .
\end{aligned}
$$

Using these two resource constraints together with (84) - (85) and (86) - (87) it must follow that domestic and foreign aggregate output supply can be expressed in the following terms,

$$
\begin{aligned}
Y_{t}^{H} & =\Xi_{t}^{H} A_{t}\left(K_{t}\right)^{1-\psi}\left(L_{t}\right)^{\psi}, \\
Y_{t}^{F *} & =\Xi_{t}^{F *} A_{t}^{*}\left(K_{t}^{*}\right)^{1-\psi}\left(L_{t}^{*}\right)^{1-\psi},
\end{aligned}
$$

where I measure the impact of the relative price dispersion on aggregate output with the following two wedges,

$$
\begin{aligned}
\Xi_{t}^{H} & \equiv\left[\left(\frac{\widetilde{P}_{t}^{H}}{P_{t}^{H}}\right)^{-\theta}\left(\frac{C_{t}^{H}+X_{t}^{H}}{C_{t}^{H}+X_{t}^{H}+C_{t}^{H *}+X_{t}^{H *}}\right)+\left(\frac{\widetilde{P}_{t}^{H *}}{P_{t}^{H *}}\right)^{-\theta}\left(\frac{C_{t}^{H *}+X_{t}^{H *}}{C_{t}^{H}+X_{t}^{H}+C_{t}^{H *}+X_{t}^{H *}}\right)\right]^{-1} \\
\Xi_{t}^{F *} & \equiv\left[\left(\frac{\widetilde{P}_{t}^{F}}{P_{t}^{F}}\right)^{-\theta}\left(\frac{C_{t}^{F}+X_{t}^{F}}{C_{t}^{F}+X_{t}^{F}+C_{t}^{F *}+X_{t}^{F *}}\right)+\left(\frac{\widetilde{P}_{t}^{F *}}{P_{t}^{F *}}\right)^{-\theta}\left(\frac{C_{t}^{F *}+X_{t}^{F *}}{C_{t}^{F}+X_{t}^{F}+C_{t}^{F *}+X_{t}^{F *}}\right)\right]^{-1} .
\end{aligned}
$$

Whenever the law of one price holds, then it is possible to re-write these wedges as,

$$
\Xi_{t}^{H} \equiv\left(\frac{\widetilde{P}_{t}^{H}}{P_{t}^{H}}\right)^{\theta}, \Xi_{t}^{F *} \equiv\left(\frac{\widetilde{P}_{t}^{F *}}{P_{t}^{F *}}\right)^{\theta} .
$$

Otherwise, the wedges have to explicitly incorporate the effects of the deviations of the law of one price, i.e.

$$
\begin{gathered}
\Xi_{t}^{H} \equiv\left(\frac{\widetilde{P}_{t}^{H}}{P_{t}^{H}}\right)^{\theta}\left[\left(\frac{\frac{S_{t} \widetilde{P}_{t}^{H *}}{\widetilde{P}_{t}^{H}}}{\frac{S_{t} P_{t}^{H *}}{P_{t}^{H}}}\right)^{-\theta}+\left(\frac{C_{t}^{H}+X_{t}^{H}}{C_{t}^{H}+X_{t}^{H}+C_{t}^{H *}+X_{t}^{H *}}\right)\left(1-\left(\frac{\frac{S_{t} \widetilde{P}_{t}^{H *}}{\widetilde{P}_{t}^{H}}}{\frac{S_{t} P_{t}^{H *}}{P_{t}^{H}}}\right)^{-\theta}\right)\right]^{-1}, \\
\Xi_{t}^{F *} \equiv\left(\frac{\widetilde{P}_{t}^{F *}}{P_{t}^{F *}}\right)^{\theta}\left[\left(\frac{\frac{\widetilde{P}_{t}^{F}}{S_{t} \tilde{P}_{t}^{F *}}}{\frac{P_{t}^{F}}{S_{t} P_{t}^{F *}}}\right)^{-\theta}+\left(1-\left(\frac{\frac{\widetilde{P}_{t}^{F}}{S_{t} \widetilde{P}_{t}^{F *}}}{\frac{P_{t}^{F}}{S_{t} P_{t}^{F *}}}\right)^{-\theta}\right)\left(\frac{C_{t}^{F *}+X_{t}^{F *}}{C_{t}^{F}+X_{t}^{F}+C_{t}^{F *}+X_{t}^{F *}}\right)\right]^{-1} .
\end{gathered}
$$

These wedges are often interpreted as a measure of the 'efficiency distortion' caused by nominal rigidities (whether the law of one price fails or holds). In the absence of those nominal rigidities there should be no price differences across firms and the wedges ought to be equal to one. However, if prices are different across 
firms due to nominal rigidities, then the resulting relative price effect leads to resources (and demand) being missallocated.

The wedges $\Xi_{t}^{H}$ and $\Xi_{t}^{F *}$ in (98) - (99) combine two measures of relative price dispersion corresponding one to the domestic market and the other to the foreign market (expressed in their respective local currencies) for the varieties included in the bundle of goods produced by each country. The wedges weight the relative price dispersion of the same bundle of varieties produced by a country in the two markets in which it is distributed based on the share that domestic and foreign sources of demand have on the aggregate demand of the bundle. Those relative price dispersion measures can be re-written using (17) - (20) and (88) - (91) as follows,

$$
\begin{gathered}
\left(\frac{\widetilde{P}_{t}^{H}}{P_{t}^{H}}\right)^{\theta}=\left(\frac{\left[\int_{0}^{1}\left(P_{t}(h)\right)^{-\theta} d h\right]^{-\frac{1}{\theta}}}{\left[\int_{0}^{1}\left(P_{t}(h)\right)^{1-\theta} d h\right]^{\frac{1}{1-\theta}}}\right)^{\theta}=\left(\frac{\left[\int_{0}^{1}\left(P_{t}(h)\right)^{1-(\theta+1)} d h\right]^{\frac{1}{1-(\theta+1)}}}{\left[\int_{0}^{1}\left(P_{t}(h)\right)^{1-\theta} d h\right]^{\frac{1}{1-\theta}}}\right)^{\theta}, \\
\left(\frac{\left[\int_{t}^{1}\left(P_{t}^{*}(h)\right)^{-\theta} d h\right]^{-\frac{1}{\theta}}}{P_{t}^{H *}}\right)^{\theta}=\left(\frac{\left[\int_{0}^{1}\left(P_{t}^{*}(h)\right)^{1-(\theta+1)} d h\right]^{\frac{1}{1-(\theta+1)}}}{\left[\int_{0}^{1}\left(P_{t}^{*}(h)\right)^{1-\theta} d h\right]^{\frac{1}{1-\theta}}}\right)^{\theta}, \\
\left(\frac{\left[\int_{0}^{1}\left(P_{t}^{*}(h)\right)^{1-\theta} d h\right]^{\frac{1}{1-\theta}}}{\widetilde{P}_{t}^{F}}\right)^{\theta}=\left(\frac{\left[\int_{0}^{1}\left(P_{t}(f)\right)^{-\theta} d f\right]^{-\frac{1}{\theta}}}{\left[\int_{0}^{1}\left(P_{t}(f)\right)^{1-\theta} d f\right]^{\frac{1}{1-\theta}}}\right)^{\theta}=\left(\frac{\left.\left[P_{0}^{1}(f)\right)^{1-(\theta+1)} d f\right]^{\frac{1}{1-(\theta+1)}}}{\left[\int_{0}^{1}\left(P_{t}(f)\right)^{1-\theta} d f\right]^{\frac{1}{1-\theta}}}\right)^{\theta}, \\
\left(\frac{\left[\int_{t}^{1}\left(P_{t}^{*}(f)\right)^{-\theta} d f\right]^{-\frac{1}{\theta}}}{\widetilde{P}_{t}^{F *}}\right)^{\theta}=\left(\frac{\left[\int_{0}^{1}\left(P_{t}^{*}(f)\right)^{1-(\theta+1)} d f\right]^{\frac{1}{1-(\theta+1)}}}{\left[\int_{0}^{1}\left(P_{t}^{*}(f)\right)^{1-\theta} d f\right]^{\frac{1}{1-\theta}}}\right)^{\theta} .
\end{gathered}
$$

If the prices of all varieties are identical, i.e. $P_{t}(h)=P_{t}^{H}$ and $P_{t}^{*}(h)=P_{t}^{H *}$ for all $h \in[0,1]$ and $P_{t}(f)=P_{t}^{F}$ and $P_{t}^{*}(f)=P_{t}^{F *}$ for all $f \in[0,1]$, then the numerator and denominator in each expression in $(102)-(105)$ must be equal too, i.e. $\widetilde{P}_{t}^{H}=P_{t}^{H}, \widetilde{P}_{t}^{H *}=P_{t}^{H *}, \widetilde{P}_{t}^{F}=P_{t}^{F}$, and $\widetilde{P}_{t}^{F *}=P_{t}^{F *}$. Moreover, both the numerator and denominator are of the CES form and special cases of a generalized mean often referred to as the weighted power mean. It can be verified by the general means inequality proven below that $\widetilde{P}_{t}^{H} \leq P_{t}^{H}$, $\widetilde{P}_{t}^{H *} \leq P_{t}^{H *}, \widetilde{P}_{t}^{F} \leq P_{t}^{F}$, and $\widetilde{P}_{t}^{F *} \leq P_{t}^{F *}$ and that the equality holds only whenever the prices of all varieties are the same. Therefore, it holds true that,

$$
\Xi_{t}^{H} \leq 1, \Xi_{t}^{F *} \leq 1
$$

In other words, these wedges conveniently characterize the output 'distortion' caused by the relative price dispersion resulting from nominal rigidities and the possibility of deviations from the law of one price - since, absent those nominal rigidities, the prices of all varieties within the domestic bundle should be equal and the same should be true for the prices of all varieties within the foreign bundle. 
Remark 2 Given the nonlinear structure of the model, the wedges $\Xi_{t}^{H} \leq 1$ and $\Xi_{t}^{F *} \leq 1$ also introduce a source of asymmetry in the model. In response to any given shock (whether positive or negative in sign), nominal rigidities imply that prices of varieties within the same bundle will diverge. Hence, the wedges would be less than one and the supply of the bundle of those varieties (i.e. $Y_{t}^{H}$ and $Y_{t}^{F *}$ ) will be lowered (taking as given the amount of aggregate capital and labor, as can be seen from (96) - (97)). Simultaneously, the supply of the bundles of varieties may go up or down depending on the sign of the shock-resulting in an asymetric effect on output. Let me consider a productivity shock for illustration purposes. On one hand, a positive productivity shock will likely drive production up, but the distortion caused by the relative price dispersion will attenuate that increase. On the other hand, a negative productivity shock will likely drive production down, while the distortion caused by the relative price dispersion further aggravates the fall. It is the fact that the supply of output is unequivocally lowered by the relative price 'distortion' that accounts for the apparent asymmetry in the output response to a shock.

Finally, I want to point out that this relative price 'distortion' is of second-order importance and, therefore, does not 'appear' in the log-linearized equilibrium conditions of the model. In turn, it can be of major importance for welfare analysis where a second-order approximation of the welfare function is often needed.

Notation 1 Any of the price aggregators in (17) - (20) and (88) - (91) can be re-written as follows,

$$
\begin{aligned}
{\left[\int_{0}^{1}\left(P_{t}(h)\right)^{r} d h\right]^{\frac{1}{r}} } & =\left[\int_{a_{h}}^{b_{h}}(\underbrace{f_{t}(i)}_{\text {mass of firms with price } P(i) \text { at time } t}(P(i))^{r}) d i\right], \\
\text { where } P\left(a_{h}\right) & \equiv \min \left\{P_{t}(h) \forall h \in[0,1]\right\}, P\left(b_{h}\right) \equiv \max \left\{P_{t}(h) \forall h \in[0,1]\right\}, a_{h} \leq b_{h}, \\
{\left[\int_{0}^{1}\left(P_{t}(f)\right)^{r} d f\right]^{\frac{1}{r}} } & =\left[\int_{a_{f}}^{b_{f}}(\underbrace{f_{t}(i)}_{\text {mass of firms with price } P(i) \text { at time } t})^{r}\right]^{\frac{1}{r}}, \\
\text { where } P\left(a_{f}\right) & \equiv \min \left\{P_{t}(f) \forall f \in[0,1]\right\}, P\left(b_{f}\right) \equiv \max \left\{P_{t}(f) \forall f \in[0,1]\right\}, a_{f} \leq b_{f},
\end{aligned}
$$

where $r$ can take the value of $-\theta$, it can take the value of $1-\theta$, or it can be given any other real number value.

Definition 1 The weighted power mean of degree $r$. Let $f(i)$ be a positive real number for all $i \in[a, b]$ such that $\int_{a}^{b} f(i) d i=1$. For any real number $r \neq 0$, the weighted power mean of degree $r$ of the positive real numbers $P(i) \in[P(a), P(b)]$ (such that $P(a)>0$ ) with respect to their mass $f(i)$ can be defined as,

$$
M_{f}^{r}(P(i) \forall i \in[a, b]) \equiv\left[\int_{a}^{b} f(i)(P(i))^{r} d i\right]^{\frac{1}{r}} .
$$

For the case $r=0$, the weighted geometric mean can be defined as,

$$
M_{f}^{0}(P(i) \forall i \in[a, b]) \equiv \prod_{a}^{b}(P(i))^{f(i) d i},
$$

which comes from taking the limit $r \rightarrow 0$ on the weighted power mean $M_{f}^{r}(P(i) \forall i \in[a, b])$. 
Proof. Using the Taylor series expansion $e^{x}=1+x+\mathcal{O}\left(x^{2}\right)$, where $\mathcal{O}\left(x^{2}\right)$ represents terms of second order and higher, I can write,

$$
(P(i))^{r}=e^{r \ln (P(i))}=1+r \ln (P(i))+\mathcal{O}\left(r^{2}\right) .
$$

By substituting this Taylor expansion into the definition of $M_{f}^{r}(P(i) \forall i \in[a, b])$ I obtain that,

$$
\begin{aligned}
M_{f}^{r}(P(i) \forall i \in[a, b]) & =\left[\int_{a}^{b} f(i)(1+r \ln (P(i))) d i+\mathcal{O}\left(r^{2}\right)\right]^{\frac{1}{r}} \\
& =\left[1+r \int_{a}^{b} f(i) \ln P(i) d i+\mathcal{O}\left(r^{2}\right)\right]^{\frac{1}{r}} \\
& =\left[1+r \int_{a}^{b} \ln (P(i))^{f(i)} d i+\mathcal{O}\left(r^{2}\right)\right]^{\frac{1}{r}} \\
& =\left[1+r \ln \left(\prod_{a}^{b}(P(i))^{f(i) d i}\right)+\mathcal{O}\left(r^{2}\right)\right]^{\frac{1}{r}} \\
& =\exp \left\{\frac{1}{r} \ln \left[1+r \ln \left(\prod_{a}^{b}(P(i))^{f(i) d i}\right)+\mathcal{O}\left(r^{2}\right)\right]\right\},
\end{aligned}
$$

where $\prod$ denotes the product integral (the continuous counterpart of the discrete product operator). Using now the Taylor series expansion $\ln (1+x)=x+\mathcal{O}\left(x^{2}\right)$, I easily obtain that,

$$
\begin{aligned}
M_{f}^{r}(P(i) \forall i \in[a, b]) & =\exp \left\{\frac{1}{r} \ln \left[1+r \ln \left(\prod_{a}^{b}(P(i))^{f(i) d i}\right)+\mathcal{O}\left(r^{2}\right)\right]\right\} \\
& =\exp \left\{\frac{1}{r}\left[r \ln \left(\prod_{a}^{b}(P(i))^{f(i) d i}\right)+\mathcal{O}\left(r^{2}\right)\right]\right\} \\
& =\exp \left\{\ln \left(\prod_{a}^{b}(P(i))^{f(i) d i}\right)+\mathcal{O}(r)\right\}
\end{aligned}
$$

Taking the limit as $r \rightarrow 0$, then I get that,

$$
\begin{aligned}
M_{f}^{0}(P(i) \forall i \in[a, b]) & =\exp \left\{\ln \left(\prod_{a}^{b}(P(i))^{f(i) d i}\right)\right\} \\
& =\prod_{a}^{b}(P(i))^{f(i) d i} .
\end{aligned}
$$

Proposition 1 General means inequality. For any two real numbers $r<s$, the weighted power means of orders $r$ and $s$ of the positive real numbers $P(i) \in[P(a), P(b)]$ defined in (109) - (110) satisfy the inequality,

$$
M_{f}^{r}(P(i) \forall i \in[a, b]) \leq M_{f}^{s}(P(i) \forall i \in[a, b]),
$$


with equality if and only if all the $P(i)$ for all $i \in[a, b]$ are equal.

Proof. Case (a): I assume that $0<r<s$. I write $x=\frac{s}{r}>1$, and define $y(i) \equiv(P(i))^{r}$ for all $i \in[a, b]$ and $(y(i))^{x} \equiv(P(i))^{s}$ for all $i \in[a, b]$. The function $g(z)=z^{x}$ satisfies that its second derivative is $g^{\prime \prime}(z)=x(x-1) z^{x-2}>0$ for all $z(z$ being a positive real number) since $x>1$. Therefore, $g(z)$ is strictly convex and according to Jensen's inequality it must follow that,

$$
\begin{aligned}
{\left[\int_{a}^{b} f(i) y(i) d i\right]^{x} } & =g\left(\int_{a}^{b} f(i) y(i) d i\right) \\
& \leq \int_{a}^{b} f(i) g(y(i)) d i \\
& =\int_{a}^{b} f(i)(y(i))^{x} d i
\end{aligned}
$$

with equality if and only if $y(i)=y$ for all $i \in[a, b]$. By substituting $x=\frac{s}{r}$ and $y(i) \equiv(P(i))^{r}$ back into this inequality, I get that,

$$
\left[\int_{a}^{b} f(i)(P(i))^{r} d i\right]^{\frac{s}{r}} \leq \int_{a}^{b} f(i)(P(i))^{s} d i
$$

with equality if and only if $P(i)=P$ for all $i \in[a, b]$. Since $s$ is positive, the function $z^{\frac{1}{s}}$ is strictly increasing and raising both sides to the power $\frac{1}{s}$ preserves the inequality, i.e.

$$
\left[\int_{a}^{b} f(i)(P(i))^{r} d i\right]^{\frac{1}{r}} \leq\left[\int_{a}^{b} f(i)(P(i))^{s} d i\right]^{\frac{1}{s}} .
$$

Equality holds if and only if all $P(i)$ are equal.

Case (b): I assume that $r=0<s . M_{f}^{0}(P(i) \forall i \in[a, b])$ is defined as $\lim _{r \rightarrow 0} M_{f}^{r}(P(i) \forall i \in[a, b])$. Since $M_{f}^{r}(P(i) \forall i \in[a, b]) \leq M_{f}^{s}(P(i) \forall i \in[a, b])$ for all $r<s$ with $r \neq 0$ but arbitrarily close, then the same inequality must hold for the limit when $r \rightarrow 0$.

Case (c): I assume that $r<0<s$. I write $x=\frac{s}{r}<0$, and define $y(i) \equiv(P(i))^{r}$ for all $i \in[a, b]$ and $(y(i))^{x} \equiv(P(i))^{s}$ for all $i \in[a, b]$. The function $g(z)=z^{x}$ satisfies that its second derivative is $g^{\prime \prime}(z)=x(x-1) z^{x-2}>0$ for all $z(z$ being a positive real number) since $x<0$. Therefore, $g(z)$ is strictly convex and according to Jensen's inequality it must follow that,

$$
\begin{aligned}
{\left[\int_{a}^{b} f(i) y(i) d i\right]^{x} } & =g\left(\int_{a}^{b} f(i) y(i) d i\right) \\
& \leq \int_{a}^{b} f(i) g(y(i)) d i \\
& =\int_{a}^{b} f(i)(y(i))^{x} d i
\end{aligned}
$$

with equality if and only if $y(i)=y$ for all $i \in[a, b]$. By substituting $x=\frac{s}{r}$ and $y(i) \equiv(P(i))^{r}$ back into 
this inequality, I get that,

$$
\left[\int_{a}^{b} f(i)(P(i))^{r} d i\right]^{\frac{s}{r}} \leq \int_{a}^{b} f(i)(P(i))^{s} d i
$$

with equality if and only if $P(i)=P$ for all $i \in[a, b]$. Since $s$ is positive, the function $z^{\frac{1}{s}}$ is strictly increasing and raising both sides to the power $\frac{1}{s}$ preserves the inequality, i.e.

$$
\left[\int_{a}^{b} f(i)(P(i))^{r} d i\right]^{\frac{1}{r}} \leq\left[\int_{a}^{b} f(i)(P(i))^{s} d i\right]^{\frac{1}{s}} .
$$

Equality holds if and only if all $P(i)$ are equal.

Case (d): I assume that $r<s=0 . M_{f}^{0}(P(i) \forall i \in[a, b])$ is defined as $\lim _{s \rightarrow 0} M_{f}^{s}(P(i) \forall i \in[a, b])$. Since $M_{f}^{r}(P(i) \forall i \in[a, b]) \leq M_{f}^{s}(P(i) \forall i \in[a, b])$ for all $r<s$ with $s \neq 0$ but arbitrarily close, then the same inequality must hold for the limit when $s \rightarrow 0$.

Case (e): I assume that $r<s<0$. I write $0<x=\frac{s}{r}<1$, and define $y(i) \equiv(P(i))^{r}$ for all $i \in[a, b]$ and $(y(i))^{x} \equiv(P(i))^{s}$ for all $i \in[a, b]$. The function $g(z)=z^{x}$ satisfies that its second derivative is $g^{\prime \prime}(z)=x(x-1) z^{x-2}<0$ for all $z(z$ being a positive real number) since $0<x<1$. Therefore, $g(z)$ is strictly concave and according to Jensen's inequality it must follow that,

$$
\begin{aligned}
{\left[\int_{a}^{b} f(i) y(i) d i\right]^{x} } & =g\left(\int_{a}^{b} f(i) y(i) d i\right) \\
& \geq \int_{a}^{b} f(i) g(y(i)) d i \\
& =\int_{a}^{b} f(i)(y(i))^{x} d i
\end{aligned}
$$

with equality if and only if $y(i)=y$ for all $i \in[a, b]$. By substituting $x=\frac{s}{r}$ and $y(i) \equiv(P(i))^{r}$ back into this inequality, I get that,

$$
\left[\int_{a}^{b} f(i)(P(i))^{r} d i\right]^{\frac{s}{r}} \geq \int_{a}^{b} f(i)(P(i))^{s} d i
$$

or simply,

$$
\left[\int_{a}^{b} f(i)(P(i))^{r} d i\right]^{-\frac{s}{r}} \leq\left[\int_{a}^{b} f(i)(P(i))^{s} d i\right]^{-1},
$$

with equality if and only if $P(i)=P$ for all $i \in[a, b]$. Since $s$ is negative, the function $z^{-\frac{1}{s}}$ is strictly increasing and raising both sides to the power $-\frac{1}{s}$ preserves the inequality, i.e.

$$
\left[\int_{a}^{b} f(i)(P(i))^{r} d i\right]^{\frac{1}{r}} \leq\left[\int_{a}^{b} f(i)(P(i))^{s} d i\right]^{\frac{1}{s}} .
$$

Equality holds if and only if all $P(i)$ are equal. 
Real Rental Rates of Capital and the Firm's Efficiency Conditions. Combining the implied production equations in (84) - (85) with the efficiency conditions for firms in (24) - (25) and the labor supply equations from the households' problem (in equations (54) - (55)), I can express the real rental rate of capital in terms of productivity shocks, aggregate consumption, output and physical capital, i.e.

$$
\begin{aligned}
\frac{Z_{t}}{P_{t}} & =\frac{1-\psi}{\psi} \frac{W_{t}}{P_{t}} \frac{L_{t}}{K_{t}}=\frac{1-\psi}{\psi}\left(A_{t}\right)^{-\frac{1+\varphi}{\psi}}\left(C_{t}\right)^{\sigma^{-1}}\left(Y_{t}\right)^{\frac{1+\varphi}{\psi}}\left(K_{t}\right)^{-\left(\frac{1+(1-\psi) \varphi}{\psi}\right)}, \\
\frac{Z_{t}^{*}}{P_{t}^{*}} & =\frac{1-\psi}{\psi} \frac{W_{t}^{*}}{P_{t}^{*}} \frac{L_{t}^{*}}{K_{t}^{*}}=\frac{1-\psi}{\psi}\left(A_{t}^{*}\right)^{-\frac{1+\varphi}{\psi}}\left(C_{t}^{*}\right)^{\sigma^{-1}}\left(Y_{t}^{*}\right)^{\frac{1+\varphi}{\psi}}\left(K_{t}^{*}\right)^{-\left(\frac{1+(1-\psi) \varphi}{\psi}\right)} .
\end{aligned}
$$

These two equations summarize the efficiency condition that requires the capital-to-labor ratios to be proportional to the factor price ratios. This characterization is also convenient because it implies that I do not need to keep track of wages or labor in the simulation of this equilibrium. Manipulating the same set of equilibrium conditions a little bit more also allows me to re-write the real wages in terms of the real rental rate of capital, the productivity shock, aggregate consumption, output and physical capital, i.e.

$$
\begin{aligned}
\frac{W_{t}}{P_{t}} & =\left(\frac{1-\psi}{\psi}\right)^{-\frac{(1-\psi) \varphi}{1+(1-\psi) \varphi}}\left(A_{t}\right)^{-\frac{\varphi}{1+(1-\psi) \varphi}}\left(C_{t}\right)^{\frac{\sigma^{-1}}{1+(1-\psi) \varphi}}\left(Y_{t}\right)^{\frac{\varphi}{1+(1-\psi) \varphi}}\left(\frac{Z_{t}}{P_{t}}\right)^{\frac{(1-\psi) \varphi}{1+(1-\psi) \varphi}}, \\
\frac{W_{t}^{*}}{P_{t}^{*}} & =\left(\frac{1-\psi}{\psi}\right)^{-\frac{(1-\psi)}{1+(1-\psi) \varphi} \varphi}\left(A_{t}^{*}\right)^{-\frac{\varphi}{1+(1-\psi) \varphi}}\left(C_{t}^{*}\right)^{\frac{\sigma}{1+(1-\psi) \varphi}}\left(Y_{t}^{*}\right)^{\frac{\varphi}{1+(1-\psi) \varphi}}\left(\frac{Z_{t}^{*}}{P_{t}^{*}}\right)^{\frac{(1-\psi) \varphi}{1+(1-\psi) \varphi}} .
\end{aligned}
$$

These two equations suffice for the purpose of replacing real wages out of the marginal cost equations.

The Resource Constraint. The budget constraint in (2) of the domestic household can be expressed in equilibrium (under equality) as,

$$
P_{t}\left(C_{t}+X_{t}\right)+T_{t}+\frac{1}{I_{t}} B_{t+1}+\frac{1}{I_{t}^{*}} S_{t} B_{t+1}^{F *}+\frac{\mu}{2} \frac{P_{t}}{I_{t}^{*}}\left(\frac{S_{t} B_{t+1}^{F *}}{P_{t}}-\bar{a}\right)^{2}=B_{t}+S_{t} B_{t}^{F *}+W_{t} L_{t}+Z_{t} K_{t}+P r_{t} .
$$

Using the domestic bond market clearing condition in (6), the budget constraint can be reduced as follows,

$$
C_{t}+X_{t}+\frac{T_{t}}{P_{t}}+\frac{1}{I_{t}^{*}} \frac{S_{t} B_{t+1}^{F *}}{P_{t}}+\frac{1}{I_{t}^{*}} \frac{\mu}{2}\left(\frac{S_{t} B_{t+1}^{F *}}{P_{t}}-\bar{a}\right)^{2}=\frac{S_{t} B_{t}^{F *}}{P_{t}}+\frac{W_{t}}{P_{t}} L_{t}+\frac{Z_{t}}{P_{t}} K_{t}+P r_{t}
$$

The per-period profits of the domestic firms distributed to the domestic households can be calculated as follows (including the labor subsidies),

$$
\begin{aligned}
\operatorname{Pr}_{t} & \equiv \int_{0}^{1}\left[P_{t}(h)\left(C_{t}(h)+X_{t}(h)\right)+S_{t} P_{t}^{*}(h)\left(C_{t}^{*}(h)+X_{t}^{*}(h)\right)\right] d h-\left(1-\xi_{t}\right) W_{t} L_{t}-\left(1-\xi_{t}\right) Z_{t} K_{t} \\
& =\int_{0}^{1}\left[P_{t}(h)\left(C_{t}(h)+X_{t}(h)\right)+S_{t} P_{t}^{*}(h)\left(C_{t}^{*}(h)+X_{t}^{*}(h)\right)\right] d h+\xi_{t} M C_{t} Y_{t}-W_{t} L_{t}-Z_{t} K_{t} .
\end{aligned}
$$


Therefore, replacing the expression for per-period profits inside the budget constraint of the domestic household I get that,

$$
\begin{aligned}
C_{t} & +X_{t}+\frac{T_{t}}{P_{t}}+\frac{1}{I_{t}^{*}}\left[\frac{S_{t} B_{t+1}^{F *}}{P_{t}}+\frac{\mu}{2}\left(\frac{S_{t} B_{t+1}^{F *}}{P_{t}}-\bar{a}\right)^{2}\right] \\
& =\frac{S_{t} B_{t}^{F *}}{P_{t}}+\frac{\int_{0}^{1}\left[P_{t}(h)\left(C_{t}(h)+X_{t}(h)\right)+S_{t} P_{t}^{*}(h)\left(C_{t}^{*}(h)+X_{t}^{*}(h)\right)\right] d h+\xi_{t} M C_{t} Y_{t}}{P_{t}} .
\end{aligned}
$$

Using the domestic government balanced-budget rule in (40), I obtain that,

$$
\begin{aligned}
C_{t} & +X_{t}+\frac{1}{I_{t}^{*}}\left[\frac{S_{t} B_{t+1}^{F *}}{P_{t}}+\frac{\mu}{2}\left(\frac{S_{t} B_{t+1}^{F *}}{P_{t}}-\bar{a}\right)^{2}\right] \\
& =\frac{S_{t} B_{t}^{F *}}{P_{t}}+\frac{\int_{0}^{1}\left[P_{t}(h)\left(C_{t}(h)+X_{t}(h)\right)+S_{t} P_{t}^{*}(h)\left(C_{t}^{*}(h)+X_{t}^{*}(h)\right)\right] d h}{P_{t}} .
\end{aligned}
$$

Then, I can use the demand equations in (46) - (49) and (50) - (53) to derive the following expression for the resource constraint,

$$
\begin{aligned}
\frac{1}{I_{t}^{*}}[ & \left.\frac{S_{t} B_{t+1}^{F *}}{P_{t}}+\frac{\mu}{2}\left(\frac{S_{t} B_{t+1}^{F *}}{P_{t}^{*}}-\bar{a}\right)^{2}\right]= \\
= & \frac{S_{t} B_{t}^{F *}}{P_{t}}+\frac{\int_{0}^{1}\left[P_{t}(h) \phi_{H}\left(\frac{P_{t}(h)}{P_{t}^{H}}\right)^{-\theta}\left(\frac{P_{t}^{H}}{P_{t}}\right)^{-\eta}\left(C_{t}+X_{t}\right)+S_{t} P_{t}^{*}(h) \phi_{F}\left(\frac{P_{t}^{*}(h)}{P_{t}^{H *}}\right)^{-\theta}\left(\frac{P_{t}^{H *}}{P_{t}^{* *}}\right)^{-\eta}\left(C_{t}^{*}+X_{t}^{*}\right)\right] d h}{P_{t}}-\left(C_{t}+X_{t}\right) \\
= & \frac{S_{t} B_{t}^{F *}}{P_{t}}+\phi_{H} \frac{P_{t}^{H}}{P_{t}}\left[\int_{0}^{1}\left(\frac{P_{t}(h)}{P_{t}^{H}}\right)^{1-\theta} d h\right]\left(\frac{P_{t}^{H}}{P_{t}}\right)^{-\eta}\left(C_{t}+X_{t}\right)+\ldots \\
& \phi_{F} \frac{S_{t} P_{t}^{H *}}{P_{t}}\left[\int_{0}^{1}\left(\frac{P_{t}^{*}(h)}{P_{t}^{H *}}\right)^{1-\theta} d h\right]\left(\frac{P_{t}^{H *}}{P_{t}^{*}}\right)^{-\eta}\left(C_{t}^{*}+X_{t}^{*}\right)-\left(C_{t}+X_{t}\right),
\end{aligned}
$$

Using the price indexes in (17) - (20) I infer that the relative price terms that enter into the resource constraint must be equal to one, i.e.

$$
\begin{aligned}
& \int_{0}^{1}\left(\frac{P_{t}(h)}{P_{t}^{H}}\right)^{1-\theta} d h=1 \\
& \int_{0}^{1}\left(\frac{P_{t}^{*}(h)}{P_{t}^{H *}}\right)^{1-\theta} d h=1
\end{aligned}
$$

and so I can re-write the resource constraint itself as,

$$
\begin{aligned}
\frac{1}{I_{t}^{*}} & {\left[\frac{S_{t} B_{t+1}^{F *}}{P_{t}}+\frac{\mu}{2}\left(\frac{S_{t} B_{t+1}^{F *}}{P_{t}}-\bar{a}\right)^{2}\right]=} \\
& =\frac{S_{t} B_{t}^{F *}}{P_{t}}+\phi_{H}\left(\frac{P_{t}^{H}}{P_{t}}\right)^{1-\eta}\left(C_{t}+X_{t}\right)+\phi_{F} \frac{S_{t} P_{t}^{*}}{P_{t}}\left(\frac{P_{t}^{H *}}{P_{t}^{*}}\right)^{1-\eta}\left(C_{t}^{*}+X_{t}^{*}\right)-\left(C_{t}+X_{t}\right) \\
& =\frac{S_{t} B_{t}^{F *}}{P_{t}}+\left[\phi_{H}\left(\frac{P_{t}^{H}}{P_{t}}\right)^{1-\eta}\left(C_{t}+X_{t}\right)+\phi_{F} R S_{t}\left(\frac{P_{t}^{H *}}{P_{t}^{*}}\right)^{1-\eta}\left(C_{t}^{*}+X_{t}^{*}\right)-\left(C_{t}+X_{t}\right)\right],
\end{aligned}
$$

where I have also made use of the definition of the real exchange rate in (21). Defining now the real net 
foreign asset position of the domestic household as $N F A_{t+1} \equiv \frac{S_{t} B_{t+1}^{F *}}{P_{t}}$, I can re-write equation (116) more conveniently as,

$$
\begin{aligned}
& \frac{1}{I_{t}^{*}}\left[N F A_{t+1}+\frac{\mu}{2}\left(N F A_{t+1}-\bar{a}\right)^{2}\right]= \\
& \quad=\frac{S_{t}}{S_{t-1}} \frac{P_{t-1}}{P_{t}} N F A_{t}+\left[\phi_{H}\left(\frac{P_{t}^{H}}{P_{t}}\right)^{1-\eta}\left(C_{t}+X_{t}\right)+\phi_{F} R S_{t}\left(\frac{P_{t}^{H *}}{P_{t}^{*}}\right)^{1-\eta}\left(C_{t}^{*}+X_{t}^{*}\right)-\left(C_{t}+X_{t}\right)\right] .
\end{aligned}
$$

Equation (117) is the resource constraint equation that is needed to close down the model with incomplete asset markets.

\section{The Deterministic, Zero-Inflation Steady State}

I postulate a zero-inflation steady state where the nominal exchange rate equals one, $\bar{S}=1$, and where absorption in both countries differs only by a factor related to the steady state real exchange rate, i.e. $(\bar{C}+\bar{X})=(\overline{R S})^{\eta}\left(\bar{C}^{*}+\bar{X}^{*}\right)$. This normalization facilitates the log-linearization of the equilibrium conditions of the model. However, the normalization relating the absorption of both countries to the real exchange rate imposes additional restrictions on the steady state aggregate productivity across countries that need to be explored in greater detail. Moreover, it does not necessarily imply that consumption and investment equalize across countries in steady state. For the purpose of this paper, suffices to consider the implications of this normalization when also adding capital and non-zero real net foreign assets in the steady state.

First Step. I look at the steady state investment-to-capital ratio. The domestic capital accumulation equation in (3) (and its foreign counterpart) require the following relationships to hold in steady state,

$$
\begin{aligned}
\bar{K} & =(1-\delta) \bar{K}+\bar{V} \Phi(\bar{X}, \bar{X}, \bar{K}) \bar{X} \\
\bar{K}^{*} & =(1-\delta) \bar{K}^{*}+\bar{V}^{*} \Phi\left(\bar{X}^{*}, \bar{X}^{*}, \bar{K}^{*}\right) \bar{X}^{*}
\end{aligned}
$$

Operating on the steady state capital accumulation equations it follows that,

$$
\delta=\bar{V} \Phi(\bar{X}, \bar{X}, \bar{K}) \frac{\bar{X}}{\bar{K}}=\bar{V}^{*} \Phi\left(\bar{X}^{*}, \bar{X}^{*}, \bar{K}^{*}\right) \frac{\bar{X}^{*}}{\bar{K}^{*}} .
$$

The two equalities in (120) simplify to $\frac{\bar{X}}{\bar{K}}=\frac{\bar{X}^{*}}{\bar{K}^{*}}=\delta$ because I assume that the steady state IST shock is normalized to one in both countries, i.e. $\bar{V}=\bar{V}^{*}=1$, and because I know that: (a) under the specification of the capital adjustment cost (CAC) function, it holds that $\Phi\left(\frac{\bar{X}}{\bar{K}}\right)=\Phi\left(\frac{\bar{X}^{*}}{\bar{K}^{*}}\right)=\Phi(\delta)=1$; and (b) under the specification of the investment adjustment cost (IAC) function, it holds that $\Phi\left(\frac{\bar{X}}{\bar{X}}\right)=\Phi\left(\frac{\bar{X}^{*}}{\bar{X}^{*}}\right)=\Phi(1)=1$. Therefore, these adjustment cost functions have no steady state implications, and investment purely replaces the depreciated stock of capital (i.e. $\bar{X}=\delta \bar{K}$ and $\bar{X}^{*}=\delta \bar{K}^{*}$ ).

Second Step. I look at the nominal and real interest rates as well as the real rental rate of capital and Tobin's q. Using the fact that the investment-to-capital ratio is pined down by the depreciation rate (from (120)) and the properties of the adjustment cost function $\Phi(\cdot)$ (in (9) or (10)), I can write the steady state household efficiency conditions - in either (66) - (69) or (70) - (73) - that summarize Tobin's q and the real 
rental rate of capital as follows,

$$
\begin{aligned}
\bar{Q} & =\beta\left[\frac{\bar{Z}}{\bar{P}}+\bar{Q}(1-\delta)\right], \\
\bar{Q}^{*} & =\beta\left[\frac{\bar{Z}^{*}}{\bar{P}^{*}}+\bar{Q}^{*}(1-\delta)\right], \\
\bar{Q} & =\bar{Q}^{*}=1,
\end{aligned}
$$

where I explicitly used the fact that $\Phi^{\prime}\left(\frac{\bar{X}}{\bar{K}}\right)=\Phi^{\prime}\left(\frac{\bar{X}^{*}}{\bar{K}^{*}}\right)=\Phi^{\prime}(\delta)=0$ and $\Phi^{\prime}\left(\frac{\bar{X}}{\bar{X}}\right)=\Phi^{\prime}\left(\frac{\bar{X}^{*}}{\bar{X}^{*}}\right)=\Phi^{\prime}(1)=0$, as well as the steady state normalization of the IST shocks to $\bar{V}=\bar{V}^{*}=1$. These equations are satisfied trivially in the NAC model without adjustment costs. Naturally, the steady state real rental rate of capital is,

$$
\frac{\bar{Z}}{\bar{P}}=\frac{\bar{Z}^{*}}{\bar{P}^{*}}=\beta^{-1}-(1-\delta) .
$$

The steady state interest rates given by equations (58) and (60) imply that,

$$
\begin{aligned}
\beta \bar{I} & =1, \\
\beta \bar{I}^{*} & =1 .
\end{aligned}
$$

Nominal and real interest rates must be equal in this (deterministic) zero-inflation steady state. These results say that Tobin's q is equal to 1 in steady state, and that the real rental rates on capital must be equal to the real returns on bonds (where the real returns on bonds are $\bar{I}=\bar{I}^{*}=\beta^{-1}$ as given by (125) - (126)) after taking into account the effect of the capital depreciation, $\delta$.

Moreover, from equation (59) on international risk-sharing, I get that,

$$
\beta \bar{I}^{*}=1+\mu\left(\frac{\bar{B}^{F *}}{\bar{P}}-\bar{a}\right),
$$

where I have implicitly used that the steady state nominal exchange rate is $\bar{S}=1$. Whenever $\mu>0$ and (126) is satisfied, this expression holds true only if $\overline{N F A} \equiv \frac{\overline{S B}^{F *}}{\bar{P}}=\bar{a}$. Naturally, given the foreign bond market clearing condition in (7), it must follow that $\frac{\overline{S B}^{*}}{\bar{P}}=-\frac{\overline{S B}^{F *}}{\bar{P}}=-\bar{a}$. In other words, in steady state there are no costs associated with trading in foreign bonds because the domestic households set their real net foreign asset position at the reference level of $\bar{a}$.

Equations (38) and (39) give me the specification of the monetary policy rules in the context of this model. In steady state, it must follow that,

$$
\begin{aligned}
\bar{I} & =\bar{M}(\bar{I})^{\rho_{i}}\left[\bar{I}\left(\frac{\bar{\Pi}}{\bar{\Pi}}\right)^{\psi_{\pi}}(\overline{\bar{Y}})^{\psi_{y}}\right]^{1-\rho_{i}}, \\
\bar{I}^{*} & =\bar{M}^{*}\left(\bar{I}^{*}\right)^{\rho_{i}}\left[\bar{I}^{*}\left(\frac{\bar{\Pi}^{*}}{\bar{\Pi}^{*}}\right)^{\psi_{\pi}}\left(\frac{\bar{Y}^{*}}{\bar{Y}^{*}}\right)^{\psi_{y}}\right]^{1-\rho_{i}},
\end{aligned}
$$


which immediately reduces to,

$$
\begin{aligned}
\bar{M} & =1, \\
\bar{M}^{*} & =1 .
\end{aligned}
$$

This gives a conventional normalization for the steady state monetary policy shocks in both countries.

Third Step. I look at the steady state investment shares $\gamma_{x}^{y} \equiv \frac{\bar{X}}{\bar{Y}}$ and $\gamma_{x^{*}}^{y} \equiv \frac{\bar{X}^{*}}{\bar{Y}^{*}}$. Using the fact noted in (120) that in steady state the investment-to-capital ratio is determined by the depreciation rate, $\delta, I$ can write the investment shares as proportional to the capital-to-output ratios in each country,

$$
\begin{aligned}
\gamma_{x}^{y} & \equiv \delta \frac{\bar{K}}{\bar{Y}}, \\
\gamma_{x^{*}}^{y} & \equiv \delta \frac{\bar{K}^{*}}{\bar{Y}^{*}} .
\end{aligned}
$$

From the Cobb-Douglas specification of the firm production functions in (22) - (23), the efficiency conditions on the firm's optimization problem in (24) - (25), and the aggregate production functions derived in (84) (85), I get that the capital-to-output ratio can be expressed as a function of the productivity shocks and the factor price ratios in each country. Hence, it follows that,

$$
\begin{aligned}
\gamma_{x}^{y} & =\delta\left(\frac{1-\psi}{\psi}\right)^{\psi} \frac{1}{\bar{A}}\left(\frac{\bar{W}}{\bar{Z}}\right)^{\psi}=\delta\left(\frac{1-\psi}{\psi}\right)^{\psi}\left(\frac{\bar{Z}}{\bar{P}}\right)^{-\psi}\left[\frac{1}{\bar{A}}\left(\frac{\bar{W}}{\bar{P}}\right)^{\psi}\right], \\
\gamma_{x^{*}}^{y} & =\delta\left(\frac{1-\psi}{\psi}\right)^{\psi} \frac{1}{\bar{A}^{*}}\left(\frac{\bar{W}^{*}}{\bar{Z}^{*}}\right)^{\psi}=\delta\left(\frac{1-\psi}{\psi}\right)^{\psi}\left(\frac{\bar{Z}^{*}}{\bar{P}^{*}}\right)^{-\psi}\left[\frac{1}{\bar{A}^{*}}\left(\frac{\bar{W}^{*}}{\bar{P}^{*}}\right)^{\psi}\right],
\end{aligned}
$$

where $\bar{A}$ and $\bar{A}^{*}$ are the steady state domestic and foreign productivity shocks.

I also know that in a deterministic zero-inflation steady state all firms charge the same price (the Calvo parameter is irrelevant), the law of one price holds and the standard price setting formula under monopolistic competition determines the pricing decision for firms. In other words, prices of the same goods in diferent markets are equalized when expressed in units of the same currency, and prices must be equal to a mark-up over marginal costs. Then, the pricing equations in (74) and (77) in conjunction with the marginal cost functions in $(26)-(27)$ reduce to,

$$
\begin{aligned}
\bar{P}^{H} & =\frac{\theta(1-\bar{\xi})}{\theta-1} \frac{1}{\bar{A}} \frac{1}{\psi^{\psi}(1-\psi)^{1-\psi}}(\bar{W})^{\psi}(\bar{Z})^{1-\psi} \\
\bar{P}^{F *} & =\frac{\theta\left(1-\bar{\xi}^{*}\right)}{\theta-1} \frac{1}{\bar{A}^{*}} \frac{1}{\psi^{\psi}(1-\psi)^{1-\psi}}\left(\bar{W}^{*}\right)^{\psi}\left(\bar{Z}^{*}\right)^{1-\psi}
\end{aligned}
$$

where the domestic firms' subsidy in steady state, $\bar{\xi}$, and the foreign firms' subsidy in steady state, $\bar{\xi}^{*}$, are also incorporated. I assume that the steady state subsidy is the same in both countries, i.e. $\bar{\xi}=\bar{\xi}^{*}$. Dividing these expressions by $\bar{P}$ and $\bar{P}^{*}$ respectively and then re-arranging terms, I get that real wages are linked to 
real rental rates on capital by,

$$
\begin{aligned}
\frac{1}{\bar{A}}\left(\frac{\bar{W}}{\bar{P}}\right)^{\psi} & =\frac{\theta-1}{\theta(1-\bar{\xi})}\left[\psi^{\psi}(1-\psi)^{1-\psi} \frac{\bar{P}^{H}}{\bar{P}}\left(\frac{\bar{Z}}{\bar{P}}\right)^{\psi-1}\right] \\
\frac{1}{\bar{A}^{*}}\left(\frac{\bar{W}^{*}}{\bar{P}^{*}}\right)^{\psi} & =\frac{\theta-1}{\theta\left(1-\bar{\xi}^{*}\right)}\left[\psi^{\psi}(1-\psi)^{1-\psi} \frac{\bar{P}^{F *}}{\bar{P}^{*}}\left(\frac{\bar{Z}^{*}}{\bar{P}^{*}}\right)^{\psi-1}\right] .
\end{aligned}
$$

If I replace the expressions in (138) and (139) inside the formula for the domestic and foreign investment shares obtained before in (134) and (135), I get that,

$$
\begin{aligned}
\gamma_{x}^{y} & =\delta\left(\frac{1-\psi}{\psi}\right)^{\psi}\left(\frac{\bar{Z}}{\bar{P}}\right)^{-\psi}\left[\frac{\theta-1}{\theta(1-\bar{\xi})}\left(\psi^{\psi}(1-\psi)^{1-\psi} \frac{\bar{P}^{H}}{\bar{P}}\left(\frac{\bar{Z}}{\bar{P}}\right)^{\psi-1}\right)\right] \\
& =\delta(1-\psi)\left[\left(\frac{\theta(1-\bar{\xi})}{\theta-1}\right)\left(\frac{\bar{P}}{\bar{P}^{H}}\right)\left(\frac{\bar{Z}}{\bar{P}}\right)\right]^{-1}, \\
\gamma_{x^{*}}^{y} & =\delta\left(\frac{1-\psi}{\psi}\right)^{\psi}\left(\frac{\bar{Z}^{*}}{\bar{P}^{*}}\right)^{-\psi}\left[\frac{\theta-1}{\theta\left(1-\bar{\xi}^{*}\right)}\left(\psi^{\psi}(1-\psi)^{1-\psi} \frac{\bar{P}^{F *}}{\bar{P}^{*}}\left(\frac{\bar{Z}^{*}}{\bar{P}^{*}}\right)^{\psi-1}\right)\right] \\
& =\delta(1-\psi)\left[\left(\frac{\theta\left(1-\bar{\xi}^{*}\right)}{\theta-1}\right)\left(\frac{\bar{P}^{*}}{\bar{P}^{F *}}\right)\left(\frac{\bar{Z}^{*}}{\bar{P}^{*}}\right)\right]^{-1} .
\end{aligned}
$$

Then, using the steady state real rental rates on capital derived in (124), I can say that,

$$
\begin{aligned}
\gamma_{x}^{y}=\delta\left[\frac{1-\psi}{\left(\frac{\theta(1-\bar{\xi})}{\theta-1}\right)\left(\beta^{-1}-(1-\delta)\right)}\right]\left(\frac{\bar{P}^{H}}{\bar{P}}\right), \\
\gamma_{x^{*}}^{y}=\delta\left[\frac{1-\psi}{\left(\frac{\theta\left(1-\bar{\xi}^{*}\right)}{\theta-1}\right)\left(\beta^{-1}-(1-\delta)\right)}\right]\left(\frac{\bar{P}^{F *}}{\bar{P}^{*}}\right) .
\end{aligned}
$$

In other words, the domestic and foreign investment shares in steady state depend directly on the depreciation rate of capital, $\delta$, and the capital share in the production function, $1-\psi$, and depend indirectly on the markup, $\frac{\theta}{\theta-1}$, the government subsidy, $\bar{\xi}$ and $\bar{\xi}^{*}$, and the real rental rate on capital, $\beta^{-1}-(1-\delta)$. The domestic investment share also depends on the relative price $\left(\frac{\bar{P}^{H}}{\bar{P}}\right)$, while the foreign investment share depends on the relative price $\left(\frac{\bar{P}^{F *}}{\bar{P}^{*}}\right)$.

Hence, the steady state investment shares will not differ across countries, i.e.,

$$
\gamma_{x}^{y} \equiv \delta \frac{\bar{K}}{\bar{Y}}=\delta\left[\frac{1-\psi}{\left(\frac{\theta(1-\bar{\xi})}{\theta-1}\right)\left(\beta^{-1}-(1-\delta)\right)}\right]=\delta \frac{\bar{K}^{*}}{\bar{Y}^{*}} \equiv \gamma_{x^{*}}^{y},
$$


only if the steady state government subsidies are the same for all countries (i.e. $\bar{\xi}=\bar{\xi}^{*}$ ) and prices are equalized for all bundles of goods (i.e. $\bar{P}=\bar{P}^{H}=\bar{P}^{F}$ and $\bar{P}^{*}=\bar{P}^{H *}=\bar{P}^{F *}$ ). Otherwise, the steady state investment shares can only depend on the steady state real net foreign asset position of the domestic country $\bar{a}$ through its impact on the steady state relative prices $\left(\frac{\bar{P}^{H}}{\bar{P}}\right)$ and $\left(\frac{\bar{P}^{F *}}{\bar{P}^{*}}\right)$.

Fourth Step. I look at the steady state price indexes and sub-indexes as well as the real exchange rate. I write the steady state price sub-indexes in equations $(17)-(20)$ as follows,

$$
\begin{aligned}
\bar{P}^{H} & =\overline{\widetilde{P}}(h), \bar{P}^{F}=\overline{\widetilde{P}}(f), \\
\bar{P}^{H *} & =\overline{\widetilde{P}}^{*}(h), \bar{P}^{F *}=\overline{\widetilde{P}}^{*}(f),
\end{aligned}
$$

and,

$$
\bar{P}^{H}=\bar{P}^{H *} \bar{S}, \bar{P}^{F}=\bar{P}^{F *} \bar{S}
$$

since the law of one price holds in the deterministic zero-inflation steady state. These results are derived looking at the pricing equations in $(74)-(77)$ as well. Using the consumption price indexes in equations (15) - (16) evaluated at their steady state, I also can infer that the real exchange rate should be equal to,

$$
\begin{aligned}
\overline{R S} & =\frac{\overline{S P}^{*}}{\bar{P}}=\left[\frac{\phi_{F}\left(\overline{S P}^{H *}\right)^{1-\eta}+\phi_{H}\left(\overline{S P}^{F *}\right)^{1-\eta}}{\phi_{H}\left(\bar{P}^{H}\right)^{1-\eta}+\phi_{F}\left(\bar{P}^{F}\right)^{1-\eta}}\right]^{\frac{1}{1-\eta}} \\
& =\left[\frac{\phi_{F}+\phi_{H}\left(\frac{\bar{P}^{F}}{\bar{P}^{H}}\right)^{1-\eta}}{\phi_{H}+\phi_{F}\left(\frac{\bar{P}^{F}}{\bar{P}^{H}}\right)^{1-\eta}}\right]^{\frac{1}{1-\eta}},
\end{aligned}
$$

which is a function of the steady state terms of trade $\left(\frac{\bar{P}^{F}}{\bar{P}^{H}}\right)$. The steady state real exchange rate will be equal to one if it holds that $\bar{P}=\bar{P}^{F}=\bar{P}^{H}$. Moreover, given the definitions of the consumption price indexes in $(15)-(16)$, I can also note that,

$$
\begin{aligned}
\frac{\bar{P}^{H}}{\bar{P}} & =\frac{\bar{P}^{H}}{\left[\phi_{H}\left(\bar{P}^{H}\right)^{1-\eta}+\phi_{F}\left(\bar{P}^{F}\right)^{1-\eta}\right]^{\frac{1}{1-\eta}}}=\left[\frac{1}{\phi_{H}+\phi_{F}\left(\bar{P}^{F}\right)^{1-\eta}}\right]^{\frac{1}{1-\eta}}, \\
\frac{\bar{P}^{F *}}{\bar{P}^{*}} & =\frac{\bar{P}^{F *}}{\left[\phi_{F}\left(\bar{P}^{H *}\right)^{1-\eta}+\phi_{H}\left(\bar{P}^{F *}\right)^{1-\eta}\right]^{\frac{1}{1-\eta}}}=\left[\frac{\left(\overline{S P}^{F *}\right)^{1-\eta}}{\phi_{F}\left(\overline{S P}^{H *}\right)^{1-\eta}+\phi_{H}\left(\overline{S P}^{F *}\right)^{1-\eta}}\right]^{\frac{1}{1-\eta}} \\
& =\left[\frac{\left(\frac{\bar{P}^{F}}{\bar{P}^{H}}\right)^{1-\eta}}{\phi_{F}+\phi_{H}\left(\frac{\bar{P}^{F}}{\bar{P}^{H}}\right)^{1-\eta}}\right]^{\frac{1}{1-\eta}}
\end{aligned}
$$

which ties both relative price expressions (which enter into the derivation of the investment shares $\gamma_{x}^{y}$ and $\left.\gamma_{x^{*}}^{y}\right)$ to the terms of trade $\left(\frac{\bar{P}^{F}}{\bar{P}^{H}}\right)$. Naturally, since $\phi_{H}+\phi_{F}=1$, then $\frac{\bar{P}^{H}}{\bar{P}}=1$ and $\frac{\bar{P}^{F *}}{\bar{P}^{*}}=1$ if $\bar{P}=\bar{P} F=\bar{P}^{H}$. 
Fifth Step. I look at aggregate output demand and the resource constraint. The aggregate output demands in (92) - (93) implies in steady state that,

$$
\begin{aligned}
\bar{Y} & =\phi_{H}\left(\frac{\bar{P}^{H}}{\bar{P}}\right)^{-\eta}(\bar{C}+\bar{X})+\phi_{F}\left(\frac{\bar{P}^{H *}}{\bar{P}^{*}}\right)^{-\eta}\left(\bar{C}^{*}+\bar{X}^{*}\right) \\
& =\phi_{H}\left(\frac{\bar{P}^{H}}{\bar{P}}\right)^{-\eta}(\bar{C}+\bar{X})+\phi_{F}\left(\frac{1}{\overline{R S}} \frac{\bar{P}^{H}}{\bar{P}}\right)^{-\eta}\left(\bar{C}^{*}+\bar{X}^{*}\right) \\
& =\phi_{H}\left(\frac{\bar{P}^{H}}{\bar{P}}\right)^{-\eta}(\bar{C}+\bar{X})+\phi_{F}(\overline{R S})^{\eta}\left(\frac{\bar{P}^{H}}{\bar{P}}\right)^{-\eta}\left(\bar{C}^{*}+\bar{X}^{*}\right), \\
\bar{Y}^{*} & \phi_{F}\left(\frac{\bar{P}^{F}}{\bar{P}}\right)^{-\eta}(\bar{C}+\bar{X})+\phi_{H}\left(\frac{\bar{P}^{F *}}{\bar{P}^{*}}\right)^{-\eta}\left(\bar{C}^{*}+\bar{X}^{*}\right) \\
& =\phi_{F}\left(\frac{\bar{P}^{F}}{\bar{P}}\right)^{-\eta}(\bar{C}+\bar{X})+\phi_{H}\left(\frac{1}{\overline{R S}} \frac{\bar{P}}{\bar{P}}\right)^{-\eta}\left(\bar{C}^{*}+\bar{X}^{*}\right) \\
& =\phi_{F}\left(\frac{\bar{P}^{F}}{\bar{P}}\right)^{-\eta}(\bar{C}+\bar{X})+\phi_{H}(\overline{R S})^{\eta}\left(\frac{\bar{P}}{\bar{P}}\right)^{-\eta}\left(\bar{C}^{*}+\bar{X}^{*}\right) .
\end{aligned}
$$

Moreover, the resource constraint in (117) can be characterized as follows,

$$
\begin{aligned}
\frac{1}{\bar{I}^{*} \bar{a}} & =\bar{a}+\left[\phi_{H}\left(\frac{\bar{P}^{H}}{\bar{P}}\right)^{1-\eta}(\bar{C}+\bar{X})+\phi_{F} \overline{R S}\left(\frac{\bar{P}^{H *}}{\bar{P}^{*}}\right)^{1-\eta}\left(\bar{C}^{*}+\bar{X}^{*}\right)-(\bar{C}+\bar{X})\right] \\
& =\bar{a}+\left[\phi_{H}\left(\frac{\bar{P}^{H}}{\bar{P}}\right)^{1-\eta}(\bar{C}+\bar{X})+\phi_{F} \overline{R S}\left(\frac{\bar{P}}{\overline{S P}} \frac{\bar{P}^{H}}{\bar{P}}\right)^{1-\eta}\left(\bar{C}^{*}+\bar{X}^{*}\right)-(\bar{C}+\bar{X})\right] \\
& =\bar{a}+\left[\phi_{H}\left(\frac{\bar{P}^{H}}{\bar{P}}\right)^{1-\eta}(\bar{C}+\bar{X})+\phi_{F}(\overline{R S})^{\eta}\left(\frac{\bar{P}^{H}}{\bar{P}}\right)^{1-\eta}\left(\bar{C}^{*}+\bar{X}^{*}\right)-(\bar{C}+\bar{X})\right],
\end{aligned}
$$

and, alternatively, re-written as,

$$
\left(\frac{1-\bar{I}^{*}}{\bar{I}^{*}}\right) \bar{a}=\left[\left(\frac{\bar{P}^{H}}{\bar{P}}\right) \bar{Y}-(\bar{C}+\bar{X})\right],
$$

where I have already used the domestic aggregate output demand equation obtained in (150). Obviously, if the steady state real net foreign asset position of the domestic household is equal to zero (i.e. $\bar{a}=0$ ), then I obtain the standard result implying that $\bar{P}^{H} \bar{Y}=\bar{P}(\bar{C}+\bar{X})$. In turn, $\bar{Y}=\bar{C}+\bar{X}$ holds when it also happens to be true that the law of one price holds and $\bar{P}=\bar{P}^{H}=\bar{P}^{F}$. In the more general case that I am exploring here, however, the relationship in (153) implies that,

$$
\bar{P}^{H} \bar{Y}=\bar{P}\left[(\bar{C}+\bar{X})+\left(\frac{1-\bar{I}^{*}}{\bar{I}^{*}}\right) \bar{a}\right],
$$


where the real net foreign asset position of the domestic household, $\bar{a}$, is expressed in units of domestic consumption. Then, the resource constraint in (152) can be written as,

$$
\begin{aligned}
\left(\frac{1-\bar{I}^{*}}{\bar{I}^{*}}\right) \bar{a} & =\left[\left(\phi_{H}\left(\frac{\bar{P}^{H}}{\bar{P}}\right)^{1-\eta}-1\right)(\bar{C}+\bar{X})+\phi_{F}(\overline{R S})^{\eta}\left(\frac{\bar{P}^{H}}{\bar{P}}\right)^{1-\eta}\left(\bar{C}^{*}+\bar{X}^{*}\right)\right] \\
& =\phi_{F}(\overline{R S})^{\eta}\left(\frac{\bar{P}^{H}}{\bar{P}}\right)^{1-\eta}\left(\bar{C}^{*}+\bar{X}^{*}\right)-\phi_{F}\left(\frac{\bar{P}^{F}}{\bar{P}}\right)^{1-\eta}(\bar{C}+\bar{X}),
\end{aligned}
$$

where I have used the definition of the domestic consumption price index in (15), i.e.,

$$
\bar{P}=\left[\phi_{H}\left(\bar{P}^{H}\right)^{1-\eta}+\phi_{F}\left(\bar{P}^{F}\right)^{1-\eta}\right]^{\frac{1}{1-\eta}} .
$$

The expression for the foreign aggregate output demand in (151) can be expressed now as,

$$
\bar{Y}^{*}=\left(\frac{\bar{P}^{F}}{\bar{P}}\right)^{-\eta}\left[\phi_{F}(\bar{C}+\bar{X})+\phi_{H}(\overline{R S})^{\eta}\left(\bar{C}^{*}+\bar{X}^{*}\right)\right] .
$$

Hence, combining this foreign aggregate demand equation with the second version of the resource constraint in (155), it follows that,

$$
\begin{aligned}
\bar{Y}^{*} & =\left(\frac{\bar{P}}{\bar{P}^{F}}\right)\left[\phi_{F}\left(\frac{\bar{P}^{F}}{\bar{P}}\right)^{1-\eta}(\bar{C}+\bar{X})\right]+\phi_{H}\left(\frac{\bar{P}^{F}}{\bar{P}}\right)^{-\eta}(\overline{R S})^{\eta}\left(\bar{C}^{*}+\bar{X}^{*}\right) \\
& =\left(\frac{\bar{P}}{\bar{P}^{F}}\right)\left[\phi_{F}(\overline{R S})^{\eta}\left(\frac{\bar{P}^{H}}{\bar{P}}\right)^{1-\eta}\left(\bar{C}^{*}+\bar{X}^{*}\right)-\left(\frac{1-\bar{I}^{*}}{\bar{I}^{*}}\right) \bar{a}\right]+\phi_{H}\left(\frac{\bar{P}^{F}}{\bar{P}}\right)^{-\eta}\left(\overline{R S}^{\eta}\left(\bar{C}^{*}+\bar{X}^{*}\right)\right. \\
& =\left[\phi_{F}\left(\frac{\bar{P}}{\bar{P}^{F}}\right)\left(\frac{\bar{P}^{H}}{\bar{P}}\right)^{1-\eta}+\phi_{H}\left(\frac{\bar{P}^{F}}{\bar{P}}\right)^{-\eta}\right](\overline{R S})^{\eta}\left(\bar{C}^{*}+\bar{X}^{*}\right)-\left(\frac{1-\bar{I}^{*}}{\bar{I}^{*}}\right)\left(\frac{\bar{P}}{\bar{P}^{F}}\right) \bar{a} \\
& =\left[\phi_{F}\left(\frac{\bar{P}^{H}}{\bar{P}}\right)^{1-\eta}+\phi_{H}\left(\frac{\bar{P}^{F}}{\bar{P}}\right)^{1-\eta}\right](\overline{R S})^{\eta}\left(\frac{\bar{P}}{\bar{P}^{F}}\right)\left(\bar{C}^{*}+\bar{X}^{*}\right)-\left(\frac{1-\bar{I}^{*}}{\bar{I}^{*}}\right)\left(\frac{\bar{P}}{\bar{P}^{F}}\right) \bar{a} .
\end{aligned}
$$

Then, using the definition of the foreign consumption price index in (16), i.e.,

$$
\bar{P}^{*}=\left[\phi_{F}\left(\bar{P}^{H *}\right)^{1-\eta}+\phi_{H}\left(\bar{P}^{F *}\right)^{1-\eta}\right]^{\frac{1}{1-\eta}},
$$

the implications of the law of one price, and the definition of the real exchange rate in (21) (i.e. $\overline{R S}=\frac{\overline{S P}^{*}}{\bar{P}}$ ), 
it follows that,

$$
\begin{aligned}
\bar{Y}^{*} & =\left[\phi_{F}\left(\frac{\bar{S}^{-1} \bar{P}^{H}}{\bar{S}^{-1} \bar{P}}\right)^{1-\eta}+\phi_{H}\left(\frac{\bar{S}^{-1} \bar{P}^{F}}{\bar{S}^{-1} \bar{P}}\right)^{1-\eta}\right](\overline{R S})^{\eta}\left(\frac{\bar{P}}{\bar{P}^{F}}\right)\left(\bar{C}^{*}+\bar{X}^{*}\right)-\left(\frac{1-\bar{I}^{*}}{\bar{I}^{*}}\right)\left(\frac{\bar{P}}{\bar{P}^{F}}\right) \bar{a} \\
& =\left[\phi_{F}\left(\frac{\bar{P}^{H *}}{\bar{P}^{*}} \frac{\bar{P}^{*}}{\bar{S}^{-1} \bar{P}}\right)^{1-\eta}+\phi_{H}\left(\frac{\bar{P}^{F *}}{\bar{P}^{*}} \frac{\bar{P}^{*}}{\bar{S}^{-1} \bar{P}}\right)^{1-\eta}\right](\overline{R S})^{\eta}\left(\frac{\bar{P}}{\bar{P}^{F}}\right)\left(\bar{C}^{*}+\bar{X}^{*}\right)-\left(\frac{1-\bar{I}^{*}}{\bar{I}^{*}}\right)\left(\frac{\bar{P}}{\bar{P}^{F}}\right) \bar{a} \\
& =\left[\phi_{F}\left(\frac{\bar{P}^{H *}}{\bar{P}^{*}}\right)^{1-\eta}+\phi_{H}\left(\frac{\bar{P}^{F *}}{\bar{P}^{*}}\right)^{1-\eta}\right]\left(\frac{\overline{S P}^{*}}{\bar{P}}\right)^{1-\eta}(\overline{R S})^{\eta}\left(\frac{\bar{P}}{\bar{P}^{F}}\right)\left(\bar{C}^{*}+\bar{X}^{*}\right)-\left(\frac{1-\bar{I}^{*}}{\bar{I}^{*}}\right)\left(\frac{\bar{P}}{\bar{P}^{F}}\right) \bar{a} \\
& =(\overline{R S})^{1-\eta}(\overline{R S})^{\eta}\left(\frac{\bar{P}}{\bar{P}^{F}}\right)\left(\bar{C}^{*}+\bar{X}^{*}\right)-\left(\frac{1-\bar{I}^{*}}{\bar{I}^{*}}\right)\left(\frac{\bar{P}}{\bar{P}^{F}}\right) \bar{a},
\end{aligned}
$$

and,

$$
\begin{aligned}
\bar{Y}^{*} & =\overline{R S}\left(\frac{\bar{P}}{\bar{P}^{F}}\right)\left(\bar{C}^{*}+\bar{X}^{*}\right)-\left(\frac{1-\bar{I}^{*}}{\bar{I}^{*}}\right)\left(\frac{\bar{P}}{\bar{P}^{F}}\right) \bar{a} \\
& =\left(\frac{\bar{P}^{*}}{\bar{P}^{F *}}\right)\left(\bar{C}^{*}+\bar{X}^{*}\right)-\left(\frac{\bar{P}}{\bar{P}^{F}}\right)\left(\frac{1-\bar{I}^{*}}{\bar{I}^{*}}\right) \bar{a} \\
& =\left(\frac{\bar{P}^{*}}{\bar{P}^{F *}}\right)\left(\bar{C}^{*}+\bar{X}^{*}\right)-\left(\frac{\overline{S P}^{*}}{\bar{P}^{F}} \frac{\bar{P}}{\overline{S P}^{*}}\right)\left(\frac{1-\bar{I}^{*}}{\bar{I}^{*}}\right) \bar{a} \\
& =\left(\frac{\bar{P}^{*}}{\bar{P}^{F *}}\right)\left(\bar{C}^{*}+\bar{X}^{*}\right)-\left(\frac{\bar{P}^{*}}{\bar{P}^{F *}} \frac{1}{\overline{R S}}\right)\left(\frac{1-\bar{I}^{*}}{\bar{I}^{*}}\right) \bar{a} .
\end{aligned}
$$

Obviously, if the steady state real net foreign asset position of the domestic household is equal to zero (i.e. $\bar{a}=0)$, then I obtain the standard result implying that $\bar{P}^{F *} \bar{Y}^{*}=\bar{P}^{*}\left(\bar{C}^{*}+\bar{X}^{*}\right)$. In turn, $\bar{Y}^{*}=\left(\bar{C}^{*}+\bar{X}^{*}\right)$ holds only whenever it also holds true that $\bar{P}^{*}=\bar{P}^{H *}=\bar{P}^{F *}$. In the more general case that I am exploring here, however, the relationship in (158) implies that,

$$
\bar{P}^{F *} \bar{Y}^{*}=\bar{P}^{*}\left[\left(\bar{C}^{*}+\bar{X}^{*}\right)-\left(\frac{1-\bar{I}^{*}}{\bar{I}^{*}}\right)\left(\frac{1}{\overline{R S}}\right) \bar{a}\right] .
$$

This expression differs slightly from the expression I derived for the domestic output demand in (154) because the real net foreign asset position of the domestic household, $\bar{a}$, is expressed in units of domestic consumption. Hence, the real exchange rate needs to be introduced in the formula in (159) in order to express everything in consistent (comparable) units.

Using the second version of the resource constraint in (155) again, I get a steady state expression in terms of domestic and foreign absorption,

$$
\phi_{F}\left(\frac{\bar{P}^{F}}{\bar{P}}\right)^{1-\eta}(\bar{C}+\bar{X})=\phi_{F}(\overline{R S})^{\eta}\left(\frac{\bar{P}^{H}}{\bar{P}}\right)^{1-\eta}\left(\bar{C}^{*}+\bar{X}^{*}\right)-\left(\frac{1-\bar{I}^{*}}{\bar{I}^{*}}\right) \bar{a}
$$


This implies that $(\bar{C}+\bar{X})=(\overline{R S})^{\eta}\left(\frac{\bar{P}^{H}}{\bar{P}^{F}}\right)^{1-\eta}\left(\bar{C}^{*}+\bar{X}^{*}\right)$ holds only if the real net foreign asset position of the domestic household becomes equal to zero in steady state, i.e. $\bar{a}=0$. In turn, $(\bar{C}+\bar{X})=\left(\bar{C}^{*}+\bar{X}^{*}\right)$ holds if it is also the case that prices equalize, i.e. $\bar{P}=\bar{P}^{H}=\bar{P}^{F}$ and $\bar{P}^{*}=\bar{P}^{H *}=\bar{P}^{F *}$. Furthermore, if prices and absorption equalize across countries, then the consumption shares will also be equal across countries because the investment shares must be identical (as discussed before for (142) - (143)).

In summary, I have obtained the following three key equations in (154), (159), and (160), i.e.

$$
\begin{aligned}
& \bar{Y}=\left(\frac{\bar{P}}{\bar{P}^{H}}\right)[(\bar{C}+\bar{X})-(1-\beta) \bar{a}] \\
& \bar{Y}^{*}=\left(\frac{\bar{P}^{*}}{\bar{P}^{F *}}\right)\left[\left(\bar{C}^{*}+\bar{X}^{*}\right)+(1-\beta)\left(\frac{1}{\overline{R S}}\right) \bar{a}\right] \\
& \phi_{F}\left(\frac{\bar{P}^{F}}{\bar{P}}\right)^{1-\eta}(\bar{C}+\bar{X})=\phi_{F}(\overline{R S})^{\eta}\left(\frac{\bar{P}^{H}}{\bar{P}}\right)^{1-\eta}\left(\bar{C}^{*}+\bar{X}^{*}\right)+(1-\beta) \bar{a},
\end{aligned}
$$

after replacing the steady state real interest rate derived in (125) and (126) (i.e. $\bar{I}=\bar{I}^{*}=\beta^{-1}$ ). In a more general setting where the real net foreign asset position is potentially different from zero (i.e. $\bar{a} \neq 0$ ), then the assumption that absorption in both countries differs only by a factor related to the steady state real exchange rate (i.e. $\left.(\bar{C}+\bar{X})=(\overline{R S})^{\eta}\left(\bar{C}^{*}+\bar{X}^{*}\right)\right)$ requires the following condition to be satisfied,

$$
\phi_{F}\left(\frac{\bar{P}^{F}}{\bar{P}}\right)^{1-\eta}(\bar{C}+\bar{X})=\phi_{F}\left(\frac{\bar{P}^{H}}{\bar{P}}\right)^{1-\eta}(\bar{C}+\bar{X})+(1-\beta) \bar{a},
$$

which follows from imposing the assumption on absorption to equation (163).

The real net foreign asset position of the domestic household $\bar{a}$ can be written as a fraction of the domestic absorption, $\bar{a}^{a}$, and alternatively as a fraction of the domestic output, $\bar{a}^{y}$, i.e.,

$$
\begin{aligned}
\bar{a} & \equiv \bar{a}^{a}(\bar{C}+\bar{X}), \\
\bar{a} & \equiv \bar{a}^{y} \bar{Y} .
\end{aligned}
$$

I have characterized the domestic output demand, $\bar{Y}$, in (161), so it is possible to re-write the real net foreign asset position of the domestic household relative to domestic output demand, $\bar{a}^{y}$, in the following terms,

$$
\begin{aligned}
\bar{a}^{y} & \equiv \frac{\bar{a}}{\bar{Y}}=\frac{\bar{a}}{\bar{C}+\bar{X} \frac{\bar{C}+\bar{X}}{\bar{Y}}} \\
& =\frac{\bar{a}}{\bar{C}+\bar{X}} \frac{\bar{C}+\bar{X}}{\left(\frac{\bar{P}}{\bar{P}^{H}}\right)[(\bar{C}+\bar{X})-(1-\beta) \bar{a}]}=\left(\frac{\bar{a}^{a}}{1-(1-\beta) \bar{a}^{a}}\right)\left(\frac{\bar{P}^{H}}{\bar{P}}\right)
\end{aligned}
$$

where $\left(\frac{\bar{P}^{H}}{\bar{P}}\right)$ is again a function of the terms of trade ratio $\left(\frac{\bar{P}^{F}}{\bar{P}^{H}}\right)$. In fact, given (148) I can write $\bar{a}^{y}$ as 
follows,

$$
\bar{a}^{y}=\left(\frac{\bar{a}^{a}}{1-(1-\beta) \bar{a}^{a}}\right)\left[\frac{1}{\phi_{H}+\phi_{F}\left(\frac{\bar{P}^{F}}{\bar{P}^{H}}\right)^{1-\eta}}\right]^{\frac{1}{1-\eta}} .
$$

Hence, if I choose the real net foreign asset position relative to domestic absorption, $\bar{a}^{a}$, to (uniquely) characterize the steady state terms of trade, then I can pin down the real net foreign asset position relative to domestic output demand, $\bar{a}^{y}$, from (168). I characterize the share relative to domestic absorption, $\bar{a}^{a}$, as the reference level of the real net foreign asset position in the model, but I will use the alternative share representation, $\bar{a}^{y}$, whenever it is more appropriate (or convenient) to describe the steady state or to define the parameterization of the model.

Condition (164) above - which links domestic and foreign absorption together under the proposed normalization of the steady state - can be expressed more compactly combined with (165) as,

$$
\phi_{F}\left(\frac{\bar{P}^{F}}{\bar{P}}\right)^{1-\eta}=\phi_{F}\left(\frac{\bar{P}^{H}}{\bar{P}}\right)^{1-\eta}+(1-\beta) \bar{a}^{a},
$$

or simply,

$$
\phi_{F}\left(\frac{\bar{P}^{F}}{\bar{P}^{H}}\right)^{1-\eta}=\phi_{F}+\frac{1}{\left(\frac{\bar{P}^{H}}{\bar{P}}\right)^{1-\eta}}(1-\beta) \bar{a}^{a} .
$$

I concentrate on this expression in order to derive the terms of trade ratio $\left(\frac{\bar{P}^{F}}{\bar{P}^{H}}\right)$ as a function of $\bar{a}^{a}$.

The price ratio $\left(\frac{\bar{P}^{H}}{\bar{P}}\right)$ can be expressed as a function of the terms of trade $\left(\frac{\bar{P}^{F}}{\bar{P}^{H}}\right)$ as given in (148), so operating on equation (170) I immediately obtain that,

$$
\phi_{F}\left(\frac{\bar{P}^{F}}{\bar{P}^{H}}\right)^{1-\eta}=\phi_{F}+\left(\phi_{H}+\phi_{F}\left(\frac{\bar{P}^{F}}{\bar{P}^{H}}\right)^{1-\eta}\right)(1-\beta) \bar{a}^{a},
$$

or simply,

$$
\left(\frac{\bar{P}^{F}}{\bar{P}^{H}}\right)^{1-\eta} \phi_{F}\left(1-(1-\beta) \bar{a}^{a}\right)=\phi_{F}+\phi_{H}(1-\beta) \bar{a}^{a} .
$$

In the extreme case in which $\frac{\bar{P}^{F}}{\bar{P}^{H}}=1$ whenever $\bar{P}=\bar{P}^{H}=\bar{P}^{F}$, then it must follow from this condition that,

$$
0=(1-\beta) \bar{a}^{a}
$$

Instances in which $\bar{a}^{a}$ is non-zero can be characterized through the condition above in (171). In turn, that would imply that the terms of trade are different from one, i.e. $\frac{\bar{P}^{F}}{\bar{P}^{H}} \neq 1$, and so is the real exchange rate, i.e. $\overline{R S} \neq 1$.

In fact, after a little bit of algebra with (171), I obtain a closed form solution for the terms of trade $\left(\frac{\bar{P}^{F}}{\bar{P}^{H}}\right)$ as a function of $\bar{a}^{a}$ as follows,

$$
\frac{\bar{P}^{F}}{\bar{P}^{H}}=\left(\frac{\phi_{F}+\phi_{H}(1-\beta) \bar{a}^{a}}{\phi_{F}-\phi_{F}(1-\beta) \bar{a}^{a}}\right)^{\frac{1}{1-\eta}} .
$$


Terms of trade $\left(\frac{\bar{P}^{F}}{\bar{P}^{H}}\right)$ are tied to the real net foreign asset position of the domestic households as a share of domestic absorption, $\bar{a}^{a}$, as well as to other structural parameters of the model (i.e. the intertemporal discount factor, $\beta$, the share of the home goods in the domestic aggregators, $\phi_{H}$, and the share of foreign goods, $\phi_{F}$ ). Replacing expression (173) into the expression for the real net foreign asset position of the domestic households as a share of domestic output demand in (168), I obtain that,

$$
\begin{aligned}
\bar{a}^{y} & =\left(\frac{\bar{a}^{a}}{1-(1-\beta) \bar{a}^{a}}\right)\left[\frac{1}{\phi_{H}+\phi_{F}\left(\frac{\phi_{F}+\phi_{H}(1-\beta) \bar{a}^{a}}{\phi_{F}-\phi_{F}(1-\beta) \bar{a}^{a}}\right)}\right]^{\frac{1}{1-\eta}} \\
& =\left(\frac{\bar{a}^{a}}{1-(1-\beta) \bar{a}^{a}}\right)\left[\frac{\phi_{F}\left(1-(1-\beta) \bar{a}^{a}\right)}{\phi_{H} \phi_{F}-\phi_{H} \phi_{F}(1-\beta) \bar{a}^{a}+\phi_{F} \phi_{F}+\phi_{F} \phi_{H}(1-\beta) \bar{a}^{a}}\right]^{\frac{1}{1-\eta}} \\
& =\left(\frac{\bar{a}^{a}}{1-(1-\beta) \bar{a}^{a}}\right)\left[\frac{\phi_{F}\left(1-(1-\beta) \bar{a}^{a}\right)}{\left(\phi_{H}+\phi_{F}\right) \phi_{F}}\right]^{\frac{1}{1-\eta}}=\bar{a}^{a}\left(1-(1-\beta) \bar{a}^{a}\right)^{\frac{\eta}{1-\eta}}
\end{aligned}
$$

In other words, there is a very straightforward mapping between the domestic real net foreign asset share on domestic absorption and on domestic output demand, $\bar{a}^{a}$ and $\bar{a}^{y}$ respectively.

Similarly, I can use the formula for steady state terms of trade $\left(\frac{\bar{P}^{F}}{\bar{P}^{H}}\right)$ in (173) to re-write the steady state expressions for $\overline{R S}, \frac{\bar{P}^{H}}{\bar{P}}$ and $\frac{\bar{P}^{F *}}{\bar{P}^{*}}$ obtained in (147), (148), (149) as follows,

$$
\begin{aligned}
\overline{R S} & =\frac{\overline{S P}^{*}}{\bar{P}}=\left[\frac{\phi_{F}+\phi_{H}\left(\frac{\bar{P}^{F}}{\bar{P}^{H}}\right)^{1-\eta}}{\phi_{H}+\phi_{F}\left(\frac{\bar{P}^{F}}{\bar{P}^{H}}\right)^{1-\eta}}\right]^{\frac{1}{1-\eta}}=\left[\frac{\phi_{F}+\phi_{H}\left(\frac{\phi_{F}+\phi_{H}(1-\beta) \bar{a}^{a}}{\phi_{F}-\phi_{F}(1-\beta) \bar{a}^{a}}\right)}{\phi_{H}+\phi_{F}\left(\frac{\phi_{F}+\phi_{H}(1-\beta) \bar{a}^{a}}{\phi_{F}-\phi_{F}(1-\beta) \bar{a}^{a}}\right)}\right]^{\frac{1}{1-\eta}} \\
& =\left[1+\left(\frac{\phi_{H} \phi_{H}-\phi_{F} \phi_{F}}{\phi_{F}}\right)(1-\beta) \bar{a}^{a}\right]^{\frac{1}{1-\eta}}, \\
\frac{\bar{P}^{H}}{\bar{P}} & =\left[\frac{1}{\phi_{H}+\phi_{F}\left(\frac{\bar{P}^{F}}{\bar{P}^{H}}\right)^{1-\eta}}\right]^{\frac{1}{1-\eta}}=\left[\frac{1}{\phi_{H}+\phi_{F}\left(\frac{\phi_{F}+\phi_{H}(1-\beta) \bar{a}^{a}}{\phi_{F}-\phi_{F}(1-\beta) \bar{a}^{a}}\right)}\right]^{\frac{1}{1-\eta}} \\
& =\left[1-(1-\beta) \bar{a}^{a}\right]^{\frac{1}{1-\eta}}, \\
\bar{P}^{F *} & =\left[\frac{\left(\frac{\bar{P}^{F}}{\bar{P}^{H}}\right)^{1-\eta}}{\bar{P}_{F}+\phi_{H}\left(\frac{\bar{P}^{F}}{\bar{P}^{H}}\right)^{1-\eta}}\right]^{\frac{1}{1-\eta}}=\left[\frac{\left(\frac{\phi_{F}+\phi_{H}(1-\beta) \bar{a}^{a}}{\phi_{F}-\phi_{F}(1-\beta) \bar{a}^{a}}\right)}{\phi_{F}+\phi_{H}\left(\frac{\phi_{F}+\phi_{H}(1-\beta) \bar{a}^{a}}{\phi_{F}-\phi_{F}(1-\beta) \bar{a}^{a}}\right)}\right]^{\frac{1}{1-\eta}} \\
& =\left[\frac{\phi_{F}+\phi_{H}(1-\beta) \bar{a}^{a}}{\phi_{F}+\left(\phi_{H} \phi_{H}-\phi_{F} \phi_{F}\right)(1-\beta) \bar{a}^{a}}\right]^{\frac{1}{1-\eta}} \cdot
\end{aligned}
$$

Interestingly, a non-zero domestic real net foreign asset position in steady state (i.e. $\bar{a}^{a} \neq 0$ ) does not imply that the real exchange rate must inevitably differ from one. In the special case where the consumption baskets are identical across countries (i.e. $\phi_{H}=\phi_{F}$ ), then I get that $\overline{R S}=1$ independently of $\bar{a}^{a}$. Therefore, if the consumption baskets are truly identical, then the assumption that absorption across countries must be related to the real exchange rate in steady state (i.e. $\left.(\bar{C}+\bar{X})=(\overline{R S})^{\eta}\left(\bar{C}^{*}+\bar{X}^{*}\right)\right)$ reduces to the conventional assumption that absorption equalizes across countries (i.e. $(\bar{C}+\bar{X})=\left(\bar{C}^{*}+\bar{X}^{*}\right)$ ). 
Sixth Step. I revisit the cross-country relationship of aggregate output and the investment shares. It now follows that a relationship can be derived between domestic and foreign output from the three relationships summarized before in (161) - (163), i.e.,

$\phi_{F}\left(\frac{\bar{P}^{F}}{\bar{P}}\right)^{1-\eta}\left(\left(\frac{\bar{P}^{H}}{\bar{P}}\right) \bar{Y}+(1-\beta) \bar{a}\right)=\phi_{F}(\overline{R S})^{\eta}\left(\frac{\bar{P}^{H}}{\bar{P}}\right)^{1-\eta}\left(\left(\frac{\bar{P}^{F *}}{\bar{P}^{*}}\right) \bar{Y}^{*}-(1-\beta)\left(\frac{1}{\overline{R S}}\right) \bar{a}\right)+(1-\beta) \bar{a}$.

After some more algebra, I get that,

$\phi_{F}\left(\frac{\bar{P}^{F}}{\bar{P}}\right)^{1-\eta}\left(\frac{\bar{P}^{H}}{\bar{P}}\right) \bar{Y}=\phi_{F}(\overline{R S})^{\eta}\left(\frac{\bar{P}^{H}}{\bar{P}}\right)^{1-\eta}\left(\frac{\bar{P}^{F *}}{\bar{P}^{*}}\right) \bar{Y}^{*}+\left[1-\phi_{F}\left(\frac{1}{\overline{R S}} \frac{\bar{P}^{H}}{\bar{P}}\right)^{1-\eta}-\phi_{F}\left(\frac{\bar{P}^{F}}{\bar{P}}\right)^{1-\eta}\right](1-\beta) \bar{a}$.

By the definition of the domestic consumption price index in (15) it must follow that $\phi_{F}\left(\frac{\bar{P}^{F}}{\bar{P}}\right)^{1-\eta}=$ $1-\phi_{H}\left(\frac{\bar{P}^{H}}{\bar{P}}\right)^{1-\eta}$, so I can write this relationship as,

$\phi_{F}\left(\frac{\bar{P}^{F}}{\bar{P}}\right)^{1-\eta}\left(\frac{\bar{P}^{H}}{\bar{P}}\right) \bar{Y}=\phi_{F}\left(\frac{1}{\overline{R S}} \frac{\bar{P}^{H}}{\bar{P}}\right)^{1-\eta}\left(\overline{R S} \frac{\bar{P}^{F *}}{\bar{P}^{*}}\right) \bar{Y}^{*}+\left[\phi_{H}\left(\frac{\bar{P}^{H}}{\bar{P}}\right)^{1-\eta}-\phi_{F}\left(\frac{1}{\overline{R S}} \frac{\bar{P}^{H}}{\bar{P}}\right)^{1-\eta}\right](1-\beta) \bar{a}$,

and given the definition of the real exchange rate in (21) (i.e. $\overline{R S} \equiv \frac{\overline{S P}^{*}}{\bar{P}}$ ) and the law of one price (specifically, $\left.\bar{P}^{F}=\overline{S P}^{F *}\right)$, it must follow that,

$\phi_{F}\left(\frac{\bar{P}^{F}}{\bar{P}}\right)^{1-\eta}\left(\frac{\bar{P}^{H}}{\bar{P}}\right) \bar{Y}=\phi_{F}\left(\frac{1}{\overline{R S}} \frac{\bar{P}^{H}}{\bar{P}}\right)^{1-\eta}\left(\frac{\bar{P}^{F}}{\bar{P}}\right) \bar{Y}^{*}+\left[\phi_{H}\left(\frac{\bar{P}^{H}}{\bar{P}}\right)^{1-\eta}-\phi_{F}\left(\frac{1}{\overline{R S}} \frac{\bar{P}^{H}}{\bar{P}}\right)^{1-\eta}\right](1-\beta) \bar{a}$.

Then, after some more algebra it must follow that,

$$
\left(\frac{\bar{P}^{H}}{\bar{P}}\right) \bar{Y}=\left(\frac{1}{\overline{R S}} \frac{\bar{P}^{H}}{\bar{P}^{F}}\right)^{1-\eta}\left[\left(\frac{\bar{P}^{F}}{\bar{P}}\right) \bar{Y}^{*}+\left(\frac{\phi_{H}(\overline{R S})^{1-\eta}-\phi_{F}}{\phi_{F}}\right)(1-\beta) \bar{a}\right] .
$$

Equation (179) implies that in steady state the output of both countries would satisfy that $\bar{P}^{H} \bar{Y}=$ $\left(\frac{1}{\overline{R S}} \frac{\bar{P}^{H}}{\bar{P}^{F}}\right)^{1-\eta} \bar{P}^{F} \bar{Y}^{*}$ only if the real net foreign asset position of the domestic household is zero (i.e. $\bar{a}=0$ ) or in the knife-edge case when $\overline{R S}=\left(\frac{\phi_{F}}{\phi_{H}}\right)^{\frac{1}{1-\eta}}$ (which also implies that $\overline{R S}=1$ if $\phi_{H}=\phi_{F}=\frac{1}{2}$ ). Furthermore, the output of both countries equalizes in steady state if: (a) the real net foreign asset position $\bar{a}$ is zero (or, alternatively, if the consumption baskets are equal across countries because $\phi_{H}=\phi_{F}=\frac{1}{2}$ ), and (b) prices equalize (i.e. $\bar{P}=\bar{P}^{H}=\bar{P}^{F}$ and $\bar{P}^{*}=\bar{P}^{H *}=\bar{P}^{F *}$ ). In that case, moreover, the consumption and investment shares equalize across countries as well and that must imply that aggregate consumption and investment must be the same in both countries (i.e. $\bar{C}=\bar{C}^{*}$ and $\bar{X}=\bar{X}^{*}$ ). 
Since $\frac{\bar{P}^{F}}{\bar{P}}=\overline{R S}\left(\frac{\bar{P}^{F *}}{\bar{P}^{*}}\right)$, I can also infer from (179) that,

$$
\left(\frac{\bar{P}^{H}}{\bar{P}}\right) \bar{Y}=\left(\frac{1}{\overline{R S}} \frac{\bar{P}^{H}}{\bar{P}^{F}}\right)^{1-\eta}\left[\left(\overline{R S}\left(\frac{\bar{P}^{F *}}{\bar{P}^{*}}\right)\right) \bar{Y}^{*}+\left(\frac{\phi_{H}(\overline{R S})^{1-\eta}-\phi_{F}}{\phi_{F}}\right)(1-\beta) \bar{a}\right]
$$

where I already know from $(147),(148)$, and (149) that $\overline{R S}, \frac{\bar{P}^{H}}{\bar{P}}$, and $\frac{\bar{P}^{F *}}{\bar{P}^{*}}$ are all functions of the steady state terms of trade $\left(\frac{\bar{P}^{F}}{\bar{P}^{H}}\right)$ and from $(175),(176)$, and $(177)$ that $\overline{R S}, \frac{\bar{P}^{H}}{\bar{P}}$, and $\frac{\bar{P}^{F *}}{\bar{P}^{*}}$ are all functions of $\bar{a}^{a}$. Furthermore, I can express the real net foreign asset position of the domestic household in terms of the domestic output as in (166), so the equation above can be re-expressed as,

$$
\left[\left(\frac{\bar{P}^{H}}{\bar{P}}\right)-\left(\frac{1}{\overline{R S}} \frac{\bar{P}^{H}}{\bar{P}^{F}}\right)^{1-\eta}\left(\frac{\phi_{H}(\overline{R S})^{1-\eta}-\phi_{F}}{\phi_{F}}\right)(1-\beta) \bar{a}^{y}\right] \bar{Y}=\left[( \frac { 1 } { \overline { R S } } \frac { \overline { P } ^ { H } } { \overline { P } ^ { F } } ) ^ { 1 - \eta } \left(\overline{R S}^{\left.\left.\left(\frac{P^{F *}}{\bar{P}^{*}}\right)\right)\right]} \bar{Y}^{*}\right.\right.
$$

I know that $\bar{a}^{y}$ is a function of $\bar{a}^{a}$, the intertemporal discount factor $\beta$ and the elasticity of intratemporal substitution between the home and foreign bundles of varieties $\eta$ by (174). Therefore, for any given value of $\bar{a}^{a}$ I can use equation (181) to determine how different the steady state aggregate output is going to be across these two countries as follows,

$$
\begin{aligned}
& \left(1-(1-\beta) \bar{a}^{a}\right)^{\frac{1}{1-\eta}}\left[1-\left(\frac{1}{(\overline{R S})^{1-\eta}}\right)\left(\frac{\phi_{H}(\overline{R S})^{1-\eta}-\phi_{F}}{\phi_{F}+\phi_{H}(1-\beta) \bar{a}^{a}}\right)(1-\beta) \bar{a}^{a}\right] \bar{Y} \\
& \quad=\left[\left(\frac{1}{(\overline{R S})^{1-\eta}}\right)\left(\frac{\phi_{F}-\phi_{F}(1-\beta) \bar{a}^{a}}{\phi_{F}+\phi_{H}(1-\beta) \bar{a}^{a}}\right)(\overline{R S})\left(\frac{\phi_{F}+\phi_{H}(1-\beta) \bar{a}^{a}}{\phi_{F}+\left(\phi_{H} \phi_{H}-\phi_{F} \phi_{F}\right)(1-\beta) \bar{a}^{a}}\right)^{\frac{1}{1-\eta}}\right] \bar{Y}^{*},
\end{aligned}
$$

and,

$$
\bar{Y}=\left[\frac{\left(\frac{\phi_{F}-\phi_{F}(1-\beta) \bar{a}^{a}}{\phi_{F}+\left(\phi_{H} \phi_{H}-\phi_{F} \phi_{F}\right)(1-\beta) \bar{a}^{a}}\right)\left(\frac{\phi_{F}+\phi_{H}(1-\beta) \bar{a}^{a}}{\phi_{F}}\right)^{\frac{\eta}{1-\eta}}\left(1-(1-\beta) \bar{a}^{a}\right)^{-\frac{1}{1-\eta}}}{1-\left(\frac{1}{\phi_{F}+\left(\phi_{H} \phi_{H}-\phi_{F} \phi_{F}\right)(1-\beta) \bar{a}^{a}}\right)\left(\frac{\phi_{F}\left(\phi_{H}-\phi_{F}\right)+\phi_{H}\left(\phi_{H} \phi_{H}-\phi_{F} \phi_{F}\right)(1-\beta) \bar{a}^{a}}{\phi_{F}+\phi_{H}(1-\beta) \bar{a}^{a}}\right)(1-\beta) \bar{a}^{a}}\right] \bar{Y}^{*},
$$

which expresses (181) in terms of structural parameters only.

I define the domestic and foreign steady state investment shares relative to aggregate output (i.e. $\gamma_{x^{*}}^{y}$ in (142) and $\gamma_{x^{*}}^{y}$ in (143)), while the investment and consumption shares relative to absorption can be defined 
as,

$$
\begin{aligned}
& \gamma_{x}^{a} \equiv \frac{\bar{X}}{\bar{C}+\bar{X}}=\frac{\bar{X}}{\bar{Y}} \frac{\bar{Y}}{\bar{C}+\bar{X}}=\delta\left[\frac{1-\psi}{\left(\frac{\theta(1-\bar{\xi})}{\theta-1}\right)\left(\beta^{-1}-(1-\delta)\right)}\right]\left(\frac{\bar{P}^{H}}{\bar{P}}\right)\left(\frac{\bar{Y}}{\bar{C}+\bar{X}}\right) \\
& =\delta\left[\frac{1-\psi}{\left(\frac{\theta(1-\bar{\xi})}{\theta-1}\right)\left(\beta^{-1}-(1-\delta)\right)}\right]\left[\frac{(\bar{C}+\bar{X})-(1-\beta) \bar{a}}{\bar{C}+\bar{X}}\right] \text {, } \\
& \gamma_{c}^{a} \equiv \frac{\bar{C}}{\bar{C}+\bar{X}}=1-\gamma_{x}^{a} \text {, } \\
& \gamma_{x^{*}}^{a} \equiv \frac{\bar{X}^{*}}{\bar{C}^{*}+\bar{X}^{*}}=\frac{\bar{X}^{*}}{\bar{Y}^{*}} \frac{\bar{Y}^{*}}{\bar{C}^{*}+\bar{X}^{*}}=\delta\left[\frac{1-\psi}{\left(\frac{\theta\left(1-\bar{\xi}^{*}\right)}{\theta-1}\right)\left(\beta^{-1}-(1-\delta)\right)}\right]\left(\frac{\bar{P}^{F *}}{\bar{P}^{*}}\right)\left(\frac{\bar{Y}^{*}}{\bar{C}^{*}+\bar{X}^{*}}\right) \\
& =\delta\left[\frac{1-\psi}{\left(\frac{\theta\left(1-\bar{\xi}^{*}\right)}{\theta-1}\right)\left(\beta^{-1}-(1-\delta)\right)}\right]\left[\frac{\left(\bar{C}^{*}+\bar{X}^{*}\right)+(1-\beta)\left(\frac{1}{\overline{R S}}\right) \bar{a}}{\bar{C}^{*}+\bar{X}^{*}}\right], \\
& \gamma_{c^{*}}^{a} \equiv \frac{\bar{C}^{*}}{\bar{C}^{*}+\bar{X}^{*}}=1-\gamma_{x^{*}}^{a},
\end{aligned}
$$

after having used the aggregate output equations derived in (161) and (162). From here, using the definition of the real net foreign asset position of the domestic household as a share of domestic absorption $\bar{a}^{a}$ found in (165) and the assumption that in steady state it must hold that $(\bar{C}+\bar{X})=(\overline{R S})^{\eta}\left(\bar{C}^{*}+\bar{X}^{*}\right)$, I can argue that the consumption and investment shares relative to absorption must be equal to,

$$
\begin{aligned}
\gamma_{x}^{a} & \equiv \frac{\bar{X}}{\bar{C}+\bar{X}}=\delta\left[\frac{1-\psi}{\left(\frac{\theta(1-\bar{\xi})}{\theta-1}\right)\left(\beta^{-1}-(1-\delta)\right)}\right]\left(1-(1-\beta) \bar{a}^{a}\right), \\
\gamma_{c}^{a} & \equiv \frac{\bar{C}}{\bar{C}+\bar{X}}=1-\gamma_{x}^{a}, \\
\gamma_{x^{*}}^{a} \equiv & \frac{\bar{X}^{*}}{\bar{C}^{*}+\bar{X}^{*}}=\delta\left[\frac{1-\psi}{\left(\frac{\theta\left(1-\bar{\xi}^{*}\right)}{\theta-1}\right)\left(\beta^{-1}-(1-\delta)\right)}\right]\left(1+\left(\frac{1}{\overline{R S}}\right)^{1-\eta}(1-\beta) \bar{a}^{a}\right) \\
& =\delta\left[\frac{1-\psi}{\left(\frac{\theta\left(1-\bar{\xi}^{*}\right)}{\theta-1}\right)\left(\beta^{-1}-(1-\delta)\right)}\right]\left(\frac{\phi_{F}+\phi_{H}(1-\beta) \bar{a}^{a}}{\phi_{F}+\left(\phi_{H} \phi_{H}-\phi_{F} \phi_{F}\right)(1-\beta) \bar{a}^{a}}\right), \\
\gamma_{c^{*}}^{a} & \equiv \frac{\bar{C}^{*}}{\bar{C}^{*}+\bar{X}^{*}}=1-\gamma_{x^{*}}^{a} .
\end{aligned}
$$

Let me denote the consumption and investment shares in the special case with a zero net foreign asset 
position, i.e. with $\bar{a}^{a}=0$, as follows,

$$
\begin{aligned}
& \gamma_{x}=\delta\left[\frac{1-\psi}{\left(\frac{\theta(1-\bar{\xi})}{\theta-1}\right)\left(\beta^{-1}-(1-\delta)\right)}\right], \gamma_{c}=1-\gamma_{x}, \\
& \gamma_{x^{*}}=\delta\left[\frac{1-\psi}{\left(\frac{\theta\left(1-\bar{\xi}^{*}\right)}{\theta-1}\right)\left(\beta^{-1}-(1-\delta)\right)}\right], \gamma_{c^{*}}=1-\gamma_{x^{*}} .
\end{aligned}
$$

Then, when the steady state real net foreign asset position is zero and the steady state subsidies are equal across countries, the steady state consumption and investment shares in (187) - (189) reduce to the conventional case where the absorption shares are equal across countries and equal to the shares expressed relative to output, i.e. if $\bar{a}^{a}=0$ and $\bar{\xi}=\bar{\xi}^{*}$ then $\gamma_{x}^{a}=\gamma_{x^{*}}^{a}=\gamma_{x}^{y}=\gamma_{x^{*}}^{y}=\gamma_{x}=\gamma_{x^{*}}$ and $\gamma_{c}^{a}=\gamma_{c^{*}}^{a}=\gamma_{c}^{y}=\gamma_{c^{*}}^{y}=\gamma_{c}=\gamma_{c^{*}}$.

Seventh Step. I look at the pricing equations from the firms' optimization problem. I can write the monopolistic competition price-setting rule for the domestic firms with their marginal cost function combining (26) and (74) and the monopolistic competition price-setting rule for the foreign firms with their marginal cost function combining (27) and (75) as in (136) and (137), i.e.,

$$
\begin{aligned}
\frac{\bar{P}^{H}}{\bar{P}} & =\frac{\theta(1-\bar{\xi})}{\theta-1} \frac{1}{\bar{A}} \frac{1}{\psi^{\psi}(1-\psi)^{1-\psi}}\left(\frac{\bar{W}}{\bar{P}}\right)^{\psi}\left(\frac{\bar{Z}}{\bar{P}}\right)^{1-\psi} \\
& =\frac{\theta(1-\bar{\xi})}{\theta-1} \frac{1}{\bar{A}} \frac{1}{\psi^{\psi}(1-\psi)^{1-\psi}}\left(\frac{\bar{W}}{\bar{P}}\right)^{\psi}\left(\beta^{-1}-(1-\delta)\right)^{1-\psi} \\
\frac{\bar{P}^{F *}}{\bar{P}^{*}} & =\frac{\theta\left(1-\bar{\xi}^{*}\right)}{\theta-1} \overline{\bar{A}}^{*} \frac{1}{\psi^{\psi}(1-\psi)^{1-\psi}}\left(\frac{\bar{W}^{*}}{\bar{P}^{*}}\right)^{\psi}\left(\frac{\bar{Z}^{*}}{\bar{P}^{*}}\right)^{1-\psi} \\
& =\frac{\theta\left(1-\bar{\xi}^{*}\right)}{\theta-1} \frac{1}{\bar{A}^{*}} \frac{1}{\psi^{\psi}(1-\psi)^{1-\psi}}\left(\frac{\bar{W}^{*}}{\bar{P}^{*}}\right)^{\psi}\left(\beta^{-1}-(1-\delta)\right)^{1-\psi},
\end{aligned}
$$

where I have divided each equation by the consumption price level of its respective country and I have appropriately replaced the real rental rate on capital derived before in (124). The prices of all domestic varieties are equalized, and so are the prices of all foreign varieties.

From the labor supply equations in (54) and (55), I obtain that,

$$
\begin{aligned}
\frac{\bar{W}}{\bar{P}} & =(\bar{C})^{\sigma^{-1}}\left(\bar{L}^{s}\right)^{\varphi}, \\
\frac{\bar{W}^{*}}{\bar{P}^{*}} & =\left(\bar{C}^{*}\right)^{\sigma^{-1}}\left(\bar{L}^{s *}\right)^{\varphi} .
\end{aligned}
$$

Given the firm production functions in (22) and (23) and the fact that capital-to-labor ratios are equalized across firms within a country, it is possible to write the following steady state domestic and foreign aggregate 
output demand equations from (84) and (85), ${ }^{7}$ i.e.,

$$
\begin{aligned}
\bar{Y} & =\bar{A}(\bar{K})^{1-\psi}(\bar{L})^{\psi}, \\
\bar{Y}^{*} & =\bar{A}^{*}\left(\bar{K}^{*}\right)^{1-\psi}\left(\bar{L}^{*}\right)^{\psi},
\end{aligned}
$$

and, most importantly, I can establish that,

$$
\begin{aligned}
& 1=\bar{A}\left(\frac{\bar{K}}{\bar{Y}}\right)^{1-\psi}\left(\frac{\bar{L}}{\bar{Y}}\right)^{\psi}=\bar{A}\left(\frac{\gamma_{x}^{y}}{\delta}\right)^{1-\psi}\left(\frac{\bar{L}}{\bar{Y}}\right)^{\psi}, \\
& 1=\bar{A}^{*}\left(\frac{\bar{K}^{*}}{\bar{Y}^{*}}\right)^{1-\psi}\left(\frac{\bar{L}^{*}}{\bar{Y}^{*}}\right)^{\psi}=\bar{A}^{*}\left(\frac{\gamma_{x^{*}}^{y}}{\delta}\right)^{1-\psi}\left(\frac{\bar{L}^{*}}{\bar{Y}^{*}}\right)^{\psi},
\end{aligned}
$$

where the second equality uses the fact that the capital-to-output ratio is proportional to the investment share over output in each country as noted in (132) and (133), i.e. $\gamma_{x}^{y} \equiv \delta \frac{\bar{K}}{\bar{Y}}$ and $\gamma_{x^{*}}^{y} \equiv \delta \frac{\bar{K}^{*}}{\bar{Y}^{*}}$.

Using the labor market clearing conditions, i.e. $\bar{L}^{s}=\bar{L}$ and $\bar{L}^{s *}=\bar{L}^{*}$, I can express the domestic and foreign labor supply equations as,

$$
\begin{aligned}
\overline{\bar{P}} & =(\bar{C})^{\sigma^{-1}}(\bar{Y})^{\varphi}\left(\frac{\bar{L}}{\bar{Y}}\right)^{\varphi}=(\bar{C})^{\sigma^{-1}}(\bar{Y})^{\varphi}\left[\frac{1}{\bar{A}}\left(\frac{\gamma_{x}^{y}}{\delta}\right)^{\psi-1}\right]^{\frac{\varphi}{\psi}}, \\
\frac{\bar{W}^{*}}{\bar{P}^{*}} & =\left(\bar{C}^{*}\right)^{\sigma^{-1}}\left(\bar{Y}^{*}\right)^{\varphi}\left(\frac{\bar{L}^{*}}{\bar{Y}^{*}}\right)^{\varphi}=\left(\bar{C}^{*}\right)^{\sigma^{-1}}\left(\bar{Y}^{*}\right)^{\varphi}\left[\overline{\bar{A}}^{*}\left(\frac{\gamma_{x^{*}}^{y}}{\delta}\right)^{\psi-1}\right]^{\frac{\varphi}{\psi}},
\end{aligned}
$$

and so the domestic and foreign price-setting rules in (193) and (194) become simply,

$$
\begin{aligned}
\frac{\bar{P}^{H}}{\bar{P}} & =\frac{\theta(1-\bar{\xi})}{\theta-1} \frac{1}{\psi^{\psi}(1-\psi)^{1-\psi}}\left[\frac{1}{\bar{A}}\right]^{1+\varphi}\left(\frac{\gamma_{x}^{y}}{\delta}\right)^{(\psi-1) \varphi}\left(\beta^{-1}-(1-\delta)\right)^{1-\psi}\left((\bar{C})^{\sigma^{-1}}(\bar{Y})^{\varphi}\right)^{\psi}, \\
\frac{\bar{P}^{F *}}{\bar{P}^{*}} & =\frac{\theta\left(1-\bar{\xi}^{*}\right)}{\theta-1} \frac{1}{\psi^{\psi}(1-\psi)^{1-\psi}}\left[\frac{1}{\bar{A}^{*}}\right]^{1+\varphi}\left(\frac{\gamma_{x^{*}}^{y}}{\delta}\right)^{(\psi-1) \varphi}\left(\beta^{-1}-(1-\delta)\right)^{1-\psi}\left(\left(\bar{C}^{*}\right)^{\sigma^{-1}}\left(\bar{Y}^{*}\right)^{\varphi}\right)^{\psi}(204)
\end{aligned}
$$

Then, appropriately replacing the investment shares $\gamma_{x}^{y}$ and $\gamma_{x^{*}}^{y}$ derived in (142) and (143) it is possible to

\footnotetext{
${ }^{7}$ Given that the prices of all varieties equalize within a country in steady state, then the wedges in (98) - (99) must be equal to one in steady state, i.e. $\bar{\Xi}^{H}=\bar{\Xi}^{F *}=1$. Therefore, the supply of the aggregate output bundle (of varieties) in each country obtained in (96) - (97) must be equivalent to the aggregation of each individual variety in (84) - (85). Then, it must be the case that $\bar{Y}=\bar{Y}^{H}$ and $\bar{Y}^{*}=\bar{Y}^{F *}$ and that there is no output loss due to price dispersion. However, the steady state can still be distorted by the mark-up charged by each firm operating under monopolistic competition unless the subsidy fully eliminates this distortion as it would happen in (44).
} 
obtain the following general expression for the price ratios $\frac{\bar{P}^{H}}{\bar{P}}$ and $\frac{\bar{P}^{F *}}{\bar{P}^{*}}$, i.e.,

$$
\begin{aligned}
& \frac{\theta(1-\bar{\xi})}{\theta-1} \frac{1}{\psi^{\psi}(1-\psi)^{1-\psi}}\left(\left(\frac{1-\psi}{\left(\frac{\theta(1-\bar{\xi})}{\theta-1}\right)\left(\beta^{-1}-(1-\delta)\right)}\right)\left(\frac{\bar{P}^{H}}{\bar{P}}\right)\right)^{(\psi-1) \varphi}\left(\beta^{-1}-(1-\delta)\right)^{1-\psi}\left((\bar{C})^{\sigma^{-1}}(\bar{Y})^{\varphi}\right)^{\psi} \\
& \quad=[\bar{A}]^{1+\varphi} \frac{\bar{P}^{H}}{\bar{P}} \\
& \frac{\theta\left(1-\bar{\xi}^{*}\right)}{\theta-1} \frac{1}{\psi^{\psi}(1-\psi)^{1-\psi}}\left(\left(\frac{1-\psi}{\left(\frac{\theta\left(1-\bar{\xi}^{*}\right)}{\theta-1}\right)\left(\beta^{-1}-(1-\delta)\right)}\right)\left(\frac{\bar{P}^{F *}}{\bar{P}^{*}}\right)\right)^{(\psi-1) \varphi}\left(\beta^{-1}-(1-\delta)\right)^{1-\psi}\left(\left(\bar{C}^{*}\right)^{\sigma^{-1}}\left(\bar{Y}^{*}\right)^{\varphi}\right)^{\psi} \\
& =\left[\bar{A}^{*}\right]^{1+\varphi} \frac{\bar{P}^{F *}}{\bar{P}^{*}}
\end{aligned}
$$

or simply,

$$
\begin{aligned}
& \frac{\theta(1-\bar{\xi})}{\theta-1} \frac{1}{\psi^{\psi}(1-\psi)^{1-\psi}}\left(\frac{1-\psi}{\left(\frac{\theta(1-\bar{\xi})}{\theta-1}\right)\left(\beta^{-1}-(1-\delta)\right)}\right){ }^{(\psi-1) \varphi}\left(\beta^{-1}-(1-\delta)\right)^{1-\psi}\left((\bar{C})^{\sigma^{-1}}(\bar{Y})^{\varphi}\right)^{\psi} \\
& \quad=[\bar{A}]^{1+\varphi}\left(\frac{\bar{P}^{H}}{\bar{P}}\right)^{1+(1-\psi) \varphi}, \\
& \frac{\theta\left(1-\bar{\xi}^{*}\right)}{\theta-1} \frac{1}{\psi^{\psi}(1-\psi)^{1-\psi}}\left(\frac{1-\psi}{\left(\frac{\theta\left(1-\bar{\xi}^{*}\right)}{\theta-1}\right)\left(\beta^{-1}-(1-\delta)\right)}\right)^{(\psi-1) \varphi}\left(\beta^{-1}-(1-\delta)\right)^{1-\psi}\left(\left(\bar{C}^{*}\right)^{\sigma^{-1}}\left(\bar{Y}^{*}\right)^{\varphi}\right)^{\psi} \\
& =\left[\bar{A}^{*}\right]^{1+\varphi}\left(\frac{\bar{P}^{F *}}{\bar{P}^{*}}\right)^{1+(1-\psi) \varphi},
\end{aligned}
$$

where I explicitly maintain that the steady state productivity levels are not normalized to one, i.e. $\bar{A}$ and $\bar{A}^{*}$ may differ from one. Hence, after a little bit of additional algebra, it must follow that,

$$
\begin{aligned}
& {\left[\frac{\left(\frac{\theta(1-\bar{\xi})}{\theta-1}\right)\left(\beta^{-1}-(1-\delta)\right)^{1-\psi}}{\psi^{\psi}(1-\psi)^{1-\psi}}\left(\frac{\left(\frac{\theta(1-\bar{\xi})}{\theta-1}\right)\left(\beta^{-1}-(1-\delta)\right)}{1-\psi}\right)^{(1-\psi) \varphi}\right]^{\frac{1}{1+(1-\psi) \varphi}}\left((\bar{C})^{\sigma^{-1}}(\bar{Y})^{\varphi}\right)^{\frac{\psi}{1+(1-\psi) \varphi}}} \\
& =[\bar{A}]^{\frac{1+\varphi}{1+(1-\psi) \varphi}}\left(\frac{\bar{P}^{H}}{\bar{P}}\right), \\
& {\left[\frac{\left(\frac{\theta\left(1-\bar{\xi}^{*}\right)}{\theta-1}\right)\left(\beta^{-1}-(1-\delta)\right)^{1-\psi}}{\psi^{\psi}(1-\psi)^{1-\psi}}\left(\frac{\left(\frac{\theta\left(1-\bar{\xi}^{*}\right)}{\theta-1}\right)\left(\beta^{-1}-(1-\delta)\right)}{1-\psi}\right)^{(1-\psi) \varphi}\right]^{\frac{1}{1+(1-\psi) \varphi}}\left(\left(\bar{C}^{*}\right)^{\sigma^{-1}}\left(\bar{Y}^{*}\right)^{\varphi}\right)^{\frac{\psi}{1+(1-\psi) \varphi}}} \\
& =\left[\bar{A}^{*}\right]^{\frac{1+\varphi}{1+(1-\psi) \varphi}}\left(\frac{\bar{P}^{F *}}{\bar{P}^{*}}\right),
\end{aligned}
$$


and,

$$
\begin{aligned}
& {\left[\frac{\left(\frac{\theta(1-\bar{\xi})}{\theta-1}\right)^{1+(1-\psi) \varphi}\left(\beta^{-1}-(1-\delta)\right)^{(1-\psi)(1+\varphi)}}{\psi^{\psi}(1-\psi)^{(1-\psi)(1+\varphi)}}\right]^{\frac{1}{1+(1-\psi) \varphi}}(\bar{C})^{\frac{\psi}{1+(1-\psi) \varphi} \sigma^{-1}}(\bar{Y})^{\frac{\psi}{1+(1-\psi) \varphi} \varphi}} \\
& =[A]^{\frac{1+\varphi}{1+(1-\psi) \varphi}}\left(\frac{\bar{P}^{H}}{\bar{P}}\right), \\
& {\left[\frac{\left(\frac{\theta\left(1-\bar{\xi}^{*}\right)}{\theta-1}\right)^{1+(1-\psi) \varphi}\left(\beta^{-1}-(1-\delta)\right)^{(1-\psi)(1+\varphi)}}{\psi^{\psi}(1-\psi)^{(1-\psi)(1+\varphi)}}\right]^{\frac{1}{1+(1-\psi) \varphi}}\left(\bar{C}^{*}\right)^{\frac{\psi}{1+(1-\psi) \varphi} \sigma^{-1}}\left(\bar{Y}^{*}\right)^{\frac{\psi}{1+(1-\psi) \varphi} \varphi}} \\
& =\left[\bar{A}^{*}\right]^{\frac{1+\varphi}{1+(1-\psi) \varphi}}\left(\frac{\bar{P}^{F *}}{\bar{P}^{*}}\right),
\end{aligned}
$$

and,

$$
\begin{aligned}
& \left(\frac{\theta(1-\bar{\xi})}{\theta-1}\right)\left[\frac{\left(\beta^{-1}-(1-\delta)\right)^{(1-\psi)(1+\varphi)}}{\psi^{\psi}(1-\psi)^{(1-\psi)(1+\varphi)}}\right]^{\frac{1}{1+(1-\psi) \varphi}}(\bar{C})^{\frac{\psi}{1+(1-\psi) \varphi} \sigma^{-1}}(\bar{Y})^{\frac{\psi}{1+(1-\psi) \varphi} \varphi} \\
& \quad=[\bar{A}]^{\frac{1+\varphi}{1+(1-\psi) \varphi}}\left(\frac{\bar{P}^{H}}{\bar{P}}\right) \\
& \left(\frac{\theta\left(1-\bar{\xi}^{*}\right)}{\theta-1}\right)\left[\frac{\left(\beta^{-1}-(1-\delta)\right)^{(1-\psi)(1+\varphi)}}{\psi^{\psi}(1-\psi)^{(1-\psi)(1+\varphi)}}\right]^{\frac{1}{1+(1-\psi) \varphi}}\left(\bar{C}^{*}\right)^{\frac{\psi}{1+(1-\psi) \varphi} \sigma^{-1}}\left(\bar{Y}^{*}\right)^{\frac{\psi}{1+(1-\psi) \varphi} \varphi} \\
& =\left[\bar{A}^{*}\right]^{\frac{1+\varphi}{1+(1-\psi) \varphi}}\left(\frac{\bar{P}^{F *}}{\bar{P}^{*}}\right) .
\end{aligned}
$$

Expressions (207) and (208) tie the relative price ratios $\left(\frac{\bar{P}^{H}}{\bar{P}}\right)$ and $\left(\frac{\bar{P}^{F *}}{\bar{P}^{*}}\right)$ to aggregate consumption and output in both countries as well as to the steady state levels of productivity at home and abroad.

I know from my earlier derivations in (161) and (162) that the domestic and foreign aggregate output demands in (92) - (93) satisfy that,

$$
\begin{aligned}
\bar{Y} & =\left(\frac{\bar{P}}{\bar{P}^{H}}\right)[(\bar{C}+\bar{X})-(1-\beta) \bar{a}], \\
\bar{Y}^{*} & =\left(\frac{\bar{P}^{*}}{\bar{P}^{F *}}\right)\left[\left(\bar{C}^{*}+\bar{X}^{*}\right)+(1-\beta)\left(\frac{1}{\overline{R S}}\right) \bar{a}\right] .
\end{aligned}
$$

Hence, I can re-write the expressions above in (207) and (208) implied by the price-setting rules of the firms in the following terms,

$$
\begin{aligned}
& \left(\frac{\theta(1-\bar{\xi})}{\theta-1}\right)\left[\frac{\left(\beta^{-1}-(1-\delta)\right)^{(1-\psi)(1+\varphi)}}{\psi^{\psi}(1-\psi)^{(1-\psi)(1+\varphi)}}\right]^{\frac{1}{1+(1-\psi) \varphi}}(\bar{C})^{\frac{\psi}{1+(1-\psi) \varphi} \sigma^{-1}}\left(\left(\frac{\bar{P}}{\bar{P}^{H}}\right)((\bar{C}+\bar{X})-(1-\beta) \bar{a})\right)^{\frac{\psi}{1+(1-\psi) \varphi} \varphi} \\
& \quad=[\bar{A}]^{\frac{1+\varphi}{1+(1-\psi) \varphi}}\left(\frac{\bar{P}^{H}}{\bar{P}}\right) \\
& \left(\frac{\theta\left(1-\bar{\xi}^{*}\right)}{\theta-1}\right)\left[\frac{\left(\beta^{-1}-(1-\delta)\right)^{(1-\psi)(1+\varphi)}}{\psi^{\psi}(1-\psi)^{(1-\psi)(1+\varphi)}}\right]^{\frac{1}{1+(1-\psi) \varphi}}\left(\bar{C}^{*}\right)^{\frac{\psi}{1+(1-\psi) \varphi} \sigma^{-1}}\left(\left(\frac{\bar{P}^{*}}{\bar{P}^{F *}}\right)\left(\left(\bar{C}^{*}+\bar{X}^{*}\right)+(1-\beta)\left(\frac{1}{\overline{R S}}\right) \bar{a}\right)\right)^{\frac{\psi}{1+(1-\psi) \varphi} \varphi} \\
& \quad=\left[\bar{A}^{*}\right]^{\frac{1+\varphi}{1+(1-\psi) \varphi}}\left(\frac{\bar{P}^{F *}}{\bar{P}^{*}}\right),
\end{aligned}
$$


and,

$$
\begin{aligned}
& \left(\frac{\theta(1-\bar{\xi})}{\theta-1}\right)\left[\frac{\left(\beta^{-1}-(1-\delta)\right)^{(1-\psi)(1+\varphi)}}{\psi^{\psi}(1-\psi)^{(1-\psi)(1+\varphi)}}\right]^{\frac{1}{1+(1-\psi) \varphi}}(\bar{C})^{\frac{\psi}{1+(1-\psi) \varphi} \sigma^{-1}}((\bar{C}+\bar{X})-(1-\beta) \bar{a})^{\frac{\psi}{1+(1-\psi) \varphi} \varphi} \\
& =[\bar{A}]^{\frac{1+\varphi}{1+(1-\psi) \varphi}}\left(\frac{\bar{P}^{H}}{\bar{P}}\right)^{\frac{1+\varphi}{1+(1-\psi) \varphi}}, \\
& \left(\frac{\theta\left(1-\bar{\xi}^{*}\right)}{\theta-1}\right)\left[\frac{\left(\beta^{-1}-(1-\delta)\right)^{(1-\psi)(1+\varphi)}}{\psi^{\psi}(1-\psi)^{(1-\psi)(1+\varphi)}}\right]^{\frac{1}{1+(1-\psi) \varphi}}\left(\bar{C}^{*}\right)^{\frac{\psi}{1+(1-\psi) \varphi} \sigma^{-1}}\left(\left(\bar{C}^{*}+\bar{X}^{*}\right)+(1-\beta)\left(\frac{1}{\overline{R S}}\right) \bar{a}\right)^{\frac{\psi}{1+(1-\psi) \varphi} \varphi} \\
& =\left[\bar{A}^{*}\right]^{\frac{1+\varphi}{1+(1-\psi) \varphi}}\left(\frac{\bar{P}^{F *}}{\bar{P}^{*}}\right)^{\frac{1+\varphi}{1+(1-\psi) \varphi}},
\end{aligned}
$$

and,

$$
\begin{aligned}
& \left(\frac{\theta(1-\bar{\xi})}{\theta-1}\right)^{\frac{1+(1-\psi) \varphi}{1+\varphi}}\left(\frac{\left(\beta^{-1}-(1-\delta)\right)^{1-\psi}}{\psi^{\frac{\psi}{1+\varphi}}(1-\psi)^{1-\psi}}\right)(\bar{C})^{\frac{\psi \sigma^{-1}}{1+\varphi}}((\bar{C}+\bar{X})-(1-\beta) \bar{a})^{\frac{\psi \varphi}{1+\varphi}} \\
& \quad=\bar{A}\left(\frac{\bar{P}^{H}}{\bar{P}}\right), \\
& \left(\frac{\theta\left(1-\bar{\xi}^{*}\right)}{\theta-1}\right)^{\frac{1+(1-\psi) \varphi}{1+\varphi}}\left(\frac{\left(\beta^{-1}-(1-\delta)\right)^{1-\psi}}{\psi^{\frac{\psi}{1+\varphi}}(1-\psi)^{1-\psi}}\right)\left(\bar{C}^{*}\right)^{\frac{\psi \sigma^{-1}}{1+\varphi}}\left(\left(\bar{C}^{*}+\bar{X}^{*}\right)+(1-\beta)\left(\frac{1}{\overline{R S}}\right) \bar{a}\right)^{\frac{\psi \varphi}{1+\varphi}} \\
& \quad=\bar{A}^{*}\left(\frac{\bar{P}^{F *}}{\bar{P}^{*}}\right) .
\end{aligned}
$$

I shall recall now that the real net foreign asset position of the domestic household can be expressed as a share of the domestic absorption (consumption and investment) in the home country as in (165), i.e.,

$$
\bar{a}=\bar{a}^{a}(\bar{C}+\bar{X}) .
$$

Hence, the equations in (211) and (212) become,

$$
\begin{aligned}
& \left(\frac{\theta(1-\bar{\xi})}{\theta-1}\right)^{\frac{1+(1-\psi) \varphi}{1+\varphi}}\left(\frac{\left(\beta^{-1}-(1-\delta)\right)^{1-\psi}}{\psi^{\frac{\psi}{1+\varphi}}(1-\psi)^{1-\psi}}\right)\left(1-(1-\beta) \bar{a}^{a}\right)^{\frac{\psi \varphi}{1+\varphi}}(\bar{C})^{\frac{\psi \sigma}{1+\varphi}}(\bar{C}+\bar{X})^{\frac{\psi \varphi}{1+\varphi}} \\
& \quad=\bar{A}\left(\frac{\bar{P}^{H}}{\bar{P}}\right) \\
& \left(\frac{\theta\left(1-\bar{\xi}^{*}\right)}{\theta-1}\right)^{\frac{1+(1-\psi) \varphi}{1+\varphi}}\left(\frac{\left(\beta^{-1}-(1-\delta)\right)^{1-\psi}}{\psi^{\frac{\psi}{1+\varphi}}(1-\psi)^{1-\psi}}\right)\left(\bar{C}^{*}\right)^{\frac{\psi \sigma^{-1}}{1+\varphi}}\left(\left(\bar{C}^{*}+\bar{X}^{*}\right)+(1-\beta)\left(\frac{1}{\overline{R S}}\right) \bar{a}^{a}(\bar{C}+\bar{X})\right)^{\frac{\psi \varphi}{1+\varphi}} \\
& \quad=\bar{A}^{*}\left(\frac{\bar{P}^{F *}}{\bar{P}^{*}}\right) .
\end{aligned}
$$

Expressions (213) and (214) are going to be decisive to pin down the steady state consumption level in both countries. Implicitly I treat $\left(\frac{\bar{P}^{H}}{\bar{P}}\right),\left(\frac{\bar{P}^{F *}}{\bar{P}^{*}}\right)$, and $\overline{R S}$ as functions of the structural parameters of the model including the share of real net foreign assets relative to domestic absorption, $\bar{a}^{a}$. Therefore, at this stage, these relative prices are viewed as composite coefficients rather than as endogenous steady state variables. Under the assumption that absorption in both countries differs only by a factor related to the steady state 
real exchange rate (i.e. $(\bar{C}+\bar{X})=(\overline{R S})^{\eta}\left(\bar{C}^{*}+\bar{X}^{*}\right)$ ), I can re-write equation (214) more compactly as,

$$
\begin{aligned}
& \left(\frac{\theta\left(1-\bar{\xi}^{*}\right)}{\theta-1}\right)^{\frac{1+(1-\psi) \varphi}{1+\varphi}}\left(\frac{\left(\beta^{-1}-(1-\delta)\right)^{1-\psi}}{\psi^{\frac{\psi}{1+\varphi}}(1-\psi)^{1-\psi}}\right)\left(1+\frac{(1-\beta) \bar{a}^{a}}{(\overline{R S})^{1-\eta}}\right)^{\frac{\psi \varphi}{1+\varphi}}\left(\bar{C}^{*}\right)^{\frac{\psi \sigma}{1+\varphi}}\left(\bar{C}^{*}+\bar{X}^{*}\right)^{\frac{\psi \varphi}{1+\varphi}} \\
& \quad=\bar{A}^{*}\left(\frac{\bar{P}^{F *}}{\bar{P}^{*}}\right) .
\end{aligned}
$$

This expression offers a more precise characterization of the foreign equation in (214).

Eighth Step. I look at aggregate output, consumption and investment as well as the normalization of steady state aggregate productivity in the model. It follows from the domestic aggregate output equation derived above in (161) (i.e. $\left.\bar{Y}=\left(\frac{\bar{P}}{\bar{P}^{H}}\right)[(\bar{C}+\bar{X})-(1-\beta) \bar{a}]\right)$, the foreign aggregate output equation derived above in (162) (i.e. $\left.\bar{Y}^{*}=\left(\frac{\bar{P}^{*}}{\bar{P}^{F *}}\right)\left[\left(\bar{C}^{*}+\bar{X}^{*}\right)+(1-\beta)\left(\frac{1}{\overline{R S}}\right) \bar{a}\right]\right)$, the definition of the domestic real net foreign share over domestic absorption $\bar{a}^{a}$ in (165) (i.e. $\bar{a}=\bar{a}^{a}(\bar{C}+\bar{X})$ ) and the assumption that absorption in both countries differs only by a factor related to the steady state real exchange rate (i.e. $\left.(\bar{C}+\bar{X})=(\overline{R S})^{\eta}\left(\bar{C}^{*}+\bar{X}^{*}\right)\right)$, that I can write,

$$
\begin{aligned}
1 & =\left(1-(1-\beta) \bar{a}^{a}\right)\left(\frac{\bar{P}}{\bar{P}^{H}}\right)\left(\frac{\bar{C}}{\bar{Y}}+\frac{\bar{X}}{\bar{Y}}\right), \\
1 & =\left(1+\left(\frac{(1-\beta) \bar{a}^{a}}{(\overline{R S})^{1-\eta}}\right)\right)\left(\frac{\bar{P}^{*}}{\bar{P}^{F *}}\right)\left(\frac{\bar{C}^{*}}{\bar{Y}^{*}}+\frac{\bar{X}^{*}}{\bar{Y}^{*}}\right) .
\end{aligned}
$$

This implies that the consumption share in the domestic and foreign countries must be equal to,

$$
\begin{aligned}
& \frac{\bar{C}}{\bar{Y}}=\left(\frac{\bar{P}^{H}}{\bar{P}}\right)\left(\frac{1}{1-(1-\beta) \bar{a}^{a}}\right)-\gamma_{x}^{y} \\
& =\left(\frac{\bar{P}^{H}}{\bar{P}}\right)\left[\left(\frac{1}{1-(1-\beta) \bar{a}^{a}}\right)-\delta\left(\frac{1-\psi}{\left(\frac{\theta(1-\bar{\xi})}{\theta-1}\right)\left(\beta^{-1}-(1-\delta)\right)}\right)\right], \\
& \frac{\bar{C}^{*}}{\bar{Y}^{*}}=\left(\frac{\bar{P}^{F *}}{\bar{P}^{*}}\right)\left(\frac{1}{1+\left(\frac{(1-\beta) \bar{a}^{a}}{(\overline{R S})^{1-\eta}}\right)}\right)-\gamma_{x^{*}}^{y} \\
& =\left(\frac{\bar{P}^{F *}}{\bar{P}^{*}}\right)\left[\left(\frac{1}{1+\left(\frac{(1-\beta) \bar{a}^{a}}{(\overline{R S})^{1-\eta}}\right)}\right)-\delta\left(\frac{1-\psi}{\left(\frac{\theta\left(1-\bar{\xi}^{*}\right)}{\theta-1}\right)\left(\beta^{-1}-(1-\delta)\right)}\right)\right] \text {, }
\end{aligned}
$$

where I have replaced the domestic investment share obtained in (142) and the foreign investment share in (143). Moreover, with (216) and (217) I can also compute the domestic and foreign investment-to- 
consumption ratios in steady state as follows,

$$
\begin{aligned}
\overline{\bar{Y}} & =\left(1-(1-\beta) \bar{a}^{a}\right)\left(\frac{\bar{P}}{\bar{P}^{H}}\right)\left(1+\frac{\bar{X}}{\bar{C}}\right), \\
\bar{Y}^{*} & =\left(1+\frac{(1-\beta) \bar{a}^{a}}{(\overline{R S})^{1-\eta}}\right)\left(\frac{\bar{P}^{*}}{\bar{P}^{F *}}\right)\left(1+\frac{\bar{X}^{*}}{\bar{C}^{*}}\right) .
\end{aligned}
$$

Replacing (218) and (219) appropriately into these two expressions it follows that,

$$
\begin{aligned}
\overline{\bar{X}} & =\left(\frac{\bar{P}^{H}}{\bar{P}}\right)\left(\frac{1}{1-(1-\beta) \bar{a}^{a}}\right)\left(\frac{\bar{Y}}{\bar{C}}\right)-1 \\
& =\left(\frac{1}{1-(1-\beta) \bar{a}^{a}}\right)\left[\left(\frac{1}{1-(1-\beta) \bar{a}^{a}}\right)-\delta\left(\frac{1-\psi}{\left(\frac{\theta(1-\bar{\xi})}{\theta-1}\right)\left(\beta^{-1}-(1-\delta)\right)}\right)\right]^{-1}-1, \\
\frac{\bar{X}^{*}}{\bar{C}^{*}} & =\left(\frac{\bar{P}^{F *}}{\bar{P}^{*}}\right)\left(\frac{1}{1+\frac{(1-\beta) \bar{a}^{a}}{(\overline{R S})^{1-\eta}}}\right)\left(\frac{\bar{Y}^{*}}{\bar{C}^{*}}\right)-1 \\
& =\left(\frac{1}{1+\frac{(1-\beta) \bar{a}^{a}}{(\overline{R S})^{1-\eta}}}\right)\left[\left(\frac{1}{1+\left(\frac{(1-\beta) \bar{a}^{a}}{(\overline{R S})^{1-\eta}}\right)}\right)-\delta\left(\frac{1-\psi}{\left(\frac{\theta\left(1-\bar{\xi}^{*}\right)}{\theta-1}\right)\left(\beta^{-1}-(1-\delta)\right)}\right)\right]^{-1}-1,
\end{aligned}
$$

where $\overline{R S}, \frac{\bar{P}^{H}}{\bar{P}}$, and $\frac{\bar{P}^{F *}}{\bar{P}^{*}}$ are all functions of $\bar{a}^{a}$ based on my derivations in (175), (176), and (177).

As a result, I can infer from these calculations that the expression implied by the price-setting rule in the domestic country in (213) can be re-written as,

$$
\begin{aligned}
\bar{A} & \left(\frac{\bar{P}^{H}}{\bar{P}}\right)=\left(\frac{\theta(1-\bar{\xi})}{\theta-1}\right)^{\frac{1+(1-\psi) \varphi}{1+\varphi}}\left(\frac{\left(\beta^{-1}-(1-\delta)\right)^{1-\psi}}{\psi^{\frac{\psi}{1+\varphi}}(1-\psi)^{1-\psi}}\right)\left(1-(1-\beta) \bar{a}^{a}\right)^{\frac{\psi \varphi}{1+\varphi}}(\bar{C})^{\frac{\psi \sigma^{-1}}{1+\varphi}}\left(\left(1+\frac{\bar{X}}{\bar{C}}\right) \bar{C}\right)^{\frac{\psi \varphi}{1+\varphi}} \\
& =\left(\frac{\theta(1-\bar{\xi})}{\theta-1}\right)^{\frac{1+(1-\psi) \varphi}{1+\varphi}}\left(\frac{\left(\beta^{-1}-(1-\delta)\right)^{1-\psi}}{\psi^{\frac{\psi}{1+\varphi}}(1-\psi)^{1-\psi}}\right)\left(1-(1-\beta) \bar{a}^{a}\right)^{\frac{\psi \varphi}{1+\varphi}}\left(1+\frac{\bar{X}}{\bar{C}}\right)^{\frac{\psi \varphi}{1+\varphi}}(\bar{C})^{\frac{\psi \varphi}{1+\varphi}+\frac{\psi \sigma^{-1}}{1+\varphi}} \\
& =\left(\frac{\theta(1-\bar{\xi})}{\theta-1}\right)^{\frac{1+(1-\psi) \varphi}{1+\varphi}}\left(\frac{\left(\beta^{-1}-(1-\delta)\right)^{1-\psi}}{\psi^{\frac{\psi}{1+\varphi}}(1-\psi)^{1-\psi}}\right)\left(\frac{1}{1-(1-\beta) \bar{a}^{a}}-\delta\left(\frac{1-\psi}{\left(\frac{\theta(1-\bar{\xi})}{\theta-1}\right)\left(\beta^{-1}-(1-\delta)\right)}\right)\right)^{-\frac{\psi \varphi}{1+\varphi}}(\bar{C})^{\frac{\psi\left(\sigma^{-1}+\varphi\right.}{1+\varphi}} .
\end{aligned}
$$

I can also infer from these calculations that the expression implied by the price-setting rule in the foreign 
country in (215) can be re-written as,

$$
\begin{aligned}
\bar{A}^{*} & \left(\frac{\bar{P}^{F *}}{\bar{P}^{*}}\right)=\left(\frac{\theta\left(1-\bar{\xi}^{*}\right)}{\theta-1}\right)^{\frac{1+(1-\psi) \varphi}{1+\varphi}}\left(\frac{\left(\beta^{-1}-(1-\delta)\right)^{1-\psi}}{\psi^{\frac{\psi}{1+\varphi}}(1-\psi)^{1-\psi}}\right)\left(1+\frac{(1-\beta) \bar{a}^{a}}{(\overline{R S})^{1-\eta}}\right)^{\frac{\psi \varphi}{1+\varphi}}\left(\bar{C}^{*}\right)^{\frac{\psi \sigma}{1+\varphi}}\left(\left(1+\frac{\bar{X}^{*}}{\bar{C}^{*}}\right) \bar{C}^{*}\right)^{\frac{\psi \varphi}{1+\varphi}} \\
& =\left(\frac{\theta\left(1-\bar{\xi}^{*}\right)}{\theta-1}\right)^{\frac{1+(1-\psi) \varphi}{1+\varphi}}\left(\frac{\left(\beta^{-1}-(1-\delta)\right)^{1-\psi}}{\psi^{\frac{\psi}{1+\varphi}}(1-\psi)^{1-\psi}}\right)\left(1+\frac{(1-\beta) \bar{a}^{a}}{(\overline{R S})^{1-\eta}}\right)^{\frac{\psi \varphi}{1+\varphi}}\left(1+\frac{\bar{X}^{*}}{\bar{C}^{*}}\right)^{\frac{\psi \varphi}{1+\varphi}}\left(\bar{C}^{*}\right)^{\frac{\psi \varphi}{1+\varphi}+\frac{\psi \sigma}{1+1}} \\
& =\left(\frac{\theta\left(1-\bar{\xi}^{*}\right)}{\theta-1}\right)^{\frac{1+(1-\psi) \varphi}{1+\varphi}}\left(\frac{\left(\beta^{-1}-(1-\delta)\right)^{1-\psi}}{\psi^{\frac{\psi}{1+\varphi}}(1-\psi)^{1-\psi}}\right)\left(\frac{1}{1+\left(\frac{(1-\beta) \bar{a}^{a}}{(R S)^{1-\eta}}\right)}-\delta\left(\frac{1-\psi}{\left(\frac{\theta\left(1-\bar{\xi}^{*}\right)}{\theta-1}\right)\left(\beta^{-1}-(1-\delta)\right)}\right)\right)^{-\frac{\psi \varphi}{1+\varphi}}\left(\bar{C}^{*}\right)^{\frac{\psi \varphi}{1+\varphi}+\frac{\psi \sigma}{1+\varphi}} .
\end{aligned}
$$

From (224) and (225) it clearly follows that the domestic and foreign consumption levels, $\bar{C}$ and $\bar{C}^{*}$, can be expressed as,

$$
\begin{aligned}
& \bar{C}=(\bar{A})^{\frac{1+\varphi}{\psi\left(\sigma^{-1}+\varphi\right)}}\left(\frac{\bar{P}^{H}}{\bar{P}}\right)^{\frac{1+\varphi}{\psi\left(\sigma^{-1}+\varphi\right)}}\left(\frac{\psi^{\psi}(1-\psi)^{(1-\psi)(1+\varphi)}}{\left(\frac{\theta(1-\bar{\xi})}{\theta-1}\right)^{1+(1-\psi) \varphi}\left(\beta^{-1}-(1-\delta)\right)^{(1-\psi)(1+\varphi)}}\right)^{\frac{1}{\psi\left(\sigma^{-1}+\varphi\right)}} \times \ldots \\
& \left(\frac{1}{1-(1-\beta) \bar{a}^{a}}-\delta\left(\frac{1-\psi}{\left(\frac{\theta(1-\bar{\xi})}{\theta-1}\right)\left(\beta^{-1}-(1-\delta)\right)}\right)\right)^{\frac{\varphi}{\sigma^{-1}+\varphi}}, \\
& \bar{C}^{*}=\left(\bar{A}^{*}\right)^{\frac{1+\varphi}{\psi\left(\varphi+\sigma^{-1}\right)}}\left(\frac{\bar{P}^{F *}}{\bar{P}^{*}}\right)^{\frac{1+\varphi}{\psi\left(\varphi+\sigma^{-1}\right)}}\left(\frac{\psi^{\psi}(1-\psi)(1-\psi)(1+\varphi)}{\left(\frac{\theta\left(1-\bar{\xi}^{*}\right)}{\theta-1}\right)^{1+(1-\psi) \varphi}\left(\beta^{-1}-(1-\delta)\right)^{(1-\psi)(1+\varphi)}}\right)^{\frac{1}{\psi\left(\varphi+\sigma^{-1}\right)}} \times \ldots \\
& \left(\frac{1}{1+\left(\frac{(1-\beta) \bar{a}^{a}}{(\overline{R S})^{1-\eta}}\right)}-\delta\left(\frac{1-\psi}{\left(\frac{\theta\left(1-\bar{\xi}^{*}\right)}{\theta-1}\right)\left(\beta^{-1}-(1-\delta)\right)}\right)\right)^{\frac{\varphi}{\sigma-1+\varphi}}
\end{aligned}
$$

while the relative prices $\left(\frac{\bar{P}^{H}}{\bar{P}}\right),\left(\frac{\bar{P}^{F *}}{\bar{P}^{*}}\right)$, and $\overline{R S}$ are tied to structural parameters by $(175),(176)$ and (177). Hence, it follows that,

$$
\begin{aligned}
\bar{C}= & (\bar{A})^{\frac{1+\varphi}{\psi\left(\sigma^{-1}+\varphi\right)}}\left(1-(1-\beta) \bar{a}^{a}\right)^{\frac{1+\varphi}{(1-\eta) \psi\left(\sigma^{-1}+\varphi\right)}}\left(\frac{\psi^{\psi}(1-\psi)^{(1-\psi)(1+\varphi)}}{\left(\frac{\theta(1-\bar{\xi})}{\theta-1}\right)^{1+(1-\psi) \varphi}\left(\beta^{-1}-(1-\delta)\right)^{(1-\psi)(1+\varphi)}}\right)^{\frac{1}{\psi\left(\sigma^{-1}+\varphi\right)}} \times \ldots \\
& \left(\frac{1}{1-(1-\beta) \bar{a}^{a}}-\delta\left(\frac{1-\psi}{\left(\frac{\theta(1-\bar{\xi})}{\theta-1}\right)\left(\beta^{-1}-(1-\delta)\right)}\right)\right)^{\frac{\varphi}{\sigma^{-1}+\varphi}}, \\
\bar{C}^{*}= & \left(\bar{A}^{*}\right)^{\frac{1+\varphi}{\psi\left(\varphi+\sigma^{-1}\right)}}\left(\frac{\phi_{F}+\phi_{H}(1-\beta) \bar{a}^{a}}{\phi_{F}+\left(\phi_{H} \phi_{H}-\phi_{F} \phi_{F}\right)(1-\beta) \bar{a}^{a}}\right)^{\frac{1+\varphi}{(1-\eta) \psi\left(\varphi+\sigma^{-1}\right)}}\left(\frac{\psi^{\psi}(1-\psi)^{(1-\psi)(1+\varphi)}}{\left(\frac{\theta\left(1-\bar{\xi}^{*}\right)}{\theta-1}\right)^{1+(1-\psi) \varphi}\left(\beta^{-1}-(1-\delta)\right)^{(1-\psi)(1+\varphi)}}\right)^{\frac{1}{\psi\left(\varphi+\sigma^{-1}\right)}} \times \ldots \\
& \left(\frac{1+\left(\frac{\phi_{H} \phi_{H}-\phi_{F} \phi_{F}}{\phi_{F}}\right)(1-\beta) \bar{a}^{a}}{1+\left(\frac{\phi_{H}}{\phi_{F}}\right)(1-\beta) \bar{a}^{a}}-\delta\left(\frac{1-\psi}{\left(\frac{\theta\left(1-\bar{\xi}^{*}\right)}{\theta-1}\right)\left(\beta^{-1}-(1-\delta)\right)}\right)\right)^{\frac{\psi^{-1}+\varphi}{\sigma^{-1}}} \cdot
\end{aligned}
$$

Given the domestic consumption-to-output ratio $\frac{\bar{C}}{\bar{Y}}$ in (218) and the foreign consumption-to-output ratio $\frac{\bar{C}^{*}}{\overline{Y^{*}}}$ 
in (219), it must follow that domestic and foreign aggregate output are equal to,

$$
\begin{aligned}
& \bar{Y}=\left(\frac{\bar{P}^{H}}{\bar{P}}\right)^{-1}\left(\frac{1}{1-(1-\beta) \bar{a}^{a}}-\delta\left(\frac{1-\psi}{\left(\frac{\theta(1-\bar{\xi})}{\theta-1}\right)\left(\beta^{-1}-(1-\delta)\right)}\right)\right)^{-1} \bar{C} \\
& =(\bar{A})^{\frac{1+\varphi}{\psi\left(\sigma^{-1}+\varphi\right)}}\left(\frac{\bar{P}^{H}}{\bar{P}}\right)^{\frac{1+(1-\psi) \varphi-\psi \sigma^{-1}}{\psi\left(\sigma^{-1}+\varphi\right)}}\left(\frac{1}{1-(1-\beta) \bar{a}^{a}}-\delta\left(\frac{1-\psi}{\left(\frac{\theta(1-\bar{\xi})}{\theta-1}\right)\left(\beta^{-1}-(1-\delta)\right)}\right)\right)^{\frac{-\sigma^{-1}}{\sigma^{-1}+\varphi}} \times \ldots \\
& \left(\frac{\psi^{\psi}(1-\psi)^{(1-\psi)(1+\varphi)}}{\left(\frac{\theta(1-\bar{\xi})}{\theta-1}\right)^{1+(1-\psi) \varphi}\left(\beta^{-1}-(1-\delta)\right)^{(1-\psi)(1+\varphi)}}\right)^{\frac{1}{\psi\left(\sigma^{-1}+\varphi\right)}} \\
& \bar{Y}^{*}=\left(\frac{\bar{P}^{F *}}{\bar{P}^{*}}\right)^{-1}\left(\frac{1}{1+\left(\frac{(1-\beta) \bar{a}^{a}}{(\overline{R S})^{1-\eta}}\right)}-\delta\left(\frac{1-\psi}{\left(\frac{\theta\left(1-\bar{\xi}^{*}\right)}{\theta-1}\right)\left(\beta^{-1}-(1-\delta)\right)}\right)\right)^{-1} \bar{C}^{*} \\
& =\left(\bar{A}^{*}\right)^{\frac{1+\varphi}{\psi\left(\varphi+\sigma^{-1}\right)}}\left(\frac{\bar{P}^{F *}}{\bar{P}^{*}}\right)^{\frac{1+(1-\psi) \varphi-\psi \sigma^{-1}}{\psi\left(\varphi+\sigma^{-1}\right)}}\left(\frac{1}{1+\left(\frac{(1-\beta) \bar{a}^{a}}{(\overline{R S})^{1-\eta}}\right)}-\delta\left(\frac{1-\psi}{\left(\frac{\theta\left(1-\bar{\xi}^{*}\right)}{\theta-1}\right)\left(\beta^{-1}-(1-\delta)\right)}\right)\right)^{\frac{-\sigma^{-1}}{\sigma-1+\varphi}} \\
& \left(\frac{\psi^{\psi}(1-\psi)^{(1-\psi)(1+\varphi)}}{\left(\frac{\theta\left(1-\bar{\xi}^{*}\right)}{\theta-1}\right)^{1+(1-\psi) \varphi}\left(\beta^{-1}-(1-\delta)\right)^{(1-\psi)(1+\varphi)}}\right)^{\overline{\psi\left(\varphi+\sigma^{-1}\right)}},
\end{aligned}
$$

or simply,

$$
\begin{aligned}
\bar{Y}= & (\bar{A})^{\frac{1+\varphi}{\psi\left(\sigma^{-1}+\varphi\right)}}\left(1-(1-\beta) \bar{a}^{a}\right)^{\frac{1+(1-\psi) \varphi-\psi \sigma^{-1}}{(1-\eta) \psi\left(\sigma^{-1}+\varphi\right)}}\left(\frac{1}{1-(1-\beta) \bar{a}^{a}}-\delta\left(\frac{1-\psi}{\left(\frac{\theta(1-\bar{\xi})}{\theta-1}\right)\left(\beta^{-1}-(1-\delta)\right)}\right)\right)^{\frac{-\sigma^{-1}}{\sigma^{-1}+\varphi}} \times \ldots \\
& \left(\frac{\psi^{\psi}(1-\psi)^{(1-\psi)(1+\varphi)}}{\left(\frac{\theta(1-\bar{\xi})}{\theta-1}\right)^{1+(1-\psi) \varphi}\left(\beta^{-1}-(1-\delta)\right)^{(1-\psi)(1+\varphi)}}\right)^{\frac{1}{\psi(-1+\varphi)}}, \\
\bar{Y}^{*}= & \left(\bar{A}^{*}\right)^{\frac{1+\varphi}{\psi\left(\varphi+\sigma^{-1}\right)}}\left(\frac{\phi_{F}+\phi_{H}(1-\beta) \bar{a}^{a}}{\phi_{F}+\left(\phi_{H} \phi_{H}-\phi_{F} \phi_{F}\right)(1-\beta) \bar{a}^{a}}\right)^{\frac{1+(1-\psi) \varphi-\psi \sigma^{-1}}{(1-\eta) \psi\left(\varphi+\sigma^{-1}\right)}} \times \ldots \\
& \left(\frac{1+\left(\frac{\phi_{H} \phi_{H}-\phi_{F} \phi_{F}}{\phi_{F}}\right)(1-\beta) \bar{a}^{a}}{1+\left(\frac{\phi_{H}}{\phi_{F}}\right)(1-\beta) \bar{a}^{a}}-\delta\left(\frac{\theta\left(1-\bar{\xi}^{*}\right)}{\left(\frac{1-\psi}{\theta-1}\right)\left(\beta^{-1}-(1-\delta)\right)}\right)\right)^{\frac{-\sigma^{-1}}{\sigma-1+\varphi}} \times \ldots \\
& \left(\frac{1}{\left(\frac{\theta\left(1-\bar{\xi}^{*}\right)}{\theta-1}\right)^{1+(1-\psi) \varphi}\left(\beta^{-1}-(1-\delta)\right)^{(1-\psi)(1+\varphi)}}\right)^{\frac{\psi^{\psi}\left(\varphi+\sigma^{-1}\right)}{(1-\psi)(1+\varphi)}} \times
\end{aligned}
$$

Similarly, I infer domestic investment $\bar{X}$ from the domestic investment share $\gamma_{x}^{y}$ in (142) and the domestic aggregate output $\bar{Y}$ in (230) and foreign investment from the foreign investment share in (143) and the foreign 
aggregate output in (231) as follows,

$$
\begin{aligned}
& \bar{X}=\gamma_{x}^{y} \bar{Y}=\delta\left(\frac{1-\psi}{\left(\frac{\theta(1-\bar{\xi})}{\theta-1}\right)\left(\beta^{-1}-(1-\delta)\right)}\right)\left(\frac{\bar{P}^{H}}{\bar{P}}\right) \bar{Y} \\
& =(\bar{A})^{\frac{1+\varphi}{\psi\left(\sigma^{-1}+\varphi\right)}}\left(\frac{\bar{P}^{H}}{\bar{P}}\right)^{\frac{1+\varphi}{\psi\left(\sigma^{-1}+\varphi\right)}} \delta\left(\frac{1-\psi}{\left(\frac{\theta(1-\bar{\xi})}{\theta-1}\right)\left(\beta^{-1}-(1-\delta)\right)}\right) \times \ldots \\
& \left(\frac{1}{1-(1-\beta) \bar{a}^{a}}-\delta\left(\frac{1-\psi}{\left(\frac{\theta(1-\bar{\xi})}{\theta-1}\right)\left(\beta^{-1}-(1-\delta)\right)}\right)\right)^{\frac{-\sigma^{-1}}{\sigma^{-1}+\varphi}}\left(\frac{\psi^{\psi}(1-\psi)^{(1-\psi)(1+\varphi)}}{\left(\frac{\theta(1-\bar{\xi})}{\theta-1}\right)^{1+(1-\psi) \varphi}\left(\beta^{-1}-(1-\delta)\right)^{(1-\psi)(1+\varphi)}}\right)^{\frac{1}{\psi\left(\sigma^{-1}+\varphi\right)}}, \\
& \bar{X}^{*}=\gamma_{x^{*}}^{y} \bar{Y}^{*}=\delta\left(\frac{1-\psi}{\left(\frac{\theta\left(1-\bar{\xi}^{*}\right)}{\theta-1}\right)\left(\beta^{-1}-(1-\delta)\right)}\right)\left(\frac{\bar{P}^{F *}}{\bar{P}^{*}}\right) \bar{Y}^{*} \\
& =\left(\bar{A}^{*}\right)^{\frac{1+\varphi}{\psi\left(\varphi+\sigma^{-1}\right)}}\left(\frac{\bar{P}^{F *}}{\bar{P}^{*}}\right)^{\frac{1+\varphi}{\psi\left(\varphi+\sigma^{-1}\right)}} \delta\left(\frac{1-\psi}{\left(\frac{\theta\left(1-\bar{\xi}^{*}\right)}{\theta-1}\right)\left(\beta^{-1}-(1-\delta)\right)}\right) \times \ldots \\
& \left(\frac{1}{1+\frac{(1-\beta) \bar{\sigma}^{a}}{(\overline{R S})^{1-\eta}}}-\delta\left(\frac{1-\psi}{\left(\frac{\theta\left(1-\bar{\xi}^{*}\right)}{\theta-1}\right)\left(\beta^{-1}-(1-\delta)\right)}\right)\right)^{\frac{-\sigma^{-1}}{\sigma-1+\varphi}}\left(\frac{\psi^{\psi}(1-\psi)^{(1-\psi)(1+\varphi)}}{\left(\frac{\theta\left(1-\bar{\xi}^{*}\right)}{\theta-1}\right)^{11(1-\psi) \varphi}\left(\beta^{-1}-(1-\delta)\right)^{(1-\psi)(1+\varphi)}}\right)^{\frac{1}{\psi\left(\varphi+\sigma^{-1}\right)}},
\end{aligned}
$$

or simply,

$$
\begin{aligned}
\bar{X}= & (\bar{A})^{\frac{1+\varphi}{\psi\left(\sigma^{-1}+\varphi\right)}}\left(1-(1-\beta) \bar{a}^{a}\right)^{\frac{1+\varphi}{(1-\eta) \psi\left(\sigma^{-1}+\varphi\right)}} \delta\left(\frac{1-\psi}{\left(\frac{\theta(1-\bar{\xi})}{\theta-1}\right)\left(\beta^{-1}-(1-\delta)\right)}\right) \times \ldots \\
& \left.\left(\frac{1}{1-(1-\beta) \bar{a}^{a}}-\delta\left(\frac{1-\psi}{\left(\frac{\theta(1-\bar{\xi})}{\theta-1}\right)\left(\beta^{-1}-(1-\delta)\right)}\right)\right)^{\frac{-\sigma^{-1}}{\sigma^{-1}+\varphi}}\left(\frac{\psi^{\psi}(1-\psi)^{(1-\psi)(1+\varphi)}}{\left(\frac{\theta(1-\bar{\xi})}{\theta-1}\right)^{1+(1-\psi) \varphi}\left(\beta^{-1}-(1-\delta)\right)^{(1-\psi)(1+\varphi)}}\right)\right)^{\frac{1}{\psi\left(\sigma^{-1}+\varphi\right)}}, \\
\bar{X}^{*}= & \left(\bar{A}^{*}\right)^{\frac{1+\varphi}{\psi\left(\varphi+\sigma^{-1}\right)}}\left(\frac{\phi_{F}+\phi_{H}(1-\beta) \bar{a}^{a}}{\phi_{F}+\left(\phi_{H} \phi_{H}-\phi_{F} \phi_{F}\right)(1-\beta) \bar{a}^{a}}\right)^{\frac{1+\varphi}{(1-\eta) \psi\left(\varphi+\sigma^{-1}\right)}} \delta\left(\frac{1-\psi}{\left(\frac{\theta\left(1-\bar{\xi}^{*}\right)}{\theta-1}\right)\left(\beta^{-1}-(1-\delta)\right)}\right) \times \ldots \\
& \left(\frac{1+\left(\frac{\phi_{H} \phi_{H}-\phi_{F} \phi_{F}}{\phi_{F}}\right)(1-\beta) \bar{a}^{a}}{1+\left(\frac{\phi_{H}}{\phi_{F}}\right)(1-\beta) \bar{a}^{a}}-\delta\left(\frac{1-\psi}{\left(\frac{\theta\left(1-\bar{\xi}^{*}\right)}{\theta-1}\right)\left(\beta^{-1}-(1-\delta)\right)}\right)\right)^{\frac{-\sigma}{\sigma-1}+\varphi} \times \ldots \\
& \left(\frac{\psi^{\psi}(1-\psi)^{(1-\psi)(1+\varphi)}}{\left(\frac{\theta\left(1-\bar{\xi}^{*}\right)}{\theta-1}\right)^{1+(1-\psi) \varphi}\left(\beta^{-1}-(1-\delta)\right)^{(1-\psi)(1+\varphi)}}\right)^{\frac{1}{\psi\left(\varphi+\sigma^{-1}\right)}},
\end{aligned}
$$

The expressions in (228), (232) and (236) characterize the steady state solution for $\bar{C}, \bar{Y}$ and $\bar{X}$ from the perspective of the domestic country. Analogously, the expressions in (229), (233) and (237) characterize the steady state solution for $\bar{C}^{*}, \bar{Y}^{*}$ and $\bar{X}^{*}$ from the perspective of the foreign country.

Remark 3 Given the values for $(\bar{C}, \bar{X}, \bar{Y})$, I can alternatively derive the foreign aggregate output $\bar{Y}^{*}$ from 
the following relationship previously obtained in (179),

$$
\left(\frac{\bar{P}^{H}}{\bar{P}}\right) \bar{Y}=\left(\frac{1}{\overline{R S}}\right)^{1-\eta}\left(\frac{\bar{P}^{H}}{\bar{P}^{F}}\right)^{1-\eta}\left[\left(\frac{\bar{P}^{F}}{\bar{P}}\right) \bar{Y}^{*}+\left(\frac{\phi_{H}(\overline{R S})^{1-\eta}-\phi_{F}}{\phi_{F}}\right)(1-\beta) \bar{a}\right],
$$

which, in turn, can be re-written as,

$$
\bar{Y}^{*}=\left[(\overline{R S})^{1-\eta}\left(\frac{\bar{P}^{F}}{\bar{P}^{H}}\right)^{-\eta} \bar{Y}-\left(\frac{\phi_{H}(\overline{R S})^{1-\eta}-\phi_{F}}{\phi_{F}}\right)\left(\frac{\bar{P}^{F}}{\bar{P}}\right)^{-1}(1-\beta) \bar{a}\right] .
$$

Foreign aggregate investment $\bar{X}^{*}$ can be derived from the expression for foreign output in (238) and the foreign investment share in (143),

$$
\bar{X}^{*}=\gamma_{x^{*}}^{y} \bar{Y}^{*}=\delta\left(\frac{1-\psi}{\left(\frac{\theta\left(1-\bar{\xi}^{*}\right)}{\theta-1}\right)\left(\beta^{-1}-(1-\delta)\right)}\right)\left(\frac{\bar{P}^{F *}}{\bar{P}^{*}}\right) \bar{Y}^{*},
$$

where the level of investment may differ because the investment shares in both countries differ and/or because aggregate output is not equalized between the two countries. Aggregate consumption in the foreign country $\bar{C}^{*}$ can be infered from the following relationship (previously derived in (163)),

$$
\begin{aligned}
\phi_{F}\left(\frac{\bar{P}^{F}}{\bar{P}}\right)^{1-\eta}(\bar{C}+\bar{X}) & =\phi_{F}(\overline{R S})^{\eta}\left(\frac{\bar{P}^{H}}{\bar{P}}\right)^{1-\eta}\left(\bar{C}^{*}+\bar{X}^{*}\right)+(1-\beta) \bar{a} \\
& =\phi_{F}(\overline{R S})^{\eta}\left(\frac{\bar{P}^{H}}{\bar{P}}\right)^{1-\eta}\left(\bar{C}^{*}+\bar{X}^{*}\right)+(1-\beta) \bar{a}^{a}(\bar{C}+\bar{X}),
\end{aligned}
$$

which-after a little bit of algebra—implies that,

$$
\bar{C}^{*}=(\overline{R S})^{-\eta}\left[\left(\frac{\bar{P}^{F}}{\bar{P}^{H}}\right)^{1-\eta}-\left(\frac{\bar{P}^{H}}{\bar{P}}\right)^{-(1-\eta)} \frac{(1-\beta) \bar{a}^{a}}{\phi_{F}}\right](\bar{C}+\bar{X})-\bar{X}^{*} .
$$

The expressions in (238), (239) and (240) also characterize the solution of the model with non-trivial domestic real net foreign asset holdings in steady state.

Hence, from (216) and (230) I can now calculate the domestic absorption (consumption plus investment) 
as follows,

$$
\begin{aligned}
\bar{C}+ & \bar{X}=\frac{1}{1-(1-\beta) \bar{a}^{a}}\left(\frac{\bar{P}^{H}}{\bar{P}}\right) \bar{Y} \\
= & \frac{1}{1-(1-\beta) \bar{a}^{a}}\left(\frac{\bar{P}^{H}}{\bar{P}}\right)(\bar{A})^{\frac{1+\varphi}{\psi\left(\sigma^{-1}+\varphi\right)}}\left(\frac{\bar{P}^{H}}{\bar{P}}\right)^{\frac{1+(1-\psi) \varphi-\psi \sigma^{-1}}{\psi\left(\sigma^{-1}+\varphi\right)}}\left(\frac{1}{1-(1-\beta) \bar{a}^{a}}-\delta\left(\frac{1-\psi}{\left(\frac{\theta(1-\bar{\xi})}{\theta-1}\right)\left(\beta^{-1}-(1-\delta)\right)}\right)\right)^{\frac{\sigma^{-1}}{\sigma^{-1}+\varphi}} \times \ldots \\
& \left(\frac{\psi^{\psi}(1-\psi)^{(1-\psi)(1+\varphi)}}{\left(\frac{\theta(1-\bar{\xi})}{\theta-1}\right)^{1+(1-\psi) \varphi}\left(\beta^{-1}-(1-\delta)\right)^{(1-\psi)(1+\varphi)}}\right) \\
= & \frac{1}{1-(1-\beta) \bar{a}^{a}}(\bar{A})^{\frac{1}{\psi\left(\sigma^{-1}+\varphi\right)}}\left(\frac{\bar{P}^{H}}{\bar{P}}\right)^{\frac{1+\varphi}{\psi\left(\sigma^{-1}+\varphi\right)}}\left(\frac{1}{\frac{1-(1-\beta) \bar{a}^{a}}{1-\varphi}}-\delta\left(\frac{1-\psi}{\left(\frac{\theta(1-\bar{\xi})}{\theta-1}\right)\left(\beta^{-1}-(1-\delta)\right)}\right)\right)^{\frac{-\sigma^{-1}}{\sigma^{-1}+\varphi}} \times \ldots \\
& \left(\frac{\psi^{\psi}(1-\psi)^{(1-\psi)(1+\varphi)}}{\left(\frac{\theta(1-\bar{\xi})}{\theta-1}\right)^{1+(1-\psi) \varphi}\left(\beta^{-1}-(1-\delta)\right)^{(1-\psi)(1+\varphi)}}\right)^{\frac{1}{\psi\left(\sigma^{-1}+\varphi\right)}},
\end{aligned}
$$

which helps me pin down the exact form of the real net foreign asset position $\bar{a}$ since I know from (165) that $\bar{a}=\bar{a}^{a}(\bar{C}+\bar{X})$. Alternatively, I can combine (226) and (234) to obtain this other representation of the domestic absorption,

$$
\begin{aligned}
\bar{C}+ & \bar{X}=(\bar{A})^{\frac{1+\varphi}{\psi\left(\sigma^{-1}+\varphi\right)}}\left(\frac{\bar{P}^{H}}{\bar{P}}\right)^{\frac{1+\varphi}{\psi\left(\sigma^{-1}+\varphi\right)}}\left(\frac{\psi^{\psi}(1-\psi)^{(1-\psi)(1+\varphi)}}{\left(\frac{\theta(1-\bar{\xi})}{\theta-1}\right)^{1+(1-\psi) \varphi}\left(\beta^{-1}-(1-\delta)\right)^{(1-\psi)(1+\varphi)}}\right)^{\frac{\varphi}{\psi\left(\sigma^{-1}+\varphi\right)}} \times \ldots \\
& {\left[\left(\frac{1}{1-(1-\beta) \bar{a}^{a}}-\delta\left(\frac{1-\psi}{\left(\frac{\theta(1-\bar{\xi})}{\theta-1}\right)\left(\beta^{-1}-(1-\delta)\right)}\right)\right)^{\frac{\varphi}{\sigma^{-1}+\varphi}}+\ldots\right.} \\
& \left.\delta\left(\frac{1-\psi}{\left(\frac{\theta(1-\bar{\xi})}{\theta-1}\right)\left(\beta^{-1}-(1-\delta)\right)}\right)\left(\frac{1}{1-(1-\beta) \bar{a}^{\alpha}}-\delta\left(\frac{1-\psi}{\left(\frac{\theta(1-\bar{\xi})}{\theta-1}\right)\left(\beta^{-1}-(1-\delta)\right)}\right)\right)^{\frac{-\sigma^{-1}}{\sigma^{-1}+\varphi}}\right],
\end{aligned}
$$

although it can easily be shown that (241) and (242) are - as could be expected-identical. From (217) and 
(231) I can calculate the foreign absorption (consumption and investment) as follows,

$$
\begin{aligned}
\bar{C}^{*}+ & \bar{X}^{*}=\frac{1}{1+\frac{(1-\beta) \bar{a}^{a}}{(\overline{R S})^{-\eta}}}\left(\frac{\bar{P}^{F *}}{\bar{P}^{*}}\right) \bar{Y}^{*} \\
= & \frac{1}{1+\frac{1-\beta) \bar{a}^{a}}{(R S)^{1-\eta}}}\left(\frac{\bar{P}^{F *}}{\bar{P}^{*}}\right)\left(\bar{A}^{*}\right)^{\frac{1+\varphi}{\psi\left(\varphi+\sigma^{-1}\right)}}\left(\frac{\bar{P}^{F *}}{\bar{P}^{*}}\right)^{\frac{1+(1-\psi) \varphi-\psi \sigma^{-1}}{\psi\left(\varphi+\sigma^{-1}\right)}}\left(\frac{1}{1+\left(\frac{(1-\beta) \bar{a}^{a}}{(R S)^{1-\eta}}\right)}-\delta\left(\frac{1-\psi}{\left(\frac{\theta\left(1-\bar{\xi}^{*}\right)}{\theta-1}\right)\left(\beta^{-1}-(1-\delta)\right)}\right)\right)^{\frac{-\sigma^{-1}}{\sigma-1+\varphi}} \times \ldots \\
& \left(\frac{\psi^{\psi}(1-\psi)^{(1-\psi)(1+\varphi)}}{\left(\frac{\theta\left(1-\bar{\xi}^{*}\right)}{\theta-1}\right)^{1+(1-\psi) \varphi}\left(\beta^{-1}-(1-\delta)\right)^{(1-\psi)(1+\varphi)}}\right) \\
= & \frac{1}{1+\frac{(1-\beta) \bar{a}^{a}}{(R S)^{1-\eta}}}\left(\bar{A}^{*}\right)^{\frac{1+\varphi}{\psi\left(\varphi+\sigma^{-1}\right)}}\left(\frac{\bar{P}^{F *}}{\bar{P}^{*}}\right)^{\frac{1+\varphi}{\psi\left(\varphi+\sigma^{-1}\right)}}\left(\frac{1}{1+\left(\frac{(1-\beta) \bar{a}^{a}}{(R S)^{1-\eta}}\right)}-\delta\left(\frac{1-\psi}{\left(\frac{\theta\left(1-\bar{\xi}^{*}\right)}{\theta-1}\right)\left(\beta^{-1}-(1-\delta)\right)}\right)\right)^{\frac{-\sigma^{-1}}{\sigma^{-1}+\varphi}} \times \ldots \\
& \left(\frac{\psi^{\psi}(1-\psi)^{(1-\psi)(1+\varphi)}}{\left(\frac{\theta\left(1-\bar{\xi}^{*}\right)}{\theta-1}\right)^{1+(1-\psi) \varphi}\left(\beta^{-1}-(1-\delta)\right)^{(1-\psi)(1+\varphi)}}\right)^{\frac{1\left(\varphi+\sigma^{-1}\right)}{\cdot}}
\end{aligned}
$$

In fact, from (175), (176), and (177), I can express the domestic and foreign absorption in (241) and (243) more compactly as,

$$
\begin{aligned}
& \bar{C}+\bar{X}=(\bar{A})^{\frac{1+\varphi}{\psi\left(\sigma^{-1}+\varphi\right)}}\left(1-(1-\beta) \bar{a}^{a}\right)^{\frac{1+\varphi}{(1-\eta) \psi\left(\sigma^{-1}+\varphi\right)}}-1 \text {. } \\
& \left(\frac{1}{1-(1-\beta) \bar{a}^{a}}-\delta\left(\frac{1-\psi}{\left(\frac{\theta(1-\bar{\xi})}{\theta-1}\right)\left(\beta^{-1}-(1-\delta)\right)}\right)\right)^{\frac{-\sigma^{-1}}{\sigma-1+\varphi}} \times \ldots \\
& \left(\frac{\psi^{\psi}(1-\psi)^{(1-\psi)(1+\varphi)}}{\left(\frac{\theta(1-\bar{\xi})}{\theta-1}\right)^{1+(1-\psi) \varphi}\left(\beta^{-1}-(1-\delta)\right)^{(1-\psi)(1+\varphi)}}\right)^{\frac{1}{\psi\left(\sigma^{-1}+\varphi\right)}}, \\
& \bar{C}^{*}+\bar{X}^{*}=\left(\bar{A}^{*}\right)^{\frac{1+\varphi}{\psi\left(\varphi+\sigma^{-1}\right)}}\left(\frac{\phi_{F}+\phi_{H}(1-\beta) \bar{a}^{a}}{\phi_{F}+\left(\phi_{H} \phi_{H}-\phi_{F} \phi_{F}\right)(1-\beta) \bar{a}^{a}}\right)^{\frac{1+\varphi}{(1-\eta) \psi\left(\varphi+\sigma^{-1}\right)}}-1 \times \ldots \\
& \left(\left(\frac{\phi_{F}+\left(\phi_{H} \phi_{H}-\phi_{F} \phi_{F}\right)(1-\beta) \bar{a}^{a}}{\phi_{F}+\phi_{H}(1-\beta) \bar{a}^{a}}\right)-\delta\left(\frac{1-\psi}{\left(\frac{\theta\left(1-\bar{\xi}^{*}\right)}{\theta-1}\right)\left(\beta^{-1}-(1-\delta)\right)}\right)\right)^{\frac{-\sigma^{-1}}{\sigma-1}+\varphi} \times \ldots \\
& \left(\frac{\psi^{\psi}(1-\psi)^{(1-\psi)(1+\varphi)}}{\left(\frac{\theta\left(1-\bar{\xi}^{*}\right)}{\theta-1}\right)^{1+(1-\psi) \varphi}\left(\beta^{-1}-(1-\delta)\right)^{(1-\psi)(1+\varphi)}}\right)^{\frac{1}{\psi\left(\varphi+\sigma^{-1}\right)}},
\end{aligned}
$$

which determines absorption in both countries in terms of structural parameters only. 
Combining (241) and (243) it immediately follows that,

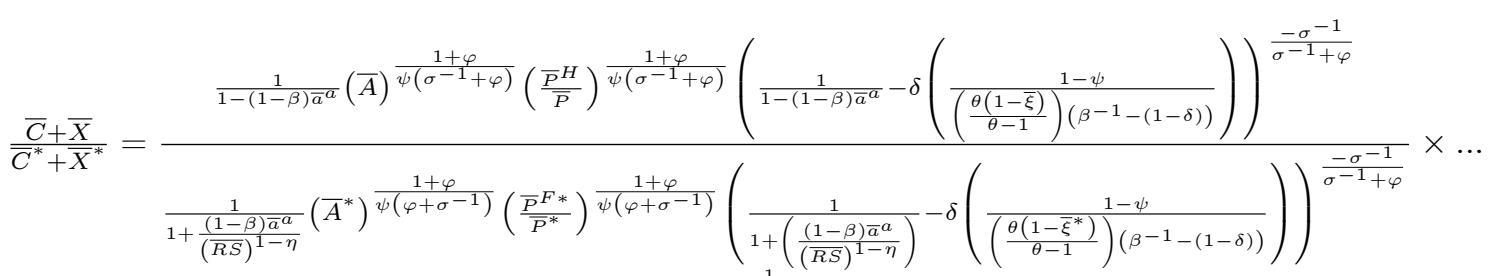

$$
\begin{aligned}
& \left(\frac{\psi^{\psi}(1-\psi)(1-\psi)(1+\varphi)}{\left(\frac{\theta(1-\bar{\xi})}{\theta-1}\right)^{1+(1-\psi) \varphi}\left(\beta^{-1}-(1-\delta)\right)^{(1-\psi)(1+\varphi)}}\right)^{\frac{1}{\psi\left(\sigma^{-1}+\varphi\right)}} \\
& \left(\frac{\psi^{\psi}(1-\psi)(1-\psi)(1+\varphi)}{\left(\frac{\theta\left(1-\bar{\xi}^{*}\right)}{\theta-1}\right)^{1+(1-\psi) \varphi}\left(\beta^{-1}-(1-\delta)\right)^{(1-\psi)(1+\varphi)}}\right)^{\frac{1}{\psi\left(\varphi+\sigma^{-1}\right)}} \\
& =\frac{1+\frac{(1-\beta) \bar{a}^{a}}{(R S)^{1-\eta}}}{1-(1-\beta) \bar{a}^{a}}\left(\frac{\bar{A}}{\bar{A}^{*}}\right)^{\frac{1+\varphi}{\psi\left(\sigma^{-1}+\varphi\right)}}\left(\frac{\frac{\bar{P} H}{\bar{P}}}{\frac{\bar{P} F *}{\bar{P}^{*}}}\right)^{\frac{1+\varphi}{\psi\left(\sigma^{-1}+\varphi\right)}}\left(\frac{\frac{1}{1-(1-\beta) \bar{a}}-\delta\left(\frac{1-\psi}{\left(\frac{\theta(1-\bar{\xi})}{\theta-1}\right)\left(\beta^{-1}-(1-\delta)\right)}\right)}{\frac{1}{1+\left(\frac{(1-\beta) \bar{a}^{a}}{(R S)^{1-\eta}}\right)}-\delta\left(\frac{1-\psi}{\left(\frac{\theta\left(1-\bar{\xi}^{*}\right)}{\theta-1}\right)\left(\beta^{-1}-(1-\delta)\right)}\right)}\right)^{\frac{-\sigma^{-1}}{\sigma^{-1}+\varphi}} \\
& =\frac{1+\frac{(1-\beta) \bar{a}^{a}}{(\overline{R S})^{-\eta}}}{1-(1-\beta) \bar{a}^{a}}\left(\frac{\bar{A}}{\bar{A}^{*}}\right)^{\frac{1+\varphi}{\psi\left(\sigma^{-1}+\varphi\right)}}\left(\frac{\bar{P}^{H}}{\overline{S P}} \frac{\overline{S P}^{*}}{\bar{P}}\right)^{\frac{1+\varphi}{\psi\left(\sigma^{-1}+\varphi\right)}}\left(\frac{\frac{1}{1-(1-\beta) \bar{a}^{a}}-\delta\left(\frac{1-\psi}{\left(\frac{\theta(1-\bar{\xi})}{\theta-1}\right)\left(\beta^{-1}-(1-\delta)\right)}\right)}{\frac{1}{1+\left(\frac{(1-\beta))^{a}}{(\overline{R S})^{1-\eta}}\right)}-\delta\left(\frac{1-\psi}{\left(\frac{\theta\left(1-\bar{\xi}^{*}\right)}{\theta-1}\right)\left(\beta^{-1}-(1-\delta)\right)}\right)}\right)^{\frac{-\sigma^{-1}}{\sigma^{-1}+\varphi}} \\
& =\frac{1+\frac{(1-\beta)^{a}}{(\overline{R S})^{1-\eta}}}{1-(1-\beta) \bar{a}^{a}}\left(\frac{\bar{A}}{\bar{A}^{*}}\right)^{\frac{1+\varphi}{\psi\left(\sigma^{-1}+\varphi\right)}}\left(\frac{\bar{P}^{H}}{\bar{P}^{F}} \overline{R S}\right)^{\frac{1+\varphi}{\psi\left(\sigma^{-1}+\varphi\right)}}\left(\frac{\frac{1}{1-(1-\beta) \bar{a}^{a}}-\delta\left(\frac{1-\psi}{\left(\frac{\theta(1-\bar{\xi})}{\theta-1}\right)\left(\beta^{-1}-(1-\delta)\right)}\right)}{\frac{1}{1+\left(\frac{(1-\beta) \bar{a}^{a}}{(\overline{R S})^{1-\eta}}\right)}-\delta\left(\frac{1-\psi}{\left(\frac{\theta\left(1-\bar{\xi}^{*}\right)}{\theta-1}\right)\left(\beta^{-1}-(1-\delta)\right)}\right)}\right)^{\frac{-\sigma^{-1}}{\sigma^{-1}+\varphi}},
\end{aligned}
$$

where I have used the definition of the real exchange rate in (21) (i.e. $\overline{R S} \equiv \frac{\overline{S P}^{*}}{\bar{P}}$ ) and the fact that in steady state the law of one price holds, i.e. $\bar{P}^{F}=\bar{P}^{F *} \bar{S}$. Then, under the assumption that absorption in both countries differs only by a factor related to the steady state real exchange rate (i.e. $(\bar{C}+\bar{X})=$ $\left.(\overline{R S})^{\eta}\left(\bar{C}^{*}+\bar{X}^{*}\right)\right)$ I can infer the following restriction on relative steady state productivity levels from the ratio above in (246),

$\left(\begin{array}{c}\bar{A} \\ \bar{A}^{*}\end{array}\right)=(\overline{R S})^{\frac{\eta \psi \sigma^{-1}+(\eta \psi-1) \varphi-1}{1+\varphi}}\left(\frac{\bar{P}^{F}}{\bar{P}^{H}}\right)\left(\frac{1-(1-\beta) \bar{a}^{a}}{1+\frac{(1-\beta) \bar{a}^{a}}{(\overline{R S})^{1-\eta}}}\right)^{\frac{\psi\left(\sigma^{-1}+\varphi\right)}{1+\varphi}}\left(\frac{\frac{1}{1-(1-\beta) \bar{a}^{a}}-\delta\left(\frac{1-\psi}{\left(\frac{\theta(1-\bar{\xi})}{\theta-1}\right)\left(\beta^{-1}-(1-\delta)\right)}\right)}{\frac{1}{1+\frac{(1-\beta) \bar{a}^{a}}{(\overline{R S})^{1-\eta}}}-\delta\left(\frac{1-\psi}{\left(\frac{\theta\left(1-\bar{\xi}^{*}\right)}{\theta-1}\right)\left(\beta^{-1}-(1-\delta)\right)}\right)}\right)^{\frac{\sigma^{-1} \psi}{1+\varphi}}$ 
or simply,

$$
\begin{aligned}
& \left(\frac{\bar{A}}{\bar{A}^{*}}\right)=\left(1+\left(\frac{\phi_{H} \phi_{H}-\phi_{F} \phi_{F}}{\phi_{F}}\right)(1-\beta) \bar{a}^{a}\right)^{\frac{\eta \psi \sigma^{-1}+(\eta \psi-1) \varphi-1}{(1-\eta)(1+\varphi)}}\left(\frac{\phi_{F}+\phi_{H}(1-\beta) \bar{a}^{a}}{\phi_{F}-\phi_{F}(1-\beta) \bar{a}^{a}}\right)^{\frac{1}{1-\eta}} \times \ldots
\end{aligned}
$$

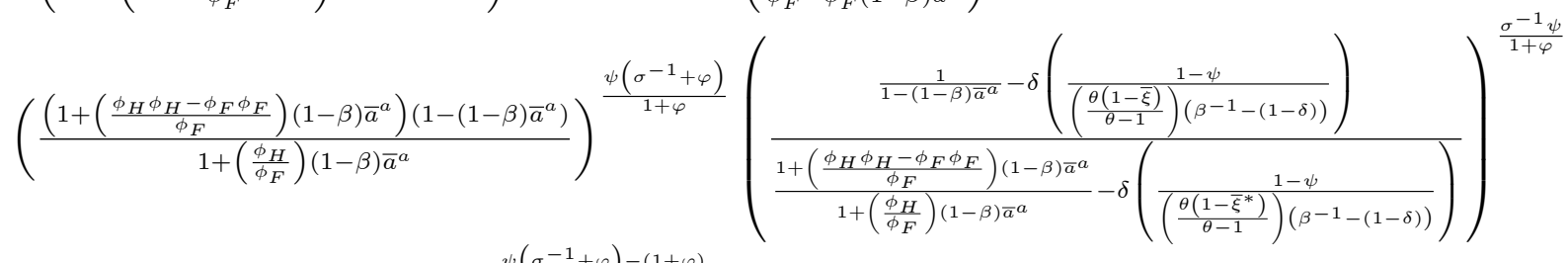

$$
\begin{aligned}
& =\left(1+\left(\frac{\phi_{H} \phi_{H}-\phi_{F} \phi_{F}}{\phi_{F}}\right)(1-\beta) \bar{a}^{a}\right)^{\frac{\psi\left(\sigma^{-1}+\varphi\right)-(1+\varphi)}{(1-\eta)(1+\varphi)}}\left(\frac{\phi_{F}+\phi_{H}(1-\beta) \bar{a}^{a}}{\phi_{F}-\phi_{F}(1-\beta) \bar{a}^{a}}\right)^{\frac{1}{1-\eta}} \times \ldots \\
& \left(\frac{1-(1-\beta) \bar{a}^{a}}{1+\left(\frac{\phi_{H}}{\phi_{F}}\right)(1-\beta) \bar{a}^{a}}\right)^{\frac{\psi\left(\sigma^{-1}+\varphi\right)}{1+\varphi}}\left(\frac{\frac{1}{1-(1-\beta) \bar{a}^{a}}-\delta\left(\frac{1-\psi}{\left(\frac{\theta(1-\bar{\xi})}{\theta-1}\right)\left(\beta^{-1}-(1-\delta)\right)}\right)^{\frac{\sigma^{-1} \psi}{1+\varphi}}}{\frac{1+\left(\frac{\phi_{H} \phi_{H}-\phi_{F} \phi_{F}}{\phi_{F}}\right)(1-\beta) \bar{a}^{a}}{1+\left(\frac{\phi_{H}}{\phi_{F}}\right)(1-\beta) \bar{a}^{a}}-\delta\left(\frac{1-\psi}{\left(\frac{\theta\left(1-\bar{\xi}^{*}\right)}{\theta-1}\right)\left(\beta^{-1}-(1-\delta)\right)}\right)}\right) \cdot
\end{aligned}
$$

The expression in (248) ties the ratio of the steady state productivities for the two countries to the structural parameters of the model. Under the assumption that the real net foreign asset position of the domestic household (relative to domestic absorption) is equal to zero, $\bar{a}^{a}=0$, then (248) reduces to simply,

$$
\left(\frac{\bar{A}}{\bar{A}^{*}}\right)=(\overline{R S})^{\frac{\eta \sigma^{-1}+(\eta \psi-1) \varphi-1}{1+\varphi}}\left(\frac{\bar{P}^{F}}{\bar{P}^{H}}\right)=1 .
$$

Since the real exchange rate is also a function of the terms of trade as noted in equation (147), then I can easily see from here that terms of trade is a function of the steady state productivity ratio $\left(\frac{\bar{A}}{\bar{A}^{*}}\right)$. However, I know that $\frac{\bar{P}^{F}}{\bar{P}^{H}}=\left(\frac{\phi_{F}+\phi_{H}(1-\beta) \bar{a}^{a}}{\phi_{F}-\phi_{F}(1-\beta) \bar{a}^{a}}\right)^{\frac{1}{1-\eta}}$ and $\overline{R S}=\left[1+\left(\frac{\phi_{H} \phi_{H}-\phi_{F} \phi_{F}}{\phi_{F}}\right)(1-\beta) \bar{a}^{a}\right]^{\frac{1}{1-\eta}}$, so the second equality in (249) follows under the assumption of zero real net foreign assets in steady state and I must conclude that,

$$
\bar{A}=\bar{A}^{*} \text {. }
$$

In general, however, I should not expect the productivity of both countries to be identical in steady state. For simplicity, I normalize the steady state productivity in the domestic country to be $\bar{A}=1$ and let equation (248) pin down the steady state productivity in the foreign country. 
Nineth Step. Finally, I look at the normalization of steady state aggregate productivity in the model one more time. I need to revisit the price-setting equations that I already derived in (213) and (215), i.e.,

$$
\begin{aligned}
\bar{A}= & \left(\frac{\bar{P}^{H}}{\bar{P}}\right)^{-1}\left(\frac{\theta(1-\bar{\xi})}{\theta-1}\right)^{\frac{1+(1-\psi) \varphi}{1+\varphi}}\left(\frac{\left(\beta^{-1}-(1-\delta)\right)^{1-\psi}}{\psi^{\frac{\psi}{1+\varphi}}(1-\psi)^{1-\psi}}\right) \times \ldots \\
& \left(1-(1-\beta) \bar{a}^{a}\right)^{\frac{\psi \varphi}{1+\varphi}}(\bar{C})^{\frac{\psi \sigma^{-1}}{1+\varphi}}(\bar{C}+\bar{X})^{\frac{\psi \varphi}{1+\varphi}}, \\
\bar{A}^{*}= & \left(\frac{\bar{P}^{F *}}{\bar{P}^{*}}\right)^{-1}\left(\frac{\theta\left(1-\bar{\xi}^{*}\right)}{\theta-1}\right)^{\frac{1+(1-\psi) \varphi}{1+\varphi}}\left(\frac{\left(\beta^{-1}-(1-\delta)\right)^{1-\psi}}{\psi^{\frac{\psi}{1+\varphi}}(1-\psi)^{1-\psi}}\right) \times \ldots \\
& \left(1+\frac{(1-\beta) \bar{a}^{a}}{(\overline{R S})^{1-\eta}}\right)^{\frac{\psi \varphi}{1+\varphi}}\left(\bar{C}^{*}\right)^{\frac{\psi \sigma^{-1}}{1+\varphi}}\left(\bar{C}^{*}+\bar{X}^{*}\right)^{\frac{\psi \varphi}{1+\varphi}} .
\end{aligned}
$$

Given my previous derivations, I know that the price ratios $\left(\frac{\bar{P}^{H}}{\bar{P}}\right)$ and $\left(\frac{\bar{P}^{F *}}{\bar{P}^{*}}\right)$, the aggregate consumption levels $\bar{C}$ and $\bar{C}^{*}$, and the absorption levels $(\bar{C}+\bar{X})$ and $\left(\bar{C}^{*}+\bar{X}^{*}\right)$, are all functions of the structural parameters of the model - including among them the real net foreign asset position of the domestic household relative to domestic absorption, $\bar{a}^{a}$. Therefore, the equations in (251) and (252) need to be satisfied in order for the steady state to be well-defined. Naturally, the long-run productivity levels of $\bar{A}$ and $\bar{A}^{*}$ must be chosen to satisfy these two conditions.

Let me assume that the real net foreign asset position is equal to zero, $\bar{a}^{a}=0$, then the conditions in (251) and (252) reduce to,

$$
\begin{aligned}
\bar{A} & =\left(\frac{\bar{P}^{H}}{\bar{P}}\right)^{-1}\left(\frac{\theta(1-\bar{\xi})}{\theta-1}\right)^{\frac{1+(1-\psi) \varphi}{1+\varphi}}\left(\frac{\left(\beta^{-1}-(1-\delta)\right)^{1-\psi}}{\psi^{\frac{\psi}{1+\varphi}}(1-\psi)^{1-\psi}}\right)(\bar{C})^{\frac{\psi \sigma}{1+\varphi}}(\bar{C}+\bar{X})^{\frac{\psi \varphi}{1+\varphi}} \\
\bar{A}^{*} & =\left(\frac{\bar{P}^{F *}}{\bar{P}^{*}}\right)^{-1}\left(\frac{\theta\left(1-\bar{\xi}^{*}\right)}{\theta-1}\right)^{\frac{1+(1-\psi) \varphi}{1+\varphi}}\left(\frac{\left(\beta^{-1}-(1-\delta)\right)^{1-\psi}}{\psi^{\frac{\psi}{1+\varphi}}(1-\psi)^{1-\psi}}\right)\left(\bar{C}^{*}\right)^{\frac{\psi \sigma}{1+\varphi}}\left(\bar{C}^{*}+\bar{X}^{*}\right)^{\frac{\psi \varphi}{1+\varphi}}
\end{aligned}
$$

However, under the same assumption that $\bar{a}^{a}=0$ it must follow that $\frac{\bar{P}^{H}}{\bar{P}}=\frac{\bar{P}^{F *}}{\bar{P}^{*}}=1$. Moreover, I can also show that absorption equalizes across countries (from $(244)-(245)$ ),

$$
\begin{aligned}
& \bar{C}+\bar{X}=(\bar{A})^{\frac{1+\varphi}{\psi\left(\sigma^{-1}+\varphi\right)}}\left(1-\delta\left(\frac{1-\psi}{\left(\frac{\theta(1-\bar{\xi})}{\theta-1}\right)\left(\beta^{-1}-(1-\delta)\right)}\right)\right)^{\frac{-\sigma^{-1}}{\sigma^{-1}+\varphi}} \times \ldots \\
& \left(\frac{\psi^{\psi}(1-\psi)^{(1-\psi)(1+\varphi)}}{\left(\frac{\theta(1-\bar{\xi})}{\theta-1}\right)^{1+(1-\psi) \varphi}\left(\beta^{-1}-(1-\delta)\right)^{(1-\psi)(1+\varphi)}}\right)^{\frac{1}{\psi\left(\sigma^{-1}+\varphi\right)}}, \\
& \bar{C}^{*}+\bar{X}^{*}=\left(\bar{A}^{*}\right)^{\frac{1+\varphi}{\psi\left(\varphi+\sigma^{-1}\right)}}\left(1-\delta\left(\frac{1-\psi}{\left(\frac{\theta\left(1-\bar{\xi}^{*}\right)}{\theta-1}\right)\left(\beta^{-1}-(1-\delta)\right)}\right)\right)^{\frac{-\sigma^{-1}}{\sigma-1}+\varphi} \times \ldots \\
& \left(\frac{\psi^{\psi}(1-\psi)^{(1-\psi)(1+\varphi)}}{\left(\frac{\theta\left(1-\bar{\xi}^{*}\right)}{\theta-1}\right)^{1+(1-\psi) \varphi}\left(\beta^{-1}-(1-\delta)\right)^{(1-\psi)(1+\varphi)}}\right)^{\frac{1}{\psi\left(\varphi+\sigma^{-1}\right)}},
\end{aligned}
$$


up to a scaling factor that depends on the productivity level in both countries (and possibly on the government subsidies), and so does consumption (from (228) - (229)),

$$
\begin{aligned}
\bar{C}= & \left.(\bar{A})^{\frac{1+\varphi}{\psi\left(\sigma^{-1}+\varphi\right)}}\left(\frac{\psi^{\psi}(1-\psi)^{(1-\psi)(1+\varphi)}}{\left(\frac{\theta(1-\bar{\xi})}{\theta-1}\right)^{1+(1-\psi) \varphi}\left(\beta^{-1}-(1-\delta)\right)^{(1-\psi)(1+\varphi)}}\right)\right)^{\frac{1}{\psi\left(\sigma^{-1}+\varphi\right)}} \times \ldots \\
& \left(1-\delta\left(\frac{1-\psi}{\left(\frac{\theta(1-\bar{\xi})}{\theta-1}\right)\left(\beta^{-1}-(1-\delta)\right)}\right)\right)^{\frac{\varphi}{\sigma-1}+\varphi}, \\
\bar{C}^{*}= & \left(\bar{A}^{*}\right)^{\frac{1+\varphi}{\psi\left(\varphi+\sigma^{-1}\right)}}\left(\frac{\psi^{\psi}(1-\psi)^{(1-\psi)(1+\varphi)}}{\left(\frac{\theta\left(1-\bar{\xi}^{*}\right)}{\theta-1}\right)^{1+(1-\psi) \varphi}\left(\beta^{-1}-(1-\delta)\right)^{(1-\psi)(1+\varphi)}}\right)^{\frac{1}{\psi\left(\varphi+\sigma^{-1}\right)}} \times \ldots \\
& \left(1-\delta\left(\frac{1-\psi}{\left(\frac{\theta\left(1-\bar{\xi}^{*}\right)}{\theta-1}\right)\left(\beta^{-1}-(1-\delta)\right)}\right)\right)^{\frac{\varphi}{\sigma^{-1}+\varphi}},
\end{aligned}
$$

up to a scaling factor that depends on the productivity level in both countries (and possibly on the government subsidies).

Recalling the domestic consumption in (226) and the domestic absorption in (241), then the price-setting condition in (251) reduces to,

$$
\begin{aligned}
& \bar{A}=\left(\frac{\bar{P}^{H}}{\bar{P}}\right)^{-1}\left(\frac{\theta(1-\bar{\xi})}{\theta-1}\right)^{\frac{1+(1-\psi) \varphi}{1+\varphi}}\left(\frac{\left(\beta^{-1}-(1-\delta)\right)^{1-\psi}}{\psi^{\frac{\psi}{1+\varphi}}(1-\psi)^{1-\psi}}\right)\left(1-(1-\beta) \bar{a}^{a}\right)^{\frac{\psi \varphi}{1+\varphi}}(\bar{C})^{\frac{\psi \sigma^{-1}}{1+\varphi}}(\bar{C}+\bar{X})^{\frac{\psi \varphi}{1+\varphi}} \\
& =(\bar{A})^{\frac{\sigma^{-1}}{\sigma^{-1}+\varphi}}\left(\frac{\bar{P}^{H}}{\bar{P}}\right)^{-\frac{\varphi}{\sigma^{-1}+\varphi}}\left(\frac{\left(\frac{\theta(1-\bar{\xi})}{\theta-1}\right)^{\frac{1+(1-\psi) \varphi}{1+\varphi}}\left(\beta^{-1}-(1-\delta)\right)^{1-\psi}}{\psi^{\frac{\psi}{1+\varphi}}(1-\psi)^{1-\psi}}\right)^{\frac{\varphi}{\sigma^{-1}+\varphi}} \times \ldots \\
& \left(\frac{1}{1-(1-\beta) \bar{a}^{a}}-\delta\left(\frac{1-\psi}{\left(\frac{\theta(1-\bar{\xi})}{\theta-1}\right)\left(\beta^{-1}-(1-\delta)\right)}\right)\right)^{\frac{\psi \varphi \sigma^{-1}}{\left(\sigma^{-1}+\varphi\right)(1+\varphi)}}\left[\left(1-(1-\beta) \bar{a}^{a}\right)(\bar{C}+\bar{X})\right]^{\frac{\psi \varphi}{1+\varphi}} \\
& =(\bar{A})^{\frac{\sigma^{-1}+\varphi}{\sigma-1}+\varphi}\left(\frac{\bar{P}^{H}}{\bar{P}}\right)^{-\frac{\varphi}{\sigma^{-1}+\varphi}}\left(\frac{\bar{P}^{H}}{\bar{P}}\right)^{\frac{\varphi}{\sigma^{-1}+\varphi}}\left(\frac{\left(\frac{\theta(1-\bar{\xi})}{\theta-1}\right)^{\frac{1+(1-\psi) \varphi}{1+\varphi}}\left(\beta^{-1}-(1-\delta)\right)^{1-\psi}}{\psi^{\frac{\psi}{1+\varphi}}(1-\psi)^{1-\psi}}\right)^{\frac{\varphi}{\sigma-1+\varphi}} \times \ldots \\
& \left(\frac{\left(\frac{\theta(1-\bar{\xi})}{\theta-1}\right)^{\frac{1+(1-\psi) \varphi}{1+\varphi}}\left(\beta^{-1}-(1-\delta)\right)^{1-\psi}}{\psi^{\frac{\psi}{1+\varphi}}(1-\psi)^{1-\psi}}\right)^{-\frac{\varphi}{\sigma^{-1}+\varphi}}\left(\frac{1}{1-(1-\beta) \bar{a}^{\alpha}}-\delta\left(\frac{1-\psi}{\left(\frac{\theta(1-\bar{\xi})}{\theta-1}\right)\left(\beta^{-1}-(1-\delta)\right)}\right)\right)^{\frac{\psi \varphi \sigma^{-1}}{\left(\sigma^{-1}+\varphi\right)(1+\varphi)}} \times \ldots \\
& \left(\frac{1}{1-(1-\beta) \bar{a}^{a}}-\delta\left(\frac{1-\psi}{\left(\frac{\theta(1-\bar{\xi})}{\theta-1}\right)\left(\beta^{-1}-(1-\delta)\right)}\right)\right)^{\frac{-\psi \varphi \sigma^{-1}}{\left(\sigma^{-1}+\varphi\right)(1+\varphi)}} \\
& =\bar{A} \text {, }
\end{aligned}
$$

which is trivially satisfied for any value of $\bar{A}$ (as expected). Recalling the foreign consumption in (227) and 
the foreign absorption in (243), then the price-setting condition in (252) reduces to,

$$
\begin{aligned}
& \bar{A}^{*}=\left(\frac{\bar{P}^{F *}}{\bar{P}^{*}}\right)^{-1}\left(\frac{\theta\left(1-\bar{\xi}^{*}\right)}{\theta-1}\right)^{\frac{1+(1-\psi) \varphi}{1+\varphi}}\left(\frac{\left(\beta^{-1}-(1-\delta)\right)^{1-\psi}}{\psi^{\frac{\psi}{1+\varphi}}(1-\psi)^{1-\psi}}\right)\left(1+\frac{(1-\beta) \bar{a}^{a}}{(\overline{R S})^{1-\eta}}\right)^{\frac{\psi \varphi}{1+\varphi}}\left(\bar{C}^{*}\right)^{\frac{\psi \sigma}{1+\varphi}}\left(\bar{C}^{*}+\bar{X}^{*}\right)^{\frac{\psi \varphi}{1+\varphi}} \\
& =\left(\bar{A}^{*}\right)^{\frac{\sigma^{-1}}{\varphi+\sigma^{-1}}}\left(\frac{\bar{P}^{F *}}{\bar{P}^{*}}\right)^{-\frac{\varphi}{\varphi+\sigma^{-1}}}\left(\frac{\left(\frac{\theta\left(1-\bar{\xi}^{*}\right)}{\theta-1}\right)^{\frac{1+(1-\psi) \varphi}{1+\varphi}}\left(\beta^{-1}-(1-\delta)\right)^{1-\psi}}{\psi^{\frac{\psi}{1+\varphi}}(1-\psi)^{1-\psi}}\right)^{\frac{\varphi}{\varphi+\sigma^{-1}}} \times \ldots \\
& \left(\frac{1}{1+\left(\frac{(1-\beta) \bar{a}^{a}}{(\overline{R S})^{1-\eta}}\right)}-\delta\left(\frac{1-\psi}{\left(\frac{\theta\left(1-\bar{\xi}^{*}\right)}{\theta-1}\right)\left(\beta^{-1}-(1-\delta)\right)}\right)\right)^{\frac{\psi \varphi \sigma^{-1}}{\left(\sigma^{-1}+\varphi\right)(1+\varphi)}}\left[\left(1+\frac{(1-\beta) \bar{a}^{a}}{(\overline{R S})^{1-\eta}}\right)\left(\bar{C}^{*}+\bar{X}^{*}\right)\right]^{\frac{\psi \varphi}{1+\varphi}} \\
& =\left(\bar{A}^{*}\right)^{\frac{\varphi+\sigma^{-1}}{\varphi+\sigma^{-1}}}\left(\frac{\bar{P}^{F *}}{\bar{P}^{*}}\right)^{\frac{\varphi}{\varphi+\sigma^{-1}}}\left(\frac{\bar{P}^{F *}}{\bar{P}^{*}}\right)^{-\frac{\varphi}{\varphi+\sigma^{-1}}}\left(\frac{\left(\frac{\theta\left(1-\bar{\xi}^{*}\right)}{\theta-1}\right)^{\frac{1+(1-\psi) \varphi}{1+\varphi}}\left(\beta^{-1}-(1-\delta)\right)^{1-\psi}}{\psi^{\frac{\psi}{1+\varphi}}(1-\psi)^{1-\psi}}\right)^{\frac{\varphi}{\varphi+\sigma^{-1}}} \times \ldots \\
& \left(\frac{\left(\frac{\theta\left(1-\bar{\xi}^{*}\right)}{\theta-1}\right)^{\frac{1+(1-\psi) \varphi}{1+\varphi}}\left(\beta^{-1}-(1-\delta)\right)^{1-\psi}}{\psi^{\frac{\psi}{1+\varphi}}(1-\psi)^{1-\psi}}\right)^{-\frac{\varphi}{\varphi+\sigma^{-1}}}\left(\frac{1}{1+\left(\frac{(1-\beta) \bar{a}^{a}}{(\overline{R S})^{1-\eta}}\right)}-\delta\left(\frac{1-\psi}{\left(\frac{\theta\left(1-\bar{\xi}^{*}\right)}{\theta-1}\right)\left(\beta^{-1}-(1-\delta)\right)}\right)\right)^{\frac{\psi \varphi \sigma^{-1}}{\left(\sigma^{-1}+\varphi\right)(1+\varphi)}} \times \ldots \\
& \left(\frac{1}{1+\left(\frac{(1-\beta) \bar{a}^{a}}{(R S)^{1-\eta}}\right)}-\delta\left(\frac{1-\psi}{\left(\frac{\theta\left(1-\bar{\xi}^{*}\right)}{\theta-1}\right)\left(\beta^{-1}-(1-\delta)\right)}\right)\right)^{\frac{-\psi \varphi \sigma^{-1}}{\left(\sigma^{-1}+\varphi\right)(1+\varphi)}} \\
& =\bar{A}^{*} \text {, }
\end{aligned}
$$

which again holds trivially for any value of $\bar{A}^{*}$ (as expected). What this implies is that I can normalize the steady state productivity level to take any value as long as the ratio satisfies (248). As I already know from (250), in the special case with $\bar{a}^{a}=0$ the productivity levels in both countries are equal and the conventional normalization implies that $\bar{A}=\bar{A}^{*}=1$. In the general case with $\bar{a}^{a} \neq 0$, however, I cannot ensure anymore that the steady state productivities in both countries would be the same. The normalization, however, still implies that the level of steady state productivity is normalized to one in one of the two countries, since the only thing that matters in order to ensure that the steady state is well-defined in the general case where $\bar{a}^{a} \neq 0$ is the ratio of the productivities as defined by condition (247) (or, more compactly, by condition $(248))$.

For the purpose of cross-validating my previous results on the steady state productivity ratio, I can take 
the ratio between the price-setting conditions in (251) and (252), i.e.,

$$
\begin{aligned}
& \bar{A} \bar{A}^{*}=\frac{\left(\frac{\bar{P}^{H}}{\bar{P}}\right)^{-1}\left(\frac{\left(\frac{\theta(1-\bar{\xi})}{\theta-1}\right)^{\frac{1+(1-\psi) \varphi}{1+\varphi}}\left(\beta^{-1}-(1-\delta)\right)^{1-\psi}}{\psi^{\frac{\psi}{1+\varphi}}(1-\psi)^{1-\psi}}\right)\left(1-(1-\beta) \bar{a}^{a}\right)^{\frac{\psi \varphi}{1+\varphi}}(\bar{C})^{\frac{\psi \sigma}{1+\varphi}}(\bar{C}+\bar{X})^{\frac{\psi \varphi}{1+\varphi}}}{\left(\frac{\bar{P}^{F *}}{\bar{P}^{*}}\right)^{-1}\left(\frac{\left(\frac{\theta\left(1-\bar{\xi}^{*}\right)}{\theta-1}\right)^{\frac{1+(1-\psi) \varphi}{1+\varphi}}\left(\beta^{-1}-(1-\delta)\right)^{1-\psi}}{\psi^{\frac{\psi}{1+\varphi}}(1-\psi)^{1-\psi}}\right)\left(1+\frac{(1-\beta) \bar{a}^{a}}{(\overline{R S})^{1-\eta}}\right)^{\frac{\psi \varphi}{1+\varphi}}\left(\bar{C}^{*}\right)^{\frac{\psi \sigma-1}{1+\varphi}}\left(\bar{C}^{*}+\bar{X}^{*}\right)^{\frac{\psi \varphi}{1+\varphi}}} \\
& =\frac{\overline{S P}^{F *}}{\bar{P}^{H}} \frac{\bar{P}}{\overline{S P}^{*}}\left(\frac{1-(1-\beta) \bar{a}^{a}}{1+\frac{(1-\beta) \bar{a}^{a}}{(\overline{R S})^{1-\eta}}}\right)^{\frac{\psi \varphi}{1+\varphi}}\left(\frac{\bar{C}}{\bar{C}^{*}}\right)^{\frac{\psi \sigma^{-1}}{1+\varphi}}\left(\frac{\bar{C}+\bar{X}}{\bar{C}^{*}+\bar{X}^{*}}\right)^{\frac{\psi \varphi}{1+\varphi}} \\
& =\frac{\overline{S P}^{F *}}{\bar{P}^{H}} \frac{\bar{P}}{\overline{S P}^{*}}\left(\frac{1-(1-\beta) \bar{a}^{a}}{1+\frac{(1-\beta) \bar{a}^{a}}{(\overline{R S})^{1-\eta}}}\right)^{\frac{\psi \varphi}{1+\varphi}}\left(\frac{\bar{C}}{\bar{C}^{*}}\right)^{\frac{\psi \sigma}{1+\varphi}}(\overline{R S})^{\eta \frac{\psi \varphi}{1+\varphi}} \\
& =\frac{\bar{P}^{F}}{\bar{P}^{H}}(\overline{R S})^{\eta \frac{\psi \varphi}{1+\varphi}-1}\left(\frac{1-(1-\beta) \bar{a}^{a}}{1+\frac{(1-\beta) \bar{a}^{a}}{(\overline{R S})^{1-\eta}}}\right)^{\frac{\psi \varphi}{1+\varphi}}\left(\frac{\bar{C}}{\bar{C}^{*}}\right)^{\frac{\psi \sigma}{1+\varphi}}
\end{aligned}
$$

where the third equality makes use of the maintained assumption that absorption in both countries differs only by a factor related to the steady state real exchange rate (i.e. $(\bar{C}+\bar{X})=(\overline{R S})^{\eta}\left(\bar{C}^{*}+\bar{X}^{*}\right)$ ). In turn, the fourth equality uses the definition of the real exchange rate in (21) (i.e. $\overline{R S} \equiv \frac{\overline{S P}^{*}}{\bar{P}}$ ) and the fact that in steady state the law of one price holds, i.e. $\bar{P}^{F}=\bar{P}^{F *} \bar{S}$. Then, using the aggregate consumption expressions 
for both countries given in (226) and (227) I obtain that,

$$
\begin{aligned}
& \frac{\bar{A}}{\bar{A}^{*}}=\bar{P}^{F}(\overline{R S})^{\frac{\eta \psi \varphi}{1+\varphi}-1}\left(\frac{1-(1-\beta) \bar{a}^{a}}{1+\frac{1-\beta \bar{a}^{a}}{(\overline{R S})^{1-\eta}}}\right)^{\frac{\psi \varphi}{1+\varphi}}\left(\frac{(\bar{A})^{\frac{1+\varphi}{\psi\left(\sigma^{-1}+\varphi\right)}}\left(\frac{\bar{P}^{H}}{\bar{P}}\right)^{\frac{1+\varphi}{\psi\left(\sigma^{-1}+\varphi\right)}}}{\left(\bar{A}^{*}\right)^{\left.\frac{1+\varphi}{\psi\left(\varphi+\sigma^{-1}\right.}\right)}\left(\frac{\bar{P}^{F *}}{\bar{P}^{*}}\right)^{\frac{1+\varphi}{\psi\left(\varphi+\sigma^{-1}\right)}}}\right)^{\frac{\psi \sigma^{-1}}{1+\varphi}} \times \ldots \\
& \left(\frac{\left(\frac{\psi^{\psi}(1-\psi)(1-\psi)(1+\varphi)}{\left(\frac{\theta(1-\bar{\xi})}{\theta-1}\right)^{1+(1-\psi) \varphi}\left(\beta^{-1}-(1-\delta)\right)^{(1-\psi)(1+\varphi)}}\right)^{\frac{1}{\psi\left(\sigma^{-1}+\varphi\right)}}\left(\frac{1}{1-(1-\beta) \bar{a}^{a}}-\delta\left(\frac{1-\psi}{\left(\frac{\theta(1-\bar{\xi})}{\theta-1}\right)\left(\beta^{-1}-(1-\delta)\right)}\right)\right)^{\frac{\varphi}{\sigma-1+\varphi}}}{\left.\left.\left.\left(\frac{\psi^{\psi(1-\psi)}(1-\psi)(1+\varphi)}{\left(\frac{\theta\left(1-\bar{\xi}^{*}\right)}{\theta-1}\right)^{1+(1-\psi) \varphi}\left(\beta^{-1}-(1-\delta)\right)^{(1-\psi)(1+\varphi)}}\right)^{\frac{1}{\psi\left(\varphi+\sigma^{-1}\right)}}\left(\frac{1}{1+\left(\frac{(1-\beta)^{a}}{\left.(\overline{R S})^{1-\eta}\right)}-\delta\left(\frac{\varphi \sigma^{-1}}{1+\varphi}\right.\right.}\right)\right)^{\frac{1}{\sigma-1+\varphi}}\right)^{\left.\frac{\theta\left(1-\bar{\xi}^{*}\right)}{\theta-1}\right)\left(\beta^{-1}-(1-\delta)\right)}\right)}\right. \\
& =\left(\frac{\bar{A}}{\bar{A}^{*}}\right)^{\frac{\sigma^{-1}}{\sigma-1}+\varphi}\left(\frac{\bar{P}^{H}}{\overline{S P}{ }^{F *}} \frac{\overline{S P}^{*}}{\bar{P}}\right)^{\frac{\sigma^{-1}}{\sigma-1}+\varphi} \frac{\bar{P}^{F}}{\bar{P}^{H}}(\overline{R S})^{\frac{\eta \psi \varphi}{1+\varphi}-1}\left(\frac{1-(1-\beta) \bar{a}^{a}}{1+\frac{(1-\beta) \bar{a}^{a}}{(\overline{R S})^{1-\eta}}}\right)^{\frac{\psi \varphi}{1+\varphi}} \times \ldots \\
& \left(\frac{\frac{1}{1-(1-\beta) \bar{a}^{a}}-\delta\left(\frac{1-\psi}{\left(\frac{\theta(1-\bar{\xi})}{\theta-1}\right)\left(\beta^{-1}-(1-\delta)\right)}\right)}{\frac{1}{1+\left(\frac{(1-\beta) \bar{a}^{a}}{(\overline{R S})^{1-\eta}}\right)}-\delta\left(\frac{1-\psi}{\left(\frac{\theta\left(1-\bar{\xi}^{*}\right)}{\theta-1}\right)\left(\beta^{-1}-(1-\delta)\right)}\right)}\right)^{\frac{\varphi \psi \sigma^{-1}}{\left(\sigma^{-1}+\varphi\right)(1+\varphi)}}
\end{aligned}
$$

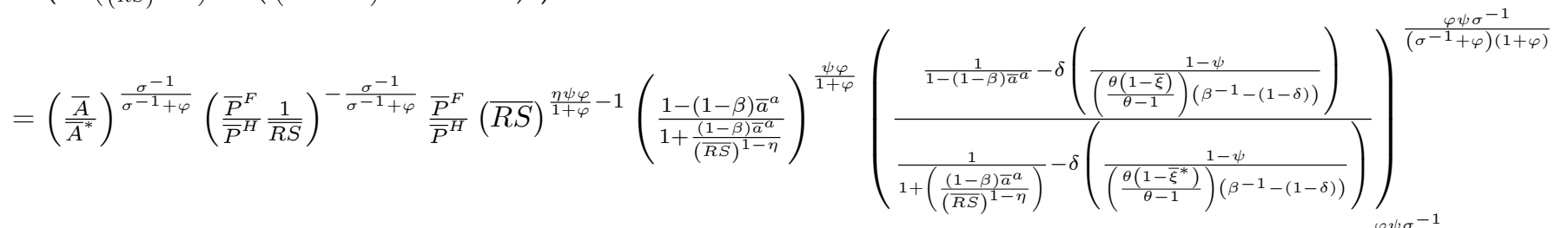

$$
\begin{aligned}
& =\left(\frac{\bar{A}}{\bar{A}^{*}}\right)^{\frac{\sigma^{-1}}{\sigma^{-1}+\varphi}}\left(\frac{\bar{P}^{F}}{\bar{P}^{H}} \frac{1}{\overline{R S}}\right)^{1-\frac{\sigma^{-1}}{\sigma^{-1}+\varphi}}(\overline{R S})^{\frac{\eta \psi \varphi}{1+\varphi}}\left(\frac{1-(1-\beta))^{a}}{1+\frac{\left(1-\beta \bar{a}^{a}\right.}{(\overline{R S})^{1-\eta}}}\right)^{\frac{\psi \varphi}{1+\varphi}}\left(\frac{\frac{1}{1-(1-\beta) \bar{a}^{a}}-\delta\left(\frac{1-\psi}{\left(\frac{\theta(1-\bar{\xi})}{\theta-1}\right)\left(\beta^{-1}-(1-\delta)\right)}\right)}{\frac{1}{1+\left(\frac{(1-\beta) \bar{a}^{a}}{(\overline{R S})^{1-\eta}}\right)}-\delta\left(\frac{1-\psi}{\left(\frac{\theta\left(1-\bar{\xi}^{*}\right)}{\theta-1}\right)\left(\beta^{-1}-(1-\delta)\right)}\right)}\right)^{\frac{\varphi \psi \sigma^{-1}}{\left(\sigma^{-1}+\varphi\right)(1+\varphi)}} \\
& =\left(\frac{\bar{A}}{\bar{A}^{*}}\right)^{\frac{\sigma^{-1}}{\sigma^{-1}+\varphi}}\left(\frac{\bar{P}^{F}}{\bar{P}^{H}} \frac{1}{\overline{R S}}\right)^{\frac{\varphi}{\sigma^{-1}+\varphi}}(\overline{R S})^{\frac{\eta \psi \varphi}{1+\varphi}}\left(\frac{1-(1-\beta)^{a}}{1+\frac{(1-\beta) \bar{a}^{a}}{(R S)^{1-\eta}}}\right)^{\frac{\psi \varphi}{1+\varphi}}\left(\frac{\frac{1}{1-(1-\beta) \bar{a}^{a}}-\delta\left(\frac{1-\psi}{\left(\frac{\theta(1-\bar{\xi})}{\theta-1}\right)\left(\beta^{-1}-(1-\delta)\right)}\right)}{\frac{1}{1+\left(\frac{(1-\beta) \bar{a}^{a}}{(R S)^{1-\eta}}\right)}-\delta\left(\frac{1-\psi}{\left(\frac{\theta\left(1-\bar{\xi}^{*}\right)}{\theta-1}\right)\left(\beta^{-1}-(1-\delta)\right)}\right)}\right)^{\frac{\varphi \psi \sigma \sigma^{-1}}{\left(\sigma^{-1}+\varphi\right)(1+\varphi)}}
\end{aligned}
$$

Then, after a little bit of extra algebra, I end up with the following condition,

$$
\left(\frac{\bar{A}}{\bar{A}^{*}}\right)^{\frac{\varphi}{\sigma^{-1}+\varphi}}=\left(\frac{\bar{P}^{F}}{\bar{P}^{H}} \frac{1}{\overline{R S}}\right)^{\frac{\varphi}{\sigma^{-1}+\varphi}}(\overline{R S})^{\frac{\eta \psi \varphi}{1+\varphi}}\left(\frac{1-(1-\beta) \bar{a}^{a}}{1+\frac{(1-\beta) \bar{a}^{a}}{(\overline{R S})^{1-\eta}}}\right)^{\frac{\psi \varphi}{1+\varphi}}\left(\frac{\frac{1}{1-(1-\beta) \bar{a}^{a}}-\delta\left(\frac{1-\psi}{\left(\frac{\theta(1-\bar{\xi})}{\theta-1}\right)\left(\beta^{-1}-(1-\delta)\right)}\right)}{\frac{1}{1+\left(\frac{(1-\beta) \bar{a}^{a}}{(\overline{R S})^{1-\eta}}\right)}-\delta\left(\frac{1-\psi}{\left(\frac{\theta\left(1-\bar{\xi}^{*}\right)}{\theta-1}\right)\left(\beta^{-1}-(1-\delta)\right)}\right)}\right)^{\frac{\varphi \psi \sigma^{-1}}{\left(\sigma^{-1}+\varphi\right)(1+\varphi)}}
$$


or simply,

$$
\left(\frac{\bar{A}}{\bar{A}^{*}}\right)=(\overline{R S})^{\frac{\eta \psi \sigma^{-1}+(\eta \psi-1) \varphi-1}{1+\varphi}}\left(\frac{\bar{P}^{F}}{\bar{P}^{H}}\right)\left(\frac{1-(1-\beta) \bar{a}^{a}}{1+\frac{(1-\beta) \bar{a}^{a}}{(\overline{R S})^{1-\eta}}}\right)^{\frac{\psi\left(\sigma^{-1}+\varphi\right)}{1+\varphi}}\left(\frac{\frac{1}{1-(1-\beta) \bar{a}^{a}}-\delta\left(\frac{1-\psi}{\left(\frac{\theta(1-\bar{\xi})}{\theta-1}\right)\left(\beta^{-1}-(1-\delta)\right)}\right)}{\frac{1}{1+\frac{(1-\beta) \bar{a}^{a}}{(\overline{R S})^{1-\eta}}}-\delta\left(\frac{1-\psi}{\left(\frac{\theta\left(1-\bar{\xi}^{*}\right)}{\theta-1}\right)\left(\beta^{-1}-(1-\delta)\right)}\right)}\right)^{\frac{\sigma^{-1} \psi}{1+\varphi}}
$$

which is exactly the same condition that I derived for the productivity ratio in (247). Therefore, this confirms that in a steady state with a real net foreign asset position for the domestic household (relative to domestic absorption) different than zero, $\bar{a}^{a} \neq 0$, it has to be the case that the productivities across countries are unequal in order to reconcile the model with the assumption that the net foreign asset position is different than zero (and, consequently, the trade balance is also different than zero).

For simplicity, I shall assume that the long-run steady state productivity of the domestic country is normalized to one, i.e. $\bar{A}=1$. Hence, the foreign productivity level would be characterized by equation (247) (or, more compactly, by condition (248)).

\section{The Log-Linearized Equilibrium Conditions}

Here, I log-linearize the equilibrium conditions around the deterministic zero-inflation steady state. I denote $\widehat{n}_{t} \equiv \ln N_{t}-\ln \bar{N}$ the deviation of a variable $N_{t}$ in $\operatorname{logs}$ from its steady state.

\subsection{The Households' Equilibrium Conditions}

The log-linearization of the Euler equations in (58)-(60) is quite standard, and characterizes the consumptionsavings decisions of the households as follows,

$$
\begin{aligned}
& \widehat{c}_{t} \approx \mathbb{E}_{t}\left[\widehat{c}_{t+1}\right]-\sigma\left(\widehat{i}_{t}-\mathbb{E}_{t}\left[\widehat{\pi}_{t+1}\right]\right), \\
& \widehat{c}_{t}^{*} \approx \mathbb{E}_{t}\left[\widehat{c}_{t+1}^{*}\right]-\sigma\left(\hat{i}_{t}^{*}-\mathbb{E}_{t}\left[\widehat{\pi}_{t+1}^{*}\right]\right) .
\end{aligned}
$$

The international risk-sharing equation comes from the log-linearization of (59), i.e.,

$$
\begin{aligned}
\mathbb{E}_{t}\left[-\sigma^{-1}\left(\widehat{c}_{t+1}-\widehat{c}_{t}\right)-\widehat{\pi}_{t+1}+\left(\widehat{s}_{t+1}-\widehat{s}_{t}\right)+\widehat{i}_{t}^{*}\right] & \approx\left(\frac{\mu \overline{N F A}}{1+\mu(\overline{N F A}-\bar{a})}\right) \widehat{n f a}_{t+1} \\
& =\mu \bar{a} \widehat{n f a}_{t+1},
\end{aligned}
$$

or more compactly,

$$
\begin{aligned}
\mathbb{E}_{t}\left[\widehat{s}_{t+1}-\widehat{s}_{t}\right] & \approx \sigma^{-1} \mathbb{E}_{t}\left[\widehat{c}_{t+1}-\widehat{c}_{t}\right]+\mathbb{E}_{t}\left[\widehat{\pi}_{t+1}\right]-\widehat{i}_{t}^{*}+\mu \bar{a} \widehat{n f a} \\
& \approx \widehat{i}_{t+1}-\widehat{i}_{t}^{*}+\mu \bar{a} \widehat{n f a} a_{t+1},
\end{aligned}
$$


where the second approximation follows from the Euler equation in (261). The steady state real net foreign asset position of the domestic household $\bar{a}$ is pined down by the definition in (165) as,

$$
\bar{a} \equiv \bar{a}^{a}(\bar{C}+\bar{X}),
$$

while the steady state domestic absorption is given by (244) as,

$$
\begin{aligned}
& \bar{C}+\bar{X}=(\bar{A})^{\frac{1+\varphi}{\psi\left(\sigma^{-1}+\varphi\right)}}\left(1-(1-\beta) \bar{a}^{a}\right)^{{\frac{1+\varphi}{(1-\eta) \psi\left(\sigma^{-1}+\varphi\right)}}^{-1}} \times \ldots \\
& \left(\frac{1}{1-(1-\beta) \bar{a}^{a}}-\delta\left(\frac{1-\psi}{\left(\frac{\theta(1-\bar{\xi})}{\theta-1}\right)\left(\beta^{-1}-(1-\delta)\right)}\right)\right)^{\frac{-\sigma^{-1}}{\sigma-1+\varphi}} \times \ldots \\
& \left(\frac{\psi^{\psi}(1-\psi)^{(1-\psi)(1+\varphi)}}{\left(\frac{\theta(1-\bar{\xi})}{\theta-1}\right)^{1+(1-\psi) \varphi}\left(\beta^{-1}-(1-\delta)\right)^{(1-\psi)(1+\varphi)}}\right)^{\frac{1}{\psi\left(\sigma^{-1}+\varphi\right)}},
\end{aligned}
$$

The steady state productivity of the domestic country is normalized to one, $\bar{A}=1$.

From here, after some easy manipulations on equation (263), I obtain that the uncovered interest rate parity condition does not hold (even for a first-order approximation) since a risk premium term tied to the real net foreign asset position of the domestic household appears now in the expression, i.e.,

$$
\widehat{i}_{t}^{*}-\widehat{i}_{t}+\mathbb{E}_{t}\left[\widehat{s}_{t+1}-\widehat{s}_{t}\right] \approx \mu \bar{a} \widehat{n f a} a_{t+1} .
$$

More precisely, I obtain that the interest rate spread plus the expected changes in the nominal exchange rate must be proportional to the real net foreign asset position of the domestic household. Alternatively, I can represent equation (264) in real terms as follows,

$$
\left(\widehat{i}_{t}^{*}-\mathbb{E}_{t}\left[\widehat{\pi}_{t+1}^{*}\right]\right)-\left(\widehat{i}_{t}-\mathbb{E}_{t}\left[\widehat{\pi}_{t+1}\right]\right)+\mathbb{E}_{t}\left[\Delta \widehat{r s}_{t+1}\right] \approx \mu \bar{a} \widehat{n f a} a_{t+1},
$$

and,

$$
\mathbb{E}_{t}\left[\widehat{c}_{t+1}^{*}-\widehat{c}_{t}^{*}\right]-\mathbb{E}_{t}\left[\widehat{c}_{t+1}-\widehat{c}_{t}\right]+\sigma \mathbb{E}_{t}\left[\Delta \widehat{r s}_{t+1}\right] \approx \sigma \mu \bar{a} \widehat{n f a}{ }_{t+1},
$$

using the log-linearization of the real exchange rate in (21), i.e. $\widehat{r s}_{t}=\widehat{s}_{t}+\widehat{p}_{t}^{*}-\widehat{p}_{t}$ and $\Delta \widehat{r s}_{t+1} \equiv \widehat{r s}_{t+1}-\widehat{r s}_{t}$, and the Euler equations in (261) and (262).

The log-linearization of the resource constraint in (117) gives me the characterization of the dynamics of the real net foreign asset position of the domestic household as follows,

$$
\begin{aligned}
& -\widehat{i}_{t}^{*}+\left(\frac{(1+\mu(\overline{N F A}-\bar{a})) \overline{N F A}}{\overline{N F A}+\frac{\mu}{2}(\overline{N F A}-\bar{a})^{2}}\right) \widehat{n f a}_{t+1} \\
& \approx\left(\frac{\overline{N F A}}{\frac{1}{\bar{I}^{*}}\left(\overline{N F A}+\frac{\mu}{2}(\overline{N F A}-\bar{a})^{2}\right)}\right)\left(\left(\widehat{s}_{t}-\widehat{s}_{t-1}\right)+\left(\widehat{p}_{t-1}-\widehat{p}_{t}\right)+\widehat{n f a}_{t}\right)+\ldots \\
& \phi_{H}(1-\eta)\left(\frac{\left(\frac{\bar{P} H}{\bar{P}}\right)^{1-\eta}(\bar{C}+\bar{X})}{\frac{1}{\bar{I}^{*}}\left(\overline{N F A}+\frac{\mu}{2}(\overline{N F A}-\bar{a})^{2}\right)}\right)\left(\widehat{p}_{t}^{H}-\widehat{p}_{t}\right)+\phi_{F}(1-\eta)\left(\frac{\overline{R S}\left(\frac{\bar{p}^{H *}}{\bar{P}^{*}}\right)^{1-\eta}\left(\bar{C}^{*}+\bar{X}^{*}\right)}{\frac{1}{\bar{I}^{*}}\left(\overline{N F A}+\frac{\mu}{2}(\overline{N F A}-\bar{a})^{2}\right)}\right)\left(\widehat{p}_{t}^{H *}-\widehat{p}_{t}^{*}\right)+\ldots
\end{aligned}
$$

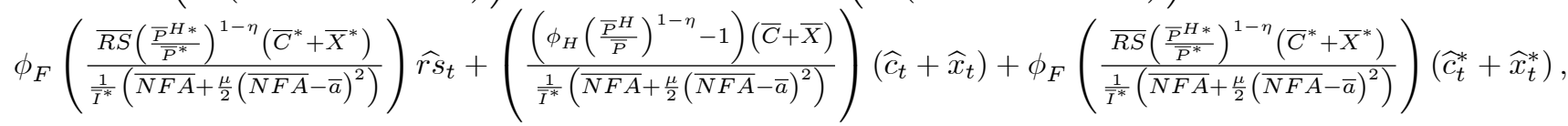


and,

$$
\begin{aligned}
-\widehat{i}_{t}^{*} & +\widehat{n f a}_{t+1} \\
& \approx \bar{I}^{*}\left(\left(\widehat{s}_{t}-\widehat{s}_{t-1}\right)+\left(\widehat{p}_{t-1}-\widehat{p}_{t}\right)+\widehat{n f a} a_{t}\right)+\ldots \\
& \phi_{H}(1-\eta) \bar{I}^{*}\left(\frac{\left(\frac{\overline{P^{H}}}{\overline{\bar{P}}}\right)^{1-\eta}(\bar{C}+\bar{X})}{\bar{a}}\right)\left(\widehat{p}_{t}^{H}-\widehat{p}_{t}\right)+\phi_{F}(1-\eta) \bar{I}^{*}\left(\frac{\overline{R S}\left(\frac{\bar{P}^{H *}}{\bar{P}^{*}}\right)^{1-\eta}\left(\bar{C}^{*}+\bar{X}^{*}\right)}{\bar{a}}\right)\left(\widehat{p}_{t}^{H *}-\widehat{p}_{t}^{*}\right)+\ldots \\
& \phi_{F} \bar{I}^{*}\left(\frac{\overline{R S}\left(\frac{\bar{P}^{H *}}{\overline{P^{*}}}\right)^{1-\eta}\left(\bar{C}^{*}+\bar{X}^{*}\right)}{\bar{a}}\right) \widehat{r s}_{t}+\bar{I}^{*}\left(\frac{\left(\phi_{H}\left(\frac{\bar{P}^{H}}{\bar{P}}\right)^{1-\eta}-1\right)(\bar{C}+\bar{X})}{\bar{a}}\right)\left(\widehat{c}_{t}+\widehat{x}_{t}\right)+\phi_{F} \bar{I}^{*}\left(\frac{\overline{R S}\left(\frac{\bar{P}^{H *}}{\bar{P}^{*}}\right)^{1-\eta}\left(\bar{C}^{*}+\bar{X}^{*}\right)}{\bar{a}}\right)\left(\widehat{c}_{t}^{*}+\widehat{x}_{t}^{*}\right) .
\end{aligned}
$$

The steady state price ratios $\left(\frac{\bar{P}^{F}}{\bar{P}}\right)$ and $\left(\frac{\bar{P}^{H}}{\bar{P}}\right)$ and the real exchange rate $\overline{R S}$ are tied to the steady state terms of trade by (147), (148) and (149) (or to structural parameters by (175), (176) and (177)), i.e.,

$$
\begin{aligned}
& \overline{R S}=\frac{\overline{S P}^{*}}{\bar{P}}=\left[\frac{\phi_{F}+\phi_{H}\left(\frac{\bar{P}^{F}}{\bar{P}^{H}}\right)^{1-\eta}}{\phi_{H}+\phi_{F}\left(\frac{\bar{P}^{F}}{\bar{P}^{H}}\right)^{1-\eta}}\right]^{\frac{1}{1-\eta}}=\left[1+\left(\frac{\phi_{H} \phi_{H}-\phi_{F} \phi_{F}}{\phi_{F}}\right)(1-\beta) \bar{a}^{a}\right]^{\frac{1}{1-\eta}}, \\
& \frac{\bar{P}^{H}}{\bar{P}}=\left[\frac{1}{\phi_{H}+\phi_{F}\left(\frac{\bar{P}^{F}}{\bar{P}^{H}}\right)^{1-\eta}}\right]^{\frac{1}{1-\eta}}=\left[1-(1-\beta) \bar{a}^{a}\right]^{\frac{1}{1-\eta}}, \\
& \frac{\bar{P}^{F *}}{\bar{P}^{*}}=\left[\frac{\left(\frac{\bar{P}^{F}}{\bar{P}^{H}}\right)^{1-\eta}}{\phi_{F}+\phi_{H}\left(\frac{\bar{P}^{F}}{\bar{P}^{H}}\right)^{1-\eta}}\right]^{\frac{1}{1-\eta}}=\left[\frac{\phi_{F}+\phi_{H}(1-\beta) \bar{a}^{a}}{\phi_{F}+\left(\phi_{H} \phi_{H}-\phi_{F} \phi_{F}\right)(1-\beta) \bar{a}^{a}}\right]^{\frac{1}{1-\eta}} .
\end{aligned}
$$

The steady state terms of trade $\left(\frac{\bar{P}^{F}}{\bar{P}^{H}}\right)$ can be derived as a function of the structural parameters of the model from condition (173), i.e.,

$$
\frac{\bar{P}^{F}}{\bar{P}^{H}}=\left(\frac{\phi_{F}+\phi_{H}(1-\beta) \bar{a}^{a}}{\phi_{F}-\phi_{F}(1-\beta) \bar{a}^{a}}\right)^{\frac{1}{1-\eta}} .
$$

All these complex expressions are derived under the assumption that steady state absorption (consumption plus investment) in both countries differs only by a factor related to the steady state real exchange rate, i.e. $(\bar{C}+\bar{X})=(\overline{R S})^{\eta}\left(\bar{C}^{*}+\bar{X}^{*}\right)$. Given the fact that $\frac{\bar{P}^{H *}}{\bar{P}^{*}}=\frac{\bar{P}}{\overline{S P}{ }^{*}} \frac{\overline{S P}^{H *}}{\bar{P}}=\frac{1}{\overline{R S}} \frac{\bar{P}^{H}}{\bar{P}}$ and replacing appropriately the steady state interest rates given by $(125)-(126)$ (i.e. $\bar{I}=\bar{I}^{*}=\beta^{-1}$ ), then it follows that the resource constraint can be re-written as,

$$
\begin{aligned}
-\widehat{i}_{t}^{*} & +\widehat{n f a}_{t+1} \\
& \approx \frac{1}{\beta}\left(\left(\widehat{s}_{t}-\widehat{s}_{t-1}\right)+\left(\widehat{p}_{t-1}-\widehat{p}_{t}\right)+\widehat{n f a}_{t}\right)+\ldots \\
& \phi_{H}(1-\eta) \frac{1}{\beta}\left(\frac{\left(\frac{\bar{P} H}{\bar{P}}\right)^{1-\eta}(\bar{C}+\bar{X})}{\bar{a}}\right)\left(\widehat{p}_{t}^{H}-\widehat{p}_{t}\right)+\phi_{F}(1-\eta) \frac{1}{\beta}\left(\frac{\left(\frac{\bar{P} H}{\bar{P}}\right)^{1-\eta}(\overline{R S})^{\eta}\left(\bar{C}^{*}+\bar{X}^{*}\right)}{\bar{a}}\right)\left(\widehat{p}_{t}^{H *}-\widehat{p}_{t}^{*}\right)+\ldots \\
& \phi_{F} \frac{1}{\beta}\left(\frac{\left(\frac{\bar{P} H}{\bar{P}}\right)^{1-\eta}(\overline{R S})^{\eta}\left(\bar{C}^{*}+\bar{X}^{*}\right)}{\bar{a}}\right) \widehat{r s}_{t}+\frac{1}{\beta}\left(\frac{\left(\phi_{H}\left(\frac{\bar{P}^{H}}{\bar{P}}\right)^{1-\eta}-1\right)(\bar{C}+\bar{X})}{\bar{a}}\right)\left(\widehat{c}_{t}+\widehat{x}_{t}\right)+\phi_{F} \frac{1}{\beta}\left(\frac{\left(\frac{\bar{P} H}{\bar{P}}\right)^{1-\eta}(\overline{R S})^{\eta}\left(\bar{C}^{*}+\bar{X}^{*}\right)}{\bar{a}}\right)\left(\widehat{c}_{t}^{*}+\widehat{x}_{t}^{*}\right)
\end{aligned}
$$


Given the steady state domestic consumption price index (i.e. $\bar{P}=\left[\phi_{H}\left(\bar{P}^{H}\right)^{1-\eta}+\phi_{F}\left(\bar{P}^{F}\right)^{1-\eta}\right]^{\frac{1}{1-\eta}}$ ) and the fact that $\frac{\bar{P}^{F}}{\bar{P}}=\overline{R S}\left(\frac{\bar{P}^{F *}}{\bar{P}^{*}}\right)$, then I can further re-write the resource constraint in the following terms,

$$
\begin{aligned}
& -\widehat{i}_{t}^{*}+\widehat{n f a}_{t+1} \\
& \quad \approx \frac{1}{\beta}\left(\left(\widehat{s}_{t}-\widehat{s}_{t-1}\right)+\left(\widehat{p}_{t-1}-\widehat{p}_{t}\right)+\widehat{n f a} \widehat{c}_{t}\right)+\ldots \\
& \phi_{H}(1-\eta) \frac{1}{\beta}\left(\frac{\left(\frac{\bar{P} H}{\bar{P}}\right)^{1-\eta}(\bar{C}+\bar{X})}{\bar{a}}\right)\left(\widehat{p}_{t}^{H}-\widehat{p}_{t}\right)+\phi_{F}(1-\eta) \frac{1}{\beta}\left(\frac{\left(\frac{\bar{P}^{H}}{\bar{P}}\right)^{1-\eta}(\overline{R S})^{\eta}\left(\bar{C}^{*}+\bar{X}^{*}\right)}{\bar{a}}\right)\left(\widehat{p}_{t}^{H *}-\widehat{p}_{t}^{*}\right)+\ldots \\
& \quad \phi_{F} \frac{1}{\beta}\left(\frac{\left(\frac{\bar{P}^{H}}{\bar{P}}\right)^{1-\eta}(\overline{R S})^{\eta}\left(\bar{C}^{*}+\bar{X}^{*}\right)}{\bar{a}}\right) \widehat{r}_{t}-\phi_{F} \frac{1}{\beta}\left(\frac{\left(\frac{\bar{P}^{F}}{\bar{P}}\right)^{1-\eta}(\bar{C}+\bar{X})}{\bar{a}}\right)\left(\widehat{c}_{t}+\widehat{x}_{t}\right)+\phi_{F} \frac{1}{\beta}\left(\frac{\left(\frac{\bar{P} H}{\bar{P}}\right)^{1-\eta}(\overline{R S})^{\eta}\left(\bar{C}^{*}+\bar{X}^{*}\right)}{\bar{a}}\right)\left(\widehat{c}_{t}^{*}+\widehat{x}_{t}^{*}\right),
\end{aligned}
$$

or more compactly as,

$$
\begin{aligned}
-\widehat{i}_{t}^{*} & +\widehat{n f a}_{t+1} \\
& \approx \frac{1}{\beta}\left(\left(\widehat{s}_{t}-\widehat{s}_{t-1}\right)+\left(\widehat{p}_{t-1}-\widehat{p}_{t}\right)+\widehat{n f a}_{t}\right)+\ldots \\
& \phi_{H}(1-\eta) \frac{1}{\beta}\left(\frac{\left(\frac{\bar{P} H}{\bar{P}}\right)^{1-\eta}(\bar{C}+\bar{X})}{\bar{a}}\right)\left(\widehat{p}_{t}^{H}-\widehat{p}_{t}\right)+\phi_{F}(1-\eta) \frac{1}{\beta}\left(\frac{\left(\frac{\bar{P} H}{\bar{P}}\right)^{1-\eta}(\overline{R S})^{\eta}\left(\bar{C}^{*}+\bar{X}^{*}\right)}{\bar{a}}\right)\left(\widehat{p}_{t}^{H *}-\widehat{p}_{t}^{*}\right)+\ldots \\
& \phi_{F} \frac{1}{\beta}\left(\frac{\left(\frac{\bar{P} H}{\bar{P}}\right)^{1-\eta}(\overline{R S})^{\eta}\left(\bar{C}^{*}+\bar{X}^{*}\right)}{\bar{a}}\right) \widehat{r s}_{t}-\phi_{F} \frac{1}{\beta}\left(\frac{\left(\overline{R S}\left(\frac{\bar{P}^{F *}}{\bar{P} *}\right)\right)^{1-\eta}(\bar{C}+\bar{X})}{\bar{a}}\right)\left(\widehat{c}_{t}+\widehat{x}_{t}\right)+\phi_{F} \frac{1}{\beta}\left(\frac{\left(\frac{\bar{P}^{H}}{\bar{P}}\right)^{1-\eta}(\overline{R S})^{\eta}\left(\bar{C}^{*}+\bar{X}^{*}\right)}{\bar{a}}\right)\left(\widehat{c}_{t}^{*}+\widehat{x}_{t}^{*}\right)
\end{aligned}
$$

Since absorption in both countries differs only by a factor related to the steady state real exchange rate, i.e. $(\bar{C}+\bar{X})=(\overline{R S})^{\eta}\left(\bar{C}^{*}+\bar{X}^{*}\right)$, then dynamics of the net foreign asset position can be expressed as,

$$
\begin{aligned}
-\widehat{i}_{t}^{*} & +\widehat{n f a}_{t+1} \\
& \approx \frac{1}{\beta}\left(\left(\widehat{s}_{t}-\widehat{s}_{t-1}\right)+\left(\widehat{p}_{t-1}-\widehat{p}_{t}\right)+\widehat{n f a}_{t}\right)+\ldots \\
& \phi_{H}(1-\eta) \frac{1}{\beta}\left(\frac{\left(\frac{\bar{P}^{H}}{\bar{P}}\right)^{1-\eta}(\bar{C}+\bar{X})}{\bar{a}}\right)\left(\widehat{p}_{t}^{H}-\widehat{p}_{t}\right)+\phi_{F}(1-\eta) \frac{1}{\beta}\left(\frac{\left(\frac{\bar{P}^{H}}{\bar{P}}\right)^{1-\eta}(\bar{C}+\bar{X})}{\bar{a}}\right)\left(\widehat{p}_{t}^{H *}-\widehat{p}_{t}^{*}\right)+\ldots \\
& \phi_{F} \frac{1}{\beta}\left(\frac{\left(\frac{\bar{P}^{H}}{\bar{P}}\right)^{1-\eta}(\bar{C}+\bar{X})}{\bar{a}}\right) \widehat{r s}_{t}-\phi_{F} \frac{1}{\beta}\left(\frac{\left(\overline{R S}\left(\frac{\bar{P}^{F *}}{\bar{P}^{*}}\right)\right)^{1-\eta}(\bar{C}+\bar{X})}{\bar{a}}\right)\left(\widehat{c}_{t}+\widehat{x}_{t}\right)+\phi_{F} \frac{1}{\beta}\left(\frac{\left(\frac{\bar{P}^{H}}{\bar{P}}\right)^{1-\eta}(\bar{C}+\bar{X})}{\bar{a}}\right)\left(\widehat{c}_{t}^{*}+\widehat{x}_{t}^{*}\right) .
\end{aligned}
$$

Moreover, given my definition of the steady state real net foreign asset position of the domestic household relative to domestic absorption in (165) as $\bar{a}=\bar{a}^{a}(\bar{C}+\bar{X})$, I can finally summarize the resource constraint as,

$$
\begin{aligned}
\widehat{n f a} & \widehat{i}_{t+1}^{*}+\frac{1}{\beta}\left(\Delta \widehat{r s}_{t}-\widehat{\pi}_{t}^{*}+\widehat{n f a}_{t}\right)+\ldots \\
& (1-\eta)\left(\frac{\bar{P}^{H}}{\bar{P}}\right)^{1-\eta} \frac{1}{\beta \bar{a}^{a}}\left[\phi_{H}\left(\widehat{p}_{t}^{H}-\widehat{p}_{t}\right)+\phi_{F}\left(\widehat{p}_{t}^{H *}-\widehat{p}_{t}^{*}\right)\right]+\ldots \\
& \phi_{F}\left(\frac{\bar{P}^{H}}{\bar{P}}\right)^{1-\eta} \frac{1}{\beta \bar{a}^{a}} \widehat{r s}_{t}+\phi_{F} \frac{1}{\beta \bar{a}^{a}}\left[\left(\frac{\bar{P}^{H}}{\bar{P}}\right)^{1-\eta}\left(\widehat{c}_{t}^{*}+\widehat{x}_{t}^{*}\right)-\left(\overline{R S}\left(\frac{\bar{P}^{F *}}{\bar{P}^{*}}\right)\right)^{1-\eta}\left(\widehat{c}_{t}+\widehat{x}_{t}\right)\right],
\end{aligned}
$$

where $\widehat{\pi}_{t} \equiv \widehat{p}_{t}-\widehat{p}_{t-1}, \widehat{r s}_{t}=\widehat{s}_{t}+\widehat{p}_{t}^{*}-\widehat{p}_{t}$ and $\Delta \widehat{r s}_{t+1} \equiv \widehat{r s}_{t+1}-\widehat{r s}_{t}$.

I define the world price sub-indexes as $\widehat{p}_{t}^{H, W} \equiv \phi_{H} \widehat{p}_{t}^{H}+\phi_{F} \widehat{p}_{t}^{H *}$ and $\widehat{p}_{t}^{F, W *} \equiv \phi_{F} \widehat{p}_{t}^{F}+\phi_{H} \widehat{p}_{t}^{F *}$, and the 
relative price sub-indexes as $\widehat{p}_{t}^{H, R} \equiv \widehat{p}_{t}^{H}-\widehat{p}_{t}^{H *}$ and $\widehat{p}_{t}^{F, R} \equiv \widehat{p}_{t}^{F}-\widehat{p}_{t}^{F *}$. Then, naturally, I can write that,

$$
\begin{aligned}
\widehat{p}_{t}^{H} & =\widehat{p}_{t}^{H, W}+\phi_{F} \widehat{p}_{t}^{H, R}, \widehat{p}_{t}^{H *}=\widehat{p}_{t}^{H, W}-\phi_{H} \widehat{p}_{t}^{H, R}, \\
\widehat{p}_{t}^{F} & =\widehat{p}_{t}^{F, W *}+\phi_{H} \widehat{p}_{t}^{F, R}, \widehat{p}_{t}^{F *}=\widehat{p}_{t}^{F, W *}-\phi_{F} \widehat{p}_{t}^{F, R} .
\end{aligned}
$$

Analogously, I have defined the world CPI as $\widehat{p}_{t}^{W} \equiv \phi_{H} \widehat{p}_{t}+\phi_{F} \widehat{p}_{t}^{*}$ and $\widehat{p}_{t}^{W *} \equiv \phi_{F} \widehat{p}_{t}+\phi_{H} \widehat{p}_{t}^{*}$, and the relative CPI as $\widehat{p}_{t}^{R} \equiv \widehat{p}_{t}-\widehat{p}_{t}^{*}$. Then, I can write that,

$$
\begin{aligned}
& \widehat{p}_{t}=\widehat{p}_{t}^{W}+\phi_{F} \widehat{p}_{t}^{R}, \widehat{p}_{t}^{*}=\widehat{p}_{t}^{W}-\phi_{H} \widehat{p}_{t}^{R}, \\
& \widehat{p}_{t}=\widehat{p}_{t}^{W *}+\phi_{H} \widehat{p}_{t}^{R}, \widehat{p}_{t}^{*}=\widehat{p}_{t}^{W *}-\phi_{F} \widehat{p}_{t}^{R} .
\end{aligned}
$$

Using these definitions, it is possible to express the relative prices $\left(\widehat{p}_{t}^{H *}-\widehat{p}_{t}^{*}\right)$ and $\left(\widehat{p}_{t}^{F}-\widehat{p}_{t}\right)$ in the following terms, i.e.,

$$
\begin{aligned}
\widehat{p}_{t}^{H *}-\widehat{p}_{t}^{*} & =\left(\widehat{p}_{t}^{H, W}-\phi_{H} \widehat{p}_{t}^{H, R}\right)-\left(\widehat{p}_{t}^{W}-\phi_{H} \widehat{p}_{t}^{R}\right) \\
& =\widehat{p}_{t}^{H, W}-\widehat{p}_{t}^{W}-\phi_{H}\left(\widehat{p}_{t}^{H, R}-\widehat{p}_{t}^{R}\right), \\
\widehat{p}_{t}^{F}-\widehat{p}_{t} & =\widehat{p}_{t}^{F, W *}+\phi_{H} \widehat{p}_{t}^{F, R}-\left(\widehat{p}_{t}^{W *}+\phi_{H} \widehat{p}_{t}^{R}\right) \\
& =\widehat{p}_{t}^{F, W *}-\widehat{p}_{t}^{W *}+\phi_{H}\left(\widehat{p}_{t}^{F, R}-\widehat{p}_{t}^{R}\right),
\end{aligned}
$$

where the world terms of trade is defined as $\widehat{t}_{t}^{W} \equiv \widehat{p}_{t}^{F, W *}-\widehat{p}_{t}^{W *}$. The definition of CPI in both countries, i.e. $\widehat{p}_{t} \approx \phi_{H} \widehat{p}_{t}^{H}+\phi_{F} \widehat{p}_{t}^{F}$ and $\widehat{p}_{t}^{*} \approx \phi_{F} \widehat{p}_{t}^{H *}+\phi_{H} \widehat{p}_{t}^{F *}$, can be written as,

$$
\begin{aligned}
\phi_{H}\left[\widehat{p}_{t}^{H}-\widehat{p}_{t}\right]+\phi_{F}\left[\widehat{p}_{t}^{F}-\widehat{p}_{t}\right] & \approx 0, \\
\phi_{F}\left[\widehat{p}_{t}^{H *}-\widehat{p}_{t}^{*}\right]+\phi_{H}\left[\widehat{p}_{t}^{F *}-\widehat{p}_{t}^{*}\right] & \approx 0 .
\end{aligned}
$$

Based on my definitions of the world aggregates, denoted with the superscripts $W$ and $W^{*}$, it is possible to argue that,

$$
\begin{aligned}
\phi_{H} & {\left[\left(\widehat{p}_{t}^{H, W}-\widehat{p}_{t}^{W}\right)+\phi_{F}\left(\widehat{p}_{t}^{H, R}-\widehat{p}_{t}^{R}\right)\right]+\phi_{F}\left[\left(\widehat{p}_{t}^{F, W *}-\widehat{p}_{t}^{W *}\right)+\phi_{H}\left(\widehat{p}_{t}^{F, R}-\widehat{p}_{t}^{R}\right)\right] } \\
& =\phi_{H}\left(\widehat{p}_{t}^{H}-\widehat{p}_{t}\right)+\phi_{F}\left(\widehat{p}_{t}^{F}-\widehat{p}_{t}\right)=0, \\
\phi_{F} & {\left[\left(\widehat{p}_{t}^{H, W}-\widehat{p}_{t}^{W}\right)-\phi_{H}\left(\widehat{p}_{t}^{H, R}-\widehat{p}_{t}^{R}\right)\right]+\phi_{H}\left[\left(\widehat{p}_{t}^{F, W *}-\widehat{p}_{t}^{W *}\right)-\phi_{F}\left(\widehat{p}_{t}^{F, R}-\widehat{p}_{t}^{R}\right)\right] } \\
& =\phi_{F}\left(\widehat{p}_{t}^{H *}-\widehat{p}_{t}^{*}\right)+\phi_{H}\left(\widehat{p}_{t}^{F *}-\widehat{p}_{t}^{*}\right)=0,
\end{aligned}
$$

since $\widehat{p}_{t}=\phi_{H} \widehat{p}_{t}^{H}+\phi_{F} \widehat{p}_{t}^{F}$ and $\widehat{p}_{t}^{*}=\phi_{F} \widehat{p}_{t}^{H *}+\phi_{H} \widehat{p}_{t}^{F *}$. Furthermore, I also know based on those same definitions, that the following result must hold true,

$$
\begin{aligned}
& \left(\widehat{p}_{t}^{H, W}-\widehat{p}_{t}^{W}\right)+\left(\widehat{p}_{t}^{F, W *}-\widehat{p}_{t}^{W *}\right)=\widehat{p}_{t}^{H, W}+\widehat{p}_{t}^{F, W *}-\left(\widehat{p}_{t}^{W}+\widehat{p}_{t}^{W *}\right) \\
& \quad=\phi_{H}\left(\widehat{p}_{t}^{H}-\widehat{p}_{t}\right)+\phi_{F}\left(\widehat{p}_{t}^{H *}-\widehat{p}_{t}^{*}\right)+\phi_{F}\left(\widehat{p}_{t}^{F}-\widehat{p}_{t}\right)+\phi_{H}\left(\widehat{p}_{t}^{F *}-\widehat{p}_{t}^{*}\right) \\
& \quad=\left[\phi_{H}\left(\widehat{p}_{t}^{H}-\widehat{p}_{t}\right)+\phi_{F}\left(\widehat{p}_{t}^{F}-\widehat{p}_{t}\right)\right]+\left[\phi_{F}\left(\widehat{p}_{t}^{H *}-\widehat{p}_{t}^{*}\right)+\phi_{H}\left(\widehat{p}_{t}^{F *}-\widehat{p}_{t}^{*}\right)\right]=0 .
\end{aligned}
$$


From here it follows that,

$$
\begin{aligned}
(1- & \eta)\left(\frac{\bar{P}^{H}}{\bar{P}}\right)^{1-\eta} \frac{1}{\beta \bar{a}^{a}}\left[\phi_{H}\left(\widehat{p}_{t}^{H}-\widehat{p}_{t}\right)+\phi_{F}\left(\widehat{p}_{t}^{H *}-\widehat{p}_{t}^{*}\right)\right] \\
& =(1-\eta)\left(\frac{\bar{P}^{H}}{\bar{P}}\right)^{1-\eta} \frac{1}{\beta \bar{a}^{a}}\left[-\phi_{F}\left(\widehat{p}_{t}^{F}-\widehat{p}_{t}\right)+\phi_{F}\left(\widehat{p}_{t}^{H *}-\widehat{p}_{t}^{*}\right)\right] \\
& =(1-\eta)\left(\frac{\bar{P}^{H}}{\bar{P}}\right)^{1-\eta} \frac{1}{\beta \bar{a}^{a}}\left[-\phi_{F}\left(\widehat{p}_{t}^{F, W *}-\widehat{p}_{t}^{W *}+\phi_{H}\left(\widehat{p}_{t}^{F, R}-\widehat{p}_{t}^{R}\right)\right)+\phi_{F}\left(\widehat{p}_{t}^{H, W}-\widehat{p}_{t}^{W}-\phi_{H}\left(\widehat{p}_{t}^{H, R}-\widehat{p}_{t}^{R}\right)\right)\right] \\
& =-(1-\eta)\left(\frac{\bar{P}^{H}}{\bar{P}}\right)^{1-\eta} \frac{1}{\beta \bar{a}^{a}}\left[\phi_{F}\left(\widehat{p}_{t}^{F, W *}-\widehat{p}_{t}^{W *}+\phi_{H}\left(\widehat{p}_{t}^{F, R}-\widehat{p}_{t}^{R}\right)\right)+\phi_{H}\left(\widehat{p}_{t}^{F, W *}-\widehat{p}_{t}^{W *}-\phi_{F}\left(\widehat{p}_{t}^{F, R}-\widehat{p}_{t}^{R}\right)\right)\right] \\
& =-(1-\eta)\left(\frac{\bar{P}^{H}}{\bar{P}}\right)^{1-\eta} \frac{1}{\beta \bar{a}^{a}}\left[\left(\phi_{F}+\phi_{H}\right)\left(\widehat{p}_{t}^{F, W *}-\widehat{p}_{t}^{W *}\right)+\phi_{F} \phi_{H}\left(\widehat{p}_{t}^{F, R}-\widehat{p}_{t}^{R}\right)-\phi_{F} \phi_{H}\left(\widehat{p}_{t}^{F, R}-\widehat{p}_{t}^{R}\right)\right] \\
& =-(1-\eta)\left(\frac{\bar{P}^{H}}{\bar{P}}\right)^{1-\eta} \frac{1}{\beta \bar{a}^{a}}\left[\widehat{p}_{t}^{F, W *}-\widehat{p}_{t}^{W *}\right] \\
& =-(1-\eta)\left(\frac{\bar{P}^{H}}{\bar{P}}\right)^{1-\eta} \frac{1}{\beta \bar{a}^{a}} \widehat{t}_{t}^{W},
\end{aligned}
$$

where the world terms of trade is defined as $\widehat{t}_{t}^{W} \equiv \widehat{p}_{t}^{F, W *}-\widehat{p}_{t}^{W *}$. Therefore, the dynamics of the real net foreign asset position of the domestic households in (267) can be re-written more compactly as,

$$
\begin{aligned}
& \widehat{n f a}_{t+1} \approx \widehat{i}_{t}^{*}+\frac{1}{\beta}\left(\Delta \widehat{r s}_{t}-\widehat{\pi}_{t}^{*}+\widehat{n f a}_{t}\right)-(1-\eta)\left(\frac{\bar{P}^{H}}{\bar{P}}\right)^{1-\eta} \frac{1}{\beta \bar{a}^{a}} \widehat{t}_{t}^{W}+\ldots \\
& \quad \phi_{F}\left(\frac{\bar{P}^{H}}{\bar{P}}\right)^{1-\eta} \frac{1}{\beta \bar{a}^{a}} \widehat{r s}_{t}+\phi_{F} \frac{1}{\beta \bar{a}^{a}}\left[\left(\frac{\bar{P}^{H}}{\bar{P}}\right)^{1-\eta}\left(\widehat{c}_{t}^{*}+\widehat{x}_{t}^{*}\right)-\left(\overline{R S}\left(\frac{\bar{P}^{F *}}{\bar{P}^{*}}\right)\right)^{1-\eta}\left(\widehat{c}_{t}+\widehat{x}_{t}\right)\right],
\end{aligned}
$$

or simply as,

$$
\begin{aligned}
& \widehat{n f a}_{t+1} \approx \widehat{i}_{t}^{*}+\frac{1}{\beta}\left(\Delta \widehat{r s}_{t}-\widehat{\pi}_{t}^{*}+\widehat{n f a}_{t}\right)-(1-\eta)\left(\frac{1-(1-\beta) \bar{a}^{a}}{\beta \bar{a}^{a}}\right) \widehat{t}_{t}^{W}+\ldots \\
& \quad \phi_{F}\left(\frac{1-(1-\beta) \bar{a}^{a}}{\beta \bar{a}^{a}}\right) \widehat{r s}_{t}+\phi_{F}\left(\frac{1-(1-\beta) \bar{a}^{a}}{\beta \bar{a}^{a}}\right)\left[\left(\widehat{c}_{t}^{*}+\widehat{x}_{t}^{*}\right)-\left(\frac{\phi_{F}+\phi_{H}(1-\beta) \bar{a}^{a}}{\phi_{F}\left(1-(1-\beta) \bar{a}^{a}\right)}\right)\left(\widehat{c}_{t}+\widehat{x}_{t}\right)\right] .
\end{aligned}
$$

This expression allows me to close down the model with incomplete asset markets since the premium that accounts for deviations of the uncovered interest rate parity condition in (264) is tied to the real net foreign asset position, and all other variables in the model are determined endogenously even in the complete asset markets case.

The Law of Motion for Capital. The log-linearization of the domestic capital accumulation formula in (3) and its foreign counterpart without adjustment costs (NAC) allows me to obtain the following set of equations,

$$
\begin{aligned}
\widehat{k}_{t+1} & \approx(1-\delta) \widehat{k}_{t}+(\bar{V} \overline{\bar{X}})\left(\widehat{x}_{t}+\widehat{v}_{t}\right) \\
& =(1-\delta) \widehat{k}_{t}+\delta\left(\widehat{x}_{t}+\widehat{v}_{t}\right) \\
\widehat{k}_{t+1}^{*} & \approx(1-\delta) \widehat{k}_{t}^{*}+\left(\bar{V}^{*} \frac{\bar{X}^{*}}{\bar{K}^{*}}\right)\left(\widehat{x}_{t}^{*}+\widehat{v}_{t}^{*}\right) \\
& =(1-\delta) \widehat{k}_{t}^{*}+\delta\left(\widehat{x}_{t}^{*}+\widehat{v}_{t}^{*}\right)
\end{aligned}
$$

where the second-equality follows from the steady state investment-to-capital ratio being tied by the depreciation rate $\delta$ and the normalization of the steady state level of the IST shocks to one (i.e. $\bar{V}=\bar{V}^{*}=1$ ). The investment-specific technological shocks (ISTs) in this model are labelled $\widehat{v}_{t}$ and $\widehat{v}_{t}^{*}$. 
The log-linearization of the domestic capital accumulation equation in (3) and its foreign counterpart under capital adjustment costs (CAC) allows me to obtain the following set of equations,

$$
\begin{aligned}
\widehat{k}_{t+1} & \approx\left[(1-\delta)-\bar{V} \Phi^{\prime}\left(\frac{\bar{X}}{\bar{K}}\right)\left(\frac{\bar{X}}{\bar{K}}\right)^{2}\right] \widehat{k}_{t}+\left[\bar{V} \Phi\left(\frac{\bar{X}}{\bar{K}}\right) \frac{\bar{X}}{\bar{K}}+\bar{V} \Phi^{\prime}\left(\frac{\bar{X}}{\bar{K}}\right)\left(\frac{\bar{X}}{\bar{K}}\right)^{2}\right] \widehat{x}_{t}+\ldots \\
& \bar{V} \Phi\left(\frac{\bar{X}}{\bar{K}}\right) \frac{\bar{X}}{\bar{K}} \widehat{v}_{t} \\
= & (1-\delta) \widehat{k}_{t}+\delta\left(\widehat{x}_{t}+\widehat{v}_{t}\right), \\
\widehat{k}_{t+1}^{*} & \approx\left[(1-\delta)-\bar{V}^{*} \Phi^{\prime}\left(\frac{\bar{X}^{*}}{\bar{K}^{*}}\right)\left(\frac{\bar{X}^{*}}{\bar{K}^{*}}\right)^{2}\right] \widehat{k}_{t}^{*}+\left[\bar{V}^{*} \Phi\left(\frac{\bar{X}^{*}}{\bar{K}^{*}}\right) \frac{\bar{X}^{*}}{\bar{K}^{*}}+\bar{V}^{*} \Phi^{\prime}\left(\frac{\bar{X}^{*}}{\bar{K}^{*}}\right)\left(\frac{\bar{X}^{*}}{\bar{K}^{*}}\right)^{2}\right] \widehat{x}_{t}^{*}+\ldots \\
& \bar{V}^{*} \Phi\left(\frac{\bar{X}^{*}}{\bar{K}^{*}}\right) \frac{\bar{X}^{*}}{\bar{K}^{*}} \widehat{v}_{t}^{*} \\
= & (1-\delta) \widehat{k}_{t}^{*}+\delta\left(\widehat{x}_{t}^{*}+\widehat{v}_{t}^{*}\right),
\end{aligned}
$$

where the second-equality follows from the steady state properties of the CAC function. The log-linearization of the capital accumulation formula in (3) and its foreign counterpart under investment adjustment costs (IAC) allows me to obtain the following set of equations,

$$
\begin{aligned}
& \widehat{k}_{t+1} \approx(1-\delta) \widehat{k}_{t}+\left[\bar{V} \Phi\left(\frac{\bar{X}}{\bar{X}}\right) \frac{\bar{X}}{\bar{K}}+\bar{V} \Phi^{\prime}\left(\frac{\bar{X}}{\bar{X}}\right)\left(\frac{\bar{X}}{\bar{X}}\right) \frac{\bar{X}}{\bar{K}}\right] \widehat{x}_{t}-\ldots \\
& {\left[\bar{V} \Phi^{\prime}\left(\frac{\bar{X}}{\bar{X}}\right)\left(\frac{\bar{X}}{\bar{X}}\right)^{2} \frac{\bar{X}}{\bar{K}}\right] \widehat{x}_{t}+\bar{V} \Phi\left(\frac{\bar{X}}{\bar{X}}\right) \frac{\bar{X}}{\bar{K}} \widehat{v}_{t} } \\
&=(1-\delta) \widehat{k}_{t}+\delta\left(\widehat{x}_{t}+\widehat{v}_{t}\right), \\
& \widehat{k}_{t+1}^{*} \approx(1-\delta) \widehat{k}_{t}^{*}+\left[\bar{V}^{*} \Phi\left(\frac{\bar{X}^{*}}{\bar{X}^{*}}\right) \frac{\bar{X}^{*}}{\bar{K}^{*}}+\bar{V}^{*} \Phi^{\prime}\left(\frac{\bar{X}^{*}}{\bar{X}^{*}}\right)\left(\frac{\bar{X}^{*}}{\bar{X}^{*}}\right) \frac{\bar{X}^{*}}{\bar{K}^{*}}\right] \widehat{x}_{t}^{*}-\ldots \\
& {\left[\bar{V}^{*} \Phi^{\prime}\left(\frac{\bar{X}^{*}}{\bar{X}^{*}}\right)\left(\frac{\bar{X}^{*}}{\bar{X}^{*}}\right)^{2} \frac{\bar{X}^{*}}{\bar{K}^{*}}\right] \widehat{x}_{t}^{*}+\bar{V}^{*} \Phi\left(\frac{\bar{X}^{*}}{\bar{X}^{*}}\right) \frac{\bar{X}^{*}}{\bar{K}^{*}} \widehat{v}_{t}^{*} } \\
&=(1-\delta) \widehat{k}_{t}^{*}+\delta\left(\widehat{x}_{t}^{*}+\widehat{v}_{t}^{*}\right),
\end{aligned}
$$

where the second-equality follows from the steady state properties of the IAC function, and the fact that in steady state $\bar{X}=\delta \bar{K}$ and $\bar{X}^{*}=\delta \bar{K}^{*}$. It is interesting to notice that in spite of the fact that I am using three different specifications for the adjustment cost function, the log-linearized law of motion for capital is the same in all cases.

However, unlike for the law of motion, the log-linearization of the equilibrium conditions on capitalinvestment is not independent of the choice of the adjustment cost function.

The Capital-Investment Decision under NAC. The log-linearization of the capital-investment conditions coming from the domestic households' problem in (62) - (63) are,

$$
\begin{aligned}
\widehat{q}_{t} & \approx \mathbb{E}_{t}\left\{-\frac{1}{\sigma}\left(\widehat{c}_{t+1}-\widehat{c}_{t}\right)+(1-(1-\delta) \beta) \widehat{r}_{t+1}^{z}+(1-\delta) \beta \widehat{q}_{t+1}\right\}, \\
\widehat{q}_{t} & \approx-\widehat{v}_{t},
\end{aligned}
$$

and, analogously for the foreign counterparts in (64) - (65), the log-linearizations give me that,

$$
\begin{aligned}
& \widehat{q}_{t}^{*} \approx \mathbb{E}_{t}\left\{-\frac{1}{\sigma}\left(\widehat{c}_{t+1}^{*}-\widehat{c}_{t}^{*}\right)+(1-(1-\delta) \beta) \widehat{r}_{t+1}^{* *}+(1-\delta) \beta \widehat{q}_{t+1}^{*}\right\}, \\
& \widehat{q}_{t}^{*} \approx-\widehat{v}_{t}^{*},
\end{aligned}
$$


where naturally $\widehat{q}_{t}$ and $\widehat{q}_{t}^{*}$ are the real shadow values of an additional unit of capital (or Tobin's q) in each country, and $\widehat{r}_{t+1}^{z} \equiv \widehat{z}_{t+1}-\widehat{p}_{t+1}$ and $\widehat{r}_{t+1}^{z *} \equiv \widehat{z}_{t+1}^{*}-\widehat{p}_{t+1}^{*}$ denote the real rental rates on capital in the domestic and foreign countries respectively. This pair of equations can be re-arranged to show that,

$$
\begin{aligned}
(1-(1-\delta) \beta) \mathbb{E}_{t}\left(\widehat{r}_{t+1}^{z}\right) & \approx\left(\widehat{i}_{t}-\mathbb{E}_{t}\left(\widehat{\pi}_{t+1}\right)\right)+(1-\delta) \beta \mathbb{E}_{t}\left(\widehat{v}_{t+1}\right)-\widehat{v}_{t} \\
(1-(1-\delta) \beta) \mathbb{E}_{t}\left(\widehat{r}_{t+1}^{z *}\right) & \approx\left(\hat{i}_{t}^{*}-\mathbb{E}_{t}\left(\widehat{\pi}_{t+1}^{*}\right)\right)+(1-\delta) \beta \mathbb{E}_{t}\left(\widehat{v}_{t+1}^{*}\right)-\widehat{v}_{t}^{*}
\end{aligned}
$$

by adding the Euler equations in (261) - (262). I could interpret this pair of equations as indicating that the real rental rate on capital (the aggregate marginal product of capital) is proportional to the real interest rate. The two rates are not equal, however, because capital depreciates over time, while borrowing and lending in the bond markets is not subject to the same physical depreciation. The two rates also differ because of the contribution of the IST shock (or Tobin's q) to the capital returns. In other words, the real interest rate should be proportional to the aggregate marginal product of capital only if there are no adjustment costs and IST shocks.

The Capital-Investment Decision under CAC. The log-linearization of the capital-investment conditions coming from the domestic households' problem in (66) - (67) are,

$$
\begin{aligned}
\widehat{q}_{t} & \approx \mathbb{E}_{t}\left\{\frac{\bar{Q}\left(\bar{V} \Phi^{\prime \prime}\left(\frac{\bar{X}}{\bar{K}}\right)\left(\frac{\bar{X}}{\bar{K}}\right)^{2}\left(\widehat{c}_{t+1}-\widehat{c}_{t}\right)+(1-(1-\delta) \beta) \widehat{r}_{t+1}^{z}+(1-\delta) \beta \widehat{q}_{t+1}-\ldots\right.}{\left.\left.\frac{\bar{Z}}{\bar{K}}\right)\left(\frac{\bar{X}}{\bar{K}}\right)\right) \frac{\bar{X}}{\bar{K}}}\left(\widehat{x}_{t+1}-\widehat{k}_{t+1}\right)-\frac{\left.\bar{Q}(1-\delta)-\bar{V} \Phi^{\prime}\left(\frac{\bar{X}}{\bar{K}}\right)\left(\frac{\bar{X}}{\bar{K}}\right)^{2}\right)\left(\frac{\bar{X}}{\bar{K}}\right)^{2}}{\frac{\bar{Z}}{\bar{P}}+\bar{Q}\left((1-\delta)-\bar{V} \Phi^{\prime}\left(\frac{\bar{X}}{\bar{K}}\right)\left(\frac{\bar{X}}{\bar{K}}\right)^{2}\right)} \widehat{v}_{t+1}\right\} \\
& =\mathbb{E}_{t}\left\{-\frac{1}{\sigma}\left(\widehat{c}_{t+1}-\widehat{c}_{t}\right)+(1-(1-\delta) \beta) \widehat{r}_{t+1}^{z}+(1-\delta) \beta \widehat{q}_{t+1}+\chi \delta^{2} \beta\left(\widehat{x}_{t+1}-\widehat{k}_{t+1}\right)\right\} \\
\widehat{q}_{t} & \approx-\left[\frac{\left(\Phi^{\prime \prime}\left(\frac{\bar{X}}{\bar{K}}\right) \frac{\bar{X}}{\bar{K}}+2 \Phi^{\prime}\left(\frac{\bar{X}}{\bar{K}}\right)\right) \frac{\bar{X}}{\bar{K}}}{\Phi\left(\frac{\bar{X}}{\bar{K}}\right)+\Phi^{\prime}\left(\frac{\bar{X}}{\bar{K}}\right) \frac{\bar{X}}{\bar{K}}}\right]\left(\widehat{x}_{t}-\widehat{k}_{t}\right)-\widehat{v}_{t} \\
& =\chi \delta\left(\widehat{x}_{t}-\widehat{k}_{t}\right)-\widehat{v}_{t},
\end{aligned}
$$

and, analogously for the foreign counterparts in (68) - (69), I obtain that,

$$
\begin{aligned}
& \widehat{q}_{t}^{*} \approx \mathbb{E}_{t}\left\{-\frac{1}{\sigma}\left(\widehat{c}_{t+1}^{*}-\widehat{c}_{t}^{*}\right)+(1-(1-\delta) \beta) \widehat{r}_{t+1}^{z *}+(1-\delta) \beta \widehat{q}_{t+1}^{*}+\chi \delta^{2} \beta\left(\widehat{x}_{t+1}^{*}-\widehat{k}_{t+1}^{*}\right)\right\} \\
& \widehat{q}_{t}^{*} \approx \chi \delta\left(\widehat{x}_{t}^{*}-\widehat{k}_{t}^{*}\right)-\widehat{v}_{t}^{*} .
\end{aligned}
$$

This pair of equations describes $\widehat{q}_{t}$ and $\widehat{q}_{t}^{*}$ as the real shadow values of an additional unit of capital (or Tobin's q) in each country, $\widehat{r}_{t+1}^{z} \equiv \widehat{z}_{t+1}-\widehat{p}_{t+1}$ and $\widehat{r}_{t+1}^{z *} \equiv \widehat{z}_{t+1}^{*}-\widehat{p}_{t+1}^{*}$ denote the real rental rates on capital in the domestic and foreign countries, while $\chi$ regulates the degree of concavity of the CAC adjustment cost function around the steady state.

The pair of equations that relate the current and expected Tobin's q to the real rental rate on capital 
and the real interest rate can be re-arranged as,

$$
\begin{aligned}
\widehat{q}_{t} & \approx \beta \mathbb{E}_{t}\left[\widehat{q}_{t+1}\right]+\left[(1-(1-\delta) \beta) \mathbb{E}_{t}\left(\widehat{r}_{t+1}^{z}\right)-\left(\widehat{i}_{t}-\mathbb{E}_{t}\left(\widehat{\pi}_{t+1}\right)\right)\right], \\
\widehat{q}_{t}^{*} & \approx \beta \mathbb{E}_{t}\left[\widehat{q}_{t+1}^{*}\right]+\left[(1-(1-\delta) \beta) \mathbb{E}_{t}\left(\widehat{r}_{t+1}^{* *}\right)-\left(\widehat{i}_{t}^{*}-\mathbb{E}_{t}\left(\widehat{\pi}_{t+1}^{*}\right)\right)\right],
\end{aligned}
$$

by adding the Euler equations in (261) - (262). I could interpret this pair of equations as indicating that the differences between the real interest rate and the real rental rate on capital (the aggregate marginal product of capital) are the result of fluctuations in Tobin's q. In the polar case where there are no adjustment costs and IST shocks (i.e., when $\chi=0$ and $\widehat{v}_{t}=\widehat{v}_{t}^{*}=0$ ), then $\widehat{q}_{t}=\widehat{q}_{t}^{*}=0$ for all $t$.

The Capital-Investment Decision under IAC. The log-linearization of the capital-investment conditions coming from the first-order conditions of the households' problem in (70) - (71) are,

$$
\begin{aligned}
& \widehat{q}_{t} \approx \mathbb{E}_{t}\left\{-\frac{1}{\sigma}\left(\widehat{c}_{t+1}-\widehat{c}_{t}\right)+\frac{\frac{\overline{\underline{Z}}}{\bar{P}}}{\frac{\bar{Z}}{P}+(1-\delta)} \widehat{r}_{t+1}^{z}+\frac{(1-\delta)}{\frac{\bar{Z}}{\bar{P}}+(1-\delta)} \widehat{q}_{t+1}\right\} \\
& =\mathbb{E}_{t}\left\{-\frac{1}{\sigma}\left(\widehat{c}_{t+1}-\widehat{c}_{t}\right)+(1-(1-\delta) \beta) \widehat{r}_{t+1}^{z}+(1-\delta) \beta \widehat{q}_{t+1}\right\} \text {, } \\
& \widehat{q}_{t} \approx-\widehat{v}_{t}-\frac{\left(2 \bar{V} \Phi^{\prime}\left(\frac{\bar{X}}{\bar{X}}\right)+\bar{V} \Phi^{\prime \prime}\left(\frac{\bar{X}}{\bar{X}}\right)\left(\frac{\bar{X}}{\bar{X}}\right)\right)\left(\frac{\bar{X}}{\bar{X}}\right)}{\bar{V} \Phi\left(\frac{\bar{X}}{\bar{X}}\right)+\bar{V} \Phi^{\prime}\left(\frac{\bar{X}}{\bar{X}}\right)\left(\frac{\bar{X}}{\bar{X}}\right)}\left(\widehat{x}_{t}-\widehat{x}_{t-1}\right)+\ldots \\
& \mathbb{E}_{t}\left\{\begin{array}{c}
-\frac{1}{\sigma} \frac{\beta \bar{V} \Phi^{\prime}\left(\frac{\bar{X}}{\bar{X}}\right)\left(\frac{\bar{x}}{\bar{X}}\right)^{2}}{1+\beta \bar{V} \Phi^{\prime}\left(\frac{\bar{X}}{\bar{X}}\right)\left(\frac{\bar{x}}{\bar{X}}\right)^{2}}\left(\widehat{c}_{t+1}-\widehat{c}_{t}\right)+\frac{\beta \bar{V} \Phi^{\prime}\left(\frac{\bar{X}}{\bar{X}}\right)\left(\frac{\bar{x}}{\bar{X}}\right)^{2}}{1+\beta \bar{V} \Phi^{\prime}\left(\frac{\bar{X}}{\bar{X}}\right)\left(\frac{\bar{x}}{\bar{X}}\right)^{2}}\left(\widehat{q}_{t+1}+\widehat{v}_{t+1}\right)+\ldots \\
\frac{\beta\left(2 \bar{V} \Phi^{\prime}\left(\frac{\bar{X}}{\bar{X}}\right)\left(\frac{\bar{X}}{\bar{X}}\right)+\bar{V} \Phi^{\prime \prime}\left(\frac{\bar{X}}{\bar{X}}\right)\left(\frac{\bar{X}}{\bar{X}}\right)^{2}\right) \frac{\bar{X}}{\bar{X}}}{1+\beta \bar{V} \Phi^{\prime}\left(\frac{\bar{X}}{\bar{X}}\right)\left(\frac{\bar{X}}{\bar{X}}\right)^{2}}\left(\widehat{x}_{t+1}-\widehat{x}_{t}\right)
\end{array}\right\} \\
& =\kappa\left[\left(\widehat{x}_{t}-\widehat{x}_{t-1}\right)-\beta \mathbb{E}_{t}\left(\widehat{x}_{t+1}-\widehat{x}_{t}\right)\right]-\widehat{v}_{t},
\end{aligned}
$$

and, analogously for the foreign counterparts in (72) - (73), I obtain that,

$$
\begin{aligned}
& \widehat{q}_{t}^{*} \approx \mathbb{E}_{t}\left\{-\frac{1}{\sigma}\left(\widehat{c}_{t+1}^{*}-\widehat{c}_{t}^{*}\right)+(1-(1-\delta) \beta) \widehat{r}_{t+1}^{* *}+(1-\delta) \beta \widehat{q}_{t+1}^{*}\right\}, \\
& \widehat{q}_{t}^{*} \approx \kappa\left[\left(\widehat{x}_{t}^{*}-\widehat{x}_{t-1}^{*}\right)-\beta \mathbb{E}_{t}\left(\widehat{x}_{t+1}^{*}-\widehat{x}_{t}^{*}\right)\right]-\widehat{v}_{t}^{*} .
\end{aligned}
$$

This pair of equations summarizes $\widehat{q}_{t}$ and $\widehat{q}_{t}^{*}$ as the real shadow values of an additional unit of capital (or Tobin's q) in each country, $\widehat{r}_{t+1}^{z} \equiv \widehat{z}_{t+1}-\widehat{p}_{t+1}$ and $\widehat{r}_{t+1}^{z *} \equiv \widehat{z}_{t+1}^{*}-\widehat{p}_{t+1}^{*}$ denote the real rental rates on capital in the domestic and foreign countries, and $\kappa$ regulates the degree of concavity of the IAC adjustment cost function around the steady state.

The pair of equations that relate the current and expected Tobin's q to the real rental rates on capital and the real interest rate can be re-arranged as,

$$
\begin{aligned}
\widehat{q}_{t} & \approx(1-\delta) \beta \mathbb{E}_{t}\left[\widehat{q}_{t+1}\right]+\left[(1-(1-\delta) \beta) \mathbb{E}_{t}\left(\widehat{r}_{t+1}^{z}\right)-\left(\widehat{i}_{t}-\mathbb{E}_{t}\left[\widehat{\pi}_{t+1}\right]\right)\right], \\
\widehat{q}_{t}^{*} & \approx(1-\delta) \beta \mathbb{E}_{t}\left[\widehat{q}_{t+1}^{*}\right]+\left[(1-(1-\delta) \beta) \mathbb{E}_{t}\left(\widehat{r}_{t+1}^{z *}\right)-\left(\widehat{i}_{t}^{*}-\mathbb{E}_{t}\left[\widehat{\pi}_{t+1}^{*}\right]\right)\right],
\end{aligned}
$$


by adding the Euler equations in (261) - (262). I could interpret this pair of equations as indicating that the differences between the real interest rate and the real rental rate on capital are the result of fluctuations in Tobin's q. These equations are almost identical to (282) and (283) except for the fact that the expectations term on the right-hand side is diminished by $(1-\delta)$. Hence, I conjecture that expectations about the future play a potentially 'smaller' role in the dynamics of Tobin's q under the IAC specification. In the polar case where there are no adjustment costs and IST shocks (i.e., when $\kappa=0$ and $\widehat{v}_{t}=\widehat{v}_{t}^{*}=0$ ), then $\widehat{q}_{t}=\widehat{q}_{t}^{*}=0$ for all $t$.

Finally, I re-write equations (285) and (287) in a more compact form as follows,

$$
\begin{aligned}
\widehat{x}_{t} & \approx \frac{1}{1+\beta} \widehat{x}_{t-1}+\frac{\beta}{1+\beta} \mathbb{E}_{t}\left[\widehat{x}_{t+1}\right]+\frac{1}{\kappa(1+\beta)}\left(\widehat{q}_{t}+\widehat{v}_{t}\right), \\
\widehat{x}_{t}^{*} & \approx \frac{1}{1+\beta} \widehat{x}_{t-1}^{*}+\frac{\beta}{1+\beta} \mathbb{E}_{t}\left[\widehat{x}_{t+1}^{*}\right]+\frac{1}{\kappa(1+\beta)}\left(\widehat{q}_{t}^{*}+\widehat{v}_{t}^{*}\right) .
\end{aligned}
$$

The presence of investment adjustment costs (IAC) changes equations (285) and (287) completely. First, it introduces an element of inertia in investment captured by the lagged terms in (290) and (291). Second, the investment decision also becomes forward-looking, captured by the expectations term, because it becomes costly to adjust the level of investment. The elasticity of investment with respect to Tobin's q (the shadow value of an additional unit of capital) is inversely related to the curvature of the IAC function (regulated by the parameter $\kappa$ ). By contrast, investment under the assumption of capital adjustment costs (CAC) responds immediately to movements in Tobin's q (as can be seen from equations (279) and (281)), while the elasticity of investment with respect to Tobin's q is inversely related to the curvature of the CAC function (regulated by the parameter $\chi$ ) and the depreciation rate (given by the parameter $\delta$ ).

\subsection{The Monetary Policy Rules}

A simple log-linearization of the Taylor indexes described in equations (38) - (39) gives me the following monetary policy rules,

$$
\begin{aligned}
\hat{i}_{t} & \approx \rho_{i} \hat{i}_{t-1}+\left(1-\rho_{i}\right)\left[\psi_{\pi} \widehat{\pi}_{t}+\psi_{y} \widehat{y}_{t}\right]+\widehat{m}_{t}, \\
\hat{i}_{t}^{*} & \approx \rho_{i} \hat{i}_{t-1}^{*}+\left(1-\rho_{i}\right)\left[\psi_{\pi} \widehat{\pi}_{t}^{*}+\psi_{y} \widehat{y}_{t}^{*}\right]+\widehat{m}_{t}^{*},
\end{aligned}
$$

where $\widehat{m}_{t}$ and $\widehat{m}_{t}^{*}$ denote a pair of monetary policy shocks (expressed in logs and relative to their unconditional expectations). The Taylor rule for each country is symmetric, has a smoothing component regulated by the parameter $\rho_{i}>0$, and it also responds to fluctuations in output and inflation with weights $\psi_{y}>1$ and $\psi_{\pi} \geq 0$, respectively.

Fiscal policy plays only a supporting role in this environment and, in the end, the balanced-budget equations in $(40)-(41)$ do not need to be explicitly log-linearized to characterize the equilibrium of the model (up to a first-order approximation).

\subsection{The Firms' Equilibrium Conditions}

Efficiency conditions. The efficiency conditions are summarized by equations (112) - (113), as reported before. The log-linearization of these conditions implies that the real rental rates on capital must be approx- 
imately equal to,

$$
\begin{aligned}
\widehat{r}_{t}^{z} & \approx \frac{1}{\sigma} \widehat{c}_{t}+\frac{1+\varphi}{\psi} \widehat{y}_{t}-\left(\frac{1+(1-\psi) \varphi}{\psi}\right) \widehat{k}_{t}-\frac{1+\varphi}{\psi} \widehat{a}_{t} \\
\widehat{r}_{t}^{z *} & \approx \frac{1}{\sigma} \widehat{c}_{t}^{*}+\frac{1+\varphi}{\psi} \widehat{y}_{t}^{*}-\left(\frac{1+(1-\psi) \varphi}{\psi}\right) \widehat{k}_{t}^{*}-\frac{1+\varphi}{\psi} \widehat{a}_{t}^{*}
\end{aligned}
$$

If I define the world consumption as $\widehat{c}_{t}^{a W} \equiv \phi_{H}\left(\frac{1-\gamma_{x}^{a}}{1-\gamma_{x}}\right) \widehat{c}_{t}+\phi_{F}\left(\frac{1-\gamma_{x^{*}}^{a}}{1-\gamma_{x}}\right) \widehat{c}_{t}^{*}$ and $\widehat{c}_{t}^{a W *} \equiv \phi_{F}\left(\frac{1-\gamma_{x}^{a}}{1-\gamma_{x}}\right) \widehat{c}_{t}+$ $\phi_{H}\left(\frac{1-\gamma_{x^{*}}^{a}}{1-\gamma_{x}}\right) \widehat{c}_{t}^{*}$ and the relative consumption as $\widehat{c}_{t}^{a R} \equiv\left(\frac{1-\gamma_{x}^{a}}{1-\gamma_{x}}\right) \widehat{c}_{t}-\left(\frac{1-\gamma_{x^{*}}^{a}}{1-\gamma_{x}}\right) \widehat{c}_{t}^{*}$, then I can write domestic and foreign consumption as,

$$
\begin{aligned}
\widehat{c}_{t} & =\left(\frac{1-\gamma_{x}}{1-\gamma_{x}^{a}}\right)\left(\widehat{c}_{t}^{a W}+\phi_{F} \widehat{c}_{t}^{a R}\right), \\
\widehat{c}_{t}^{*} & =\left(\frac{1-\gamma_{x}}{1-\gamma_{x^{*}}^{a}}\right)\left(\widehat{c}_{t}^{a W *}-\phi_{F} \widehat{c}_{t}^{a R}\right) .
\end{aligned}
$$

Then, I express the efficiency conditions in (294) - (295) as,

$$
\begin{aligned}
\widehat{r}_{t}^{z} & \approx \frac{1}{\sigma}\left(\frac{1-\gamma_{x}}{1-\gamma_{x}^{a}}\right) \widehat{c}_{t}^{a W}+\phi_{F} \frac{1}{\sigma}\left(\frac{1-\gamma_{x}}{1-\gamma_{x}^{a}}\right) \widehat{c}_{t}^{a R}+\frac{1+\varphi}{\psi} \widehat{y}_{t}-\left(\frac{1+(1-\psi) \varphi}{\psi}\right) \widehat{k}_{t}-\frac{1+\varphi}{\psi} \widehat{a}_{t} \\
\widehat{r}_{t}^{z *} & \approx \frac{1}{\sigma}\left(\frac{1-\gamma_{x}}{1-\gamma_{x^{*}}^{a}}\right) \widehat{c}_{t}^{a W *}-\phi_{F} \frac{1}{\sigma}\left(\frac{1-\gamma_{x}}{1-\gamma_{x^{*}}^{a}}\right) \widehat{c}_{t}^{a R}+\frac{1+\varphi}{\psi} \widehat{y}_{t}^{*}-\left(\frac{1+(1-\psi) \varphi}{\psi}\right) \widehat{k}_{t}^{*}-\frac{1+\varphi}{\psi} \widehat{a}_{t}^{*}
\end{aligned}
$$

These equations are necessary to close down the model without having to keep track of either labor or wages explicitly.

Aggregate Output. Using the demand constraints of the domestic firms in equations (33) - (34), the demand constraints of the foreign firms in equations (36) - (37), complemented by (50) - (53) and the corresponding foreign counterparts, I define total output demand as $Y_{t+\tau}(h) \equiv C_{t+\tau}(h)+X_{t+\tau}(h)+$ $C_{t+\tau}^{*}(h)+X_{t+\tau}^{*}(h)$ for a domestic firm $h$ and $Y_{t+\tau}^{*}(f) \equiv C_{t+\tau}(f)+X_{t+\tau}(f)+C_{t+\tau}^{*}(f)+X_{t+\tau}^{*}(f)$ for a foreign firm $f$. Then, it follows that the log-linearization around the steady state of the output demand for a given re-optimizing firm, i.e. $\widehat{y}_{t+\tau}(h)$ for a domestic firm $h$ and $\widehat{y}_{t+\tau}^{*}(f)$ for a foreign firm $f$, takes the 
following form,

$$
\begin{aligned}
& \widehat{y}_{t+\tau}(h) \approx-\theta\left(\frac{\phi_{H}\left(\frac{\bar{P} H}{\bar{P}}\right)^{-\eta}(\bar{C}+\bar{X})}{\phi_{H}\left(\frac{\bar{P} H}{\bar{P}}\right)^{-\eta}(\bar{C}+\bar{X})+\phi_{F}\left(\frac{\bar{P} \bar{P}^{*}}{\bar{P}^{*}}\right)^{-\eta}\left(\bar{C}^{*}+\bar{X}^{*}\right)}\right)\left(\widehat{p}_{t+\tau}(h)-\widehat{p}_{t+\tau}^{H}\right)-\ldots \\
& \eta\left(\frac{\phi_{H}\left(\frac{\overline{\bar{P}} H}{\bar{P}}\right)^{-\eta}(\bar{C}+\bar{X})}{\phi_{H}\left(\frac{\overline{\bar{P}} H}{\bar{P}}\right)^{-\eta}(\bar{C}+\bar{X})+\phi_{F}\left(\frac{\bar{P} \bar{P}^{*}}{\overline{\bar{P}^{*}}}\right)^{-\eta}\left(\bar{C}^{*}+\bar{X}^{*}\right)}\right)\left(\widehat{p}_{t+\tau}^{H}-\widehat{p}_{t+\tau}\right)+\ldots \\
& \left(\frac{\phi_{H}\left(\frac{\bar{P} H}{\bar{P}}\right)^{-\eta} \bar{C}}{\phi_{H}\left(\frac{\bar{P}^{H}}{\bar{P}}\right)^{-\eta}(\bar{C}+\bar{X})+\phi_{F}\left(\frac{\bar{P}^{H *}}{\bar{P}^{*}}\right)^{-\eta}\left(\bar{C}^{*}+\bar{X}^{*}\right)}\right) \widehat{c}_{t+\tau}+\ldots \\
& \left(\frac{\phi_{H}\left(\frac{\bar{P} H}{\bar{P}}\right)^{-\eta} \bar{X}}{\phi_{H}\left(\frac{\bar{P}^{H}}{\bar{P}}\right)^{-\eta}(\bar{C}+\bar{X})+\phi_{F}\left(\frac{\bar{P}^{H *}}{\bar{P}^{*}}\right)^{-\eta}\left(\bar{C}^{*}+\bar{X}^{*}\right)}\right) \widehat{x}_{t+\tau}-\ldots \\
& \theta\left(\frac{\phi_{F}\left(\frac{\bar{P}^{H *}}{\bar{P} *}\right)^{-\eta}\left(\bar{C}^{*}+\bar{X}^{*}\right)}{\phi_{H}\left(\frac{\bar{P}^{H}}{\bar{P}}\right)^{-\eta}(\bar{C}+\bar{X})+\phi_{F}\left(\frac{\bar{P}^{H *}}{\bar{P}^{*}}\right)^{-\eta}\left(\bar{C}^{*}+\bar{X}^{*}\right)}\right)\left(\widehat{p}_{t+\tau}^{*}(h)-\widehat{p}_{t+\tau}^{H *}\right)-\ldots \\
& \eta\left(\frac{\phi_{F}\left(\frac{\bar{P}^{H *}}{\overline{P^{*}}}\right)^{-\eta}\left(\bar{C}^{*}+\bar{X}^{*}\right)}{\phi_{H}\left(\frac{\bar{P}^{H}}{\bar{P}}\right)^{-\eta}(\bar{C}+\bar{X})+\phi_{F}\left(\frac{\bar{P} \bar{P}^{H *}}{\bar{P}^{*}}\right)^{-\eta}\left(\bar{C}^{*}+\bar{X}^{*}\right)}\right)\left(\widehat{p}_{t+\tau}^{H *}-\widehat{p}_{t+\tau}^{*}\right)+\ldots \\
& \left(\frac{\phi_{F}\left(\frac{\bar{P} H^{*}}{\overline{P^{*}}}\right)^{-\eta} \bar{C}^{*}}{\phi_{H}\left(\frac{\overline{\bar{P}} H}{\bar{P}}\right)^{-\eta}(\bar{C}+\bar{X})+\phi_{F}\left(\frac{\overline{P^{H *}}}{\overline{P^{*}}}\right)^{-\eta}\left(\bar{C}^{*}+\bar{X}^{*}\right)}\right) \widehat{c}_{t+\tau}^{*}+\ldots \\
& \left(\frac{\phi_{F}\left(\frac{\overline{\bar{P}}^{H *}}{\overline{\bar{P}}{ }^{*}}\right)^{-\eta} \bar{X}^{*}}{\phi_{H}\left(\frac{\bar{P}^{H}}{\overline{\bar{P}}}\right)^{-\eta}(\bar{C}+\bar{X})+\phi_{F}\left(\frac{\bar{P}^{H *}}{\bar{P}^{*}}\right)^{-\eta}\left(\bar{C}^{*}+\bar{X}^{*}\right)}\right) \widehat{x}_{t+\tau}^{*}, \\
& \widehat{y}_{t+\tau}^{*}(f) \approx-\theta\left(\frac{\phi_{F}\left(\frac{\bar{P}^{F}}{\bar{P}}\right)^{-\eta}(\bar{C}+\bar{X})}{\phi_{F}\left(\frac{\bar{P}^{F}}{\bar{P}}\right)^{-\eta}(\bar{C}+\bar{X})+\phi_{H}\left(\frac{\bar{P}^{F *}}{\bar{P}^{*}}\right)^{-\eta}\left(\bar{C}^{*}+\bar{X}^{*}\right)}\right)\left(\widehat{p}_{t+\tau}(f)-\widehat{p}_{t+\tau}^{F}\right)-\ldots \\
& \eta\left(\frac{\phi_{F}\left(\frac{\bar{P}^{F}}{\overline{\bar{P}}}\right)^{-\eta}(\bar{C}+\bar{X})}{\phi_{F}\left(\frac{\bar{P}^{F}}{\bar{P}}\right)^{-\eta}(\bar{C}+\bar{X})+\phi_{H}\left(\frac{\bar{P}^{F *}}{\bar{P}^{*}}\right)^{-\eta}\left(\bar{C}^{*}+\bar{X}^{*}\right)}\right)\left(\widehat{p}_{t+\tau}^{F}-\widehat{p}_{t+\tau}\right)+\ldots \\
& \left(\frac{\phi_{F}\left(\frac{\bar{P} F}{\bar{P}}\right)^{-\eta} \bar{C}}{\phi_{F}\left(\frac{\bar{P}^{F}}{\bar{P}}\right)^{-\eta}(\bar{C}+\bar{X})+\phi_{H}\left(\frac{\bar{P}^{F *}}{\bar{P}^{*}}\right)^{-\eta}\left(\bar{C}^{*}+\bar{X}^{*}\right)}\right) \widehat{c}_{t+\tau}+\ldots \\
& \left(\frac{\phi_{F}\left(\frac{\bar{P} F}{\bar{P}}\right)^{-\eta} \bar{X}}{\phi_{F}\left(\frac{\bar{P}^{F}}{\bar{P}}\right)^{-\eta}(\bar{C}+\bar{X})+\phi_{H}\left(\frac{\bar{P}^{F *}}{\bar{P}^{*}}\right)^{-\eta}\left(\bar{C}^{*}+\bar{X}^{*}\right)}\right) \widehat{x}_{t+\tau}-\ldots \\
& \theta\left(\frac{\phi_{H}\left(\frac{\bar{P}^{F *}}{\bar{P}^{*}}\right)^{-\eta}\left(\bar{C}^{*}+\bar{X}^{*}\right)}{\phi_{F}\left(\frac{\bar{P}^{F}}{\bar{P}}\right)^{-\eta}(\bar{C}+\bar{X})+\phi_{H}\left(\frac{\bar{P}^{F *}}{\bar{P}^{*}}\right)^{-\eta}\left(\bar{C}^{*}+\bar{X}^{*}\right)}\right)\left(\widehat{p}_{t+\tau}^{*}(f)-\widehat{p}_{t+\tau}^{F *}\right)-\ldots \\
& \eta\left(\frac{\phi_{H}\left(\frac{\bar{P}^{F *}}{\overline{\bar{N}}^{*}}\right)^{-\eta}\left(\bar{C}^{*}+\bar{X}^{*}\right)}{\phi_{F}\left(\frac{\bar{P}^{F}}{\bar{P}}\right)^{-\eta}(\bar{C}+\bar{X})+\phi_{H}\left(\frac{\bar{P}^{F *}}{\bar{P}^{*}}\right)^{-\eta}\left(\bar{C}^{*}+\bar{X}^{*}\right)}\right)\left(\widehat{p}_{t+\tau}^{F *}-\widehat{p}_{t+\tau}^{*}\right)+\ldots \\
& \left(\frac{\phi_{H}\left(\frac{\bar{P}^{F *}}{\bar{P}^{*}}\right)^{-\eta} \bar{C}^{*}}{\phi_{F}\left(\frac{\bar{P}^{F}}{\bar{P}}\right)^{-\eta}(\bar{C}+\bar{X})+\phi_{H}\left(\frac{\bar{P}^{F *}}{\bar{P}^{*}}\right)^{-\eta}\left(\bar{C}^{*}+\bar{X}^{*}\right)}\right) \widehat{c}_{t+\tau}^{*}+\ldots \\
& \left(\frac{\phi_{H}\left(\frac{\bar{P}^{F *}}{\bar{P}^{*}}\right)^{-\eta} \bar{X}^{*}}{\phi_{F}\left(\frac{\bar{P}^{F}}{\bar{P}}\right)^{-\eta}(\bar{C}+\bar{X})+\phi_{H}\left(\frac{\bar{P}^{F *}}{\bar{P}^{*}}\right)^{-\eta}\left(\bar{C}^{*}+\bar{X}^{*}\right)}\right) \widehat{x}_{t+\tau}^{*} \text {. }
\end{aligned}
$$


Using the fact that in steady state it must be the case that $\left(\frac{\bar{P}^{F *}}{\bar{P}^{*}}\right)=\frac{1}{\overline{R S}}\left(\frac{\bar{P}^{F}}{\bar{P}}\right)$ and $\left(\frac{\bar{P}^{H *}}{\bar{P}^{*}}\right)=\frac{1}{\overline{R S}}\left(\frac{\bar{P}^{H}}{\bar{P}}\right)$, then I can re-write these two expressions as follows,

$$
\begin{aligned}
& \widehat{y}_{t+\tau}(h) \approx-\theta\left(\frac{\phi_{H}(\bar{C}+\bar{X})}{\phi_{H}(\bar{C}+\bar{X})+\phi_{F}(\overline{R S})^{\eta}\left(\bar{C}^{*}+\bar{X}^{*}\right)}\right)\left(\widehat{p}_{t+\tau}(h)-\widehat{p}_{t+\tau}^{H}\right)-\eta\left(\frac{\phi_{H}(\bar{C}+\bar{X})}{\phi_{H}(\bar{C}+\bar{X})+\phi_{F}(\overline{R S})^{\eta}\left(\bar{C}^{*}+\bar{X}^{*}\right)}\right)\left(\widehat{p}_{t+\tau}^{H}-\widehat{p}_{t+\tau}\right)+\ldots \\
& \left(\frac{\phi_{H} \bar{C}}{\phi_{H}(\bar{C}+\bar{X})+\phi_{F}(\overline{R S})^{\eta}\left(\bar{C}^{*}+\bar{X}^{*}\right)}\right) \widehat{c}_{t+\tau}+\left(\frac{\phi_{H} \bar{X}}{\phi_{H}(\bar{C}+\bar{X})+\phi_{F}(\overline{R S})^{\eta}\left(\bar{C}^{*}+\bar{X}^{*}\right)}\right) \widehat{x}_{t+\tau}-\ldots \\
& \theta\left(\frac{\phi_{F}(\overline{R S})^{\eta}\left(\bar{C}^{*}+\bar{X}^{*}\right)}{\phi_{H}(\bar{C}+\bar{X})+\phi_{F}(\overline{R S})^{\eta}\left(\bar{C}^{*}+\bar{X}^{*}\right)}\right)\left(\widehat{p}_{t+\tau}^{*}(h)-\widehat{p}_{t+\tau}^{H *}\right)-\eta\left(\frac{\phi_{F}(\overline{R S})^{\eta}\left(\bar{C}^{*}+\bar{X}^{*}\right)}{\phi_{H}(\bar{C}+\bar{X})+\phi_{F}(\overline{R S})^{\eta}\left(\bar{C}^{*}+\bar{X}^{*}\right)}\right)\left(\widehat{p}_{t+\tau}^{H *}-\widehat{p}_{t+\tau}^{*}\right)+\ldots \\
& \left(\frac{\phi_{F}(\overline{R S})^{\eta} \bar{C}^{*}}{\phi_{H}(\bar{C}+\bar{X})+\phi_{F}(\overline{R S})^{\eta}\left(\bar{C}^{*}+\bar{X}^{*}\right)}\right) \widehat{c}_{t+\tau}^{*}+\left(\frac{\phi_{F}(\overline{R S})^{\eta} \bar{X}^{*}}{\phi_{H}(\bar{C}+\bar{X})+\phi_{F}(\overline{R S})^{\eta}\left(\bar{C}^{*}+\bar{X}^{*}\right)}\right) \widehat{x}_{t+\tau}^{*}, \\
& \widehat{y}_{t+\tau}^{*}(f) \approx-\theta\left(\frac{\phi_{F}(\bar{C}+\bar{X})}{\phi_{F}(\bar{C}+\bar{X})+\phi_{H}(\overline{R S})^{\eta}\left(\bar{C}^{*}+\bar{X}^{*}\right)}\right)\left(\widehat{p}_{t+\tau}(f)-\widehat{p}_{t+\tau}^{F}\right)-\eta\left(\frac{\phi_{F}(\bar{C}+\bar{X})}{\phi_{F}(\bar{C}+\bar{X})+\phi_{H}(\overline{R S})^{\eta}\left(\bar{C}^{*}+\bar{X}^{*}\right)}\right)\left(\widehat{p}_{t+\tau}^{F}-\widehat{p}_{t+\tau}\right)+\ldots \\
& \left(\frac{\phi_{F} \bar{C}}{\phi_{F}(\bar{C}+\bar{X})+\phi_{H}(\overline{R S})^{\eta}\left(\bar{C}^{*}+\bar{X}^{*}\right)}\right) \widehat{c}_{t+\tau}+\left(\frac{\phi_{F} \bar{X}}{\phi_{F}(\bar{C}+\bar{X})+\phi_{H}(\overline{R S})^{\eta}\left(\bar{C}^{*}+\bar{X}^{*}\right)}\right) \widehat{x}_{t+\tau}-\ldots \\
& \theta\left(\frac{\phi_{H}(\overline{R S})^{\eta}\left(\bar{C}^{*}+\bar{X}^{*}\right)}{\phi_{F}(\bar{C}+\bar{X})+\phi_{H}(\overline{R S})^{\eta}\left(\bar{C}^{*}+\bar{X}^{*}\right)}\right)\left(\widehat{p}_{t+\tau}^{*}(f)-\widehat{p}_{t+\tau}^{F *}\right)-\eta\left(\frac{\phi_{H}(\overline{R S})^{\eta}\left(\bar{C}^{*}+\bar{X}^{*}\right)}{\phi_{F}(\bar{C}+\bar{X})+\phi_{H}(\overline{R S})^{\eta}\left(\bar{C}^{*}+\bar{X}^{*}\right)}\right)\left(\widehat{p}_{t+\tau}^{F *}-\widehat{p}_{t+\tau}^{*}\right)+\ldots \\
& \left(\frac{\phi_{H}(\overline{R S})^{\eta} \bar{C}^{*}}{\phi_{F}(\bar{C}+\bar{X})+\phi_{H}(\overline{R S})^{\eta}\left(\bar{C}^{*}+\bar{X}^{*}\right)}\right) \widehat{c}_{t+\tau}^{*}+\left(\frac{\phi_{H}(\overline{R S})^{\eta} \bar{X}^{*}}{\phi_{F}(\bar{C}+\bar{X})+\phi_{H}(\overline{R S})^{\eta}\left(\bar{C}^{*}+\bar{X}^{*}\right)}\right) \widehat{x}_{t+\tau}^{*} \text {. }
\end{aligned}
$$

Then, in a steady state where absorption (consumption plus investment) in both countries differs only by a factor related to the steady state real exchange rate, i.e. $(\bar{C}+\bar{X})=(\overline{R S})^{\eta}\left(\bar{C}^{*}+\bar{X}^{*}\right)$, the output demand of each variety can be expressed more compactly as,

$$
\begin{gathered}
\widehat{y}_{t+\tau}(h) \approx-\theta \phi_{H}\left(\widehat{p}_{t+\tau}(h)-\widehat{p}_{t+\tau}^{H}\right)-\eta \phi_{H}\left(\widehat{p}_{t+\tau}^{H}-\widehat{p}_{t+\tau}\right)+\ldots \\
\phi_{H}\left(\frac{\bar{C}}{\overline{C+\bar{X}}}\right) \widehat{c}_{t+\tau}+\phi_{H}\left(\frac{\bar{X}}{\bar{C}+\bar{X}}\right) \widehat{x}_{t+\tau}-\ldots \\
\theta \phi_{F}\left(\widehat{p}_{t+\tau}^{*}(h)-\widehat{p}_{t+\tau}^{H *}\right)-\eta \phi_{F}\left(\widehat{p}_{t+\tau}^{H *}-\widehat{p}_{t+\tau}^{*}\right)+\ldots \\
\phi_{F}\left(\frac{\overline{\bar{C}^{*}}}{\bar{C}^{*}+\bar{X}^{*}}\right) \widehat{c}_{t+\tau}^{*}+\phi_{F}\left(\frac{\overline{X^{*}}}{\bar{C}^{*}+\bar{X}^{*}}\right) \widehat{x}_{t+\tau}^{*}, \\
\widehat{y}_{t+\tau}^{*}(f) \approx-\theta \phi_{F}\left(\widehat{p}_{t+\tau}(f)-\widehat{p}_{t+\tau}^{F}\right)-\eta \phi_{F}\left(\widehat{p}_{t+\tau}^{F}-\widehat{p}_{t+\tau}\right)+\ldots \\
\phi_{F}\left(\frac{\bar{C}}{\bar{C}+\bar{X}}\right) \widehat{c}_{t+\tau}+\phi_{F}\left(\frac{\bar{X}}{\bar{C}+\bar{X}}\right) \widehat{x}_{t+\tau}-\ldots \\
\theta \phi_{H}\left(\widehat{p}_{t+\tau}^{*}(f)-\widehat{p}_{t+\tau}^{F *}\right)-\eta \phi_{H}\left(\widehat{p}_{t+\tau}^{F}-\widehat{p}_{t+\tau}\right)+\ldots \\
\phi_{H}\left(\frac{\overline{C^{*}}}{\bar{C}^{*}+\bar{X}^{*}}\right) \widehat{c}_{t+\tau}^{*}+\phi_{H}\left(\frac{\bar{X}^{*}}{\bar{C}^{*}+\bar{X}^{*}}\right) \widehat{x}_{t+\tau}^{*}
\end{gathered}
$$


I define the steady state investment and consumption shares relative to absorption in (187) - (190) as,

$$
\begin{aligned}
\gamma_{x}^{a} & \equiv \frac{\bar{X}}{\bar{C}+\bar{X}}=\delta\left[\frac{1-\psi}{\left(\frac{\theta(1-\bar{\xi})}{\theta-1}\right)\left(\beta^{-1}-(1-\delta)\right)}\right]\left(1-(1-\beta) \bar{a}^{a}\right), \\
\gamma_{c}^{a} & \equiv \frac{\bar{C}}{\bar{C}+\bar{X}}=1-\gamma_{x}^{a}, \\
\gamma_{x^{*}}^{a} & \equiv \frac{\bar{X}^{*}}{\bar{C}^{*}+\bar{X}^{*}}=\delta\left[\frac{1-\psi}{\left(\frac{\theta\left(1-\bar{\xi}^{*}\right)}{\theta-1}\right)\left(\beta^{-1}-(1-\delta)\right)}\right]\left(\frac{\phi_{F}+\phi_{H}(1-\beta) \bar{a}^{a}}{\phi_{F}+\left(\phi_{H} \phi_{H}-\phi_{F} \phi_{F}\right)(1-\beta) \bar{a}^{a}}\right), \\
\gamma_{c^{*}}^{a} & \equiv \bar{C}^{*}+\bar{X}^{*}=1-\gamma_{x^{*}}^{a} .
\end{aligned}
$$

With these expressions at hand, I can write the log-linearized aggregate output equations as follows,

$$
\begin{gathered}
\widehat{y}_{t+\tau}(h) \approx-\theta\left[\phi_{H}\left(\widehat{p}_{t+\tau}(h)-\widehat{p}_{t+\tau}^{H}\right)+\phi_{F}\left(\widehat{p}_{t+\tau}^{*}(h)-\widehat{p}_{t+\tau}^{H *}\right)\right]-\ldots \\
\eta\left[\phi_{H}\left(\widehat{p}_{t+\tau}^{H}-\widehat{p}_{t+\tau}\right)+\phi_{F}\left(\widehat{p}_{t+\tau}^{H *}-\widehat{p}_{t+\tau}^{*}\right)\right]+\ldots \\
\phi_{H}\left(1-\gamma_{x}^{a}\right) \widehat{c}_{t+\tau}+\phi_{F}\left(1-\gamma_{x^{*}}^{a}\right) \widehat{c}_{t+\tau}^{*}+\phi_{H} \gamma_{x}^{a} \widehat{x}_{t+\tau}+\phi_{F} \gamma_{x^{*}}^{a} \widehat{x}_{t+\tau}^{*}, \\
\widehat{y}_{t+\tau}^{*}(f) \approx-\theta\left[\phi_{F}\left(\widehat{p}_{t+\tau}(f)-\widehat{p}_{t+\tau}^{F}\right)+\phi_{H}\left(\widehat{p}_{t+\tau}^{*}(f)-\widehat{p}_{t+\tau}^{F *}\right)\right]-\ldots \\
\eta\left[\phi_{F}\left(\widehat{p}_{t+\tau}^{F}-\widehat{p}_{t+\tau}\right)+\phi_{H}\left(\widehat{p}_{t+\tau}^{F}-\widehat{p}_{t+\tau}\right)\right]+\ldots \\
\phi_{F}\left(1-\gamma_{x}^{a}\right) \widehat{c}_{t+\tau}+\phi_{H}\left(1-\gamma_{x^{*}}^{a}\right) \widehat{c}_{t+\tau}^{*}+\phi_{F} \gamma_{x}^{a} \widehat{x}_{t+\tau}+\phi_{H} \gamma_{x^{*}}^{a} \widehat{x}_{t+\tau}^{*} .
\end{gathered}
$$

The consumption and investment shares in the special case with a zero net foreign asset position, i.e. with $\bar{a}^{a}=0$, are defined in (191) - (192) as follows,

$$
\begin{aligned}
& \gamma_{x}=\delta\left[\frac{1-\psi}{\left(\frac{\theta(1-\bar{\xi})}{\theta-1}\right)\left(\beta^{-1}-(1-\delta)\right)}\right], \gamma_{c}=1-\gamma_{x}, \\
& \gamma_{x^{*}}=\delta\left[\frac{1-\psi}{\left(\frac{\theta\left(1-\bar{\xi}^{*}\right)}{\theta-1}\right)\left(\beta^{-1}-(1-\delta)\right)}\right], \gamma_{c^{*}}=1-\gamma_{x^{*}} .
\end{aligned}
$$

Hence, I can re-write the expression for the output demands in the following terms,

$$
\begin{aligned}
& \widehat{y}_{t+\tau}(h) \approx-\theta\left[\widehat{p}_{t+\tau}^{W}(h)-\widehat{p}_{t+\tau}^{H, W}\right]-\eta\left[\widehat{p}_{t+\tau}^{H, W}-\widehat{p}_{t+\tau}^{W}\right]+\left(1-\gamma_{x}\right) \widehat{c}_{t+\tau}^{a W}+\gamma_{x} \widehat{x}_{t+\tau}^{a W} \\
& \widehat{y}_{t+\tau}^{*}(f) \approx-\theta\left[\widehat{p}_{t+\tau}^{W *}(f)-\widehat{p}_{t+\tau}^{F, W *}\right]-\eta\left[\widehat{p}_{t+\tau}^{F, W *}-\widehat{p}_{t+\tau}^{W *}\right]+\left(1-\gamma_{x}\right) \widehat{c}_{t+\tau}^{a W *}+\gamma_{x} \widehat{x}_{t+\tau}^{a W *},
\end{aligned}
$$


where the weighted variables are,

$$
\begin{aligned}
\widehat{c}_{t}^{a W} & \equiv \phi_{H}\left(\frac{1-\gamma_{x}^{a}}{1-\gamma_{x}}\right) \widehat{c}_{t}+\phi_{F}\left(\frac{1-\gamma_{x^{*}}^{a}}{1-\gamma_{x}}\right) \widehat{c}_{t}^{*} \\
\widehat{c}_{t}^{a W *} & \equiv \phi_{F}\left(\frac{1-\gamma_{x}^{a}}{1-\gamma_{x}}\right) \widehat{c}_{t}+\phi_{H}\left(\frac{1-\gamma_{x^{*}}^{a}}{1-\gamma_{x}}\right) \widehat{c}_{t}^{*} \\
\widehat{x}_{t}^{a W} & \equiv \phi_{H}\left(\frac{\gamma_{x}^{a}}{\gamma_{x}}\right) \widehat{x}_{t}+\phi_{F}\left(\frac{\gamma_{x^{*}}^{a}}{\gamma_{x}}\right) \widehat{x}_{t}^{*} \\
\widehat{x}_{t}^{a W *} & \equiv \phi_{F}\left(\frac{\gamma_{x}^{a}}{\gamma_{x}}\right) \widehat{x}_{t}+\phi_{H}\left(\frac{\gamma_{x^{*}}^{a}}{\gamma_{x}}\right) \widehat{x}_{t}^{*} \\
\widehat{p}_{t}^{W}(h) & \equiv \phi_{H} \widehat{p}_{t}(h)+\phi_{F} \widehat{p}_{t}^{*}(h), \widehat{p}_{t}^{W *}(f) \equiv \phi_{F} \widehat{p}_{t}(f)+\phi_{H} \widehat{p}_{t}^{*}(f), \\
\widehat{p}_{t}^{H, W} & \equiv \phi_{H} \widehat{p}_{t}^{H}+\phi_{F} \widehat{p}_{t}^{H *}, \widehat{p}_{t}^{F, W *} \equiv \phi_{F} \widehat{p}_{t}^{F}+\phi_{H} \widehat{p}_{t}^{F *}, \\
\widehat{p}_{t}^{W} & \equiv \phi_{H} \widehat{p}_{t}+\phi_{F} \widehat{p}_{t}^{*}, \widehat{p}_{t}^{W *} \equiv \phi_{F} \widehat{p}_{t}+\phi_{H} \widehat{p}_{t}^{*} .
\end{aligned}
$$

The expression above is essentially the same expression that I would expect to find in the standard case with a zero net foreign asset position in steady state, except that world aggregate consumption and investment are defined from the perspective of each country, i.e. $\widehat{c}_{t}^{a W}, \widehat{c}_{t}^{a W *}, \widehat{x}_{t}^{a W}$, and $\widehat{x}_{t}^{a W *}$. These measures are not computed based solely on the weights in the consumption basket of goods (i.e. $\phi_{H}$ and $\phi_{F}$ ), but are re-weighted depending on the gap that exists between the steady state consumption and investment shares with a non-zero net foreign asset position and the steady state consumption and investment shares whenever the net foreign asset position is zero.

Keeping that distinction in mind I can proceed ahead with the derivation of the rest of the log-linearized equilibrium conditions in a similar fashion. I can re-express the aggregate price of all domestic and foreign firms as $\widehat{p}_{t+\tau}^{H, W} \approx \int_{0}^{1} \widehat{p}_{t+\tau}^{W}(h) d h$ and $\widehat{p}_{t+\tau}^{F, W *} \approx \int_{0}^{1} \widehat{p}_{t+\tau}^{W *}(f) d f$. Adding up the output functions for all firms within each country, I obtain an expression for aggregate output in these terms,

$$
\begin{aligned}
& \widehat{y}_{t+\tau} \approx-\eta\left(\widehat{p}_{t+\tau}^{H, W}-\widehat{p}_{t+\tau}^{W}\right)+\left(1-\gamma_{x}\right) \widehat{c}_{t+\tau}^{a W}+\gamma_{x} \widehat{x}_{t+\tau}^{a W} \\
& \widehat{y}_{t+\tau}^{*} \approx-\eta\left(\widehat{p}_{t+\tau}^{F, W *}-\widehat{p}_{t+\tau}^{W *}\right)+\left(1-\gamma_{x}\right) \widehat{c}_{t+\tau}^{a W *}+\gamma_{x} \widehat{x}_{t+\tau}^{a W *}
\end{aligned}
$$

These two equations will become very important in my posterior derivations of the Phillips curves. Furthermore, if I combine the efficiency conditions in (296) - (297) with the output equations derived in (298) - (299), it follows that,

$$
\begin{aligned}
\widehat{r}_{t}^{z} & \approx\left(\frac{1}{\sigma}\left(\frac{1-\gamma_{x}}{1-\gamma_{x}^{a}}\right)+\left(1-\gamma_{x}\right) \frac{1+\varphi}{\psi}\right) \widehat{c}_{t}^{a W}+\gamma_{x} \frac{1+\varphi}{\psi} \widehat{x}_{t}^{a W}+\phi_{F} \frac{1}{\sigma}\left(\frac{1-\gamma_{x}}{1-\gamma_{x}^{a}}\right) \widehat{c}_{t}^{a R}-\ldots \\
& \eta \frac{1+\varphi}{\psi}\left(\widehat{p}_{t}^{H, W}-\widehat{p}_{t}^{W}\right)-\left(\frac{1+(1-\psi) \varphi}{\psi}\right) \widehat{k}_{t}-\frac{1+\varphi}{\psi} \widehat{a}_{t} \\
\widehat{r}_{t}^{z *} & \approx\left(\frac{1}{\sigma}\left(\frac{1-\gamma_{x}}{1-\gamma_{x *}^{a}}\right)+\left(1-\gamma_{x}\right) \frac{1+\varphi}{\psi}\right) \widehat{c}_{t}^{a W *}+\gamma_{x} \frac{1+\varphi}{\psi} \widehat{x}_{t}^{a W *}-\phi_{F} \frac{1}{\sigma}\left(\frac{1-\gamma_{x}}{1-\gamma_{x^{*}}^{a}}\right) \widehat{c}_{t}^{a R}-\ldots \\
& \eta \frac{1+\varphi}{\psi}\left(\widehat{p}_{t}^{F, W *}-\widehat{p}_{t}^{W *}\right)-\left(\frac{1+(1-\psi) \varphi}{\psi}\right) \widehat{k}_{t}^{*}-\frac{1+\varphi}{\psi} \widehat{a}_{t}^{*} .
\end{aligned}
$$

These conditions will be appropriately used to simplify the description of the Phillips curves in this model.

The Open Economy Phillips Curves. In steady state the standard pricing rule under monopolistic competiation of charging a mark-up over marginal costs holds. Accordingly, the log-linearization of the 
optimal pricing equations in (74), (75), (76) and (77) can be compactly expressed as follows,

$$
\begin{aligned}
& \widehat{\widetilde{p}}_{t}(h)-\widehat{p}_{t} \approx \mathbb{E}_{t}\left[\sum_{\tau=1}^{+\infty}(\beta \alpha)^{\tau} \widehat{\pi}_{t+\tau}\right]+(1-\beta \alpha) \mathbb{E}_{t}\left[\sum_{\tau=0}^{+\infty}(\beta \alpha)^{\tau}\left(\widehat{m c}_{t+\tau}-\widehat{p}_{t+\tau}\right)\right], \\
& \widehat{\widetilde{p}}_{t}^{*}(h)-\widehat{p}_{t}^{*} \approx \mathbb{E}_{t}\left[\sum_{\tau=1}^{+\infty}(\beta \alpha)^{\tau} \widehat{\pi}_{t+\tau}^{*}\right]+(1-\beta \alpha) \mathbb{E}_{t}\left[\sum_{\tau=0}^{+\infty}(\beta \alpha)^{\tau}\left(\widehat{m c}_{t+\tau}-\widehat{p}_{t+\tau}-\widehat{r s}_{t+\tau}\right)\right], \\
& \widehat{\widetilde{p}}_{t}(f)-\widehat{p}_{t} \approx \mathbb{E}_{t}\left[\sum_{\tau=1}^{+\infty}(\beta \alpha)^{\tau} \widehat{\pi}_{t+\tau}\right]+(1-\beta \alpha) \mathbb{E}_{t}\left[\sum_{\tau=0}^{+\infty}(\beta \alpha)^{\tau}\left(\widehat{m c}_{t+\tau}^{*}-\widehat{p}_{t+\tau}^{*}+\widehat{r s}_{t+\tau}\right)\right], \\
& \widetilde{\widetilde{p}}_{t}^{*}(f)-\widehat{p}_{t}^{*} \approx \mathbb{E}_{t}\left[\sum_{\tau=1}^{+\infty}(\beta \alpha)^{\tau} \widehat{\pi}_{t+\tau}^{*}\right]+(1-\beta \alpha) \mathbb{E}_{t}\left[\sum_{\tau=0}^{+\infty}(\beta \alpha)^{\tau}\left(\widehat{m c}_{t+\tau}^{*}-\widehat{p}_{t+\tau}^{*}\right)\right],
\end{aligned}
$$

which defines the distance between the optimal price decision of a given re-optimizing firm and the CPI level prevailing in each market as a weighted function of current and expected future CPI inflation and real marginal costs. Here I must recall the assumption that the government subsidy is time-invariant and equal to its steady state value in every period, which explains why the government subsidies do not appear in the log-linearized pricing equations. I derive the (pre-subsidy) marginal cost equations in (26) - (27), and they can be log-linearized as,

$$
\begin{aligned}
& \widehat{m c}_{t+\tau} \approx \psi \widehat{w}_{t+\tau}+(1-\psi)\left(\widehat{r}_{t+\tau}^{z}+\widehat{p}_{t+\tau}\right)-\widehat{a}_{t+\tau}, \\
& \widehat{m c}_{t+\tau}^{*} \approx \psi \widehat{w}_{t+\tau}^{*}+(1-\psi)\left(\widehat{r}_{t+\tau}^{z *}+\widehat{p}_{t+\tau}^{*}\right)-\widehat{a}_{t+\tau}^{*},
\end{aligned}
$$

while the labor market clearing conditions, which are implicit in (114) - (115), can be approximated as,

$$
\begin{aligned}
\widehat{w}_{t+\tau} & \approx-\frac{\varphi}{1+(1-\psi) \varphi} \widehat{a}_{t+\tau}+\frac{1}{1+(1-\psi) \varphi} \frac{1}{\sigma} \widehat{c}_{t+\tau}+\frac{\varphi}{1+(1-\psi) \varphi} \widehat{y}_{t+\tau}+\frac{(1-\psi) \varphi}{1+(1-\psi) \varphi}\left(\widehat{r}_{t+\tau}^{*}+\widehat{p}_{t+\tau}\right)+\frac{1}{1+(1-\psi) \varphi} \widehat{p}_{t+\tau}, \\
\widehat{w}_{t+\tau}^{*} & \approx-\frac{\varphi}{1+(1-\psi) \varphi} \widehat{a}_{t+\tau}^{*}+\frac{1}{1+(1-\psi) \varphi} \frac{1}{\sigma} \widehat{c}_{t+\tau}^{*}+\frac{\varphi}{1+(1-\psi) \varphi} \widehat{y}_{t+\tau}^{*}+\frac{(1-\psi) \varphi}{1+(1-\psi) \varphi}\left(\widehat{r}_{t+\tau}^{* *}+\widehat{p}_{t+\tau}^{*}\right)+\frac{1}{1+(1-\psi) \varphi} \widehat{p}_{t+\tau}^{*}
\end{aligned}
$$

Naturally, the labor market clearing conditions and the marginal costs reduce to the standard linear-in-labor case without capital if the labor share in the production function goes to one (i.e., $\psi \rightarrow 1$ ). If I combine these two log-linearized equations, it follows that the marginal costs can be expressed as,

$$
\begin{aligned}
\widehat{m c}_{t+\tau} & \approx \frac{\psi}{1+(1-\psi) \varphi} \frac{1}{\sigma} \widehat{c}_{t+\tau}+\frac{\varphi \psi}{1+(1-\psi) \varphi} \widehat{y}_{t+\tau}-\frac{1+\varphi}{1+(1-\psi) \varphi}\left(\widehat{a}_{t+\tau}-(1-\psi)\left(\widehat{r}_{t+\tau}^{z}+\widehat{p}_{t+\tau}\right)\right)+\frac{\psi}{1+(1-\psi) \varphi} \widehat{p}_{t+\tau}, \\
\widehat{m c}_{t+\tau}^{*} & \approx \frac{\psi}{1+(1-\psi) \varphi} \frac{1}{\sigma} \widehat{c}_{t+\tau}^{*}+\frac{\varphi \psi}{1+(1-\psi) \varphi} \widehat{y}_{t+\tau}^{*}-\frac{1+\varphi}{1+(1-\psi) \varphi}\left(\widehat{a}_{t+\tau}^{*}-(1-\psi)\left(\widehat{r}_{t+\tau}^{z *}+\widehat{p}_{t+\tau}^{*}\right)\right)+\frac{\psi}{1+(1-\psi) \varphi} \widehat{p}_{t+\tau}^{*},
\end{aligned}
$$

where $\widehat{y}_{t+\tau}$ and $\widehat{y}_{t+\tau}^{*}$ denote domestic and foreign aggregate output. Finally, if I combine the marginal cost equations with the output equations derived before in (298) - (299), it follows that,

$$
\begin{aligned}
& \widehat{m c}_{t+\tau}-\widehat{p}_{t+\tau} \approx \frac{\psi}{1+(1-\psi) \varphi} \frac{1}{\sigma} \widehat{c}_{t+\tau}-\frac{\varphi \psi}{1+(1-\psi) \varphi} \eta\left(\widehat{p}_{t+\tau}^{H, W}-\widehat{p}_{t+\tau}^{W}\right)+\frac{\varphi \psi}{1+(1-\psi) \varphi}\left(\left(1-\gamma_{x}\right) \widehat{c}_{t+\tau}^{a W}+\gamma_{x} \widehat{x}_{t+\tau}^{a W}\right)-\ldots \\
& \quad \frac{1+\varphi}{1+(1-\psi) \varphi}\left(\widehat{a}_{t+\tau}-(1-\psi) \widehat{r}_{t+\tau}^{z}\right), \\
& \widehat{m c}_{t+\tau}^{*}-\widehat{p}_{t+\tau}^{*} \approx \frac{\psi}{1+(1-\psi) \varphi} \frac{1}{\sigma} \widehat{c}_{t+\tau}^{*}-\frac{\varphi \psi}{1+(1-\psi) \varphi} \eta\left(\widehat{p}_{t+\tau}^{F, W *}-\widehat{p}_{t+\tau}^{W *}\right)+\frac{\varphi \psi}{1+(1-\psi) \varphi}\left(\left(1-\gamma_{x}\right) \widehat{c}_{t+\tau}^{a W *}+\gamma_{x} \widehat{x}_{t+\tau}^{a W *}\right)-\ldots \\
& \quad \frac{1+\varphi}{1+(1-\psi) \varphi}\left(\widehat{a}_{t+\tau}^{*}-(1-\psi) \widehat{r}_{t+\tau}^{*}\right) .
\end{aligned}
$$

I can use my characterization of the real marginal costs with the pricing formulas log-linearized before 
to write that,

$$
\begin{aligned}
& \widehat{\widetilde{p}}_{t}(h)-\widehat{p}_{t} \approx \\
& \quad(1-\beta \alpha)\left(\frac{\psi}{1+(1-\psi) \varphi}\right) \sum_{\tau=0}^{+\infty}(\beta \alpha)^{\tau} \mathbb{E}_{t}\left[\begin{array}{c}
\frac{1}{\sigma} \widehat{c}_{t+\tau}-\varphi \eta\left(\widehat{p}_{t+\tau}^{H, W}-\widehat{p}_{t+\tau}^{W}\right)+\varphi\left((1-\gamma) \widehat{c}_{t+\tau}^{a W}+\gamma_{x} \widehat{x}_{t+\tau}^{a W}\right)-\ldots \\
\left(\frac{1+\varphi}{\psi}\right)\left(\widehat{a}_{t+\tau}-(1-\psi) \widehat{r}_{t+\tau}^{z}\right)
\end{array}\right]+\ldots \\
& \quad \sum_{\tau=1}^{+\infty}(\beta \alpha)^{\tau} \mathbb{E}_{t}\left(\widehat{\pi}_{t+\tau}\right), \\
& \widehat{\widetilde{p}}_{t}^{*}(h)-\widehat{p}_{t}^{*} \approx \\
& \quad(1-\beta \alpha)\left(\frac{\psi}{1+(1-\psi) \varphi}\right) \sum_{\tau=0}^{+\infty}(\beta \alpha)^{\tau} \mathbb{E}_{t}\left[\begin{array}{c}
\frac{1}{\sigma} \widehat{c}_{t+\tau}-\varphi \eta\left(\widehat{p}_{t+\tau}^{H, W}-\widehat{p}_{t+\tau}^{W}\right)+\varphi\left(\left(1-\gamma_{x}\right) \widehat{c}_{t+\tau}^{a W}+\gamma_{x} \widehat{x}_{t+\tau}^{a W}\right)-\ldots \\
\left(\frac{1+\varphi}{\psi}\right)\left(\widehat{a}_{t+\tau}-(1-\psi) \widehat{r}_{t+\tau}^{z}\right)-\left(\frac{1+(1-\psi) \varphi}{\psi}\right) \widehat{r}_{t+\tau}
\end{array}\right]+\ldots \\
& \quad \sum_{\tau=1}^{+\infty}(\beta \alpha)^{\tau} \mathbb{E}_{t}\left(\widehat{\pi}_{t+\tau}^{*}\right), \\
& \widehat{\widetilde{p}}_{t}(f)-\widehat{p}_{t} \approx \\
& \quad(1-\beta \alpha)\left(\frac{\psi}{1+(1-\psi) \varphi}\right) \sum_{\tau=0}^{+\infty}(\beta \alpha)^{\tau} \mathbb{E}_{t}\left[\begin{array}{c}
\frac{1}{\sigma} \widehat{c}_{t+\tau}^{*}-\varphi \eta\left(\widehat{p}_{t+\tau}^{F, W *}-\widehat{p}_{t+\tau}^{W *}\right)+\varphi\left(\left(1-\gamma_{x}\right) \widehat{c}_{t+\tau}^{a W *}+\gamma_{x} \widehat{x}_{t+\tau}^{a W *}\right)-\ldots \\
\left(\frac{1+\varphi}{\psi}\right)\left(\widehat{a}_{t+\tau}^{*}-(1-\psi) \widehat{r}_{t+\tau}^{z *}\right)+\left(\frac{1+(1-\psi) \varphi}{\psi}\right) \widehat{r}_{t+\tau}
\end{array}\right]+\ldots \\
& \quad \sum_{\tau=1}^{+\infty}(\beta \alpha)^{\tau} \mathbb{E}_{t}\left(\widehat{\pi}_{t+\tau}\right), \\
& \widehat{\widetilde{p}}_{t}^{*}(f)-\widehat{p}_{t}^{*} \approx \\
& \quad(1-\beta \alpha)\left(\frac{\psi}{1+(1-\psi) \varphi}\right) \sum_{\tau=0}^{+\infty}(\beta \alpha)^{\tau} \mathbb{E}_{t}\left[\begin{array}{c}
\frac{1}{\sigma} \widehat{c}_{t+\tau}^{*}-\varphi \eta\left(\widehat{p}_{t+\tau}^{F, W *}-\widehat{p}_{t+\tau}^{W *}\right)+\varphi\left(\left(1-\gamma_{x}\right) \widehat{c}_{t+\tau}^{a W *}+\gamma_{x} \widehat{x}_{t+\tau}^{a W *}\right)-\ldots \\
\left(\frac{1+\varphi}{\psi}\right)\left(\widehat{a}_{t+\tau}^{*}-(1-\psi) \widehat{r}_{t+\tau}^{z *}\right)
\end{array}\right]+\ldots \\
& \quad \sum_{\tau=1}^{+\infty}(\beta \alpha)^{\tau} \mathbb{E}_{t}\left(\widehat{\pi}_{t+\tau}^{*}\right) .
\end{aligned}
$$

I log-linearize the price sub-indexes in $(78)-(79)$ and $(80)-(81)$ and re-arrange them to obtain that,

$$
\begin{aligned}
& \widehat{\widetilde{p}}_{t}(h)-\widehat{p}_{t} \approx\left(\widehat{p}_{t}^{H}-\widehat{p}_{t}\right)+\left(\frac{\alpha}{1-\alpha}\right) \widehat{\pi}_{t}^{H}, \\
& \widehat{\widetilde{p}}_{t}^{*}(h)-\widehat{p}_{t}^{*} \approx\left(\widehat{p}_{t}^{H *}-\widehat{p}_{t}^{*}\right)+\left(\frac{\alpha}{1-\alpha}\right) \widehat{\pi}_{t}^{H *}, \\
& \widehat{\widetilde{p}}_{t}(f)-\widehat{p}_{t} \approx\left(\widehat{p}_{t}^{F}-\widehat{p}_{t}\right)+\left(\frac{\alpha}{1-\alpha}\right) \widehat{\pi}_{t}^{F}, \\
& \widehat{\widetilde{p}}_{t}^{*}(f)-\widehat{p}_{t}^{*} \approx\left(\widehat{p}_{t}^{F *}-\widehat{p}_{t}^{*}\right)+\left(\frac{\alpha}{1-\alpha}\right) \widehat{\pi}_{t}^{F *},
\end{aligned}
$$

which is quite convenient for my purposes. I replace the isolated terms $\frac{1}{\sigma} \widehat{c}_{t}$ and $\frac{1}{\sigma} \widehat{c}_{t}^{*}$ out of the marginal cost equations. If I define the world consumption as $\widehat{c}_{t}^{a W} \equiv \phi_{H}\left(\frac{1-\gamma_{x}^{a}}{1-\gamma_{x}}\right) \widehat{c}_{t}+\phi_{F}\left(\frac{1-\gamma_{x^{*}}^{a}}{1-\gamma_{x}}\right) \widehat{c}_{t}^{*}$ and $\widehat{c}_{t}^{a W *} \equiv$ $\phi_{F}\left(\frac{1-\gamma_{x}^{a}}{1-\gamma_{x}}\right) \widehat{c}_{t}+\phi_{H}\left(\frac{1-\gamma_{x^{*}}^{a}}{1-\gamma_{x}}\right) \widehat{c}_{t}^{*}$ and the relative consumption as $\widehat{c}_{t}^{a R} \equiv\left(\frac{1-\gamma_{x}^{a}}{1-\gamma_{x}}\right) \widehat{c}_{t}-\left(\frac{1-\gamma_{x^{*}}^{a}}{1-\gamma_{x}}\right) \widehat{c}_{t}^{*}$, then I can write that,

$$
\begin{aligned}
\widehat{c}_{t} & =\left(\frac{1-\gamma_{x}}{1-\gamma_{x}^{a}}\right)\left(\widehat{c}_{t}^{a W}+\phi_{F} \widehat{c}_{t}^{a R}\right), \\
\widehat{c}_{t}^{*} & =\left(\frac{1-\gamma_{x}}{1-\gamma_{x^{*}}^{a}}\right)\left(\widehat{c}_{t}^{a W *}-\phi_{F} \widehat{c}_{t}^{a R}\right) .
\end{aligned}
$$


Hence, the pricing equations can be expressed more compactly as,

$$
\begin{aligned}
& \widehat{\pi}_{t}^{H}+\left(\frac{1-\alpha}{\alpha}\right)\left(\widehat{p}_{t}^{H}-\widehat{p}_{t}\right) \approx \\
& \Psi\left(\frac{\psi}{1+(1-\psi) \varphi}\right) \sum_{\tau=0}^{+\infty}(\beta \alpha)^{\tau} \mathbb{E}_{t}\left[\begin{array}{c}
\left(\left(\frac{1-\gamma_{x}}{1-\gamma_{x}^{a}}\right) \sigma^{-1}+\left(1-\gamma_{x}\right) \varphi\right) \widehat{c}_{t+\tau}^{a W}+\gamma_{x} \varphi \widehat{x}_{t+\tau}^{a W}+\ldots \\
\phi_{F}\left(\frac{1-\gamma_{x}}{1-\gamma_{x}^{a}}\right) \sigma^{-1} \widehat{c}_{t+\tau}^{a R}-\varphi \eta\left(\widehat{p}_{t+\tau}^{H, W}-\widehat{p}_{t+\tau}^{W}\right)-\ldots \\
\left(\frac{1+\varphi}{\psi}\right)\left(\widehat{a}_{t+\tau}-(1-\psi) \widehat{r}_{t+\tau}^{z}\right)
\end{array}\right]+\ldots \\
& \left(\frac{1-\alpha}{\alpha}\right) \sum_{\tau=1}^{+\infty}(\beta \alpha)^{\tau} \mathbb{E}_{t}\left(\widehat{\pi}_{t+\tau}\right), \\
& \widehat{\pi}_{t}^{H *}+\left(\frac{1-\alpha}{\alpha}\right)\left(\widehat{p}_{t}^{H *}-\widehat{p}_{t}^{*}\right) \approx \\
& \Psi\left(\frac{\psi}{1+(1-\psi) \varphi}\right) \sum_{\tau=0}^{+\infty}(\beta \alpha)^{\tau} \mathbb{E}_{t}\left[\begin{array}{c}
\left(\left(\frac{1-\gamma_{x}}{1-\gamma_{x}^{a}}\right) \sigma^{-1}+\left(1-\gamma_{x}\right) \varphi\right) \widehat{c}_{t+\tau}^{a W}+\gamma_{x} \varphi \widehat{x}_{t+\tau}^{a W}+\ldots \\
\phi_{F}\left(\frac{1-\gamma_{x}}{1-\gamma_{x}^{a}}\right) \sigma^{-1} \widehat{c}_{t+\tau}^{a R}-\left(\frac{1+(1-\psi) \varphi}{\psi}\right) \widehat{r s}_{t+\tau}-\varphi \eta\left(\widehat{p}_{t+\tau}^{H, W}-\widehat{p}_{t+\tau}^{W}\right)-\ldots \\
\left(\frac{1+\varphi}{\psi}\right)\left(\widehat{a}_{t+\tau}-(1-\psi) \widehat{r}_{t+\tau}^{z}\right)
\end{array}\right]+\ldots \\
& \left(\frac{1-\alpha}{\alpha}\right) \sum_{\tau=1}^{+\infty}(\beta \alpha)^{\tau} \mathbb{E}_{t}\left(\widehat{\pi}_{t+\tau}^{*}\right), \\
& \widehat{\pi}_{t}^{F}+\left(\frac{1-\alpha}{\alpha}\right)\left(\widehat{p}_{t}^{F}-\widehat{p}_{t}\right) \approx \\
& \Psi\left(\frac{\psi}{1+(1-\psi) \varphi}\right) \sum_{\tau=0}^{+\infty}(\beta \alpha)^{\tau} \mathbb{E}_{t}\left[\begin{array}{c}
\left(\left(\frac{1-\gamma_{x}}{1-\gamma_{x^{*}}}\right) \sigma^{-1}+\left(1-\gamma_{x}\right) \varphi\right) \widehat{c}_{t+\tau}^{a W *}+\gamma_{x} \varphi \widehat{x}_{t+\tau}^{a W *}-\ldots \\
\phi_{F}\left(\frac{1-\gamma_{x}}{1-\gamma_{x^{*}}^{a}}\right) \sigma^{-1} \widehat{c}_{t+\tau}^{a R}+\left(\frac{1+(1-\psi) \varphi}{\psi}\right) \widehat{r s}_{t+\tau}-\varphi \eta\left(\widehat{p}_{t+\tau}^{F, W *}-\widehat{p}_{t+\tau}^{W *}\right)-\ldots \\
\left(\frac{1+\varphi}{\psi}\right)\left(\widehat{a}_{t+\tau}^{*}-(1-\psi) \widehat{r}_{t+\tau}^{z *}\right)
\end{array}\right]+\ldots \\
& \left(\frac{1-\alpha}{\alpha}\right) \sum_{\tau=1}^{+\infty}(\beta \alpha)^{\tau} \mathbb{E}_{t}\left(\widehat{\pi}_{t+\tau}\right), \\
& \widehat{\pi}_{t}^{F *}+\left(\frac{1-\alpha}{\alpha}\right)\left(\widehat{p}_{t}^{F *}-\widehat{p}_{t}^{*}\right) \approx \\
& \Psi\left(\frac{\psi}{1+(1-\psi) \varphi}\right) \sum_{\tau=0}^{+\infty}(\beta \alpha)^{\tau} \mathbb{E}_{t}\left[\begin{array}{c}
\left(\left(\frac{1-\gamma_{x}}{1-\gamma_{x *}^{a}}\right) \sigma^{-1}+\left(1-\gamma_{x}\right) \varphi\right) \widehat{c}_{t+\tau}^{a W *}+\gamma_{x} \varphi \widehat{x}_{t+\tau}^{a W *}-\ldots \\
\phi_{F}\left(\frac{1-\gamma_{x}}{1-\gamma_{x^{*}}^{a}}\right) \sigma^{-1} \widehat{c}_{t+\tau}^{a R}-\varphi \eta\left(\widehat{p}_{t+\tau}^{F, W *}-\widehat{p}_{t+\tau}^{W *}\right)-\ldots \\
\left(\frac{1+\varphi}{\psi}\right)\left(\widehat{a}_{t+\tau}^{*}-(1-\psi) \widehat{r}_{t+\tau}^{z *}\right)
\end{array}\right]+\ldots \\
& \left(\frac{1-\alpha}{\alpha}\right) \sum_{\tau=1}^{+\infty}(\beta \alpha)^{\tau} \mathbb{E}_{t}\left(\widehat{\pi}_{t+\tau}^{*}\right),
\end{aligned}
$$

where the composite coefficient is defined as $\Psi \equiv \frac{(1-\alpha)(1-\beta \alpha)}{\alpha}$.

Furthermore, this system of pricing equations can be expressed in the form of a system of expectational difference equations. Let me focus on the first equation as an example. If I re-write the equation at time $t+1$ and take conditional expectations on information up to time $t$, it should follow that,

$$
\begin{aligned}
& \mathbb{E}_{t}\left[\widehat{\pi}_{t+1}^{H}+\left(\frac{1-\alpha}{\alpha}\right)\left(\widehat{p}_{t+1}^{H}-\widehat{p}_{t+1}\right)\right] \approx \approx \\
&\left(\frac{\psi}{1+(1-\psi) \varphi}\right) \Psi \sum_{\tau=0}^{+\infty}(\beta \alpha)^{\tau} \mathbb{E}_{t}\left[\begin{array}{c}
\left(\left(\frac{1-\gamma_{x}}{1-\gamma_{x}^{a}}\right) \sigma^{-1}+\left(1-\gamma_{x}\right) \varphi\right) \widehat{c}_{t+1+\tau}^{a W}+\gamma_{x} \varphi \widehat{x}_{t+1+\tau}^{a W}+\ldots \\
\phi_{F}\left(\frac{1-\gamma_{x}}{1-\gamma_{x}^{a}}\right) \sigma^{-1} \widehat{c}_{t+1+\tau}^{a R}-\varphi \eta\left(\widehat{p}_{t+1+\tau}^{H, W}-\widehat{p}_{t+1+\tau}^{W}\right)-\ldots \\
\left(\frac{1+\varphi}{\psi}\right)\left(\widehat{a}_{t+1+\tau}-(1-\psi) \widehat{r}_{t+1+\tau}^{z}\right)
\end{array}\right]+\ldots \\
&\left(\frac{1-\alpha}{\alpha}\right) \sum_{\tau=1}^{+\infty}(\beta \alpha)^{\tau} \mathbb{E}_{t}\left(\widehat{\pi}_{t+1+\tau}\right) .
\end{aligned}
$$


Hence, using this conditional expectation, the pricing equation can easily be decomposed in two terms as,

$$
\begin{aligned}
& \widehat{\pi}_{t}^{H}+\left(\frac{1-\alpha}{\alpha}\right)\left(\widehat{p}_{t}^{H}-\widehat{p}_{t}\right) \approx \\
& {\left[\begin{array}{c}
\left(\left(\frac{\psi}{1-\gamma_{x}}\right) \sigma^{-1}+\left(1-\gamma_{x}\right) \varphi\right) \widehat{c}_{t}^{a W}+\gamma_{x} \varphi \widehat{x}_{t}^{a W}+\ldots \\
\phi_{F}\left(\frac{1-\gamma_{x}}{1-\gamma_{x}^{a}}\right) \sigma^{-1} \widehat{c}_{t}^{a R}-\varphi \eta\left(\widehat{p}_{t}^{H, W}-\widehat{p}_{t}^{W}\right)-\ldots \\
\left(\frac{1+\varphi}{\psi}\right)\left(\widehat{a}_{t}-(1-\psi) \widehat{r}_{t}^{z}\right)
\end{array}\right]+\ldots} \\
& (1-\alpha) \beta \mathbb{E}_{t}\left(\widehat{\pi}_{t+1}\right)+\alpha \beta \mathbb{E}_{t}\left[\widehat{\pi}_{t+1}^{H}+\left(\frac{1-\alpha}{\alpha}\right)\left(\widehat{p}_{t+1}^{H}-\widehat{p}_{t+1}\right)\right] .
\end{aligned}
$$

Further re-arranging allows me to express the expectational difference equation as,

$$
\begin{aligned}
& \widehat{\pi}_{t}^{H}-\beta \mathbb{E}_{t}\left[\widehat{\pi}_{t+1}^{H}\right]+\Psi\left(\widehat{p}_{t}^{H}-\widehat{p}_{t}\right) \\
& \approx\left(\frac{\psi}{1+(1-\psi) \varphi}\right) \Psi\left[\begin{array}{c}
\left(\left(\frac{1-\gamma_{x}}{1-\gamma_{x}^{a}}\right) \sigma^{-1}+\left(1-\gamma_{x}\right) \varphi\right) \widehat{c}_{t}^{a W}+\gamma_{x} \varphi \widehat{x}_{t}^{a W}+\ldots \\
\phi_{F}\left(\frac{1-\gamma_{x}}{1-\gamma_{x}^{a}}\right) \sigma^{-1} \widehat{c}_{t}^{a R}-\varphi \eta\left(\widehat{p}_{t}^{H, W}-\widehat{p}_{t}^{W}\right)-\ldots \\
\left(\frac{1+\varphi}{\psi}\right)\left(\widehat{a}_{t}-(1-\psi) \widehat{r}_{t}^{z}\right)
\end{array}\right] .
\end{aligned}
$$

I can apply the same approach (and algebraic steps) to re-write all other pricing equations as expectational difference equations, i.e.

$$
\begin{aligned}
& \widehat{\pi}_{t}^{H *}-\beta \mathbb{E}_{t}\left(\widehat{\pi}_{t+1}^{H *}\right)+\Psi\left(\widehat{p}_{t}^{H *}-\widehat{p}_{t}^{*}\right) \\
& \approx\left(\frac{\psi}{1+(1-\psi) \varphi}\right) \Psi\left[\begin{array}{c}
\left(\left(\frac{1-\gamma_{x}}{1-\gamma_{x}^{x}}\right) \sigma^{-1}+\left(1-\gamma_{x}\right) \varphi\right) \widehat{c}_{t}^{a W}+\gamma_{x} \varphi \widehat{x}_{t}^{a W}+\ldots \\
\phi_{F}\left(\frac{1-\gamma_{x}}{1-\gamma_{x}^{a}}\right) \sigma^{-1} \widehat{c}_{t}^{a R}-\left(\frac{1+(1-\psi) \varphi}{\psi}\right) \widehat{r}_{t}-\varphi \eta\left(\widehat{p}_{t}^{H, W}-\widehat{p}_{t}^{W}\right)-\ldots \\
\left(\frac{1+\varphi}{\psi}\right)\left(\widehat{a}_{t}-(1-\psi) \widehat{r}_{t}^{z}\right)
\end{array}\right], \\
& \widehat{\pi}_{t}^{F}-\beta \mathbb{E}_{t}\left(\widehat{\pi}_{t+1}^{F}\right)+\Psi\left(\widehat{p}_{t}^{F}-\widehat{p}_{t}\right) \\
& \approx\left(\frac{\psi}{1+(1-\psi) \varphi}\right) \Psi\left[\begin{array}{c}
\left(\left(\frac{1-\gamma_{x}}{1-\gamma_{x}^{*}}\right) \sigma^{-1}+\left(1-\gamma_{x}\right) \varphi\right) \widehat{c}_{t}^{a W *}+\gamma_{x} \varphi \widehat{x}_{t}^{a W *}-\ldots \\
\phi_{F}\left(\frac{1-\gamma_{x}}{1-\gamma_{x^{*}}^{a}}\right) \sigma^{-1} \widehat{c}_{t}^{a R}+\left(\frac{1+(1-\psi) \varphi}{\psi}\right) \widehat{r s}_{t}-\varphi \eta\left(\widehat{p}_{t}^{F, W *}-\widehat{p}_{t}^{W *}\right)-\ldots \\
\left(\frac{1+\varphi}{\psi}\right)\left(\widehat{a}_{t}^{*}-(1-\psi) \widehat{r}_{t}^{z *}\right)
\end{array}\right] \\
& \widehat{\pi}_{t}^{F *}-\beta \mathbb{E}_{t}\left(\widehat{\pi}_{t+1}^{F *}\right)+\Psi\left(\widehat{p}_{t}^{F *}-\widehat{p}_{t}^{*}\right) \\
& \approx\left(\frac{\psi}{1+(1-\psi) \varphi}\right) \Psi\left[\begin{array}{c}
\left(\left(\frac{1-\gamma_{x}}{1-\gamma_{x *}^{a}}\right) \sigma^{-1}+\left(1-\gamma_{x}\right) \varphi\right) \widehat{c}_{t}^{a W *}+\gamma_{x} \varphi \widehat{x}_{t}^{a W *}-\ldots \\
\phi_{F}\left(\frac{1-\gamma_{x}}{1-\gamma_{x^{*}}^{a}}\right) \sigma^{-1} \widehat{c}_{t}^{a R}-\varphi \eta\left(\widehat{p}_{t}^{F, W *}-\widehat{p}_{t}^{W *}\right)-\ldots \\
\left(\frac{1+\varphi}{\psi}\right)\left(\widehat{a}_{t}^{*}-(1-\psi) \widehat{r}_{t}^{2 *}\right)
\end{array}\right] .
\end{aligned}
$$

These equations provide a very simple characterization of the price dynamics at the price sub-index level.

Now, I use the pricing equations described above to infer the dynamics of the relative price sub-indexes $\widehat{\pi}_{t}^{H, R} \equiv \widehat{\pi}_{t}^{H}-\widehat{\pi}_{t}^{H *}\left(\widehat{p}_{t}^{H, R} \equiv \widehat{p}_{t}^{H}-\widehat{p}_{t}^{H *}\right)$ and $\widehat{\pi}_{t}^{F, R} \equiv \widehat{\pi}_{t}^{F}-\widehat{\pi}_{t}^{F *}\left(\widehat{p}_{t}^{F, R} \equiv \widehat{p}_{t}^{F}-\widehat{p}_{t}^{F *}\right)$ as follows,

$$
\begin{aligned}
\widehat{\pi}_{t}^{H, R}-\beta \mathbb{E}_{t}\left(\widehat{\pi}_{t+1}^{H, R}\right)+\Psi\left(\widehat{p}_{t}^{H, R}-\widehat{p}_{t}^{R}\right) & \approx \Psi \widehat{r}_{t}, \\
\widehat{\pi}_{t}^{F, R}-\beta \mathbb{E}_{t}\left(\widehat{\pi}_{t+1}^{F, R}\right)+\Psi\left(\widehat{p}_{t}^{F, R}-\widehat{p}_{t}^{R}\right) & \approx \Psi \widehat{r}_{t},
\end{aligned}
$$

where $\widehat{p}_{t}^{R} \equiv \widehat{p}_{t}-\widehat{p}_{t}^{*}$ is the relative CPI across countries. Using these relative price sub-index dynamics, I can 
re-write the pricing equations further as follows,

$$
\begin{aligned}
& \widehat{\pi}_{t}^{H} \approx \beta \mathbb{E}_{t}\left(\widehat{\pi}_{t+1}^{H}\right)+\ldots \\
& \left(\frac{\psi}{1+(1-\psi) \varphi}\right) \Psi\left[\begin{array}{c}
\left(\left(\frac{1-\gamma_{x}}{1-\gamma_{x}^{a}}\right) \sigma^{-1}+\left(1-\gamma_{x}\right) \varphi\right) \widehat{c}_{t}^{a W}+\gamma_{x} \varphi \widehat{x}_{t}^{a W}+\ldots \\
\phi_{F}\left(\frac{1-\gamma_{x}}{1-\gamma_{x}^{a}}\right) \sigma^{-1} \widehat{c}_{t}^{a R}-\varphi \eta\left(\widehat{p}_{t}^{H, W}-\widehat{p}_{t}^{W}\right)-\ldots \\
\left(\frac{1+(1-\psi) \varphi}{\psi}\right)\left(\widehat{p}_{t}^{H}-\widehat{p}_{t}\right)-\left(\frac{1+\varphi}{\psi}\right)\left(\widehat{a}_{t}-(1-\psi) \widehat{r}_{t}^{z}\right)
\end{array}\right] \\
& \widehat{\pi}_{t}^{H *} \approx \beta \mathbb{E}_{t}\left(\widehat{\pi}_{t+1}^{H *}\right)+\ldots \\
& \left(\frac{\psi}{1+(1-\psi) \varphi}\right) \Psi\left[\begin{array}{c}
\left(\left(\frac{1-\gamma_{x}}{1-\gamma_{x}^{a}}\right) \sigma^{-1}+\left(1-\gamma_{x}\right) \varphi\right) \widehat{c}_{t}^{a W}+\gamma_{x} \varphi \widehat{x}_{t}^{a W}+\ldots \\
\phi_{F}\left(\frac{1-\gamma_{x}}{1-\gamma_{x}^{a}}\right) \sigma^{-1} \widehat{c}_{t}^{a R}-\left(\frac{1+(1-\psi) \varphi}{\psi}\right) \widehat{r}_{t}-\varphi \eta\left(\widehat{p}_{t}^{H, W}-\widehat{p}_{t}^{W}\right)-\ldots \\
\left(\frac{1+(1-\psi) \varphi}{\psi}\right)\left(\widehat{p}_{t}^{H *}-\widehat{p}_{t}^{*}\right)-\left(\frac{1+\varphi}{\psi}\right)\left(\widehat{a}_{t}-(1-\psi) \widehat{r}_{t}^{z}\right)
\end{array}\right], \\
& \widehat{\pi}_{t}^{F} \approx \beta \mathbb{E}_{t}\left(\widehat{\pi}_{t+1}^{F}\right)+\ldots \\
& \left(\frac{\psi}{1+(1-\psi) \varphi}\right) \Psi\left[\begin{array}{c}
\left(\left(\frac{1-\gamma_{x}}{1-\gamma_{x}^{*}}\right) \sigma^{-1}+\left(1-\gamma_{x}\right) \varphi\right) \widehat{c}_{t}^{a W *}+\gamma_{x} \varphi \widehat{x}_{t}^{a W *}-\ldots \\
\phi_{F}\left(\frac{1-\gamma_{x}}{1-\gamma_{x^{*}}^{a}}\right) \sigma^{-1} \widehat{c}_{t}^{a R}+\left(\frac{1+(1-\psi) \varphi}{\psi}\right) \widehat{r s}_{t}-\varphi \eta\left(\widehat{p}_{t}^{F, W *}-\widehat{p}_{t}^{W *}\right)-\ldots \\
\left(\frac{1+(1-\psi) \varphi}{\psi}\right)\left(\widehat{p}_{t}^{F}-\widehat{p}_{t}\right)-\left(\frac{1+\varphi}{\psi}\right)\left(\widehat{a}_{t}^{*}-(1-\psi) \widehat{r}_{t}^{z *}\right)
\end{array}\right], \\
& \widehat{\pi}_{t}^{F *} \approx \beta \mathbb{E}_{t}\left(\widehat{\pi}_{t+1}^{F *}\right)+\ldots \\
& \left(\frac{\psi}{1+(1-\psi) \varphi}\right) \Psi\left[\begin{array}{c}
\left(\left(\frac{1-\gamma_{x}}{1-\gamma_{x}^{x}}\right) \sigma^{-1}+\left(1-\gamma_{x}\right) \varphi\right) \widehat{c}_{t}^{a W *}+\gamma_{x} \varphi \widehat{x}_{t}^{a W *}-\ldots \\
\phi_{F}\left(\frac{1-\gamma_{x}}{1-\gamma_{x}^{a}}\right) \sigma^{-1} \widehat{c}_{t}^{a R}-\varphi \eta\left(\widehat{p}_{t}^{F, W *}-\widehat{p}_{t}^{W *}\right)-\ldots \\
\left(\frac{1+(1-\psi) \varphi}{\psi}\right)\left(\widehat{p}_{t}^{F *}-\widehat{p}_{t}^{*}\right)-\left(\frac{1+\varphi}{\psi}\right)\left(\widehat{a}_{t}^{*}-(1-\psi) \widehat{r}_{t}^{z *}\right)
\end{array}\right] .
\end{aligned}
$$

I have defined the world price sub-indexes as $\widehat{p}_{t}^{H, W} \equiv \phi_{H} \widehat{p}_{t}^{H}+\phi_{F} \widehat{p}_{t}^{H *}$ and $\widehat{p}_{t}^{F, W *} \equiv \phi_{F} \widehat{p}_{t}^{F}+\phi_{H} \widehat{p}_{t}^{F *}$, and the relative price sub-indexes as $\widehat{p}_{t}^{H, R} \equiv \widehat{p}_{t}^{H}-\widehat{p}_{t}^{H *}$ and $\widehat{p}_{t}^{F, R} \equiv \widehat{p}_{t}^{F}-\widehat{p}_{t}^{F *}$. Then, naturally, I can write that,

$$
\begin{aligned}
\widehat{p}_{t}^{H} & =\widehat{p}_{t}^{H, W}+\phi_{F} \widehat{p}_{t}^{H, R}, \widehat{p}_{t}^{H *}=\widehat{p}_{t}^{H, W}-\phi_{H} \widehat{p}_{t}^{H, R}, \\
\widehat{p}_{t}^{F} & =\widehat{p}_{t}^{F, W *}+\phi_{H} \widehat{p}_{t}^{F, R}, \widehat{p}_{t}^{F *}=\widehat{p}_{t}^{F, W *}-\phi_{F} \widehat{p}_{t}^{F, R} .
\end{aligned}
$$

Analogously, I have defined the world CPI as $\widehat{p}_{t}^{W} \equiv \phi_{H} \widehat{p}_{t}+\phi_{F} \widehat{p}_{t}^{*}$ and $\widehat{p}_{t}^{W *} \equiv \phi_{F} \widehat{p}_{t}+\phi_{H} \widehat{p}_{t}^{*}$, and the relative CPI as $\widehat{p}_{t}^{R} \equiv \widehat{p}_{t}-\widehat{p}_{t}^{*}$. So, I can write that,

$$
\begin{aligned}
& \widehat{p}_{t}=\widehat{p}_{t}^{W}+\phi_{F} \widehat{p}_{t}^{R}, \widehat{p}_{t}^{*}=\widehat{p}_{t}^{W}-\phi_{H} \widehat{p}_{t}^{R} \\
& \widehat{p}_{t}=\widehat{p}_{t}^{W *}+\phi_{H} \widehat{p}_{t}^{R}, \widehat{p}_{t}^{*}=\widehat{p}_{t}^{W *}-\phi_{F} \widehat{p}_{t}^{R}
\end{aligned}
$$


Therefore, I can re-arrange the pricing equations as follows,

$$
\begin{aligned}
& \widehat{\pi}_{t}^{H} \approx \beta \mathbb{E}_{t}\left(\widehat{\pi}_{t+1}^{H}\right)+\ldots \\
& \left(\frac{\psi}{1+(1-\psi) \varphi}\right) \Psi\left[\begin{array}{c}
\left(\left(\frac{1-\gamma_{x}}{1-\gamma_{x}^{a}}\right) \sigma^{-1}+\left(1-\gamma_{x}\right) \varphi\right) \widehat{c}_{t}^{a W}+\gamma_{x} \varphi \widehat{x}_{t}^{a W}+\ldots \\
\phi_{F}\left(\frac{1-\gamma_{x}}{1-\gamma_{x}^{a}}\right) \sigma^{-1} \widehat{c}_{t}^{a R}-\left(\frac{1+(1-\psi) \varphi}{\psi}+\varphi \eta\right)\left(\widehat{p}_{t}^{H, W}-\widehat{p}_{t}^{W}\right)-\ldots \\
\left(\frac{1+(1-\psi) \varphi}{\psi}\right) \phi_{F}\left(\widehat{p}_{t}^{H, R}-\widehat{p}_{t}^{R}\right)-\left(\frac{1+\varphi}{\psi}\right)\left(\widehat{a}_{t}-(1-\psi) \widehat{r}_{t}^{z}\right)
\end{array}\right], \\
& \widehat{\pi}_{t}^{H *} \approx \beta \mathbb{E}_{t}\left(\widehat{\pi}_{t+1}^{H *}\right)+\ldots \\
& \left(\frac{\psi}{1+(1-\psi) \varphi}\right) \Psi\left[\begin{array}{c}
\left(\left(\frac{1-\gamma_{x}}{1-\gamma_{x}^{a}}\right) \sigma^{-1}+\left(1-\gamma_{x}\right) \varphi\right) \widehat{c}_{t}^{a W}+\gamma_{x} \varphi \widehat{x}_{t}^{a W}+\ldots \\
\phi_{F}\left(\frac{1-\gamma_{x}}{1-\gamma_{x}^{a}}\right) \sigma^{-1} \widehat{c}_{t}^{a R}-\left(\frac{1+(1-\psi) \varphi}{\psi}\right) \widehat{r}_{t}-\ldots \\
\left(\frac{1+(1-\psi) \varphi}{\psi}+\varphi \eta\right)\left(\widehat{p}_{t}^{H, W}-\widehat{p}_{t}^{W}\right)+\ldots \\
\left(\frac{1+(1-\psi) \varphi}{\psi}\right) \phi_{H}\left(\widehat{p}_{t}^{H, R}-\widehat{p}_{t}^{R}\right)-\left(\frac{1+\varphi}{\psi}\right)\left(\widehat{a}_{t}-(1-\psi) \widehat{r}_{t}^{z}\right)
\end{array}\right], \\
& \widehat{\pi}_{t}^{F} \approx \beta \mathbb{E}_{t}\left(\widehat{\pi}_{t+1}^{F}\right)+\ldots \\
& \left(\frac{\psi}{1+(1-\psi) \varphi}\right) \Psi\left[\begin{array}{c}
\left(\left(\frac{1-\gamma_{x}}{1-\gamma_{x^{*}}^{a}}\right) \sigma^{-1}+\left(1-\gamma_{x}\right) \varphi\right) \widehat{c}_{t}^{a W *}+\gamma_{x} \varphi \widehat{x}_{t}^{a W *}-\ldots \\
\phi_{F}\left(\frac{1-\gamma_{x}}{1-\gamma_{x^{*}}}\right) \sigma^{-1} \widehat{c}_{t}^{a R}+\left(\frac{1+(1-\psi) \varphi}{\psi}\right) \widehat{r}_{t}-\ldots \\
\left(\frac{1+(1-\psi) \varphi}{\psi}+\varphi \eta\right)\left(\widehat{p}_{t}^{F, W *}-\widehat{p}_{t}^{W *}\right)-\ldots \\
\left(\frac{1+(1-\psi) \varphi}{\psi}\right) \phi_{H}\left(\widehat{p}_{t}^{F, R}-\widehat{p}_{t}^{R}\right)-\left(\frac{1+\varphi}{\psi}\right)\left(\widehat{a}_{t}^{*}-(1-\psi) \widehat{r}_{t}^{z *}\right)
\end{array}\right], \\
& \widehat{\pi}_{t}^{F *} \approx \beta \mathbb{E}_{t}\left(\widehat{\pi}_{t+1}^{F *}\right)+\ldots \\
& \left(\frac{\psi}{1+(1-\psi) \varphi}\right) \Psi\left[\begin{array}{c}
\left(\left(\frac{1-\gamma_{x}}{1-\gamma_{x}^{a}}\right) \sigma^{-1}+\left(1-\gamma_{x}\right) \varphi\right) \widehat{c}_{t}^{a W *}+\gamma_{x} \varphi \widehat{x}_{t}^{a W *}-\ldots \\
\phi_{F}\left(\frac{1-\gamma_{x}}{1-\gamma_{x *}^{a}}\right) \sigma^{-1} \widehat{c}_{t}^{a R}-\left(\frac{1+(1-\psi) \varphi}{\psi}+\varphi \eta\right)\left(\widehat{p}_{t}^{F, W *}-\widehat{p}_{t}^{W *}\right)+\ldots \\
\left(\frac{1+(1-\psi) \varphi}{\psi}\right) \phi_{F}\left(\widehat{p}_{t}^{F, R}-\widehat{p}_{t}^{R}\right)-\left(\frac{1+\varphi}{\psi}\right)\left(\widehat{a}_{t}^{*}-(1-\psi) \widehat{r}_{t}^{z *}\right)
\end{array}\right] .
\end{aligned}
$$

By appropriately replacing the efficiency conditions in (300) - (301), and after a little bit of algebra, I can 
now re-arrange the pricing equations for the sub-indexes in (304) - (307) as follows,

$$
\begin{aligned}
& \widehat{\pi}_{t}^{H} \approx \beta \mathbb{E}_{t}\left(\widehat{\pi}_{t+1}^{H}\right)+\ldots \\
& \Psi\left[\begin{array}{c}
\left(\left(\frac{1-\gamma_{x}}{1-\gamma_{x}^{a}}\right) \sigma^{-1}+\left(1-\gamma_{x}\right)\left(\frac{\psi}{1+(1-\psi) \varphi}\right)\left(\varphi+(1-\psi)\left(\frac{1+\varphi}{\psi}\right)^{2}\right)\right) \widehat{c}_{t}^{a W}+\ldots \\
\gamma_{x}\left(\frac{\psi}{1+(1-\psi) \varphi}\right)\left(\varphi+(1-\psi)\left(\frac{1+\varphi}{\psi}\right)^{2}\right) \widehat{x}_{t}^{a W}+\ldots \\
\phi_{F}\left(\frac{1-\gamma_{x}}{1-\gamma_{x}^{a}}\right) \sigma^{-1} \widehat{c}_{t}^{a R}-\ldots \\
\left(1+\eta\left(\frac{\psi}{1+(1-\psi) \varphi}\right)\left(\varphi+(1-\psi)\left(\frac{1+\varphi}{\psi}\right)^{2}\right)\right)\left(\widehat{p}_{t}^{H, W}-\widehat{p}_{t}^{W}\right)-\ldots \\
\phi_{F}\left(\widehat{p}_{t}^{H, R}-\widehat{p}_{t}^{R}\right)-(1-\psi)\left(\frac{1+\varphi}{\psi}\right) \widehat{k}_{t}-\left(\frac{1+\varphi}{\psi}\right) \widehat{a}_{t}
\end{array}\right], \\
& \widehat{\pi}_{t}^{H *} \approx \beta \mathbb{E}_{t}\left(\widehat{\pi}_{t+1}^{H *}\right)+\ldots \\
& {\left[\left(\left(\frac{1-\gamma_{x}}{1-\gamma_{x}^{a}}\right) \sigma^{-1}+\left(1-\gamma_{x}\right)\left(\frac{\psi}{1+(1-\psi) \varphi}\right)\left(\varphi+(1-\psi)\left(\frac{1+\varphi}{\psi}\right)^{2}\right)\right) \widehat{c}_{t}^{a W}+\ldots\right.} \\
& \gamma_{x}\left(\frac{\psi}{1+(1-\psi) \varphi}\right)\left(\varphi+(1-\psi)\left(\frac{1+\varphi}{\psi}\right)^{2}\right) \widehat{x}_{t}^{a W}+\ldots \\
& \Psi \quad \phi_{F}\left(\frac{1-\gamma_{x}}{1-\gamma_{x}^{a}}\right) \sigma^{-1} \widehat{c}_{t}^{a R}-\widehat{r s}_{t}-\ldots \\
& \left(1+\eta\left(\frac{\psi}{1+(1-\psi) \varphi}\right)\left(\varphi+(1-\psi)\left(\frac{1+\varphi}{\psi}\right)^{2}\right)\right)\left(\widehat{p}_{t}^{H, W}-\widehat{p}_{t}^{W}\right)+\ldots \\
& \phi_{H}\left(\widehat{p}_{t}^{H, R}-\widehat{p}_{t}^{R}\right)-(1-\psi)\left(\frac{1+\varphi}{\psi}\right) \widehat{k}_{t}-\left(\frac{1+\varphi}{\psi}\right) \widehat{a}_{t} \\
& \widehat{\pi}_{t}^{F} \approx \beta \mathbb{E}_{t}\left(\widehat{\pi}_{t+1}^{F}\right)+\ldots \\
& \Psi\left[\begin{array}{c}
\left(\left(\frac{1-\gamma_{x}}{1-\gamma_{x^{*}}^{a}}\right) \sigma^{-1}+\left(1-\gamma_{x}\right)\left(\frac{\psi}{1+(1-\psi) \varphi}\right)\left(\varphi+(1-\psi)\left(\frac{1+\varphi}{\psi}\right)^{2}\right)\right) \widehat{c}_{t}^{a W *}+\ldots \\
\gamma_{x}\left(\frac{\psi}{1+(1-\psi) \varphi}\right)\left(\varphi+(1-\psi)\left(\frac{1+\varphi}{\psi}\right)^{2}\right) \widehat{x}_{t}^{a W *}-\ldots \\
\phi_{F}\left(\frac{1-\gamma_{x}}{1-\gamma_{x^{*}}}\right) \sigma^{-1} \widehat{c}_{t}^{a R}+\widehat{r s}_{t}-\ldots \\
\left(1+\eta\left(\frac{\psi}{1+(1-\psi) \varphi}\right)\left(\varphi+(1-\psi)\left(\frac{1+\varphi}{\psi}\right)^{2}\right)\right)\left(\widehat{p}_{t}^{F, W *}-\widehat{p}_{t}^{W *}\right)-\ldots \\
\phi_{H}\left(\widehat{p}_{t}^{F, R}-\widehat{p}_{t}^{R}\right)-(1-\psi)\left(\frac{1+\varphi}{\psi}\right) \widehat{k}_{t}^{*}-\left(\frac{1+\varphi}{\psi}\right) \widehat{a}_{t}^{*}
\end{array}\right] \\
& \widehat{\pi}_{t}^{F *} \approx \beta \mathbb{E}_{t}\left(\widehat{\pi}_{t+1}^{F *}\right)+\ldots \\
& \Psi\left[\begin{array}{c}
\left(\left(\frac{1-\gamma_{x}}{1-\gamma_{x^{*}}^{a}}\right) \sigma^{-1}+\left(1-\gamma_{x}\right)\left(\frac{\psi}{1+(1-\psi) \varphi}\right)\left(\varphi+(1-\psi)\left(\frac{1+\varphi}{\psi}\right)^{2}\right)\right) \widehat{c}_{t}^{a W *}+\ldots \\
\gamma_{x}\left(\frac{\psi}{1+(1-\psi) \varphi}\right)\left(\varphi+(1-\psi)\left(\frac{1+\varphi}{\psi}\right)^{2}\right) \widehat{x}_{t}^{a W *}-\ldots \\
\phi_{F}\left(\frac{1-\gamma_{x}}{1-\gamma_{x^{*}}}\right) \sigma^{-1} \widehat{c}_{t}^{a R}-\ldots \\
\left(1+\eta\left(\frac{\psi}{1+(1-\psi) \varphi}\right)\left(\varphi+(1-\psi)\left(\frac{1+\varphi}{\psi}\right)^{2}\right)\right)\left(\widehat{p}_{t}^{F, W *}-\widehat{p}_{t}^{W *}\right)+\ldots \\
\phi_{F}\left(\widehat{p}_{t}^{F, R}-\widehat{p}_{t}^{R}\right)-(1-\psi)\left(\frac{1+\varphi}{\psi}\right) \widehat{k}_{t}^{*}-\left(\frac{1+\varphi}{\psi}\right) \widehat{a}_{t}^{*}
\end{array}\right] .
\end{aligned}
$$

I define the world price sub-indexes as $\widehat{p}_{t}^{H, W} \equiv \phi_{H} \widehat{p}_{t}^{H}+\phi_{F} \widehat{p}_{t}^{H *}$ and $\widehat{p}_{t}^{F, W *} \equiv \phi_{F} \widehat{p}_{t}^{F}+\phi_{H} \widehat{p}_{t}^{F *}$. Therefore, 
I derive the dynamics of $\widehat{\pi}_{t}^{H, W} \equiv \widehat{p}_{t}^{H, W}-\widehat{p}_{t-1}^{H, W}$ and $\widehat{\pi}_{t}^{F, W *} \equiv \widehat{p}_{t}^{F, W *}-\widehat{p}_{t-1}^{F, W *}$ from the equations above as,

$$
\begin{aligned}
& \widehat{\pi}_{t}^{H, W} \approx \beta \mathbb{E}_{t}\left(\widehat{\pi}_{t+1}^{H, W}\right)+\ldots \\
& \Psi\left[\begin{array}{c}
\left(\left(\frac{1-\gamma_{x}}{1-\gamma_{x}^{a}}\right) \sigma^{-1}+\left(1-\gamma_{x}\right)\left(\frac{\psi}{1+(1-\psi) \varphi}\right)\left(\varphi+(1-\psi)\left(\frac{1+\varphi}{\psi}\right)^{2}\right)\right) \widehat{c}_{t}^{a W}+\ldots \\
\gamma_{x}\left(\frac{\psi}{1+(1-\psi) \varphi}\right)\left(\varphi+(1-\psi)\left(\frac{1+\varphi}{\psi}\right)^{2}\right) \widehat{x}_{t}^{a W}+\ldots \\
\phi_{F}\left(\frac{1-\gamma_{x}}{1-\gamma_{x}^{a}}\right) \sigma^{-1} \widehat{c}_{t}^{a R}-\phi_{F} \widehat{r s}_{t}-\ldots \\
\left(1+\eta\left(\frac{\psi}{1+(1-\psi) \varphi}\right)\left(\varphi+(1-\psi)\left(\frac{1+\varphi}{\psi}\right)^{2}\right)\right)\left(\widehat{p}_{t}^{H, W}-\widehat{p}_{t}^{W}\right)-\ldots \\
(1-\psi)\left(\frac{1+\varphi}{\psi}\right) \widehat{k}_{t}-\left(\frac{1+\varphi}{\psi}\right) \widehat{a}_{t}
\end{array}\right], \\
& \widehat{\pi}_{t}^{F, W *} \approx \beta \mathbb{E}_{t}\left(\widehat{\pi}_{t+1}^{F, W *}\right)+\ldots \quad\left(\begin{array}{c}
\left.\left(1-\gamma_{x}\right)\left(\frac{\psi}{1+(1-\psi) \varphi}\right)\left(\varphi+(1-\psi)\left(\frac{1+\varphi}{\psi}\right)^{2}\right)\right) \widehat{c}_{t}^{a W *}+\ldots \\
\left(\left(\frac{1-\gamma_{x}}{1-\gamma_{x *}^{a}}\right) \sigma^{-1}+\left(1-\gamma_{x}\right)\left(\frac{\psi}{1+(1-\psi) \varphi}\right)\left(\varphi+(1-\psi)\left(\frac{1+\varphi}{\psi}\right)^{2}\right) \widehat{x}_{t}^{a W *}-\ldots\right. \\
\phi_{F}\left(\frac{1-\gamma_{x}}{1-\gamma_{x *}^{a}}\right) \sigma^{-1} \widehat{c}_{t}^{a R}+\phi_{F} \widehat{r s}_{t}-\ldots \\
\left(1+\eta\left(\frac{\psi}{1+(1-\psi) \varphi}\right)\left(\varphi+(1-\psi)\left(\frac{1+\varphi}{\psi}\right)^{2}\right)\right)\left(\widehat{p}_{t}^{F, W *}-\widehat{p}_{t}^{W *}\right)-\ldots \\
(1-\psi)\left(\frac{1+\varphi}{\psi}\right) \widehat{k}_{t}^{*}-\left(\frac{1+\varphi}{\psi}\right) \widehat{a}_{t}^{*}
\end{array}\right] .
\end{aligned}
$$

I define the CPI indexes of both countries as $\widehat{p}_{t} \approx \phi_{H} \widehat{p}_{t}^{H}+\phi_{F} \widehat{p}_{t}^{F}$ and $\widehat{p}_{t}^{*} \approx \phi_{F} \widehat{p}_{t}^{H *}+\phi_{H} \widehat{p}_{t}^{F *}$, respectively. Therefore, it is easy to derive the dynamics of $\widehat{\pi}_{t} \equiv \widehat{p}_{t}-\widehat{p}_{t-1}$ and $\widehat{\pi}_{t}^{*} \equiv \widehat{p}_{t}^{*}-\widehat{p}_{t-1}^{*}$ from the equations above 
as follows,

$$
\begin{aligned}
& \widehat{\pi}_{t} \approx \beta \mathbb{E}_{t}\left(\widehat{\pi}_{t+1}\right)+\ldots \\
& \Psi\left[\begin{array}{c}
\sigma^{-1}\left[\left(\frac{1-\gamma_{x}}{1-\gamma_{x}^{a}}\right) \phi_{H} \widehat{c}_{t}^{a W}+\left(\frac{1-\gamma_{x}}{1-\gamma_{x^{*}}}\right) \phi_{F} \widehat{c}_{t}^{a W *}\right]+\ldots \\
\left(1-\gamma_{x}\right)\left(\frac{\psi}{1+(1-\psi) \varphi}\right)\left(\varphi+(1-\psi)\left(\frac{1+\varphi}{\psi}\right)^{2}\right)\left[\phi_{H} \widehat{c}_{t}^{a W}+\phi_{F} \widehat{c}_{t}^{a W *}\right]+\ldots \\
\gamma_{x}\left(\frac{\psi}{1+(1-\psi) \varphi}\right)\left(\varphi+(1-\psi)\left(\frac{1+\varphi}{\psi}\right)^{2}\right)\left[\phi_{H} \widehat{x}_{t}^{a W}+\phi_{F} \widehat{x}_{t}^{a W *}\right]+\ldots \\
\phi_{F}\left(\phi_{H}\left(\frac{1-\gamma_{x}}{1-\gamma_{x}^{a}}\right)-\phi_{F}\left(\frac{1-\gamma_{x}}{1-\gamma_{x}^{a}}\right)\right) \sigma^{-1} \widehat{c}_{t}^{a R}+\phi_{F} \widehat{r s}_{t}-\ldots \\
\left(1+\eta\left(\frac{\psi}{1+(1-\psi) \varphi}\right)\left(\varphi+(1-\psi)\left(\frac{1+\varphi}{\psi}\right)^{2}\right)\right)\left[\phi_{H}\left(\widehat{p}_{t}^{H, W}-\widehat{p}_{t}^{W}\right)+\phi_{F}\left(\widehat{p}_{t}^{F, W *}-\widehat{p}_{t}^{W *}\right)\right]-\ldots \\
\phi_{F} \phi_{H}\left[\left(\widehat{p}_{t}^{H, R}-\widehat{p}_{t}^{R}\right)+\left(\widehat{p}_{t}^{F, R}-\widehat{p}_{t}^{R}\right)\right]-(1-\psi)\left(\frac{1+\varphi}{\psi}\right)\left[\phi_{H} \widehat{k}_{t}+\phi_{F} \widehat{k}_{t}^{*}\right]-\ldots \\
\left(\frac{1+\varphi}{\psi}\right)\left[\phi_{H} \widehat{a}_{t}+\phi_{F} \widehat{a}_{t}^{*}\right]
\end{array}\right], \\
& \widehat{\pi}_{t}^{*} \approx \beta \mathbb{E}_{t}\left(\widehat{\pi}_{t+1}^{*}\right)+\ldots \\
& \Psi\left[\begin{array}{c}
\sigma^{-1}\left[\left(\frac{1-\gamma_{x}}{1-\gamma_{x}^{a}}\right) \phi_{F} \widehat{c}_{t}^{a W}+\left(\frac{1-\gamma_{x}}{1-\gamma_{x}^{*}}\right) \phi_{H} \widehat{c}_{t}^{a W *}\right]+\ldots \\
\left(1-\gamma_{x}\right)\left(\frac{\psi}{1+(1-\psi) \varphi}\right)\left(\varphi+(1-\psi)\left(\frac{1+\varphi}{\psi}\right)^{2}\right)\left[\phi_{F} \widehat{c}_{t}^{a W}+\phi_{H} \widehat{c}_{t}^{a W *}\right]+\ldots \\
\gamma_{x}\left(\frac{\psi}{1+(1-\psi) \varphi}\right)\left(\varphi+(1-\psi)\left(\frac{1+\varphi}{\psi}\right)^{2}\right)\left[\phi_{F} \widehat{x}_{t}^{a W}+\phi_{H} \widehat{x}_{t}^{a W *}\right]-\ldots \\
\phi_{F}\left(\phi_{H}\left(\frac{1-\gamma_{x}}{1-\gamma_{x^{*}}}\right)-\phi_{F}\left(\frac{1-\gamma_{x}}{1-\gamma_{x}^{a}}\right)\right) \sigma^{-1} \widehat{c}_{t}^{a R}-\phi_{F} \widehat{r}_{t}-\ldots \\
\left(1+\eta\left(\frac{\psi}{1+(1-\psi) \varphi}\right)\left(\varphi+(1-\psi)\left(\frac{1+\varphi}{\psi}\right)\right)\right)\left[\phi_{F}\left(\widehat{p}_{t}^{H, W}-\widehat{p}_{t}^{W}\right)+\phi_{H}\left(\widehat{p}_{t}^{F, W *}-\widehat{p}_{t}^{W *}\right)\right]+\ldots \\
\phi_{F} \phi_{H}\left[\left(\widehat{p}_{t}^{H, R}-\widehat{p}_{t}^{R}\right)+\left(\widehat{p}_{t}^{F, R}-\widehat{p}_{t}^{R}\right)\right]-(1-\psi)\left(\frac{1+\varphi}{\psi}\right)\left[\phi_{F} \widehat{k}_{t}+\phi_{H} \widehat{k}_{t}^{*}\right]-\ldots \\
\left(\frac{1+\varphi}{\psi}\right)\left[\phi_{F} \widehat{a}_{t}+\phi_{H} \widehat{a}_{t}^{*}\right]
\end{array}\right],
\end{aligned}
$$

where world aggregate capital is defined as $\widehat{k}_{t}^{W} \equiv \phi_{H} \widehat{k}_{t}+\phi_{F} \widehat{k}_{t}^{*}$ and $\widehat{k}_{t}^{W *} \equiv \phi_{F} \widehat{k}_{t}+\phi_{H} \widehat{k}_{t}^{*}$. I can also write certain terms inside the brackets of the Phillips curves more compactly as,

$$
\begin{aligned}
- & \left(1+\eta\left(\frac{\psi}{1+(1-\psi) \varphi}\right)\left(\varphi+(1-\psi)\left(\frac{1+\varphi}{\psi}\right)^{2}\right)\right)\left[\phi_{H}\left(\widehat{p}_{t}^{H, W}-\widehat{p}_{t}^{W}\right)+\phi_{F}\left(\widehat{p}_{t}^{F, W *}-\widehat{p}_{t}^{W *}\right)\right]-\ldots \\
& \phi_{H} \phi_{F}\left[\left(\widehat{p}_{t}^{H, R}-\widehat{p}_{t}^{R}\right)+\left(\widehat{p}_{t}^{F, R}-\widehat{p}_{t}^{R}\right)\right]=\eta\left(\frac{\psi}{1+(1-\psi) \varphi}\right)\left(\varphi+(1-\psi)\left(\frac{1+\varphi}{\psi}\right)^{2}\right)\left[\phi_{H}-\phi_{F}\right]\left(\widehat{p}_{t}^{F, W *}-\widehat{p}_{t}^{W *}\right)-\ldots \\
& \eta\left(\frac{\psi}{1+(1-\psi) \varphi}\right)\left(\varphi+(1-\psi)\left(\frac{1+\varphi}{\psi}\right)^{2}\right) \phi_{H}\left[\left(\widehat{p}_{t}^{H, W}-\widehat{p}_{t}^{W}\right)+\left(\widehat{p}_{t}^{F, W *}-\widehat{p}_{t}^{W *}\right)\right]-\ldots \\
& \phi_{H}\left[\left(\widehat{p}_{t}^{H, W}-\widehat{p}_{t}^{W}\right)+\phi_{F}\left(\widehat{p}_{t}^{H, R}-\widehat{p}_{t}^{R}\right)\right]-\phi_{F}\left[\left(\widehat{p}_{t}^{F, W *}-\widehat{p}_{t}^{W *}\right)+\phi_{H}\left(\widehat{p}_{t}^{F, R}-\widehat{p}_{t}^{R}\right)\right], \\
- & \left(1+\eta\left(\frac{\psi}{1+(1-\psi) \varphi}\right)\left(\varphi+(1-\psi)\left(\frac{1+\varphi}{\psi}\right)^{2}\right)\right)\left[\phi_{F}\left(\widehat{p}_{t}^{H, W}-\widehat{p}_{t}^{W}\right)+\phi_{H}\left(\widehat{p}_{t}^{F, W *}-\widehat{p}_{t}^{W *}\right)\right]+\ldots \\
& \phi_{F} \phi_{H}\left[\left(\widehat{p}_{t}^{H, R}-\widehat{p}_{t}^{R}\right)+\left(\widehat{p}_{t}^{F, R}-\widehat{p}_{t}^{R}\right)\right]=-\eta\left(\frac{\psi}{1+(1-\psi) \varphi}\right)\left(\varphi+(1-\psi)\left(\frac{1+\varphi}{\psi}\right)^{2}\right)\left[\phi_{H}-\phi_{F}\right]\left(\widehat{p}_{t}^{F, W *}-\widehat{p}_{t}^{W *}\right)-\ldots \\
& \eta\left(\frac{\psi}{1+(1-\psi) \varphi}\right)\left(\varphi+(1-\psi)\left(\frac{1+\varphi}{\psi}\right)^{2}\right) \phi_{F}\left[\left(\widehat{p}_{t}^{H, W}-\widehat{p}_{t}^{W}\right)+\left(\widehat{p}_{t}^{F, W *}-\widehat{p}_{t}^{W *}\right)\right]-\ldots \\
& \phi_{F}\left[\left(\widehat{p}_{t}^{H, W}-\widehat{p}_{t}^{W}\right)-\phi_{H}\left(\widehat{p}_{t}^{H, R}-\widehat{p}_{t}^{R}\right)\right]-\phi_{H}\left[\left(\widehat{p}_{t}^{F, W *}-\widehat{p}_{t}^{W *}\right)-\phi_{F}\left(\widehat{p}_{t}^{F, R}-\widehat{p}_{t}^{R}\right)\right] .
\end{aligned}
$$

Based on the definitions of the world aggregates, denoted with the superscripts $W$ and $W^{*}$, it is possible for 
me to argue that,

$$
\begin{aligned}
& \phi_{H}\left[\left(\widehat{p}_{t}^{H, W}-\widehat{p}_{t}^{W}\right)+\phi_{F}\left(\widehat{p}_{t}^{H, R}-\widehat{p}_{t}^{R}\right)\right]+\phi_{F}\left[\left(\widehat{p}_{t}^{F, W *}-\widehat{p}_{t}^{W *}\right)+\phi_{H}\left(\widehat{p}_{t}^{F, R}-\widehat{p}_{t}^{R}\right)\right] \\
& \quad=\phi_{H}\left(\widehat{p}_{t}^{H}-\widehat{p}_{t}\right)+\phi_{F}\left(\widehat{p}_{t}^{F}-\widehat{p}_{t}\right)=0, \\
& \phi_{F}\left[\left(\widehat{p}_{t}^{H, W}-\widehat{p}_{t}^{W}\right)-\phi_{H}\left(\widehat{p}_{t}^{H, R}-\widehat{p}_{t}^{R}\right)\right]+\phi_{H}\left[\left(\widehat{p}_{t}^{F, W *}-\widehat{p}_{t}^{W *}\right)-\phi_{F}\left(\widehat{p}_{t}^{F, R}-\widehat{p}_{t}^{R}\right)\right] \\
& \quad=\phi_{F}\left(\widehat{p}_{t}^{H *}-\widehat{p}_{t}^{*}\right)+\phi_{H}\left(\widehat{p}_{t}^{F *}-\widehat{p}_{t}^{*}\right)=0,
\end{aligned}
$$

since $\widehat{p}_{t}=\phi_{H} \widehat{p}_{t}^{H}+\phi_{F} \widehat{p}_{t}^{F}$ and $\widehat{p}_{t}^{*}=\phi_{F} \widehat{p}_{t}^{H *}+\phi_{H} \widehat{p}_{t}^{F *}$. Furthermore, based on those same definitions I also know that the following result must hold true,

$$
\begin{aligned}
& \left(\widehat{p}_{t}^{H, W}-\widehat{p}_{t}^{W}\right)+\left(\widehat{p}_{t}^{F, W *}-\widehat{p}_{t}^{W *}\right)=\widehat{p}_{t}^{H, W}+\widehat{p}_{t}^{F, W *}-\left(\widehat{p}_{t}^{W}+\widehat{p}_{t}^{W *}\right) \\
& \quad=\phi_{H}\left(\widehat{p}_{t}^{H}-\widehat{p}_{t}\right)+\phi_{F}\left(\widehat{p}_{t}^{H *}-\widehat{p}_{t}^{*}\right)+\phi_{F}\left(\widehat{p}_{t}^{F}-\widehat{p}_{t}\right)+\phi_{H}\left(\widehat{p}_{t}^{F *}-\widehat{p}_{t}^{*}\right) \\
& \quad=\left[\phi_{H}\left(\widehat{p}_{t}^{H}-\widehat{p}_{t}\right)+\phi_{F}\left(\widehat{p}_{t}^{F}-\widehat{p}_{t}\right)\right]+\left[\phi_{F}\left(\widehat{p}_{t}^{H *}-\widehat{p}_{t}^{*}\right)+\phi_{H}\left(\widehat{p}_{t}^{F *}-\widehat{p}_{t}^{*}\right)\right]=0 .
\end{aligned}
$$

Hence, I argue that those terms inside the brackets of the Phillips curves involving relative prices can be simplified as,

$$
\begin{aligned}
- & \left(1+\eta\left(\frac{\psi}{1+(1-\psi) \varphi}\right)\left(\varphi+(1-\psi)\left(\frac{1+\varphi}{\psi}\right)^{2}\right)\right)\left[\phi_{H}\left(\widehat{p}_{t}^{H, W}-\widehat{p}_{t}^{W}\right)+\phi_{F}\left(\widehat{p}_{t}^{F, W *}-\widehat{p}_{t}^{W *}\right)\right]-\ldots \\
& \phi_{H} \phi_{F}\left[\left(\widehat{p}_{t}^{H, R}-\widehat{p}_{t}^{R}\right)+\left(\widehat{p}_{t}^{F, R}-\widehat{p}_{t}^{R}\right)\right]=\eta\left(\frac{\psi}{1+(1-\psi) \varphi}\right)\left(\varphi+(1-\psi)\left(\frac{1+\varphi}{\psi}\right)^{2}\right)\left[\phi_{H}-\phi_{F}\right]\left(\widehat{p}_{t}^{F, W *}-\widehat{p}_{t}^{W *}\right) \\
- & \left(1+\eta\left(\frac{\psi}{1+(1-\psi) \varphi}\right)\left(\varphi+(1-\psi)\left(\frac{1+\varphi}{\psi}\right)^{2}\right)\right)\left[\phi_{F}\left(\widehat{p}_{t}^{H, W}-\widehat{p}_{t}^{W}\right)+\phi_{H}\left(\widehat{p}_{t}^{F, W *}-\widehat{p}_{t}^{W *}\right)\right]+\ldots \\
& \phi_{F} \phi_{H}\left[\left(\widehat{p}_{t}^{H, R}-\widehat{p}_{t}^{R}\right)+\left(\widehat{p}_{t}^{F, R}-\widehat{p}_{t}^{R}\right)\right]=-\eta\left(\frac{\psi}{1+(1-\psi) \varphi}\right)\left(\varphi+(1-\psi)\left(\frac{1+\varphi}{\psi}\right)^{2}\right)\left[\phi_{H}-\phi_{F}\right]\left(\widehat{p}_{t}^{F, W *}-\widehat{p}_{t}^{W *}\right) .
\end{aligned}
$$


I conclude that both Phillips curves in the model take the following form,

$$
\begin{aligned}
& \widehat{\pi}_{t} \approx \beta \mathbb{E}_{t}\left(\widehat{\pi}_{t+1}\right)+\ldots \\
& \Psi\left[\begin{array}{c}
\sigma^{-1}\left[\left(\frac{1-\gamma_{x}}{1-\gamma_{x}^{a}}\right) \phi_{H} \widehat{c}_{t}^{a W}+\left(\frac{1-\gamma_{x}}{1-\gamma_{x *}^{a}}\right) \phi_{F} \widehat{c}_{t}^{a W *}\right]+\ldots \\
\left(1-\gamma_{x}\right)\left(\frac{\psi}{1+(1-\psi) \varphi}\right)\left(\varphi+(1-\psi)\left(\frac{1+\varphi}{\psi}\right)^{2}\right)\left[\phi_{H} \widehat{c}_{t}^{a W}+\phi_{F} \widehat{c}_{t}^{a W *}\right]+\ldots \\
\gamma_{x}\left(\frac{\psi}{1+(1-\psi) \varphi}\right)\left(\varphi+(1-\psi)\left(\frac{1+\varphi}{\psi}\right)^{2}\right)\left[\phi_{H} \widehat{x}_{t}^{a W}+\phi_{F} \widehat{x}_{t}^{a W *}\right]+\ldots \\
\phi_{F}\left(\phi_{H}\left(\frac{1-\gamma_{x}}{1-\gamma_{x}^{a}}\right)-\phi_{F}\left(\frac{1-\gamma_{x}}{1-\gamma_{x}^{a}}\right)\right) \sigma^{-1} \widehat{c}_{t}^{a R}+\phi_{F} \widehat{r s}_{t}+\ldots \\
\left(\phi_{H}-\phi_{F}\right) \eta\left(\frac{\psi}{1+(1-\psi) \varphi}\right)\left(\varphi+(1-\psi)\left(\frac{1+\varphi}{\psi}\right)^{2}\right)\left(\widehat{p}_{t}^{F, W *}-\widehat{p}_{t}^{W *}\right)-\ldots \\
(1-\psi)\left(\frac{1+\varphi}{\psi}\right) \widehat{k}_{t}^{W}-\left(\frac{1+\varphi}{\psi}\right)\left[\phi_{H} \widehat{a}_{t}+\phi_{F} \widehat{a}_{t}^{*}\right]
\end{array}\right] \\
& \widehat{\pi}_{t}^{*} \approx \beta \mathbb{E}_{t}\left(\widehat{\pi}_{t+1}^{*}\right)+\ldots \\
& \Psi\left[\begin{array}{c}
\sigma^{-1}\left[\left(\frac{1-\gamma_{x}}{1-\gamma_{x}^{a}}\right) \phi_{F} \widehat{c}_{t}^{a W}+\left(\frac{1-\gamma_{x}}{1-\gamma_{x^{*}}}\right) \phi_{H} \widehat{c}_{t}^{a W *}\right]+\ldots \\
\left(1-\gamma_{x}\right)\left(\frac{\psi}{1+(1-\psi) \varphi}\right)\left(\varphi+(1-\psi)\left(\frac{1+\varphi}{\psi}\right)^{2}\right)\left[\phi_{F} \widehat{c}_{t}^{a W}+\phi_{H} \widehat{c}_{t}^{a W *}\right]+\ldots \\
\gamma_{x}\left(\frac{\psi}{1+(1-\psi) \varphi}\right)\left(\varphi+(1-\psi)\left(\frac{1+\varphi}{\psi}\right)^{2}\right)\left[\phi_{F} \widehat{x}_{t}^{a W}+\phi_{H} \widehat{x}_{t}^{a W *}\right]-\ldots \\
\phi_{F}\left(\phi_{H}\left(\frac{1-\gamma_{x}}{1-\gamma_{x *}^{a}}\right)-\phi_{F}\left(\frac{1-\gamma_{x}}{1-\gamma_{x}^{a}}\right)\right) \sigma^{-1} \widehat{c}_{t}^{a R}-\phi_{F} \widehat{r s}_{t}-\ldots \\
\left(\phi_{H}-\phi_{F}\right) \eta\left(\frac{\psi}{1+(1-\psi) \varphi}\right)\left(\varphi+(1-\psi)\left(\frac{1+\varphi}{\psi}\right)^{2}\right)\left(\widehat{p}_{t}^{F, W *}-\widehat{p}_{t}^{W *}\right)-\ldots \\
(1-\psi)\left(\frac{1+\varphi}{\psi}\right) \widehat{k}_{t}^{W *}-\left(\frac{1+\varphi}{\psi}\right)\left[\phi_{F} \widehat{a}_{t}+\phi_{H} \widehat{a}_{t}^{*}\right]
\end{array}\right]
\end{aligned}
$$

which extends the characterization of the inflation dynamics in models like those of Steinsson (2008) by adding capital and investment.

With a little bit of additional algebra, it is possible to obtain simply that,

$$
\begin{aligned}
& \widehat{\pi}_{t}^{H, W} \approx \beta \mathbb{E}_{t}\left(\widehat{\pi}_{t+1}^{H, W}\right)+\ldots \\
& \Psi\left[\begin{array}{c}
\left(\left(\frac{1-\gamma_{x}}{1-\gamma_{x}^{a}}\right) \sigma^{-1}+\left(1-\gamma_{x}\right) \varphi\left(\frac{\varphi \psi^{2}+(1-\psi)(1+\varphi)^{2}}{\varphi \psi+(1-\psi) \psi \varphi^{2}}\right)\right) \widehat{c}_{t}^{a W}+\ldots \\
\gamma_{x} \varphi\left(\frac{\varphi \psi^{2}+(1-\psi)(1+\varphi)^{2}}{\varphi \psi+(1-\psi) \psi \varphi^{2}}\right) \widehat{x}_{t}^{a W}+\ldots \\
\phi_{F}\left(\frac{1-\gamma_{x}}{1-\gamma_{x}^{a}}\right) \sigma^{-1} \widehat{c}_{t}^{a R}-\phi_{F} \widehat{r s}_{t}-\ldots \\
\left(1+\eta \varphi\left(\frac{\varphi \psi^{2}+(1-\psi)(1+\varphi)^{2}}{\varphi \psi+(1-\psi) \psi \varphi^{2}}\right)\right)\left(\widehat{p}_{t}^{H, W}-\widehat{p}_{t}^{W}\right)-\ldots \\
\left(\frac{(1-\psi)(1+\varphi)}{\psi}\right) \widehat{k}_{t}-\left(\frac{1+\varphi}{\psi}\right) \widehat{a}_{t}
\end{array}\right], \\
& \widehat{\pi}_{t}^{F, W *} \approx \beta \mathbb{E}_{t}\left(\widehat{\pi}_{t+1}^{F, W *}\right)+\ldots \\
& \Psi\left[\begin{array}{c}
\left(\left(\frac{1-\gamma_{x}}{1-\gamma_{x^{*}}^{a}}\right) \sigma^{-1}+\left(1-\gamma_{x}\right) \varphi\left(\frac{\varphi \psi^{2}+(1-\psi)(1+\varphi)^{2}}{\varphi \psi+(1-\psi) \psi \varphi^{2}}\right)\right) \widehat{c}_{t}^{a W *}+\ldots \\
\gamma_{x} \varphi\left(\frac{\varphi \psi^{2}+(1-\psi)(1+\varphi)^{2}}{\varphi \psi+(1-\psi) \psi \varphi^{2}}\right) \widehat{x}_{t}^{a W *}-\ldots \\
\phi_{F}\left(\frac{1-\gamma_{x}}{1-\gamma_{x}^{a}}\right) \sigma^{-1} \widehat{c}_{t}^{a R}+\phi_{F} \widehat{r s}_{t}-\ldots \\
\left(1+\eta \varphi\left(\frac{\varphi \psi^{2}+(1-\psi)(1+\varphi)^{2}}{\varphi \psi+(1-\psi) \psi \varphi^{2}}\right)\right)\left(\widehat{p}_{t}^{F, W *}-\widehat{p}_{t}^{W *}\right)-\ldots \\
\left(\frac{(1-\psi)(1+\varphi)}{\psi}\right) \widehat{k}_{t}^{*}-\left(\frac{1+\varphi}{\psi}\right) \widehat{a}_{t}^{*}
\end{array}\right],
\end{aligned}
$$


and, naturally,

$$
\begin{aligned}
& \widehat{\pi}_{t} \approx \beta \mathbb{E}_{t}\left(\widehat{\pi}_{t+1}\right)+\ldots \\
& \Psi\left[\begin{array}{c}
\sigma^{-1}\left[\left(\frac{1-\gamma_{x}}{1-\gamma_{x}^{a}}\right) \phi_{H} \widehat{c}_{t}^{a W}+\left(\frac{1-\gamma_{x}}{1-\gamma_{x}^{a}}\right) \phi_{F} \widehat{c}_{t}^{a W *}\right]+\ldots \\
\left(1-\gamma_{x}\right) \varphi\left(\frac{\varphi \psi^{2}+(1-\psi)(1+\varphi)^{2}}{\varphi \psi+(1-\psi) \psi \varphi^{2}}\right)\left[\phi_{H} \widehat{c}_{t}^{a W}+\phi_{F} \widehat{c}_{t}^{a W *}\right]+\ldots \\
\gamma_{x} \varphi\left(\frac{\varphi \psi^{2}+(1-\psi)(1+\varphi)^{2}}{\varphi \psi+(1-\psi) \psi \varphi^{2}}\right)\left[\phi_{H} \widehat{x}_{t}^{a W}+\phi_{F} \widehat{x}_{t}^{a W *}\right]+\ldots \\
\phi_{F}\left(\phi_{H}\left(\frac{1-\gamma_{x}}{1-\gamma_{x}^{a}}\right)-\phi_{F}\left(\frac{1-\gamma_{x}}{1-\gamma_{x}^{a}}\right)\right) \sigma^{-1} \widehat{c}_{t}^{a R}+\phi_{F} \widehat{r s}_{t}+\ldots \\
\left(\phi_{H}-\phi_{F}\right) \eta \varphi\left(\frac{\varphi \psi^{2}+(1-\psi)(1+\varphi)^{2}}{\varphi \psi+(1-\psi) \psi \varphi^{2}}\right)\left(\widehat{p}_{t}^{F, W *}-\widehat{p}_{t}^{W *}\right)-\ldots \\
\left(\frac{(1-\psi)(1+\varphi)}{\psi}\right) \widehat{k}_{t}^{W}-\left(\frac{1+\varphi}{\psi}\right)\left[\phi_{H} \widehat{a}_{t}+\phi_{F} \widehat{a}_{t}^{*}\right]
\end{array}\right], \\
& \widehat{\pi}_{t}^{*} \approx \beta \mathbb{E}_{t}\left(\widehat{\pi}_{t+1}^{*}\right)+\ldots \\
& \Psi\left[\begin{array}{c}
\sigma^{-1}\left[\left(\frac{1-\gamma_{x}}{1-\gamma_{x}^{a}}\right) \phi_{F} \widehat{c}_{t}^{a W}+\left(\frac{1-\gamma_{x}}{1-\gamma_{x^{*}}^{a}}\right) \phi_{H} \widehat{c}_{t}^{a W *}\right]+\ldots \\
\left(1-\gamma_{x}\right) \varphi\left(\frac{\varphi \psi^{2}+(1-\psi)(1+\varphi)^{2}}{\varphi \psi+(1-\psi) \psi \varphi^{2}}\right)\left[\phi_{F} \widehat{c}_{t}^{a W}+\phi_{H} \widehat{c}_{t}^{a W *}\right]+\ldots \\
\gamma_{x} \varphi\left(\frac{\varphi \psi^{2}+(1-\psi)(1+\varphi)^{2}}{\varphi \psi+(1-\psi) \psi \varphi^{2}}\right)\left[\phi_{F} \widehat{x}_{t}^{a W}+\phi_{H} \widehat{x}_{t}^{a W *}\right]-\ldots \\
\phi_{F}\left(\phi_{H}\left(\frac{1-\gamma_{x}}{1-\gamma_{x *}^{a}}\right)-\phi_{F}\left(\frac{1-\gamma_{x}}{1-\gamma_{x}^{a}}\right)\right) \sigma^{-1} \widehat{c}_{t}^{a R}-\phi_{F} \widehat{r s}_{t}-\ldots \\
\left(\phi_{H}-\phi_{F}\right) \eta \varphi\left(\frac{\varphi \psi^{2}+(1-\psi)(1+\varphi)^{2}}{\varphi \psi+(1-\psi) \psi \varphi^{2}}\right)\left(\widehat{p}_{t}^{F, W *}-\widehat{p}_{t}^{W *}\right)-\ldots \\
\left(\frac{(1-\psi)(1+\varphi)}{\psi}\right) \widehat{k}_{t}^{W *}-\left(\frac{1+\varphi}{\psi}\right)\left[\phi_{F} \widehat{a}_{t}+\phi_{H} \widehat{a}_{t}^{*}\right]
\end{array}\right] .
\end{aligned}
$$

Capital appears in the equation dynamics because it captures the impact of the efficiency conditions on the marginal costs of firms.

Let me define $\widehat{t}_{t}^{W} \equiv \widehat{p}_{t}^{F, W *}-\widehat{p}_{t}^{W *}$ as the world measure of terms of trade in the model. Then, the Phillips curves under price stickiness and local-currency pricing (LCP) can be re-expressed as,

$$
\begin{aligned}
& \widehat{\pi}_{t} \approx \beta \mathbb{E}_{t}\left(\widehat{\pi}_{t+1}\right)+\ldots \\
& \Psi\left[\begin{array}{c}
\sigma^{-1}\left[\left(\frac{1-\gamma_{x}}{1-\gamma_{x}^{a}}\right) \phi_{H} \widehat{c}_{t}^{a W}+\left(\frac{1-\gamma_{x}}{1-\gamma_{x^{*}}}\right) \phi_{F} \widehat{c}_{t}^{a W *}\right]+\ldots \\
\left(1-\gamma_{x}\right) \varphi\left(\frac{\varphi \psi^{2}+(1-\psi)(1+\varphi)^{2}}{\varphi \psi+(1-\psi) \psi \varphi^{2}}\right)\left[\phi_{H} \widehat{c}_{t}^{a W}+\phi_{F} \widehat{c}_{t}^{a W *}\right]+\ldots \\
\gamma_{x} \varphi\left(\frac{\varphi \psi^{2}+(1-\psi)(1+\varphi)^{2}}{\varphi \psi+(1-\psi) \psi \varphi^{2}}\right)\left[\phi_{H} \widehat{x}_{t}^{a W}+\phi_{F} \widehat{x}_{t}^{a W *}\right]+\ldots \\
\phi_{F}\left(\phi_{H}\left(\frac{1-\gamma_{x}}{1-\gamma_{x}^{a}}\right)-\phi_{F}\left(\frac{1-\gamma_{x}}{1-\gamma_{x}^{a}}\right)\right) \sigma^{-1} \widehat{c}_{t}^{a R}+\phi_{F} \widehat{r s}_{t}+\ldots \\
\left(\phi_{H}-\phi_{F}\right) \eta \varphi\left(\frac{\varphi \psi^{2}+(1-\psi)(1+\varphi)^{2}}{\varphi \psi+(1-\psi) \psi \varphi^{2}}\right) \widehat{t}_{t}^{W}-\ldots \\
\left(\frac{(1-\psi)(1+\varphi)}{\psi}\right) \widehat{k}_{t}^{W}-\left(\frac{1+\varphi}{\psi}\right)\left[\phi_{H} \widehat{a}_{t}+\phi_{F} \widehat{a}_{t}^{*}\right]
\end{array}\right], \\
& \widehat{\pi}_{t}^{*} \approx \beta \mathbb{E}_{t}\left(\widehat{\pi}_{t+1}^{*}\right)+\ldots \\
& \Psi\left[\begin{array}{c}
\sigma^{-1}\left[\left(\frac{1-\gamma_{x}}{1-\gamma_{x}^{a}}\right) \phi_{F} \widehat{c}_{t}^{a W}+\left(\frac{1-\gamma_{x}}{1-\gamma_{x^{*}}^{a}}\right) \phi_{H} \widehat{c}_{t}^{a W *}\right]+\ldots \\
\left(1-\gamma_{x}\right) \varphi\left(\frac{\varphi \psi^{2}+(1-\psi)(1+\varphi)^{2}}{\varphi \psi+(1-\psi) \psi \varphi^{2}}\right)\left[\phi_{F} \widehat{c}_{t}^{a W}+\phi_{H} \widehat{c}_{t}^{a W *}\right]+\ldots \\
\gamma_{x} \varphi\left(\frac{\varphi \psi^{2}+(1-\psi)(1+\varphi)^{2}}{\varphi \psi+(1-\psi) \psi \varphi^{2}}\right)\left[\phi_{F} \widehat{x}_{t}^{a W}+\phi_{H} \widehat{x}_{t}^{a W *}\right]-\ldots \\
\phi_{F}\left(\phi_{H}\left(\frac{1-\gamma_{x}}{1-\gamma_{x^{*}}^{a}}\right)-\phi_{F}\left(\frac{1-\gamma_{x}}{1-\gamma_{x}^{a}}\right)\right) \sigma^{-1} \widehat{c}_{t}^{a R}-\phi_{F} \widehat{r}_{t}-\ldots \\
\left(\phi_{H}-\phi_{F}\right) \eta \varphi\left(\frac{\varphi \psi^{2}+(1-\psi)(1+\varphi)^{2}}{\varphi \psi+(1-\psi) \psi \varphi^{2}}\right) \widehat{t}_{t}^{W}-\ldots \\
\left(\frac{(1-\psi)(1+\varphi)}{\psi}\right) \widehat{k}_{t}^{W *}-\left(\frac{1+\varphi}{\psi}\right)\left[\phi_{F} \widehat{a}_{t}+\phi_{H} \widehat{a}_{t}^{*}\right]
\end{array}\right] .
\end{aligned}
$$


These equations constitute the aggregate supply block in this environment.

The International Relative Prices. I have defined the world price sub-indexes as $\widehat{p}_{t}^{H, W} \equiv \phi_{H} \widehat{p}_{t}^{H}+\phi_{F} \widehat{p}_{t}^{H *}$ and $\widehat{p}_{t}^{F, W *} \equiv \phi_{F} \widehat{p}_{t}^{F}+\phi_{H} \widehat{p}_{t}^{F *}$, and the relative price sub-indexes as $\widehat{p}_{t}^{H, R} \equiv \widehat{p}_{t}^{H}-\widehat{p}_{t}^{H *}$ and $\widehat{p}_{t}^{F, R} \equiv \widehat{p}_{t}^{F}-\widehat{p}_{t}^{F *}$. Then, naturally, I can write that,

$$
\begin{aligned}
\widehat{p}_{t}^{H} & =\widehat{p}_{t}^{H, W}+\phi_{F} \widehat{p}_{t}^{H, R}, \widehat{p}_{t}^{H *}=\widehat{p}_{t}^{H, W}-\phi_{H} \widehat{p}_{t}^{H, R}, \\
\widehat{p}_{t}^{F} & =\widehat{p}_{t}^{F, W *}+\phi_{H} \widehat{p}_{t}^{F, R}, \widehat{p}_{t}^{F *}=\widehat{p}_{t}^{F, W *}-\phi_{F} \widehat{p}_{t}^{F, R} .
\end{aligned}
$$

Analogously, I have defined the world CPI as $\widehat{p}_{t}^{W} \equiv \phi_{H} \widehat{p}_{t}+\phi_{F} \widehat{p}_{t}^{*}$ and $\widehat{p}_{t}^{W *} \equiv \phi_{F} \widehat{p}_{t}+\phi_{H} \widehat{p}_{t}^{*}$, and the relative CPI as $\widehat{p}_{t}^{R} \equiv \widehat{p}_{t}-\widehat{p}_{t}^{*}$. Then, I can write that,

$$
\begin{aligned}
& \widehat{p}_{t}=\widehat{p}_{t}^{W}+\phi_{F} \widehat{p}_{t}^{R}, \widehat{p}_{t}^{*}=\widehat{p}_{t}^{W}-\phi_{H} \widehat{p}_{t}^{R} \\
& \widehat{p}_{t}=\widehat{p}_{t}^{W *}+\phi_{H} \widehat{p}_{t}^{R}, \widehat{p}_{t}^{*}=\widehat{p}_{t}^{W *}-\phi_{F} \widehat{p}_{t}^{R}
\end{aligned}
$$

The definition of CPI in both countries, i.e. $\widehat{p}_{t} \approx \phi_{H} \widehat{p}_{t}^{H}+\phi_{F} \widehat{p}_{t}^{F}$ and $\widehat{p}_{t}^{*} \approx \phi_{F} \widehat{p}_{t}^{H *}+\phi_{H} \widehat{p}_{t}^{F *}$, can be written as,

$$
\begin{aligned}
\phi_{H}\left[\widehat{p}_{t}^{H}-\widehat{p}_{t}\right]+\phi_{F}\left[\widehat{p}_{t}^{F}-\widehat{p}_{t}\right] & \approx 0, \\
\phi_{F}\left[\widehat{p}_{t}^{H *}-\widehat{p}_{t}^{*}\right]+\phi_{H}\left[\widehat{p}_{t}^{F *}-\widehat{p}_{t}^{*}\right] & \approx 0 .
\end{aligned}
$$

Then, based on the relationships described before, I can re-write the definitions of the CPI indexes as,

$$
\begin{aligned}
& \phi_{H}\left[\left(\widehat{p}_{t}^{H, W}-\widehat{p}_{t}^{W}\right)+\phi_{F}\left(\widehat{p}_{t}^{H, R}-\widehat{p}_{t}^{R}\right)\right]+\phi_{F}\left[\left(\widehat{p}_{t}^{F, W *}-\widehat{p}_{t}^{W *}\right)+\phi_{H}\left(\widehat{p}_{t}^{F, R}-\widehat{p}_{t}^{R}\right)\right] \approx 0, \\
& \phi_{F}\left[\left(\widehat{p}_{t}^{H, W}-\widehat{p}_{t}^{W}\right)-\phi_{H}\left(\widehat{p}_{t}^{H, R}-\widehat{p}_{t}^{R}\right)\right]+\phi_{H}\left[\left(\widehat{p}_{t}^{F, W *}-\widehat{p}_{t}^{W *}\right)-\phi_{F}\left(\widehat{p}_{t}^{F, R}-\widehat{p}_{t}^{R}\right)\right] \approx 0 .
\end{aligned}
$$

Let me define $\widehat{t}_{t}^{W} \equiv \widehat{p}_{t}^{F, W *}-\widehat{p}_{t}^{W *}$ as the world measure of terms of trade in this model. World terms of trade are implicitly characterized by the previous pair of equations.

I already know that by construction $\left(\widehat{p}_{t}^{H, W}-\widehat{p}_{t}^{W}\right)+\left(\widehat{p}_{t}^{F, W *}-\widehat{p}_{t}^{W *}\right) \approx 0$ (see equation (312) for a demonstration), hence the two expressions above become simply,

$$
\begin{aligned}
\left(\phi_{H}-\phi_{F}\right)\left(\widehat{p}_{t}^{F, W *}-\widehat{p}_{t}^{W *}\right) & \approx \phi_{H} \phi_{F}\left[\left(\widehat{p}_{t}^{H, R}-\widehat{p}_{t}^{R}\right)+\left(\widehat{p}_{t}^{F, R}-\widehat{p}_{t}^{R}\right)\right], \\
\left(\phi_{H}-\phi_{F}\right)\left(\widehat{p}_{t}^{F, W *}-\widehat{p}_{t}^{W *}\right) & \approx \phi_{H} \phi_{F}\left[\left(\widehat{p}_{t}^{H, R}-\widehat{p}_{t}^{R}\right)+\left(\widehat{p}_{t}^{F, R}-\widehat{p}_{t}^{R}\right)\right] .
\end{aligned}
$$

In summary, the only constraint that pins down the world terms of trade in this environment is given by,

$$
\left(\phi_{H}-\phi_{F}\right)\left(\widehat{p}_{t}^{F, W *}-\widehat{p}_{t}^{W *}\right) \approx \phi_{H} \phi_{F}\left[\left(\widehat{p}_{t}^{H, R}-\widehat{p}_{t}^{R}\right)+\left(\widehat{p}_{t}^{F, R}-\widehat{p}_{t}^{R}\right)\right]
$$

If the model has no home-product bias in consumption and investment, i.e. $\phi_{H}=\phi_{F}$, then $\left(\widehat{p}_{t}^{F, W *}-\widehat{p}_{t}^{W *}\right)$ only matters because it affects output and output enters into the specification of the Taylor rules in (292) (293). Therefore, it must follow from (321) that $\left(\widehat{p}_{t}^{H, R}-\widehat{p}_{t}^{R}\right)+\left(\widehat{p}_{t}^{F, R}-\widehat{p}_{t}^{R}\right) \approx 0$. In that case, this constraint imposes no restriction on the world terms of trade $\widehat{t}_{t}^{W} \equiv \widehat{p}_{t}^{F, W *}-\widehat{p}_{t}^{W *}$, and I would need to keep track of the 
price sub-indexes in order to close down the model.

If the model assumes home-product bias in consumption and investment, i.e. $\phi_{H} \neq \phi_{F}$, then $\widehat{t}_{t}^{W} \equiv$ $\left(\widehat{p}_{t}^{F, W *}-\widehat{p}_{t}^{W *}\right)$ matters because it affects output in both countries and it also matters because it affects the inflation dynamics directly through the Phillips curves. Moreover, I can write the world terms of trade as follows,

$$
\widehat{t}_{t}^{W} \equiv \widehat{p}_{t}^{F, W *}-\widehat{p}_{t}^{W *}=\frac{\phi_{H} \phi_{F}}{\phi_{H}-\phi_{F}}\left[\left(\widehat{p}_{t}^{H, R}-\widehat{p}_{t}^{R}\right)+\left(\widehat{p}_{t}^{F, R}-\widehat{p}_{t}^{R}\right)\right] .
$$

In equations (302) and (303) I already derive a simple characterization for the relative price sub-indexes $\widehat{\pi}_{t}^{H, R}$ and $\widehat{\pi}_{t}^{F, R}$, i.e.,

$$
\begin{aligned}
\widehat{\pi}_{t}^{H, R}-\beta \mathbb{E}_{t}\left(\widehat{\pi}_{t+1}^{H, R}\right)+\Psi\left(\widehat{p}_{t}^{H, R}-\widehat{p}_{t}^{R}\right) & \approx \Psi \widehat{r s}_{t}, \\
\widehat{\pi}_{t}^{F, R}-\beta \mathbb{E}_{t}\left(\widehat{\pi}_{t+1}^{F, R}\right)+\Psi\left(\widehat{p}_{t}^{F, R}-\widehat{p}_{t}^{R}\right) & \approx \Psi \widehat{r s}_{t} .
\end{aligned}
$$

Simple manipulations allow me to write this pair of equations as,

$$
\begin{aligned}
\left(\widehat{\pi}_{t}^{H, R}-\widehat{\pi}_{t}^{R}\right)-\beta \mathbb{E}_{t}\left(\widehat{\pi}_{t+1}^{H, R}-\widehat{\pi}_{t+1}^{R}\right)+\Psi\left(\widehat{p}_{t}^{H, R}-\widehat{p}_{t}^{R}\right) & \approx \Psi \widehat{r s}_{t}-\widehat{\pi}_{t}^{R}+\beta \mathbb{E}_{t}\left(\widehat{\pi}_{t+1}^{R}\right), \\
\left(\widehat{\pi}_{t}^{F, R}-\widehat{\pi}_{t}^{R}\right)-\beta \mathbb{E}_{t}\left(\widehat{\pi}_{t+1}^{F, R}-\widehat{\pi}_{t+1}^{R}\right)+\Psi\left(\widehat{p}_{t}^{F, R}-\widehat{p}_{t}^{R}\right) & \approx \Psi \widehat{r s s}_{t}-\widehat{\pi}_{t}^{R}+\beta \mathbb{E}_{t}\left(\widehat{\pi}_{t+1}^{R}\right),
\end{aligned}
$$

where the relative CPI is defined as $\widehat{p}_{t}^{R} \equiv \widehat{p}_{t}-\widehat{p}_{t}^{*}$. If I use the definition of world terms of trade $\widehat{t}_{t}^{W} \equiv$ $\widehat{p}_{t}^{F, W *}-\widehat{p}_{t}^{W *}$ and I combine it with these two equations, I can write the dynamics of $\widehat{t}_{t}^{W}$ as,

$$
\Delta \widehat{t}_{t}^{W}-\beta \mathbb{E}_{t}\left(\Delta \widehat{t}_{t+1}^{W}\right)+\Psi \widehat{t}_{t}^{W} \approx \frac{\phi_{H} \phi_{F}}{\phi_{H}-\phi_{F}}\left[\Psi \widehat{r}_{t}-\widehat{\pi}_{t}^{R}+\beta \mathbb{E}_{t}\left(\widehat{\pi}_{t+1}^{R}\right)\right]
$$

where I define the first-difference of world terms of trade as $\Delta \widehat{t}_{t}^{W} \equiv \widehat{t}_{t}^{W}-\widehat{t}_{t-1}^{W}$. This suffices to close down my model.

Following on Engel (forthcoming), I can show that when the degree of price stickiness is the same across firms and markets then the relative prices in each country must be equalized even if the law of one price fails to hold, i.e. $\left(\widehat{p}_{t}^{F}-\widehat{p}_{t}^{H}\right) \approx\left(\widehat{p}_{t}^{F *}-\widehat{p}_{t}^{H *}\right)$ must hold true. To show this, I start by computing the inflation rate for the relative prices in each country $\left(\widehat{\pi}_{t}^{F}-\widehat{\pi}_{t}^{H}\right)$ and $\left(\widehat{\pi}_{t}^{F *}-\widehat{\pi}_{t}^{H *}\right)$ from the dynamics of the price 
sub-indexes in (308) - (311) as follows,

$$
\begin{aligned}
& \widehat{\pi}_{t}^{F}-\widehat{\pi}_{t}^{H} \approx \beta \mathbb{E}_{t}\left(\widehat{\pi}_{t+1}^{F}-\widehat{\pi}_{t+1}^{H}\right)+\ldots \\
& \Psi\left[\begin{array}{c}
\sigma^{-1}\left(\left(\frac{1-\gamma_{x}}{1-\gamma_{x}^{a}}\right) \widehat{c}_{t}^{a W *}-\left(\frac{1-\gamma_{x}}{1-\gamma_{x}^{a}}\right) \widehat{c}_{t}^{a W}\right)+\ldots \\
\left(1-\gamma_{x}\right)\left(\frac{\psi}{1+(1-\psi) \varphi}\right)\left(\varphi+(1-\psi)\left(\frac{1+\varphi}{\psi}\right)^{2}\right)\left(\widehat{c}_{t}^{a W *}-\widehat{c}_{t}^{a W}\right)+\ldots \\
\gamma_{x}\left(\frac{\psi}{1+(1-\psi) \varphi}\right)\left(\varphi+(1-\psi)\left(\frac{1+\varphi}{\psi}\right)^{2}\right)\left(\widehat{x}_{t}^{a W *}-\widehat{x}_{t}^{a W}\right)-\ldots \\
\phi_{F} \sigma^{-1}\left(\left(\frac{1-\gamma_{x}}{1-\gamma_{x *}^{a}}\right)+\left(\frac{1-\gamma_{x}}{1-\gamma_{x}^{a}}\right)\right) \widehat{c}_{t}^{a R}+\widehat{r s}_{t}-\ldots \\
\left(1+\eta\left(\frac{\psi}{1+(1-\psi) \varphi}\right)\left(\varphi+(1-\psi)\left(\frac{1+\varphi}{\psi}\right)^{2}\right)\right)\left(\left(\widehat{p}_{t}^{F, W *}-\widehat{p}_{t}^{W *}\right)-\left(\widehat{p}_{t}^{H, W}-\widehat{p}_{t}^{W}\right)\right)-\ldots \\
\left(\phi_{H}\left(\widehat{p}_{t}^{F, R}-\widehat{p}_{t}^{R}\right)-\phi_{F}\left(\widehat{p}_{t}^{H, R}-\widehat{p}_{t}^{R}\right)\right)-(1-\psi)\left(\frac{1+\varphi}{\psi}\right)\left(\widehat{k}_{t}^{*}-\widehat{k}_{t}\right)-\left(\frac{1+\varphi}{\psi}\right)\left(\widehat{a}_{t}^{*}-\widehat{a}_{t}\right)
\end{array}\right], \\
& \widehat{\pi}_{t}^{F *}-\widehat{\pi}_{t}^{H *} \approx \beta \mathbb{E}_{t}\left(\widehat{\pi}_{t+1}^{F *}-\widehat{\pi}_{t+1}^{H *}\right)+\ldots \\
& \Psi\left[\begin{array}{c}
\sigma^{-1}\left(\left(\frac{1-\gamma_{x}}{1-\gamma_{x}^{x}}\right) \widehat{c}_{t}^{a W *}-\left(\frac{1-\gamma_{x}}{1-\gamma_{x}^{a}}\right) \widehat{c}_{t}^{a W}\right)+\ldots \\
\left(1-\gamma_{x}\right)\left(\frac{\psi}{1+(1-\psi) \varphi}\right)\left(\varphi+(1-\psi)\left(\frac{1+\varphi}{\psi}\right)^{2}\right)\left(\widehat{c}_{t}^{a W *}-\widehat{c}_{t}^{a W}\right)+\ldots \\
\gamma_{x}\left(\frac{\psi}{1+(1-\psi) \varphi}\right)\left(\varphi+(1-\psi)\left(\frac{1+\varphi}{\psi}\right)^{2}\right)\left(\widehat{x}_{t}^{a W *}-\widehat{x}_{t}^{a W}\right)-\ldots \\
\phi_{F} \sigma^{-1}\left(\left(\frac{1-\gamma_{x}}{1-\gamma_{x}^{a}}\right)+\left(\frac{1-\gamma_{x}}{1-\gamma_{x}^{a}}\right)\right) \widehat{c}_{t}^{a R}+\widehat{r}_{t}-\ldots \\
\left(1+\eta\left(\frac{\psi}{1+(1-\psi) \varphi}\right)\left(\varphi+(1-\psi)\left(\frac{1+\varphi}{\psi}\right)^{2}\right)\right)\left(\left(\widehat{p}_{t}^{F, W *}-\widehat{p}_{t}^{W *}\right)-\left(\widehat{p}_{t}^{H, W}-\widehat{p}_{t}^{W}\right)\right)+\ldots \\
\left(\phi_{F}\left(\widehat{p}_{t}^{F, R}-\widehat{p}_{t}^{R}\right)-\phi_{H}\left(\widehat{p}_{t}^{H, R}-\widehat{p}_{t}^{R}\right)\right)-(1-\psi)\left(\frac{1+\varphi}{\psi}\right)\left(\widehat{k}_{t}^{*}-\widehat{k}_{t}\right)-\left(\frac{1+\varphi}{\psi}\right)\left(\widehat{a}_{t}^{*}-\widehat{a}_{t}\right)
\end{array}\right] .
\end{aligned}
$$

I noted already that by construction $\left(\widehat{p}_{t}^{H, W}-\widehat{p}_{t}^{W}\right)+\left(\widehat{p}_{t}^{F, W *}-\widehat{p}_{t}^{W *}\right) \approx 0$, hence the two expressions for the 
relative prices above become simply,

$$
\begin{aligned}
& \widehat{\pi}_{t}^{F}-\widehat{\pi}_{t}^{H} \approx \beta \mathbb{E}_{t}\left(\widehat{\pi}_{t+1}^{F}-\widehat{\pi}_{t+1}^{H}\right)+\ldots \\
& \Psi\left[\begin{array}{c}
\sigma^{-1}\left(\left(\frac{1-\gamma_{x}}{1-\gamma_{x}^{a}}\right) \widehat{c}_{t}^{a W *}-\left(\frac{1-\gamma_{x}}{1-\gamma_{x}^{a}}\right) \widehat{c}_{t}^{a W}\right)+\ldots \\
\left(1-\gamma_{x}\right)\left(\frac{\psi}{1+(1-\psi) \varphi}\right)\left(\varphi+(1-\psi)\left(\frac{1+\varphi}{\psi}\right)^{2}\right)\left(\widehat{c}_{t}^{a W *}-\widehat{c}_{t}^{a W}\right)+\ldots \\
\gamma_{x}\left(\frac{\psi}{1+(1-\psi) \varphi}\right)\left(\varphi+(1-\psi)\left(\frac{1+\varphi}{\psi}\right)^{2}\right)\left(\widehat{x}_{t}^{a W *}-\widehat{x}_{t}^{a W}\right)-\ldots \\
\phi_{F} \sigma^{-1}\left(\left(\frac{1-\gamma_{x}}{1-\gamma_{x^{*}}^{a}}\right)+\left(\frac{1-\gamma_{x}}{1-\gamma_{x}^{a}}\right)\right) \widehat{c}_{t}^{a R}+\widehat{r}_{t}-\ldots \\
2\left(1+\eta\left(\frac{\psi}{1+(1-\psi) \varphi}\right)\left(\varphi+(1-\psi)\left(\frac{1+\varphi}{\psi}\right)^{2}\right)\right)\left(\widehat{p}_{t}^{F, W *}-\widehat{p}_{t}^{W *}\right)-\ldots \\
\left(\phi_{H}\left(\widehat{p}_{t}^{F, R}-\widehat{p}_{t}^{R}\right)-\phi_{F}\left(\widehat{p}_{t}^{H, R}-\widehat{p}_{t}^{R}\right)\right)-(1-\psi)\left(\frac{1+\varphi}{\psi}\right)\left(\widehat{k}_{t}^{*}-\widehat{k}_{t}\right)-\left(\frac{1+\varphi}{\psi}\right)\left(\widehat{a}_{t}^{*}-\widehat{a}_{t}\right)
\end{array}\right], \\
& \widehat{\pi}_{t}^{F *}-\widehat{\pi}_{t}^{H *} \approx \beta \mathbb{E}_{t}\left(\widehat{\pi}_{t+1}^{F *}-\widehat{\pi}_{t+1}^{H *}\right)+\ldots \\
& \Psi\left[\begin{array}{c}
\sigma^{-1}\left(\left(\frac{1-\gamma_{x}}{1-\gamma^{*}}\right) \widehat{c}_{t}^{a W *}-\left(\frac{1-\gamma_{x}}{1-\gamma_{x}^{a}}\right) \widehat{c}_{t}^{a W}\right)+\ldots \\
\left(1-\gamma_{x}\right)\left(\frac{\psi}{1+(1-\psi) \varphi}\right)\left(\varphi+(1-\psi)\left(\frac{1+\varphi}{\psi}\right)^{2}\right)\left(\widehat{c}_{t}^{a W *}-\widehat{c}_{t}^{a W}\right)+\ldots \\
\gamma_{x}\left(\frac{\psi}{1+(1-\psi) \varphi}\right)\left(\varphi+(1-\psi)\left(\frac{1+\varphi}{\psi}\right)^{2}\right)\left(\widehat{x}_{t}^{a W *}-\widehat{x}_{t}^{a W}\right)-\ldots \\
\phi_{F} \sigma^{-1}\left(\left(\frac{1-\gamma_{x}}{1-\gamma_{x^{*}}}\right)+\left(\frac{1-\gamma_{x}}{1-\gamma_{x}^{a}}\right)\right) \widehat{c}_{t}^{a R}+\widehat{r}_{t}-\ldots \\
2\left(1+\eta\left(\frac{\psi}{1+(1-\psi) \varphi}\right)\left(\varphi+(1-\psi)\left(\frac{1+\varphi}{\psi}\right)^{2}\right)\right)\left(\widehat{p}_{t}^{F, W *}-\widehat{p}_{t}^{W *}\right)+\ldots \\
\left(\phi_{F}\left(\widehat{p}_{t}^{F, R}-\widehat{p}_{t}^{R}\right)-\phi_{H}\left(\widehat{p}_{t}^{H, R}-\widehat{p}_{t}^{R}\right)\right)-(1-\psi)\left(\frac{1+\varphi}{\psi}\right)\left(\widehat{k}_{t}^{*}-\widehat{k}_{t}\right)-\left(\frac{1+\varphi}{\psi}\right)\left(\widehat{a}_{t}^{*}-\widehat{a}_{t}\right)
\end{array}\right] .
\end{aligned}
$$

Let me define the variable $\widehat{z}_{t}$ as the difference between the relative prices in both countries, i.e. $\widehat{z}_{t} \equiv$ $\left(\widehat{p}_{t}^{F}-\widehat{p}_{t}^{H}\right)-\left(\widehat{p}_{t}^{F *}-\widehat{p}_{t}^{H *}\right)$, and the first-difference of $\widehat{z}_{t}$ as $\Delta \widehat{z}_{t} \equiv\left(\widehat{\pi}_{t}^{F}-\widehat{\pi}_{t}^{H}\right)-\left(\widehat{\pi}_{t}^{F *}-\widehat{\pi}_{t}^{H *}\right)$. Using the two equations I derived previously for $\left(\widehat{\pi}_{t}^{F}-\widehat{\pi}_{t}^{H}\right)$ and $\left(\widehat{\pi}_{t}^{F *}-\widehat{\pi}_{t}^{H *}\right)$, it immediately follows that,

$$
\begin{aligned}
& \left(\widehat{\pi}_{t}^{F}-\widehat{\pi}_{t}^{H}\right)-\left(\widehat{\pi}_{t}^{F *}-\widehat{\pi}_{t}^{H *}\right) \approx \beta \mathbb{E}_{t}\left(\left(\widehat{\pi}_{t+1}^{F}-\widehat{\pi}_{t+1}^{H}\right)-\left(\widehat{\pi}_{t+1}^{F *}-\widehat{\pi}_{t+1}^{H *}\right)\right)-\ldots \\
& \quad \Psi\left[\left(\phi_{H}\left(\widehat{p}_{t}^{F, R}-\widehat{p}_{t}^{R}\right)-\phi_{F}\left(\widehat{p}_{t}^{H, R}-\widehat{p}_{t}^{R}\right)\right)+\left(\phi_{F}\left(\widehat{p}_{t}^{F, R}-\widehat{p}_{t}^{R}\right)-\phi_{H}\left(\widehat{p}_{t}^{H, R}-\widehat{p}_{t}^{R}\right)\right)\right] \\
& \quad \approx \beta \mathbb{E}_{t}\left(\left(\widehat{\pi}_{t+1}^{F}-\widehat{\pi}_{t+1}^{H}\right)-\left(\widehat{\pi}_{t+1}^{F *}-\widehat{\pi}_{t+1}^{H *}\right)\right)-\Psi\left[\left(\widehat{p}_{t}^{F, R}-\widehat{p}_{t}^{R}\right)-\left(\widehat{p}_{t}^{H, R}-\widehat{p}_{t}^{R}\right)\right] \\
& \quad \approx \beta \mathbb{E}_{t}\left(\left(\widehat{\pi}_{t+1}^{F}-\widehat{\pi}_{t+1}^{H}\right)-\left(\widehat{\pi}_{t+1}^{F *}-\widehat{\pi}_{t+1}^{H *}\right)\right)-\Psi\left[\widehat{p}_{t}^{F, R}-\widehat{p}_{t}^{H, R}\right] .
\end{aligned}
$$

Finally, since I have already defined $\widehat{p}_{t}^{H, R} \equiv\left(\widehat{p}_{t}^{H}-\widehat{p}_{t}^{H *}\right)$ and $\widehat{p}_{t}^{F, R} \equiv\left(\widehat{p}_{t}^{F}-\widehat{p}_{t}^{F *}\right)$, I can also infer that,

$$
\left[\widehat{p}_{t}^{F, R}-\widehat{p}_{t}^{H, R}\right]=\left(\widehat{p}_{t}^{F}-\widehat{p}_{t}^{F *}\right)-\left(\widehat{p}_{t}^{H}-\widehat{p}_{t}^{H *}\right)=\left(\widehat{p}_{t}^{F}-\widehat{p}_{t}^{H}\right)-\left(\widehat{p}_{t}^{F *}-\widehat{p}_{t}^{H *}\right)=\widehat{z}_{t},
$$

and, accordingly, I can re-write the expression above for the dynamics of $\Delta \widehat{z}_{t}$ as,

$$
\Delta \widehat{z}_{t} \approx \beta \mathbb{E}_{t}\left(\Delta \widehat{z}_{t+1}\right)-\Psi \widehat{z}_{t}
$$

Naturally, as Engel (forthcoming) emphasizes, if I combine equation (325) with the initial condition $\widehat{z}_{0}=0$, then it has to be the case that the solution implies that the relative prices in both countries ought to equalize 
as I postulated initially, i.e.,

$$
\widehat{z}_{t} \equiv\left(\widehat{p}_{t}^{F}-\widehat{p}_{t}^{H}\right)-\left(\widehat{p}_{t}^{F *}-\widehat{p}_{t}^{H *}\right) \approx 0
$$

or simply that,

$$
\left(\widehat{p}_{t}^{F}-\widehat{p}_{t}^{H}\right) \approx\left(\widehat{p}_{t}^{F *}-\widehat{p}_{t}^{H *}\right) .
$$

Furthermore, if I combine equations (324) with the solution in (326), I obtain the following result,

$$
\left(\widehat{p}_{t}^{F, R}-\widehat{p}_{t}^{R}\right)-\left(\widehat{p}_{t}^{H, R}-\widehat{p}_{t}^{R}\right) \approx 0
$$

or simply,

$$
\left(\widehat{p}_{t}^{F, R}-\widehat{p}_{t}^{R}\right) \approx\left(\widehat{p}_{t}^{H, R}-\widehat{p}_{t}^{R}\right) .
$$

Therefore, I can re-write the world terms of trade defined in (322) as follows,

$$
\widehat{t}_{t}^{W} \equiv \widehat{p}_{t}^{F, W *}-\widehat{p}_{t}^{W *}=2 \frac{\phi_{H} \phi_{F}}{\phi_{H}-\phi_{F}}\left(\widehat{p}_{t}^{F, R}-\widehat{p}_{t}^{R}\right)
$$

Equation (329) is going to be particularly helpful to simplify the log-linearized equilibrium conditions of the model later on.

\subsection{Other Relationships}

On Aggregate Output. I have shown in equations (298) and (299) that the aggregate output in each country can be expressed as,

$$
\begin{aligned}
\widehat{y}_{t} & \approx-\eta\left(\widehat{p}_{t}^{H, W}-\widehat{p}_{t}^{W}\right)+\left(1-\gamma_{x}\right) \widehat{c}_{t}^{a W}+\gamma_{x} \widehat{x}_{t}^{a W} \\
& =\eta\left(\widehat{p}_{t}^{F, W *}-\widehat{p}_{t}^{W *}\right)+\left(1-\gamma_{x}\right) \widehat{c}_{t}^{a W}+\gamma_{x} \widehat{x}_{t}^{a W}, \\
\widehat{y}_{t}^{*} & \approx-\eta\left(\widehat{p}_{t}^{F, W *}-\widehat{p}_{t}^{W *}\right)+\left(1-\gamma_{x}\right) \widehat{c}_{t}^{a W *}+\gamma_{x} \widehat{x}_{t}^{a W *},
\end{aligned}
$$

since $\left(\widehat{p}_{t}^{H, W}-\widehat{p}_{t}^{W}\right)+\left(\widehat{p}_{t}^{F, W *}-\widehat{p}_{t}^{W *}\right) \approx 0$. Most notably, I can write both output equations as functions of world terms of trade without having to keep track of any other international relative price. Using the world terms of trade definition I can write aggregate output as,

$$
\begin{aligned}
\widehat{y}_{t} & \approx \eta \widehat{t}_{t}^{W}+\left(1-\gamma_{x}\right) \widehat{c}_{t}^{a W}+\gamma_{x} \widehat{x}_{t}^{a W}, \\
\widehat{y}_{t}^{*} & \approx-\eta \widehat{t}_{t}^{W}+\left(1-\gamma_{x}\right) \widehat{c}_{t}^{a W *}+\gamma_{x} \widehat{x}_{t}^{a W *} .
\end{aligned}
$$


This also means that world aggregate and world relative output must satisfy the following conditions,

$$
\begin{aligned}
& \widehat{y}_{t}^{W} \equiv \phi_{H} \widehat{y}_{t}+\phi_{F} \widehat{y}_{t}^{*} \approx-\eta\left[\phi_{H}\left(\widehat{p}_{t}^{H, W}-\widehat{p}_{t}^{W}\right)+\phi_{F}\left(\widehat{p}_{t}^{F, W *}-\widehat{p}_{t}^{W *}\right)\right]+\ldots \\
&\left(1-\gamma_{x}\right)\left[\phi_{H} \widehat{c}_{t}^{a W}+\phi_{F} \widehat{c}_{t}^{a W *}\right]+\gamma_{x}\left[\phi_{H} \widehat{x}_{t}^{a W}+\phi_{F} \widehat{x}_{t}^{a W *}\right] \\
&=\eta\left(\phi_{H}-\phi_{F}\right)\left[\widehat{p}_{t}^{F, W *}-\widehat{p}_{t}^{W *}\right]+\left(1-\gamma_{x}\right)\left[\phi_{H} \widehat{c}_{t}^{a W}+\phi_{F} \widehat{c}_{t}^{a W *}\right]+\gamma_{x}\left[\phi_{H} \widehat{x}_{t}^{a W}+\phi_{F} \widehat{x}_{t}^{a W *}\right] \\
&=\eta\left(\phi_{H}-\phi_{F}\right) \widehat{t}_{t}^{W}+\left(1-\gamma_{x}\right)\left[\phi_{H} \widehat{c}_{t}^{a W}+\phi_{F} \widehat{c}_{t}^{a W *}\right]+\gamma_{x}\left[\phi_{H} \widehat{x}_{t}^{a W}+\phi_{F} \widehat{x}_{t}^{a W *}\right], \\
& \widehat{y}_{t}^{W *} \equiv \phi_{F} \widehat{y}_{t}+\phi_{H} \widehat{y}_{t}^{*} \approx-\eta\left[\phi_{F}\left(\widehat{p}_{t}^{H, W}-\widehat{p}_{t}^{W}\right)+\phi_{H}\left(\widehat{p}_{t}^{F, W *}-\widehat{p}_{t}^{W *}\right)\right]+\ldots \\
&\left(1-\gamma_{x}\right)\left[\phi_{F} \widehat{c}_{t}^{a W}+\phi_{H} \widehat{c}_{t}^{a W *}\right]+\gamma_{x}\left[\phi_{F} \widehat{x}_{t}^{a W}+\phi_{H} \widehat{x}_{t}^{a W *}\right] \\
&=-\eta\left(\phi_{H}-\phi_{F}\right)\left[\widehat{p}_{t}^{F, W *}-\widehat{p}_{t}^{W *}\right]+\left(1-\gamma_{x}\right)\left[\phi_{F} \widehat{c}_{t}^{a W}+\phi_{H} \widehat{c}_{t}^{a W *}\right]+\gamma_{x}\left[\phi_{F} \widehat{x}_{t}^{a W}+\phi_{H} \widehat{x}_{t}^{a W *}\right] \\
&=-\eta\left(\phi_{H}-\phi_{F}\right) \widehat{t}_{t}^{W}+\left(1-\gamma_{x}\right)\left[\phi_{F} \widehat{c}_{t}^{a W}+\phi_{H} \widehat{c}_{t}^{a W *}\right]+\gamma_{x}\left[\phi_{F} \widehat{x}_{t}^{a W}+\phi_{H} \widehat{x}_{t}^{a W *}\right], \\
& \widehat{y}_{t}^{R} \equiv \widehat{y}_{t}-\widehat{y}_{t}^{*} \approx-\eta\left[\left(\widehat{p}_{t}^{H, W}-\widehat{p}_{t}^{W}\right)-\left(\widehat{p}_{t}^{F, W *}-\widehat{p}_{t}^{W *}\right)\right]+\ldots \\
&\left(1-\gamma_{x}\right)\left[\widehat{c}_{t}^{a W}-\widehat{c}_{t}^{a W *}\right]+\gamma_{x}\left[\widehat{x}_{t}^{a W}-\widehat{x}_{t}^{a W *}\right] \\
&= 2 \eta\left[\widehat{p}_{t}^{F, W *}-\widehat{p}_{t}^{W *}\right]+\left(1-\gamma_{x}\right)\left[\widehat{c}_{t}^{a W}-\widehat{c}_{t}^{a W *}\right]+\gamma_{x}\left[\widehat{x}_{t}^{a W}-\widehat{x}_{t}^{a W *}\right] \\
&= 2 \eta \hat{t}_{t}^{W}+\left(1-\gamma_{x}\right)\left[\widehat{c}_{t}^{a W}-\widehat{c}_{t}^{a W *}\right]+\gamma_{x}\left[\widehat{x}_{t}^{a W}-\widehat{x}_{t}^{a W *}\right] .
\end{aligned}
$$

I could use the equations derived before for $\widehat{y}_{t}^{W}$ and $\widehat{y}_{t}^{W *}$ in order to substitute out consumption in the Phillips curves, so I can write everything in terms of output instead of consumption. I can also replace $\widehat{t}_{t}^{W} \equiv\left(\widehat{p}_{t}^{F, W *}-\widehat{p}_{t}^{W *}\right)$ out using the equation for relative output.

On the Efficiency Conditions. Using the efficiency conditions in (300) and (301) together with the definition of the world terms of trade $\widehat{t}_{t}^{W} \equiv\left(\widehat{p}_{t}^{F, W *}-\widehat{p}_{t}^{W *}\right)$, it follows after a little bit of algebra that the real rental rates on capital can be expressed as,

$$
\begin{aligned}
& \widehat{r}_{t}^{z} \approx\left(\frac{1}{\sigma}\left(\frac{1-\gamma_{x}}{1-\gamma_{x}^{a}}\right)+\left(1-\gamma_{x}\right) \frac{1+\varphi}{\psi}\right) \widehat{c}_{t}^{a W}+\gamma_{x} \frac{1+\varphi}{\psi} \widehat{x}_{t}^{a W}+\phi_{F}\left(\frac{1-\gamma_{x}}{1-\gamma_{x}^{a}}\right) \sigma^{-1} \widehat{c}_{t}^{a R}+\eta \frac{1+\varphi}{\psi} \widehat{t}_{t}^{W}-\ldots \\
& \quad\left(\frac{1+(1-\psi) \varphi}{\psi}\right) \widehat{k}_{t}-\frac{1+\varphi}{\psi} \widehat{a}_{t}, \\
& \widehat{r}_{t}^{z *} \approx\left(\frac{1}{\sigma}\left(\frac{1-\gamma_{x}}{1-\gamma_{x *}^{a}}\right)+\left(1-\gamma_{x}\right) \frac{1+\varphi}{\psi}\right) \widehat{c}_{t}^{a W *}+\gamma_{x} \frac{1+\varphi}{\psi} \widehat{x}_{t}^{a W *}-\phi_{F}\left(\frac{1-\gamma_{x}}{1-\gamma_{x^{*}}^{a}}\right) \sigma^{-1} \widehat{c}_{t}^{a R}-\eta \frac{1+\varphi}{\psi} \widehat{t}_{t}^{W}-\ldots \\
& \quad\left(\frac{1+(1-\psi) \varphi}{\psi}\right) \widehat{k}_{t}^{*}-\frac{1+\varphi}{\psi} \widehat{a}_{t}^{*} .
\end{aligned}
$$

This simply re-writes the previous conditions replacing the international relative prices with the definition of world terms of trade. However, for the purpose of simulating the model, suffices to use the expressions derived in $(294)-(295)$ or in $(296)-(297)$.

On Aggregate Employment. The aggregate employment can be easily derived by log-linearizing the aggregate production equations in (84) and (85) as,

$$
\begin{aligned}
\widehat{y}_{t} & \approx \widehat{a}_{t}+(1-\psi) \widehat{k}_{t}+\psi \widehat{l}_{t}, \\
\widehat{y}_{t}^{*} & \approx \widehat{a}_{t}^{*}+(1-\psi) \widehat{k}_{t}^{*}+\psi \widehat{l}_{t}^{*} .
\end{aligned}
$$

Naturally, the linear-in-labor case for employment can be derived as a special case of this log-linearized aggregate production equation in which the labor share converges to one, i.e. $\psi \rightarrow 1$. 
On Real Exports, Real Imports, and the Net Exports Share. In a two-country model, suffice to determine the net exports share of the domestic country. Let me denote the deviation of net exports / GDP from its steady state as $\widehat{t b}_{t} .{ }^{8}$ Then, because the trade balance is easily computed as the difference between domestic aggregate output and domestic aggregate consumption and investment in real terms (or domestic absorption) (see, e.g., Galí and Monacelli (2005)), I obtain that,

$$
\widehat{t b}_{t} \equiv \widehat{y}_{t}-\left(1-\gamma_{x}^{a}\right) \widehat{c}_{t}-\gamma_{x}^{a} \widehat{x}_{t}
$$

I have defined the world consumption as $\widehat{c}_{t}^{a W} \equiv \phi_{H}\left(\frac{1-\gamma_{x}^{a}}{1-\gamma_{x}}\right) \widehat{c}_{t}+\phi_{F}\left(\frac{1-\gamma_{x}^{a}}{1-\gamma_{x}}\right) \widehat{c}_{t}^{*}$ and $\widehat{c}_{t}^{a W *} \equiv \phi_{F}\left(\frac{1-\gamma_{x}^{a}}{1-\gamma_{x}}\right) \widehat{c}_{t}+$ $\phi_{H}\left(\frac{1-\gamma_{x^{*}}^{a}}{1-\gamma_{x}}\right) \widehat{c}_{t}^{*}$ and the relative consumption as $\widehat{c}_{t}^{a R} \equiv\left(\frac{1-\gamma_{x}^{a}}{1-\gamma_{x}}\right) \widehat{c}_{t}-\left(\frac{1-\gamma_{x^{*}}^{a}}{1-\gamma_{x}}\right) \widehat{c}_{t}^{*}$. I have defined the world investment as $\widehat{x}_{t}^{a W} \equiv \phi_{H}\left(\frac{\gamma_{x}^{a}}{\gamma_{x}}\right) \widehat{x}_{t}+\phi_{F}\left(\frac{\gamma_{x^{*}}^{a}}{\gamma_{x}}\right) \widehat{x}_{t}^{*}$ and $\widehat{x}_{t}^{a W *} \equiv \phi_{F}\left(\frac{\gamma_{x}^{a}}{\gamma_{x}}\right) \widehat{x}_{t}+\phi_{H}\left(\frac{\gamma_{x^{*}}^{a}}{\gamma_{x}}\right) \widehat{x}_{t}^{*}$ and the relative investment as $\widehat{x}_{t}^{a R} \equiv\left(\frac{\gamma_{x}^{a}}{\gamma_{x}}\right) \widehat{x}_{t}-\left(\frac{\gamma_{x^{*}}^{a}}{\gamma_{x}}\right) \widehat{x}_{t}^{*}$. Then, I can write domestic and foreign consumption and investment as follows,

$$
\begin{aligned}
\widehat{c}_{t} & =\left(\frac{1-\gamma_{x}}{1-\gamma_{x}^{a}}\right)\left(\widehat{c}_{t}^{a W}+\phi_{F} \widehat{c}_{t}^{a R}\right), \\
\widehat{c}_{t}^{*} & =\left(\frac{1-\gamma_{x}}{1-\gamma_{x^{*}}^{a}}\right)\left(\widehat{c}_{t}^{a W *}-\phi_{F} \widehat{c}_{t}^{a R}\right), \\
\widehat{x}_{t} & =\left(\frac{\gamma_{x}}{\gamma_{x}^{a}}\right)\left(\widehat{x}_{t}^{a W}+\phi_{F} \widehat{x}_{t}^{a R}\right), \\
\widehat{x}_{t}^{*} & =\left(\frac{\gamma_{x}}{\gamma_{x^{*}}^{a}}\right)\left(\widehat{x}_{t}^{a W *}-\phi_{F} \widehat{x}_{t}^{a R}\right) .
\end{aligned}
$$

Using the formula derived above for domestic aggregate output in (330) and the expressions above for the net exports share, I obtain the following equation for the trade balance,

$$
\begin{aligned}
\widehat{t b}_{t} & \approx \eta \widehat{t}_{t}^{W}+\left(1-\gamma_{x}\right) \widehat{c}_{t}^{a W}+\gamma_{x} \widehat{x}_{t}^{a W}-\left(1-\gamma_{x}^{a}\right) \widehat{c}_{t}-\gamma_{x}^{a} \widehat{x}_{t} \\
& =\eta \widehat{t}_{t}^{W}+\left(1-\gamma_{x}\right)\left(\widehat{c}_{t}^{a W}-\left(\frac{1-\gamma_{x}^{a}}{1-\gamma_{x}}\right) \widehat{c}_{t}\right)+\gamma_{x}\left(\widehat{x}_{t}^{a W}-\left(\frac{\gamma_{x}^{a}}{\gamma_{x}}\right) \widehat{x}_{t}\right) \\
& =\eta \widehat{t}_{t}^{W}+\left(1-\gamma_{x}\right)\left(\widehat{c}_{t}^{a W}-\left(\frac{1-\gamma_{x}^{a}}{1-\gamma_{x}}\right)\left(\frac{1-\gamma_{x}}{1-\gamma_{x}^{a}}\right)\left(\widehat{c}_{t}^{a W}+\phi_{F} \widehat{c}_{t}^{a R}\right)\right)+\gamma_{x}\left(\widehat{x}_{t}^{a W}-\left(\frac{\gamma_{x}^{a}}{\gamma_{x}}\right)\left(\frac{\gamma_{x}}{\gamma_{x}^{a}}\right)\left(\widehat{x}_{t}^{a W}+\phi_{F} \widehat{x}_{t}^{a R}\right)\right) \\
& =\eta \widehat{t}_{t}^{W}-\left(1-\gamma_{x}\right) \phi_{F} \widehat{c}_{t}^{a R}-\gamma_{x} \phi_{F} \widehat{x}_{t}^{a R} .
\end{aligned}
$$

In other words, adjustment in the domestic trade balance comes through movements in the world terms of trade $\hat{t}_{t}^{W}$, or from relative adjustments in either the consumption or investment paths.

The real exports and imports of domestic goods in the model can be inferred from equations (46) - (53) as follows,

$$
\begin{aligned}
E X P_{t} & \equiv \int_{0}^{1}\left[C_{t}^{*}(h)+X_{t}^{*}(h)\right] d h=\phi_{H}^{*} \int_{0}^{1}\left(\frac{P_{t}^{*}(h)}{P_{t}^{H *}}\right)^{-\theta}\left(\frac{P_{t}^{H *}}{P_{t}^{*}}\right)^{-\eta}\left[C_{t}^{*}+X_{t}^{*}\right] d h, \\
I M P_{t} & \equiv \int_{0}^{1}\left[C_{t}(f)+X_{t}(f)\right] d f=\phi_{F} \int_{0}^{1}\left(\frac{P_{t}(f)}{P_{t}^{F}}\right)^{-\theta}\left(\frac{P_{t}^{F}}{P_{t}}\right)^{-\eta}\left[C_{t}+X_{t}\right] d f,
\end{aligned}
$$

\footnotetext{
${ }^{8}$ I use $\widehat{t b}_{t}$ instead of the more conventional $\widehat{n x}_{t}$ notation in order to avoid possible confusion with the investment variable.
} 
where $\phi_{H}^{*}=\phi_{F}$ under my assumption of (symmetric) home-product bias in consumption and investment. A simple log-linearization of both definitions allows me to obtain the following pair of equations,

$$
\begin{aligned}
& \widehat{\exp }_{t} \approx-\theta\left(\int_{0}^{1} \widehat{p}_{t}^{*}(h) d h-\widehat{p}_{t}^{H *}\right)-\eta\left(\widehat{p}_{t}^{H *}-\widehat{p}_{t}^{*}\right)+\left(\frac{\bar{C}^{*}}{\bar{C}^{*}+\bar{X}^{*}}\right) \widehat{c}_{t}^{*}+\left(\frac{\bar{X}^{*}}{\bar{C}^{*}+\bar{X}^{*}}\right) \widehat{x}_{t}^{*}, \\
& \widehat{\operatorname{imp}}_{t} \approx-\theta\left(\int_{0}^{1} \widehat{p}_{t}(f) d f-\widehat{p}_{t}^{F}\right)-\eta\left(\widehat{p}_{t}^{F}-\widehat{p}_{t}\right)+\left(\frac{\bar{C}}{\bar{C}+\bar{X}}\right) \widehat{c}_{t}+\left(\frac{\bar{X}}{\bar{C}+\bar{X}}\right) \widehat{x}_{t} .
\end{aligned}
$$

I define the steady state investment and consumption shares relative to absorption in (187) - (190) as,

$$
\begin{aligned}
& \gamma_{x}^{a} \equiv \frac{\bar{X}}{\bar{C}+\bar{X}}=\delta\left[\frac{1-\psi}{\left(\frac{\theta(1-\bar{\xi})}{\theta-1}\right)\left(\beta^{-1}-(1-\delta)\right)}\right]\left(1-(1-\beta) \bar{a}^{a}\right), \\
& \gamma_{c}^{a} \equiv \frac{\bar{C}}{\bar{C}+\bar{X}}=1-\gamma_{x}^{a}, \\
& \gamma_{x^{*}}^{a} \equiv \frac{\bar{X}^{*}}{\bar{C}^{*}+\bar{X}^{*}}=\delta\left[\frac{1-\psi}{\left(\frac{\theta\left(1-\bar{\xi}^{*}\right)}{\theta-1}\right)\left(\beta^{-1}-(1-\delta)\right)}\right]\left(\frac{\phi_{F}+\phi_{H}(1-\beta) \bar{a}^{a}}{\phi_{F}+\left(\phi_{H} \phi_{H}-\phi_{F} \phi_{F}\right)(1-\beta) \bar{a}^{a}}\right), \\
& \gamma_{c^{*}}^{a} \equiv \frac{\bar{C}^{*}}{\bar{C}^{*}+\bar{X}^{*}}=1-\gamma_{x^{*}}^{a} .
\end{aligned}
$$

With these expressions at hand, I can write the log-linearized import and export equations as follows,

$$
\begin{aligned}
& \widehat{\exp }_{t} \approx-\theta\left(\int_{0}^{1} \widehat{p}_{t}^{*}(h) d h-\widehat{p}_{t}^{H *}\right)-\eta\left(\widehat{p}_{t}^{H *}-\widehat{p}_{t}^{*}\right)+\left(1-\gamma_{x^{*}}^{a}\right) \widehat{c}_{t}^{*}+\gamma_{x^{*}}^{a} \widehat{x}_{t}^{*}, \\
& \widehat{i m p}_{t} \approx-\theta\left(\int_{0}^{1} \widehat{p}_{t}(f) d f-\widehat{p}_{t}^{F}\right)-\eta\left(\widehat{p}_{t}^{F}-\widehat{p}_{t}\right)+\left(1-\gamma_{x}^{a}\right) \widehat{c}_{t}+\gamma_{x}^{a} \widehat{x}_{t} .
\end{aligned}
$$

Recall that the log-linearization of the price sub-indexes in $(17)-(20)$ clearly implies that $\int_{0}^{1} \widehat{p}_{t}^{*}(h) d h \approx \widehat{p}_{t}^{H *}$ and $\int_{0}^{1} \widehat{p}_{t}(f) d h \approx \widehat{p}_{t}^{F}$. Therefore, the first-order effects of relative price dispersion at the variety level are negligible, and I can re-write the export and import equations as,

$$
\begin{aligned}
\widehat{\exp }_{t} & \approx-\eta\left(\widehat{p}_{t}^{H *}-\widehat{p}_{t}^{*}\right)+\left(1-\gamma_{x^{*}}^{a}\right) \widehat{c}_{t}^{*}+\gamma_{x^{*}}^{a} \widehat{x}_{t}^{*} \\
\widehat{i m p}_{t} & \approx-\eta\left(\widehat{p}_{t}^{F}-\widehat{p}_{t}\right)+\left(1-\gamma_{x}^{a}\right) \widehat{c}_{t}+\gamma_{x}^{a} \widehat{x}_{t} .
\end{aligned}
$$

These expressions for the export and import equations are rather convenient.

I have defined the world price sub-indexes as $\widehat{p}_{t}^{H, W} \equiv \phi_{H} \widehat{p}_{t}^{H}+\phi_{F} \widehat{p}_{t}^{H *}$ and $\widehat{p}_{t}^{F, W *} \equiv \phi_{F} \widehat{p}_{t}^{F}+\phi_{H} \widehat{p}_{t}^{F *}$, and the relative price sub-indexes as $\widehat{p}_{t}^{H, R} \equiv \widehat{p}_{t}^{H}-\widehat{p}_{t}^{H *}$ and $\widehat{p}_{t}^{F, R} \equiv \widehat{p}_{t}^{F}-\widehat{p}_{t}^{F *}$. Then, naturally, I can write that,

$$
\begin{aligned}
\widehat{p}_{t}^{H} & =\widehat{p}_{t}^{H, W}+\phi_{F} \widehat{p}_{t}^{H, R}, \widehat{p}_{t}^{H *}=\widehat{p}_{t}^{H, W}-\phi_{H} \widehat{p}_{t}^{H, R}, \\
\widehat{p}_{t}^{F} & =\widehat{p}_{t}^{F, W *}+\phi_{H} \widehat{p}_{t}^{F, R}, \widehat{p}_{t}^{F *}=\widehat{p}_{t}^{F, W *}-\phi_{F} \widehat{p}_{t}^{F, R} .
\end{aligned}
$$

Analogously, I have defined the world CPI as $\widehat{p}_{t}^{W} \equiv \phi_{H} \widehat{p}_{t}+\phi_{F} \widehat{p}_{t}^{*}$ and $\widehat{p}_{t}^{W *} \equiv \phi_{F} \widehat{p}_{t}+\phi_{H} \widehat{p}_{t}^{*}$, and the relative 
CPI as $\widehat{p}_{t}^{R} \equiv \widehat{p}_{t}-\widehat{p}_{t}^{*}$. Then, I can write that,

$$
\begin{aligned}
& \widehat{p}_{t}=\widehat{p}_{t}^{W}+\phi_{F} \widehat{p}_{t}^{R}, \widehat{p}_{t}^{*}=\widehat{p}_{t}^{W}-\phi_{H} \widehat{p}_{t}^{R}, \\
& \widehat{p}_{t}=\widehat{p}_{t}^{W *}+\phi_{H} \widehat{p}_{t}^{R}, \widehat{p}_{t}^{*}=\widehat{p}_{t}^{W *}-\phi_{F} \widehat{p}_{t}^{R} .
\end{aligned}
$$

Using these definitions, it is possible to express the relative prices embedded in the definition of real exports and imports in the following terms, i.e.,

$$
\begin{aligned}
\widehat{p}_{t}^{H *}-\widehat{p}_{t}^{*} & =\left(\widehat{p}_{t}^{H, W}-\phi_{H} \widehat{p}_{t}^{H, R}\right)-\left(\widehat{p}_{t}^{W}-\phi_{H} \widehat{p}_{t}^{R}\right) \\
& =\widehat{p}_{t}^{H, W}-\widehat{p}_{t}^{W}-\phi_{H}\left(\widehat{p}_{t}^{H, R}-\widehat{p}_{t}^{R}\right), \\
\widehat{p}_{t}^{F}-\widehat{p}_{t} & =\widehat{p}_{t}^{F, W *}+\phi_{H} \widehat{p}_{t}^{F, R}-\left(\widehat{p}_{t}^{W *}+\phi_{H} \widehat{p}_{t}^{R}\right) \\
& =\widehat{p}_{t}^{F, W *}-\widehat{p}_{t}^{W *}+\phi_{H}\left(\widehat{p}_{t}^{F, R}-\widehat{p}_{t}^{R}\right),
\end{aligned}
$$

where the world terms of trade is defined as $\widehat{t}_{t}^{W} \equiv \widehat{p}_{t}^{F, W *}-\widehat{p}_{t}^{W *}$. The definition of CPI in both countries, i.e. $\widehat{p}_{t} \approx \phi_{H} \widehat{p}_{t}^{H}+\phi_{F} \widehat{p}_{t}^{F}$ and $\widehat{p}_{t}^{*} \approx \phi_{F} \widehat{p}_{t}^{H *}+\phi_{H} \widehat{p}_{t}^{F *}$, can be re-written as,

$$
\begin{aligned}
\phi_{H}\left[\widehat{p}_{t}^{H}-\widehat{p}_{t}\right]+\phi_{F}\left[\widehat{p}_{t}^{F}-\widehat{p}_{t}\right] & \approx 0, \\
\phi_{F}\left[\widehat{p}_{t}^{H *}-\widehat{p}_{t}^{*}\right]+\phi_{H}\left[\hat{p}_{t}^{F *}-\widehat{p}_{t}^{*}\right] & \approx 0 .
\end{aligned}
$$

Then, based on the relationships described before, I can further re-write the definitions of the CPI indexes as,

$$
\begin{aligned}
& \phi_{H}\left[\left(\widehat{p}_{t}^{H, W}-\widehat{p}_{t}^{W}\right)+\phi_{F}\left(\widehat{p}_{t}^{H, R}-\widehat{p}_{t}^{R}\right)\right]+\phi_{F}\left[\left(\widehat{p}_{t}^{F, W *}-\widehat{p}_{t}^{W *}\right)+\phi_{H}\left(\widehat{p}_{t}^{F, R}-\widehat{p}_{t}^{R}\right)\right] \approx 0, \\
& \phi_{F}\left[\left(\widehat{p}_{t}^{H, W}-\widehat{p}_{t}^{W}\right)-\phi_{H}\left(\widehat{p}_{t}^{H, R}-\widehat{p}_{t}^{R}\right)\right]+\phi_{H}\left[\left(\widehat{p}_{t}^{F, W *}-\widehat{p}_{t}^{W *}\right)-\phi_{F}\left(\widehat{p}_{t}^{F, R}-\widehat{p}_{t}^{R}\right)\right] \approx 0 .
\end{aligned}
$$

Using the second equality derived above and the definition of the world terms of trade $\widehat{t}_{t}^{W} \equiv \widehat{p}_{t}^{F, W *}-\widehat{p}_{t}^{W *}$, I can write the relative prices embedded in the definition of real exports and imports in the following terms, i.e.,

$$
\begin{aligned}
\widehat{p}_{t}^{H *}-\widehat{p}_{t}^{*} & \approx-\frac{\phi_{H}}{\phi_{F}}\left[\widehat{t}_{t}^{W}-\phi_{F}\left(\widehat{p}_{t}^{F, R}-\widehat{p}_{t}^{R}\right)\right], \\
\widehat{p}_{t}^{F}-\widehat{p}_{t} & \approx \widehat{t}_{t}^{W}+\phi_{H}\left(\widehat{p}_{t}^{F, R}-\widehat{p}_{t}^{R}\right)
\end{aligned}
$$

and,

$$
\begin{aligned}
\phi_{F}\left(\widehat{p}_{t}^{H *}-\widehat{p}_{t}^{*}\right) & \approx-\phi_{H}\left[\widehat{t}_{t}^{W}-\phi_{F}\left(\widehat{p}_{t}^{F, R}-\widehat{p}_{t}^{R}\right)\right], \\
\phi_{F}\left(\widehat{p}_{t}^{F}-\widehat{p}_{t}\right) & \approx \phi_{F}\left[\widehat{t}_{t}^{W}+\phi_{H}\left(\widehat{p}_{t}^{F, R}-\widehat{p}_{t}^{R}\right)\right] .
\end{aligned}
$$


Under these conditions it naturally follows that,

$$
\begin{aligned}
\phi_{F}\left[\left(\widehat{p}_{t}^{H *}-\widehat{p}_{t}^{*}\right)-\left(\widehat{p}_{t}^{F}-\widehat{p}_{t}\right)\right] & \approx-\phi_{H}\left[\widehat{t}_{t}^{W}-\phi_{F}\left(\widehat{p}_{t}^{F, R}-\widehat{p}_{t}^{R}\right)\right]-\phi_{F}\left[\widehat{t}_{t}^{W}+\phi_{H}\left(\widehat{p}_{t}^{F, R}-\widehat{p}_{t}^{R}\right)\right] \\
& =-\left(\phi_{H}+\phi_{F}\right) \widehat{t}_{t}^{W}=-\widehat{t}_{t}^{W}
\end{aligned}
$$

and, based on equation (329), I also get that,

$$
\begin{aligned}
\widehat{p}_{t}^{H *}-\widehat{p}_{t}^{*} & \approx-\frac{\phi_{H}}{\phi_{F}}\left[\widehat{t}_{t}^{W}-\phi_{F}\left(\widehat{p}_{t}^{F, R}-\widehat{p}_{t}^{R}\right)\right]=\left[-\frac{\phi_{H}}{\phi_{F}}+\left(\frac{\phi_{H}-\phi_{F}}{2 \phi_{F}}\right)\right] \widehat{t}_{t}^{W}=-\left(\frac{1}{2 \phi_{F}}\right) \widehat{t}_{t}^{W}, \\
\hat{p}_{t}^{F}-\widehat{p}_{t} & \approx \widehat{t}_{t}^{W}+\phi_{H}\left(\widehat{p}_{t}^{F, R}-\widehat{p}_{t}^{R}\right)=\left[1+\left(\frac{\phi_{H}-\phi_{F}}{2 \phi_{F}}\right)\right] \widehat{t}_{t}^{W}=\left(\frac{1}{2 \phi_{F}}\right) \widehat{t}_{t}^{W},
\end{aligned}
$$

Hence, the import and export equations can be re-written as follows,

$$
\begin{aligned}
& \widehat{\exp }_{t} \approx \eta\left(\frac{1}{2 \phi_{F}}\right) \widehat{t}_{t}^{W}+\left(1-\gamma_{x^{*}}^{a}\right) \widehat{c}_{t}^{*}+\gamma_{x^{*}}^{a} \widehat{x}_{t}^{*} \\
& \widehat{i m p}_{t} \approx-\eta\left(\frac{1}{2 \phi_{F}}\right) \widehat{t}_{t}^{W}+\left(1-\gamma_{x}^{a}\right) \widehat{c}_{t}+\gamma_{x}^{a} \widehat{x}_{t} .
\end{aligned}
$$

These two equations show that the strength of the demand for consumption and investment purposes is likely to have a major impact on both exports and imports. However, they also show that exports and imports depend on world terms of trade, $\widehat{t}_{t}^{W}$, which is the sole variable that summarizes the impact of international relative prices on both real exports and real imports in the context of this model.

Therefore, I can compute the real trade balance (relative to steady state domestic output) in this model straight from the definitions of real exports and imports in (335) and (336) as,

$$
\begin{aligned}
\phi_{F}\left(\widehat{\exp }_{t}-\widehat{i m p}_{t}\right) & \approx \phi_{F}\left(\eta\left(\frac{1}{2 \phi_{F}}\right) \widehat{t}_{t}^{W}+\left(1-\gamma_{x^{*}}^{a}\right) \widehat{c}_{t}^{*}+\gamma_{x^{*}}^{a} \widehat{x}_{t}^{*}\right)-\phi_{F}\left(-\eta\left(\frac{1}{2 \phi_{F}}\right) \widehat{t}_{t}^{W}+\left(1-\gamma_{x}^{a}\right) \widehat{c}_{t}+\gamma_{x}^{a} \widehat{x}_{t}\right) \\
& =\eta \widehat{t}_{t}^{W}+\phi_{F}\left(\left(1-\gamma_{x^{*}}^{a}\right) \widehat{c}_{t}^{*}+\gamma_{x^{*}}^{a} \widehat{x}_{t}^{*}\right)-\phi_{F}\left(\left(1-\gamma_{x}^{a}\right) \widehat{c}_{t}+\gamma_{x}^{a} \widehat{x}_{t}\right) \\
& =\eta \widehat{t}_{t}^{W}+\phi_{F}\left(\left(1-\gamma_{x^{*}}^{a}\right) \widehat{c}_{t}^{*}-\left(1-\gamma_{x}^{a}\right) \widehat{c}_{t}\right)+\phi_{F}\left(\gamma_{x^{*}}^{a} \widehat{x}_{t}^{*}-\gamma_{x}^{a} \widehat{x}_{t}\right) \\
& =\eta \widehat{t}_{t}^{W}-\left(1-\gamma_{x}\right) \phi_{F}\left(\left(\frac{1-\gamma_{x}^{a}}{1-\gamma_{x}}\right) \widehat{c}_{t}-\left(\frac{1-\gamma_{x^{*}}^{a}}{1-\gamma_{x}}\right) \widehat{c}_{t}^{*}\right)-\gamma_{x} \phi_{F}\left(\frac{\gamma_{x}^{a}}{\gamma_{x}} \widehat{x}_{t}-\frac{\gamma_{x^{*}}^{a}}{\gamma_{x}} \widehat{x}_{t}^{*}\right) \\
& =\eta \widehat{t}_{t}^{W}-\left(1-\gamma_{x}\right) \phi_{F} \widehat{c}_{t}^{a R}-\gamma_{x} \phi_{F} \widehat{x}_{t}^{a R} \approx \widehat{t b}_{t},
\end{aligned}
$$

where $\widehat{c}_{t}^{a R} \equiv\left(\frac{1-\gamma_{x}^{a}}{1-\gamma_{x}}\right) \widehat{c}_{t}-\left(\frac{1-\gamma_{x^{*}}^{a}}{1-\gamma_{x}}\right) \widehat{c}_{t}^{*}$ and $\widehat{x}_{t}^{a R} \equiv\left(\frac{\gamma_{x}^{a}}{\gamma_{x}}\right) \widehat{x}_{t}-\left(\frac{\gamma_{x^{*}}^{a}}{\gamma_{x}}\right) \widehat{x}_{t}^{*}$. In other words, my measure of domestic the trade balance in (334) is equivalent to the difference between the log of real exports and real imports (in deviations relative to their respective steady states) scaled by the parameter $\phi_{F}$. In the deterministic 
steady state of the model, it follows easily from equations (46) - (53) that,

$$
\begin{aligned}
\bar{C}^{H} & =\phi_{H}\left(\frac{\bar{P}^{H}}{\bar{P}}\right)^{-\eta} \bar{C}, \bar{X}^{H}=\phi_{H}\left(\frac{\bar{P}^{H}}{\bar{P}}\right)^{-\eta} \bar{X}, \\
\bar{C}^{F} & =\phi_{F}\left(\frac{\bar{P}^{F}}{\bar{P}}\right)^{-\eta} \bar{C}, \bar{X}^{F}=\phi_{F}\left(\frac{\bar{P}^{F}}{\bar{P}}\right)^{-\eta} \bar{X}, \\
\bar{C}^{H *} & =\phi_{F}\left(\frac{\bar{P}^{H *}}{\bar{P}^{*}}\right)^{-\eta} \bar{C}^{*}, \bar{X}^{H *}=\phi_{F}\left(\frac{\bar{P}^{H *}}{\bar{P}^{*}}\right)^{-\eta} \bar{X}^{*}, \\
\bar{C}^{F *} & =\phi_{H}\left(\frac{\bar{P}^{F *}}{\bar{P}^{*}}\right)^{-\eta} \bar{C}^{*}, \bar{X}^{F *}=\phi_{H}\left(\frac{\bar{P}^{F *}}{\bar{P}^{*}}\right)^{-\eta} \bar{X}^{*} .
\end{aligned}
$$

Under the assumption of (symmetric) home-product bias in consumption and investment (i.e., $\phi_{H}^{*}=\phi_{F}$ ), I write the domestic real import and real export shares over domestic output as,

$$
\begin{aligned}
\frac{\overline{E X P}}{\bar{Y}}=\frac{\bar{C}^{H *}+\bar{X}^{H *}}{\bar{C}^{H}+\bar{X}^{H}+\bar{C}^{H *}+\bar{X}^{H *}}=\left(\frac{\phi_{F}\left(\frac{\bar{P}^{H *}}{\bar{P}^{*}}\right)^{-\eta}\left(\bar{C}^{*}+\bar{X}^{*}\right)}{\phi_{H}\left(\frac{\bar{P}^{H}}{\bar{P}}\right)^{-\eta}(\bar{C}+\bar{X})+\phi_{F}\left(\frac{\bar{P}^{H *}}{\bar{P}^{*}}\right)^{-\eta}\left(\bar{C}^{*}+\bar{X}^{*}\right)}\right), \\
\frac{\overline{I M P}}{\bar{Y}}=\frac{\left.\bar{C}_{F}^{F}+\bar{X}^{F} \overline{\bar{P}}\right)^{-\eta}(\bar{C}+\bar{X})}{\bar{C}^{F}+\bar{X}^{F}+\bar{C}^{F *}+\bar{X}^{F *}}=\left(\frac{\bar{C}^{F}}{\phi_{F}\left(\frac{\bar{P}^{F}}{\bar{P}}\right)^{-\eta}(\bar{C}+\bar{X})+\phi_{H}\left(\frac{\bar{P}^{F *}}{\bar{P}^{*}}\right)^{-\eta}\left(\bar{C}^{*}+\bar{X}^{*}\right)}\right),
\end{aligned}
$$

where the first equality follows from the steady state goods market clearing condition (and the fact that in steady state there is no price disperion at the variety level and $\bar{Y}=\bar{Y}^{H}$ ). Using the equivalences that $\left(\frac{\bar{P}^{F *}}{\bar{P}^{*}}\right)=\frac{1}{\overline{R S}}\left(\frac{\bar{P}^{F}}{\bar{P}}\right)$ and $\left(\frac{\bar{P}^{H *}}{\bar{P}^{*}}\right)=\frac{1}{\overline{R S}}\left(\frac{\bar{P}^{H}}{\bar{P}}\right)$, then I can re-write these two steady state shares as follows,

$$
\begin{aligned}
& \frac{\overline{E X P}}{\bar{Y}}=\left(\frac{\phi_{F}(\overline{R S})^{\eta}\left(\bar{C}^{*}+\bar{X}^{*}\right)}{\phi_{H}(\bar{C}+\bar{X})+\phi_{F}(\overline{R S})^{\eta}\left(\bar{C}^{*}+\bar{X}^{*}\right)}\right), \\
& \frac{\overline{I M P}}{\bar{Y}}=\left(\frac{\phi_{F}(\bar{C}+\bar{X})}{\phi_{F}(\bar{C}+\bar{X})+\phi_{H}(\overline{R S})^{\eta}\left(\bar{C}^{*}+\bar{X}^{*}\right)}\right) .
\end{aligned}
$$

Then, in a steady state where $\phi_{H}+\phi_{F}=1$ and where absorption (consumption plus investment) in both countries differs only by a factor related to the steady state real exchange rate, i.e. $(\bar{C}+\bar{X})=(\overline{R S})^{\eta}\left(\bar{C}^{*}+\bar{X}^{*}\right)$, the import and export shares can be expressed more compactly as,

$$
\begin{aligned}
& \frac{\overline{E X P}}{\bar{Y}}=\left(\frac{\phi_{F}(\bar{C}+\bar{X})}{\phi_{H}(\bar{C}+\bar{X})+\phi_{F}(\bar{C}+\bar{X})}\right)=\phi_{F}, \\
& \frac{\overline{I M P}}{\bar{Y}}=\left(\frac{\phi_{F}(\bar{C}+\bar{X})}{\phi_{F}(\bar{C}+\bar{X})+\phi_{H}(\bar{C}+\bar{X})}\right)=\phi_{F} .
\end{aligned}
$$

Indeed, the parameter $\phi_{F}$ denotes the share of real imports and real exports for consumption and investment purposes relative to domestic output in steady state. 
The world terms of trade, $\widehat{t}_{t}^{W}$, can be expressed in terms of the relative price of each country as follows,

$$
\begin{aligned}
\widehat{t}_{t}^{W} & \equiv \widehat{p}_{t}^{F, W *}-\widehat{p}_{t}^{W *}=\phi_{F} \widehat{p}_{t}^{F}+\phi_{H} \widehat{p}_{t}^{F *}-\phi_{F} \widehat{p}_{t}-\phi_{H} \widehat{p}_{t}^{*} \\
& =\phi_{F}\left(\widehat{p}_{t}^{F}-\widehat{p}_{t}\right)+\phi_{H}\left(\widehat{p}_{t}^{F *}-\widehat{p}_{t}^{*}\right) \\
& \approx \phi_{F}\left(\widehat{p}_{t}^{F}-\phi_{H} \widehat{p}_{t}^{H}-\phi_{F} \widehat{p}_{t}^{F}\right)+\phi_{H}\left(\widehat{p}_{t}^{F *}-\phi_{F} \widehat{p}_{t}^{H *}-\phi_{H} \widehat{p}_{t}^{F *}\right) \\
& =\phi_{F} \phi_{H}\left(\widehat{p}_{t}^{F}-\widehat{p}_{t}^{H}\right)+\phi_{F} \phi_{H}\left(\widehat{p}_{t}^{F *}-\widehat{p}_{t}^{H *}\right) \\
& =\phi_{F} \phi_{H}\left[\left(\widehat{p}_{t}^{F}-\widehat{p}_{t}^{H}\right)+\left(\widehat{p}_{t}^{F *}-\widehat{p}_{t}^{H *}\right)\right]
\end{aligned}
$$

while using the definition of the CPI for both countries, i.e. $\widehat{p}_{t} \approx \phi_{H} \widehat{p}_{t}^{H}+\phi_{F} \widehat{p}_{t}^{F}$ and $\widehat{p}_{t}^{*} \approx \phi_{F} \widehat{p}_{t}^{H *}+\phi_{H} \widehat{p}_{t}^{F *}$. I can re-write the real exchange rate, $\widehat{r} s_{t}$, as,

$$
\begin{aligned}
\widehat{r s}_{t} & =\widehat{s}_{t}+\widehat{p}_{t}^{*}-\widehat{p}_{t} \\
& \approx \widehat{s}_{t}+\left(\phi_{F} \widehat{p}_{t}^{H *}+\phi_{H} \widehat{p}_{t}^{F *}\right)-\left(\phi_{H} \widehat{p}_{t}^{H}+\phi_{F} \widehat{p}_{t}^{F}\right) \\
& =\widehat{s}_{t}+\left(\left(1-\phi_{H}\right) \widehat{p}_{t}^{H *}+\phi_{H} \widehat{p}_{t}^{F *}\right)-\left(\phi_{H} \widehat{p}_{t}^{H}+\left(1-\phi_{H}\right) \widehat{p}_{t}^{F}\right) \\
& =\left(\widehat{s}_{t}+\widehat{p}_{t}^{H *}-\widehat{p}_{t}^{F}\right)+\phi_{H}\left[\left(\widehat{p}_{t}^{F}-\widehat{p}_{t}^{H}\right)+\left(\widehat{p}_{t}^{F *}-\widehat{p}_{t}^{H *}\right)\right] .
\end{aligned}
$$

The international relative price effect on trade can be partly captured by the cost of replacing one unit of the foreign good with one unit of the exported domestic good, i.e. it is in part a function of the domestic terms of trade $\widehat{t o t}_{t} \equiv\left(\widehat{p}_{t}^{F}-\widehat{s}_{t}-\widehat{p}_{t}^{H *}\right)$. If the law of one price holds (as it is the case under producer-currency pricing (PCP)), then I can express domestic terms of trade as the opportunity cost of replacing one unit of the foreign good with one unit of the domestic good sold locally, $\widehat{t o t}_{t} \equiv\left(\widehat{p}_{t}^{F}-\widehat{p}_{t}^{H}\right)$, since $\widehat{p}_{t}^{H} \approx \widehat{s}_{t}+\widehat{p}_{t}^{H *}$. However, in the local-currency pricing (LCP) case with deviations of the law of one price considered in this model I have to use the definition of world terms of trade, $\widehat{t}_{t}^{W}$, to re-write the real exchange rate, $\widehat{r}_{t}$, as a function of domestic and world terms of trade as follows,

$$
\widehat{r s}_{t} \approx-\widehat{t o t}_{t}+\left(\frac{1}{\phi_{F}}\right) \widehat{t}_{t}^{W}
$$

World terms of trade, $\widehat{t}_{t}^{W}$, can be expressed now as a function of the domestic terms of trade, $\widehat{\text { tot }}_{t} \equiv$ $\left(\widehat{p}_{t}^{F}-\widehat{s}_{t}-\widehat{p}_{t}^{H *}\right)$, and the real exchange rate, $\widehat{r s}_{t}$, as,

$$
\widehat{t}_{t}^{W} \approx \phi_{F}\left(\widehat{t o t}_{t}+\widehat{r s}_{t}\right)
$$

The advantage of this transformation is that the world terms of trade can be expressed as a linear function of domestic terms of trade and the real exchange rate which are both measurable in the data-unlike world terms of trade itself. Hence, the trade balance, the real export and the real import equations can be all re-expressed in terms of international relative prices that are easier to match with the data even in the presence of nominal rigidities and LCP pricing (that is, even when the law of one price does not hold).

\section{An Extension: The Role of Capacity Utilization}

For this extension, I follow the definition of capacity utilization as presented in Christiano, et al. (2005). 


\subsection{The Structure of the Model}

\subsubsection{The Intertemporal Consumption and Savings Problem}

I assume that both countries operate in the same environment described before under incomplete asset markets. The domestic household maximizes its lifetime utility in (1) subject to the sequence of budget constraints described by,

$P_{t}\left(C_{t}+X_{t}+A\left(U_{t}\right) \widetilde{K}_{t}\right)+T_{t}+\frac{1}{I_{t}} B_{t+1}+\frac{1}{I_{t}^{*}} S_{t} B_{t+1}^{F *}+\frac{\mu}{2} \frac{P_{t}}{I_{t}^{*}}\left(\frac{S_{t} B_{t+1}^{F *}}{P_{t}}-\bar{a}\right)^{2} \leq B_{t}+S_{t} B_{t}^{F *}+W_{t} L_{t}+Z_{t} U_{t} \widetilde{K}_{t}+P r_{t}$,

and the law of motion for physical capital,

$$
\widetilde{K}_{t+1} \leq(1-\delta) \widetilde{K}_{t}+V_{t} \Phi\left(X_{t}, X_{t-1}, K_{t}\right) X_{t}
$$

while the foreign household maximizes its lifetime utility (the foreign counterpart of (1)) subject to the sequence of budget constraints described by,

$$
P_{t}^{*}\left(C_{t}^{*}+X_{t}^{*}+A\left(U_{t}^{*}\right) \widetilde{K}_{t}^{*}\right)+T_{t}^{*}+\frac{1}{I_{t}^{*}} B_{t+1}^{*} \leq B_{t}^{*}+W_{t}^{*} L_{t}^{*}+Z_{t}^{*} U_{t}^{*} \widetilde{K}_{t}^{*}+P r_{t}^{*}+\operatorname{Tr}_{t}^{*},
$$

a law of motion for capital analogous to the one described in (339), and a transfer function $T r_{t}^{*}$ that implies that foreign households receive all the revenues from the international borrowing costs paid by the domestic households in trading foreign bonds, i.e.,

$$
\operatorname{Tr}_{t}^{*}=\frac{\mu}{2} \frac{P_{t}}{S_{t} I_{t}^{*}}\left(\frac{S_{t} B_{t+1}^{F *}}{P_{t}}-\bar{a}\right)^{2}
$$

Here, $W_{t}$ and $W_{t}^{*}$ are the domestic and foreign nominal wages respectively, $P_{t}$ and $P_{t}^{*}$ are the domestic and foreign CPI indexes, and $T_{t}$ and $T_{t}^{*}$ are domestic and foreign nominal (lump-sum) taxes. Moreover, $X_{t}$ and $X_{t}^{*}$ are domestic and foreign real investment, $\widetilde{K}_{t}$ and $\widetilde{K}_{t}^{*}$ stand for domestic and foreign physical capital, $Z_{t}$ and $Z_{t}^{*}$ define the nominal rental rate on capital in the domestic and foreign country, $P r_{t}$ and $P r_{t}^{*}$ are the nominal profits generated by the domestic firms and by the foreign firms respectively, and $V_{t}$ and $V_{t}^{*}$ are the exogenous IST shocks in the domestic and foreign country.

Moreover, $B_{t+1}$ is the domestic demand for the (uncontingent) risk-free one-period bond denominated in domestic currency (maturing at time $t+1), B_{t+1}^{F *}$ is the domestic demand for the (uncontingent) risk-free one-period bond denominated in foreign currency (maturing at time $t+1$ ), and $B_{t+1}^{*}$ is the foreign demand for the (uncontingent) risk-free one-period bond denominated in foreign currency (maturing at time $t+1$ ). The nominal gross interest rate on the domestic and foreign bonds are $I_{t}$ and $I_{t}^{*}$ respectively, while $S_{t}$ denotes the nominal exchange rate. As in Benigno (2009), I have assumed that there is a quadratic cost function (i.e. an international borrowing cost) that penalizes changes in the real net foreign asset position of the domestic household, $\frac{S_{t} B_{t+1}^{F *}}{P_{t}}$, away from a constant real reference value of $\bar{a}$. The parameter $\mu>0$ measures the size of this international borrowing cost in units of the consumption good, which is then re-scaled by $\frac{P_{t}}{I_{t}^{*}}$ for analytical convenience.

Capital services in both countries, $K_{t}$ and $K_{t}^{*}$, are related to physical capital, $\widetilde{K}_{t}$ and $\widetilde{K}_{t}^{*}$, by the following 
expressions,

$$
\begin{aligned}
K_{t} & =U_{t} \widetilde{K}_{t}, \\
K_{t}^{*} & =U_{t}^{*} \widetilde{K}_{t}^{*} .
\end{aligned}
$$

Here, $U_{t}$ and $U_{t}^{*}$ denote the domestic and foreign utilization rate of capital - which I assume is set by the households in each country. Hence, $Z_{t} U_{t} \widetilde{K}_{t}$ represents the domestic households' earnings from supplying capital services to the domestic firms and $Z_{t}^{*} U_{t}^{*} \widetilde{K}_{t}^{*}$ represents the foreign households' earnings from supplying capital services to the foreign firms. The increasing, convex functions, $A\left(U_{t}\right) \widetilde{K}_{t}$ and $A\left(U_{t}^{*}\right) \widetilde{K}_{t}^{*}$, denote the cost, in units of their respective consumption goods, of setting the utilization rate in each country. Hence, I allow capital services to be different from physical capital as in Christiano, et al. (2005).

Physical capital accumulation may be subject to adjustment costs too. I consider three special cases: the capital adjustment cost (CAC) case in (9), the investment adjustment cost (IAC) in (10), and the case with no adjustment costs (NAC). I define capital adjustment costs in terms of capital services rather than physical capital because I want to capture the idea that the intensity of capital utilization can also influence how costly it becomes to accumulate physical capital. However, this distinction does not matter under IAC adjustment costs. All other assumptions of the model are maintained in this extension.

The home and foreign consumption bundles of the domestic household, $C_{t}^{H}$ and $C_{t}^{F}$, as well as the investment bundles, $X_{t}^{H}$ and $X_{t}^{F}$, are aggregated by means of the CES indexes in (11) - (12), while aggregate domestic consumption and investment, $C_{t}$ and $X_{t}$, are defined with the CES indexes in (13) - (14). Under standard results on functional separability, the indexes which correspond to my specification of the aggregators for consumption and investment are (15) - (16), and the price sub-indexes are (17) - (20). An analogous set of consumption and investment aggregators for the foreign household and price indexes and sub-indexes for the foreign market apply. I still define the real exchange rate as in (21).

\subsubsection{The Price-Setting Problem and Monetary Policy}

Neither the problem of the firms' nor the simple monetary policy rules à la Taylor (1993) change in this environment with variable capital utilization. The only point that is worth emphasizing is that firms in this model rent capital services rather than physical capital. Therefore, the capital utilization rate set by the households and the physical capital they accumulate will have an impact on the firms' marginal costs by influencing the overall amount of capital services supplied in equilibrium. Everything else is unchanged.

I assume that production employs a (homogeneous of degree one) Cobb-Douglas technology as in (22) (23). Solving the cost-minimization problem of each individual firm yields an efficiency condition linking the capital-services-to-labor ratios to factor price ratios as in (24) - (25), as well as a characterization for the (pre-subsidy) nominal marginal costs as in (26) - (27). The government subsidizes firms as in (28) - (29), and each country fully finances its subsidies with a lump-sum tax on households as specified in the government budget constraints in (40) - (41). The firm subsidy is time-invariant and common across countries as in (45).

A re-optimizing domestic firm $h$ under local-currency pricing (LCP) chooses a domestic and a foreign

price, $\widetilde{P}_{t}(h)$ and $\widetilde{P}_{t}^{*}(h)$, to maximize the expected discounted value of its net profits in (32), subject to a pair of demand constraints in each goods market in (33) - (34). Similarly, I characterize the objective of the foreign firm $f$ under LCP pricing as in (35), subject to the demand constraints in (36) - (37). 
The Taylor rule is often defined as the trademark of modern monetary policy. I assume that the monetary authorities set short-term nominal interest rates according to Taylor (1993) type rules as in (38) - (39).

\subsection{The Optimality Conditions}

Here, I present the relevant equilibrium conditions of the model only when they differ from those reported before.

The Optimality Conditions from the Households' Problem. Given the structure described in (11) (14), the solution to the sub-utility maximization problem implies that the home and foreign households' demands for each variety are given by (46) - (49), while the demands for the bundles of home and foreign goods are given by $(50)-(53)$. The intertemporal first-order conditions result in the equilibrium conditions reported in (58), (59) and (60), i.e.,

$$
\begin{aligned}
\beta \mathbb{E}_{t}\left[\left(\frac{C_{t+1}}{C_{t}}\right)^{-\sigma^{-1}} \frac{P_{t}}{P_{t+1}} I_{t}\right] & =1, \\
\beta \mathbb{E}_{t}\left[\left(\frac{C_{t+1}}{C_{t}}\right)^{-\sigma^{-1}} \frac{P_{t}}{P_{t+1}} \frac{S_{t+1}}{S_{t}} I_{t}^{*}\right] & =1+\mu\left(N F A_{t+1}-\bar{a}\right), \\
\beta \mathbb{E}_{t}\left[\left(\frac{C_{t+1}^{*}}{C_{t}^{*}}\right)^{-\sigma^{-1}} \frac{P_{t}^{*}}{P_{t+1}^{*}} I_{t}^{*}\right] & =1,
\end{aligned}
$$

where I define the real net foreign asset position of the domestic household as $N F A_{t+1} \equiv \frac{S_{t} B_{t+1}^{F *}}{P_{t}}$. Combining the Euler equations of the domestic household in (58) and (59), the following relationship holds true,

$$
\beta \mathbb{E}_{t}\left[\left(\frac{C_{t+1}}{C_{t}}\right)^{-\sigma^{-1}} \frac{P_{t}}{P_{t+1}}\left(\frac{S_{t+1}}{S_{t}} I_{t}^{*}-I_{t}\right)\right]=\mu\left(N F A_{t+1}-\bar{a}\right),
$$

which gives me a variant of the uncovered interest rate parity condition with a premium tied to the real net foreign asset position of the domestic household. This equilibrium condition governs the international risk-sharing in this environment with incomplete asset markets.

The equilibrium conditions of the households' problem also include a pair of labor supply functions (the intratemporal first-order conditions) which can be expressed as in (54) - (55), plus the appropriate no-Ponzi games, transversality conditions, the budget constraints in (338) and (340), the transformation functions between physical capital and capital services in (342) and (343), and the laws of motion for physical capital in both countries (analogous to (339)). Finally, the equilibrium conditions are completed with a number of equations that account for the capital-investment decisions of households. The capital-investment decisions, however, depend on the choice of the adjustment cost function $\Phi(\cdot)$ and the the capital utilization rates.

The maximization problem of the domestic household can be summarized generically in the following 
terms,

$$
\sum_{\tau=0}^{+\infty} \beta^{\tau}\left[\mathbb{E}_{t}\left[\begin{array}{c}
\frac{1}{1-\sigma^{-1}}\left(C_{t+\tau}\right)^{1-\sigma^{-1}}-\frac{1}{1+\varphi}\left(L_{t+\tau}\right)^{1+\varphi}-\ldots \\
\lambda_{t+\tau}\left[\begin{array}{c}
P_{t+\tau}\left(C_{t+\tau}+X_{t+\tau}+A\left(U_{t+\tau}\right) \widetilde{K}_{t+\tau}\right)+T_{t+\tau}+\ldots \\
\frac{1}{I_{t+\tau}} B_{t+\tau+1}+\frac{1}{I_{t+\tau}^{*}} S_{t+\tau} B_{t+\tau+1}^{F *}+\frac{\mu}{2} \frac{P_{t+\tau}}{I_{t+\tau}^{*}}\left(\frac{S_{t+\tau} B_{t+\tau+1}^{F *}}{P_{t+\tau}}-\bar{a}\right)^{2}-\ldots \\
B_{t+\tau}-S_{t+\tau} B_{t+\tau}^{F *}-W_{t+\tau} L_{t+\tau}-Z_{t+\tau} U_{t+\tau} \widetilde{K}_{t+\tau}-P r_{t+\tau} \\
\lambda_{t+\tau} \Lambda_{t+\tau}\left[\widetilde{K}_{t+\tau+1}-(1-\delta) \widetilde{K}_{t+\tau}-V_{t+\tau} \Phi\left(X_{t+\tau}, X_{t+\tau-1}, U_{t+\tau} \widetilde{K}_{t+\tau}\right)\right.
\end{array}\right]-\ldots \\
\widetilde{X}_{t+\tau}
\end{array}\right]\right]
$$

while the maximization problem of the foreign household can be summarized generically in the following terms,

$$
\sum_{\tau=0}^{+\infty} \beta^{\tau}\left[\mathbb{E}_{t}\left[\begin{array}{c}
\frac{1}{1-\sigma^{-1}}\left(C_{t+\tau}^{*}\right)^{1-\sigma^{-1}}-\frac{1}{1+\varphi}\left(L_{t+\tau}^{*}\right)^{1+\varphi}-\ldots \\
\lambda_{t+\tau}^{*}\left[\begin{array}{c}
P_{t+\tau}^{*}\left(C_{t+\tau}^{*}+X_{t+\tau}^{*}+A\left(U_{t+\tau}^{*}\right) \widetilde{K}_{t+\tau}^{*}\right)+T_{t+\tau}^{*}+\frac{1}{I_{t+\tau}^{*}} B_{t+\tau+1}^{*}-\ldots \\
B_{t+\tau}^{*}-W_{t+\tau}^{*} L_{t+\tau}^{*}-Z_{t+\tau}^{*} U_{t+\tau}^{*} \widetilde{K}_{t+\tau}^{*}-P r_{t+\tau}^{*}-T r_{t+\tau}^{*} \\
\lambda_{t+\tau}^{*} \Lambda_{t+\tau}^{*}\left[\widetilde{K}_{t+\tau+1}^{*}-(1-\delta) \widetilde{K}_{t+\tau}^{*}-V_{t+\tau}^{*} \Phi\left(X_{t+\tau}^{*}, X_{t+\tau-1}^{*}, U_{t+\tau}^{*} \widetilde{K}_{t+\tau}^{*}\right)\right.
\end{array}\right]-\ldots \\
X_{t+\tau}^{*}
\end{array}\right]\right]
$$

Then, I derive the following set of equilibrium conditions from the domestic household's problem,

$$
\begin{aligned}
& C_{t} \quad: \quad \lambda_{t} P_{t}=\left(C_{t}\right)^{-\sigma^{-1}} \text {, } \\
& \widetilde{K}_{t+1} \quad: \quad \Lambda_{t}=\beta \mathbb{E}_{t}\left[\begin{array}{c}
\frac{\lambda_{t+1}}{\lambda_{t}}\left(Z_{t+1} U_{t+1}-P_{t+1} A\left(U_{t+1}\right)\right)+\ldots \\
\frac{\lambda_{t+1}}{\lambda_{t}} \Lambda_{t+1}\left((1-\delta)+V_{t+1} \frac{\partial \Phi\left(X_{t+1}, X_{t}, U_{t+1} \widetilde{K}_{t+1}\right)}{\partial \widetilde{K}_{t+1}} X_{t+1}\right)
\end{array}\right] \text {, } \\
& X_{t} \quad: \quad 1=\frac{\Lambda_{t}}{P_{t}} V_{t}\left[\Phi\left(X_{t}, X_{t-1}, U_{t} \widetilde{K}_{t}\right)+\frac{\partial \Phi\left(X_{t}, X_{t-1}, U_{t} \widetilde{K}_{t}\right)}{\partial X_{t}} X_{t}\right]+\ldots \\
& \beta \mathbb{E}_{t}\left[\frac{\lambda_{t+1} \Lambda_{t+1}}{\lambda_{t} P_{t}} V_{t+1} \frac{\partial \Phi\left(X_{t+1}, X_{t}, U_{t+1} \widetilde{K}_{t+1}\right)}{\partial X_{t}} X_{t+1}\right] \text {, } \\
& U_{t+1} \quad: \quad \beta \mathbb{E}_{t}\left[\frac{\lambda_{t+1}}{\lambda_{t} P_{t}}\left(\begin{array}{c}
P_{t+1} A^{\prime}\left(U_{t+1}\right) \widetilde{K}_{t+1}-Z_{t+1} \widetilde{K}_{t+1}-\ldots \\
\Lambda_{t+1} V_{t+1} \frac{\partial \Phi\left(X_{t+1}, X_{t}, U_{t+1} \widetilde{K}_{t+1}\right)}{\partial U_{t+1}} X_{t+1}
\end{array}\right)\right]=0 .
\end{aligned}
$$

After further manipulation on those first-order conditions, it is possible to re-write the equilibrium conditions 
as,

$$
\begin{aligned}
& Q_{t}=\beta \mathbb{E}_{t}\left\{\left(\frac{C_{t+1}}{C_{t}}\right)^{-\sigma^{-1}}\left[\begin{array}{c}
\frac{Z_{t+1}}{P_{t+1}} U_{t+1}-A\left(U_{t+1}\right)+\ldots \\
Q_{t+1}\left((1-\delta)+V_{t+1} \frac{\partial \Phi\left(X_{t+1}, X_{t}, U_{t+1} \widetilde{K}_{t+1}\right)}{\partial \widetilde{K}_{t+1}} X_{t+1}\right)
\end{array}\right]\right\}, \\
& Q_{t}=\frac{1-\beta \mathbb{E}_{t}\left\{\left(\frac{C_{t+1}}{C_{t}}\right)^{-\sigma^{-1}}\left[Q_{t+1} V_{t+1} \frac{\partial \Phi\left(X_{t+1}, X_{t}, U_{t+1} \widetilde{K}_{t+1}\right)}{\partial X_{t}} X_{t+1}\right]\right\}}{V_{t}\left[\Phi\left(X_{t}, X_{t-1}, U_{t} \widetilde{K}_{t}\right)+\frac{\partial \Phi\left(X_{t}, X_{t-1}, U_{t} \widetilde{K}_{t}\right)}{\partial X_{t}} X_{t}\right]}, \\
& \beta \mathbb{E}_{t}\left\{\left(\frac{C_{t+1}}{C_{t}}\right)^{-\sigma^{-1}} \widetilde{K}_{t+1}\left[\frac{Z_{t+1}}{P_{t+1}}-A^{\prime}\left(U_{t+1}\right)+Q_{t+1} V_{t+1} \frac{\partial \Phi\left(X_{t+1}, X_{t}, U_{t+1} \widetilde{K}_{t+1}\right)}{\partial U_{t+1}} \frac{X_{t+1}}{\widetilde{K}_{t+1}}\right]\right\}=0,
\end{aligned}
$$

where I define the domestic Tobin's q in terms of the Lagrange multipliers as $Q_{t} \equiv \frac{\Lambda_{t}}{P_{t}}$. A similar set of derivations implies that the equilibrium conditions for the foreign country can be expressed as,

$$
\begin{aligned}
& Q_{t}^{*}=\beta \mathbb{E}_{t}\left\{\left(\frac{C_{t+1}^{*}}{C_{t}^{*}}\right)^{-\sigma^{-1}}\left[\begin{array}{c}
\frac{Z_{t+1}^{*}}{P_{t+1}^{*}} U_{t+1}^{*}-A\left(U_{t+1}^{*}\right)+\ldots \\
Q_{t+1}^{*}\left((1-\delta)+V_{t+1}^{*} \frac{\partial \Phi\left(X_{t+1}^{*}, X_{t}^{*}, U_{t+1}^{*} \widetilde{K}_{t+1}^{*}\right)}{\partial \widetilde{K}_{t+1}^{*}} X_{t+1}^{*}\right)
\end{array}\right]\right\} \\
& Q_{t}^{*}=\frac{1-\beta \mathbb{E}_{t}\left\{\left(\frac{C_{t+1}^{*}}{C_{t}^{*}}\right)^{-\sigma^{-1}}\left[Q_{t+1}^{*} V_{t+1}^{*} \frac{\partial \Phi\left(X_{t+1}^{*}, X_{t}^{*}, U_{t+1}^{*} \widetilde{K}_{t+1}^{*}\right)}{\partial X_{t}^{*}} X_{t+1}^{*}\right]\right\}}{V_{t}^{*}\left[\Phi\left(X_{t}^{*}, X_{t-1}^{*}, U_{t}^{*} \widetilde{K}_{t}^{*}\right)+\frac{\partial \Phi\left(X_{t}^{*}, X_{t-1}^{*}, U_{t}^{*} \widetilde{K}_{t}^{*}\right)}{\partial X_{t}^{*}} X_{t}^{*}\right]}, \\
& \beta \mathbb{E}_{t}\left\{\left(\frac{C_{t+1}^{*}}{C_{t}^{*}}\right)^{-\sigma^{-1}} \widetilde{K}_{t+1}^{*}\left[\frac{Z_{t+1}^{*}}{P_{t+1}^{*}}-A^{\prime}\left(U_{t+1}^{*}\right)+Q_{t+1}^{*} V_{t+1}^{*} \frac{\partial \Phi\left(X_{t+1}^{*}, X_{t}^{*}, U_{t+1}^{*} \widetilde{K}_{t+1}^{*}\right)}{\partial U_{t+1}^{*}} \frac{X_{t+1}^{*}}{\widetilde{K}_{t+1}^{*}}\right]\right\}=0
\end{aligned}
$$

where I define the foreign Tobin's q in terms of the Lagrange multipliers as $Q_{t}^{*} \equiv \frac{\Lambda_{t}^{*}}{P_{t}^{*}}$. These conditions describe the equilibrium generically, for any well-defined adjustment cost function $\Phi(\cdot)$ and utilization cost function $A(\cdot)$.

Under no adjustment costs (NAC), the set of conditions added to account for the capital-investment decisions of households are summarized as,

$$
\begin{aligned}
& Q_{t}=\beta \mathbb{E}_{t}\left\{\left(\frac{C_{t+1}}{C_{t}}\right)^{-\sigma^{-1}}\left[\frac{Z_{t+1}}{P_{t+1}} U_{t+1}-A\left(U_{t+1}\right)+Q_{t+1}(1-\delta)\right]\right\}, \\
& Q_{t}=\frac{1}{V_{t}} \\
& \beta \mathbb{E}_{t}\left\{\left(\frac{C_{t+1}}{C_{t}}\right)^{-\sigma^{-1}} \widetilde{K}_{t+1}\left[\frac{Z_{t+1}}{P_{t+1}}-A^{\prime}\left(U_{t+1}\right)\right]\right\}=0 .
\end{aligned}
$$


A similar set of derivations allows me to write the following system of equations for the foreign country,

$$
\begin{aligned}
& Q_{t}^{*}=\beta \mathbb{E}_{t}\left\{\left(\frac{C_{t+1}^{*}}{C_{t}^{*}}\right)^{-\sigma^{-1}}\left[\frac{Z_{t+1}^{*}}{P_{t+1}^{*}} U_{t+1}^{*}-A\left(U_{t+1}^{*}\right)+Q_{t+1}^{*}(1-\delta)\right]\right\}, \\
& Q_{t}^{*}=\frac{1}{V_{t}^{*}}, \\
& \beta \mathbb{E}_{t}\left\{\left(\frac{C_{t+1}^{*}}{C_{t}^{*}}\right)^{-\sigma^{-1}} \widetilde{K}_{t+1}^{*}\left[\frac{Z_{t+1}^{*}}{P_{t+1}^{*}}-A^{\prime}\left(U_{t+1}^{*}\right)\right]\right\}=0 .
\end{aligned}
$$

The Lagrange multiplier on the law of motion for capital relative to the Lagrange multiplier on the budget constraint expressed in real terms, denoted $Q_{t}$ and $Q_{t}^{*}$ respectively, has the interpretation of being the real shadow price of an additional unit of capital (or Tobin's q). In the case without adjustment costs (NAC) Tobin's q is exactly equal to one, if there are no IST shocks.

Under capital adjustment costs (CAC), the set of conditions added to account for the capital-investment decisions of domestic households are,

$$
\begin{aligned}
& Q_{t}=\beta \mathbb{E}_{t}\left\{\left(\frac{C_{t+1}}{C_{t}}\right)^{-\sigma^{-1}}\left[\begin{array}{c}
\frac{Z_{t+1}}{P_{t+1}} U_{t+1}-A\left(U_{t+1}\right)+\ldots \\
Q_{t+1}\left((1-\delta)-V_{t+1} \Phi^{\prime}\left(\frac{X_{t+1}}{U_{t+1} \widetilde{K}_{t+1}}\right)\left(\frac{X_{t+1}}{U_{t+1} \widetilde{K}_{t+1}}\right)^{2} U_{t+1}\right)
\end{array}\right]\right\} \\
& Q_{t}=\frac{1}{V_{t}}\left[\Phi\left(\frac{X_{t}}{U_{t} \widetilde{K}_{t}}\right)+\Phi^{\prime}\left(\frac{X_{t}}{U_{t} \widetilde{K}_{t}}\right) \frac{X_{t}}{U_{t} \widetilde{K}_{t}}\right]^{-1}, \\
& \beta \mathbb{E}_{t}\left\{\left(\frac{C_{t+1}}{C_{t}}\right)^{-\sigma^{-1}} \widetilde{K}_{t+1}\left[\begin{array}{c}
\frac{Z_{t+1}}{P_{t+1}}-A^{\prime}\left(U_{t+1}\right)-\ldots \\
Q_{t+1} V_{t+1} \Phi^{\prime}\left(\frac{X_{t+1}}{U_{t+1} \widetilde{K}_{t+1}}\right)\left(\frac{X_{t+1}}{U_{t+1} \widetilde{K}_{t+1}}\right)^{2}
\end{array}\right]\right\}=0 .
\end{aligned}
$$

A similar set of derivations allows me to write the following system of equations for the foreign country,

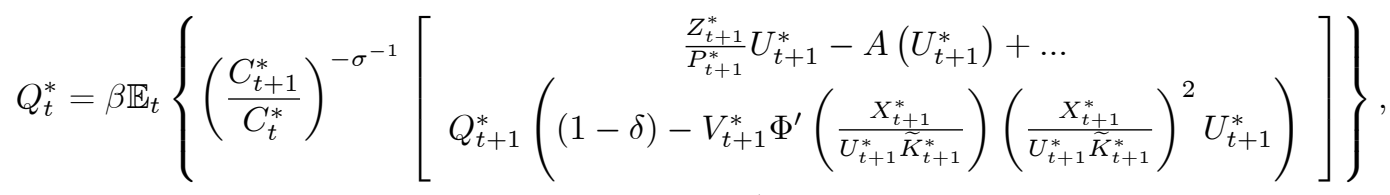

$$
\begin{aligned}
& Q_{t}^{*}=\frac{1}{V_{t}^{*}}\left[\Phi\left(\frac{X_{t}^{*}}{U_{t}^{*} \widetilde{K}_{t}^{*}}\right)+\Phi^{\prime}\left(\frac{X_{t}^{*}}{U_{t}^{*} \widetilde{K}_{t}^{*}}\right) \frac{X_{t}^{*}}{U_{t}^{*} \widetilde{K}_{t}^{*}}\right]^{-1}, \\
& \beta \mathbb{E}_{t}\left\{\left(\frac{C_{t+1}^{*}}{C_{t}^{*}}\right)^{-\sigma^{-1}} \widetilde{K}_{t+1}^{*}\left[\begin{array}{c}
\frac{Z_{t+1}^{*}}{P_{t+1}^{*}}-A^{\prime}\left(U_{t+1}^{*}\right)-\ldots \\
Q_{t+1}^{*} V_{t+1}^{*} \Phi^{\prime}\left(\frac{X_{t+1}^{*}}{U_{t+1}^{*} \widetilde{K}_{t+1}^{*}}\right)\left(\frac{X_{t+1}^{*}}{U_{t+1}^{*} \widetilde{K}_{t+1}^{*}}\right)^{2}
\end{array}\right]\right\}=0 .
\end{aligned}
$$

The Lagrange multiplier on the law of motion for physical capital relative to the Lagrange multiplier on the budget constraint expressed in real terms, denoted $Q_{t}$ and $Q_{t}^{*}$ respectively, has the interpretation of being the real shadow price of an additional unit of capital (or Tobin's q).

Under investment adjustment costs (IAC), the set of conditions added to account for the capital- 
investment decisions of domestic households are,

$$
\begin{aligned}
& Q_{t}=\beta \mathbb{E}_{t}\left\{\left(\frac{C_{t+1}}{C_{t}}\right)^{-\sigma^{-1}}\left[\frac{Z_{t+1}}{P_{t+1}} U_{t+1}-A\left(U_{t+1}\right)+Q_{t+1}(1-\delta)\right]\right\}, \\
& Q_{t}=\frac{1+\beta \mathbb{E}_{t}\left\{\left(\frac{C_{t+1}}{C_{t}}\right)^{-\sigma^{-1}}\left[Q_{t+1} V_{t+1} \Phi^{\prime}\left(\frac{X_{t+1}}{X_{t}}\right)\left(\frac{X_{t+1}}{X_{t}}\right)^{2}\right]\right\}}{V_{t}\left[\Phi\left(\frac{X_{t}}{X_{t-1}}\right)+\Phi^{\prime}\left(\frac{X_{t}}{X_{t-1}}\right)\left(\frac{X_{t}}{X_{t-1}}\right)\right]} \\
& \beta \mathbb{E}_{t}\left\{\left(\frac{C_{t+1}}{C_{t}}\right)^{-\sigma^{-1}} \widetilde{K}_{t+1}\left[\frac{Z_{t+1}}{P_{t+1}}-A^{\prime}\left(U_{t+1}\right)\right]\right\}=0 .
\end{aligned}
$$

A similar set of derivations allows me to write the following system of equations for the foreign country,

$$
\begin{aligned}
& Q_{t}^{*}=\beta \mathbb{E}_{t}\left\{\left(\frac{C_{t+1}^{*}}{C_{t}^{*}}\right)^{-\sigma^{-1}}\left[\frac{Z_{t+1}^{*}}{P_{t+1}^{*}} U_{t+1}^{*}-A\left(U_{t+1}^{*}\right)+Q_{t+1}^{*}(1-\delta)\right]\right\}, \\
& Q_{t}^{*}=\frac{1+\beta \mathbb{E}_{t}\left\{\left(\frac{C_{t+1}^{*}}{C_{t}^{*}}\right)^{-\sigma^{-1}}\left[Q_{t+1}^{*} V_{t+1}^{*} \Phi^{\prime}\left(\frac{X_{t+1}^{*}}{X_{t}^{*}}\right)\left(\frac{X_{t+1}^{*}}{X_{t}^{*}}\right)^{2}\right]\right\}}{V_{t}^{*}\left[\Phi\left(\frac{X_{t}^{*}}{X_{t-1}^{*}}\right)+\Phi^{\prime}\left(\frac{X_{t}^{*}}{X_{t-1}^{*}}\right)\left(\frac{X_{t}^{*}}{X_{t-1}^{*}}\right)\right]} \\
& \beta \mathbb{E}_{t}\left\{\left(\frac{C_{t+1}^{*}}{C_{t}^{*}}\right)^{-\sigma^{-1}} \widetilde{K}_{t+1}^{*}\left[\frac{Z_{t+1}^{*}}{P_{t+1}^{*}}-A^{\prime}\left(U_{t+1}^{*}\right)\right]\right\}=0 .
\end{aligned}
$$

Once again, the Lagrange multiplier on the law of motion for physical capital relative to the Lagrange multiplier on the budget constraint expressed in real terms, denoted $Q_{t}$ and $Q_{t}^{*}$ respectively, has the interpretation of being the real shadow price of an additional unit of capital (or Tobin's q).

The Optimality Conditions from the Firms' Problem. The necessary and sufficient first-order conditions for the domestic firm producing variety $h$ under local-currency pricing (LCP) give me the pair of price-setting formulas in $(74)-(75)$. Similarly, the first-order conditions for the foreign firm producing variety $f$ under LCP pricing give me the pair of price-setting formulas in (76) - (77). Using the law of large numbers and the inherent symmetry of the firms' problem, the price sub-indexes on domestic and foreign varieties, $P_{t}^{H}, P_{t}^{H *}, P_{t}^{F}$ and $P_{t}^{F *}$, become equal to those reported in equations $(78)-(81)$.

Aggregate Output and Rental Rates on Capital. Equations (46) - (53) determine the demand function for each variety. Those demand functions coupled with the market clearing conditions at the variety level allow me to calculate the aggregate output demand as follows,

$$
\begin{aligned}
Y_{t} & -A\left(U_{t}\right) \widetilde{K}_{t}=\int_{0}^{1}\left[C_{t}(h)+X_{t}(h)+C_{t}^{*}(h)+X_{t}^{*}(h)\right] d h \\
& =\left[\int_{0}^{1}\left(\frac{P_{t}(h)}{P_{t}^{H}}\right)^{-\theta} d h\right] \phi_{H}\left(\frac{P_{t}^{H}}{P_{t}}\right)^{-\eta}\left(C_{t}+X_{t}\right)+\left[\int_{0}^{1}\left(\frac{P_{t}^{*}(h)}{P_{t}^{H *}}\right)^{-\theta} d h\right] \phi_{H}^{*}\left(\frac{P_{t}^{H *}}{P_{t}^{*}}\right)^{-\eta}\left(C_{t}^{*}+X_{t}^{*}\right), \\
Y_{t}^{*} & -A\left(U_{t}^{*}\right) \widetilde{K}_{t}^{*}=\int_{0}^{1}\left[C_{t}(f)+X_{t}(f)+C_{t}^{*}(f)+X_{t}^{*}(f)\right] d f \\
& =\left[\int_{0}^{1}\left(\frac{P_{t}(f)}{P_{t}^{F}}\right)^{-\theta} d f\right] \phi_{F}\left(\frac{P_{t}^{F}}{P_{t}}\right)^{-\eta}\left(C_{t}+X_{t}\right)+\left[\int_{0}^{1}\left(\frac{P_{t}^{*}(f)}{P_{t}^{F *}}\right)^{-\theta} d f\right] \phi_{F}^{*}\left(\frac{P_{t}^{F *}}{P_{t}^{*}}\right)^{-\eta}\left(C_{t}^{*}+X_{t}^{*}\right)
\end{aligned}
$$


Equations (368) - (369) tie the aggregate output demand in both countries to consumption as well as to relative prices, after adjusting for the costs of capital utilization. When the utilization costs are set equal to zero, these aggregate output equations correspond exactly to those reported in (92) - (93).

Given the production functions in (22) - (23) and the fact that capital-services-to-labor ratios are equalized across firms within each country, it is possible to write the aggregate output equations as in (84) - (85). Combining these aggregate production functions with the efficiency conditions in (24) - (25) and the labor supply equations from the households' problem (as in equations (54) - (55)), I can express the real rental rates on capital services in terms of productivity shocks, consumption, output and capital as in equations (112) - (113). Manipulating the same set of conditions a little bit further also allows me to re-write the real wages in terms of real rental rates on capital services as well as productivity shocks, consumption, output and capital services as in equations (114) - (115). Those two equations suffice for the purpose of replacing real wages out of the marginal cost equations, as in the model without variable capacity utilization.

\subsection{The Deterministic Steady State}

I impose two restrictions on the capital utilization cost function $A(\cdot)$ in steady state. First, I require that the rate of utilization be set at $\bar{U}=\bar{U}^{*}=1$ in steady state. Naturally, this also implies that $\bar{K}=\bar{K}$ and $\widetilde{\widetilde{K}}^{*}=\bar{K}^{*}$. In other words, physical capital and capital services are equated in steady state. Second, I assume that in steady state $A(\bar{U})=A\left(\bar{U}^{*}\right)=0$. Hence, in steady state, the capital utilization cost drops from the first-order conditions (350) and (353), (356) and (359), and (362) and (365). Furthermore, it also drops from the steady state market clearing conditions implied by (368) - (369).

By the first-order conditions in (352) and (355), I can easily derive that,

$$
\begin{aligned}
A^{\prime}(\bar{U}) & =\frac{\bar{Z}}{\overline{\bar{P}}}, \\
A^{\prime}\left(\bar{U}^{*}\right) & =\frac{\bar{Z}^{*}}{\bar{P}^{*}} .
\end{aligned}
$$

These expressions are derived under the NAC version of the model without capital adjustment costs. For the CAC case, I can derive exactly the same steady state conditions from equations (358) and (361), and the same can be said for the IAC case based on equations (364) and (367). Hence, the steady state with capital utilization is identical to that without capital utilization, as described earlier in section 4 .

The only difference is that the first derivative of the capital utilization cost function must satisfy that,

$$
\begin{aligned}
A^{\prime}(\bar{U}) & =\frac{\bar{Z}}{\bar{P}}=\beta^{-1}-(1-\delta), \\
A^{\prime}\left(\bar{U}^{*}\right) & =\frac{\bar{Z}^{*}}{\bar{P}^{*}}=\beta^{-1}-(1-\delta),
\end{aligned}
$$

where the second equality follows from my derivations of the steady state real rental rates on capital services in (124). 


\subsection{The Log-Linearized Equilibrium Conditions}

Here, I log-linearize the equilibrium conditions around the deterministic zero-inflation steady state. I only report those equations that differ from my previous derivations. For instance, I maintain the exact same specification of the Taylor (1993) rules described in (292) - (293) as my benchmark for monetary policy.

\subsubsection{The Households' Equilibrium Conditions}

The log-linearization of the Euler equations and the international risk-sharing condition under incomplete markets is the same as reported in equations (261), (262), and (263). The dynamics of the real net foreign asset position of the domestic household can still be written as in (268). The log-linearization of the domestic capital accumulation formula in (339) and its foreign counterpart in the case without adjustment costs (NAC) is unaffected by the addition of variable capital utilization. Hence, simple re-labeling allows me to write that,

$$
\begin{aligned}
& \widehat{\widetilde{k}}_{t+1} \approx(1-\delta) \widehat{\widetilde{k}}_{t}+\delta\left(\widehat{x}_{t}+\widehat{v}_{t}\right), \\
& \widehat{\widetilde{k}}_{t+1}^{*} \approx(1-\delta) \widehat{\widetilde{k}}_{t}^{*}+\delta\left(\widehat{x}_{t}^{*}+\widehat{v}_{t}^{*}\right),
\end{aligned}
$$

where $\widehat{\widetilde{k}}_{t}$ and $\widehat{\widetilde{k}}_{t}^{*}$ denote the physical capital in both countries, and $\widehat{v}_{t}$ and $\widehat{v}_{t}^{*}$ are the IST shocks.

The log-linearization of the domestic capital accumulation formula in (339) and its foreign counterpart under capital adjustment costs (CAC) allows me to obtain the following set of equations,

$$
\begin{aligned}
\widehat{\widetilde{k}}_{t+1} \approx & {\left[(1-\delta)-\bar{V} \Phi^{\prime}\left(\frac{\bar{X}}{\bar{U} \overline{\widetilde{K}}}\right)\left(\frac{\bar{X}}{\overline{\widetilde{K}}}\right)^{2} \frac{1}{\bar{U}}\right] \widehat{\widetilde{k}}_{t}+\ldots } \\
& {\left[\bar{V} \Phi\left(\frac{\bar{X}}{\bar{U} \overline{\widetilde{K}}}\right) \frac{\bar{X}}{\overline{\widetilde{K}}}+\bar{V} \Phi^{\prime}\left(\frac{\bar{X}}{\bar{U} \overline{\widetilde{K}}}\right)\left(\frac{\bar{X}}{\widetilde{\widetilde{K}}}\right)^{2} \frac{1}{\bar{U}}\right]\left(\widehat{x}_{t}+\widehat{v}_{t}\right)-\bar{V} \Phi^{\prime}\left(\frac{\bar{X}}{\bar{U} \overline{\widetilde{K}}}\right)\left(\frac{\bar{X}}{\widetilde{\widetilde{K}}}\right)^{2} \frac{1}{\bar{U}} \widehat{u}_{t} } \\
= & (1-\delta) \widehat{\tilde{k}}_{t}+\delta\left(\widehat{x}_{t}+\widehat{v}_{t}\right), \\
\widehat{\widetilde{k}}_{t+1}^{*} \approx & {\left[(1-\delta)-\bar{V}^{*} \Phi^{\prime}\left(\frac{\bar{X}^{*}}{\bar{U}^{*} \bar{K}^{*}}\right)\left(\frac{\bar{X}^{*}}{\bar{K}^{*}}\right)^{2} \frac{1}{\bar{U}^{*}}\right] \widehat{\widetilde{k}}_{t}^{*}+\ldots } \\
& {\left[\bar{V}^{*} \Phi\left(\frac{\bar{X}^{*}}{\bar{U}^{*} \bar{K}^{*}}\right) \frac{\bar{X}^{*}}{\bar{K}^{*}}+\bar{V}^{*} \Phi^{\prime}\left(\frac{\bar{X}^{*}}{\bar{U}^{*} \bar{K}^{*}}\right)\left(\frac{\bar{X}^{*}}{\bar{K}^{*}}\right)^{2} \frac{1}{\bar{U}^{*}}\right]\left(\widehat{x}_{t}^{*}+\widehat{v}_{t}^{*}\right)-\bar{V}^{*} \Phi^{\prime}\left(\frac{\bar{X}^{*}}{\bar{U}^{*} \widetilde{K}^{*}}\right)\left(\frac{\bar{X}^{*}}{\widetilde{K}^{*}}\right)^{2} \frac{1}{\bar{U}^{*}} \widehat{u}_{t}^{*} } \\
= & (1-\delta) \widehat{\widetilde{k}}_{t}^{*}+\delta\left(\widehat{x}_{t}^{*}+\widehat{v}_{t}^{*}\right),
\end{aligned}
$$

where the second-equality follows from the steady state properties of the CAC function, and the fact that $\bar{X}=\delta \bar{K}, \bar{X}^{*}=\delta \bar{K}^{*}$ and $\bar{V}=\bar{V}^{*}=1$. The log-linearization of the capital accumulation formula in (339) and its foreign counterpart under investment adjustment costs (IAC) allows me to obtain the following set 
of equations,

$$
\begin{aligned}
& \widehat{\widetilde{k}}_{t+1} \approx(1-\delta) \widehat{\widetilde{k}}_{t}+\left[\bar{V} \Phi\left(\frac{\bar{X}}{\bar{X}}\right) \frac{\bar{X}}{\overline{\widetilde{K}}}+\bar{V} \Phi^{\prime}\left(\frac{\bar{X}}{\bar{X}}\right)\left(\frac{\bar{X}}{\bar{X}}\right) \frac{\bar{X}}{\widetilde{\widetilde{K}}}\right] \widehat{x}_{t}-\ldots \\
& {\left[\bar{V} \Phi^{\prime}\left(\frac{\bar{X}}{\bar{X}}\right)\left(\frac{\bar{X}}{\bar{X}}\right)^{2} \frac{\bar{X}}{\widetilde{\widetilde{K}}}\right] \widehat{x}_{t}+\bar{V} \Phi\left(\frac{\bar{X}}{\bar{X}}\right) \frac{\bar{X}}{\widetilde{\widetilde{K}}} \widehat{v}_{t} } \\
&=(1-\delta) \widehat{\widetilde{k}}_{t}+\delta\left(\widehat{x}_{t}+\widehat{v}_{t}\right), \\
& \widehat{\widetilde{k}}_{t+1}^{*} \approx(1-\delta) \widehat{\widetilde{k}}_{t}^{*}+\left[\bar{V}^{*} \Phi\left(\frac{\bar{X}^{*}}{\bar{X}^{*}}\right) \frac{\bar{X}^{*}}{\widetilde{\widetilde{K}}^{*}}+\bar{V}^{*} \Phi^{\prime}\left(\frac{\bar{X}^{*}}{\bar{X}^{*}}\right)\left(\frac{\bar{X}^{*}}{\bar{X}^{*}}\right) \frac{\bar{X}^{*}}{\widetilde{\widetilde{K}}^{*}}\right] \widehat{x}_{t}^{*}-\ldots \\
& {\left[\bar{V}^{*} \Phi^{\prime}\left(\frac{\bar{X}^{*}}{\bar{X}^{*}}\right)\left(\frac{\bar{X}^{*}}{\bar{X}^{*}}\right)^{2} \frac{\bar{X}^{*}}{\widetilde{\bar{K}}^{*}}\right] \widehat{x}_{t}^{*}+\bar{V}^{*} \Phi\left(\frac{\bar{X}^{*}}{\bar{X}^{*}}\right) \frac{\bar{X}^{*}}{\widetilde{\widetilde{K}}^{*}} \widehat{v}_{t}^{*} } \\
&=(1-\delta) \widehat{\widetilde{k}}_{t}^{*}+\delta\left(\widehat{x}_{t}^{*}+\widehat{v}_{t}^{*}\right),
\end{aligned}
$$

where the second-equality follows from the steady state properties of the IAC function, and the fact that $\bar{X}=\delta \overline{\widetilde{K}}, \bar{X}^{*}=\delta \overline{\widetilde{K}}^{*}$ and $\bar{V}=\bar{V}^{*}=1$. In spite of the fact that I am using three different specifications for the adjustment cost function $\Phi(\cdot)$ and introducing capital utilization, the log-linearized law of motion for physical capital is still the same in all cases.

A first-order approximation of the link between capital services and physical capital in (342) and (343) also gives me the following relationships between these two variables,

$$
\begin{aligned}
& \widehat{k}_{t} \approx \widehat{u}_{t}+\widehat{\widetilde{k}}_{t}, \\
& \widehat{k}_{t}^{*} \approx \widehat{\widetilde{u}}_{t}^{*}+\widehat{\widetilde{k}}_{t}^{*},
\end{aligned}
$$

where $\widehat{k}_{t}$ and $\widehat{k}_{t}^{*}$ denote the capital services in the domestic and foreign country, respectively.

The Capital-Investment Decision under NAC. The log-linearization of the capital-investment equilibrium conditions coming from the domestic households' problem in (350) - (351) is as follows,

$$
\begin{aligned}
\widehat{q}_{t} & \approx \mathbb{E}_{t}\left\{-\frac{1}{\sigma}\left(\widehat{c}_{t+1}-\widehat{c}_{t}\right)+\beta\left(\frac{\bar{Z}}{\bar{P}}\right) \widehat{r}_{t+1}^{z}+\beta\left[\frac{\bar{Z}}{\bar{P}}-A^{\prime}(\bar{U})\right] \bar{U} \widehat{u}_{t+1}+\beta(1-\delta) \widehat{q}_{t+1}\right\} \\
& =\mathbb{E}_{t}\left\{-\frac{1}{\sigma}\left(\widehat{c}_{t+1}-\widehat{c}_{t}\right)+(1-\beta(1-\delta)) \widehat{r}_{t+1}^{z}+\beta(1-\delta) \widehat{q}_{t+1}\right\} \\
\widehat{q}_{t} & \approx-\widehat{v}_{t}
\end{aligned}
$$

and, analogously, for the foreign counterparts in (353) - (354),

$$
\begin{aligned}
& \widehat{q}_{t}^{*} \approx \mathbb{E}_{t}\left\{-\frac{1}{\sigma}\left(\widehat{c}_{t+1}^{*}-\widehat{c}_{t}^{*}\right)+(1-\beta(1-\delta)) \widehat{r}_{t+1}^{z *}+\beta(1-\delta) \widehat{q}_{t+1}^{*}\right\} \\
& \widehat{q}_{t}^{*} \approx-\widehat{v}_{t}^{*}
\end{aligned}
$$

where $\widehat{q}_{t}$ and $\widehat{q}_{t}^{*}$ are the real shadow prices of an additional unit of capital (or Tobin's q) in each country. These are the same equations derived under no capital utilization. These capital-investment equations can 
be re-arranged to show that,

$$
\begin{aligned}
(1-\beta(1-\delta)) \mathbb{E}_{t}\left(\widehat{r}_{t+1}^{z}\right) & \approx\left(\widehat{i}_{t}-\mathbb{E}_{t}\left(\widehat{\pi}_{t+1}\right)\right)+\beta(1-\delta) \mathbb{E}_{t}\left[\widehat{v}_{t+1}\right]-\widehat{v}_{t} \\
(1-\beta(1-\delta)) \mathbb{E}_{t}\left(\widehat{r}_{t+1}^{z *}\right) & \approx\left(\widehat{i}_{t}^{*}-\mathbb{E}_{t}\left(\widehat{\pi}_{t+1}^{*}\right)\right)+\beta(1-\delta) \mathbb{E}_{t}\left[\widehat{v}_{t+1}^{*}\right]-\widehat{v}_{t}^{*}
\end{aligned}
$$

by adding the Euler equations in (261) - (262). Finally, I must add the log-linearization of (352) and (355) as follows,

$$
\begin{aligned}
& \mathbb{E}_{t}\left(\widehat{r}_{t+1}^{z}\right) \approx \lambda \mathbb{E}_{t}\left(\widehat{u}_{t+1}\right), \\
& \mathbb{E}_{t}\left(\widehat{r}_{t+1}^{z *}\right) \approx \lambda \mathbb{E}_{t}\left(\widehat{u}_{t+1}^{*}\right),
\end{aligned}
$$

where $\lambda \equiv \frac{A^{\prime \prime}(\bar{U}) \bar{U}}{A^{\prime}(\bar{U})}=\frac{A^{\prime \prime}\left(\bar{U}^{*}\right) \bar{U}^{*}}{A^{\prime}\left(\bar{U}^{*}\right)}$ and $\bar{U}=\bar{U}^{*}=1$. These two first-order conditions link the real rental rate on capital services to the capital utilization choice made by the households.

The Capital-Investment Decision under CAC. The log-linearization of the capital-investment equilibrium conditions coming from the domestic households' problem in (356) - (357) are as follows,

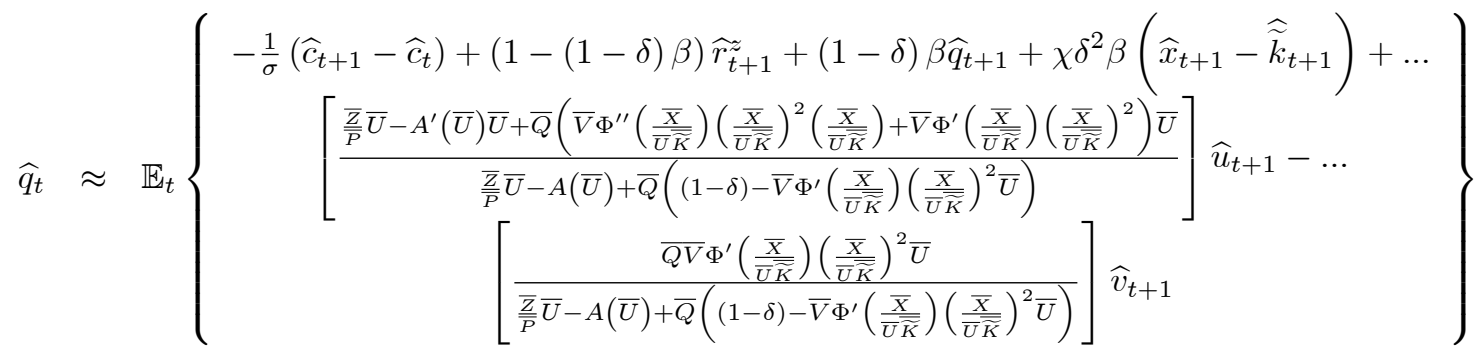

$$
\begin{aligned}
& =\mathbb{E}_{t}\left\{-\frac{1}{\sigma}\left(\widehat{c}_{t+1}-\widehat{c}_{t}\right)+(1-(1-\delta) \beta) \widehat{r}_{t+1}^{z}+(1-\delta) \beta \widehat{q}_{t+1}+\chi \delta^{2} \beta\left(\widehat{x}_{t+1}-\widehat{\widetilde{k}}_{t+1}-\widehat{u}_{t+1}\right)\right\},
\end{aligned}
$$

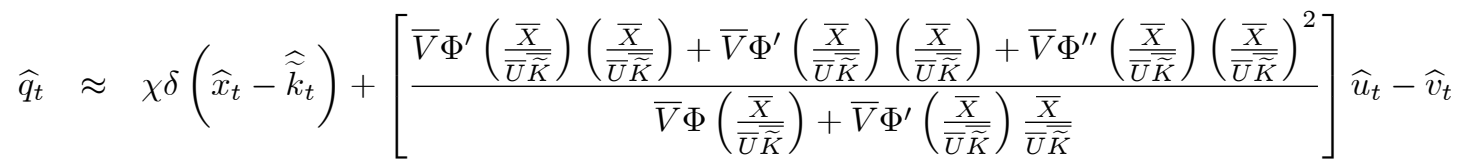

$$
\begin{aligned}
& =\chi \delta\left(\widehat{x}_{t}-\widehat{\widetilde{k}}_{t}-\widehat{u}_{t}\right)-\widehat{v}_{t},
\end{aligned}
$$

and, analogously, for the foreign counterparts in (359) - (360),

$$
\begin{aligned}
& \widehat{q}_{t}^{*} \approx \mathbb{E}_{t}\left\{-\frac{1}{\sigma}\left(\widehat{c}_{t+1}^{*}-\widehat{c}_{t}^{*}\right)+(1-(1-\delta) \beta) \widehat{r}_{t+1}^{z *}+(1-\delta) \beta \widehat{q}_{t+1}^{*}+\chi \delta^{2} \beta\left(\widehat{x}_{t+1}^{*}-\widehat{\widetilde{k}}_{t+1}^{*}-\widehat{u}_{t+1}^{*}\right)\right\} \\
& \widehat{q}_{t}^{*} \approx \chi \delta\left(\widehat{x}_{t}^{*}-\widehat{\widetilde{k}}_{t}^{*}-\widehat{u}_{t}^{*}\right)-\widehat{v}_{t}^{*}
\end{aligned}
$$

This system of equations describes $\widehat{q}_{t}$ and $\widehat{q}_{t}^{*}$ as the real shadow prices of an additional unit of capital (or Tobin's q) in each country, while $\chi$ regulates the degree of concavity of the CAC function around the steady state.

The pair of equations that relate the current and expected Tobin's q to the real rental rate on capital 
and the real interest rate can be re-arranged as,

$$
\begin{aligned}
& \widehat{q}_{t} \approx \beta \mathbb{E}_{t}\left[\widehat{q}_{t+1}\right]+\left[(1-(1-\delta) \beta) \mathbb{E}_{t}\left(\widehat{r}_{t+1}^{z}\right)-\left(\widehat{i}_{t}-\mathbb{E}_{t}\left(\widehat{\pi}_{t+1}\right)\right)\right], \\
& \widehat{q}_{t}^{*} \approx \beta \mathbb{E}_{t}\left[\widehat{q}_{t+1}^{*}\right]+\left[(1-(1-\delta) \beta) \mathbb{E}_{t}\left(\widehat{r}_{t+1}^{z *}\right)-\left(\hat{i}_{t}^{*}-\mathbb{E}_{t}\left(\widehat{\pi}_{t+1}^{*}\right)\right)\right],
\end{aligned}
$$

by adding the Euler equations in (261) - (262). These equations are exactly the same ones that I found in (282) - (283) without variable capacity utilization. I can re-write (383) and (385) in terms of capital services as,

$$
\begin{aligned}
\widehat{q}_{t} & \approx \chi \delta\left(\widehat{x}_{t}-\widehat{k}_{t}\right)-\widehat{v}_{t}, \\
\widehat{q}_{t}^{*} & \approx \chi \delta\left(\widehat{x}_{t}^{*}-\widehat{k}_{t}^{*}\right)-\widehat{v}_{t}^{*} .
\end{aligned}
$$

These equations are effectively the same ones that I found in (279) and (281), although capital is here interpreted as capital services rather than physical capital. Finally, I approximate the first-order conditions on capital utilization in (358) and (361) as follows,

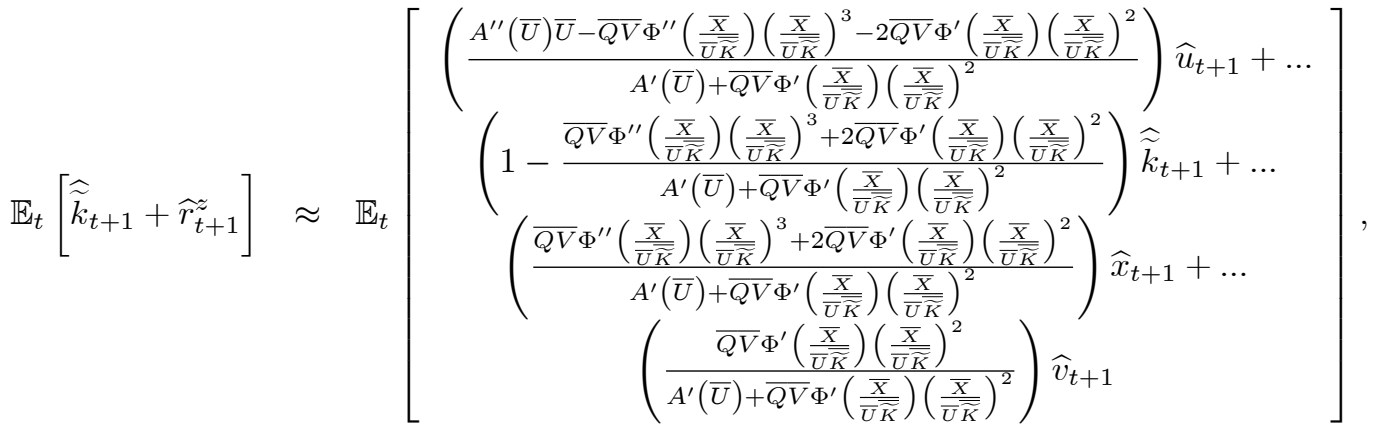

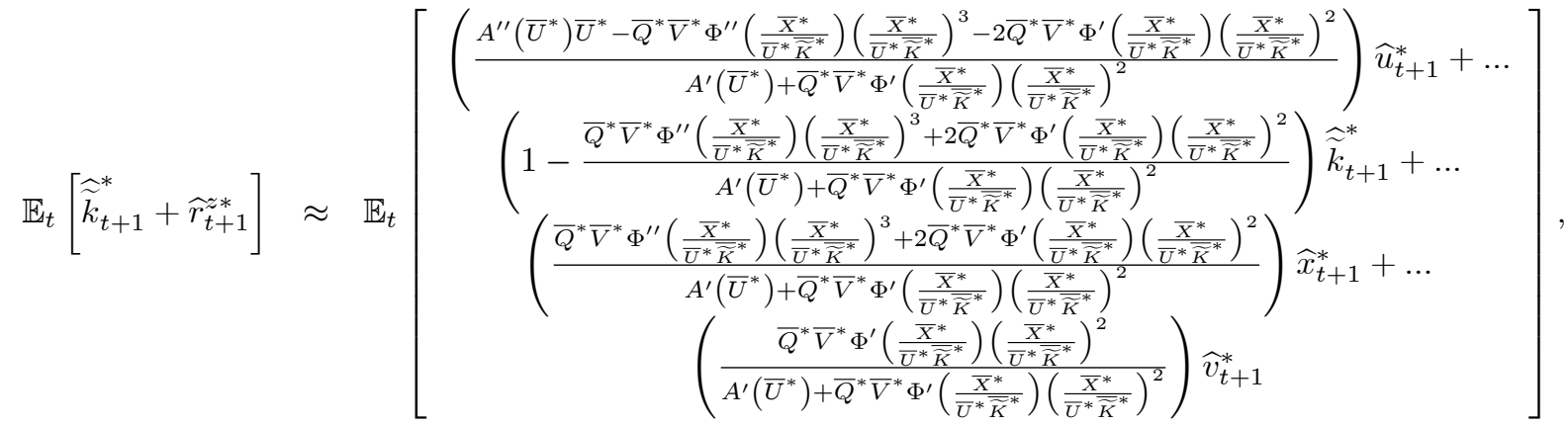


so I obtain that,

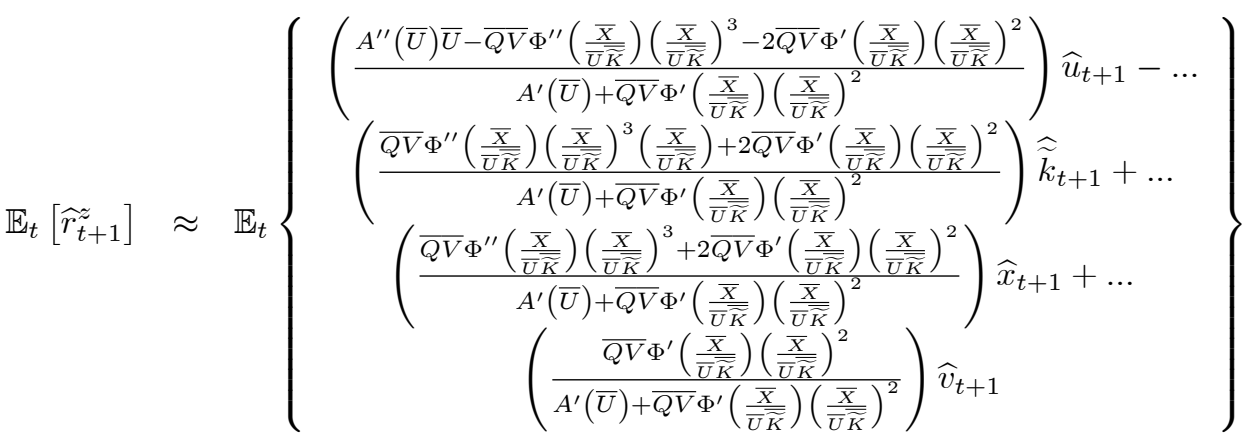

$$
\begin{aligned}
& =\mathbb{E}_{t}\left\{\begin{array}{c}
\left(\frac{A^{\prime \prime}(\bar{U}) \bar{U}}{A^{\prime}(\bar{U})+\overline{Q V} \Phi^{\prime}\left(\frac{\bar{X}}{\bar{U} \bar{K}}\right)\left(\frac{\bar{X}}{\overline{\bar{K}}}\right)^{2}}\right) \widehat{u}_{t+1}+\ldots \\
\left(\frac{\overline{Q V} \Phi^{\prime \prime}\left(\frac{\bar{X}}{\bar{U} \bar{K}}\right)\left(\frac{\bar{X}}{\bar{U} \overline{\bar{K}}}\right)^{3}+2 \overline{Q V} \Phi^{\prime}\left(\frac{\bar{X}}{\bar{U} \bar{K}}\right)\left(\frac{X}{\bar{U} \bar{K}}\right)^{2}}{A^{\prime}(\bar{U})+\overline{Q V} \Phi^{\prime}\left(\frac{\bar{X}}{\bar{U} \bar{K}}\right)\left(\frac{\bar{X}}{\bar{U} \bar{K}}\right)^{2}}\right)\left(\widehat{x}_{t+1}-\widehat{\widetilde{k}}_{t+1}-\widehat{u}_{t+1}\right)
\end{array}\right\} \\
& =\mathbb{E}_{t}\left\{\left(\frac{A^{\prime \prime}(\bar{U}) \bar{U}}{A^{\prime}(\bar{U})}\right) \widehat{u}_{t+1}+\left(\frac{\overline{Q V} \Phi^{\prime \prime}\left(\frac{\bar{X}}{\bar{U} \overline{\bar{K}}}\right)\left(\frac{\bar{X}}{\bar{U} \overline{\widetilde{K}}}\right)^{3}}{A^{\prime}(\bar{U})}\right)\left(\widehat{x}_{t+1}-\widehat{\widetilde{k}}_{t+1}-\widehat{u}_{t+1}\right)\right\} \\
& =\mathbb{E}_{t}\left\{\lambda \widehat{u}_{t+1}+\left(\frac{\Phi^{\prime \prime}(\delta) \delta^{3}}{\beta^{-1}-(1-\delta)}\right)\left(\widehat{x}_{t+1}-\widehat{\widetilde{k}}_{t+1}-\widehat{u}_{t+1}\right)\right\} \\
& =\mathbb{E}_{t}\left\{\lambda \widehat{u}_{t+1}-\left(\frac{\chi \delta^{2} \beta}{1-(1-\delta) \beta}\right)\left(\widehat{x}_{t+1}-\widehat{\widetilde{k}}_{t+1}-\widehat{u}_{t+1}\right)\right\}, \\
& \mathbb{E}_{t}\left(\widehat{r}_{t+1}^{z *}\right) \approx \mathbb{E}_{t}\left\{\lambda \widehat{u}_{t+1}^{*}-\left(\frac{\chi \delta^{2} \beta}{1-(1-\delta) \beta}\right)\left(\widehat{x}_{t+1}^{*}-\widehat{\widetilde{k}}_{t+1}^{*}-\widehat{u}_{t+1}^{*}\right)\right\} .
\end{aligned}
$$

Using equations (383) and (385) I obtain that,

$$
\begin{aligned}
& \mathbb{E}_{t}\left[\widehat{r}_{t+1}^{z}\right] \approx \mathbb{E}_{t}\left\{\lambda \widehat{u}_{t+1}-\left(\frac{\delta \beta}{1-(1-\delta) \beta}\right)\left(\widehat{q}_{t+1}+\widehat{v}_{t+1}\right)\right\}, \\
& \mathbb{E}_{t}\left[\widehat{r}_{t+1}^{z *}\right] \approx \mathbb{E}_{t}\left\{\lambda \widehat{u}_{t+1}^{*}-\left(\frac{\delta \beta}{1-(1-\delta) \beta}\right)\left(\widehat{q}_{t+1}^{*}+\widehat{v}_{t+1}^{*}\right)\right\},
\end{aligned}
$$

where $\lambda \equiv \frac{A^{\prime \prime}(\bar{U}) \bar{U}}{A^{\prime}(\bar{U})}=\frac{A^{\prime \prime}\left(\bar{U}^{*}\right) \bar{U}^{*}}{A^{\prime}\left(\bar{U}^{*}\right)}$ and $\bar{U}=\bar{U}^{*}=1$. These first-order conditions on capital utilization are similar to those derived in the case without adjustment costs (NAC), but they show that the real rental rates on capital services are tied to capital utilization, Tobin's q and the IST shocks as well.

The Capital-Investment Decision under IAC. The log-linearization of the capital-investment equilibrium conditions coming from the first-order conditions of the households' problem in (362) - (364) are as 
follows,

$$
\begin{aligned}
& \widehat{q}_{t} \approx \mathbb{E}_{t}\left\{-\frac{1}{\sigma}\left(\widehat{c}_{t+1}-\widehat{c}_{t}\right)+(1-(1-\delta) \beta) \widehat{r}_{t+1}^{z}+(1-\delta) \beta \widehat{q}_{t+1}+\frac{\left[\overline{\bar{Z}}-A^{\prime}(\bar{U})\right] \bar{U}}{\left[\frac{\bar{Z}}{\bar{P}} \bar{U}-A(\bar{U})+\bar{Q}(1-\delta)\right]} \widehat{u}_{t+1}\right\} \\
& =\mathbb{E}_{t}\left\{-\frac{1}{\sigma}\left(\widehat{c}_{t+1}-\widehat{c}_{t}\right)+(1-(1-\delta) \beta) \widehat{r}_{t+1}^{z}+(1-\delta) \beta \widehat{q}_{t+1}\right\} \text {, } \\
& \widehat{q}_{t} \approx-\widehat{v}_{t}-\frac{\left(2 \bar{V} \Phi^{\prime}\left(\frac{\bar{X}}{\bar{X}}\right)+\bar{V} \Phi^{\prime \prime}\left(\frac{\bar{X}}{\bar{X}}\right)\left(\frac{\bar{X}}{\bar{X}}\right)\right)\left(\frac{\bar{X}}{\bar{X}}\right)}{\bar{V} \Phi\left(\frac{\bar{X}}{\bar{X}}\right)+\bar{V} \Phi^{\prime}\left(\frac{\bar{X}}{\bar{X}}\right)\left(\frac{\bar{X}}{\bar{X}}\right)}\left(\widehat{x}_{t}-\widehat{x}_{t-1}\right)+\ldots
\end{aligned}
$$

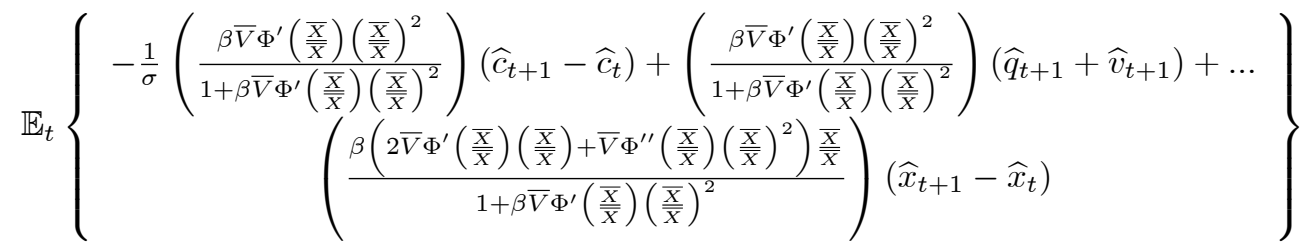

$$
\begin{aligned}
& =\kappa\left[\left(\widehat{x}_{t}-\widehat{x}_{t-1}\right)-\beta \mathbb{E}_{t}\left(\widehat{x}_{t+1}-\widehat{x}_{t}\right)\right]-\widehat{v}_{t},
\end{aligned}
$$

and, analogously, for the foreign counterparts in (365) - (366),

$$
\begin{aligned}
& \widehat{q}_{t}^{*} \approx \mathbb{E}_{t}\left\{-\frac{1}{\sigma}\left(\widehat{c}_{t+1}^{*}-\widehat{c}_{t}^{*}\right)+(1-(1-\delta) \beta) \widehat{r}_{t+1}^{z *}+(1-\delta) \beta \widehat{q}_{t+1}^{*}\right\}, \\
& \widehat{q}_{t}^{*} \approx \kappa\left[\left(\widehat{x}_{t}^{*}-\widehat{x}_{t-1}^{*}\right)-\beta \mathbb{E}_{t}\left(\widehat{x}_{t+1}^{*}-\widehat{x}_{t}^{*}\right)\right]-\widehat{v}_{t}^{*}
\end{aligned}
$$

This system of equations summarizes $\widehat{q}_{t}$ and $\widehat{q}_{t}^{*}$ as the real shadow prices of an additional unit of capital (or Tobin's q) in each country, and $\kappa$ regulates the degree of concavity of the IAC function around the steady state.

The pair of equations that relate the current and expected Tobin's q to the real rental rates on capital and the real interest rate can be re-arranged as,

$$
\begin{aligned}
\widehat{q}_{t} & \approx(1-\delta) \beta \mathbb{E}_{t}\left[\widehat{q}_{t+1}\right]+\left[(1-(1-\delta) \beta) \mathbb{E}_{t}\left(\widehat{r}_{t+1}^{z}\right)-\left(\widehat{i}_{t}-\mathbb{E}_{t}\left[\widehat{\pi}_{t+1}\right]\right)\right], \\
\widehat{q}_{t}^{*} & \approx(1-\delta) \beta \mathbb{E}_{t}\left[\widehat{q}_{t+1}^{*}\right]+\left[(1-(1-\delta) \beta) \mathbb{E}_{t}\left(\widehat{r}_{t+1}^{z *}\right)-\left(\widehat{i}_{t}^{*}-\mathbb{E}_{t}\left[\widehat{\pi}_{t+1}^{*}\right]\right)\right],
\end{aligned}
$$

by adding the Euler equations in (261) - (262). Finally, I re-write equations (391) and (393) in a more compact form as follows,

$$
\begin{aligned}
& \widehat{x}_{t} \approx \frac{1}{1+\beta} \widehat{x}_{t-1}+\frac{\beta}{1+\beta} \mathbb{E}_{t}\left[\widehat{x}_{t+1}\right]+\frac{1}{\kappa(1+\beta)}\left(\widehat{q}_{t}+\widehat{v}_{t}\right), \\
& \widehat{x}_{t}^{*} \approx \frac{1}{1+\beta} \widehat{x}_{t-1}^{*}+\frac{\beta}{1+\beta} \mathbb{E}_{t}\left[\widehat{x}_{t+1}^{*}\right]+\frac{1}{\kappa(1+\beta)}\left(\widehat{q}_{t}^{*}+\widehat{v}_{t}^{*}\right) .
\end{aligned}
$$

The presence of investment adjustment costs (IAC) makes equations (391) and (393) different from those under capital adjustment cost (CAC). These equations are the same equations derived before in the model without capital utilization. Capital utilization, however, adds an additional pair of equations to the system 
of equilibrium conditions, i.e.,

$$
\begin{aligned}
& \mathbb{E}_{t}\left(\widehat{r}_{t+1}^{z}\right) \approx \lambda \mathbb{E}_{t}\left(\widehat{u}_{t+1}\right), \\
& \mathbb{E}_{t}\left(\widehat{r}_{t+1}^{z *}\right) \approx \lambda \mathbb{E}_{t}\left(\widehat{u}_{t+1}^{*}\right),
\end{aligned}
$$

where $\lambda \equiv \frac{A^{\prime \prime}(\bar{U}) \bar{U}}{A^{\prime}(\bar{U})}=\frac{A^{\prime \prime}\left(\bar{U}^{*}\right) \bar{U}^{*}}{A^{\prime}\left(\bar{U}^{*}\right)}$ and $\bar{U}=\bar{U}^{*}=1$. These conditions link the real rental rates on capital to the capital utilization choice of the households, and they are identical to the conditions derived in the case without adjustment costs (NAC).

\subsubsection{The Firms' Equilibrium Conditions}

Efficiency conditions and Aggregate Output. The efficiency conditions can be described by the same pair of equations reported in (294) - (295) (or in (300) - (301)). Those efficiency conditions are necessary to close down the model without having to keep track of either labor or wages explicitly. Using the demand constraints of the domestic firm in equations (33) - (34), the demand constraints of the foreign firm in equations (36) - (37), complemented by (50) - (53), it follows that the log-linearization around the steady state of the output demand for a given re-optimizing firm, i.e. $\widehat{y}_{t+\tau}(h)$ for the domestic variety $h$ and $\widehat{y}_{t+\tau}^{*}(f)$ for the foreign variety $f$, takes the following form,

$$
\begin{aligned}
& \widehat{y}_{t+\tau}(h) \approx-\theta\left[\widehat{p}_{t+\tau}^{W}(h)-\widehat{p}_{t+\tau}^{H, W}\right]-\eta\left[\widehat{p}_{t+\tau}^{H, W}-\widehat{p}_{t+\tau}^{W}\right]+\left(1-\gamma_{x}\right) \widehat{c}_{t+\tau}^{a W}+\gamma_{x} \widehat{x}_{t+\tau}^{a W}, \\
& \widehat{y}_{t+\tau}^{*}(f) \approx-\theta\left[\widehat{p}_{t+\tau}^{W *}(f)-\widehat{p}_{t+\tau}^{F, W *}\right]-\eta\left[\widehat{p}_{t+\tau}^{F, W *}-\widehat{p}_{t+\tau}^{W *}\right]+\left(1-\gamma_{x}\right) \widehat{c}_{t+\tau}^{a W *}+\gamma_{x} \widehat{x}_{t+\tau}^{a W *},
\end{aligned}
$$

where the weighted variables are,

$$
\begin{aligned}
\widehat{c}_{t}^{a W} & \equiv \phi_{H}\left(\frac{1-\gamma_{x}^{a}}{1-\gamma_{x}}\right) \widehat{c}_{t}+\phi_{F}\left(\frac{1-\gamma_{x^{*}}^{a}}{1-\gamma_{x}}\right) \widehat{c}_{t}^{*} \\
\widehat{c}_{t}^{a W *} & \equiv \phi_{F}\left(\frac{1-\gamma_{x}^{a}}{1-\gamma_{x}}\right) \widehat{c}_{t}+\phi_{H}\left(\frac{1-\gamma_{x^{*}}^{a}}{1-\gamma_{x}}\right) \widehat{c}_{t}^{*} \\
\widehat{x}_{t}^{a W} & \equiv \phi_{H}\left(\frac{\gamma_{x}^{a}}{\gamma_{x}}\right) \widehat{x}_{t}+\phi_{F}\left(\frac{\gamma_{x^{*}}^{a}}{\gamma_{x}}\right) \widehat{x}_{t}^{*} \\
\widehat{x}_{t}^{a W *} & \equiv \phi_{F}\left(\frac{\gamma_{x}^{a}}{\gamma_{x}}\right) \widehat{x}_{t}+\phi_{H}\left(\frac{\gamma_{x^{*}}^{a}}{\gamma_{x}}\right) \widehat{x}_{t}^{*} \\
\widehat{p}_{t}^{W}(h) & \equiv \phi_{H} \widehat{p}_{t}(h)+\phi_{F} \widehat{p}_{t}^{*}(h), \widehat{p}_{t}^{W *}(f) \equiv \phi_{F} \widehat{p}_{t}(f)+\phi_{H} \widehat{p}_{t}^{*}(f), \\
\widehat{p}_{t}^{H, W} & \equiv \phi_{H} \widehat{p}_{t}^{H}+\phi_{F} \widehat{p}_{t}^{H *}, \widehat{p}_{t}^{F, W *} \equiv \phi_{F} \widehat{p}_{t}^{F}+\phi_{H} \widehat{p}_{t}^{F *}, \\
\widehat{p}_{t}^{W} & \equiv \phi_{H} \widehat{p}_{t}+\phi_{F} \widehat{p}_{t}^{*}, \widehat{p}_{t}^{W *} \equiv \phi_{F} \widehat{p}_{t}+\phi_{H} \widehat{p}_{t}^{*} .
\end{aligned}
$$

I can re-express the aggregate price of all domestic and foreign firms as $\widehat{p}_{t+\tau}^{H, W} \approx \int_{0}^{1} \widehat{p}_{t+\tau}^{W}(h) d h$ and $\widehat{p}_{t+\tau}^{F, W *} \approx$ $\int_{0}^{1} \widehat{p}_{t+\tau}^{W *}(f) d f$. Adding up the output demand equations for all varieties (firms) within a country, I obtain 
an expression for aggregate output in these terms,

$$
\begin{aligned}
\int_{0}^{1} \widehat{y}_{t+\tau}(h) d h & \approx-\eta\left(\widehat{p}_{t+\tau}^{H, W}-\widehat{p}_{t+\tau}^{W}\right)+\left(1-\gamma_{x}\right) \widehat{c}_{t+\tau}^{a W}+\gamma_{x} \widehat{x}_{t+\tau}^{a W} \\
\int_{0}^{1} \widehat{y}_{t+\tau}^{*}(f) d f & \approx-\eta\left(\widehat{p}_{t+\tau}^{F, W *}-\widehat{p}_{t+\tau}^{W *}\right)+\left(1-\gamma_{x}\right) \widehat{c}_{t+\tau}^{a W *}+\gamma_{x} \widehat{x}_{t+\tau}^{a W *} .
\end{aligned}
$$

These two equations are essentially the same ones derived in (298) and (299).

Adjusting aggregate output to account for the costs due to variable capital utilization, as per equations (368) - (369), I obtain an expression for aggregate output in these terms,

$$
\begin{aligned}
& \left(\frac{\bar{Y}}{\bar{Y}-A(\bar{U})}\right) \widehat{\widetilde{K}}_{t+\tau}-\left(\frac{A^{\prime}(\bar{U}) \overline{\widetilde{K}} \bar{U}}{\bar{Y}-A(\bar{U}) \overline{\widetilde{K}}}\right) \widehat{u}_{t+\tau}-\left(\frac{A(\bar{U}) \overline{\widetilde{K}}}{\bar{Y}-A(\bar{U}) \overline{\widetilde{K}}}\right) \widehat{\widetilde{k}}_{t+\tau} \\
& \quad=\widehat{y}_{t+\tau}-\left(\frac{A^{\prime}(\bar{U}) \widetilde{K} \bar{U}}{\bar{Y}}\right) \widehat{u}_{t+\tau} \approx \int_{0}^{1} \widehat{y}_{t+\tau}(h) d h \\
& \approx-\eta\left(\widehat{p}_{t+\tau}^{H, W}-\widehat{p}_{t+\tau}^{W}\right)+\left(1-\gamma_{x}\right) \widehat{c}_{t+\tau}^{a W}+\gamma_{x} \widehat{x}_{t+\tau}^{a W}, \\
& \left(\frac{\bar{Y}^{*}}{\bar{Y}^{*}-A\left(\bar{U}^{*}\right) \overline{\widetilde{K}}^{*}}\right) \widehat{y}_{t+\tau}^{*}-\left(\frac{A^{\prime}\left(\bar{U}^{*}\right) \overline{\widetilde{K}}^{*} \bar{U}^{*}}{\bar{Y}^{*}-A\left(\bar{U}^{*}\right) \widetilde{K}^{*}}\right) \widehat{u}_{t+\tau}^{*}-\left(\frac{A\left(\bar{U}^{*}\right) \widetilde{\widetilde{K}}^{*}}{\bar{Y}^{*}-A\left(\bar{U}^{*}\right) \widetilde{K}^{*}}\right) \widehat{\widetilde{k}}_{t+\tau}^{*} \\
& =\widehat{y}_{t+\tau}^{*}-\left(\frac{A^{\prime}\left(\bar{U}^{*}\right) \overline{\widetilde{K}}^{*} \bar{U}^{*}}{\bar{Y}^{*}}\right) \widehat{u}_{t+\tau}^{*} \approx \int_{0}^{1} \widehat{y}_{t+\tau}^{*}(f) d f \\
& \approx-\eta\left(\widehat{p}_{t+\tau}^{F, W *}-\widehat{p}_{t+\tau}^{W *}\right)+\left(1-\gamma_{x}\right) \widehat{c}_{t+\tau}^{a W *}+\gamma_{x} \widehat{x}_{t+\tau}^{a W *},
\end{aligned}
$$

since $A(\bar{U})=A\left(\bar{U}^{*}\right)=0$. Using my characterization of the steady state, these output equations can be further simplified as follows,

$$
\begin{gathered}
\widehat{y}_{t+\tau}-\left(\beta^{-1}-(1-\delta)\right)\left(\frac{\bar{K}}{\bar{Y}}\right) \widehat{u}_{t+\tau} \approx-\eta\left(\widehat{p}_{t+\tau}^{H, W}-\widehat{p}_{t+\tau}^{W}\right)+\left(1-\gamma_{x}\right) \widehat{c}_{t+\tau}^{a W}+\gamma_{x} \widehat{x}_{t+\tau}^{a W}, \\
\widehat{y}_{t+\tau}^{*}-\left(\beta^{-1}-(1-\delta)\right)\left(\frac{\bar{K}^{*}}{\bar{Y}^{*}}\right) \widehat{u}_{t+\tau}^{*} \approx-\eta\left(\widehat{p}_{t+\tau}^{F, W *}-\widehat{p}_{t+\tau}^{W *}\right)+\left(1-\gamma_{x}\right) \widehat{c}_{t+\tau}^{a W *}+\gamma_{x} \widehat{x}_{t+\tau}^{a W *} .
\end{gathered}
$$

I know from (132) and (133) that in steady state I can write the investment shares as proportional to the capital-to-output ratios in each country, i.e.,

$$
\gamma_{x}^{y} \equiv \delta \frac{\bar{K}}{\bar{Y}}, \gamma_{x^{*}}^{y} \equiv \delta \frac{\bar{K}^{*}}{\bar{Y}^{*}}
$$

where physical capital and capital services are equated because the utilization rate is set to $\bar{U}=\bar{U}^{*}=1$. Therefore, I can re-express the aggregate output equations in the following terms,

$$
\begin{aligned}
& \widehat{y}_{t+\tau} \approx-\eta\left(\widehat{p}_{t+\tau}^{H, W}-\widehat{p}_{t+\tau}^{W}\right)+\left(1-\gamma_{x}\right) \widehat{c}_{t+\tau}^{a W}+\gamma_{x} \widehat{x}_{t+\tau}^{a W}+\gamma_{x}^{y}\left(\frac{1-\beta(1-\delta)}{\beta \delta}\right) \widehat{u}_{t+\tau}, \\
& \widehat{y}_{t+\tau}^{*} \approx-\eta\left(\widehat{p}_{t+\tau}^{F, W *}-\widehat{p}_{t+\tau}^{W *}\right)+\left(1-\gamma_{x}\right) \widehat{c}_{t+\tau}^{a W *}+\gamma_{x} \widehat{x}_{t+\tau}^{a W *}+\gamma_{x^{*}}^{y}\left(\frac{1-\beta(1-\delta)}{\beta \delta}\right) \widehat{u}_{t+\tau}^{*} .
\end{aligned}
$$

These equations become very important in my posterior derivations of the Phillips curves. Equations (400) (401) are identical to those derived in the model without variable capital utilization in (298) - (299) only 
if $\widehat{u}_{t}=\widehat{u}_{t}^{*}=0$ for all $t$. These equations compute aggregate output from the demand-side by incorporating the capital utilization costs at the same time. I also can write both output equations as functions of world terms of trade $\widehat{t}_{t}^{W} \equiv\left(\widehat{p}_{t}^{F, W *}-\widehat{p}_{t}^{W *}\right)$ without having to keep track of any other international relative price following the same logic behind the derivation of equations (330) - (331).

I define the world consumption as $\widehat{c}_{t}^{a W} \equiv \phi_{H}\left(\frac{1-\gamma_{x}^{a}}{1-\gamma_{x}}\right) \widehat{c}_{t}+\phi_{F}\left(\frac{1-\gamma_{x^{*}}^{a}}{1-\gamma_{x}}\right) \widehat{c}_{t}^{*}$ and $\widehat{c}_{t}^{a W *} \equiv \phi_{F}\left(\frac{1-\gamma_{x}^{a}}{1-\gamma_{x}}\right) \widehat{c}_{t}+$ $\phi_{H}\left(\frac{1-\gamma_{x *}^{a}}{1-\gamma_{x}}\right) \widehat{c}_{t}^{*}$ and the relative consumption as $\widehat{c}_{t}^{a R} \equiv\left(\frac{1-\gamma_{x}^{a}}{1-\gamma_{x}}\right) \widehat{c}_{t}-\left(\frac{1-\gamma_{x^{*}}^{a}}{1-\gamma_{x}}\right) \widehat{c}_{t}^{*}$, then I can write that,

$$
\begin{aligned}
\widehat{c}_{t} & =\left(\frac{1-\gamma_{x}}{1-\gamma_{x}^{a}}\right)\left(\widehat{c}_{t}^{a W}+\phi_{F} \widehat{c}_{t}^{a R}\right), \\
\widehat{c}_{t}^{*} & =\left(\frac{1-\gamma_{x}}{1-\gamma_{x^{*}}^{a}}\right)\left(\widehat{c}_{t}^{a W *}-\phi_{F} \widehat{c}_{t}^{a R}\right) .
\end{aligned}
$$

As a result, I can express the efficiency conditions in (294) - (295) as,

$$
\begin{aligned}
\widehat{r}_{t}^{z} & \approx-\frac{1+\varphi}{\psi} \widehat{a}_{t}+\frac{1}{\sigma}\left(\frac{1-\gamma_{x}}{1-\gamma_{x}^{a}}\right) \widehat{c}_{t}^{a W}+\phi_{F} \frac{1}{\sigma}\left(\frac{1-\gamma_{x}}{1-\gamma_{x}^{a}}\right) \widehat{c}_{t}^{a R}+\frac{1+\varphi}{\psi} \widehat{y}_{t}-\left(\frac{1+(1-\psi) \varphi}{\psi}\right) \widehat{k}_{t}, \\
\widehat{r}_{t}^{z *} & \approx-\frac{1+\varphi}{\psi} \widehat{a}_{t}^{*}+\frac{1}{\sigma}\left(\frac{1-\gamma_{x}}{1-\gamma_{x^{*}}^{a}}\right) \widehat{c}_{t}^{a W *}-\phi_{F} \frac{1}{\sigma}\left(\frac{1-\gamma_{x}}{1-\gamma_{x^{*}}^{a}}\right) \widehat{c}_{t}^{a R}+\frac{1+\varphi}{\psi} \widehat{y}_{t}^{*}-\left(\frac{1+(1-\psi) \varphi}{\psi}\right) \widehat{k}_{t}^{*} .
\end{aligned}
$$

Furthermore, if I combine the efficiency conditions with the output equations derived in (400) - (401), it follows that,

$$
\begin{aligned}
\widehat{r}_{t}^{z} & \approx\left(\frac{1}{\sigma}\left(\frac{1-\gamma_{x}}{1-\gamma_{x}^{a}}\right)+\left(1-\gamma_{x}\right) \frac{1+\varphi}{\psi}\right) \widehat{c}_{t}^{a W}+\gamma_{x} \frac{1+\varphi}{\psi} \widehat{x}_{t}^{a W}+\gamma_{x}^{y} \frac{1+\varphi}{\psi}\left(\frac{1-\beta(1-\delta)}{\beta \delta}\right) \widehat{u}_{t}+\phi_{F} \frac{1}{\sigma}\left(\frac{1-\gamma_{x}}{1-\gamma_{x}^{a}}\right) \widehat{c}_{t}^{a R}-\ldots \\
& \eta \frac{1+\varphi}{\psi}\left(\widehat{p}_{t}^{H, W}-\widehat{p}_{t}^{W}\right)-\left(\frac{1+(1-\psi) \varphi}{\psi}\right) \widehat{k}_{t}-\frac{1+\varphi}{\psi} \widehat{a}_{t}, \\
\widehat{r}_{t}^{z *} & \approx\left(\frac{1}{\sigma}\left(\frac{1-\gamma_{x}}{1-\gamma_{x}^{a}}\right)+\left(1-\gamma_{x}\right) \frac{1+\varphi}{\psi}\right) \widehat{c}_{t}^{a W *}+\gamma_{x} \frac{1+\varphi}{\psi} \widehat{x}_{t}^{a W *}+\gamma_{x^{*}}^{y} \frac{1+\varphi}{\psi}\left(\frac{1-\beta(1-\delta)}{\beta \delta}\right) \widehat{u}_{t}^{*}-\phi_{F} \frac{1}{\sigma}\left(\frac{1-\gamma_{x}}{1-\gamma_{x^{*}}^{a}}\right) \widehat{c}_{t}^{a R}-\ldots \\
& \eta \frac{1+\varphi}{\psi}\left(\widehat{p}_{t}^{F, W *}-\widehat{p}_{t}^{W *}\right)-\left(\frac{1+(1-\psi) \varphi}{\psi}\right) \widehat{k}_{t}^{*}-\frac{1+\varphi}{\psi} \widehat{a}_{t}^{*} .
\end{aligned}
$$

These conditions can be used to simplify the derivation of the Phillips curves.

The Optimal Pricing Equations In steady state the monopolistic competition pricing rule of charging a mark-up over marginal costs holds. Accordingly, the log-linearization of the optimal pricing equations in (74), (75), (76) and (77) can be compactly expressed as follows,

$$
\begin{aligned}
& \widehat{\widetilde{p}}_{t}(h)-\widehat{p}_{t} \approx \mathbb{E}_{t}\left[\sum_{\tau=1}^{+\infty}(\beta \alpha)^{\tau} \widehat{\pi}_{t+\tau}\right]+(1-\beta \alpha) \mathbb{E}_{t}\left[\sum_{\tau=0}^{+\infty}(\beta \alpha)^{\tau}\left(\widehat{m c}_{t+\tau}-\widehat{p}_{t+\tau}\right)\right], \\
& \widehat{\widetilde{p}}_{t}^{*}(h)-\widehat{p}_{t}^{*} \approx \mathbb{E}_{t}\left[\sum_{\tau=1}^{+\infty}(\beta \alpha)^{\tau} \widehat{\pi}_{t+\tau}^{*}\right]+(1-\beta \alpha) \mathbb{E}_{t}\left[\sum_{\tau=0}^{+\infty}(\beta \alpha)^{\tau}\left(\widehat{m c}_{t+\tau}-\widehat{p}_{t+\tau}-\widehat{r s}_{t+\tau}\right)\right], \\
& \widehat{\widetilde{p}}_{t}(f)-\widehat{p}_{t} \approx \mathbb{E}_{t}\left[\sum_{\tau=1}^{+\infty}(\beta \alpha)^{\tau} \widehat{\pi}_{t+\tau}\right]+(1-\beta \alpha) \mathbb{E}_{t}\left[\sum_{\tau=0}^{+\infty}(\beta \alpha)^{\tau}\left(\widehat{m c}_{t+\tau}^{*}-\widehat{p}_{t+\tau}^{*}+\widehat{r s}_{t+\tau}\right)\right], \\
& \widehat{\widetilde{p}}_{t}^{*}(f)-\widehat{p}_{t}^{*} \approx \mathbb{E}_{t}\left[\sum_{\tau=1}^{+\infty}(\beta \alpha)^{\tau} \widehat{\pi}_{t+\tau}^{*}\right]+(1-\beta \alpha) \mathbb{E}_{t}\left[\sum_{\tau=0}^{+\infty}(\beta \alpha)^{\tau}\left(\widehat{m c}_{t+\tau}^{*}-\widehat{p}_{t+\tau}^{*}\right)\right],
\end{aligned}
$$

which are the same pricing formulas that I obtained without variable capital utilization. Here I must recall the assumption that the government subsidy is time-invariant and equal to its steady state value in every period, which explains why the government subsidies do not appear in the log-linearized pricing equations. 
I derive the (pre-subsidy) marginal cost equations in (26) - (27), and they can be log-linearized as,

$$
\begin{aligned}
& \widehat{m c}_{t+\tau} \approx \psi \widehat{w}_{t+\tau}+(1-\psi)\left(\widehat{r}_{t+\tau}^{z}+\widehat{p}_{t+\tau}\right)-\widehat{a}_{t+\tau}, \\
& \widehat{m c}_{t+\tau}^{*} \approx \psi \widehat{w}_{t+\tau}^{*}+(1-\psi)\left(\widehat{r}_{t+\tau}^{* *}+\widehat{p}_{t+\tau}^{*}\right)-\widehat{a}_{t+\tau}^{*},
\end{aligned}
$$

while the labor market clearing conditions, which are implicit in (114) - (115), can be approximated as,

$$
\begin{aligned}
& \widehat{w}_{t+\tau} \approx-\frac{\varphi}{1+(1-\psi) \varphi} \widehat{a}_{t+\tau}+\frac{1}{1+(1-\psi) \varphi} \frac{1}{\sigma} \widehat{c}_{t+\tau}+\frac{\varphi}{1+(1-\psi) \varphi} \widehat{y}_{t+\tau}+\frac{(1-\psi) \varphi}{1+(1-\psi) \varphi}\left(\widehat{r}_{t+\tau}^{z}+\widehat{p}_{t+\tau}\right)+\frac{1}{1+(1-\psi) \varphi} \widehat{p}_{t+\tau}, \\
& \widehat{w}_{t+\tau}^{*} \approx-\frac{\varphi}{1+(1-\psi) \varphi} \widehat{a}_{t+\tau}^{*}+\frac{1}{1+(1-\psi) \varphi} \frac{1}{\sigma} \widehat{c}_{t+\tau}^{*}+\frac{\varphi}{1+(1-\psi) \varphi} \widehat{y}_{t+\tau}^{*}+\frac{(1-\psi) \varphi}{1+(1-\psi) \varphi}\left(\widehat{r}_{t+\tau}^{z *}+\widehat{p}_{t+\tau}^{*}\right)+\frac{1}{1+(1-\psi) \varphi} \widehat{p}_{t+\tau}^{*} .
\end{aligned}
$$

If I combine these two log-linearized equations, it follows that the marginal costs can be expressed as,

$$
\begin{aligned}
& \widehat{m c}_{t+\tau} \approx \frac{\psi}{1+(1-\psi) \varphi} \frac{1}{\sigma} \widehat{c}_{t+\tau}+\frac{\varphi \psi}{1+(1-\psi) \varphi} \widehat{y}_{t+\tau}-\frac{1+\varphi}{1+(1-\psi) \varphi}\left(\widehat{a}_{t+\tau}-(1-\psi)\left(\widehat{r}_{t+\tau}^{z}+\widehat{p}_{t+\tau}\right)\right)+\frac{\psi}{1+(1-\psi) \varphi} \widehat{p}_{t+\tau}, \\
& \widehat{m c}_{t+\tau}^{*} \approx \frac{\psi}{1+(1-\psi) \varphi} \frac{1}{\sigma} \widehat{c}_{t+\tau}^{*}+\frac{\varphi \psi}{1+(1-\psi) \varphi} \widehat{y}_{t+\tau}^{*}-\frac{1+\varphi}{1+(1-\psi) \varphi}\left(\widehat{a}_{t+\tau}^{*}-(1-\psi)\left(\widehat{r}_{t+\tau}^{* *}+\widehat{p}_{t+\tau}^{*}\right)\right)+\frac{\psi}{1+(1-\psi) \varphi} \widehat{p}_{t+\tau}^{*},
\end{aligned}
$$

where $\widehat{y}_{t+\tau}$ and $\widehat{y}_{t+\tau}^{*}$ reflect domestic and foreign aggregate output, respectively. Up to this point, the derivation of marginal costs is the same independently of whether the model allows for variable capital utilization or not. If I combine the marginal cost equations with the aggregate output equations derived before in (400) - (401), it follows that,

$$
\begin{aligned}
& \widehat{m c}_{t+\tau}-\widehat{p}_{t+\tau} \approx \frac{\psi}{1+(1-\psi) \varphi} \frac{1}{\sigma} \widehat{c}_{t+\tau}-\frac{\varphi \psi}{1+(1-\psi) \varphi} \eta\left(\widehat{p}_{t+\tau}^{H, W}-\widehat{p}_{t+\tau}^{W}\right)+\ldots \\
& \quad \frac{\varphi \psi}{1+(1-\psi) \varphi}\left(\left(1-\gamma_{x}\right) \widehat{c}_{t+\tau}^{a W}+\gamma_{x} \widehat{x}_{t+\tau}^{a W}+\gamma_{x}^{y}\left(\frac{1-\beta(1-\delta)}{\beta \delta}\right) \widehat{u}_{t+\tau}\right)-\ldots \\
& \frac{1+\varphi}{1+(1-\psi) \varphi}\left(\widehat{a}_{t+\tau}-(1-\psi) \widehat{r}_{t+\tau}^{z}\right), \\
& \widehat{m c}_{t+\tau}^{*}-\widehat{p}_{t+\tau}^{*} \approx \frac{\psi}{1+(1-\psi) \varphi} \frac{1}{\sigma} \widehat{c}_{t+\tau}^{*}-\frac{\varphi \psi}{1+(1-\psi) \varphi} \eta\left(\widehat{p}_{t+\tau}^{F, W *}-\widehat{p}_{t+\tau}^{W *}\right)+\ldots \\
& \quad \frac{\varphi \psi}{1+(1-\psi) \varphi}\left(\left(1-\gamma_{x}\right) \widehat{c}_{t+\tau}^{a W *}+\gamma_{x} \widehat{x}_{t+\tau}^{a W *}+\gamma_{x^{*}}^{y}\left(\frac{1-\beta(1-\delta)}{\beta \delta}\right) \widehat{u}_{t+\tau}^{*}\right)-\ldots \\
& \quad \frac{1+\varphi}{1+(1-\psi) \varphi}\left(\widehat{a}_{t+\tau}^{*}-(1-\psi) \widehat{r}_{t+\tau}^{z *}\right) .
\end{aligned}
$$

This characterization of the marginal costs is central to my derivations of the Phillips curve. It naturally shows that now marginal costs have to account for the costs of variable capital utilization.

I can use my characterization of the real marginal costs with the pricing formulas log-linearized before 
to write that,

$$
\begin{aligned}
& \widehat{\widetilde{p}}_{t}(h)-\widehat{p}_{t} \approx \\
& \left(\frac{(1-\beta \alpha) \psi}{1+(1-\psi) \varphi}\right) \sum_{\tau=0}^{+\infty}(\beta \alpha)^{\tau} \mathbb{E}_{t}\left[\begin{array}{c}
\frac{1}{\sigma} \widehat{c}_{t+\tau}-\varphi \eta\left(\widehat{p}_{t+\tau}^{H, W}-\widehat{p}_{t+\tau}^{W}\right)+\ldots \\
\varphi\left(\left(1-\gamma_{x}\right) \widehat{c}_{t+\tau}^{a W}+\gamma_{x} \widehat{x}_{t+\tau}^{a W}+\gamma_{x}^{y}\left(\frac{1-\beta(1-\delta)}{\beta \delta}\right) \widehat{u}_{t+\tau}\right)-\ldots \\
\left(\frac{1+\varphi}{\psi}\right)\left(\widehat{a}_{t+\tau}-(1-\psi) \widehat{r}_{t+\tau}^{z}\right)
\end{array}\right]+\ldots \\
& \sum_{\tau=1}^{+\infty}(\beta \alpha)^{\tau} \mathbb{E}_{t}\left(\widehat{\pi}_{t+\tau}\right), \\
& \widehat{\widetilde{p}}_{t}^{*}(h)-\widehat{p}_{t}^{*} \approx \\
& \left(\frac{(1-\beta \alpha) \psi}{1+(1-\psi) \varphi}\right) \sum_{\tau=0}^{+\infty}(\beta \alpha)^{\tau} \mathbb{E}_{t}\left[\begin{array}{c}
\frac{1}{\sigma} \widehat{c}_{t+\tau}-\varphi \eta\left(\widehat{p}_{t+\tau}^{H, W}-\widehat{p}_{t+\tau}^{W}\right)+\ldots \\
\varphi\left(\left(1-\gamma_{x}\right) \widehat{c}_{t+\tau}^{a W}+\gamma_{x} \widehat{x}_{t+\tau}^{a W}+\gamma_{x}^{y}\left(\frac{1-\beta(1-\delta)}{\beta \delta}\right) \widehat{u}_{t+\tau}\right)-\ldots \\
\left(\frac{1+\varphi}{\psi}\right)\left(\widehat{a}_{t+\tau}-(1-\psi) \widehat{r}_{t+\tau}^{z}\right)-\left(\frac{1+(1-\psi) \varphi}{\psi}\right) \widehat{r s}_{t+\tau}
\end{array}\right]+\ldots \\
& \sum_{\tau=1}^{+\infty}(\beta \alpha)^{\tau} \mathbb{E}_{t}\left(\widehat{\pi}_{t+\tau}^{*}\right), \\
& \widehat{\widetilde{p}}_{t}(f)-\widehat{p}_{t} \approx \\
& \left(\frac{(1-\beta \alpha) \psi}{1+(1-\psi) \varphi}\right) \sum_{\tau=0}^{+\infty}(\beta \alpha)^{\tau} \mathbb{E}_{t}\left[\begin{array}{c}
\frac{1}{\sigma} \widehat{c}_{t+\tau}^{*}-\varphi \eta\left(\widehat{p}_{t+\tau}^{F, W *}-\widehat{p}_{t+\tau}^{W *}\right)+\ldots \\
\varphi\left(\left(1-\gamma_{x}\right) \widehat{c}_{t+\tau}^{a W *}+\gamma_{x} \widehat{x}_{t+\tau}^{a W *}+\gamma_{x^{*}}^{y}\left(\frac{1-\beta(1-\delta)}{\beta \delta}\right) \widehat{u}_{t+\tau}^{*}\right)-\ldots \\
\left(\frac{1+\varphi}{\psi}\right)\left(\widehat{a}_{t+\tau}^{*}-(1-\psi) \widehat{r}_{t+\tau}^{z *}\right)+\left(\frac{1+(1-\psi) \varphi}{\psi}\right) \widehat{r s}_{t+\tau}
\end{array}\right]+\ldots \\
& \sum_{\tau=1}^{+\infty}(\beta \alpha)^{\tau} \mathbb{E}_{t}\left(\widehat{\pi}_{t+\tau}\right), \\
& \widehat{\widetilde{p}}_{t}^{*}(f)-\widehat{p}_{t}^{*} \approx \\
& \left(\frac{(1-\beta \alpha) \psi}{1+(1-\psi) \varphi}\right) \sum_{\tau=0}^{+\infty}(\beta \alpha)^{\tau} \mathbb{E}_{t}\left[\begin{array}{c}
\varphi\left(\frac{1}{\sigma} \widehat{c}_{t+\tau}^{*}-\varphi \eta\left(\widehat{p}_{t+\tau}^{F, W *}-\widehat{p}_{t+\tau}^{W *}\right)+\ldots\right. \\
\left.\left(1-\gamma_{x}\right) \widehat{c}_{t+\tau}^{a W *}+\gamma_{x} \widehat{x}_{t+\tau}^{a W *}+\gamma_{x^{*}}^{y}\left(\frac{1-\beta(1-\delta)}{\beta \delta}\right) \widehat{u}_{t+\tau}^{*}\right)-\ldots \\
\left(\frac{1+\varphi}{\psi}\right)\left(\widehat{a}_{t+\tau}^{*}-(1-\psi) \widehat{r}_{t+\tau}^{z *}\right)
\end{array}\right]+\ldots \\
& \sum_{\tau=1}^{+\infty}(\beta \alpha)^{\tau} \mathbb{E}_{t}\left(\hat{\pi}_{t+\tau}^{*}\right) .
\end{aligned}
$$

I log-linearize the price sub-indexes in (78) - (79) and (80) - (81) and re-arrange them to obtain that,

$$
\begin{aligned}
& \widehat{\widetilde{p}}_{t}(h)-\widehat{p}_{t} \approx\left(\widehat{p}_{t}^{H}-\widehat{p}_{t}\right)+\left(\frac{\alpha}{1-\alpha}\right) \widehat{\pi}_{t}^{H}, \\
& \widehat{\widetilde{p}}_{t}^{*}(h)-\widehat{p}_{t}^{*} \approx\left(\widehat{p}_{t}^{H *}-\widehat{p}_{t}^{*}\right)+\left(\frac{\alpha}{1-\alpha}\right) \widehat{\pi}_{t}^{H *}, \\
& \widehat{\widetilde{p}}_{t}(f)-\widehat{p}_{t} \approx\left(\widehat{p}_{t}^{F}-\widehat{p}_{t}\right)+\left(\frac{\alpha}{1-\alpha}\right) \widehat{\pi}_{t}^{F}, \\
& \widehat{\widetilde{p}}_{t}^{*}(f)-\widehat{p}_{t}^{*} \approx\left(\widehat{p}_{t}^{F *}-\widehat{p}_{t}^{*}\right)+\left(\frac{\alpha}{1-\alpha}\right) \widehat{\pi}_{t}^{F *} .
\end{aligned}
$$

I replace the isolated terms $\frac{1}{\sigma} \widehat{c}_{t}$ and $\frac{1}{\sigma} \widehat{c}_{t}^{*}$ out of the marginal cost equations. If I define the world consumption as $\widehat{c}_{t}^{a W} \equiv \phi_{H}\left(\frac{1-\gamma_{x}^{a}}{1-\gamma_{x}}\right) \widehat{c}_{t}+\phi_{F}\left(\frac{1-\gamma_{x^{*}}^{a}}{1-\gamma_{x}}\right) \widehat{c}_{t}^{*}$ and $\widehat{c}_{t}^{a W *} \equiv \phi_{F}\left(\frac{1-\gamma_{x}^{a}}{1-\gamma_{x}}\right) \widehat{c}_{t}+\phi_{H}\left(\frac{1-\gamma_{x^{*}}^{a}}{1-\gamma_{x}}\right) \widehat{c}_{t}^{*}$ and the relative 
consumption as $\widehat{c}_{t}^{a R} \equiv\left(\frac{1-\gamma_{x}^{a}}{1-\gamma_{x}}\right) \widehat{c}_{t}-\left(\frac{1-\gamma_{x^{*}}^{a}}{1-\gamma_{x}}\right) \widehat{c}_{t}^{*}$, then I can write that,

$$
\begin{aligned}
\widehat{c}_{t} & =\left(\frac{1-\gamma_{x}}{1-\gamma_{x}^{a}}\right)\left(\widehat{c}_{t}^{a W}+\phi_{F} \widehat{c}_{t}^{a R}\right), \\
\widehat{c}_{t}^{*} & =\left(\frac{1-\gamma_{x}}{1-\gamma_{x^{*}}^{a}}\right)\left(\widehat{c}_{t}^{a W *}-\phi_{F} \widehat{c}_{t}^{a R}\right) .
\end{aligned}
$$

Hence, the optimal pricing equations can be expressed more compactly as,

$$
\begin{aligned}
& \widehat{\pi}_{t}^{H}+\left(\frac{1-\alpha}{\alpha}\right)\left(\widehat{p}_{t}^{H}-\widehat{p}_{t}\right) \approx \\
& \Psi\left(\frac{\psi}{1+(1-\psi) \varphi}\right) \sum_{\tau=0}^{+\infty}(\beta \alpha)^{\tau} \mathbb{E}_{t}\left[\begin{array}{c}
\left(\left(\frac{1-\gamma_{x}}{1-\gamma_{x}^{a}}\right) \sigma^{-1}+\left(1-\gamma_{x}\right) \varphi\right) \widehat{c}_{t+\tau}^{a W}+\gamma_{x} \varphi \widehat{x}_{t+\tau}^{a W}+\varphi \gamma_{x}^{y}\left(\frac{1-\beta(1-\delta)}{\beta \delta}\right) \widehat{u}_{t+\tau}+\ldots \\
\phi_{F}\left(\frac{1-\gamma_{x}}{1-\gamma_{x}^{a}}\right) \sigma^{-1} \widehat{c}_{t+\tau}^{a R}-\varphi \eta\left(\widehat{p}_{t+\tau}^{H, W}-\widehat{p}_{t+\tau}^{W}\right)-\ldots \\
\left(\frac{1+\varphi}{\psi}\right)\left(\widehat{a}_{t+\tau}-(1-\psi) \widehat{r}_{t+\tau}^{z}\right)
\end{array}\right]+\ldots \\
& \left(\frac{1-\alpha}{\alpha}\right) \sum_{\tau=1}^{+\infty}(\beta \alpha)^{\tau} \mathbb{E}_{t}\left(\widehat{\pi}_{t+\tau}\right), \\
& \widehat{\pi}_{t}^{H *}+\left(\frac{1-\alpha}{\alpha}\right)\left(\widehat{p}_{t}^{H *}-\widehat{p}_{t}^{*}\right) \approx \\
& \Psi\left(\frac{\psi}{1+(1-\psi) \varphi}\right) \sum_{\tau=0}^{+\infty}(\beta \alpha)^{\tau} \mathbb{E}_{t}\left[\begin{array}{c}
\left(\left(\frac{1-\gamma_{x}}{1-\gamma_{x}^{a}}\right) \sigma^{-1}+\left(1-\gamma_{x}\right) \varphi\right) \widehat{c}_{t+\tau}^{a W}+\gamma_{x} \varphi \widehat{x}_{t+\tau}^{a W}+\varphi \gamma_{x}^{y}\left(\frac{1-\beta(1-\delta)}{\beta \delta}\right) \widehat{u}_{t+\tau}+\ldots \\
\phi_{F}\left(\frac{1-\gamma_{x}}{1-\gamma_{x}^{a}}\right) \sigma^{-1} \widehat{c}_{t+\tau}^{a R}-\left(\frac{1+(1-\psi) \varphi}{\psi}\right) \widehat{r s}_{t+\tau}-\varphi \eta\left(\widehat{p}_{t+\tau}^{H, W}-\widehat{p}_{t+\tau}^{W}\right)-\ldots \\
\left(\frac{1+\varphi}{\psi}\right)\left(\widehat{a}_{t+\tau}-(1-\psi) \widehat{r}_{t+\tau}^{z}\right)
\end{array}\right]+\ldots \\
& \left(\frac{1-\alpha}{\alpha}\right) \sum_{\tau=1}^{+\infty}(\beta \alpha)^{\tau} \mathbb{E}_{t}\left(\widehat{\pi}_{t+\tau}^{*}\right), \\
& \widehat{\pi}_{t}^{F}+\left(\frac{1-\alpha}{\alpha}\right)\left(\widehat{p}_{t}^{F}-\widehat{p}_{t}\right) \approx \\
& \Psi\left(\frac{\psi}{1+(1-\psi) \varphi}\right) \sum_{\tau=0}^{+\infty}(\beta \alpha)^{\tau} \mathbb{E}_{t}\left[\begin{array}{c}
\left(\left(\frac{1-\gamma_{x}}{1-\gamma_{x *}^{a}}\right) \sigma^{-1}+\left(1-\gamma_{x}\right) \varphi\right) \widehat{c}_{t+\tau}^{a W *}+\gamma_{x} \varphi \widehat{x}_{t+\tau}^{a W *}+\varphi \gamma_{x^{*}}^{y}\left(\frac{1-\beta(1-\delta)}{\beta \delta}\right) \widehat{u}_{t+\tau}^{*}-\ldots \\
\phi_{F}\left(\frac{1-\gamma_{x}}{1-\gamma_{x^{*}}^{\alpha}}\right) \sigma^{-1} \widehat{c}_{t+\tau}^{a R}+\left(\frac{1+(1-\psi) \varphi}{\psi}\right) \widehat{r s}_{t+\tau}-\varphi \eta\left(\widehat{p}_{t+\tau}^{F, W *}-\widehat{p}_{t+\tau}^{W *}\right)-\ldots \\
\left(\frac{1+\varphi}{\psi}\right)\left(\widehat{a}_{t+\tau}^{*}-(1-\psi) \widehat{r}_{t+\tau}^{2 *}\right)
\end{array}\right]+ \\
& \left(\frac{1-\alpha}{\alpha}\right) \sum_{\tau=1}^{+\infty}(\beta \alpha)^{\tau} \mathbb{E}_{t}\left(\widehat{\pi}_{t+\tau}\right), \\
& \widehat{\pi}_{t}^{F *}+\left(\frac{1-\alpha}{\alpha}\right)\left(\widehat{p}_{t}^{F *}-\widehat{p}_{t}^{*}\right) \approx \\
& \Psi\left(\frac{\psi}{1+(1-\psi) \varphi}\right) \sum_{\tau=0}^{+\infty}(\beta \alpha)^{\tau} \mathbb{E}_{t} \\
& \left(\frac{1-\alpha}{\alpha}\right) \sum_{\tau=1}^{+\infty}(\beta \alpha)^{\tau} \mathbb{E}_{t}\left(\widehat{\pi}_{t+\tau}^{*}\right), \\
& \left.\begin{array}{c}
\left(\left(\frac{1-\gamma_{x}}{1-\gamma_{x^{*}}^{a}}\right) \sigma^{-1}+\left(1-\gamma_{x}\right) \varphi\right) \widehat{c}_{t+\tau}^{a W *}+\gamma_{x} \varphi \widehat{x}_{t+\tau}^{a W *}+\varphi \gamma_{x^{*}}^{y}\left(\frac{1-\beta(1-\delta)}{\beta \delta}\right) \widehat{u}_{t+\tau}^{*}-\ldots \\
\phi_{F}\left(\frac{1-\gamma_{x}}{1-\gamma_{x^{*}}^{a}}\right) \sigma^{-1} \widehat{c}_{t+\tau}^{a R}-\varphi \eta\left(\widehat{p}_{t+\tau}^{F, W *}-\widehat{p}_{t+\tau}^{W *}\right)-\ldots \\
\left(\frac{1+\varphi}{\psi}\right)\left(\widehat{a}_{t+\tau}^{*}-(1-\psi) \widehat{r}_{t+\tau}^{z *}\right)
\end{array}\right]+\ldots
\end{aligned}
$$

where the composite coefficient is defined as $\Psi \equiv \frac{(1-\alpha)(1-\beta \alpha)}{\alpha}$.

Furthermore, these pricing equations can be expressed in the form of a system of expectational difference equations. Let me focus on the first equation as an example. If I re-write the equation at time $t+1$ and 
take conditional expectations with all the information available up to time $t$, it should follow that,

$$
\begin{array}{r}
\mathbb{E}_{t}\left[\widehat{\pi}_{t+1}^{H}+\left(\frac{1-\alpha}{\alpha}\right)\left(\widehat{p}_{t+1}^{H}-\widehat{p}_{t+1}\right)\right] \approx \\
\left(\frac{\psi}{1+(1-\psi) \varphi}\right) \Psi \sum_{\tau=0}^{+\infty}(\beta \alpha)^{\tau} \mathbb{E}_{t} \\
\left(\frac{1-\alpha}{\alpha}\right) \sum_{\tau=1}^{+\infty}(\beta \alpha)^{\tau} \mathbb{E}_{t}\left(\widehat{\pi}_{t+1+\tau}\right) .
\end{array}
$$

$$
\begin{gathered}
\left(\left(\frac{1-\gamma_{x}}{1-\gamma_{x}^{a}}\right) \sigma^{-1}+\left(1-\gamma_{x}\right) \varphi\right) \widehat{c}_{t+1+\tau}^{a W}+\gamma_{x} \varphi \widehat{x}_{t+1+\tau}^{a W}+\varphi \gamma_{x}^{y}\left(\frac{1-\beta(1-\delta)}{\beta \delta}\right) \widehat{u}_{t+1+\tau}+\ldots \\
\phi_{F}\left(\frac{1-\gamma_{x}}{1-\gamma_{x}^{a}}\right) \sigma^{-1} \widehat{c}_{t+1+\tau}^{a R}-\varphi \eta\left(\widehat{p}_{t+1+\tau}^{H, W}-\widehat{p}_{t+1+\tau}^{W}\right)-\ldots
\end{gathered}
$$$$
\left(\frac{1+\varphi}{\psi}\right)\left(\widehat{a}_{t+1+\tau}-(1-\psi) \widehat{r}_{t+1+\tau}^{z}\right)
$$

Hence, using the properties of the conditional expectation, the pricing equation can easily be decomposed in two terms as,

$$
\begin{aligned}
& \widehat{\pi}_{t}^{H}+\left(\frac{1-\alpha}{\alpha}\right)\left(\widehat{p}_{t}^{H}-\widehat{p}_{t}\right) \approx \\
& \left(\frac{\psi}{1+(1-\psi) \varphi}\right) \Psi\left[\begin{array}{c}
\left(\left(\frac{1-\gamma_{x}}{1-\gamma_{x}^{a}}\right) \sigma^{-1}+\left(1-\gamma_{x}\right) \varphi\right) \widehat{c}_{t}^{a W}+\gamma_{x} \varphi \widehat{x}_{t}^{a W}+\varphi \gamma_{x}^{y}\left(\frac{1-\beta(1-\delta)}{\beta \delta}\right) \widehat{u}_{t}+\ldots \\
\phi_{F}\left(\frac{1-\gamma_{x}}{1-\gamma_{x}^{a}}\right) \sigma^{-1} \widehat{c}_{t}^{a R}-\varphi \eta\left(\widehat{p}_{t}^{H, W}-\widehat{p}_{t}^{W}\right)-\ldots \\
\left(\frac{1+\varphi}{\psi}\right)\left(\widehat{a}_{t}-(1-\psi) \widehat{r}_{t}^{z}\right)
\end{array}\right]+\ldots \\
& (1-\alpha) \beta \mathbb{E}_{t}\left(\widehat{\pi}_{t+1}\right)+\alpha \beta \mathbb{E}_{t}\left[\widehat{\pi}_{t+1}^{H}+\left(\frac{1-\alpha}{\alpha}\right)\left(\widehat{p}_{t+1}^{H}-\widehat{p}_{t+1}\right)\right] .
\end{aligned}
$$

Further re-arranging allows me to express the expectational difference equation as,

$$
\begin{gathered}
\widehat{\pi}_{t}^{H}-\beta \mathbb{E}_{t}\left(\widehat{\pi}_{t+1}^{H}\right)+\Psi\left(\widehat{p}_{t}^{H}-\widehat{p}_{t}\right) \\
\approx\left(\frac{\psi}{1+(1-\psi) \varphi}\right) \Psi\left[\begin{array}{c}
\left(\left(\frac{1-\gamma_{x}}{1-\gamma_{x}^{a}}\right) \sigma^{-1}+\left(1-\gamma_{x}\right) \varphi\right) \widehat{c}_{t}^{a W}+\gamma_{x} \varphi \widehat{x}_{t}^{a W}+\varphi \gamma_{x}^{y}\left(\frac{1-\beta(1-\delta)}{\beta \delta}\right) \widehat{u}_{t}+\ldots \\
\phi_{F}\left(\frac{1-\gamma_{x}}{1-\gamma_{x}^{a}}\right) \sigma^{-1} \widehat{c}_{t}^{a R}-\varphi \eta\left(\widehat{p}_{t}^{H, W}-\widehat{p}_{t}^{W}\right)-\ldots \\
\left(\frac{1+\varphi}{\psi}\right)\left(\widehat{a}_{t}-(1-\psi) \widehat{r}_{t}^{z}\right)
\end{array}\right] .
\end{gathered}
$$

I can apply the same approach (and algebraic steps) to re-write all other pricing equations as expectational 
difference equations, i.e.,

$$
\begin{aligned}
& \widehat{\pi}_{t}^{H *}-\beta \mathbb{E}_{t}\left(\widehat{\pi}_{t+1}^{H *}\right)+\Psi\left(\widehat{p}_{t}^{H *}-\widehat{p}_{t}^{*}\right) \\
& \approx\left(\frac{\psi}{1+(1-\psi) \varphi}\right) \Psi\left[\begin{array}{c}
\left(\left(\frac{1-\gamma_{x}}{1-\gamma_{x}^{a}}\right) \sigma^{-1}+\left(1-\gamma_{x}\right) \varphi\right) \widehat{c}_{t}^{a W}+\gamma_{x} \varphi \widehat{x}_{t}^{a W}+\varphi \gamma_{x}^{y}\left(\frac{1-\beta(1-\delta)}{\beta \delta}\right) \widehat{u}_{t}+\ldots \\
\phi_{F}\left(\frac{1-\gamma_{x}}{1-\gamma_{x}^{a}}\right) \sigma^{-1} \widehat{c}_{t}^{a R}-\left(\frac{1+(1-\psi) \varphi}{\psi}\right) \widehat{r s}_{t}-\varphi \eta\left(\widehat{p}_{t}^{H, W}-\widehat{p}_{t}^{W}\right)-\ldots \\
\left(\frac{1+\varphi}{\psi}\right)\left(\widehat{a}_{t}-(1-\psi) \widehat{r}_{t}^{z}\right)
\end{array}\right], \\
& \widehat{\pi}_{t}^{F}-\beta \mathbb{E}_{t}\left(\widehat{\pi}_{t+1}^{F}\right)+\Psi\left(\widehat{p}_{t}^{F}-\widehat{p}_{t}\right) \\
& \approx\left(\frac{\psi}{1+(1-\psi) \varphi}\right) \Psi\left[\begin{array}{c}
\left(\left(\frac{1-\gamma_{x}}{1-\gamma_{x *}^{a}}\right) \sigma^{-1}+\left(1-\gamma_{x}\right) \varphi\right) \widehat{c}_{t}^{a W *}+\gamma_{x} \varphi \widehat{x}_{t}^{a W *}+\varphi \gamma_{x^{*}}^{y}\left(\frac{1-\beta(1-\delta)}{\beta \delta}\right) \widehat{u}_{t}^{*}-\ldots \\
\phi_{F}\left(\frac{1-\gamma_{x}}{1-\gamma_{x^{*}}^{a}}\right) \sigma^{-1} \widehat{c}_{t}^{a R}+\left(\frac{1+(1-\psi) \varphi}{\psi}\right) \widehat{r s}_{t}-\varphi \eta\left(\widehat{p}_{t}^{F, W *}-\widehat{p}_{t}^{W *}\right)-\ldots \\
\left(\frac{1+\varphi}{\psi}\right)\left(\widehat{a}_{t}^{*}-(1-\psi) \widehat{r}_{t}^{z *}\right)
\end{array}\right] \\
& \widehat{\pi}_{t}^{F *}-\beta \mathbb{E}_{t}\left(\widehat{\pi}_{t+1}^{F *}\right)+\Psi\left(\widehat{p}_{t}^{F *}-\widehat{p}_{t}^{*}\right) \\
& \approx\left(\frac{\psi}{1+(1-\psi) \varphi}\right) \Psi\left[\begin{array}{c}
\left(\left(\frac{1-\gamma_{x}}{1-\gamma_{x^{*}}^{a}}\right) \sigma^{-1}+\left(1-\gamma_{x}\right) \varphi\right) \widehat{c}_{t}^{a W *}+\gamma_{x} \varphi \widehat{x}_{t}^{a W *}+\varphi \gamma_{x^{*}}^{y}\left(\frac{1-\beta(1-\delta)}{\beta \delta}\right) \widehat{u}_{t}^{*}-\ldots \\
\phi_{F}\left(\frac{1-\gamma_{x}}{1-\gamma_{x^{*}}^{a}}\right) \sigma^{-1} \widehat{c}_{t}^{a R}-\varphi \eta\left(\widehat{p}_{t}^{F, W *}-\widehat{p}_{t}^{W *}\right)-\ldots \\
\left(\frac{1+\varphi}{\psi}\right)\left(\widehat{a}_{t}^{*}-(1-\psi) \widehat{r}_{t}^{z *}\right)
\end{array}\right] .
\end{aligned}
$$

These equations provide a very simple characterization of the dynamics at the price sub-index level.

Now, I use the pricing equations described above to infer the dynamics of the relative price sub-indexes $\widehat{\pi}_{t}^{H, R} \equiv \widehat{\pi}_{t}^{H}-\widehat{\pi}_{t}^{H *}\left(\widehat{p}_{t}^{H, R} \equiv \widehat{p}_{t}^{H}-\widehat{p}_{t}^{H *}\right)$ and $\widehat{\pi}_{t}^{F, R} \equiv \widehat{\pi}_{t}^{F}-\widehat{\pi}_{t}^{F *}\left(\widehat{p}_{t}^{F, R} \equiv \widehat{p}_{t}^{F}-\widehat{p}_{t}^{F *}\right)$ as follows,

$$
\begin{gathered}
\widehat{\pi}_{t}^{H, R}-\beta \mathbb{E}_{t}\left(\widehat{\pi}_{t+1}^{H, R}\right)+\Psi\left(\widehat{p}_{t}^{H, R}-\widehat{p}_{t}^{R}\right) \approx \Psi \widehat{r s}_{t}, \\
\widehat{\pi}_{t}^{F, R}-\beta \mathbb{E}_{t}\left(\widehat{\pi}_{t+1}^{F, R}\right)+\Psi\left(\widehat{p}_{t}^{F, R}-\widehat{p}_{t}^{R}\right) \approx \Psi \widehat{r s}_{t},
\end{gathered}
$$

where $\widehat{p}_{t}^{R} \equiv \widehat{p}_{t}-\widehat{p}_{t}^{*}$ is the relative CPI. I can re-write the pricing equations at the price sub-index level 
further as follows,

$$
\begin{aligned}
& \widehat{\pi}_{t}^{H} \approx \beta \mathbb{E}_{t}\left(\widehat{\pi}_{t+1}^{H}\right)+\ldots \\
& \left(\frac{\psi}{1+(1-\psi) \varphi}\right) \Psi\left[\begin{array}{c}
\left(\left(\frac{1-\gamma_{x}}{1-\gamma_{x}^{a}}\right) \sigma^{-1}+\left(1-\gamma_{x}\right) \varphi\right) \widehat{c}_{t}^{a W}+\gamma_{x} \varphi \widehat{x}_{t}^{a W}+\varphi \gamma_{x}^{y}\left(\frac{1-\beta(1-\delta)}{\beta \delta}\right) \widehat{u}_{t}+\ldots \\
\phi_{F}\left(\frac{1-\gamma_{x}}{1-\gamma_{x}^{a}}\right) \sigma^{-1} \widehat{c}_{t}^{a R}-\varphi \eta\left(\widehat{p}_{t}^{H, W}-\widehat{p}_{t}^{W}\right)-\ldots \\
\left(\frac{1+(1-\psi) \varphi}{\psi}\right)\left(\widehat{p}_{t}^{H}-\widehat{p}_{t}\right)-\left(\frac{1+\varphi}{\psi}\right)\left(\widehat{a}_{t}-(1-\psi) \widehat{r}_{t}^{z}\right)
\end{array}\right], \\
& \widehat{\pi}_{t}^{H *} \approx \beta \mathbb{E}_{t}\left(\widehat{\pi}_{t+1}^{H *}\right)+\ldots \\
& \left(\frac{\psi}{1+(1-\psi) \varphi}\right) \Psi\left[\begin{array}{c}
\left(\left(\frac{1-\gamma_{x}}{1-\gamma_{x}^{a}}\right) \sigma^{-1}+\left(1-\gamma_{x}\right) \varphi\right) \widehat{c}_{t}^{a W}+\gamma_{x} \varphi \widehat{x}_{t}^{a W}+\varphi \gamma_{x}^{y}\left(\frac{1-\beta(1-\delta)}{\beta \delta}\right) \widehat{u}_{t}+\ldots \\
\phi_{F}\left(\frac{1-\gamma_{x}}{1-\gamma_{x}^{a}}\right) \sigma^{-1} \widehat{c}_{t}^{a R}-\left(\frac{1+(1-\psi) \varphi}{\psi}\right) \widehat{r s}_{t}-\varphi \eta\left(\widehat{p}_{t}^{H, W}-\widehat{p}_{t}^{W}\right)-\ldots \\
\left(\frac{1+(1-\psi) \varphi}{\psi}\right)\left(\widehat{p}_{t}^{H *}-\widehat{p}_{t}^{*}\right)-\left(\frac{1+\varphi}{\psi}\right)\left(\widehat{a}_{t}-(1-\psi) \widehat{r}_{t}^{z}\right)
\end{array}\right], \\
& \widehat{\pi}_{t}^{F} \approx \beta \mathbb{E}_{t}\left(\widehat{\pi}_{t+1}^{F}\right)+\ldots \\
& \left(\frac{\psi}{1+(1-\psi) \varphi}\right) \Psi\left[\begin{array}{c}
\left(\left(\frac{1-\gamma_{x}}{1-\gamma_{x}^{\alpha}}\right) \sigma^{-1}+\left(1-\gamma_{x}\right) \varphi\right) \widehat{c}_{t}^{a W *}+\gamma_{x} \varphi \widehat{x}_{t}^{a W *}+\varphi \gamma_{x^{*}}^{y}\left(\frac{1-\beta(1-\delta)}{\beta \delta}\right) \widehat{u}_{t}^{*}-\ldots \\
\phi_{F}\left(\frac{1-\gamma_{x}}{1-\gamma_{x^{*}}}\right) \sigma^{-1} \widehat{c}_{t}^{a R}+\left(\frac{1+(1-\psi) \varphi}{\psi}\right) \widehat{r s}_{t}-\varphi \eta\left(\widehat{p}_{t}^{F, W *}-\widehat{p}_{t}^{W *}\right)-\ldots \\
\left(\frac{1+(1-\psi) \varphi}{\psi}\right)\left(\widehat{p}_{t}^{F}-\widehat{p}_{t}\right)-\left(\frac{1+\varphi}{\psi}\right)\left(\widehat{a}_{t}^{*}-(1-\psi) \widehat{r}_{t}^{z *}\right)
\end{array}\right], \\
& \widehat{\pi}_{t}^{F *} \approx \beta \mathbb{E}_{t}\left(\widehat{\pi}_{t+1}^{F *}\right)+\ldots \\
& \left(\frac{\psi}{1+(1-\psi) \varphi}\right) \Psi\left[\begin{array}{c}
\left(\left(\frac{1-\gamma_{x}}{1-\gamma_{x}^{x}}\right) \sigma^{-1}+\left(1-\gamma_{x}\right) \varphi\right) \widehat{c}_{t}^{a W *}+\gamma_{x} \varphi \widehat{x}_{t}^{a W *}+\varphi \gamma_{x^{*}}^{y}\left(\frac{1-\beta(1-\delta)}{\beta \delta}\right) \widehat{u}_{t}^{*}-\ldots \\
\phi_{F}\left(\frac{1-\gamma_{x}}{1-\gamma_{x *}^{a}}\right) \sigma^{-1} \widehat{c}_{t}^{a R}-\varphi \eta\left(\widehat{p}_{t}^{F, W *}-\widehat{p}_{t}^{W *}\right)-\ldots \\
\left(\frac{1+(1-\psi) \varphi}{\psi}\right)\left(\widehat{p}_{t}^{F *}-\widehat{p}_{t}^{*}\right)-\left(\frac{1+\varphi}{\psi}\right)\left(\widehat{a}_{t}^{*}-(1-\psi) \widehat{r}_{t}^{* *}\right)
\end{array}\right] .
\end{aligned}
$$

I have defined the world price sub-indexes as $\widehat{p}_{t}^{H, W} \equiv \phi_{H} \widehat{p}_{t}^{H}+\phi_{F} \widehat{p}_{t}^{H *}$ and $\widehat{p}_{t}^{F, W *} \equiv \phi_{F} \widehat{p}_{t}^{F}+\phi_{H} \widehat{p}_{t}^{F *}$, and the relative price sub-indexes as $\widehat{p}_{t}^{H, R} \equiv \widehat{p}_{t}^{H}-\widehat{p}_{t}^{H *}$ and $\widehat{p}_{t}^{F, R} \equiv \widehat{p}_{t}^{F}-\widehat{p}_{t}^{F *}$. Then, naturally, I can write that,

$$
\begin{aligned}
\widehat{p}_{t}^{H} & =\widehat{p}_{t}^{H, W}+\phi_{F} \widehat{p}_{t}^{H, R}, \widehat{p}_{t}^{H *}=\widehat{p}_{t}^{H, W}-\phi_{H} \widehat{p}_{t}^{H, R}, \\
\widehat{p}_{t}^{F} & =\widehat{p}_{t}^{F, W *}+\phi_{H} \widehat{p}_{t}^{F, R}, \widehat{p}_{t}^{F *}=\widehat{p}_{t}^{F, W *}-\phi_{F} \widehat{p}_{t}^{F, R} .
\end{aligned}
$$

Analogously, I have defined the world CPI as $\widehat{p}_{t}^{W} \equiv \phi_{H} \widehat{p}_{t}+\phi_{F} \widehat{p}_{t}^{*}$ and $\widehat{p}_{t}^{W *} \equiv \phi_{F} \widehat{p}_{t}+\phi_{H} \widehat{p}_{t}^{*}$, and the relative CPI as $\widehat{p}_{t}^{R} \equiv \widehat{p}_{t}-\widehat{p}_{t}^{*}$. Then, I can write that,

$$
\begin{aligned}
& \widehat{p}_{t}=\widehat{p}_{t}^{W}+\phi_{F} \widehat{p}_{t}^{R}, \widehat{p}_{t}^{*}=\widehat{p}_{t}^{W}-\phi_{H} \widehat{p}_{t}^{R} \\
& \widehat{p}_{t}=\widehat{p}_{t}^{W *}+\phi_{H} \widehat{p}_{t}^{R}, \widehat{p}_{t}^{*}=\widehat{p}_{t}^{W *}-\phi_{F} \widehat{p}_{t}^{R}
\end{aligned}
$$


Therefore, I can re-arrange the pricing equations as follows,

$$
\begin{aligned}
& \widehat{\pi}_{t}^{H} \approx \beta \mathbb{E}_{t}\left(\widehat{\pi}_{t+1}^{H}\right)+\ldots \\
& \left(\frac{\psi}{1+(1-\psi) \varphi}\right) \Psi\left[\begin{array}{c}
\left(\left(\frac{1-\gamma_{x}}{1-\gamma_{x}^{a}}\right) \sigma^{-1}+\left(1-\gamma_{x}\right) \varphi\right) \widehat{c}_{t}^{a W}+\gamma_{x} \varphi \widehat{x}_{t}^{a W}+\varphi \gamma_{x}^{y}\left(\frac{1-\beta(1-\delta)}{\beta \delta}\right) \widehat{u}_{t}+\ldots \\
\phi_{F}\left(\frac{1-\gamma_{x}}{1-\gamma_{x}^{a}}\right) \sigma^{-1} \widehat{c}_{t}^{a R}-\left(\frac{1+(1-\psi) \varphi}{\psi}+\varphi \eta\right)\left(\widehat{p}_{t}^{H, W}-\widehat{p}_{t}^{W}\right)-\ldots \\
\left(\frac{1+(1-\psi) \varphi}{\psi}\right) \phi_{F}\left(\widehat{p}_{t}^{H, R}-\widehat{p}_{t}^{R}\right)-\left(\frac{1+\varphi}{\psi}\right)\left(\widehat{a}_{t}-(1-\psi) \widehat{r}_{t}^{z}\right)
\end{array}\right], \\
& \widehat{\pi}_{t}^{H *} \approx \beta \mathbb{E}_{t}\left(\widehat{\pi}_{t+1}^{H *}\right)+\ldots \\
& \left(\frac{\psi}{1+(1-\psi) \varphi}\right) \Psi\left[\begin{array}{c}
\left(\left(\frac{1-\gamma_{x}}{1-\gamma_{x}^{a}}\right) \sigma^{-1}+\left(1-\gamma_{x}\right) \varphi\right) \widehat{c}_{t}^{a W}+\gamma_{x} \varphi \widehat{x}_{t}^{a W}+\varphi \gamma_{x}^{y}\left(\frac{1-\beta(1-\delta)}{\beta \delta}\right) \widehat{u}_{t}+\ldots \\
\phi_{F}\left(\frac{1-\gamma_{x}}{1-\gamma_{x}^{a}}\right) \sigma^{-1} \widehat{c}_{t}^{a R}-\left(\frac{1+(1-\psi) \varphi}{\psi}\right) \widehat{r s}_{t}-\left(\frac{1+(1-\psi) \varphi}{\psi}+\varphi \eta\right)\left(\widehat{p}_{t}^{H, W}-\widehat{p}_{t}^{W}\right)+\ldots \\
\left(\frac{1+(1-\psi) \varphi}{\psi}\right) \phi_{H}\left(\widehat{p}_{t}^{H, R}-\widehat{p}_{t}^{R}\right)-\left(\frac{1+\varphi}{\psi}\right)\left(\widehat{a}_{t}-(1-\psi) \widehat{r}_{t}^{z}\right)
\end{array}\right] \\
& \widehat{\pi}_{t}^{F} \approx \beta \mathbb{E}_{t}\left(\widehat{\pi}_{t+1}^{F}\right)+\ldots \\
& \left(\frac{\psi}{1+(1-\psi) \varphi}\right) \Psi\left[\begin{array}{c}
\left(\left(\frac{1-\gamma_{x}}{1-\gamma_{x}^{a}}\right) \sigma^{-1}+\left(1-\gamma_{x}\right) \varphi\right) \widehat{c}_{t}^{a W *}+\gamma_{x} \varphi \widehat{x}_{t}^{a W *}+\varphi \gamma_{x^{*}}^{y}\left(\frac{1-\beta(1-\delta)}{\beta \delta}\right) \widehat{u}_{t}^{*}-\ldots \\
\phi_{F}\left(\frac{1-\gamma_{x}}{1-\gamma_{x *}^{a}}\right) \sigma^{-1} \widehat{c}_{t}^{a R}+\left(\frac{1+(1-\psi) \varphi}{\psi}\right) \widehat{r s}_{t}-\left(\frac{1+(1-\psi) \varphi}{\psi}+\varphi \eta\right)\left(\widehat{p}_{t}^{F, W *}-\widehat{p}_{t}^{W *}\right)-\ldots \\
\left(\frac{1+(1-\psi) \varphi}{\psi}\right) \phi_{H}\left(\widehat{p}_{t}^{F, R}-\widehat{p}_{t}^{R}\right)-\left(\frac{1+\varphi}{\psi}\right)\left(\widehat{a}_{t}^{*}-(1-\psi) \widehat{r}_{t}^{z *}\right)
\end{array}\right], \\
& \widehat{\pi}_{t}^{F *} \approx \beta \mathbb{E}_{t}\left(\widehat{\pi}_{t+1}^{F *}\right)+\ldots \\
& \left(\frac{\psi}{1+(1-\psi) \varphi}\right) \Psi\left[\begin{array}{c}
\left(\left(\frac{1-\gamma_{x}}{1-\gamma_{x^{*}}^{a}}\right) \sigma^{-1}+\left(1-\gamma_{x}\right) \varphi\right) \widehat{c}_{t}^{a W *}+\gamma_{x} \varphi \widehat{x}_{t}^{a W *}+\varphi \gamma_{x^{*}}^{y}\left(\frac{1-\beta(1-\delta)}{\beta \delta}\right) \widehat{u}_{t}^{*}-\ldots \\
\phi_{F}\left(\frac{1-\gamma_{x}}{1-\gamma_{x^{*}}^{a}}\right) \sigma^{-1} \widehat{c}_{t}^{a R}-\left(\frac{1+(1-\psi) \varphi}{\psi}+\varphi \eta\right)\left(\widehat{p}_{t}^{F, W *}-\widehat{p}_{t}^{W *}\right)+\ldots \\
\left(\frac{1+(1-\psi) \varphi}{\psi}\right) \phi_{F}\left(\widehat{p}_{t}^{F, R}-\widehat{p}_{t}^{R}\right)-\left(\frac{1+\varphi}{\psi}\right)\left(\widehat{a}_{t}^{*}-(1-\psi) \widehat{r}_{t}^{z *}\right)
\end{array}\right] .
\end{aligned}
$$

By appropriately replacing the efficiency conditions in (402) - (403), and after a little bit of algebra, I can 
now re-arrange the pricing equations for the price sub-indexes in (406) - (409) as follows,

$$
\begin{aligned}
& \widehat{\pi}_{t}^{H} \approx \beta \mathbb{E}_{t}\left(\widehat{\pi}_{t+1}^{H}\right)+\ldots \\
& \Psi\left[\begin{array}{c}
\left(\left(\frac{1-\gamma_{x}}{1-\gamma_{x}^{a}}\right) \sigma^{-1}+\left(1-\gamma_{x}\right)\left(\frac{\psi}{1+(1-\psi) \varphi}\right)\left(\varphi+(1-\psi)\left(\frac{1+\varphi}{\psi}\right)^{2}\right)\right) \widehat{c}_{t}^{a W}+\ldots \\
\gamma_{x}\left(\frac{\psi}{1+(1-\psi) \varphi}\right)\left(\varphi+(1-\psi)\left(\frac{1+\varphi}{\psi}\right)^{2}\right) \widehat{x}_{t}^{a W}+\ldots \\
\gamma_{x}^{y}\left(\frac{\psi}{1+(1-\psi) \varphi}\right)\left(\varphi+(1-\psi)\left(\frac{1+\varphi}{\psi}\right)^{2}\right)\left(\frac{1-\beta(1-\delta)}{\beta \delta}\right) \widehat{u}_{t}+\ldots \\
\phi_{F}\left(\frac{1-\gamma_{x}}{1-\gamma_{x}^{a}}\right) \sigma^{-1} \widehat{c}_{t}^{a R}-\left(1+\eta\left(\frac{\psi}{1+(1-\psi) \varphi}\right)\left(\varphi+(1-\psi)\left(\frac{1+\varphi}{\psi}\right)^{2}\right)\right)\left(\widehat{p}_{t}^{H, W}-\widehat{p}_{t}^{W}\right)-\ldots \\
\phi_{F}\left(\widehat{p}_{t}^{H, R}-\widehat{p}_{t}^{R}\right)-(1-\psi)\left(\frac{1+\varphi}{\psi}\right) \widehat{k}_{t}-\left(\frac{1+\varphi}{\psi}\right) \widehat{a}_{t}
\end{array}\right], \\
& \widehat{\pi}_{t}^{H *} \approx \beta \mathbb{E}_{t}\left(\widehat{\pi}_{t+1}^{H *}\right)+\ldots \\
& {\left[\left(\left(\frac{1-\gamma_{x}}{1-\gamma_{x}^{a}}\right) \sigma^{-1}+\left(1-\gamma_{x}\right)\left(\frac{\psi}{1+(1-\psi) \varphi}\right)\left(\varphi+(1-\psi)\left(\frac{1+\varphi}{\psi}\right)^{2}\right)\right) \widehat{c}_{t}^{a W}+\ldots\right.} \\
& \gamma_{x}\left(\frac{\psi}{1+(1-\psi) \varphi}\right)\left(\varphi+(1-\psi)\left(\frac{1+\varphi}{\psi}\right)^{2}\right) \widehat{x}_{t}^{a W}+\ldots \\
& \Psi \quad \begin{array}{c}
\gamma_{x}^{y}\left(\frac{\psi}{1+(1-\psi) \varphi}\right)\left(\varphi+(1-\psi)\left(\frac{1+\varphi}{\psi}\right)^{2}\right)\left(\frac{1-\beta(1-\delta)}{\beta \delta}\right) \widehat{u}_{t}+\ldots \\
\phi_{F}\left(\frac{1-\gamma_{x}}{1-\gamma_{x}^{a}}\right) \sigma^{-1} \widehat{c}_{t}^{a R}-\widehat{r s}_{t}-\left(1+\eta\left(\frac{\psi}{1+(1-\psi) \varphi}\right)\left(\varphi+(1-\psi)\left(\frac{1+\varphi}{\psi}\right)^{2}\right)\right)\left(\widehat{p}_{t}^{H, W}-\widehat{p}_{t}^{W}\right)+\ldots \\
\phi_{H}\left(\widehat{p}_{t}^{H, R}-\widehat{p}_{t}\right)-(1-\psi)(1+\varphi) \widehat{k}_{t}-\left(\frac{1+\varphi}{\psi}\right) \widehat{a}_{t}
\end{array} \\
& \widehat{\pi}_{t}^{F} \approx \beta \mathbb{E}_{t}\left(\widehat{\pi}_{t+1}^{F}\right)+\ldots \\
& {\left[\left(\left(\frac{1-\gamma_{x}}{1-\gamma_{x^{*}}^{a}}\right) \sigma^{-1}+\left(1-\gamma_{x}\right)\left(\frac{\psi}{1+(1-\psi) \varphi}\right)\left(\varphi+(1-\psi)\left(\frac{1+\varphi}{\psi}\right)^{2}\right)\right) \widehat{c}_{t}^{a W *}+\ldots\right.} \\
& \gamma_{x}\left(\frac{\psi}{1+(1-\psi) \varphi}\right)\left(\varphi+(1-\psi)\left(\frac{1+\varphi}{\psi}\right)^{2}\right) \widehat{x}_{t}^{a W *}+\ldots \\
& \Psi \quad \gamma_{x^{*}}^{y}\left(\frac{\psi}{1+(1-\psi) \varphi}\right)\left(\varphi+(1-\psi)\left(\frac{1+\varphi}{\psi}\right)^{2}\right)\left(\frac{1-\beta(1-\delta)}{\beta \delta}\right) \widehat{u}_{t}^{*}-\ldots \\
& \phi_{F}\left(\frac{1-\gamma_{x}}{1-\gamma_{x^{*}}^{a}}\right) \sigma^{-1} \widehat{c}_{t}^{a R}+\widehat{r s}_{t}-\left(1+\eta\left(\frac{\psi}{1+(1-\psi) \varphi}\right)\left(\varphi+(1-\psi)\left(\frac{1+\varphi}{\psi}\right)^{2}\right)\right)\left(\widehat{p}_{t}^{F, W *}-\widehat{p}_{t}^{W *}\right)-\ldots \\
& \phi_{H}\left(\widehat{p}_{t}^{F, R}-\widehat{p}_{t}^{R}\right)-(1-\psi)\left(\frac{1+\varphi}{\psi}\right) \widehat{k}_{t}^{*}-\left(\frac{1+\varphi}{\psi}\right) \widehat{a}_{t}^{*} \\
& \widehat{\pi}_{t}^{F *} \approx \beta \mathbb{E}_{t}\left(\widehat{\pi}_{t+1}^{F *}\right)+\ldots \\
& \Psi\left[\begin{array}{c}
\left(\left(\frac{1-\gamma_{x}}{1-\gamma_{x^{*}}^{a}}\right) \sigma^{-1}+\left(1-\gamma_{x}\right)\left(\frac{\psi}{1+(1-\psi) \varphi}\right)\left(\varphi+(1-\psi)\left(\frac{1+\varphi}{\psi}\right)^{2}\right)\right) \widehat{c}_{t}^{a W *}+\ldots \\
\gamma_{x}\left(\frac{\psi}{1+(1-\psi) \varphi}\right)\left(\varphi+(1-\psi)\left(\frac{1+\varphi}{\psi}\right)^{2}\right) \widehat{x}_{t}^{a W *}+\ldots \\
\gamma_{x^{*}}^{y}\left(\frac{\psi}{1+(1-\psi) \varphi}\right)\left(\varphi+(1-\psi)\left(\frac{1+\varphi}{\psi}\right)^{2}\right)\left(\frac{1-\beta(1-\delta)}{\beta \delta}\right) \widehat{u}_{t}^{*}-\ldots \\
\phi_{F}\left(\frac{1-\gamma_{x}}{1-\gamma_{x^{*}}^{a}}\right) \sigma^{-1} \widehat{c}_{t}^{a R}-\left(1+\eta\left(\frac{\psi}{1+(1-\psi) \varphi}\right)\left(\varphi+(1-\psi)\left(\frac{1+\varphi}{\psi}\right)^{2}\right)\right)\left(\widehat{p}_{t}^{F, W *}-\widehat{p}_{t}^{W *}\right)+\ldots \\
\phi_{F}\left(\widehat{p}_{t}^{F, R}-\widehat{p}_{t}^{R}\right)-(1-\psi)\left(\frac{1+\varphi}{\psi}\right) \widehat{k}_{t}^{*}-\left(\frac{1+\varphi}{\psi}\right) \widehat{a}_{t}^{*}
\end{array}\right] .
\end{aligned}
$$

I define the world price sub-indexes as $\widehat{p}_{t}^{H, W} \equiv \phi_{H} \widehat{p}_{t}^{H}+\phi_{F} \widehat{p}_{t}^{H *}$ and $\widehat{p}_{t}^{F, W *} \equiv \phi_{F} \widehat{p}_{t}^{F}+\phi_{H} \widehat{p}_{t}^{F *}$. Therefore, I can derive the dynamics of $\widehat{\pi}_{t}^{H, W} \equiv \widehat{p}_{t}^{H, W}-\widehat{p}_{t-1}^{H, W}$ and $\widehat{\pi}_{t}^{F, W *} \equiv \widehat{p}_{t}^{F, W *}-\widehat{p}_{t-1}^{F, W *}$ from the equations above 
as,

$$
\begin{aligned}
& \widehat{\pi}_{t}^{H, W} \approx \beta \mathbb{E}_{t}\left(\widehat{\pi}_{t+1}^{H, W}\right)+\ldots \\
& \Psi\left[\begin{array}{c}
\left(\left(\frac{1-\gamma_{x}}{1-\gamma_{x}^{a}}\right) \sigma^{-1}+\left(1-\gamma_{x}\right)\left(\frac{\psi}{1+(1-\psi) \varphi}\right)\left(\varphi+(1-\psi)\left(\frac{1+\varphi}{\psi}\right)^{2}\right)\right) \widehat{c}_{t}^{a W}+\ldots \\
\gamma_{x}\left(\frac{\psi}{1+(1-\psi) \varphi}\right)\left(\varphi+(1-\psi)\left(\frac{1+\varphi}{\psi}\right)^{2}\right) \widehat{x}_{t}^{a W}+\ldots \\
\gamma_{x}^{y}\left(\frac{\psi}{1+(1-\psi) \varphi}\right)\left(\varphi+(1-\psi)\left(\frac{1+\varphi}{\psi}\right)^{2}\right)\left(\frac{1-\beta(1-\delta)}{\beta \delta}\right) \widehat{u}_{t}+\ldots \\
\phi_{F}\left(\frac{1-\gamma_{x}}{1-\gamma_{x}^{a}}\right) \sigma^{-1} \widehat{c}_{t}^{a R}-\phi_{F} \widehat{r}_{t}-\ldots \\
\left(1+\eta\left(\frac{\psi}{1+(1-\psi) \varphi}\right)\left(\varphi+(1-\psi)\left(\frac{1+\varphi}{\psi}\right)^{2}\right)\right)\left(\widehat{p}_{t}^{H, W}-\widehat{p}_{t}^{W}\right)-\ldots \\
(1-\psi)\left(\frac{1+\varphi}{\psi}\right) \widehat{k}_{t}-\left(\frac{1+\varphi}{\psi}\right) \widehat{a}_{t}
\end{array}\right], \\
& \widehat{\pi}_{t}^{F, W *} \approx \beta \mathbb{E}_{t}\left(\widehat{\pi}_{t+1}^{F, W *}\right)+\ldots \\
& \Psi\left[\begin{array}{c}
\left(\left(\frac{1-\gamma_{x}}{1-\gamma_{x^{*}}^{a}}\right) \sigma^{-1}+\left(1-\gamma_{x}\right)\left(\frac{\psi}{1+(1-\psi) \varphi}\right)\left(\varphi+(1-\psi)\left(\frac{1+\varphi}{\psi}\right)^{2}\right)\right) \widehat{c}_{t}^{a W *}+\ldots \\
\gamma_{x}\left(\frac{\psi}{1+(1-\psi) \varphi}\right)\left(\varphi+(1-\psi)\left(\frac{1+\varphi}{\psi}\right)^{2}\right) \widehat{x}_{t}^{a W *}+\ldots \\
\gamma_{x^{*}}^{y}\left(\frac{\psi}{1+(1-\psi) \varphi}\right)\left(\varphi+(1-\psi)\left(\frac{1+\varphi}{\psi}\right)^{2}\right)\left(\frac{1-\beta(1-\delta)}{\beta \delta}\right) \widehat{u}_{t}^{*}-\ldots \\
\phi_{F}\left(\frac{1-\gamma_{x}}{1-\gamma_{x^{*}}^{a}}\right) \sigma^{-1} \widehat{c}_{t}^{a R}+\phi_{F} \widehat{r}_{t}-\ldots \\
\left(1+\eta\left(\frac{\psi}{1+(1-\psi) \varphi}\right)\left(\varphi+(1-\psi)\left(\frac{1+\varphi}{\psi}\right)^{2}\right)\right)\left(\widehat{p}_{t}^{F, W *}-\widehat{p}_{t}^{W *}\right)-\ldots \\
(1-\psi)\left(\frac{1+\varphi}{\psi}\right) \widehat{k}_{t}^{*}-\left(\frac{1+\varphi}{\psi}\right) \widehat{a}_{t}^{*}
\end{array}\right] .
\end{aligned}
$$

I define the domestic and foreign CPI indexes as $\widehat{p}_{t} \approx \phi_{H} \widehat{p}_{t}^{H}+\phi_{F} \widehat{p}_{t}^{F}$ and $\widehat{p}_{t}^{*} \approx \phi_{F} \widehat{p}_{t}^{H *}+\phi_{H} \widehat{p}_{t}^{F *}$, respectively. Therefore, it is easy to derive the dynamics of $\widehat{\pi}_{t} \equiv \widehat{p}_{t}-\widehat{p}_{t-1}$ and $\widehat{\pi}_{t}^{*} \equiv \widehat{p}_{t}^{*}-\widehat{p}_{t-1}^{*}$ from the pricing equations 
above as follows,

$$
\begin{aligned}
& \widehat{\pi}_{t} \approx \beta \mathbb{E}_{t}\left(\widehat{\pi}_{t+1}\right)+\ldots \\
& \Psi\left[\begin{array}{c}
\sigma^{-1}\left[\left(\frac{1-\gamma_{x}}{1-\gamma_{x}^{a}}\right) \phi_{H} \widehat{c}_{t}^{a W}+\left(\frac{1-\gamma_{x}}{1-\gamma_{x^{*}}}\right) \phi_{F} \widehat{c}_{t}^{a W *}\right]+\ldots \\
\left(1-\gamma_{x}\right)\left(\frac{\psi}{1+(1-\psi) \varphi}\right)\left(\varphi+(1-\psi)\left(\frac{1+\varphi}{\psi}\right)^{2}\right)\left[\phi_{H} \widehat{c}_{t}^{a W}+\phi_{F} \widehat{c}_{t}^{a W *}\right]+\ldots \\
\gamma_{x}\left(\frac{\psi}{1+(1-\psi) \varphi}\right)\left(\varphi+(1-\psi)\left(\frac{1+\varphi}{\psi}\right)^{2}\right)\left[\phi_{H} \widehat{x}_{t}^{a W}+\phi_{F} \widehat{x}_{t}^{a W *}\right]+\ldots \\
\gamma_{x}\left(\frac{\psi}{1+(1-\psi) \varphi}\right)\left(\varphi+(1-\psi)\left(\frac{1+\varphi}{\psi}\right)^{2}\right)\left(\frac{1-\beta(1-\delta)}{\beta \delta}\right)\left[\left(\frac{\gamma_{x}^{y}}{\gamma_{x}}\right) \phi_{H} \widehat{u}_{t}+\left(\frac{\gamma_{x^{*}}^{y}}{\gamma_{x}}\right) \phi_{F} \widehat{u}_{t}^{*}\right]+\ldots \\
\phi_{F}\left(\phi_{H}\left(\frac{1-\gamma_{x}}{1-\gamma_{x}^{a}}\right)-\phi_{F}\left(\frac{1-\gamma_{x}}{1-\gamma_{x^{*}}^{a}}\right)\right) \sigma^{-1} \widehat{c}_{t}^{a R}+\phi_{F} \widehat{r s}_{t}-\ldots \\
\left(1+\eta\left(\frac{\psi}{1+(1-\psi) \varphi}\right)\left(\varphi+(1-\psi)\left(\frac{1+\varphi}{\psi}\right)^{2}\right)\right)\left[\phi_{H}\left(\widehat{p}_{t}^{H, W}-\widehat{p}_{t}^{W}\right)+\phi_{F}\left(\widehat{p}_{t}^{F, W *}-\widehat{p}_{t}^{W *}\right)\right]-\ldots \\
\phi_{F} \phi_{H}\left[\left(\widehat{p}_{t}^{H, R}-\widehat{p}_{t}^{R}\right)+\left(\widehat{p}_{t}^{F, R}-\widehat{p}_{t}^{R}\right)\right]-(1-\psi)\left(\frac{1+\varphi}{\psi}\right)\left[\phi_{H} \widehat{k}_{t}+\phi_{F} \widehat{k}_{t}^{*}\right]-\ldots \\
\left(\frac{1+\varphi}{\psi}\right)\left[\phi_{H} \widehat{a}_{t}+\phi_{F} \widehat{a}_{t}^{*}\right]
\end{array}\right], \\
& \widehat{\pi}_{t}^{*} \approx \beta \mathbb{E}_{t}\left(\widehat{\pi}_{t+1}^{*}\right)+\ldots \\
& \Psi\left[\begin{array}{c}
\sigma^{-1}\left[\left(\frac{1-\gamma_{x}}{1-\gamma_{x}^{a}}\right) \phi_{F} \widehat{c}_{t}^{a W}+\left(\frac{1-\gamma_{x}}{1-\gamma_{x}^{a}}\right) \phi_{H} \widehat{c}_{t}^{a W *}\right]+\ldots \\
\left(1-\gamma_{x}\right)\left(\frac{\psi}{1+(1-\psi) \varphi}\right)\left(\varphi+(1-\psi)\left(\frac{1+\varphi}{\psi}\right)^{2}\right)\left[\phi_{F} \widehat{c}_{t}^{a W}+\phi_{H} \widehat{c}_{t}^{a W *}\right]+\ldots \\
\gamma_{x}\left(\frac{\psi}{1+(1-\psi) \varphi}\right)\left(\varphi+(1-\psi)\left(\frac{1+\varphi}{\psi}\right)^{2}\right)\left[\phi_{F} \widehat{x}_{t}^{a W}+\phi_{H} \widehat{x}_{t}^{a W *}\right]+\ldots \\
\gamma_{x}\left(\frac{\psi}{1+(1-\psi) \varphi}\right)\left(\varphi+(1-\psi)\left(\frac{1+\varphi}{\psi}\right)^{2}\right)\left(\frac{1-\beta(1-\delta)}{\beta \delta}\right)\left[\left(\frac{\gamma_{x}^{y}}{\gamma_{x}}\right) \phi_{F} \widehat{u}_{t}+\left(\frac{\gamma_{x^{*}}^{y}}{\gamma_{x}}\right) \phi_{H} \widehat{u}_{t}^{*}\right]-\ldots \\
\phi_{F}\left(\phi_{H}\left(\frac{1-\gamma_{x}}{1-\gamma_{x^{*}}^{a}}\right)-\phi_{F}\left(\frac{1-\gamma_{x}}{1-\gamma_{x}^{a}}\right)\right) \sigma^{-1} \widehat{c}_{t}^{a R}-\phi_{F} \widehat{r s s}_{t}-\ldots \\
\left(1+\eta\left(\frac{\psi}{1+(1-\psi) \varphi}\right)\left(\varphi+(1-\psi)\left(\frac{1+\varphi}{\psi}\right)^{2}\right)\right)\left[\phi_{F}\left(\widehat{p}_{t}^{H, W}-\widehat{p}_{t}^{W}\right)+\phi_{H}\left(\widehat{p}_{t}^{F, W *}-\widehat{p}_{t}^{W *}\right)\right]+\ldots \\
\phi_{F} \phi_{H}\left[\left(\widehat{p}_{t}^{H, R}-\widehat{p}_{t}^{R}\right)+\left(\widehat{p}_{t}^{F, R}-\widehat{p}_{t}^{R}\right)\right]-(1-\psi)\left(\frac{1+\varphi}{\psi}\right)\left[\phi_{F} \widehat{k}_{t}+\phi_{H} \widehat{k}_{t}^{*}\right]-\ldots \\
\left(\frac{1+\varphi}{\psi}\right)\left[\phi_{F} \widehat{a}_{t}+\phi_{H} \widehat{a}_{t}^{*}\right]
\end{array}\right],
\end{aligned}
$$

where world aggregate capital is defined as $\widehat{k}_{t}^{W} \equiv \phi_{H} \widehat{k}_{t}+\phi_{F} \widehat{k}_{t}^{*}$ and $\widehat{k}_{t}^{W *} \equiv \phi_{F} \widehat{k}_{t}+\phi_{H} \widehat{k}_{t}^{*}$. I can also write 
certain terms inside the brackets of the Phillips curves more compactly as,

$$
\begin{aligned}
- & \left(1+\eta\left(\frac{\psi}{1+(1-\psi) \varphi}\right)\left(\varphi+(1-\psi)\left(\frac{1+\varphi}{\psi}\right)^{2}\right)\right)\left[\phi_{H}\left(\widehat{p}_{t}^{H, W}-\widehat{p}_{t}^{W}\right)+\phi_{F}\left(\widehat{p}_{t}^{F, W *}-\widehat{p}_{t}^{W *}\right)\right]-\ldots \\
& \phi_{H} \phi_{F}\left[\left(\widehat{p}_{t}^{H, R}-\widehat{p}_{t}^{R}\right)+\left(\widehat{p}_{t}^{F, R}-\widehat{p}_{t}^{R}\right)\right]=\eta\left(\frac{\psi}{1+(1-\psi) \varphi}\right)\left(\varphi+(1-\psi)\left(\frac{1+\varphi}{\psi}\right)^{2}\right)\left[\phi_{H}-\phi_{F}\right]\left(\widehat{p}_{t}^{F, W *}-\widehat{p}_{t}^{W *}\right)-\ldots \\
& \eta\left(\frac{\psi}{1+(1-\psi) \varphi}\right)\left(\varphi+(1-\psi)\left(\frac{1+\varphi}{\psi}\right)^{2}\right) \phi_{H}\left[\left(\widehat{p}_{t}^{H, W}-\widehat{p}_{t}^{W}\right)+\left(\widehat{p}_{t}^{F, W *}-\widehat{p}_{t}^{W *}\right)\right]-\ldots \\
& \phi_{H}\left[\left(\widehat{p}_{t}^{H, W}-\widehat{p}_{t}^{W}\right)+\phi_{F}\left(\widehat{p}_{t}^{H, R}-\widehat{p}_{t}^{R}\right)\right]-\phi_{F}\left[\left(\widehat{p}_{t}^{F, W *}-\widehat{p}_{t}^{W *}\right)+\phi_{H}\left(\widehat{p}_{t}^{F, R}-\widehat{p}_{t}^{R}\right)\right], \\
- & \left(1+\eta\left(\frac{\psi}{1+(1-\psi) \varphi}\right)\left(\varphi+(1-\psi)\left(\frac{1+\varphi}{\psi}\right)^{2}\right)\right)\left[\phi_{F}\left(\widehat{p}_{t}^{H, W}-\widehat{p}_{t}^{W}\right)+\phi_{H}\left(\widehat{p}_{t}^{F, W *}-\widehat{p}_{t}^{W *}\right)\right]+\ldots \\
& \phi_{F} \phi_{H}\left[\left(\widehat{p}_{t}^{H, R}-\widehat{p}_{t}^{R}\right)+\left(\widehat{p}_{t}^{F, R}-\widehat{p}_{t}^{R}\right)\right]=-\eta\left(\frac{\psi}{1+(1-\psi) \varphi}\right)\left(\varphi+(1-\psi)\left(\frac{1+\varphi}{\psi}\right)^{2}\right)\left[\phi_{H}-\phi_{F}\right]\left(\widehat{p}_{t}^{F, W *}-\widehat{p}_{t}^{W *}\right)-\ldots \\
& \eta\left(\frac{\psi}{1+(1-\psi) \varphi}\right)\left(\varphi+(1-\psi)\left(\frac{1+\varphi}{\psi}\right)^{2}\right) \phi_{F}\left[\left(\widehat{p}_{t}^{H, W}-\widehat{p}_{t}^{W}\right)+\left(\widehat{p}_{t}^{F, W *}-\widehat{p}_{t}^{W *}\right)\right]-\ldots \\
& \phi_{F}\left[\left(\widehat{p}_{t}^{H, W}-\widehat{p}_{t}^{W}\right)-\phi_{H}\left(\widehat{p}_{t}^{H, R}-\widehat{p}_{t}^{R}\right)\right]-\phi_{H}\left[\left(\widehat{p}_{t}^{F, W *}-\widehat{p}_{t}^{W *}\right)-\phi_{F}\left(\widehat{p}_{t}^{F, R}-\widehat{p}_{t}^{R}\right)\right] .
\end{aligned}
$$

Based on my definitions of the world aggregates, denoted with the superscripts $W$ and $W^{*}$, it is possible to argue that,

$$
\begin{aligned}
& \phi_{H}\left[\left(\widehat{p}_{t}^{H, W}-\widehat{p}_{t}^{W}\right)+\phi_{F}\left(\widehat{p}_{t}^{H, R}-\widehat{p}_{t}^{R}\right)\right]+\phi_{F}\left[\left(\widehat{p}_{t}^{F, W *}-\widehat{p}_{t}^{W *}\right)+\phi_{H}\left(\widehat{p}_{t}^{F, R}-\widehat{p}_{t}^{R}\right)\right] \\
& \quad=\phi_{H}\left(\widehat{p}_{t}^{H}-\widehat{p}_{t}\right)+\phi_{F}\left(\widehat{p}_{t}^{F}-\widehat{p}_{t}\right)=0, \\
& \phi_{F}\left[\left(\widehat{p}_{t}^{H, W}-\widehat{p}_{t}^{W}\right)-\phi_{H}\left(\widehat{p}_{t}^{H, R}-\widehat{p}_{t}^{R}\right)\right]+\phi_{H}\left[\left(\widehat{p}_{t}^{F, W *}-\widehat{p}_{t}^{W *}\right)-\phi_{F}\left(\widehat{p}_{t}^{F, R}-\widehat{p}_{t}^{R}\right)\right] \\
& \quad=\phi_{F}\left(\widehat{p}_{t}^{H *}-\widehat{p}_{t}^{*}\right)+\phi_{H}\left(\widehat{p}_{t}^{F *}-\widehat{p}_{t}^{*}\right)=0,
\end{aligned}
$$

since $\widehat{p}_{t}=\phi_{H} \widehat{p}_{t}^{H}+\phi_{F} \widehat{p}_{t}^{F}$ and $\widehat{p}_{t}^{*}=\phi_{F} \widehat{p}_{t}^{H *}+\phi_{H} \widehat{p}_{t}^{F *}$. Furthermore, I also know based on those same definitions, that the following result must hold true,

$$
\begin{aligned}
& \left(\widehat{p}_{t}^{H, W}-\widehat{p}_{t}^{W}\right)+\left(\widehat{p}_{t}^{F, W *}-\widehat{p}_{t}^{W *}\right)=\widehat{p}_{t}^{H, W}+\widehat{p}_{t}^{F, W *}-\left(\widehat{p}_{t}^{W}+\widehat{p}_{t}^{W *}\right) \\
& \quad=\phi_{H}\left(\widehat{p}_{t}^{H}-\widehat{p}_{t}\right)+\phi_{F}\left(\widehat{p}_{t}^{H *}-\widehat{p}_{t}^{*}\right)+\phi_{F}\left(\widehat{p}_{t}^{F}-\widehat{p}_{t}\right)+\phi_{H}\left(\widehat{p}_{t}^{F *}-\widehat{p}_{t}^{*}\right) \\
& \quad=\left[\phi_{H}\left(\widehat{p}_{t}^{H}-\widehat{p}_{t}\right)+\phi_{F}\left(\widehat{p}_{t}^{F}-\widehat{p}_{t}\right)\right]+\left[\phi_{F}\left(\widehat{p}_{t}^{H *}-\widehat{p}_{t}^{*}\right)+\phi_{H}\left(\widehat{p}_{t}^{F *}-\widehat{p}_{t}^{*}\right)\right]=0 .
\end{aligned}
$$

Hence, I argue that those particular terms inside the brackets of the Phillips curves can be simplified as,

$$
\begin{aligned}
- & \left(1+\eta\left(\frac{\psi}{1+(1-\psi) \varphi}\right)\left(\varphi+(1-\psi)\left(\frac{1+\varphi}{\psi}\right)^{2}\right)\right)\left[\phi_{H}\left(\widehat{p}_{t}^{H, W}-\widehat{p}_{t}^{W}\right)+\phi_{F}\left(\widehat{p}_{t}^{F, W *}-\widehat{p}_{t}^{W *}\right)\right]-\ldots \\
& \phi_{H} \phi_{F}\left[\left(\widehat{p}_{t}^{H, R}-\widehat{p}_{t}^{R}\right)+\left(\widehat{p}_{t}^{F, R}-\widehat{p}_{t}^{R}\right)\right]=\eta\left(\frac{\psi}{1+(1-\psi) \varphi}\right)\left(\varphi+(1-\psi)\left(\frac{1+\varphi}{\psi}\right)^{2}\right)\left[\phi_{H}-\phi_{F}\right]\left(\widehat{p}_{t}^{F, W *}-\widehat{p}_{t}^{W *}\right), \\
- & \left(1+\eta\left(\frac{\psi}{1+(1-\psi) \varphi}\right)\left(\varphi+(1-\psi)\left(\frac{1+\varphi}{\psi}\right)^{2}\right)\right)\left[\phi_{F}\left(\widehat{p}_{t}^{H, W}-\widehat{p}_{t}^{W}\right)+\phi_{H}\left(\widehat{p}_{t}^{F, W *}-\widehat{p}_{t}^{W *}\right)\right]+\ldots \\
& \phi_{F} \phi_{H}\left[\left(\widehat{p}_{t}^{H, R}-\widehat{p}_{t}^{R}\right)+\left(\widehat{p}_{t}^{F, R}-\widehat{p}_{t}^{R}\right)\right]=-\eta\left(\frac{\psi}{1+(1-\psi) \varphi}\right)\left(\varphi+(1-\psi)\left(\frac{1+\varphi}{\psi}\right)^{2}\right)\left[\phi_{H}-\phi_{F}\right]\left(\widehat{p}_{t}^{F, W *}-\widehat{p}_{t}^{W *}\right) .
\end{aligned}
$$


I conclude that both Phillips curves in the model take the following form,

$$
\begin{aligned}
& \widehat{\pi}_{t} \approx \beta \mathbb{E}_{t}\left(\widehat{\pi}_{t+1}\right)+\ldots \\
& \Psi\left[\begin{array}{c}
\sigma^{-1}\left[\left(\frac{1-\gamma_{x}}{1-\gamma_{x}^{a}}\right) \phi_{H} \widehat{c}_{t}^{a W}+\left(\frac{1-\gamma_{x}}{1-\gamma_{x^{*}}}\right) \phi_{F} \widehat{c}_{t}^{a W *}\right]+\ldots \\
\left(1-\gamma_{x}\right)\left(\frac{\psi}{1+(1-\psi) \varphi}\right)\left(\varphi+(1-\psi)\left(\frac{1+\varphi}{\psi}\right)^{2}\right)\left[\phi_{H} \widehat{c}_{t}^{a W}+\phi_{F} \widehat{c}_{t}^{a W *}\right]+\ldots \\
\gamma_{x}\left(\frac{\psi}{1+(1-\psi) \varphi}\right)\left(\varphi+(1-\psi)\left(\frac{1+\varphi}{\psi}\right)^{2}\right)\left[\phi_{H} \widehat{x}_{t}^{a W}+\phi_{F} \widehat{x}_{t}^{a W *}\right]+\ldots \\
\gamma_{x}\left(\frac{\psi}{1+(1-\psi) \varphi}\right)\left(\varphi+(1-\psi)\left(\frac{1+\varphi}{\psi}\right)^{2}\right)\left(\frac{1-\beta(1-\delta)}{\beta \delta}\right)\left[\left(\frac{\gamma_{x}^{y}}{\gamma_{x}}\right) \phi_{H} \widehat{u}_{t}+\left(\frac{\gamma_{x^{*}}^{y}}{\gamma_{x}}\right) \phi_{F} \widehat{u}_{t}^{*}\right]+\ldots \\
\phi_{F}\left(\phi_{H}\left(\frac{1-\gamma_{x}}{1-\gamma_{x}^{a}}\right)-\phi_{F}\left(\frac{1-\gamma_{x}}{1-\gamma_{x^{*}}^{a}}\right)\right) \sigma^{-1} \widehat{c}_{t}^{a R}+\phi_{F} \widehat{r}_{t}+\ldots \\
\left(\phi_{H}-\phi_{F}\right) \eta\left(\frac{\psi}{1+(1-\psi) \varphi}\right)\left(\varphi+(1-\psi)\left(\frac{1+\varphi}{\psi}\right)^{2}\right)\left(\widehat{p}_{t}^{F, W *}-\widehat{p}_{t}^{W *}\right)-\ldots \\
(1-\psi)\left(\frac{1+\varphi}{\psi}\right) \widehat{k}_{t}^{W}-\left(\frac{1+\varphi}{\psi}\right)\left[\phi_{H} \widehat{a}_{t}+\phi_{F} \widehat{a}_{t}^{*}\right]
\end{array}\right], \\
& \widehat{\pi}_{t}^{*} \approx \beta \mathbb{E}_{t}\left(\widehat{\pi}_{t+1}^{*}\right)+\ldots \\
& \Psi\left[\begin{array}{c}
\sigma^{-1}\left[\left(\frac{1-\gamma_{x}}{1-\gamma_{x}^{a}}\right) \phi_{F} \widehat{c}_{t}^{a W}+\left(\frac{1-\gamma_{x}}{1-\gamma_{x^{*}}}\right) \phi_{H} \widehat{c}_{t}^{a W *}\right]+\ldots \\
\left(1-\gamma_{x}\right)\left(\frac{\psi}{1+(1-\psi) \varphi}\right)\left(\varphi+(1-\psi)\left(\frac{1+\varphi}{\psi}\right)^{2}\right)\left[\phi_{F} \widehat{c}_{t}^{a W}+\phi_{H} \widehat{c}_{t}^{a W *}\right]+\ldots \\
\gamma_{x}\left(\frac{\psi}{1+(1-\psi) \varphi}\right)\left(\varphi+(1-\psi)\left(\frac{1+\varphi}{\psi}\right)^{2}\right)\left[\phi_{F} \widehat{x}_{t}^{a W}+\phi_{H} \widehat{x}_{t}^{a W *}\right]+\ldots \\
\gamma_{x}\left(\frac{\psi}{1+(1-\psi) \varphi}\right)\left(\varphi+(1-\psi)\left(\frac{1+\varphi}{\psi}\right)^{2}\right)\left(\frac{1-\beta(1-\delta)}{\beta \delta}\right)\left[\left(\frac{\gamma_{x}^{y}}{\gamma_{x}}\right) \phi_{F} \widehat{u}_{t}+\left(\frac{\gamma_{x}^{y}}{\gamma_{x}}\right) \phi_{H} \widehat{u}_{t}^{*}\right]-\ldots \\
\phi_{F}\left(\phi_{H}\left(\frac{1-\gamma_{x}}{1-\gamma_{x *}^{a}}\right)-\phi_{F}\left(\frac{1-\gamma_{x}}{1-\gamma_{x}^{a}}\right)\right) \sigma^{-1} \widehat{c}_{t}^{a R}-\phi_{F} \widehat{r s}_{t}-\ldots \\
\left(\phi_{H}-\phi_{F}\right) \eta\left(\frac{\psi}{1+(1-\psi) \varphi}\right)\left(\varphi+(1-\psi)\left(\frac{1+\varphi}{\psi}\right)^{2}\right)\left(\widehat{p}_{t}^{F, W *}-\widehat{p}_{t}^{W *}\right)-\ldots \\
(1-\psi)\left(\frac{1+\varphi}{\psi}\right) \widehat{k}_{t}^{W *}-\left(\frac{1+\varphi}{\psi}\right)\left[\phi_{F} \widehat{a}_{t}+\phi_{H} \widehat{a}_{t}^{*}\right]
\end{array}\right]
\end{aligned}
$$

which extends the specification in models like those of Steinsson (2008) by adding capital, investment, and variable capital utilization.

With a little bit of additional algebra, it is possible to obtain a simpler characterization of the Phillips curves as,

$$
\begin{aligned}
& \widehat{\pi}_{t}^{H, W} \approx \beta \mathbb{E}_{t}\left(\widehat{\pi}_{t+1}^{H, W}\right)+\ldots \\
& \Psi\left[\begin{array}{c}
\left(\left(\frac{1-\gamma_{x}}{1-\gamma_{x}^{a}}\right) \sigma^{-1}+\left(1-\gamma_{x}\right) \varphi\left(\frac{\varphi \psi^{2}+(1-\psi)(1+\varphi)^{2}}{\varphi \psi+(1-\psi) \psi \varphi^{2}}\right)\right) \widehat{c}_{t}^{a W}+\gamma_{x} \varphi\left(\frac{\varphi \psi^{2}+(1-\psi)(1+\varphi)^{2}}{\varphi \psi+(1-\psi) \psi \varphi^{2}}\right) \widehat{x}_{t}^{a W}+\ldots \\
\gamma_{x}^{y} \varphi\left(\frac{\varphi \psi^{2}+(1-\psi)(1+\varphi)^{2}}{\varphi \psi+(1-\psi) \psi \varphi^{2}}\right)\left(\frac{1-\beta(1-\delta)}{\beta \delta}\right) \widehat{u}_{t}+\ldots \\
\phi_{F}\left(\frac{1-\gamma_{x}}{1-\gamma_{x}^{a}}\right) \sigma^{-1} \widehat{c}_{t}^{a R}-\phi_{F} \widehat{r s}_{t}-\left(1+\eta \varphi\left(\frac{\varphi \psi^{2}+(1-\psi)(1+\varphi)^{2}}{\varphi \psi+(1-\psi) \psi \varphi^{2}}\right)\right)\left(\widehat{p}_{t}^{H, W}-\widehat{p}_{t}^{W}\right)-\ldots \\
\left(\frac{(1-\psi)(1+\varphi)}{\psi}\right) \widehat{k}_{t}-\left(\frac{1+\varphi}{\psi}\right) \widehat{a}_{t}
\end{array}\right] \\
& \widehat{\pi}_{t}^{F, W *} \approx \beta \mathbb{E}_{t}\left(\widehat{\pi}_{t+1}^{F, W *}\right)+\ldots \\
& \Psi\left[\begin{array}{c}
\left(\left(\frac{1-\gamma_{x}}{1-\gamma_{x}^{a}}\right) \sigma^{-1}+\left(1-\gamma_{x}\right) \varphi\left(\frac{\varphi \psi^{2}+(1-\psi)(1+\varphi)^{2}}{\varphi \psi+(1-\psi) \psi \varphi^{2}}\right)\right) \widehat{c}_{t}^{a W *}+\gamma_{x} \varphi\left(\frac{\varphi \psi^{2}+(1-\psi)(1+\varphi)^{2}}{\varphi \psi+(1-\psi) \psi \varphi^{2}}\right) \widehat{x}_{t}^{a W *}+\ldots \\
\gamma_{x^{*}}^{y} \varphi\left(\frac{\varphi \psi^{2}+(1-\psi)(1+\varphi)^{2}}{\varphi \psi+(1-\psi) \psi \varphi^{2}}\right)\left(\frac{1-\beta(1-\delta)}{\beta \delta}\right) \widehat{u}_{t}^{*}-\ldots \\
\phi_{F}\left(\frac{1-\gamma_{x}}{1-\gamma_{x^{*}}}\right) \sigma^{-1} \widehat{c}_{t}^{a R}+\phi_{F} \widehat{r s}_{t}-\left(1+\eta \varphi\left(\frac{\varphi \psi^{2}+(1-\psi)(1+\varphi)^{2}}{\varphi \psi+(1-\psi) \psi \varphi^{2}}\right)\right)\left(\widehat{p}_{t}^{F, W *}-\widehat{p}_{t}^{W *}\right)-\ldots \\
\left(\frac{(1-\psi)(1+\varphi)}{\psi}\right) \widehat{k}_{t}^{*}-\left(\frac{1+\varphi}{\psi}\right) \widehat{a}_{t}^{*}
\end{array}\right],
\end{aligned}
$$


and, naturally,

$$
\begin{aligned}
& \widehat{\pi}_{t} \approx \beta \mathbb{E}_{t}\left(\widehat{\pi}_{t+1}\right)+\ldots \\
& \Psi\left[\begin{array}{c}
\sigma^{-1}\left[\left(\frac{1-\gamma_{x}}{1-\gamma_{x}^{a}}\right) \phi_{H} \widehat{c}_{t}^{a W}+\left(\frac{1-\gamma_{x}}{1-\gamma_{x}^{a}}\right) \phi_{F} \widehat{c}_{t}^{a W *}\right]+\ldots \\
\left(1-\gamma_{x}\right) \varphi\left(\frac{\varphi \psi^{2}+(1-\psi)(1+\varphi)^{2}}{\varphi \psi+(1-\psi) \psi \varphi^{2}}\right)\left[\phi_{H} \widehat{c}_{t}^{a W}+\phi_{F} \widehat{c}_{t}^{a W *}\right]+\ldots \\
\gamma_{x} \varphi\left(\frac{\varphi \psi^{2}+(1-\psi)(1+\varphi)^{2}}{\varphi \psi+(1-\psi) \psi \varphi^{2}}\right)\left[\phi_{H} \widehat{x}_{t}^{a W}+\phi_{F} \widehat{x}_{t}^{a W *}\right]+\ldots \\
\gamma_{x} \varphi\left(\frac{\varphi \psi^{2}+(1-\psi)(1+\varphi)^{2}}{\varphi \psi+(1-\psi) \psi \varphi^{2}}\right)\left(\frac{1-\beta(1-\delta)}{\beta \delta}\right)\left[\left(\frac{\gamma_{x}^{y}}{\gamma_{x}}\right) \phi_{H} \widehat{u}_{t}+\left(\frac{\gamma_{x^{*}}^{y}}{\gamma_{x}}\right) \phi_{F} \widehat{u}_{t}^{*}\right]+\ldots \\
\phi_{F}\left(\phi_{H}\left(\frac{1-\gamma_{x}}{1-\gamma_{x}^{a}}\right)-\phi_{F}\left(\frac{1-\gamma_{x}}{1-\gamma_{x}^{a}}\right)\right) \sigma^{-1} \widehat{c}_{t}^{a R}+\phi_{F} \widehat{r s}_{t}+\ldots \\
\left(\phi_{H}-\phi_{F}\right) \eta \varphi\left(\frac{\varphi \psi^{2}+(1-\psi)(1+\varphi)^{2}}{\varphi \psi+(1-\psi) \psi \varphi^{2}}\right)\left(\widehat{p}_{t}^{F, W *}-\widehat{p}_{t}^{W *}\right)-\ldots \\
\left(\frac{(1-\psi)(1+\varphi)}{\psi}\right) \widehat{k}_{t}^{W}-\left(\frac{1+\varphi}{\psi}\right)\left[\phi_{H} \widehat{a}_{t}+\phi_{F} \widehat{a}_{t}^{*}\right]
\end{array}\right], \\
& \widehat{\pi}_{t}^{*} \approx \beta \mathbb{E}_{t}\left(\widehat{\pi}_{t+1}^{*}\right)+\ldots \\
& \Psi\left[\begin{array}{c}
\sigma^{-1}\left[\left(\frac{1-\gamma_{x}}{1-\gamma_{x}^{a}}\right) \phi_{F} \widehat{c}_{t}^{a W}+\left(\frac{1-\gamma_{x}}{1-\gamma_{x}^{a}}\right) \phi_{H} \widehat{c}_{t}^{a W *}\right]+\ldots \\
\left(1-\gamma_{x}\right) \varphi\left(\frac{\varphi \psi^{2}+(1-\psi)(1+\varphi)^{2}}{\varphi \psi+(1-\psi) \psi \varphi^{2}}\right)\left[\phi_{F} \widehat{c}_{t}^{a W}+\phi_{H} \widehat{c}_{t}^{a W *}\right]+\ldots \\
\gamma_{x} \varphi\left(\frac{\varphi \psi^{2}+(1-\psi)(1+\varphi)^{2}}{\varphi \psi+(1-\psi) \psi \varphi^{2}}\right)\left[\phi_{F} \widehat{x}_{t}^{a W}+\phi_{H} \widehat{x}_{t}^{a W *}\right]+\ldots \\
\gamma_{x} \varphi\left(\frac{\varphi \psi^{2}+(1-\psi)(1+\varphi)^{2}}{\varphi \psi+(1-\psi) \psi \varphi^{2}}\right)\left(\frac{1-\beta(1-\delta)}{\beta \delta}\right)\left[\left(\frac{\gamma_{x}^{y}}{\gamma_{x}}\right) \phi_{F} \widehat{u}_{t}+\left(\frac{\gamma_{x}^{y}}{\gamma_{x}}\right) \phi_{H} \widehat{u}_{t}^{*}\right]-\ldots \\
\phi_{F}\left(\phi_{H}\left(\frac{1-\gamma_{x}}{1-\gamma_{x}^{a}}\right)-\phi_{F}\left(\frac{1-\gamma_{x}}{1-\gamma_{x}^{a}}\right)\right) \sigma^{-1} \widehat{c}_{t}^{a R}-\phi_{F} \widehat{r s}_{t}-\ldots \\
\left(\phi_{H}-\phi_{F}\right) \eta \varphi\left(\frac{\varphi \psi^{2}+(1-\psi)(1+\varphi)^{2}}{\varphi \psi+(1-\psi) \psi \varphi^{2}}\right)\left(\widehat{p}_{t}^{F, W *}-\widehat{p}_{t}^{W *}\right)-\ldots \\
\left(\frac{(1-\psi)(1+\varphi)}{\psi}\right) \widehat{k}_{t}^{W *}-\left(\frac{1+\varphi}{\psi}\right)\left[\phi_{F} \widehat{a}_{t}+\phi_{H} \widehat{a}_{t}^{*}\right]
\end{array}\right] .
\end{aligned}
$$

Capital appears in the Phillips curves because it captures the impact of the efficiency conditions on the marginal costs of firms. A similar argument can be made regarding the role of capital utilization on the amount of capital services made available to firms. Let me define $\widehat{t}_{t}^{W} \equiv \widehat{p}_{t}^{F, W *}-\widehat{p}_{t}^{W *}$ as the world measure 
of terms of trade in the model. Then, the Phillips curves can be re-expressed as,

$$
\begin{aligned}
& \widehat{\pi}_{t} \approx \beta \mathbb{E}_{t}\left(\widehat{\pi}_{t+1}\right)+\ldots \\
& \Psi\left[\begin{array}{c}
\sigma^{-1}\left[\left(\frac{1-\gamma_{x}}{1-\gamma_{x}^{a}}\right) \phi_{H} \widehat{c}_{t}^{a W}+\left(\frac{1-\gamma_{x}}{1-\gamma_{x}^{a}}\right) \phi_{F} \widehat{c}_{t}^{a W *}\right]+\ldots \\
\left(1-\gamma_{x}\right) \varphi\left(\frac{\varphi \psi^{2}+(1-\psi)(1+\varphi)^{2}}{\varphi \psi+(1-\psi) \psi \varphi^{2}}\right)\left[\phi_{H} \widehat{c}_{t}^{a W}+\phi_{F} \widehat{c}_{t}^{a W *}\right]+\ldots \\
\gamma_{x} \varphi\left(\frac{\varphi \psi^{2}+(1-\psi)(1+\varphi)^{2}}{\varphi \psi+(1-\psi) \psi \varphi^{2}}\right)\left[\phi_{H} \widehat{x}_{t}^{a W}+\phi_{F} \widehat{x}_{t}^{a W *}\right]+\ldots \\
\gamma_{x} \varphi\left(\frac{\varphi \psi^{2}+(1-\psi)(1+\varphi)^{2}}{\varphi \psi+(1-\psi) \psi \varphi^{2}}\right)\left(\frac{1-\beta(1-\delta)}{\beta \delta}\right)\left[\left(\frac{\gamma_{x}^{y}}{\gamma_{x}}\right) \phi_{H} \widehat{u}_{t}+\left(\frac{\gamma_{x^{*}}^{y}}{\gamma_{x}}\right) \phi_{F} \widehat{u}_{t}^{*}\right]+\ldots \\
\phi_{F}\left(\phi_{H}\left(\frac{1-\gamma_{x}}{1-\gamma_{x}^{a}}\right)-\phi_{F}\left(\frac{1-\gamma_{x}}{1-\gamma_{x}^{*}}\right)\right) \sigma^{-1} \widehat{c}_{t}^{a}+\phi_{F} \widehat{r s}_{t}+\ldots \\
\left(\phi_{H}-\phi_{F}\right) \eta \varphi\left(\frac{\varphi \psi^{2}+(1-\psi)(1+\varphi)^{2}}{\varphi \psi+(1-\psi) \psi \varphi^{2}}\right) \widehat{t}_{t}^{W}-\ldots \\
\left(\frac{(1-\psi)(1+\varphi)}{\psi}\right) \widehat{k}_{t}^{W}-\left(\frac{1+\varphi}{\psi}\right)\left[\phi_{H} \widehat{a}_{t}+\phi_{F} \widehat{a}_{t}^{*}\right]
\end{array}\right], \\
& \widehat{\pi}_{t}^{*} \approx \beta \mathbb{E}_{t}\left(\widehat{\pi}_{t+1}^{*}\right)+\ldots \\
& \Psi\left[\begin{array}{c}
\sigma^{-1}\left[\left(\frac{1-\gamma_{x}}{1-\gamma_{x}^{a}}\right) \phi_{F} \widehat{c}_{t}^{a W}+\left(\frac{1-\gamma_{x}}{1-\gamma_{x^{*}}^{a}}\right) \phi_{H} \widehat{c}_{t}^{a W *}\right]+\ldots \\
\left(1-\gamma_{x}\right) \varphi\left(\frac{\varphi \psi^{2}+(1-\psi)(1+\varphi)^{2}}{\varphi \psi+(1-\psi) \psi \varphi^{2}}\right)\left[\phi_{F} \widehat{c}_{t}^{a W}+\phi_{H} \widehat{c}_{t}^{a W *}\right]+\ldots \\
\gamma_{x} \varphi\left(\frac{\varphi \psi^{2}+(1-\psi)(1+\varphi)^{2}}{\varphi \psi+(1-\psi) \psi \varphi^{2}}\right)\left[\phi_{F} \widehat{x}_{t}^{a W}+\phi_{H} \widehat{x}_{t}^{a W *}\right]+\ldots \\
\gamma_{x} \varphi\left(\frac{\varphi \psi^{2}+(1-\psi)(1+\varphi)^{2}}{\varphi \psi+(1-\psi) \psi \varphi^{2}}\right)\left(\frac{1-\beta(1-\delta)}{\beta \delta}\right)\left[\left(\frac{\gamma_{x}^{y}}{\gamma_{x}}\right) \phi_{F} \widehat{u}_{t}+\left(\frac{\gamma_{x *}^{y}}{\gamma_{x}}\right) \phi_{H} \widehat{u}_{t}^{*}\right]-\ldots \\
\phi_{F}\left(\phi_{H}\left(\frac{1-\gamma_{x}}{1-\gamma_{x^{*}}}\right)-\phi_{F}\left(\frac{1-\gamma_{x}}{1-\gamma_{x}^{x}}\right)\right) \sigma^{-1} \widehat{c}_{t}^{a R}-\phi_{F} \widehat{r s}_{t}-\ldots \\
\left(\phi_{H}-\phi_{F}\right) \eta \varphi\left(\frac{\varphi \psi^{2}+(1-\psi)(1+\varphi)^{2}}{\varphi \psi+(1-\psi) \psi \varphi^{2}}\right) \widehat{t}_{t}^{W}-\ldots \\
\left(\frac{(1-\psi)(1+\varphi)}{\psi}\right) \widehat{k}_{t}^{W *}-\left(\frac{1+\varphi}{\psi}\right)\left[\phi_{F} \widehat{a}_{t}+\phi_{H} \widehat{a}_{t}^{*}\right]
\end{array}\right]
\end{aligned}
$$

These equations constitute the aggregate supply block in this economy.

The International Relative Prices. I define the world measure of terms of trade in the model as $\widehat{t}_{t}^{W} \equiv\left(\widehat{p}_{t}^{F, W *}-\widehat{p}_{t}^{W *}\right)$. As in the variant of the model without variabla capital utilization in (321), the only constraint that determines the world terms of trade is given by,

$$
\left(\phi_{H}-\phi_{F}\right)\left(\widehat{p}_{t}^{F, W *}-\widehat{p}_{t}^{W *}\right) \approx \phi_{H} \phi_{F}\left[\left(\widehat{p}_{t}^{H, R}-\widehat{p}_{t}^{R}\right)+\left(\widehat{p}_{t}^{F, R}-\widehat{p}_{t}^{R}\right)\right]
$$

If the model has no home-product bias in consumption and investment, i.e. $\phi_{H}=\phi_{F}$, then $\widehat{t}_{t}^{W} \equiv$ $\left(\widehat{p}_{t}^{F, W *}-\widehat{p}_{t}^{W *}\right)$ only matters because it affects aggregate output and aggregate output enters into the specification of the monetary policy rules. Therefore, it must follow from (423) that $\left(\widehat{p}_{t}^{H, R}-\widehat{p}_{t}^{R}\right)+$ $\left(\widehat{p}_{t}^{F, R}-\widehat{p}_{t}^{R}\right) \approx 0$. In that case, the constraint imposes no practical restriction on the world terms of trade $\widehat{t}_{t}^{W} \equiv\left(\widehat{p}_{t}^{F, W *}-\widehat{p}_{t}^{W *}\right)$, and I need to keep track of the price sub-indexes in some other way in order to close down the model.

If the model has a home-product bias in consumption and investment, i.e. $\phi_{H} \neq \phi_{F}$, then $\hat{t}_{t}^{W} \equiv$ $\left(\widehat{p}_{t}^{F, W *}-\widehat{p}_{t}^{W *}\right)$ matters because it affects aggregate output in both countries, and it also matters because it affects the real marginal costs of firms. Moreover, I can write the world terms of trade as follows,

$$
\widehat{t}_{t}^{W} \equiv \widehat{p}_{t}^{F, W *}-\widehat{p}_{t}^{W *}=\frac{\phi_{H} \phi_{F}}{\phi_{H}-\phi_{F}}\left[\left(\widehat{p}_{t}^{H, R}-\widehat{p}_{t}^{R}\right)+\left(\widehat{p}_{t}^{F, R}-\widehat{p}_{t}^{R}\right)\right] .
$$


This expression is crucial to derive the dynamics of world terms of trade. In equations (404) and (405) I already derived a simple characterization for the relative price sub-indexes $\widehat{\pi}_{t}^{H, R}$ and $\widehat{\pi}_{t}^{F, R}$, i.e.,

$$
\begin{aligned}
\widehat{\pi}_{t}^{H, R}-\beta \mathbb{E}_{t}\left(\widehat{\pi}_{t+1}^{H, R}\right)+\Psi\left(\widehat{p}_{t}^{H, R}-\widehat{p}_{t}^{R}\right) & \approx \Psi \widehat{r}_{t}, \\
\widehat{\pi}_{t}^{F, R}-\beta \mathbb{E}_{t}\left(\widehat{\pi}_{t+1}^{F, R}\right)+\Psi\left(\widehat{p}_{t}^{F, R}-\widehat{p}_{t}^{R}\right) & \approx \Psi \widehat{r s}_{t} .
\end{aligned}
$$

Simple manipulations allow me to write this pair of equations as,

$$
\begin{aligned}
\left(\widehat{\pi}_{t}^{H, R}-\widehat{\pi}_{t}^{R}\right)-\beta \mathbb{E}_{t}\left(\widehat{\pi}_{t+1}^{H, R}-\widehat{\pi}_{t+1}^{R}\right)+\Psi\left(\widehat{p}_{t}^{H, R}-\widehat{p}_{t}^{R}\right) & \approx \Psi \widehat{r s}_{t}-\widehat{\pi}_{t}^{R}+\beta \mathbb{E}_{t}\left(\widehat{\pi}_{t+1}^{R}\right), \\
\left(\widehat{\pi}_{t}^{F, R}-\widehat{\pi}_{t}^{R}\right)-\beta \mathbb{E}_{t}\left(\widehat{\pi}_{t+1}^{F, R}-\widehat{\pi}_{t+1}^{R}\right)+\Psi\left(\widehat{p}_{t}^{F, R}-\widehat{p}_{t}^{R}\right) & \approx \Psi \widehat{r}_{t}-\widehat{\pi}_{t}^{R}+\beta \mathbb{E}_{t}\left(\widehat{\pi}_{t+1}^{R}\right),
\end{aligned}
$$

where the relative CPI is defined as $\widehat{p}_{t}^{R} \equiv \widehat{p}_{t}-\widehat{p}_{t}^{*}$. If I use the definition of world terms of trade and I combine it with these two equations, I can write the dynamics of $\hat{t}_{t}^{W}$ as,

$$
\Delta \widehat{t}_{t}^{W}-\beta \mathbb{E}_{t}\left(\Delta \widehat{t}_{t+1}^{W}\right)+\Psi \widehat{t}_{t}^{W} \approx \frac{\phi_{H} \phi_{F}}{\phi_{H}-\phi_{F}}\left[\Psi \widehat{r s}_{t}-\widehat{\pi}_{t}^{R}+\beta \mathbb{E}_{t}\left(\widehat{\pi}_{t+1}^{R}\right)\right]
$$

where I define the first-difference of the world terms of trade as $\Delta \widehat{t}_{t}^{W} \equiv \widehat{t}_{t}^{W}-\widehat{t}_{t-1}^{W}$. This suffices to close down the model, but it is the same equation as in (323) without variable capital utilization.

Following on Engel (forthcoming), I can show that when the degree of price stickiness is the same across firms and markets then the relative prices in each country must be equalized even if the law of one price fails to hold, i.e. $\left(\widehat{p}_{t}^{F}-\widehat{p}_{t}^{H}\right) \approx\left(\widehat{p}_{t}^{F *}-\widehat{p}_{t}^{H *}\right)$. To show this, I start by computing the inflation for the relative prices in each country $\left(\widehat{\pi}_{t}^{F}-\widehat{\pi}_{t}^{H}\right)$ and $\left(\widehat{\pi}_{t}^{F *}-\widehat{\pi}_{t}^{H *}\right)$ from the dynamics of the price sub-indexes in $(410)-(413)$ 
as follows,

$$
\begin{aligned}
& \widehat{\pi}_{t}^{F}-\widehat{\pi}_{t}^{H} \approx \beta \mathbb{E}_{t}\left(\widehat{\pi}_{t+1}^{F}-\widehat{\pi}_{t+1}^{H}\right)+\ldots \\
& \Psi\left[\begin{array}{c}
\sigma^{-1}\left(\left(\frac{1-\gamma_{x}}{1-\gamma_{x}^{a}}\right) \widehat{c}_{t}^{a W *}-\left(\frac{1-\gamma_{x}}{1-\gamma_{x}^{a}}\right) \widehat{c}_{t}^{a W}\right)+\ldots \\
\left(1-\gamma_{x}\right)\left(\frac{\psi}{1+(1-\psi) \varphi}\right)\left(\varphi+(1-\psi)\left(\frac{1+\varphi}{\psi}\right)^{2}\right)\left(\widehat{c}_{t}^{a W *}-\widehat{c}_{t}^{a W}\right)+\ldots \\
\gamma_{x}\left(\frac{\psi}{1+(1-\psi) \varphi}\right)\left(\varphi+(1-\psi)\left(\frac{1+\varphi}{\psi}\right)^{2}\right)\left(\widehat{x}_{t}^{a W *}-\widehat{x}_{t}^{a W}\right)+\ldots \\
\left(\frac{\psi}{1+(1-\psi) \varphi}\right)\left(\varphi+(1-\psi)\left(\frac{1+\varphi}{\psi}\right)^{2}\right)\left(\frac{1-\beta(1-\delta)}{\beta \delta}\right)\left(\gamma_{x^{*}}^{y} \widehat{u}_{t}^{*}-\gamma_{x}^{y} \widehat{u}_{t}\right)-\ldots \\
\phi_{F} \sigma^{-1}\left(\left(\frac{1-\gamma_{x}}{1-\gamma_{x *}^{a}}\right)+\left(\frac{1-\gamma_{x}}{1-\gamma_{x}^{a}}\right)\right) \widehat{c}_{t}^{a R}+\widehat{r s}_{t}-\ldots \\
\left(1+\eta\left(\frac{\psi}{1+(1-\psi) \varphi}\right)\left(\varphi+(1-\psi)\left(\frac{1+\varphi}{\psi}\right)^{2}\right)\right)\left(\left(\widehat{p}_{t}^{F, W *}-\widehat{p}_{t}^{W *}\right)-\left(\widehat{p}_{t}^{H, W}-\widehat{p}_{t}^{W}\right)\right)-\ldots \\
\left(\phi_{H}\left(\widehat{p}_{t}^{F, R}-\widehat{p}_{t}^{R}\right)-\phi_{F}\left(\widehat{p}_{t}^{H, R}-\widehat{p}_{t}^{R}\right)\right)-(1-\psi)\left(\frac{1+\varphi}{\psi}\right)\left(\widehat{k}_{t}^{*}-\widehat{k}_{t}\right)-\left(\frac{1+\varphi}{\psi}\right)\left(\widehat{a}_{t}^{*}-\widehat{a}_{t}\right)
\end{array}\right], \\
& \widehat{\pi}_{t}^{F *}-\widehat{\pi}_{t}^{H *} \approx \beta \mathbb{E}_{t}\left(\widehat{\pi}_{t+1}^{F *}-\widehat{\pi}_{t+1}^{H *}\right)+\ldots \\
& \Psi\left[\begin{array}{c}
\sigma^{-1}\left(\left(\frac{1-\gamma_{x}}{1-\gamma_{x}^{a *}}\right) \widehat{c}_{t}^{a W *}-\left(\frac{1-\gamma_{x}}{1-\gamma_{x}^{a}}\right) \widehat{c}_{t}^{a W}\right)+\ldots \\
\left(1-\gamma_{x}\right)\left(\frac{\psi}{1+(1-\psi) \varphi}\right)\left(\varphi+(1-\psi)\left(\frac{1+\varphi}{\psi}\right)^{2}\right)\left(\widehat{c}_{t}^{a W *}-\widehat{c}_{t}^{a W}\right)+\ldots \\
\gamma_{x}\left(\frac{\psi}{1+(1-\psi) \varphi}\right)\left(\varphi+(1-\psi)\left(\frac{1+\varphi}{\psi}\right)^{2}\right)\left(\widehat{x}_{t}^{a W *}-\widehat{x}_{t}^{a W}\right)+\ldots \\
\left(\frac{\psi}{1+(1-\psi) \varphi}\right)\left(\varphi+(1-\psi)\left(\frac{1+\varphi}{\psi}\right)^{2}\right)\left(\frac{1-\beta(1-\delta)}{\beta \delta}\right)\left(\gamma_{x^{*}}^{y} \widehat{u}_{t}^{*}-\gamma_{x}^{y} \widehat{u}_{t}\right)-\ldots \\
\phi_{F} \sigma^{-1}\left(\left(\frac{1-\gamma_{x}}{1-\gamma_{x^{*}}}\right)+\left(\frac{1-\gamma_{x}}{1-\gamma_{x}^{a}}\right)\right) \widehat{c}_{t}^{a R}+\widehat{r}_{t}-\ldots \\
\left(1+\eta\left(\frac{\psi}{1+(1-\psi) \varphi}\right)\left(\varphi+(1-\psi)\left(\frac{1+\varphi}{\psi}\right)^{2}\right)\right)\left(\left(\widehat{p}_{t}^{F, W *}-\widehat{p}_{t}^{W *}\right)-\left(\widehat{p}_{t}^{H, W}-\widehat{p}_{t}^{W}\right)\right)+\ldots \\
\left(\phi_{F}\left(\widehat{p}_{t}^{F, R}-\widehat{p}_{t}^{R}\right)-\phi_{H}\left(\widehat{p}_{t}^{H, R}-\widehat{p}_{t}^{R}\right)\right)-(1-\psi)\left(\frac{1+\varphi}{\psi}\right)\left(\widehat{k}_{t}^{*}-\widehat{k}_{t}\right)-\left(\frac{1+\varphi}{\psi}\right)\left(\widehat{a}_{t}^{*}-\widehat{a}_{t}\right)
\end{array}\right] .
\end{aligned}
$$

I noted already that by construction $\left(\widehat{p}_{t}^{H, W}-\widehat{p}_{t}^{W}\right)+\left(\widehat{p}_{t}^{F, W *}-\widehat{p}_{t}^{W *}\right) \approx 0$, hence the two expressions for the 
relative prices above become simply,

$$
\begin{aligned}
& \widehat{\pi}_{t}^{F}-\widehat{\pi}_{t}^{H} \approx \beta \mathbb{E}_{t}\left(\widehat{\pi}_{t+1}^{F}-\widehat{\pi}_{t+1}^{H}\right)+\ldots \\
& \Psi\left[\begin{array}{c}
\sigma^{-1}\left(\left(\frac{1-\gamma_{x}}{1-\gamma_{x^{*}}}\right) \widehat{c}_{t}^{a W *}-\left(\frac{1-\gamma_{x}}{1-\gamma_{x}^{a}}\right) \widehat{c}_{t}^{a W}\right)+\ldots \\
\left(1-\gamma_{x}\right)\left(\frac{\psi}{1+(1-\psi) \varphi}\right)\left(\varphi+(1-\psi)\left(\frac{1+\varphi}{\psi}\right)^{2}\right)\left(\widehat{c}_{t}^{a W *}-\widehat{c}_{t}^{a W}\right)+\ldots \\
\gamma_{x}\left(\frac{\psi}{1+(1-\psi) \varphi}\right)\left(\varphi+(1-\psi)\left(\frac{1+\varphi}{\psi}\right)^{2}\right)\left(\widehat{x}_{t}^{a W *}-\widehat{x}_{t}^{a W}\right)+\ldots \\
\left(\frac{\psi}{1+(1-\psi) \varphi}\right)\left(\varphi+(1-\psi)\left(\frac{1+\varphi}{\psi}\right)^{2}\right)\left(\frac{1-\beta(1-\delta)}{\beta \delta}\right)\left(\gamma_{x^{*}}^{y} \widehat{u}_{t}^{*}-\gamma_{x}^{y} \widehat{u}_{t}\right)-\ldots \\
\phi_{F} \sigma^{-1}\left(\left(\frac{1-\gamma_{x}}{1-\gamma_{x}^{a}}\right)+\left(\frac{1-\gamma_{x}}{1-\gamma_{x}^{a}}\right)\right) \widehat{c}_{t}^{a R}+\widehat{r s}_{t}-\ldots \\
2\left(1+\eta\left(\frac{\psi}{1+(1-\psi) \varphi}\right)\left(\varphi+(1-\psi)\left(\frac{1+\varphi}{\psi}\right)^{2}\right)\right)\left(\widehat{p}_{t}^{F, W *}-\widehat{p}_{t}^{W *}\right)-\ldots \\
\left(\phi_{H}\left(\widehat{p}_{t}^{F, R}-\widehat{p}_{t}^{R}\right)-\phi_{F}\left(\widehat{p}_{t}^{H, R}-\widehat{p}_{t}^{R}\right)\right)-(1-\psi)\left(\frac{1+\varphi}{\psi}\right)\left(\widehat{k}_{t}^{*}-\widehat{k}_{t}\right)-\left(\frac{1+\varphi}{\psi}\right)\left(\widehat{a}_{t}^{*}-\widehat{a}_{t}\right)
\end{array}\right], \\
& \widehat{\pi}_{t}^{F *}-\widehat{\pi}_{t}^{H *} \approx \beta \mathbb{E}_{t}\left(\widehat{\pi}_{t+1}^{F *}-\widehat{\pi}_{t+1}^{H *}\right)+\ldots \\
& \Psi\left[\begin{array}{c}
\sigma^{-1}\left(\left(\frac{1-\gamma_{x}}{1-\gamma_{x *}^{a *}}\right) \widehat{c}_{t}^{a W *}-\left(\frac{1-\gamma_{x}}{1-\gamma_{x}^{a}}\right) \widehat{c}_{t}^{a W}\right)+\ldots \\
\left(1-\gamma_{x}\right)\left(\frac{\psi}{1+(1-\psi) \varphi}\right)\left(\varphi+(1-\psi)\left(\frac{1+\varphi}{\psi}\right)^{2}\right)\left(\widehat{c}_{t}^{a W *}-\widehat{c}_{t}^{a W}\right)+\ldots \\
\gamma_{x}\left(\frac{\psi}{1+(1-\psi) \varphi}\right)\left(\varphi+(1-\psi)\left(\frac{1+\varphi}{\psi}\right)^{2}\right)\left(\widehat{x}_{t}^{a W *}-\widehat{x}_{t}^{a W}\right)+\ldots \\
\left(\frac{\psi}{1+(1-\psi) \varphi}\right)\left(\varphi+(1-\psi)\left(\frac{1+\varphi}{\psi}\right)^{2}\right)\left(\frac{1-\beta(1-\delta)}{\beta \delta}\right)\left(\gamma_{x^{*}}^{y} \widehat{u}_{t}^{*}-\gamma_{x}^{y} \widehat{u}_{t}\right)-\ldots \\
\phi_{F} \sigma^{-1}\left(\left(\frac{1-\gamma_{x}}{1-\gamma_{x}^{a}}\right)+\left(\frac{1-\gamma_{x}}{1-\gamma_{x}^{a}}\right)\right) \widehat{c}_{t}^{a R}+\widehat{r s}_{t}-\ldots \\
2\left(1+\eta\left(\frac{\psi}{1+(1-\psi) \varphi}\right)\left(\varphi+(1-\psi)\left(\frac{1+\varphi}{\psi}\right)^{2}\right)\right)\left(\widehat{p}_{t}^{F, W *}-\widehat{p}_{t}^{W *}\right)+\ldots \\
\left(\phi_{F}\left(\widehat{p}_{t}^{F, R}-\widehat{p}_{t}^{R}\right)-\phi_{H}\left(\widehat{p}_{t}^{H, R}-\widehat{p}_{t}^{R}\right)\right)-(1-\psi)\left(\frac{1+\varphi}{\psi}\right)\left(\widehat{k}_{t}^{*}-\widehat{k}_{t}\right)-\left(\frac{1+\varphi}{\psi}\right)\left(\widehat{a}_{t}^{*}-\widehat{a}_{t}\right)
\end{array}\right] .
\end{aligned}
$$

Let me define the variable $\widehat{z}_{t}$ as the difference between the relative prices in both countries, i.e. $\widehat{z}_{t} \equiv$ $\left(\widehat{p}_{t}^{F}-\widehat{p}_{t}^{H}\right)-\left(\widehat{p}_{t}^{F *}-\widehat{p}_{t}^{H *}\right)$, and the first-difference of $\widehat{z}_{t}$ as $\Delta \widehat{z}_{t} \equiv\left(\widehat{\pi}_{t}^{F}-\widehat{\pi}_{t}^{H}\right)-\left(\widehat{\pi}_{t}^{F *}-\widehat{\pi}_{t}^{H *}\right)$. Using the two equations I derived previously for $\left(\widehat{\pi}_{t}^{F}-\widehat{\pi}_{t}^{H}\right)$ and $\left(\widehat{\pi}_{t}^{F *}-\widehat{\pi}_{t}^{H *}\right)$, it immediately follows that,

$$
\begin{aligned}
\left(\widehat{\pi}_{t}^{F}-\widehat{\pi}_{t}^{H}\right)-\left(\widehat{\pi}_{t}^{F *}-\widehat{\pi}_{t}^{H *}\right) \approx \beta \mathbb{E}_{t}\left(\left(\widehat{\pi}_{t+1}^{F}-\widehat{\pi}_{t+1}^{H}\right)-\left(\widehat{\pi}_{t+1}^{F *}-\widehat{\pi}_{t+1}^{H *}\right)\right)-\ldots \\
\\
\quad \Psi\left[\left(\phi_{H}\left(\widehat{p}_{t}^{F, R}-\widehat{p}_{t}^{R}\right)-\phi_{F}\left(\widehat{p}_{t}^{H, R}-\widehat{p}_{t}^{R}\right)\right)+\left(\phi_{F}\left(\widehat{p}_{t}^{F, R}-\widehat{p}_{t}^{R}\right)-\phi_{H}\left(\widehat{p}_{t}^{H, R}-\widehat{p}_{t}^{R}\right)\right)\right] \\
\approx \beta \mathbb{E}_{t}\left(\left(\widehat{\pi}_{t+1}^{F}-\widehat{\pi}_{t+1}^{H}\right)-\left(\widehat{\pi}_{t+1}^{F *}-\widehat{\pi}_{t+1}^{H *}\right)\right)-\Psi\left[\left(\widehat{p}_{t}^{F, R}-\widehat{p}_{t}^{R}\right)-\left(\widehat{p}_{t}^{H, R}-\widehat{p}_{t}^{R}\right)\right] \\
\approx \beta \mathbb{E}_{t}\left(\left(\widehat{\pi}_{t+1}^{F}-\widehat{\pi}_{t+1}^{H}\right)-\left(\widehat{\pi}_{t+1}^{F *}-\widehat{\pi}_{t+1}^{H *}\right)\right)-\Psi\left[\widehat{p}_{t}^{F, R}-\widehat{p}_{t}^{H, R}\right] .
\end{aligned}
$$

Finally, since I have already defined $\widehat{p}_{t}^{H, R} \equiv\left(\widehat{p}_{t}^{H}-\widehat{p}_{t}^{H *}\right)$ and $\widehat{p}_{t}^{F, R} \equiv\left(\widehat{p}_{t}^{F}-\widehat{p}_{t}^{F *}\right)$, I can also infer that,

$$
\left[\widehat{p}_{t}^{F, R}-\widehat{p}_{t}^{H, R}\right]=\left(\widehat{p}_{t}^{F}-\widehat{p}_{t}^{F *}\right)-\left(\widehat{p}_{t}^{H}-\widehat{p}_{t}^{H *}\right)=\left(\widehat{p}_{t}^{F}-\widehat{p}_{t}^{H}\right)-\left(\widehat{p}_{t}^{F *}-\widehat{p}_{t}^{H *}\right)=\widehat{z}_{t},
$$

and, accordingly, I can re-write the expression above for the dynamics of $\Delta \widehat{z}_{t}$ as,

$$
\Delta \widehat{z}_{t} \approx \beta \mathbb{E}_{t}\left(\Delta \widehat{z}_{t+1}\right)-\Psi \widehat{z}_{t}
$$


Naturally, as Engel (forthcoming) emphasizes, if I combine equation (427) with the initial condition $\widehat{z}_{0}=0$, then it has to be the case that the solution implies that the relative prices in both countries ought to equalize as I postulated initially, i.e.,

$$
\widehat{z}_{t} \equiv\left(\widehat{p}_{t}^{F}-\widehat{p}_{t}^{H}\right)-\left(\widehat{p}_{t}^{F *}-\widehat{p}_{t}^{H *}\right) \approx 0
$$

or simply that,

$$
\left(\widehat{p}_{t}^{F}-\widehat{p}_{t}^{H}\right) \approx\left(\widehat{p}_{t}^{F *}-\widehat{p}_{t}^{H *}\right) .
$$

Furthermore, if I combine equations (426) with the solution in (428), I obtain the following result,

$$
\left(\widehat{p}_{t}^{F, R}-\widehat{p}_{t}^{R}\right)-\left(\widehat{p}_{t}^{H, R}-\widehat{p}_{t}^{R}\right) \approx 0
$$

or simply,

$$
\left(\widehat{p}_{t}^{F, R}-\widehat{p}_{t}^{R}\right) \approx\left(\widehat{p}_{t}^{H, R}-\widehat{p}_{t}^{R}\right) .
$$

Therefore, I can re-write the world terms of trade defined in (424) as follows,

$$
\widehat{t}_{t}^{W} \equiv \widehat{p}_{t}^{F, W *}-\widehat{p}_{t}^{W *}=2 \frac{\phi_{H} \phi_{F}}{\phi_{H}-\phi_{F}}\left(\widehat{p}_{t}^{F, R}-\widehat{p}_{t}^{R}\right)
$$

Equation (431) is going to be particularly helpful to simplify the log-linearized equilibrium conditions of the model later on. This simplification is exactly the same one that I derived in a model without variable capacity utilization in (329), indicating that this result is not sensitive to the addition of variable capital utilization into the model.

\subsubsection{Other Relationships}

On Aggregate Output and the Efficiency Conditions. Using the definition of world terms of trade and the fact that by construction $\left(\widehat{p}_{t}^{H, W}-\widehat{p}_{t}^{W}\right)+\left(\widehat{p}_{t}^{F, W *}-\widehat{p}_{t}^{W *}\right) \approx 0$ (see equation (414) for a demonstration) I can write aggregate output from equations (400) - (401) as,

$$
\begin{aligned}
& \widehat{y}_{t} \approx \eta \widehat{t}_{t}^{W}+\left(1-\gamma_{x}\right) \widehat{c}_{t}^{a W}+\gamma_{x} \widehat{x}_{t}^{a W}+\gamma_{x}^{y}\left(\frac{1-\beta(1-\delta)}{\beta \delta}\right) \widehat{u}_{t}, \\
& \widehat{y}_{t}^{*} \approx-\eta \widehat{t}_{t}^{W}+\left(1-\gamma_{x}\right) \widehat{c}_{t}^{a W *}+\gamma_{x} \widehat{x}_{t}^{a W *}+\gamma_{x^{*}}^{y}\left(\frac{1-\beta(1-\delta)}{\beta \delta}\right) \widehat{u}_{t}^{*} .
\end{aligned}
$$

Using the efficiency conditions in (402) and (403) and, after a little bit of algebra, it follows that the real rental rate on capital can be expressed as,

$$
\begin{aligned}
\widehat{r}_{t}^{z} & \approx\left(\frac{1}{\sigma}\left(\frac{1-\gamma_{x}}{1-\gamma_{x}^{a}}\right)+\left(1-\gamma_{x}\right) \frac{1+\varphi}{\psi}\right) \widehat{c}_{t}^{a W}+\gamma_{x} \frac{1+\varphi}{\psi} \widehat{x}_{t}^{a W}+\gamma_{x}^{y} \frac{1+\varphi}{\psi}\left(\frac{1-\beta(1-\delta)}{\beta \delta}\right) \widehat{u}_{t}+\ldots \\
& \phi_{F} \frac{1}{\sigma}\left(\frac{1-\gamma_{x}}{1-\gamma_{x}^{a}}\right) \widehat{c}_{t}^{a R}+\eta \frac{1+\varphi}{\psi} \widehat{t}_{t}^{W}-\left(\frac{1+(1-\psi) \varphi}{\psi}\right) \widehat{k}_{t}-\frac{1+\varphi}{\psi} \widehat{a}_{t}, \\
\widehat{r}_{t}^{z *} & \approx\left(\frac{1}{\sigma}\left(\frac{1-\gamma_{x}}{1-\gamma_{x^{*}}}\right)+\left(1-\gamma_{x}\right) \frac{1+\varphi}{\psi}\right) \widehat{c}_{t}^{a W *}+\gamma_{x} \frac{1+\varphi}{\psi} \widehat{x}_{t}^{a W *}+\gamma_{x^{*}}^{y} \frac{1+\varphi}{\psi}\left(\frac{1-\beta(1-\delta)}{\beta \delta}\right) \widehat{u}_{t}^{*}-\ldots \\
& \phi_{F} \frac{1}{\sigma}\left(\frac{1-\gamma_{x}}{1-\gamma_{x^{*}}^{a}}\right) \widehat{c}_{t}^{a R}-\eta \frac{1+\varphi}{\psi} \widehat{t}_{t}^{W}-\left(\frac{1+(1-\psi) \varphi}{\psi}\right) \widehat{k}_{t}^{*}-\frac{1+\varphi}{\psi} \widehat{a}_{t}^{*} .
\end{aligned}
$$

This simply re-writes the previous conditions replacing the relative prices with the definition of world terms of trade $\widehat{t}_{t}^{W}$. 
On Aggregate Employment. The aggregate employment can be easily derived from the production functions in (84) and (85) as,

$$
\begin{aligned}
\widehat{y}_{t} & \approx \widehat{a}_{t}+(1-\psi) \widehat{k}_{t}+\psi \widehat{l}_{t}, \\
\widehat{y}_{t}^{*} & \approx \widehat{a}_{t}^{*}+(1-\psi) \widehat{k}_{t}^{*}+\psi \widehat{l}_{t}^{*} .
\end{aligned}
$$

These are the same equations that I obtained in the model without variable capital utilization. However, $\widehat{k}_{t}$ denotes now capital services rather than physical capital as before.

On Real Exports, Real Imports, and the Net Exports Share. In a two-country model, suffice to determine the net exports share of the domestic country, $\widehat{t b}_{t}$. The net exports share can be easily computed as the difference between domestic aggregate output and domestic aggregate consumption, investment and capital utilization costs (domestic absorption) in real terms, i.e.,

$$
\widehat{t b}_{t} \equiv \widehat{y}_{t}-\left(1-\gamma_{x}^{a}\right) \widehat{c}_{t}-\gamma_{x}^{a} \widehat{x}_{t}-\gamma_{x}^{y}\left(\frac{1-\beta(1-\delta)}{\beta \delta}\right) \widehat{u}_{t} .
$$

I have defined the world consumption as $\widehat{c}_{t}^{a W} \equiv \phi_{H}\left(\frac{1-\gamma_{x}^{a}}{1-\gamma_{x}}\right) \widehat{c}_{t}+\phi_{F}\left(\frac{1-\gamma_{x^{*}}^{a}}{1-\gamma_{x}}\right) \widehat{c}_{t}^{*}$ and $\widehat{c}_{t}^{a W *} \equiv \phi_{F}\left(\frac{1-\gamma_{x}^{a}}{1-\gamma_{x}}\right) \widehat{c}_{t}+$ $\phi_{H}\left(\frac{1-\gamma_{x^{*}}^{a}}{1-\gamma_{x}}\right) \widehat{c}_{t}^{*}$ and the relative consumption as $\widehat{c}_{t}^{a R} \equiv\left(\frac{1-\gamma_{x}^{a}}{1-\gamma_{x}}\right) \widehat{c}_{t}-\left(\frac{1-\gamma_{x^{*}}^{a}}{1-\gamma_{x}}\right) \widehat{c}_{t}^{*}$. I have defined the world investment as $\widehat{x}_{t}^{a W} \equiv \phi_{H}\left(\frac{\gamma_{x}^{a}}{\gamma_{x}}\right) \widehat{x}_{t}+\phi_{F}\left(\frac{\gamma_{x^{*}}^{a}}{\gamma_{x}}\right) \widehat{x}_{t}^{*}$ and $\widehat{x}_{t}^{a W *} \equiv \phi_{F}\left(\frac{\gamma_{x}^{a}}{\gamma_{x}}\right) \widehat{x}_{t}+\phi_{H}\left(\frac{\gamma_{x^{*}}^{a}}{\gamma_{x}}\right) \widehat{x}_{t}^{*}$ and the relative investment as $\widehat{x}_{t}^{a R} \equiv\left(\frac{\gamma_{x}^{a}}{\gamma_{x}}\right) \widehat{x}_{t}-\left(\frac{\gamma_{x^{*}}^{a}}{\gamma_{x}}\right) \widehat{x}_{t}^{*}$. Then, I can write domestic and foreign consumption and investment as follows,

$$
\begin{aligned}
\widehat{c}_{t} & =\left(\frac{1-\gamma_{x}}{1-\gamma_{x}^{a}}\right)\left(\widehat{c}_{t}^{a W}+\phi_{F} \widehat{c}_{t}^{a R}\right), \\
\widehat{c}_{t}^{*} & =\left(\frac{1-\gamma_{x}}{1-\gamma_{x^{*}}^{a}}\right)\left(\widehat{c}_{t}^{a W *}-\phi_{F} \widehat{c}_{t}^{a R}\right), \\
\widehat{x}_{t} & =\left(\frac{\gamma_{x}}{\gamma_{x}^{a}}\right)\left(\widehat{x}_{t}^{a W}+\phi_{F} \widehat{x}_{t}^{a R}\right), \\
\widehat{x}_{t}^{*} & =\left(\frac{\gamma_{x}}{\gamma_{x^{*}}^{a}}\right)\left(\widehat{x}_{t}^{a W *}-\phi_{F} \widehat{x}_{t}^{a R}\right) .
\end{aligned}
$$

Using the formula derived above for domestic aggregate output in (432) and the expressions above for the net exports share, I obtain the following equation for the trade balance,

$$
\begin{aligned}
\widehat{t b}_{t} & \approx \eta \widehat{t}_{t}^{W}+\left(1-\gamma_{x}\right) \widehat{c}_{t}^{a W}+\gamma_{x} \widehat{x}_{t}^{a W}+\gamma_{x}^{y}\left(\frac{1-\beta(1-\delta)}{\beta \delta}\right) \widehat{u}_{t}-\left(1-\gamma_{x}^{a}\right) \widehat{c}_{t}-\gamma_{x}^{a} \widehat{x}_{t}-\gamma_{x}^{y}\left(\frac{1-\beta(1-\delta)}{\beta \delta}\right) \widehat{u}_{t} \\
& =\eta \widehat{t}_{t}^{W}+\left(1-\gamma_{x}\right)\left(\widehat{c}_{t}^{a W}-\left(\frac{1-\gamma_{x}^{a}}{1-\gamma_{x}}\right) \widehat{c}_{t}\right)+\gamma_{x}\left(\widehat{x}_{t}^{a W}-\left(\frac{\gamma_{x}^{a}}{\gamma_{x}}\right) \widehat{x}_{t}\right) \\
& =\eta \widehat{t}_{t}^{W}+\left(1-\gamma_{x}\right)\left(\widehat{c}_{t}^{a W}-\left(\frac{1-\gamma_{x}^{a}}{1-\gamma_{x}}\right)\left(\frac{1-\gamma_{x}}{1-\gamma_{x}^{a}}\right)\left(\widehat{c}_{t}^{a W}+\phi_{F} \widehat{c}_{t}^{a R}\right)\right)+\gamma_{x}\left(\widehat{x}_{t}^{a W}-\left(\frac{\gamma_{x}^{a}}{\gamma_{x}}\right)\left(\frac{\gamma_{x}}{\gamma_{x}^{a}}\right)\left(\widehat{x}_{t}^{a W}+\phi_{F} \widehat{x}_{t}^{a R}\right)\right) \\
& =\eta \widehat{t}_{t}^{W}-\left(1-\gamma_{x}\right) \phi_{F} \widehat{c}_{t}^{a R}-\gamma_{x} \phi_{F} \widehat{x}_{t}^{a R} .
\end{aligned}
$$


In other words, adjustment in the domestic trade balance comes through movements in the world terms of trade $\widehat{t}_{t}^{W}$, or from relative adjustments in either the consumption or investment paths. The trade balance is not directly affected by capital utilization costs because aggregate capital cannot be traded across countries (only varieties are tradable). Therefore, the capital utilization term appears on the domestic aggregate output demand and it also appears on the domestic absorption, so it cancels out.

Naturally, the derivation of the real export and real import equations, $\widehat{\exp }_{t}$ and $\widehat{i m p}_{t}$, is the same as in (335) - (336). The same logic applies in this context to the derivation of the transformation of the world terms of trade, $\widehat{t}_{t}^{W}$, as a linear function of the domestic terms of trade, $\widehat{t o t}_{t}$, and the real exchange rate, $\widehat{r s}_{t}$, as in (337).

\section{Model Parameterization}

Table 1 summarizes the model parameters adopted in my simulations. The parameterization is roughly similar to that in Chari et al. (2002), except where otherwise noted.

\section{[Insert Table 1 about here]}

The intertemporal discount factor, $\beta$, equals 0.99 and the intertemporal elasticity of substitution, $\sigma$, is $1 / 5$. The share of foreign goods, $\phi_{F}$, is set to 0.06 . The elasticity of substitution across varieties, $\theta$, is chosen to equal 10. The choice of $\theta$ is consistent with a price mark-up of $11 \%$. Moreover, $\theta$ serves to pin down the steady state investment share (over GDP), $\gamma_{x}$, at 0.203 . I set the government subsidy, $\bar{\xi}$, at zero, the labor share in the production function, $\psi$, equal to $2 / 3$ and the depreciation rate, $\delta$, equal to 0.021 .

I choose the intratemporal elasticity of substitution, $\eta$, to be equal to 1.5 , which is similar to Chari et al. (2002), but significantly lower than in Steinsson (2008). The inverse of the Frisch elasticity of labor supply, $\varphi$, is set at 3 (see the micro evidence in Browning et al. (1999) and Blundell and MaCurdy (1999)). When appropriate, I set the elasticity of the capital utilization cost, $\lambda$, at 5.80 .

The Calvo price stickiness parameter, $\alpha$, is assumed to be 0.75 . This implies that the average price duration in the model is 4 quarters - the same average duration as in Chari et al. (2002) and Steinsson (2008). The interest rate inertia parameter, $\rho_{i}$, equals 0.85 , while the sensitivity of the nominal policy rate to the inflation target, $\psi_{\pi}$, equals 2 , and the sensitivity to the output target, $\psi_{y}$, is 0.5 as in Steinsson (2008).

As in Ghironi, et al. (2009) and Benigno (2009), I assume that the costs of adjusting the foreign bond holdings with respect to the steady state are such that $\mu=0.01$. This parameterization is also consistent with the estimates of Kollman (2003), which imply a value of 0.012 as noted in Benigno (2009, footnote 9). I choose $\bar{a}$ to match the $1970-2007$ average of the U.S. annual ratio of net foreign assets over GDP of $-4.06 \%$ from the Lane and Milesi-Ferretti (2007) dataset.

Remark 4 For a parameterization of the model that implies $\eta=1.5, \phi_{F}=0.06, \phi_{H}=1-\phi_{F}=0.94$, $\beta=0.99$ and $\bar{a}^{a}=-0.04065$, the numerical solution to equation (173) gives me that steady state terms of trade are equal to $\frac{\bar{P}^{F}}{\bar{P}^{H}}=1.0137$. This implies that this steady state with the domestic country holding a negative amount of real net foreign assets (i.e. $\bar{a}^{a}<0$ ) can only occur if the terms of trade are higher than one (i.e. $\frac{\bar{P}^{F}}{\bar{P}^{H}}>1$ ). In other words, only if import prices are a bit more expensive in steady state than export prices can I reconcile the fact that in steady state the domestic country is a net borrower from the foreign country. 
Based on (174) and the same parameterization, I can say that the ratio of real net foreign assets of the domestic household over domestic output must be equal to,

$$
\bar{a}^{y}=\bar{a}^{a}\left(1-(1-\beta) \bar{a}^{a}\right)^{\frac{\eta}{1-\eta}}=-0.0406 .
$$

Therefore, the parameterization is consistent with real net foreign assets over output around $-4.06 \%$, which corresponds to the average annual ratio for the U.S. during the 1970-2007 period based on the data compiled by Lane and Milesi-Ferretti (2007).

Shock Processes and Adjustment Costs. I assume that the persistence of the productivity shock, $\rho_{a}$, is fixed at 0.9 as in Steinsson (2008). Likewise, I set the persistence of the IST shock, $\rho_{v}$, at 0.9. I set the other parameters of the stochastic processes to match certain features of the U.S. real GDP data. The aim is to investigate the properties of consumption, investment and the RER under different assumptions on capital accumulation while replicating key empirical moments of U.S. real GDP.

I set the standard deviation of all shocks to match the U.S. real GDP volatility (1.54\%). In addition, I parameterize the cross-country correlation of the innovations to replicate the observed cross-correlation of U.S. and Euro area real GDP (0.44). In experiments where productivity shocks drive the business cycle in combination with either monetary or IST shocks, I set the standard deviation of the productivity shock innovation always to $0.7 \%$ and the cross-country correlation to 0.25 (e.g., Heathcote and Perri (2002) and Chari et al. (2002)). In turn, I parameterize the volatility and the cross-correlation of the innovations of the other shock - the monetary or IST shock - to match the volatility and cross-country correlation of U.S. real GDP. When appropriate, I select the adjustment cost parameter, either $\chi(\mathrm{CAC})$ or $\kappa$ (IAC), to ensure that the volatility of investment relative to output roughly matches the data (3.38 times the volatility of U.S. real GDP).

In the simulations with IST shocks an exact match of the investment and output volatilities cannot be attained without pushing the adjustment cost and the shock volatility parameters beyond a reasonable range of values. In that case, I match the volatility of U.S. real GDP with the volatility of the IST shock bounded to be below $10 \%$, and I pick the adjustment cost to keep the volatility of investment low.

\section{Quantitative Findings (Highlights)}

The model described previously in the paper incorporates the basic features of the NOEM literatureprice stickiness and local-currency pricing - while departing from the conventional assumption of complete international asset markets. Moreover, the model nests a wide range of alternative capital specifications from linear-in-labor technologies and no capital to different model variants with capital accumulation, adjustment costs of different types and variable capital utilization rates.

I start by revisit the conventional case under complete international asset markets that was originally covered in Martínez-García and Søndergaard (2008a, 2008b). Tables 2 and 3 collect the results for different variants of the model with capital accumulation. The case with no capital $(\mathrm{NoC})$, which is closer to Steinsson (2008), is compared against a variant of the model with capital but no adjustment costs (NAC), a variant with investment adjustment costs (IAC), and another alternative with capital adjustment costs (CAC). I 
report all those simulations in Columns $3-6$. I conduct some sensitivity analysis in Columns $7-10 .{ }^{9}$ I also contemplate different scenarios in which the business cycles are alternatively driven by productivity shocks (Panel 1 of Table 2), monetary shocks (Panel 2 of Table 2), a combination of productivity shocks and investment-specific technology (IST) shocks (Panel 1 of Table 3) as well as a combination of productivity shocks and monetary shocks (Panel 2 of Table 3 ).

\section{[Insert Tables 2 and 3 about here]}

Capital accumulation contributes to significantly lower the RER volatility in the NOEM model - irrespective of the shocks driving the cycle, as noted in Martínez-García and Søndergaard (2008a, 2008b). In a similar setting, Chari et al. (2002) showed that volatile RERs required monetary shocks to interact with nominal rigidities. However, if prices were held fixed for at least a year, the elasticity of intertemporal substitution was low, and preferences were separable in leisure, then the real exchange rate fluctuations generated by the model would approximate the volatility observed in the data but still not be able to match the empirical persistence.

In response to monetary shocks or a combination of productivity and monetary shocks where the latter is the main driver of the cycle, a variant with capital and adjustment costs that penalizes the growth rate of investment - as proposed in Christiano et al. (2005) - rather than the investment-to-capital ratio - as used in Chari et al. (2002) - is better to account for the volatility of the RER as well as the fluctuations in output, consumption and investment observed in the data, but it still falls short in terms of RER persistence.

I note that high endogenous persistence tends to occur in response to persistent productivity shocks or a combination of persistent productivity and investment-specific technology (IST) shocks. However, neither one of the two scenarios is capable of simultaneously generating enough volatility to match the empirical RER volatility unless very high adjustment costs (or no capital) are imposed on the model. Figures 1 through 6 illustrate the sensitivity of the results reported in Tables 2 and 3 to the parameterization of the adjustment cost function and the Taylor (1993) monetary policy rule. Interestingly, I document how the interaction between the monetary policy regime and the costs of intertemporal consumption smoothing through capital accumulation are critically dependent on the type of shocks driving the cycle.

\section{[Insert Figures 1, 2, 3, 4, 5 and 6 about here]}

The findings derived under local-currency pricing and complete international asset markets appear broadly — but not entirely - robust to departure from those two core assumptions of the workhorse NOEM model. I re-establish the law of one price by replacing the assumption of local-currency pricing in international goods markets with producer-currency pricing. Then, the RER moves in tandem with terms of trade and solely because of differences in the consumption baskets across countries. The extension of the model under producer-currency pricing is extensively discussed in Martínez-García and Søndergaard (2008a) and of particular relevance to investigate the quantitative effects of monetary shocks.

I also depart - and that is the main contribution of the paper - from the assumption of complete international asset markets, which imposes perfect international risk-sharing and a tight link between the RER and relative consumption, by adding a quadratic cost on international borrowing tied to the real net foreign

\footnotetext{
${ }^{9}$ Columns $7-8$ show the results whenever the adjustment costs are set to match the volatility of consumption rather than investment. Columns $9-10$ present the simulations with variable capital utilization.
} 
asset position of the home country (see, e.g., Benigno and Thoenissen (2008) and Benigno (2009)). I find that a bond economy with international borrowing costs and the workhorse NOEM model with complete international asset markets generates very similar international business cycle patterns in response to productivity and monetary shocks (see also Baxter and Crucini (1995), Heathcote and Perri (2002) and Chari et al. (2002)), but significant differences arise with investment-specific technology (IST) shocks. Hence, I closely examine the role of IST shocks in the context of this model with incomplete international asset markets.

\subsection{Producer-Currency Pricing and the Law of One Price}

Price stickiness alone does not imply that the law of one price fails in the NOEM model. For that, market segmentation and the assumption of local-currency pricing are also needed. Hence, under producer-currency pricing all prices must equalize across countries when expressed in the same currency - that is, the law of one price must hold - and the RER fluctuates simply because I also assume different consumption baskets for the two countries. Engel (1999) provides empirical evidence supporting the view that deviations of the law of one price on traded goods account for most of the movements in the U.S. real exchange rate. While Engel (1999) also considers the possibility that traded-goods are weighted differently in the consumption basket of each country, he concludes that RER fluctuations tied to terms-of-trade movements through this channel are not very important in the data.

Indeed, the results reported in Tables 4 and 5 complement Engel's (1999) data analysis by suggesting that consumption basket differences alone are not able to explain overall RER movements through the lens of the NOEM model with capital. The simulated model is based on the extension of the NOEM model with capital and producer-currency pricing discussed in Martínez-García and Søndergaard (2008a). The case with no capital (NoC) is compared against a variant of the model with capital but no adjustment costs (NAC), a variant with investment adjustment costs (IAC), and another alternative with capital adjustment costs (CAC). I report all those simulations in Columns 3-6. I conduct some sensitivity analysis in Columns $7-10 .{ }^{10}$ I also contemplate different scenarios in which the business cycles are alternatively driven by productivity shocks (Panel 1 of Table 4), monetary shocks (Panel 2 of Table 4), a combination of productivity shocks and investment-specific technology (IST) shocks (Panel 1 of Table 5) as well as a combination of productivity shocks and monetary shocks (Panel 2 of Table 5).

[Insert Tables 4 and 5 about here]

Betts and Devereux (2000) argue that local-currency pricing and staggered prices can magnify the response of the RER and distort the international transmission mechanism of monetary policy shocks resulting in lower consumption comovement across countries. I observe the same pattern emerge irrespective of the way capital is modelled by comparing Panel 2 of Tables 2 and 3 (under local-currency pricing) with Panel 2 of Tables 4 and 5 (under producer-currency pricing) where monetary shocks are the dominant source of business cycles. Endogenous persistence tends to be slightly higher with local-currency pricing than in the experiments with producer-currency pricing, but the RER volatility ratio is definitely larger aided by a large decline in the cross-country consumption correlation and by a small increase in consumption volatility.

\footnotetext{
${ }^{10}$ Columns $7-8$ show the results whenever the adjustment costs are set to match the volatility of consumption rather than investment. Columns $9-10$ present the simulations with variable capital utilization.
} 
By contrast, however, the RER volatility amplification attained with local-currency pricing and deviations of the law of one price is much smaller with either productivity shocks (Panel 1 of Table 2 vs. Panel 1 of Table 4) or a combination of productivity and IST shocks (Panel 1 of Table 3 vs. Panel 1 of Table 5). The effect on the endogenous RER persistence of the assumption of local-currency pricing or producer-currency pricing is still rather modest. What these findings illustrate is that large and distortionary deviations of the law of one price depend on the nature of the shocks. Not surprisingly, most of the NOEM models that investigate the RER dynamics through this channel have focused their attention primarily on the connection between nominal rigidities, local-currency pricing and monetary shocks (see Betts and Devereux (2000) and Chari et al. (2002)).

Figures 7 through 12 illustrate the sensitivity of the results reported in Tables 4 and 5 to the parameterization of the adjustment cost function and the Taylor (1993) monetary policy rule. Interestingly, inspecting these results more closely one realizes that the assumption of producer-currency pricing may have a more significant effect than our previous results would suggest whenever the business cycle is primarily driven by either productivity shocks or investment-specific technology (IST) shocks. A case in point with the productivity shock is that lower monetary policy inertia appears to be associated with higher RER volatility whenever I assume local-currency pricing than when I assume producer-currency pricing.

\section{[Insert Figures 7, 8, 9, 10, 11 and 12 about here]}

However, irrespective of whether I assume local-currency pricing or producer-currency pricing, RERs still tend to be less volatile the easier it gets for households to utilize capital accumulation to intertemporally smooth their consumption - except when the cycle is primarily driven by investment-specific technology (IST) shocks.

\subsection{IST Shocks and International Asset Market Incompleteness}

The functioning of international asset markets determines the extent to which households can efficiently insure amongst themselves to smooth their consumption in the presence of country-specific shocks. Asset markets are viewed as crucial for the propagation and transmission of business cycle fluctuations across countries, but most of the NOEM literature has often abstracted from asset market frictions of any sort to focus instead on understanding the role of frictions in the goods markets. I observe that a standard bond economy with international borrowing costs still replicates closely the persistence and volatility of the RER under complete international asset markets (see, e.g., Baxter and Crucini (1995) and Chari et al. (2002)). In turn, that is no longer the case whenever IST shocks are the primary driver of the business cycle.

I adopt a standard extension of the NOEM model with capital (under local-currency pricing) that restricts the financial assets available to just two uncontingent nominal bonds in zero-net supply (issued in the two different currencies) adding a quadratic cost on international borrowing tied to the real net foreign asset position of the home country (see, e.g., Benigno and Thoenissen (2008) and Benigno (2009)). A more in-depth exploration of the complex role of asset markets goes beyond the scope of this paper and is left for future research. I use this bond economy in order to investigate the robustness of the findings on the volatility and persistence of the RER to the assumption of complete international asset markets. The main implication is that the imperfect international risk-sharing condition in equation (59) (or (345)) introducesup to a first-order approximation - deviations in the uncovered interest rate parity condition linked to bond 
trading costs and the evolution of the domestic real net foreign asset position.

The full results under incomplete international asset markets are reported in Tables 6 and 7, and can be compared against the complete asset market results in Tables 2 and 3. The case with no capital (NoC) is compared against a variant of the model with capital but no adjustment costs (NAC), a variant with investment adjustment costs (IAC), and another alternative with capital adjustment costs (CAC). I report all those simulations in Columns $3-6$. I conduct some sensitivity analysis in Columns $7-10 .{ }^{11}$ I also contemplate different scenarios in which the business cycles are alternatively driven by productivity shocks (Panel 1 of Table 6), monetary shocks (Panel 2 of Table 6), a combination of productivity shocks and investment-specific technology (IST) shocks (Panel 1 of Table 7) as well as a combination of productivity and monetary shocks (Panel 2 of Table 7).

\section{[Insert Tables 6 and 7 about here]}

My results illustrate that the complete and incomplete international asset markets models are practically indistinguishable whenever persistent productivity shocks or non-persistent monetary shocks drive the business cycle. The international real business cycle literature without nominal rigidities also shows that a bond economy closely approximates the complete asset markets allocation when driven by persistent productivity shocks - unless, for instance, productivity shocks are permanent without spill-overs or stricter financial autarky is imposed (see, e.g., Baxter and Crucini (1995) and Heathcote and Perri (2002)). Chari et al. (2002) document a similar result in a model with nominal rigidities and non-persistent monetary shocks as the main driver of the cycle.

In turn, Panel 1 of Table 7 compared to Panel 1 of Table 3 shows that with IST shocks the RER can become somewhat more persistent but tends to be significantly less volatile. This is a powerful fact that has gone largely unnoticed in the literature until now. Chari et al. (2002) also find that a bond economy has the potential to weaken the link between the RER and relative consumption, but show that in fact this avenue is not very successful in eliminating the consumption-real exchange rate anomaly (the Backus-Smith puzzle). The consumption-real exchange rate correlation remains closer to one with conventional preferences, while the empirical counterpart lies somewhere around -0.35 (which is the value reported by Chari et al. (2002, Table 6)). Not surprisingly, I also find that the correlation between relative consumption and the real exchange rate is close to one in models with persistent productivity shocks, with non-persistent monetary shocks or with a combination of both. Only with IST shocks I am able to lower this correlation significantly, although the exploration of the Backus-Smith puzzle would be left for future research.

IST and Productivity Shocks. Raffo (2010) shows that IST shocks can help reconcile the international real business cycle model with certain hard-to-match stylized facts - the negative correlation between the RER and relative consumption (the Backus-Smith puzzle) and the volatility of terms of trade and trade flows - while preserving countercyclical trade balances. Raffo (2010) does not feature nominal rigidities or other imperfections in the goods markets, so RER fluctuations are solely due to differences in the consumption baskets across countries (a channel also present in my model). Raffo (2010) suggests dependence on that one channel makes it difficult for the international real business cycle model driven by investment-specific

\footnotetext{
${ }^{11}$ Columns $7-8$ show the results whenever the adjustment costs are set to match the volatility of consumption rather than investment. Columns $9-10$ present the simulations with variable capital utilization.
} 
technology (IST) shocks to account for the volatility and persistence of the RER. In turn, incorporating a richer market structure that allows for pricing-to-market-local-currency pricing - and large deviations of the law of one price could help reconcile the model with the data.

Here, I offer a framework with which to evaluate Raffo's (2010) conjecture. My findings, reported in Tables 3 and 5, suggest that whether the law of one price holds (under producer-currency pricing) or not (under local-currency pricing) may have limited effects on the ability of the model driven primarily by investment-specific technology (IST) shocks to account for the volatility and persistence of the RERs. However, Table 7 indicates that the structure of the international asset markets has a significantly large effect on the dynamics of the RER (especially its volatility). Figures 13 through 18 illustrate the sensitivity of the results reported in Tables 6 and 7 to the parameterization of the adjustment cost function and the Taylor (1993) monetary policy rule. In so doing, they also illustrate that those effects are also noticeable on the endogenous RER persistence generated by the model. Moreover, the figures also indicate that the impact of incomplete international asset markets is not the product of an 'unlucky' set of parameters values, but prevalent across a wide range in the parameter space.

[Insert Figures 13, 14, 15, 16, 17 and 18 about here]

In general, adding persistent IST shocks tends to imply fairly persistent endogenous RERs - but less than with persistent productivity shocks alone. Moreover, it often implies smaller consumption cross-correlations and higher consumption and RER volatilities than with persistent productivity shocks alone - although not enough to resolve the quantity puzzle or match the empirical RER volatility. In fact, the simulated consumption cross-correlation is systematically higher than the cross-country output correlation of 0.44 found in my data (and matched in all my simulations), while the empirical consumption cross-correlation tends to be smaller (0.33 in my data).

A positive IST shock makes investment temporarily more productive. Households invest more to take advantage of that, but do so partly by working and producing more and partly by sacrificing consumption in the short-run. As a result, consumption becomes countercyclical due to the strong intrinsic incentives to invest now and consume later. ${ }^{12}$ Interestingly, the incentive to postpone consumption in response to a domestic IST shock is more pronounced in the home country leading to a short-run appreciation of the RER - which reverses itself over time - in spite of the fact that domestic output is rising more than foreign output. In contrast, the RER unequivocally depreciates in response to a (positive) domestic productivity shock or an expansionary (negative) domestic monetary shock that makes domestic goods temporarily more abundant than foreign goods.

Adding even small adjustment costs is generally counterproductive to match the data. Doing so requires an even larger IST shock volatility to replicate the standard deviation of U.S. real GDP, which - in turnusually increases the endogenous volatility of investment. However, adjustment costs give households an incentive to invest more gradually and the RER persistence tends to go up as a result. The internal tension that investment-specific technology (IST) shocks bring into the model shows up in investment volatility being much larger than in the data and in consumption being countercyclical.

These findings suggest that incorporating investment-specific technology (IST) shocks as the primary

\footnotetext{
${ }^{12}$ See Raffo (2010) for a discussion on the role of the preference specification and the wealth effects on labor supply on this and other counterfactual predictions (including the Backus-Smith puzzle).
} 
driver of the business cycle makes it harder to balance the competing goals of accounting for RER (and consumption) fluctuations while fitting the volatilities of output and investment. With conventional (additively separable and isoelastic) preferences and investment-specific technology (IST) shocks, introducing large deviations of the law of one price - through price stickiness and local-currency pricing - does not suffice to reconcile the NOEM model with capital with the empirical evidence on RERs, and less so under incomplete asset markets.

\section{Concluding Remarks}

Martínez-García and Søndergaard (2008a, 2008b), among others, have extensively investigated how NOEM models generate volatility and persistence of the real exchange rate (RER). Often the NOEM literature takes for granted the assumption of complete international asset markets. This paper provides a detailed discussion of how to extend the Martínez-García and Søndergaard (2008a, 2008b) model with capital accumulation and nominal rigidities in a tractable manner to break away from that assumption asset market completeness. To do so, I set-up a bond economy with costs on domestic international borrowing (see, e.g., Benigno (2009)).

I find that irrespective of whether the model has capital or not, productivity shocks trigger highly persistent RERs while monetary shocks generally do not - although the amount of endogenous persistence is often sensitive to the specification of the adjustment cost function and the Taylor (1993) monetary policy rule. Conversely, monetary shocks trigger highly volatile RERs while monetary shocks generally do notsubject to the same caveats on the sensitivity of the results to the adjustment cost function and the monetary policy rule. These findings are consistent with conventional wisdom (see, e.g., Chari et al. (2002)).

I also find that the bond economy setting with incomplete asset markets is almost indistinguishable from the conventional specification of complete international asset markets whenever the cycle is driven primarily by either non-persistent monetary shocks or persistent productivity shocks. In turn, asset market incompleteness results into significantly lower RER volatility in response to persistent investment-specific technology (IST) shocks. I illustrate that the NOEM model with IST shocks as the primary driver of the business cycle can approximate the observed RER dynamics better than with productivity shocks alone under complete asset markets. But, even then, I also point out that the excessive investment volatility and

the countercyclical consumption associated with IST shocks can be a potential drawback of this shock-based explanation of the RER. 


\section{References}

[1] Baxter, Marianne and Mario J. Crucini (1995): "Business Cycles and the Asset Structure of Foreign Trade." International Economic Review, vol. 36 (4), pp. 821-854.

[2] Benigno, Gianluca and Christoph Thoenissen (2008): "Consumption and Real Exchange Rates with Incomplete Markets and Non-traded Goods." Journal of International Money and Finance, vol. 27 (6), pp. 926-948.

[3] Benigno, Pierpaolo (2009): "Price Stability with Imperfect Financial Integration." Journal of Money, Credit and Banking, vol. 41 (s1), pp. 121-149.

[4] Betts, Caroline and Michael B. Devereux (2000): "Exchange Rate Dynamics in a Model of Pricing-toMarket." Journal of International Economics, vol. 50 (1), pp. 215-244.

[5] Blundell, Richard and Thomas E. MaCurdy (1999): "Labor Supply: A Review of Alternative Approaches." In Orley Ashenfelter and David Card (eds.), Handbook of Labor Economics, vol. 3 (1), pp. 1559-1665. Amsterdam: Elsevier Science B.V., North Holland.

[6] Browning, Martin, Lars Peter Hansen and James J. Heckman (1999): "Micro Data and General Equilibrium Models." In John B. Taylor and Michael Woodford (eds.), Handbook of Macroeconomics, vol. 1 (1), pp. 543-633. Amsterdam: Elsevier Science B.V., North Holland.

[7] Calvo, Guillermo A. (1983): "Staggered Prices in a Utility-Maximizing Framework." Journal of Monetary Economics, vol. 12 (3), pp. 383-398.

[8] Chari, V. V., Patrick J. Kehoe and Ellen R. McGrattan (2002): "Can Sticky Price Models Generate Volatile and Persistent Real Exchange Rates?" Review of Economic Studies, vol. 69 (3), pp. 533-563.

[9] Christiano, Lawrence J., Martin Eichenbaum and Charles Evans (2005): "Nominal Rigidities and the Dynamic Effects of a Shock to Monetary Policy." Journal of Political Economy, vol. 113 (1), pp. 1-45.

[10] Engel, Charles M. (1999): "Accounting for U.S. Real Exchange Rate Changes." Journal of Political Economy, vol. 107 (3), pp. 507-538.

[11] Engel, Charles M. (forthcoming): "Currency Misalignments and Optimal Monetary Policy: A Reexamination." American Economic Review.

[12] Galí, Jordi and Tommaso Monacelli (2005): "Monetary Policy and Exchange Rate Volatility in a Small Open Economy." Review of Economic Studies, vol. 72 (3), pp. 707-734.

[13] Ghironi, Fabio, Jaewoo Lee, and Alessandro Rebucci (2009): "The Valuation Channel of External Adjustment." IMF Working Papers 09/275, International Monetary Fund.

[14] Heathcote, Jonathan and Fabrizio Perri (2002): "Financial Autarky and International Business Cycles." Journal of Monetary Economics, vol. 49 (3), pp. 601-627.

[15] Kollmann, Robert (2003): "Monetary Policy Rules in an Interdependent World." CEPR Discussion Paper No. 4012. 
[16] Lane, Philip R. and Gian Maria Milesi-Ferretti (2007): "The External Wealth of Nations Mark II: Revised and Extended Estimates of Foreign Assets and Liabilities, 1970-2004." Journal of International Economics, vol. 73 (2), pp. 223-250. The (updated) dataset can be found at: http://www.philiplane.org/EWN.html

[17] Martínez-García, Enrique and Jens Søndergaard (2008a): "Technical Note on 'The Real Exchange Rate in Sticky Price Models: Does Investment Matter?" GMPI Working Paper No. 16, Federal Reserve Bank of Dallas.

[18] Martínez-García, Enrique and Jens Søndergaard (2008b): "The Real Exchange Rate in Sticky Price Models: Does Investment Matter?" GMPI Working Paper No. 17, Federal Reserve Bank of Dallas.

[19] Raffo, Andrea (2010): "Technology Shocks: Novel Implications for International Business Cycles." International Finance Discussion Papers 992, Board of Governors of the Federal Reserve System.

[20] Schmitt-Grohé, Stephanie and Martín Uribe (2003): "Closing Small Open Economy Models." Journal of International Economics, vol. 61 (1), pp. 163-185.

[21] Smets, Frank and Raf Wouters (2003): "An Estimated Dynamic Stochastic General Equilibrium Model of the Euro Area." Journal of the European Economic Association, vol. 1 (5), pp. 1123-1175.

[22] Steinsson, Jón (2008): "The Dynamic Behavior of the Real Exchange Rate in Sticky Price Models." American Economic Review, vol. 98 (1), pp. 519-533.

[23] Taylor, John B. (1993): "Discretion Versus Policy Rules in Practice." Carnegie-Rochester Conference Series, vol. 39, pp. 195-214.

[24] Warnock, Francis E. (2003): "Exchange Rate Dynamics and the Welfare Effects of Monetary Policy in a Two-Country Model with Home-Product Bias." Journal of International Money and Finance, vol. 22 (3), pp. 343-363. 


\section{Appendix}

\section{A The Linearized Equilibrium Conditions: A Summary}

Here, I report the system of equations derived after log-linearizing the equilibrium conditions of the model. ${ }^{13}$

\section{A.1 The Model Without Capital}

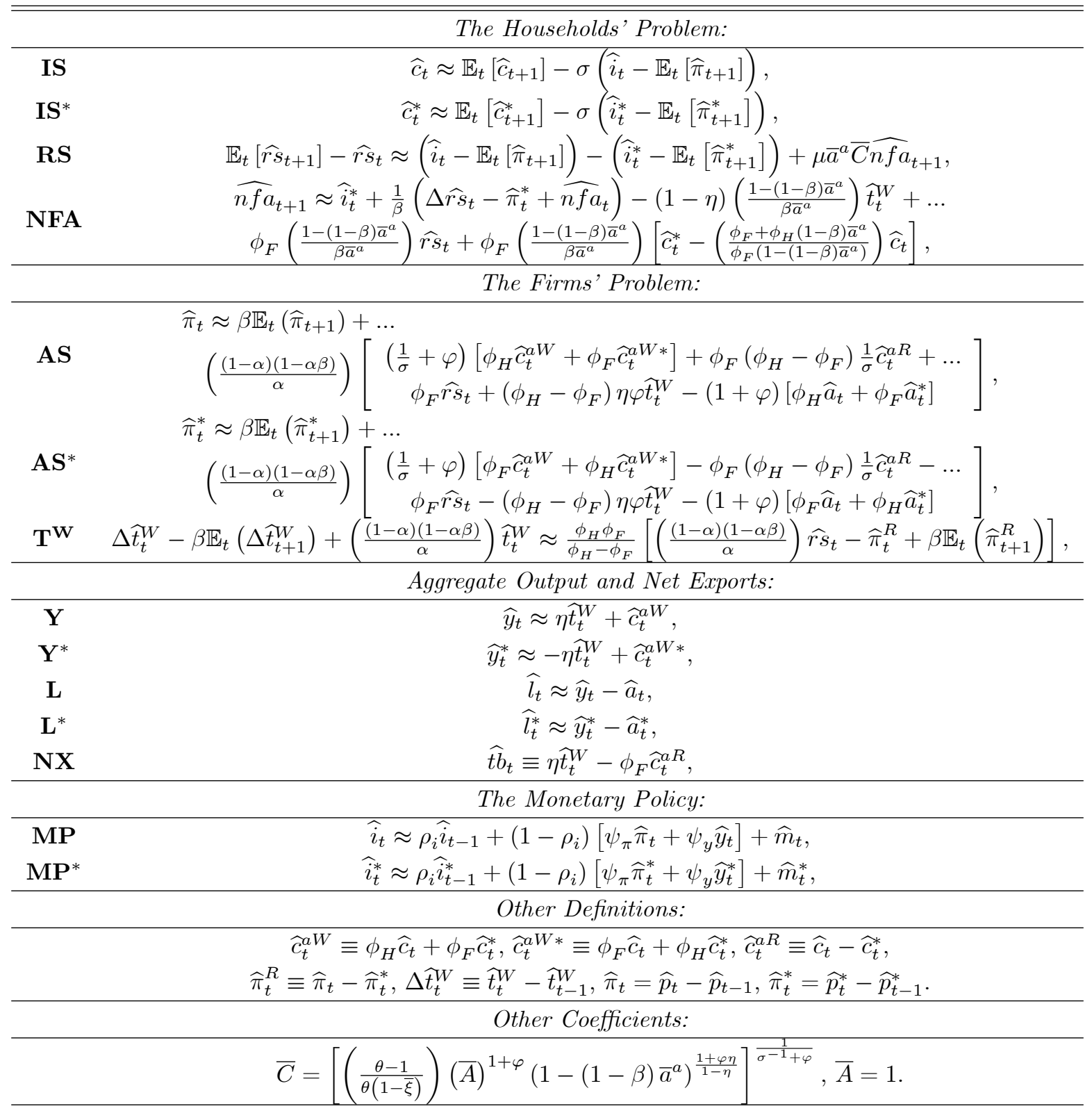

\footnotetext{
${ }^{13}$ Any variable identified with lower-case letters and a caret on top represents a transformation (expressed in log deviations relative to its steady state) of the corresponding variable in upper-case letters.
} 


\section{A.2 The Model With Capital, Without Capital Utilization - NAC}

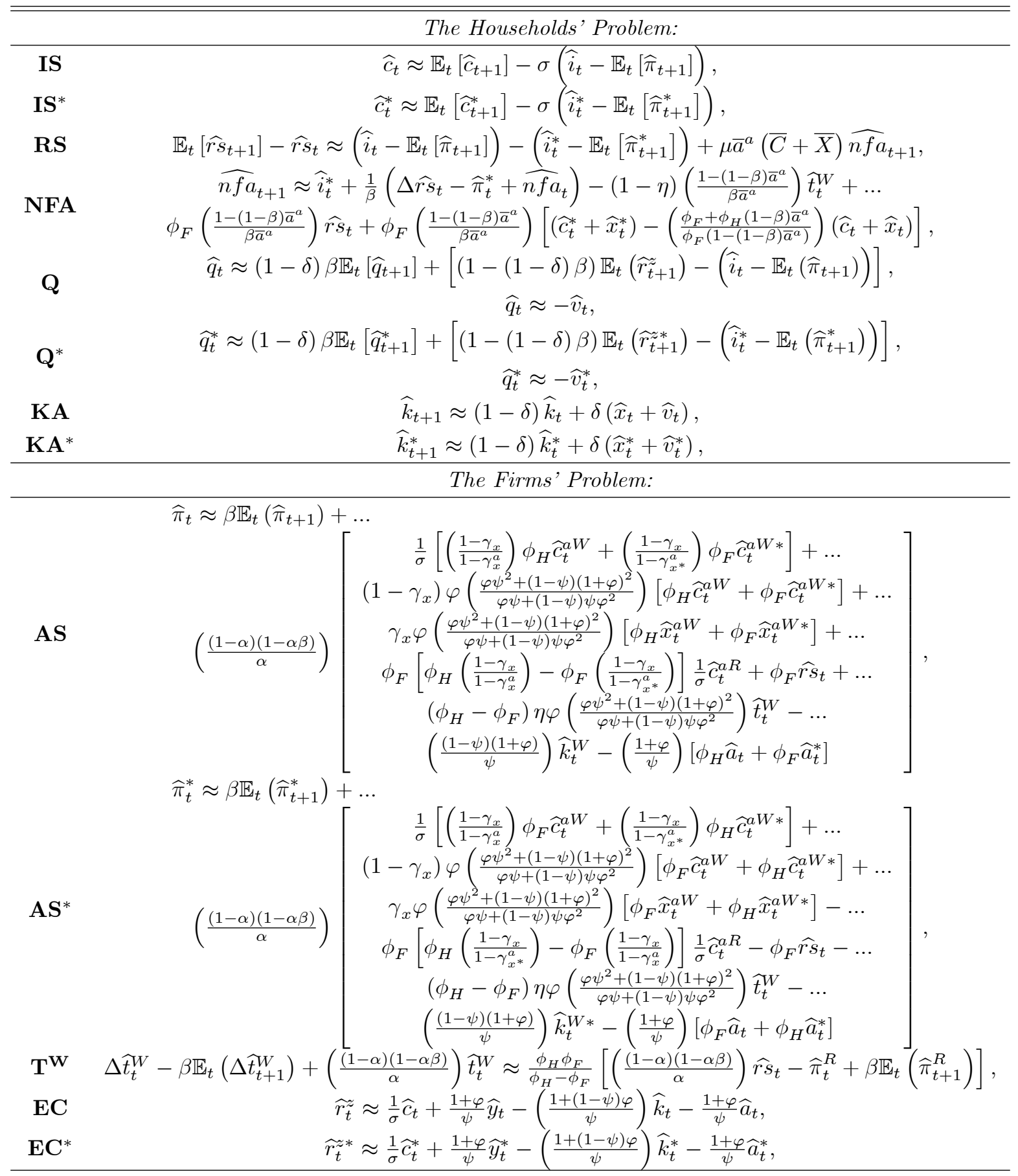




\section{A.3 The Model With Capital, Without Capital Utilization - CAC}

\begin{tabular}{|c|c|}
\hline & The Households' Problem: \\
\hline IS & $\widehat{c}_{t} \approx \mathbb{E}_{t}\left[\widehat{c}_{t+1}\right]-\sigma\left(\widehat{i}_{t}-\mathbb{E}_{t}\left[\widehat{\pi}_{t+1}\right]\right)$, \\
\hline IS* & $\widehat{c}_{t}^{*} \approx \mathbb{E}_{t}\left[\widehat{c}_{t+1}^{*}\right]-\sigma\left(\hat{i}_{t}^{*}-\mathbb{E}_{t}\left[\widehat{\pi}_{t+1}^{*}\right]\right)$ \\
\hline RS & $\mathbb{E}_{t}\left[\widehat{r s}_{t+1}\right]-\widehat{r}_{t} \approx\left(\widehat{i}_{t}-\mathbb{E}_{t}\left[\widehat{\pi}_{t+1}\right]\right)-\left(\widehat{i}_{t}^{*}-\mathbb{E}_{t}\left[\widehat{\pi}_{t+1}^{*}\right]\right)+\mu \bar{a}^{a}(\bar{C}+\bar{X}) \widehat{n f a} a_{t+1}$ \\
\hline NFA & $\begin{array}{c}\widehat{n f a}_{t+1} \approx \widehat{i}_{t}^{*}+\frac{1}{\beta}\left(\Delta \widehat{r s}_{t}-\widehat{\pi}_{t}^{*}+\widehat{n f a}_{t}\right)-(1-\eta)\left(\frac{1-(1-\beta) \bar{a}^{a}}{\beta \bar{a}^{a}}\right) \widehat{t}_{t}^{W}+\ldots \\
\phi_{F}\left(\frac{1-(1-\beta) \bar{a}^{a}}{\beta \bar{a}^{a}}\right) \widehat{r s}_{t}+\phi_{F}\left(\frac{1-(1-\beta) \bar{a}^{a}}{\beta \bar{a} \bar{a}^{a}}\right)\left[\left(\widehat{c}_{t}^{*}+\widehat{x}_{t}^{*}\right)-\left(\frac{\phi_{F}+\phi_{H}(1-\beta) \bar{a}^{a}}{\phi_{F}\left(1-(1-\beta) \bar{a}^{a}\right)}\right)\left(\widehat{c}_{t}+\widehat{x}_{t}\right)\right],\end{array}$ \\
\hline Q & $\begin{array}{c}\widehat{q}_{t} \approx \beta \mathbb{E}_{t}\left[\widehat{q}_{t+1}\right]+\left[(1-(1-\delta) \beta) \mathbb{E}_{t}\left(\widehat{r}_{t+1}^{z}\right)-\left(\widehat{i}_{t}-\mathbb{E}_{t}\left(\widehat{\pi}_{t+1}\right)\right)\right] \\
\widehat{q}_{t} \approx \chi \delta\left(\widehat{x}_{t}-\widehat{k}_{t}\right)-\widehat{v}_{t}\end{array}$ \\
\hline $\mathbf{Q}^{*}$ & $\begin{array}{c}\widehat{q}_{t}^{*} \approx \beta \mathbb{E}_{t}\left[\widehat{q}_{t+1}^{*}\right]+[ \\
{\left[(1-(1-\delta) \beta) \mathbb{E}_{t}\left(\widehat{r}_{t+1}^{z *}\right)-\left(\widehat{i}_{t}^{*}-\mathbb{E}_{t}\left(\widehat{\pi}_{t+1}^{*}\right)\right)\right]} \\
\widehat{q}_{t}^{*} \approx \chi \delta\left(\widehat{x}_{t}^{*}-\widehat{k}_{t}^{*}\right)-\widehat{v}_{t}^{*}\end{array}$ \\
\hline KA & $\widehat{k}_{t+1} \approx(1-\delta) \widehat{k}_{t}+\delta\left(\widehat{x}_{t}+\widehat{v}_{t}\right)$ \\
\hline $\mathbf{K A}^{*}$ & $\widehat{k}_{t+1}^{*} \approx(1-\delta) \widehat{k}_{t}^{*}+\delta\left(\widehat{x}_{t}^{*}+\widehat{v}_{t}^{*}\right)$ \\
\hline & The Firms' Problem: \\
\hline AS & $\begin{array}{l}\widehat{\pi}_{t} \approx \beta \mathbb{E}_{t}\left(\widehat{\pi}_{t+1}\right)+\ldots \\
{\left[\frac{(1-\alpha)(1-\alpha \beta)}{\alpha}\right)\left[\begin{array}{c}\frac{1}{\sigma}\left[\left(\frac{1-\gamma_{x}}{1-\gamma_{x}^{a}}\right) \phi_{H} \widehat{c}_{t}^{a W}+\left(\frac{1-\gamma_{x}}{1-\gamma_{x^{*}}^{a}}\right) \phi_{F} \widehat{c}_{t}^{a W *}\right]+\ldots \\
\left(1-\gamma_{x}\right) \varphi\left(\frac{\varphi \psi^{2}+(1-\psi)(1+\varphi)^{2}}{\varphi \psi+(1-\psi) \psi \varphi^{2}}\right)\left[\phi_{H} \widehat{c}_{t}^{a W}+\phi_{F} \widehat{c}_{t}^{a W *}\right]+\ldots \\
\gamma_{x} \varphi\left(\frac{\varphi \psi^{2}+(1-\psi)(1+\varphi)^{2}}{\varphi \psi+(1-\psi) \psi \varphi^{2}}\right)\left[\phi_{H} \widehat{x}_{t}^{a W}+\phi_{F} \widehat{x}_{t}^{a W *}\right]+\ldots \\
\phi_{F}\left[\phi_{H}\left(\frac{1-\gamma_{x}}{1-\gamma_{x}^{a}}\right)-\phi_{F}\left(\frac{1-\gamma_{x}}{1-\gamma_{x^{*}}^{a}}\right)\right] \frac{1}{\sigma} \widehat{c}_{t}^{a R}+\phi_{F} \widehat{r s}_{t}+\ldots \\
\left(\phi_{H}-\phi_{F}\right) \eta \varphi\left(\frac{\varphi \psi^{2}+(1-\psi)(1+\varphi)^{2}}{\varphi \psi+(1-\psi) \psi \varphi^{2}}\right) \widehat{t}_{t}^{W}-\ldots \\
\left(\frac{(1-\psi)(1+\varphi)}{\psi}\right) \widehat{k}_{t}^{W}-\left(\frac{1+\varphi}{\psi}\right)\left[\phi_{H} \widehat{a}_{t}+\phi_{F} \widehat{a}_{t}^{*}\right]\end{array}\right.}\end{array}$ \\
\hline $\mathbf{A} \mathbf{S}^{*}$ & 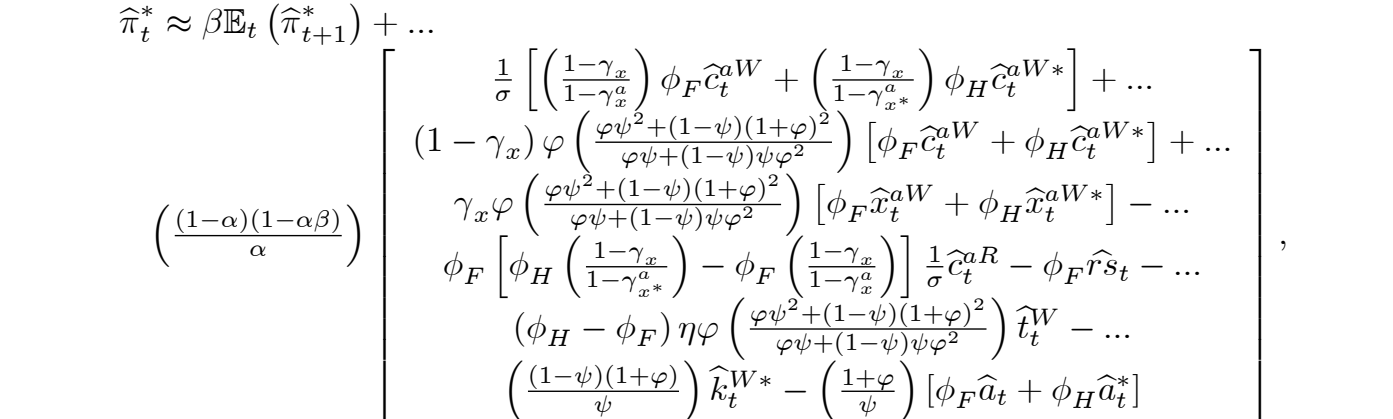 \\
\hline $\mathrm{T}^{\mathrm{W}}$ & $\Delta \widehat{t}_{t}^{W}-\beta \mathbb{E}_{t}\left(\Delta \widehat{t}_{t+1}^{W}\right)+\left(\frac{(1-\alpha)(1-\alpha \beta)}{\alpha}\right) \widehat{t}_{t}^{W} \approx \frac{\phi_{H} \phi_{F}}{\phi_{H}-\phi_{F}}\left[\left(\frac{(1-\alpha)(1-\alpha \beta)}{\alpha}\right) \widehat{r s}_{t}-\widehat{\pi}_{t}^{R}+\beta \mathbb{E}_{t}\left(\widehat{\pi}_{t+1}^{R}\right)\right.$ \\
\hline EC & $\widehat{r}_{t}^{z} \approx \frac{1}{\sigma} \widehat{c}_{t}+\frac{1+\varphi}{\psi} \widehat{y}_{t}-\left(\frac{1+(1-\psi) \varphi}{\psi}\right) \widehat{k}_{t}-\frac{1+\varphi}{\psi} \widehat{a}_{t}$ \\
\hline $\mathbf{E C}^{*}$ & $\widehat{r}_{t}^{z *} \approx \frac{1}{\sigma} \widehat{c}_{t}^{*}+\frac{1+\varphi}{\psi} \widehat{y}_{t}^{*}-\left(\frac{1+(1-\psi) \varphi}{\psi}\right) \widehat{k}_{t}^{*}-\frac{1+\varphi}{\psi} \widehat{a}_{t}^{*}$ \\
\hline
\end{tabular}




\section{A.4 The Model With Capital, Without Capital Utilization - IAC}

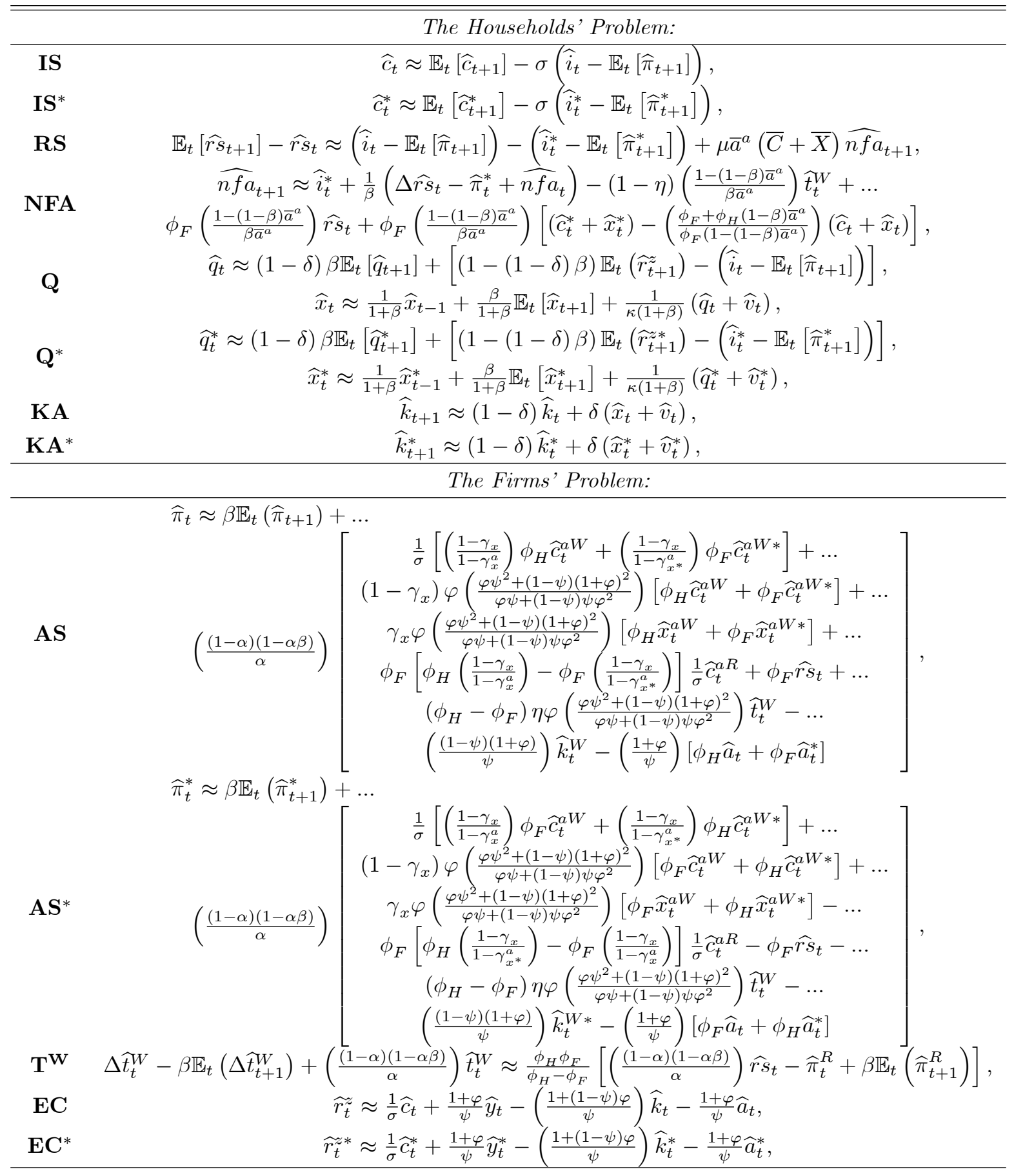




\section{A.5 Other Relationships - All Models With Capital, Without Capacity Utiliza-}

tion

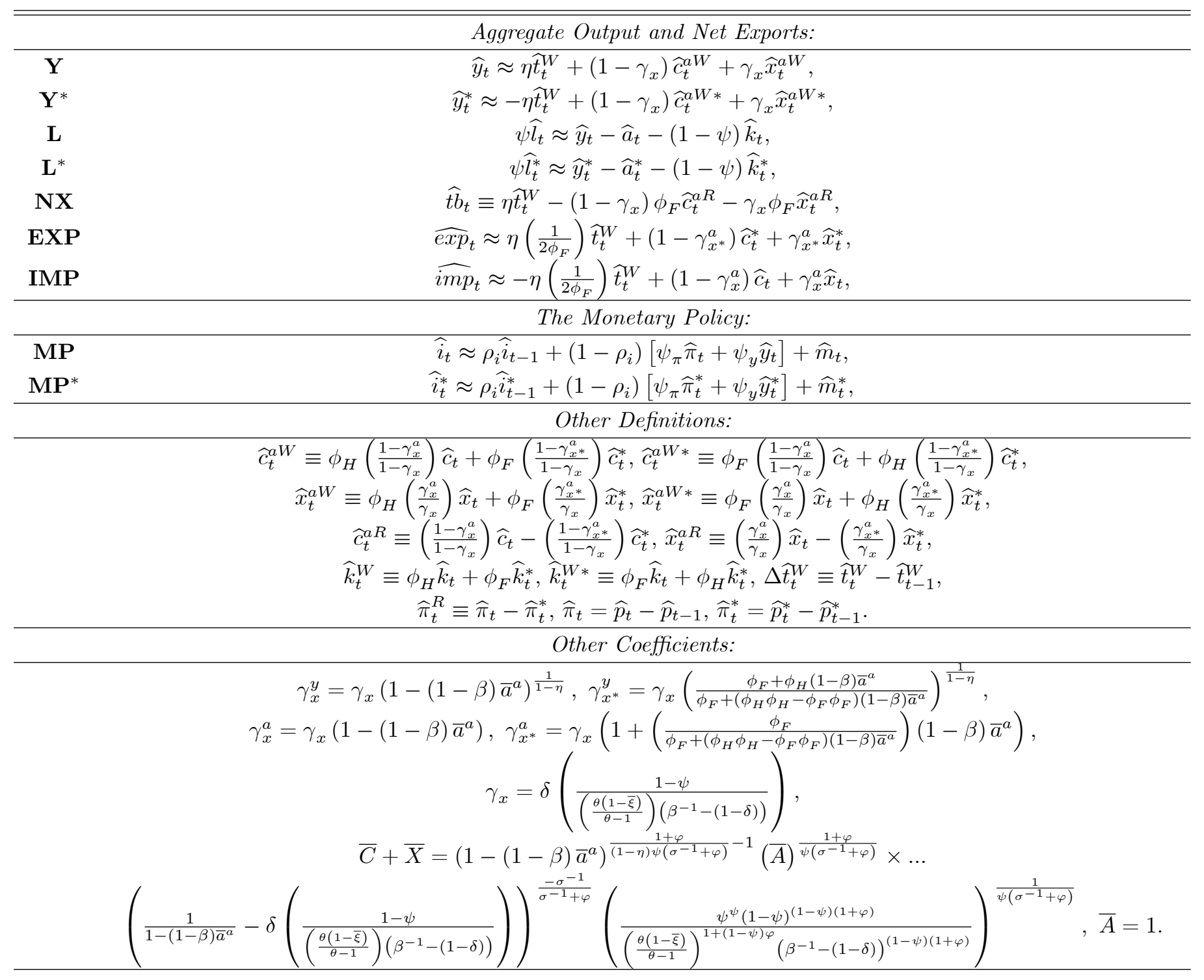




\section{A.6 The Model With Capital, With Capital Utilization - NAC}

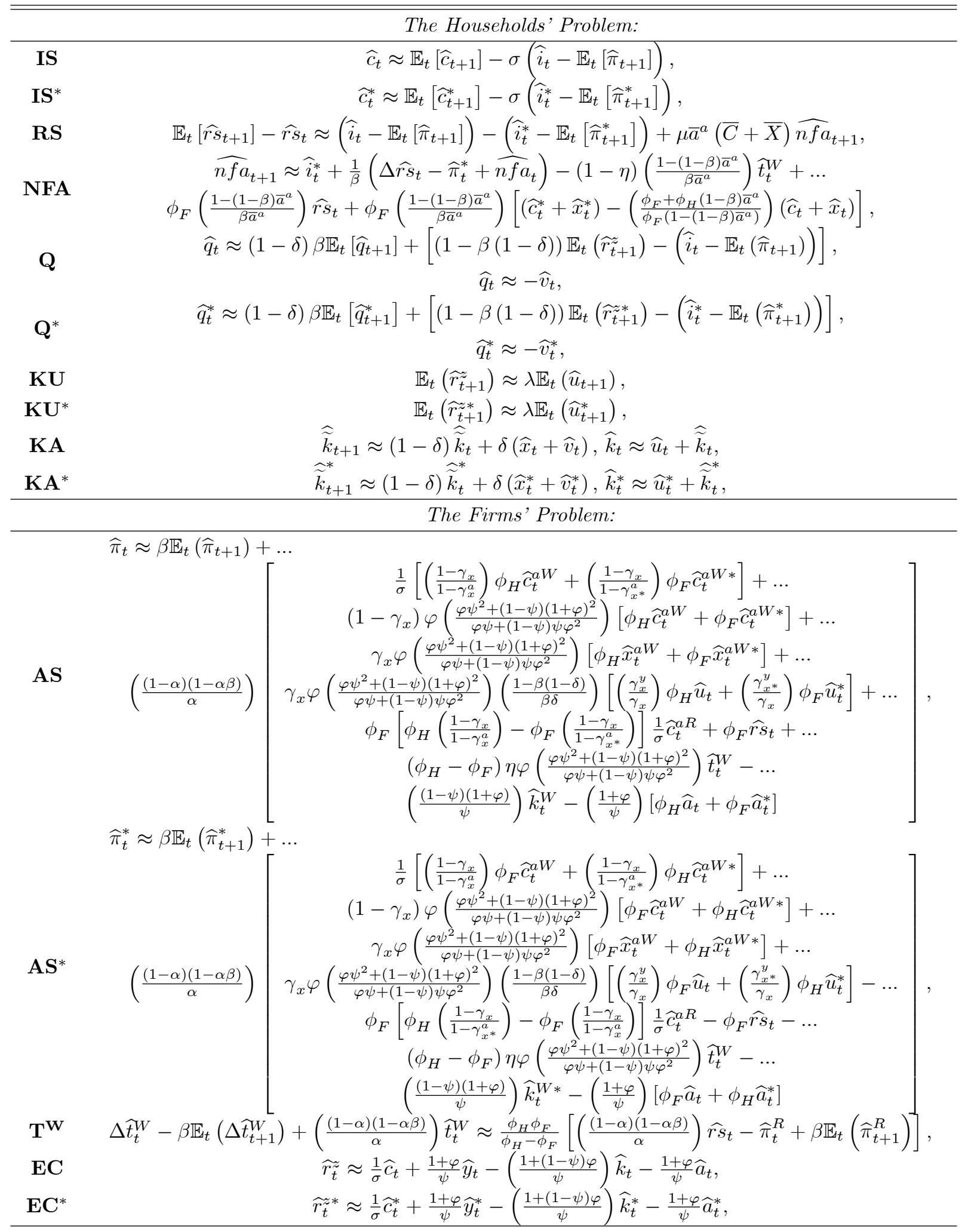




\section{A.7 The Model With Capital, With Capital Utilization - CAC}

\begin{tabular}{|c|c|}
\hline & The Households' Problem: \\
\hline IS & $\widehat{c}_{t} \approx \mathbb{E}_{t}\left[\widehat{c}_{t+1}\right]-\sigma\left(\widehat{i}_{t}-\mathbb{E}_{t}\left[\widehat{\pi}_{t+1}\right]\right)$ \\
\hline IS $^{*}$ & $\widehat{c}_{t}^{*} \approx \mathbb{E}_{t}\left[\widehat{c}_{t+1}^{*}\right]-\sigma\left(\hat{i}_{t}^{*}-\mathbb{E}_{t}\left[\widehat{\pi}_{t+1}^{*}\right]\right)$ \\
\hline $\mathbf{R S}$ & $\mathbb{E}_{t}\left[\widehat{r s}_{t+1}\right]-\widehat{r s}_{t} \approx\left(\widehat{i}_{t}-\mathbb{E}_{t}\left[\widehat{\pi}_{t+1}\right]\right)-\left(\widehat{i}_{t}^{*}-\mathbb{E}_{t}\left[\widehat{\pi}_{t+1}^{*}\right]\right)+\mu \bar{a}^{a}(\bar{C}+\bar{X}) \widehat{n f a} a_{t+1}$ \\
\hline NFA & $\begin{array}{c}\widehat{n f a}_{t+1} \approx \widehat{i}_{t}^{*}+\frac{1}{\beta}\left(\Delta \widehat{r s}_{t}-\widehat{\pi}_{t}^{*}+\widehat{n f a}_{t}\right)-(1-\eta)\left(\frac{1-(1-\beta) \bar{a}^{a}}{\beta \bar{a}^{a}}\right) \widehat{t}_{t}^{W}+\ldots \\
\phi_{F}\left(\frac{1-(1-\beta) \bar{a}^{a}}{\beta \bar{a}^{a}}\right) \widehat{r s}_{t}+\phi_{F}\left(\frac{1-(1-\beta) \bar{a}^{a}}{\beta \bar{a}^{a}}\right)\left[\left(\widehat{c}_{t}^{*}+\widehat{x}_{t}^{*}\right)-\left(\frac{\phi_{F}+\phi_{H}(1-\beta) \bar{a}^{a}}{\phi_{F}\left(1-(1-\beta) \bar{a}^{a}\right)}\right)\left(\widehat{c}_{t}+\widehat{x}_{t}\right)\right],\end{array}$ \\
\hline Q & $\begin{array}{c}\widehat{q}_{t} \approx \beta \mathbb{E}_{t}\left[\widehat{q}_{t+1}\right]+[ \\
{\left[(1-(1-\delta) \beta) \mathbb{E}_{t}\left(\widehat{r}_{t+1}^{z}\right)-\left(\widehat{i}_{t}-\mathbb{E}_{t}\left(\widehat{\pi}_{t+1}\right)\right)\right]} \\
\widehat{q}_{t} \approx \chi \delta\left(\widehat{x}_{t}-\widehat{k}_{t}\right)-\widehat{v}_{t}\end{array}$ \\
\hline $\mathrm{Q}^{*}$ & $\begin{aligned} & \widehat{q}_{t}^{*} \approx \beta \mathbb{E}_{t}\left[\widehat{q}_{t+1}^{*}\right]+[\left.(1-(1-\delta) \beta) \mathbb{E}_{t}\left(\widehat{r}_{t+1}^{z *}\right)-\left(\widehat{i}_{t}^{*}-\mathbb{E}_{t}\left(\widehat{\pi}_{t+1}^{*}\right)\right)\right], \\
& \widehat{q}_{t}^{*} \approx \chi \delta\left(\widehat{x}_{t}^{*}-\widehat{k}_{t}^{*}\right)-\widehat{v}_{t}^{*}\end{aligned}$ \\
\hline KU & $\mathbb{E}_{t}\left[\widehat{r}_{t+1}^{z}\right] \approx \mathbb{E}_{t}\left[\lambda \widehat{u}_{t+1}-\left(\frac{\delta \beta}{1-(1-\delta) \beta}\right)\left(\widehat{q}_{t+1}+\widehat{v}_{t+1}\right)\right]$ \\
\hline $\mathbf{K U}^{*}$ & $\mathbb{E}_{t}\left[\widehat{r}_{t+1}^{z *}\right] \approx \mathbb{E}_{t}\left[\lambda \widehat{u}_{t+1}^{*}-\left(\frac{\delta \beta}{1-(1-\delta) \beta}\right)\left(\widehat{q}_{t+1}^{*}+\widehat{v}_{t+1}^{*}\right)\right]$, \\
\hline KA & $\widehat{\widetilde{k}}_{t+1} \approx(1-\delta) \widehat{\widetilde{k}}_{t}+\delta\left(\widehat{x}_{t}+\widehat{v}_{t}\right), \widehat{k}_{t} \approx \widehat{u}_{t}+\widehat{\widetilde{k}}_{t}$ \\
\hline $\mathbf{K A}^{*}$ & $\widehat{\widehat{k}}_{t+1}^{*} \approx(1-\delta) \widehat{\widetilde{k}}_{t}^{*}+\delta\left(\widehat{x}_{t}^{*}+\widehat{v}_{t}^{*}\right), \widehat{k}_{t}^{*} \approx \widehat{u}_{t}^{*}+\widehat{\widehat{k}}_{t}^{*}$ \\
\hline
\end{tabular}

The Firms' Problem:

$\widehat{\pi}_{t} \approx \beta \mathbb{E}_{t}\left(\widehat{\pi}_{t+1}\right)+\ldots$

$$
\left(\frac{(1-\alpha)(1-\alpha \beta)}{\alpha}\right)\left[\begin{array}{c}
\gamma_{x} \varphi\left(\frac{\varphi \psi^{2}+(1-\psi)(1+\varphi)^{2}}{\varphi \psi+(1-\psi) \psi \varphi^{2}}\right)\left(\frac{1-\beta(1-\delta)}{\beta \delta}\right)\left[\left(\frac{\gamma_{x}^{y}}{\gamma_{x}}\right) \phi_{H} \widehat{u}_{t}+\left(\frac{\gamma_{x *}^{y}}{\gamma_{x}}\right) \phi_{F} \widehat{u}_{t}^{*}\right]+\ldots \\
\phi_{F}\left[\phi_{H}\left(\frac{1-\gamma_{x}}{1-\gamma_{x}^{a}}\right)-\phi_{F}\left(\frac{1-\gamma_{x}}{1-\gamma_{x}^{*}}\right)\right] \frac{1}{\sigma} \widehat{c}_{t}^{a R}+\phi_{F} \widehat{r}_{t}+\ldots \\
\left(\phi_{H}-\phi_{F}\right) \eta \varphi\left(\frac{\varphi \psi^{2}+(1-\psi)(1+\varphi)^{2}}{\varphi \psi+(1-\psi) \psi \varphi^{2}}\right) \widehat{t}_{t}^{W}-\ldots \\
\left(\frac{(1-\psi)(1+\varphi)}{\psi}\right) \widehat{k}_{t}^{W}-\left(\frac{1+\varphi}{\psi}\right)\left[\phi_{H} \widehat{a}_{t}+\phi_{F} \widehat{a}_{t}^{*}\right]
\end{array}\right]
$$

$\widehat{\pi}_{t}^{*} \approx \beta \mathbb{E}_{t}\left(\widehat{\pi}_{t+1}^{*}\right)+\ldots$

$\mathbf{A S}^{*}$

$$
\left(\frac{(1-\alpha)(1-\alpha \beta)}{\alpha}\right)\left[\begin{array}{c}
\frac{1}{\sigma}\left[\left(\frac{1-\gamma_{x}}{1-\gamma_{x}^{a}}\right) \phi_{F} \widehat{c}_{t}^{a W}+\left(\frac{1-\gamma_{x}}{1-\gamma_{x}^{a}}\right) \phi_{H} \widehat{c}_{t}^{a W *}\right]+\ldots \\
\left(1-\gamma_{x}\right) \varphi\left(\frac{\varphi \psi^{2}+(1-\psi)(1+\varphi)^{2}}{\varphi \psi+(1-\psi) \psi \varphi^{2}}\right)\left[\phi_{F} \widehat{c}_{t}^{a W}+\phi_{H} \widehat{c}_{t}^{a W *}\right]+\ldots \\
\gamma_{x} \varphi\left(\frac{\varphi \psi^{2}+(1-\psi)(1+\varphi)^{2}}{\varphi \psi+(1-\psi) \psi \varphi^{2}}\right)\left[\phi_{F} \widehat{x}_{t}^{a W}+\phi_{H} \widehat{x}_{t}^{a W *}\right]+\ldots \\
\gamma_{x} \varphi\left(\frac{\varphi \psi^{2}+(1-\psi)(1+\varphi)^{2}}{\varphi \psi+(1-\psi) \psi \varphi^{2}}\right)\left(\frac{1-\beta(1-\delta)}{\beta \delta}\right)\left[\left(\frac{\gamma_{x}^{y}}{\gamma_{x}}\right) \phi_{F} \widehat{u}_{t}+\left(\frac{\gamma_{x *}^{y}}{\gamma_{x}}\right) \phi_{H} \widehat{u}_{t}^{*}\right]-\ldots
\end{array}\right],
$$$$
\phi_{F}\left[\phi_{H}\left(\frac{1-\gamma_{x}}{1-\gamma_{x^{*}}^{a}}\right)-\phi_{F}\left(\frac{1-\gamma_{x}}{1-\gamma_{x}^{a}}\right)\right] \frac{1}{\sigma} \widehat{c}_{t}^{a R}-\phi_{F} \widehat{r s}_{t}-\ldots
$$$$
\left(\phi_{H}-\phi_{F}\right) \eta \varphi\left(\frac{\varphi \psi^{2}+(1-\psi)(1+\varphi)^{2}}{\varphi \psi+(1-\psi) \psi \varphi^{2}}\right) \hat{t}_{t}^{W}-\ldots
$$$$
\left(\frac{(1-\psi)(1+\varphi)}{\psi}\right) \widehat{k}_{t}^{W *}-\left(\frac{1+\varphi}{\psi}\right)\left[\phi_{F} \widehat{a}_{t}+\phi_{H} \widehat{a}_{t}^{*}\right]
$$

$\mathbf{T} \mathbf{W} \quad \Delta \widehat{t}_{t}^{W}-\beta \mathbb{E}_{t}\left(\Delta \widehat{t}_{t+1}^{W}\right)+\left(\frac{(1-\alpha)(1-\alpha \beta)}{\alpha}\right) \widehat{t}_{t}^{W} \approx \frac{\phi_{H} \phi_{F}}{\phi_{H}-\phi_{F}}\left[\left(\frac{(1-\alpha)(1-\alpha \beta)}{\alpha}\right) \widehat{r s}_{t}-\widehat{\pi}_{t}^{R}+\beta \mathbb{E}_{t}\left(\widehat{\pi}_{t+1}^{R}\right)\right]$,

EC $\quad \widehat{r}_{t}^{z} \approx \frac{1}{\sigma} \widehat{c}_{t}+\frac{1+\varphi}{\psi} \widehat{y}_{t}-\left(\frac{1+(1-\psi) \varphi}{\psi}\right) \widehat{k}_{t}-\frac{1+\varphi}{\psi} \widehat{a}_{t}$,

$\mathbf{E C}^{*} \quad \widehat{r}_{t}^{z *} \approx \frac{1}{\sigma} \widehat{c}_{t}^{*}+\frac{1+\varphi}{\psi} \widehat{y}_{t}^{*}-\left(\frac{1+(1-\psi) \varphi}{\psi}\right) \widehat{k}_{t}^{*}-\frac{1+\varphi}{\psi} \widehat{a}_{t}^{*}$, 


\section{A.8 The Model With Capital, With Capital Utilization - IAC}

\begin{tabular}{|c|c|}
\hline & The Households' Problem: \\
\hline IS & $\widehat{c}_{t} \approx \mathbb{E}_{t}\left[\widehat{c}_{t+1}\right]-\sigma\left(\widehat{i}_{t}-\mathbb{E}_{t}\left[\widehat{\pi}_{t+1}\right]\right)$ \\
\hline $\mathbf{I S}^{*}$ & $\widehat{c}_{t}^{*} \approx \mathbb{E}_{t}\left[\widehat{c}_{t+1}^{*}\right]-\sigma\left(\hat{i}_{t}^{*}-\mathbb{E}_{t}\left[\widehat{\pi}_{t+1}^{*}\right]\right)$ \\
\hline RS & $\mathbb{E}_{t}\left[\widehat{r s}_{t+1}\right]-\widehat{r s}_{t} \approx\left(\widehat{i}_{t}-\mathbb{E}_{t}\left[\widehat{\pi}_{t+1}\right]\right)-\left(\widehat{i}_{t}^{*}-\mathbb{E}_{t}\left[\widehat{\pi}_{t+1}^{*}\right]\right)+\mu \bar{a}^{a}(\bar{C}+\bar{X}) \widehat{n f a}_{t+1}$ \\
\hline NFA & $\begin{array}{c}\widehat{n f a}_{t+1} \approx \widehat{i}_{t}^{*}+\frac{1}{\beta}\left(\Delta \widehat{r s}_{t}-\widehat{\pi}_{t}^{*}+\widehat{n f a}_{t}\right)-(1-\eta)\left(\frac{1-(1-\beta) \bar{a}^{a}}{\beta \bar{a}^{a}}\right) \widehat{t}_{t}^{W}+\ldots \\
\phi_{F}\left(\frac{1-(1-\beta) \bar{a}^{a}}{\beta \bar{a}^{a}}\right) \widehat{r s}_{t}+\phi_{F}\left(\frac{1-(1-\beta) \bar{a}^{a}}{\beta \bar{a}^{a}}\right)\left[\left(\widehat{c}_{t}^{*}+\widehat{x}_{t}^{*}\right)-\left(\frac{\phi_{F}+\phi_{H}(1-\beta) \bar{a}^{a}}{\phi_{F}\left(1-(1-\beta) \bar{a}^{a}\right)}\right)\left(\widehat{c}_{t}+\widehat{x}_{t}\right)\right],\end{array}$ \\
\hline Q & $\begin{array}{c}\widehat{q}_{t} \approx(1-\delta) \beta \mathbb{E}_{t}\left[\widehat{q}_{t+1}\right]+\left[(1-(1-\delta) \beta) \mathbb{E}_{t}\left(\widehat{r}_{t+1}^{z}\right)-\left(\widehat{i}_{t}-\mathbb{E}_{t}\left[\widehat{\pi}_{t+1}\right]\right)\right] \\
\widehat{x}_{t} \approx \frac{1}{1+\beta} \widehat{x}_{t-1}+\frac{\beta}{1+\beta} \mathbb{E}_{t}\left[\widehat{x}_{t+1}\right]+\frac{1}{\kappa(1+\beta)}\left(\widehat{q}_{t}+\widehat{v}_{t}\right)\end{array}$ \\
\hline $\mathbf{Q}^{*}$ & $\begin{array}{c}\widehat{q}_{t}^{*} \approx(1-\delta) \beta \mathbb{E}_{t}\left[\widehat{q}_{t+1}^{*}\right]+\left[(1-(1-\delta) \beta) \mathbb{E}_{t}\left(\widehat{r}_{t+1}^{z *}\right)-\left(\widehat{i}_{t}^{*}-\mathbb{E}_{t}\left[\widehat{\pi}_{t+1}^{*}\right]\right)\right] \\
\widehat{x}_{t}^{*} \approx \frac{1}{1+\beta} \widehat{x}_{t-1}^{*}+\frac{\beta}{1+\beta} \mathbb{E}_{t}\left[\widehat{x}_{t+1}^{*}\right]+\frac{1}{\kappa(1+\beta)}\left(\widehat{q}_{t}^{*}+\widehat{v}_{t}^{*}\right)\end{array}$ \\
\hline KU & $\mathbb{E}_{t}\left[\widehat{r}_{t+1}^{z}\right] \approx \lambda \mathbb{E}_{t}\left[\widehat{u}_{t+1}\right]$ \\
\hline $\mathbf{K U}^{*}$ & $\mathbb{E}_{t}\left[\underset{\stackrel{\widehat{r}_{t+1}^{z *}}{\approx}}{\approx} \approx \lambda \mathbb{E}_{t}\left[\widehat{u}_{t+1}^{*}\right]\right.$ \\
\hline KA & $\widehat{\widetilde{k}}_{t+1} \approx(1-\delta) \widehat{\widetilde{k}}_{t}+\delta\left(\widehat{x}_{t}+\widehat{v}_{t}\right), \widehat{k}_{t} \approx \widehat{u}_{t}+\widehat{\widetilde{k}}_{t}$, \\
\hline $\mathbf{K A}^{*}$ & $\widehat{\widehat{k}}_{t+1}^{*} \approx(1-\delta) \widehat{\widetilde{k}}_{t}+\delta\left(\widehat{x}_{t}^{*}+\widehat{v}_{t}^{*}\right), \widehat{k}_{t}^{*} \approx \widehat{u}_{t}^{*}+\widetilde{\widetilde{k}}_{t}$, \\
\hline
\end{tabular}

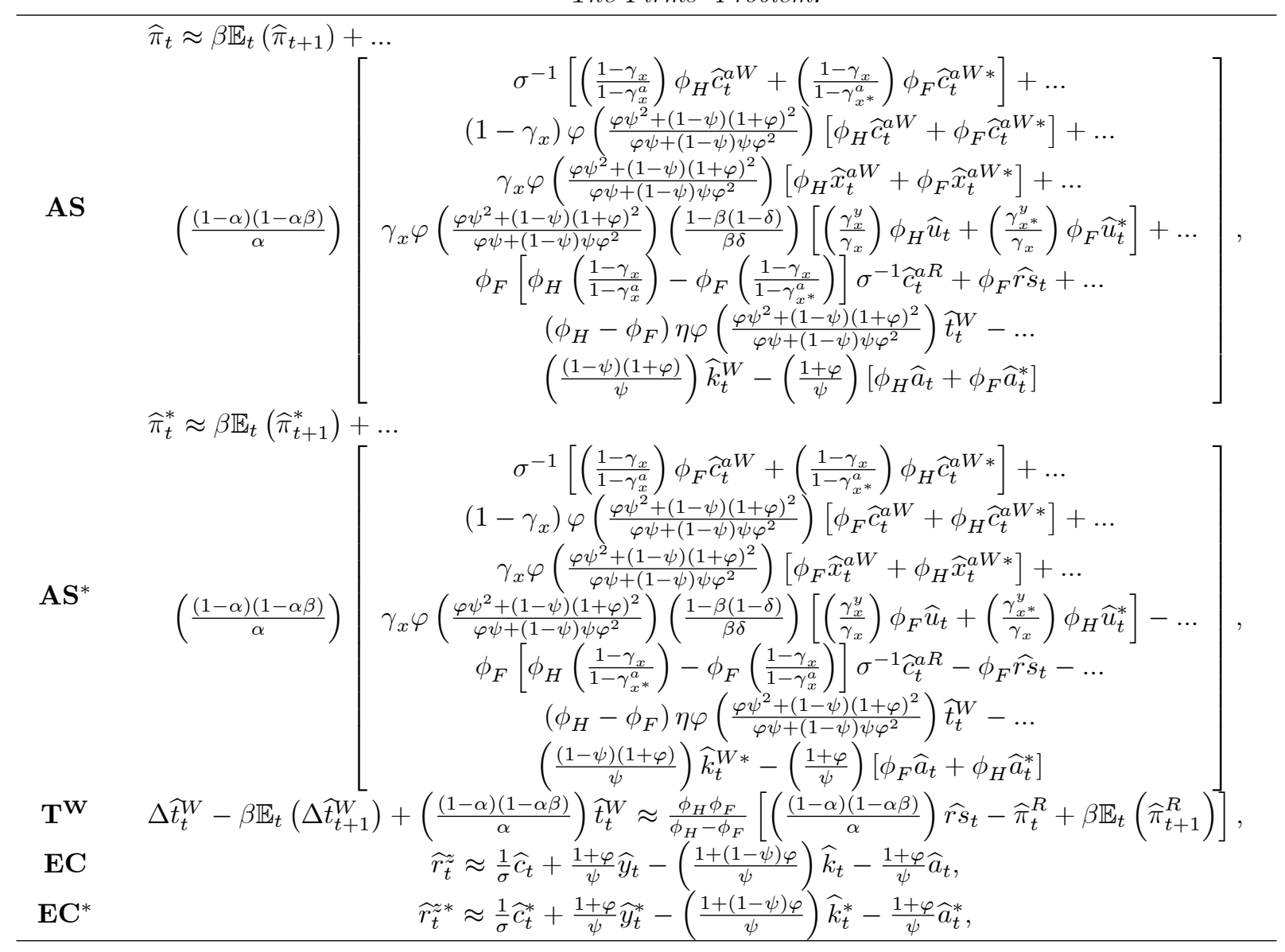




\section{A.9 Other Relationships - All Models With Capital, With Capacity Utilization}

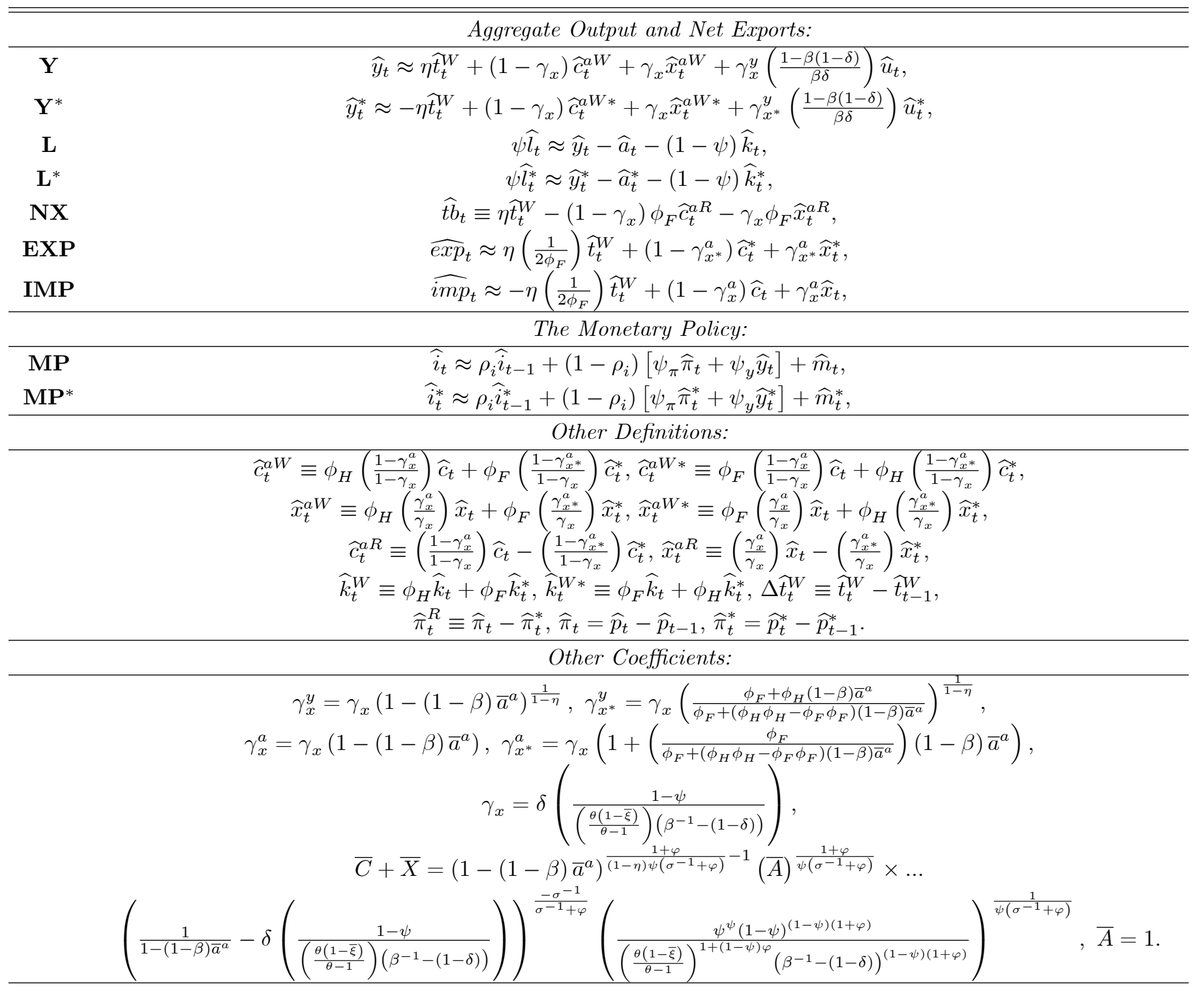




\section{B Tables}

\begin{tabular}{|c|c|c|}
\hline \multicolumn{3}{|l|}{ Structural Parameters: } \\
\hline Intertemporal Discount Factor & $\beta$ & 0.99 \\
\hline Elasticity of Intratemporal Substitution & $\eta$ & 1.5 \\
\hline Elasticity of Substitution across Varieties & $\theta$ & 10 \\
\hline Elasticity of Intertemporal Substitution & $\sigma$ & $1 / 5$ \\
\hline (Inverse) Frisch Elasticity of Labor Supply & $\varphi$ & 3 \\
\hline Share of Foreign Goods & $\phi_{F}$ & 0.06 \\
\hline Calvo Price Stickiness & $\alpha$ & 0.75 \\
\hline Depreciation Rate & $\delta$ & 0.021 \\
\hline Capital/Investment Adjustment Cost & $\chi, \kappa$ & varies \\
\hline Elasticity of Capital Utilization Cost & $\lambda$ & 5.80 \\
\hline Labor Share & $\psi$ & $2 / 3$ \\
\hline Cost of adjusting foreign bondholdings & $\mu$ & 0.01 \\
\hline Reference real net foreign assets over absorption & $\bar{a}^{a}$ & -0.04065 \\
\hline \multicolumn{3}{|l|}{ Taylor Rule Parameters and Others: } \\
\hline Interest Rate Inertia & $\rho_{i}$ & 0.85 \\
\hline Sensitivity to Inflation Target & $\psi_{\pi}$ & 2 \\
\hline Sensitivity to Output Target & $\psi_{y}$ & 0.5 \\
\hline Steady State Government Subsidy & $\bar{\xi}$ & 0 \\
\hline \multicolumn{3}{|l|}{ Exogenous Shock Parameters: } \\
\hline Productivity Shock Persistence & $\rho_{a}$ & 0.9 \\
\hline Productivity Shock Cross-Correlation & $\operatorname{corr}\left(\widehat{\varepsilon}_{t}^{a}, \widehat{\varepsilon}_{t}^{a *}\right)$ & {$[0.43,0.57]$} \\
\hline Productivity Shock Volatility & $\sigma\left(\widehat{\varepsilon}_{t}^{a}\right)=\sigma\left(\widehat{\varepsilon}_{t}^{a *}\right)$ & {$[1.27,3.41]$} \\
\hline IST Shock Persistence & $\rho_{v}$ & 0.9 \\
\hline IST Shock Cross-Correlation & $\operatorname{corr}\left(\widehat{\varepsilon}_{t}^{v}, \widehat{\varepsilon}_{t}^{v *}\right)$ & {$[0.42,0.61]$} \\
\hline IST Shock Volatility & $\sigma\left(\widehat{\varepsilon}_{t}^{v}\right)=\sigma\left(\widehat{\varepsilon}_{t}^{v *}\right)$ & {$[2.47,10]$} \\
\hline Monetary Shock Cross-Correlation & $\operatorname{corr}\left(\widehat{\varepsilon}_{t}^{m}, \widehat{\varepsilon}_{t}^{m *}\right)$ & {$[0.32,0.64]$} \\
\hline Monetary Shock Volatility & $\sigma\left(\widehat{\varepsilon}_{t}^{m}\right)=\sigma\left(\widehat{\varepsilon}_{t}^{m *}\right)$ & {$[0.29,2.63]$} \\
\hline
\end{tabular}

This table summarizes my parameterization. The choice of the capital adjustment cost parameter or the investment adjustment cost parameter is explicitly stated in the Tables. Whenever certain features of the model are not being incorporated (in particular the variable capital utilization and the incomplete international asset markets structure), I set the corresponding structural parameters to zero. The exact parameterization of the shock processes in each experiment can be obtained upon request. 


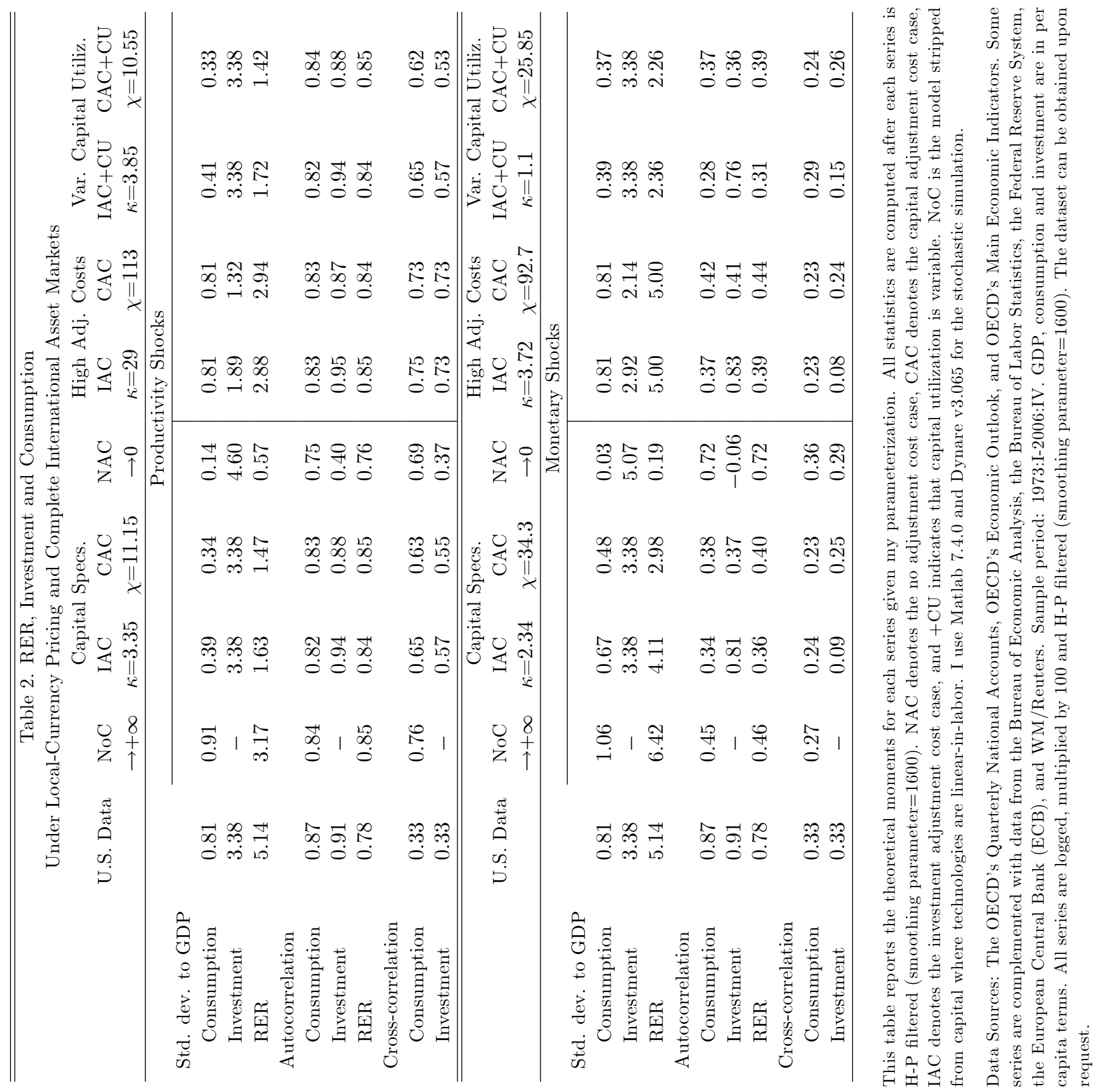




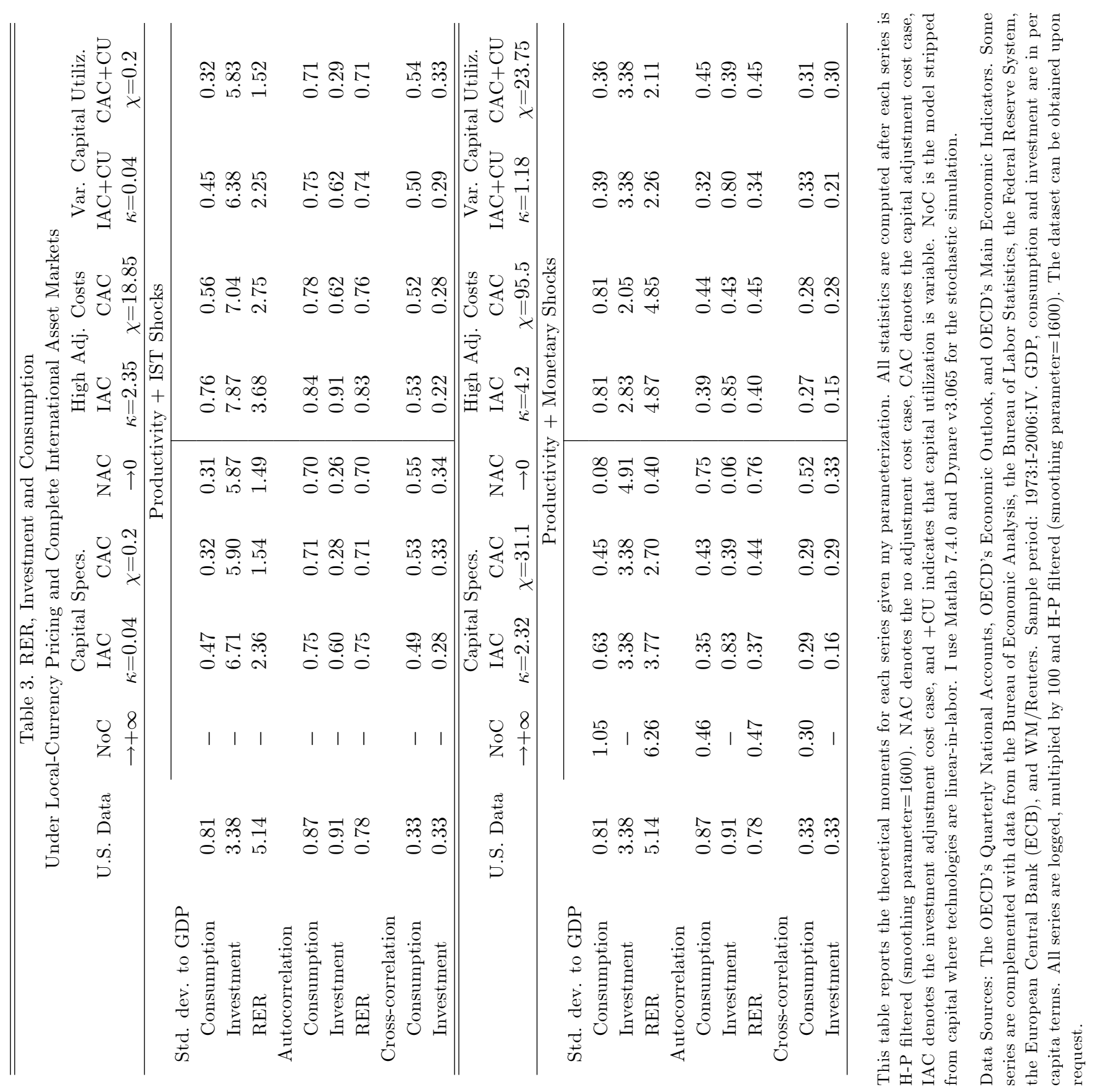




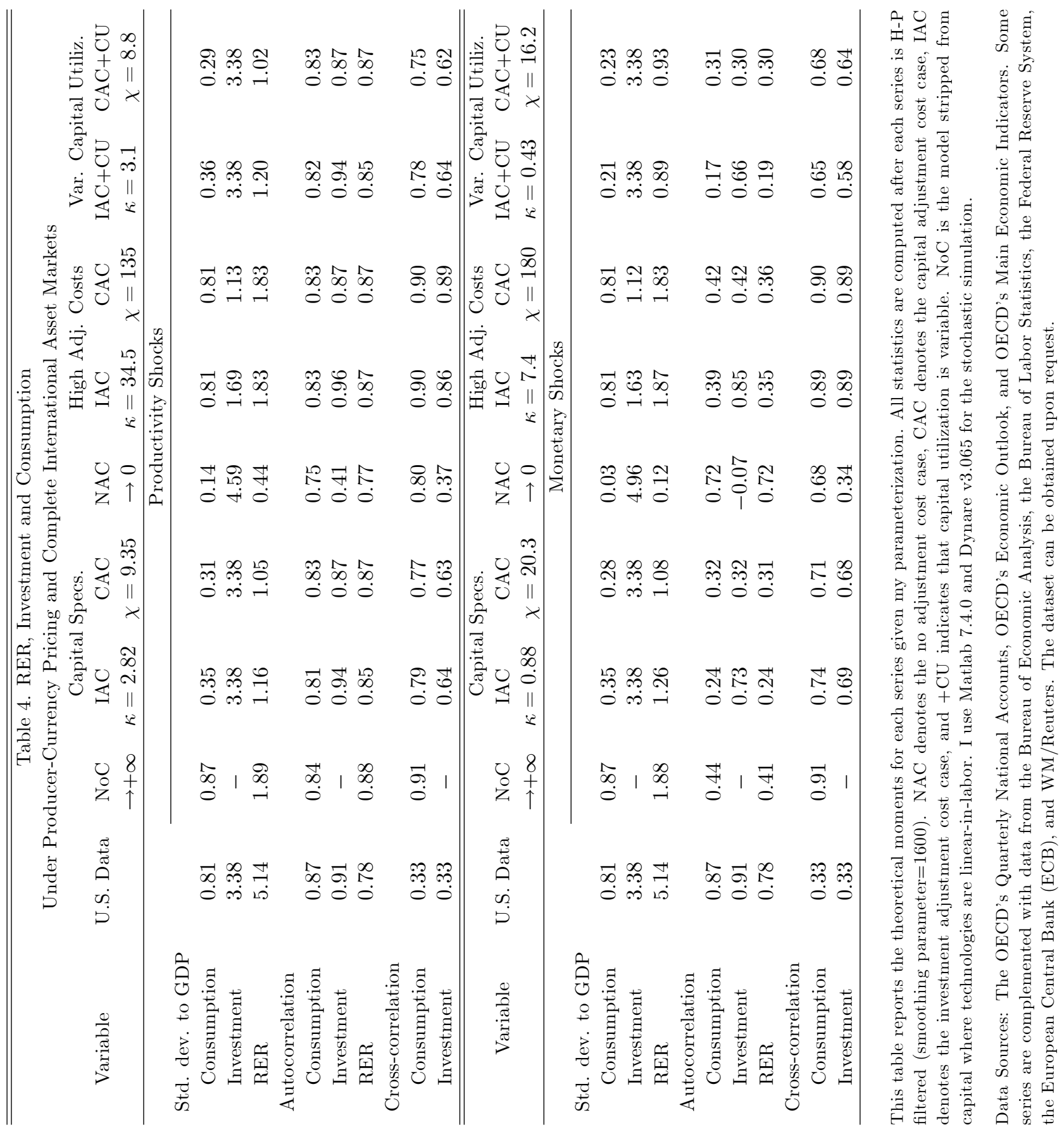




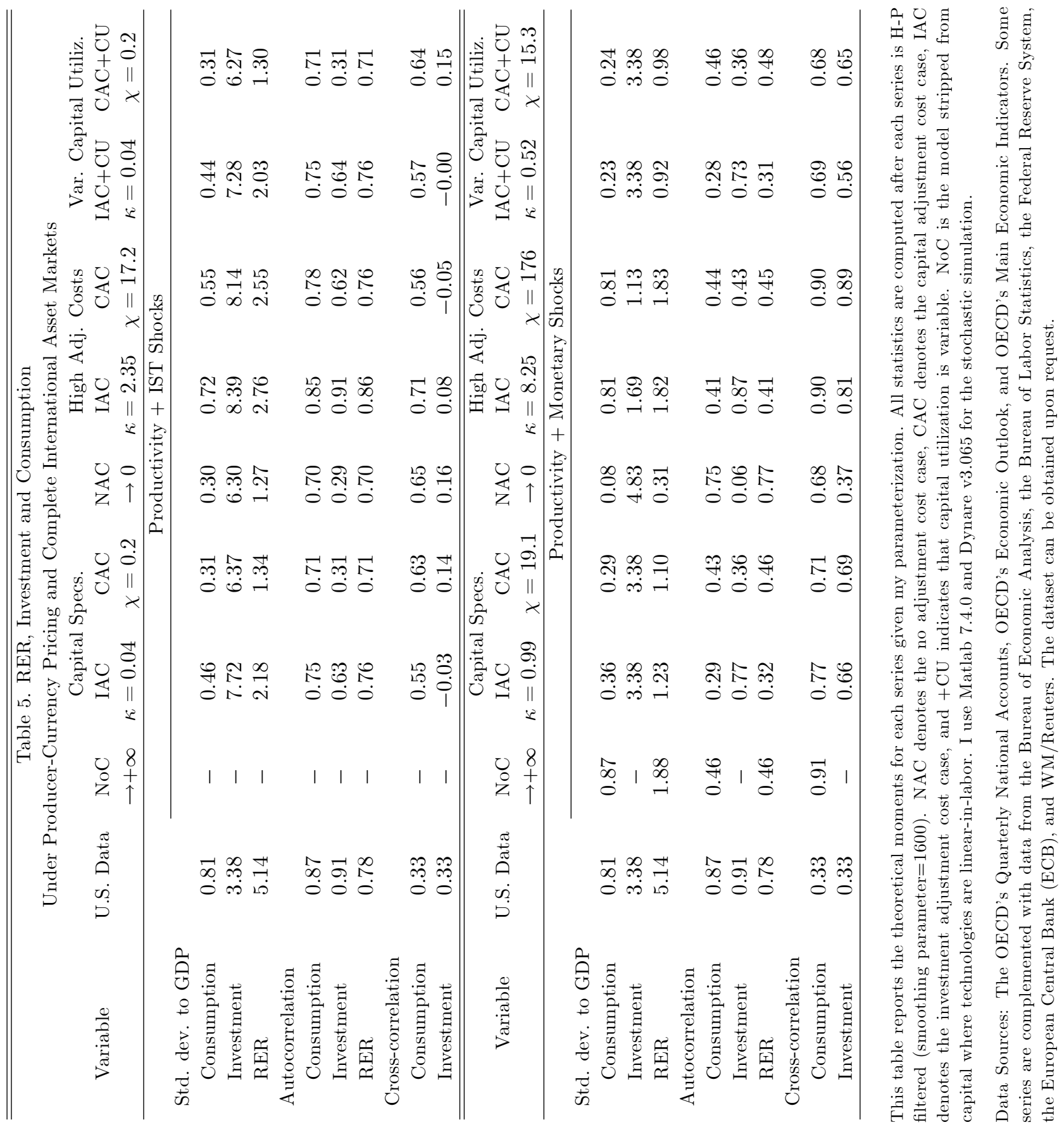




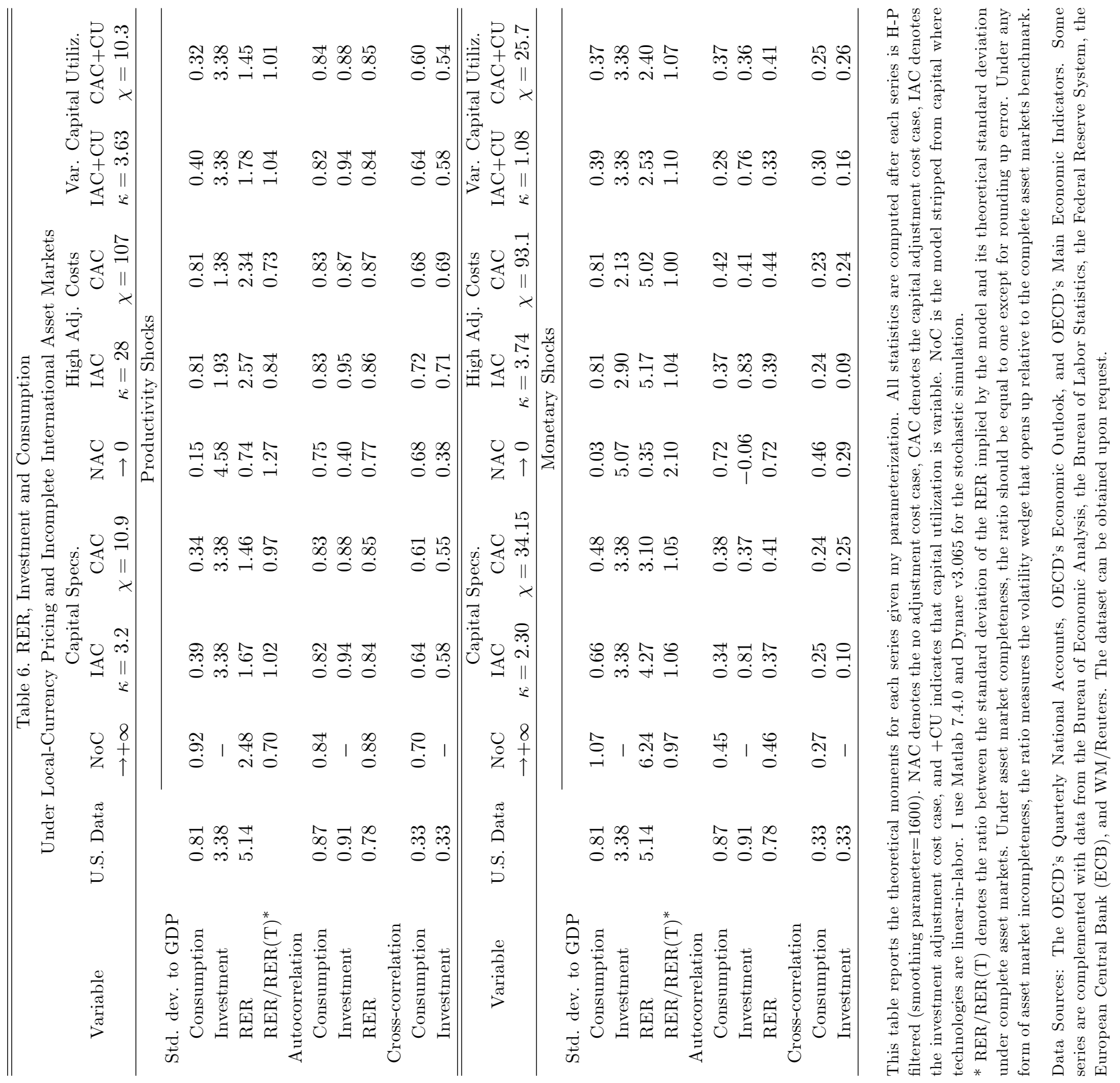




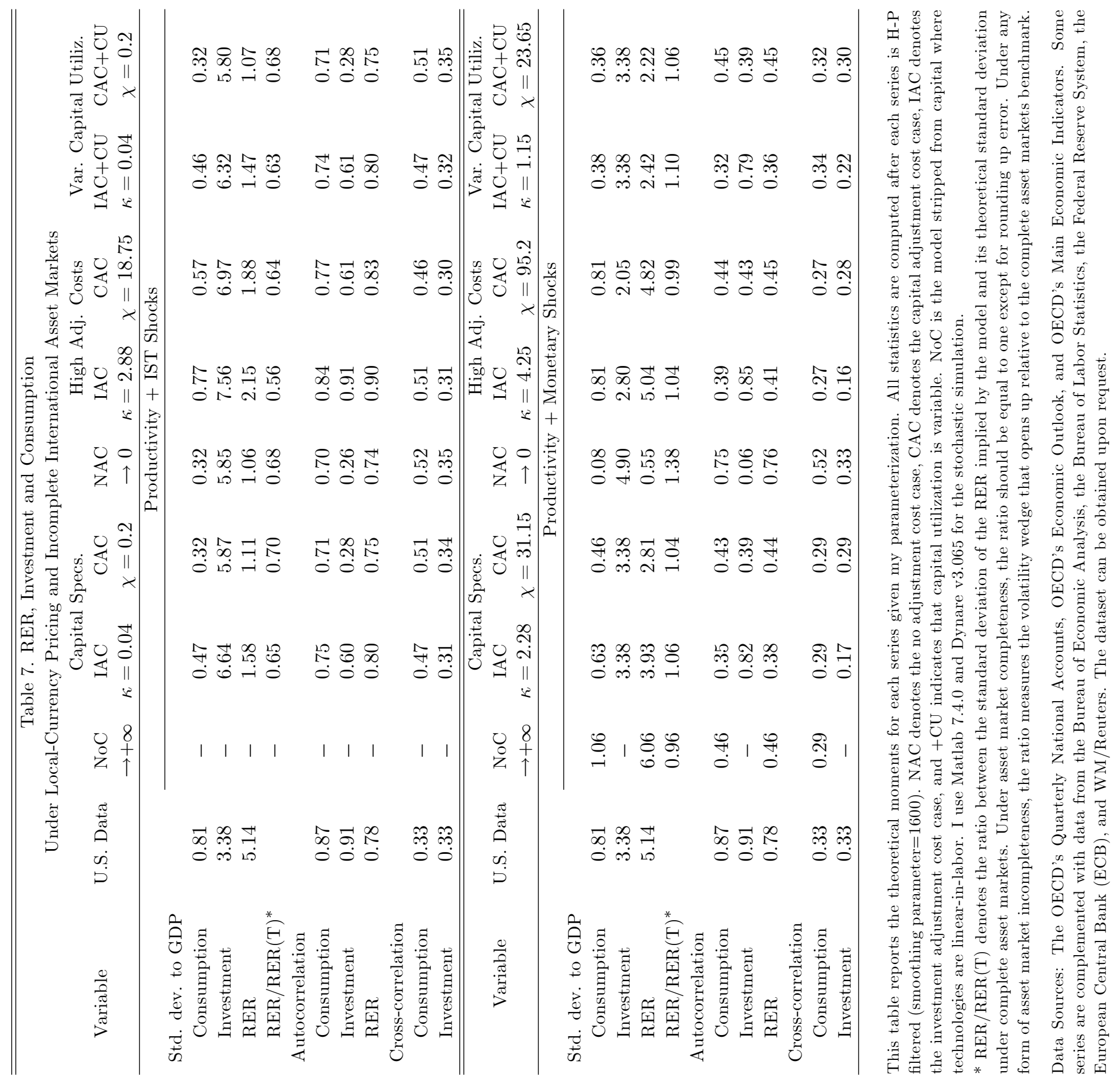




\section{Figures}

\section{C.1 Figures under Local-Currency Pricing and Complete International Asset Markets}

Figure 1. RER Volatility in Relation to the Adjustment Costs and Monetary Policy Inertia
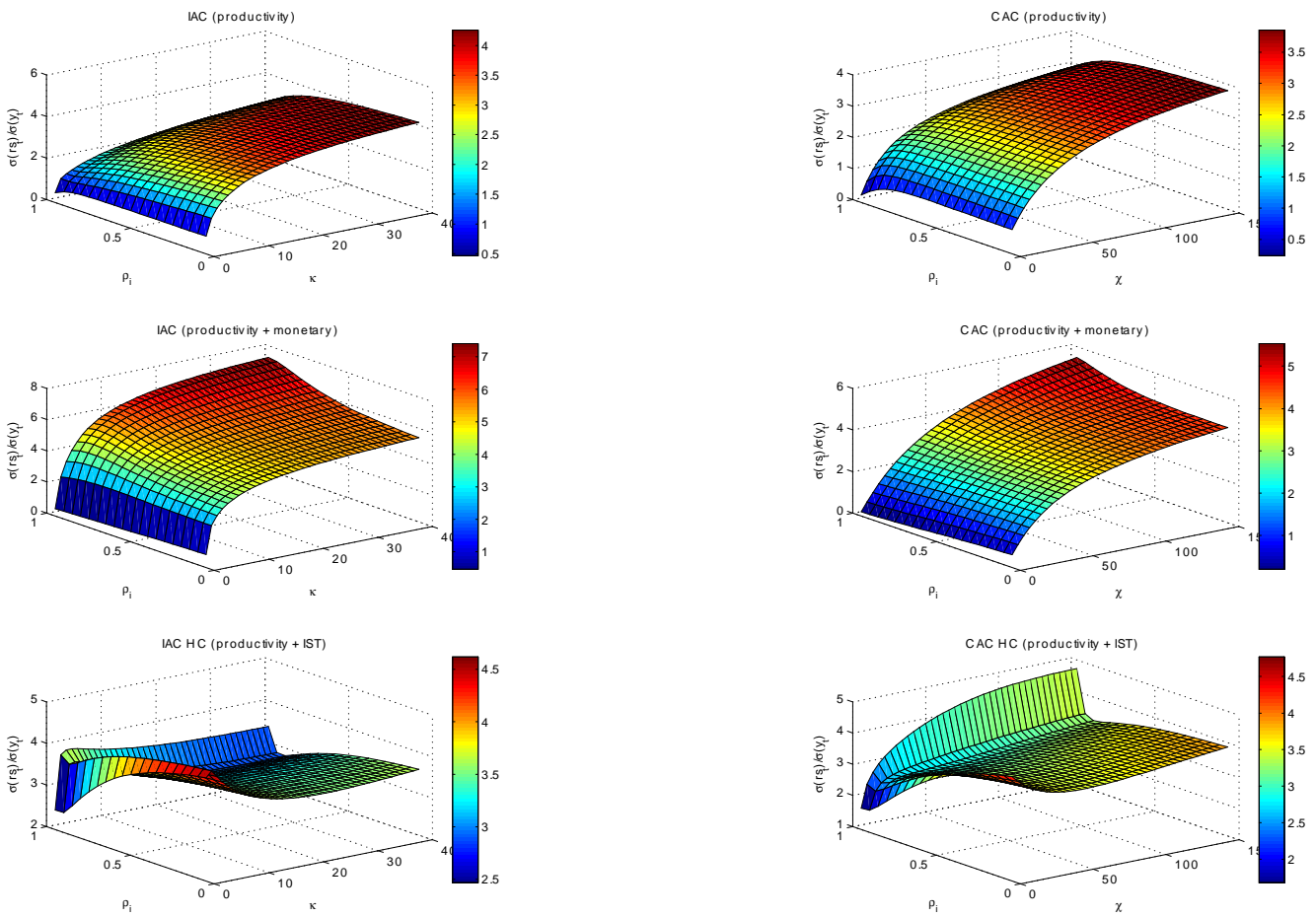

These graphs report the volatility of the RER whenever I allow the monetary policy inertia and the adjustment cost parameter to vary within a range that includes the baseline parameterization. All other structural parameters remain invariant. The statistics are computed after each series is H-P filtered (smoothing parameter=1600). CAC denotes the capital adjustment cost case, and IAC denotes the investment adjustment cost case. The baseline parameterization is unmarked and aims at matching the investment volatility ratio, while HC indicates the alternative case where I approximate the consumption volatility ratio. Within parenthesis, 'productivity' refers to the experiment where business cycles are entirely driven by productivity shocks, 'productivity + monetary' indicates the experiment where monetary and productivity shocks jointly determine the cycle, while 'productivity + IST' indicates the experiment where investment-specific technology (IST) and productivity shocks jointly determine the cycle. I use Matlab 7.4.0 and Dynare v3.065 for the stochastic simulation. 
Figure 2. RER Persistence in Relation to the Adjustment Costs and Monetary Policy Inertia
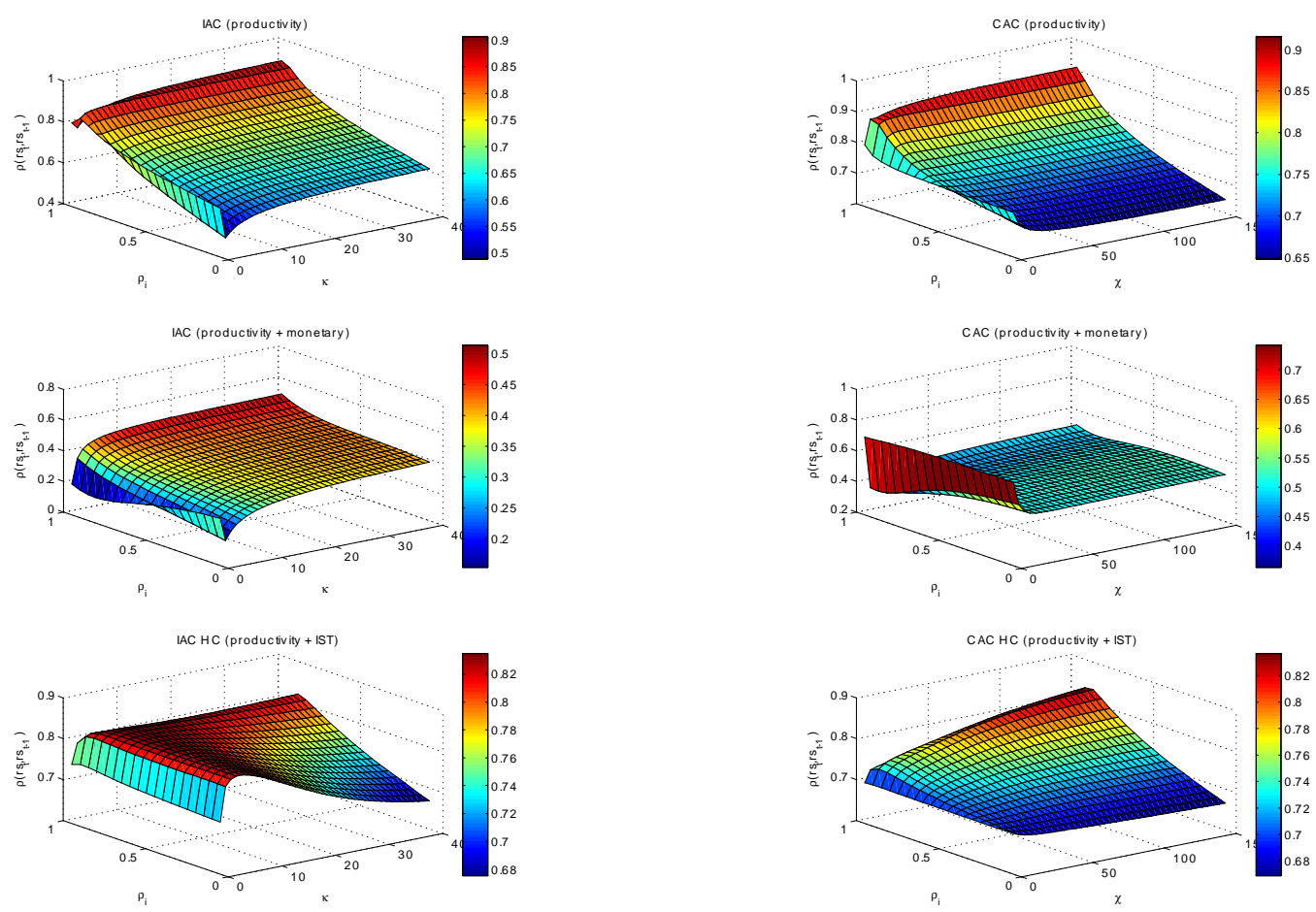

These graphs report the persistence of the RER whenever I allow the monetary policy inertia and the adjustment cost parameter to vary within a range that includes the baseline parameterization. All other structural parameters remain invariant. The statistics are computed after each series is H-P filtered (smoothing parameter $=1600$ ). The baseline parameterization is unmarked and aims at matching the investment volatility ratio, while $\mathrm{HC}$ indicates the alternative case where I approximate the consumption volatility ratio. CAC denotes the capital adjustment cost case, and IAC denotes the investment adjustment cost case. Within parenthesis, 'productivity' refers to the experiment where business cycles are entirely driven by productivity shocks, 'productivity + monetary' indicates the experiment where monetary and productivity shocks jointly determine the cycle, while 'productivity + IST' indicates the experiment where productivity and investment-specific technology (IST) shocks jointly determine the cycle. I use Matlab 7.4.0 and Dynare v3.065 for the stochastic simulation. 
Figure 3. RER Volatility in Relation to the Adjustment Costs and Policy Response to Inflation Deviations
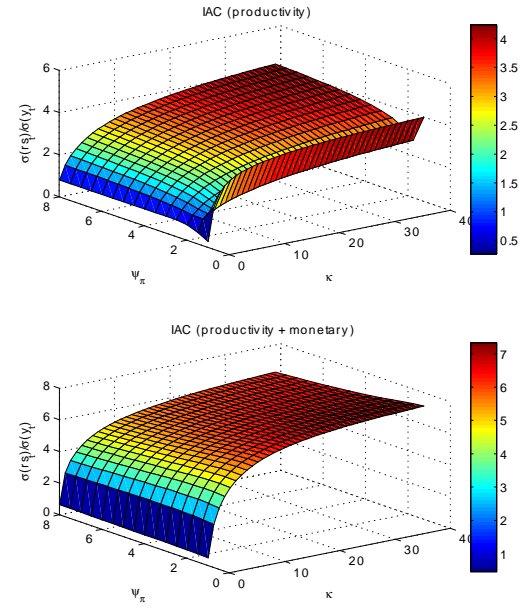

IAC HC (productivity + IST)

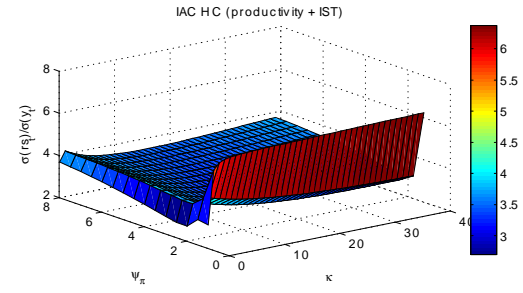

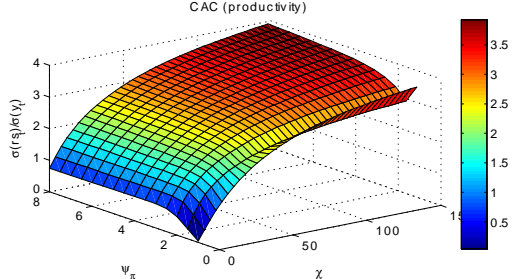
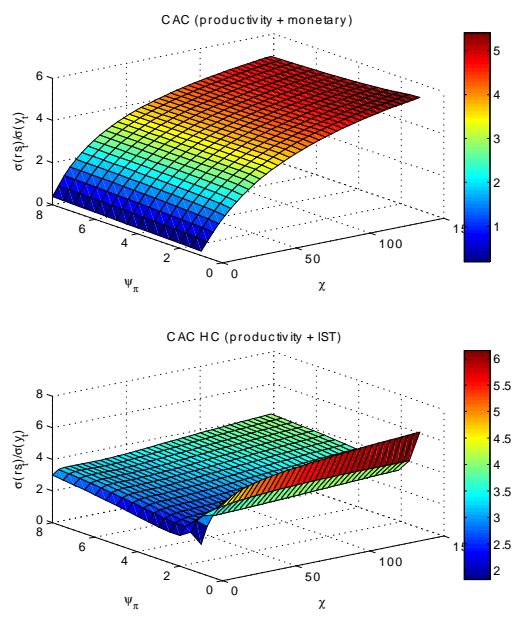

These graphs report the volatility of the RER whenever I allow the monetary policy response to inflation deviations and the adjustment cost parameter to vary within a range that includes the baseline parameterization. The weight on inflation deviations in the Taylor rule is always kept above one. All other structural parameters remain invariant. The statistics are computed after each series is H-P filtered (smoothing parameter $=1600$ ). The baseline parameterization is unmarked and aims at matching the investment volatility ratio, while $\mathrm{HC}$ indicates the alternative case where I approximate the consumption volatility ratio. CAC denotes the capital adjustment cost case, and IAC denotes the investment adjustment cost case. Within parenthesis, 'productivity' refers to the experiment where business cycles are entirely driven by productivity shocks, 'productivity + monetary' indicates the experiment where monetary and productivity shocks jointly determine the cycle, while 'productivity + IST' indicates the experiment where productivity and investment-specific technology (IST) shocks jointly determine the cycle. I use Matlab 7.4.0 and Dynare v3.065 for the stochastic simulation. 
Figure 4. RER Persistence in Relation to the Adjustment Costs and Policy Response to Inflation Deviations
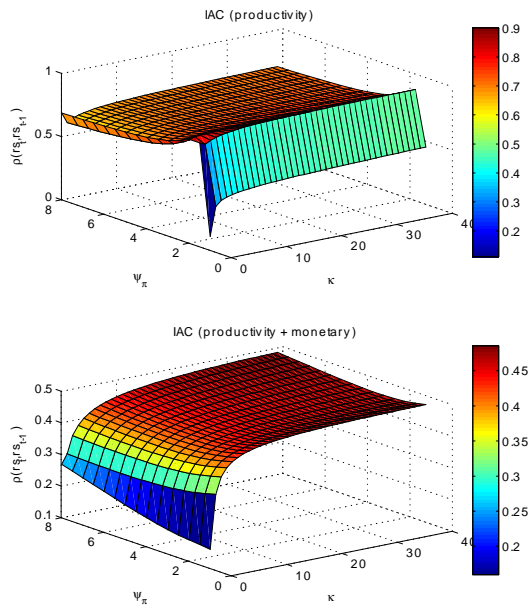

IAC HC (productivity + IST)

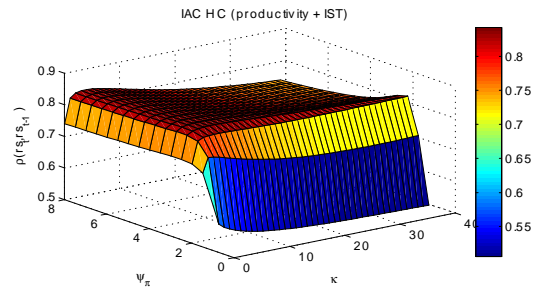

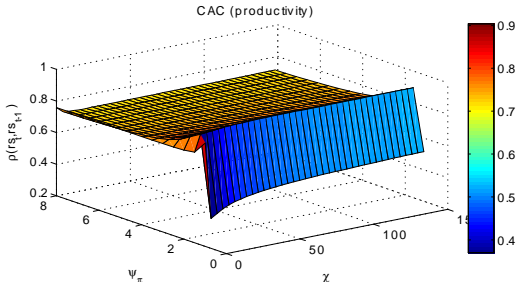
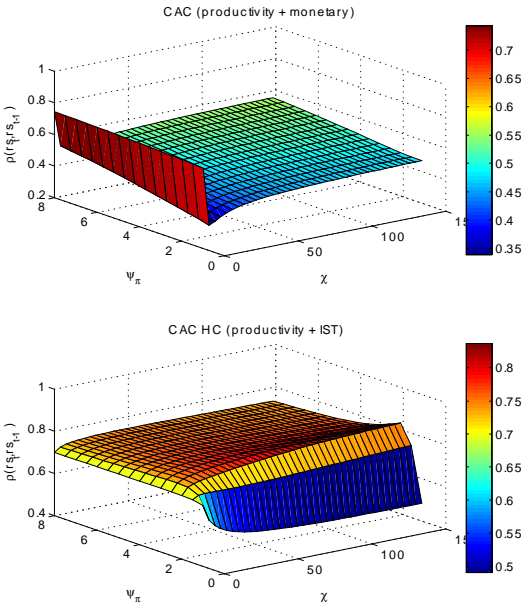

These graphs report the persistence of the RER whenever I allow the monetary policy response to inflation deviations and the adjustment cost parameter to vary within a range that includes the baseline parameterization. The weight on inflation deviations in the Taylor rule is always kept above one. All other structural parameters remain invariant. The statistics are computed after each series is H-P filtered (smoothing parameter $=1600$ ). The baseline parameterization is unmarked and aims at matching the investment volatility ratio, while $\mathrm{HC}$ indicates the alternative case where I approximate the consumption volatility ratio. CAC denotes the capital adjustment cost case, and IAC denotes the investment adjustment cost case. Within parenthesis, 'productivity' refers to the experiment where business cycles are entirely driven by productivity shocks, 'productivity + monetary' indicates the experiment where monetary and productivity shocks jointly determine the cycle, while 'productivity + IST' indicates the experiment where productivity and investment-specific technology (IST) shocks jointly determine the cycle. I use Matlab 7.4.0 and Dynare v3.065 for the stochastic simulation. 
Figure 5. RER Volatility in Relation to the Adjustment Costs and Policy Response to Output Deviations
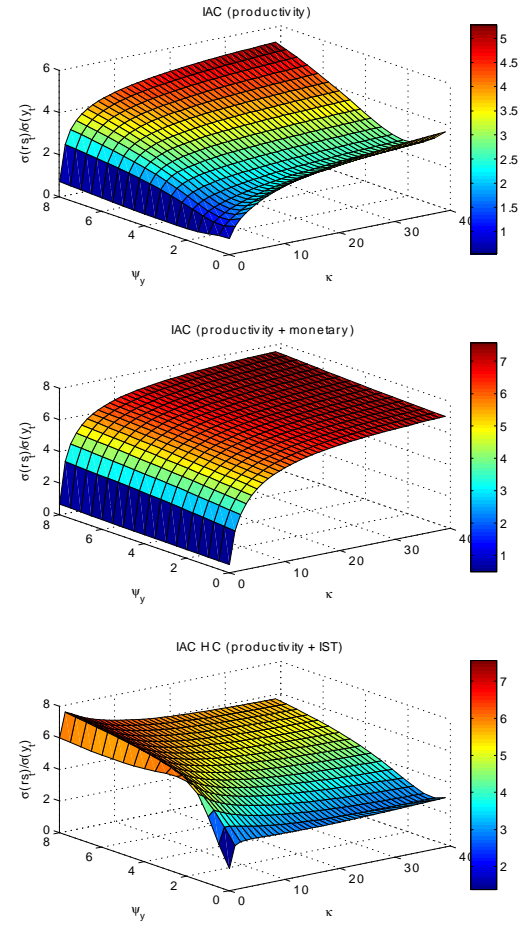
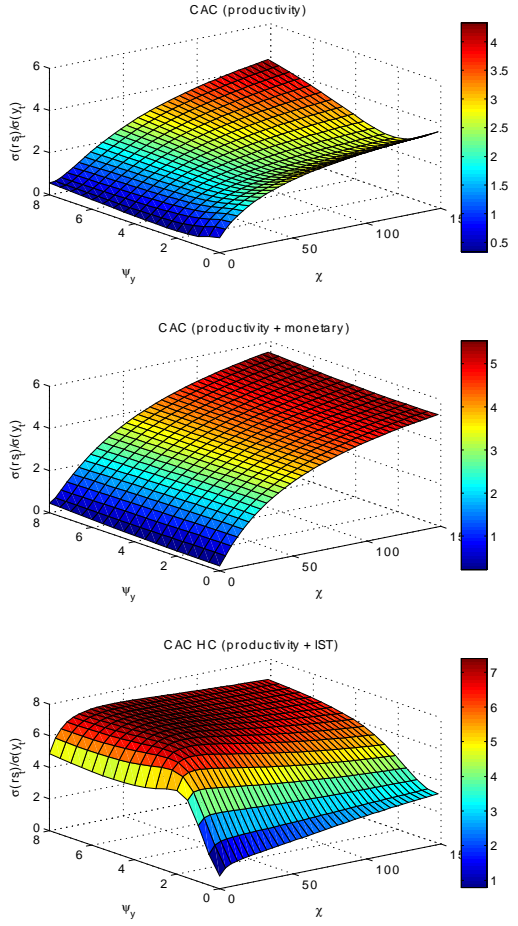

These graphs report the volatility of the RER whenever I allow the monetary policy response to output deviations and the adjustment cost parameter to vary within a range that includes the baseline parameterization. All other structural parameters remain invariant. The statistics are computed after each series is H-P filtered (smoothing parameter $=1600)$. The baseline parameterization is unmarked and aims at matching the investment volatility ratio, while $\mathrm{HC}$ indicates the alternative case where I approximate the consumption volatility ratio. CAC denotes the capital adjustment cost case, and IAC denotes the investment adjustment cost case. Within parenthesis, 'productivity' refers to the experiment where business cycles are entirely driven by productivity shocks, 'productivity + monetary' indicates the experiment where monetary and productivity shocks jointly determine the cycle, while 'productivity + IST' indicates the experiment where productivity and investment-specific technology (IST) shocks jointly determine the cycle. I use Matlab 7.4.0 and Dynare v3.065 for the stochastic simulation. 
Figure 6. RER Persistence in Relation to the Adjustment Costs and Policy Response to Output Deviations
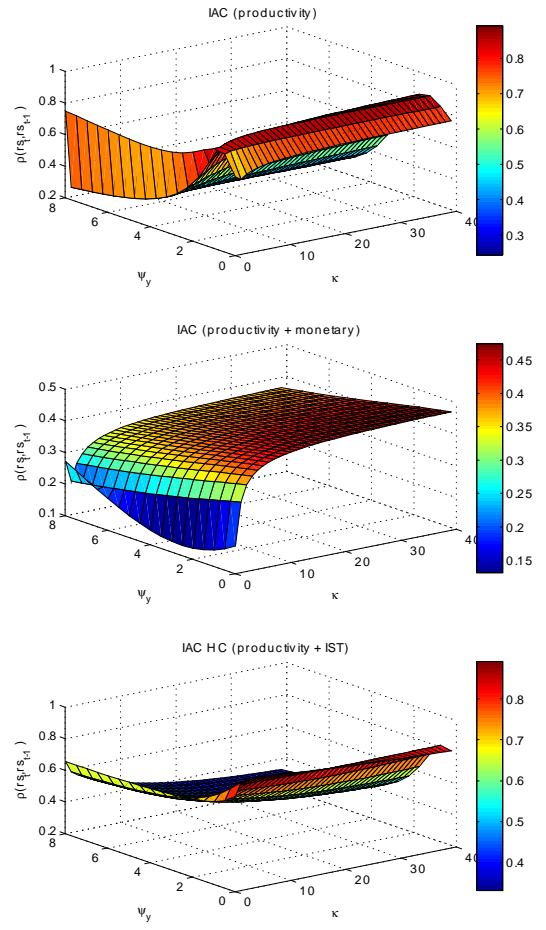
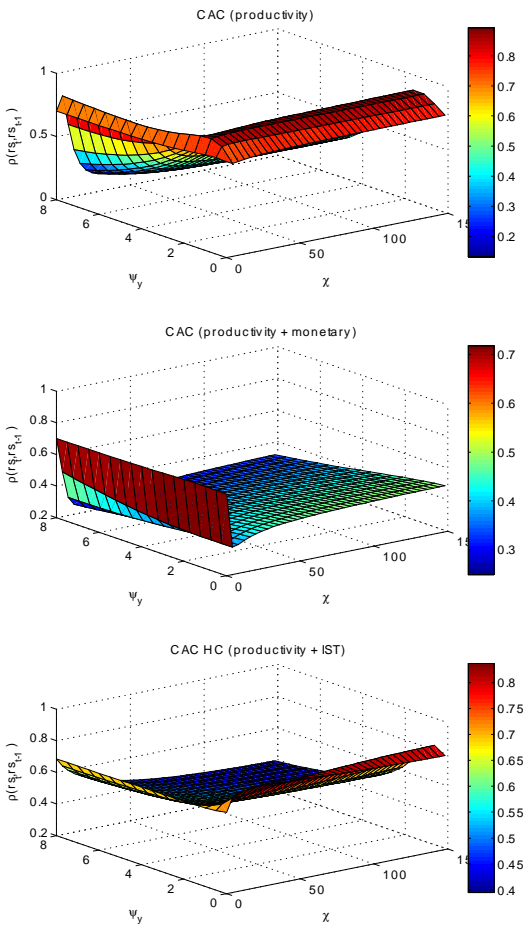

These graphs report the persistence of the RER whenever I allow the monetary policy response to output deviations and the adjustment cost parameter to vary within a range that includes the baseline parameterization. All other structural parameters remain invariant. The statistics are computed after each series is H-P filtered (smoothing parameter $=1600)$. The baseline parameterization is unmarked and aims at matching the investment volatility ratio, while $\mathrm{HC}$ indicates the alternative case where I approximate the consumption volatility ratio. CAC denotes the capital adjustment cost case, and IAC denotes the investment adjustment cost case. Within parenthesis, 'productivity' refers to the experiment where business cycles are entirely driven by productivity shocks, 'productivity + monetary' indicates the experiment where monetary and productivity shocks jointly determine the cycle, while 'productivity + IST' indicates the experiment where productivity and investment-specific technology (IST) shocks jointly determine the cycle. I use Matlab 7.4.0 and Dynare v3.065 for the stochastic simulation. 


\section{C.2 Figures under Producer-Currency Pricing and Complete International As- set Markets}

Figure 7. RER Volatility in Relation to the Adjustment Costs and Monetary Policy Inertia
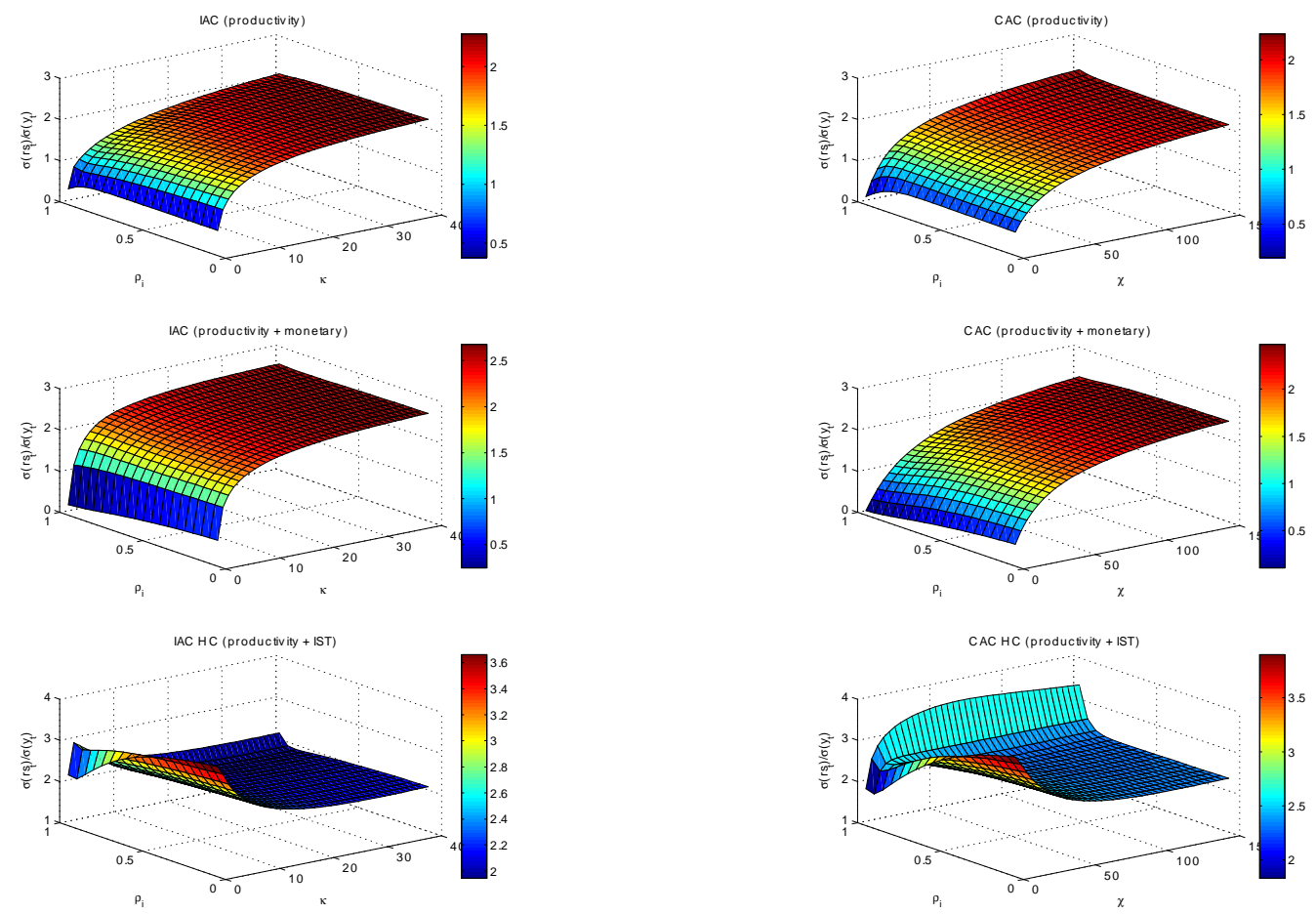

These graphs report the volatility of the RER whenever I allow the monetary policy inertia and the adjustment cost parameter to vary within a range that includes the baseline parameterization. All other structural parameters remain invariant. The statistics are computed after each series is H-P filtered (smoothing parameter $=1600$ ). CAC denotes the capital adjustment cost case, and IAC denotes the investment adjustment cost case. The baseline parameterization is unmarked and aims at matching the investment volatility ratio, while HC indicates the alternative case where I approximate the consumption volatility ratio. Within parenthesis, 'productivity' refers to the experiment where business cycles are entirely driven by productivity shocks, 'productivity + monetary' indicates the experiment where monetary and productivity shocks jointly determine the cycle, while 'productivity + IST' indicates the experiment where investment-specific technology (IST) and productivity shocks jointly determine the cycle. I use Matlab 7.4.0 and Dynare v3.065 for the stochastic simulation. 
Figure 8. RER Persistence in Relation to the Adjustment Costs and Monetary Policy Inertia
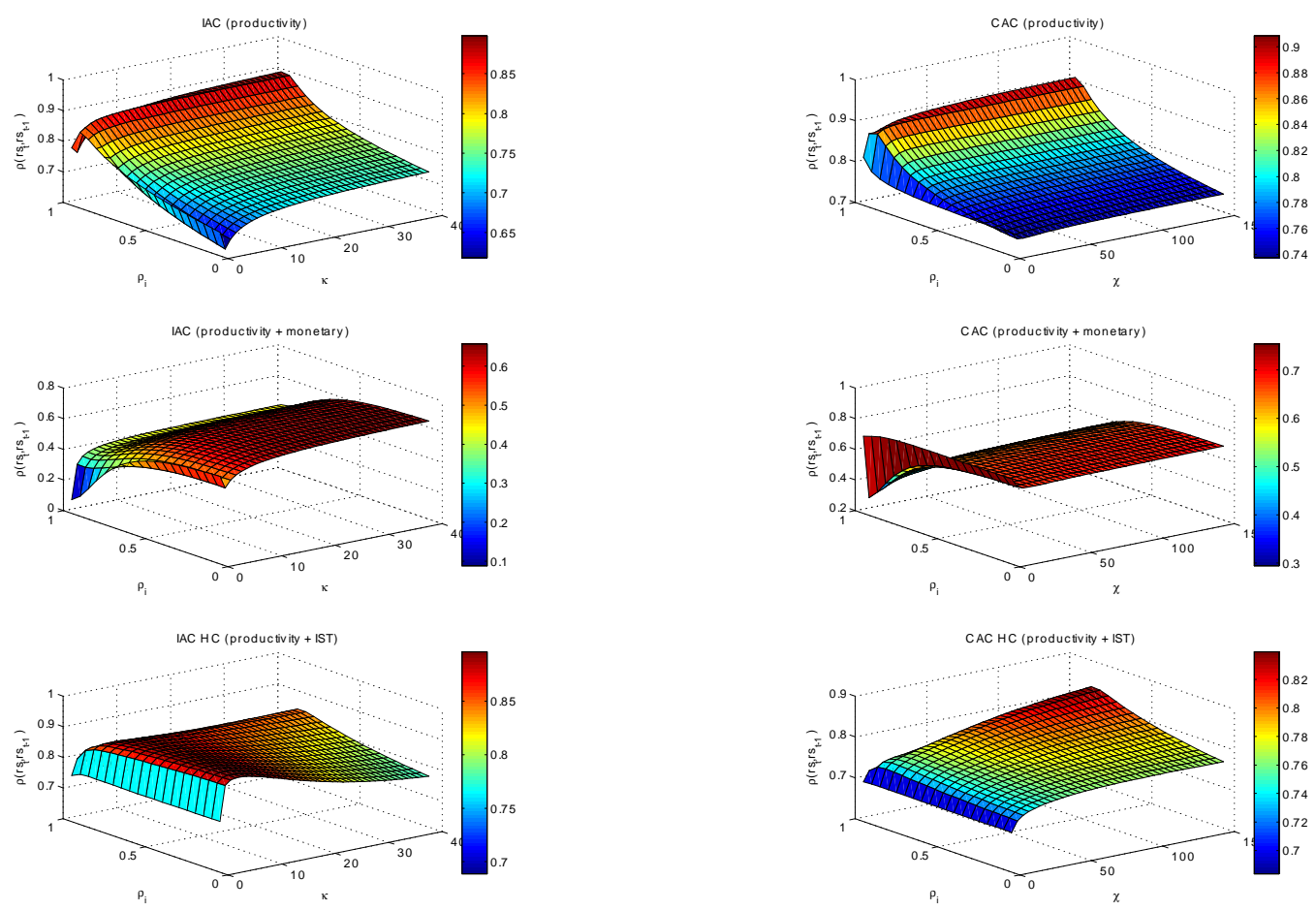

These graphs report the persistence of the RER whenever I allow the monetary policy inertia and the adjustment cost parameter to vary within a range that includes the baseline parameterization. All other structural parameters remain invariant. The statistics are computed after each series is H-P filtered (smoothing parameter $=1600$ ). The baseline parameterization is unmarked and aims at matching the investment volatility ratio, while $\mathrm{HC}$ indicates the alternative case where I approximate the consumption volatility ratio. CAC denotes the capital adjustment cost case, and IAC denotes the investment adjustment cost case. Within parenthesis, 'productivity' refers to the experiment where business cycles are entirely driven by productivity shocks, 'productivity + monetary' indicates the experiment where monetary and productivity shocks jointly determine the cycle, while 'productivity + IST' indicates the experiment where productivity and investment-specific technology (IST) shocks jointly determine the cycle. I use Matlab 7.4.0 and Dynare v3.065 for the stochastic simulation. 
Figure 9. RER Volatility in Relation to the Adjustment Costs and Policy Response to Inflation Deviations
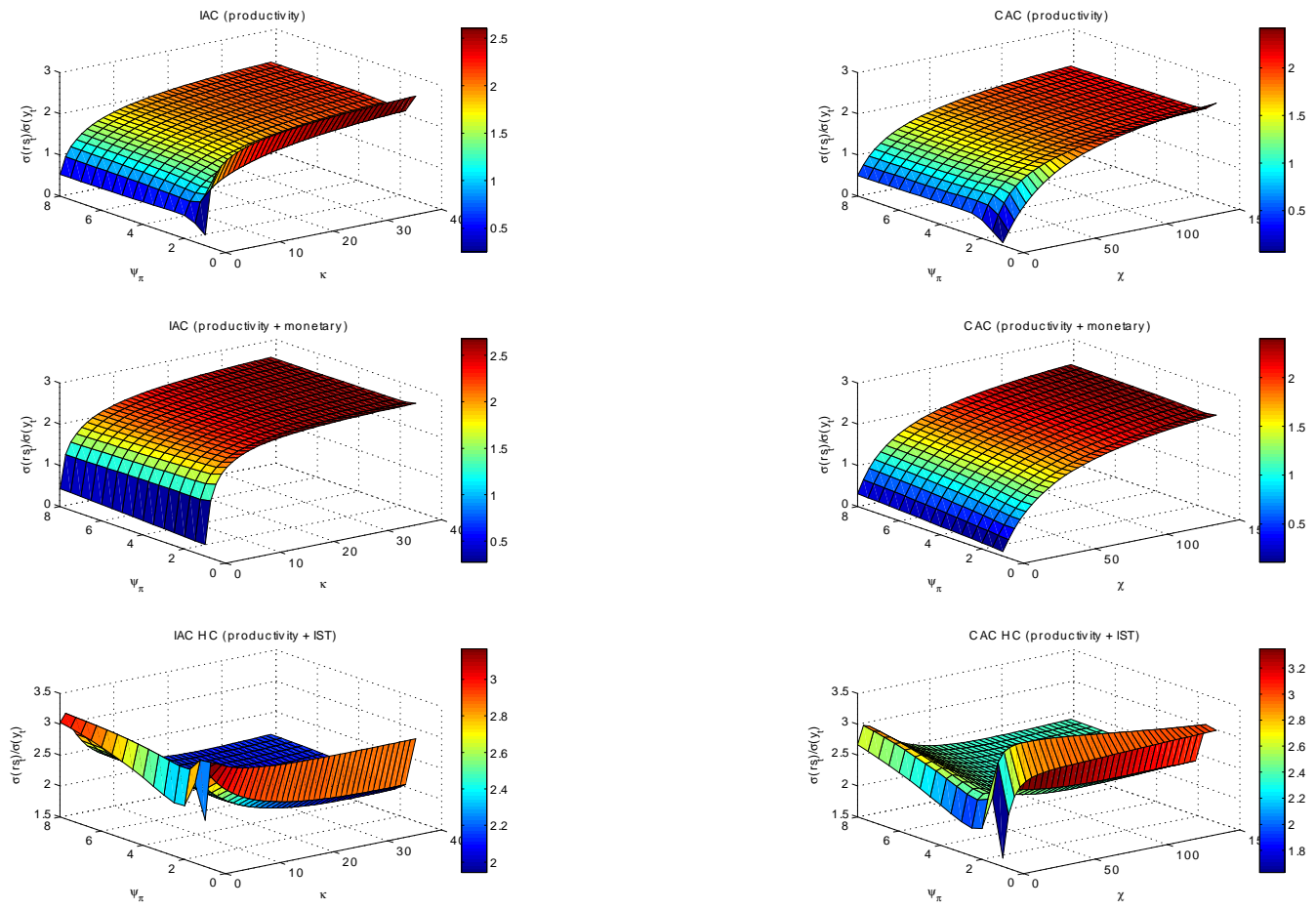

These graphs report the volatility of the RER whenever I allow the monetary policy response to inflation deviations and the adjustment cost parameter to vary within a range that includes the baseline parameterization. The weight on inflation deviations in the Taylor rule is always kept above one. All other structural parameters remain invariant. The statistics are computed after each series is H-P filtered (smoothing parameter $=1600$ ). The baseline parameterization is unmarked and aims at matching the investment volatility ratio, while $\mathrm{HC}$ indicates the alternative case where I approximate the consumption volatility ratio. CAC denotes the capital adjustment cost case, and IAC denotes the investment adjustment cost case. Within parenthesis, 'productivity' refers to the experiment where business cycles are entirely driven by productivity shocks, 'productivity + monetary' indicates the experiment where monetary and productivity shocks jointly determine the cycle, while 'productivity + IST' indicates the experiment where productivity and investment-specific technology (IST) shocks jointly determine the cycle. I use Matlab 7.4.0 and Dynare v3.065 for the stochastic simulation. 


\section{Figure 10. RER Persistence in Relation to the Adjustment Costs and Policy Response to Inflation Deviations}
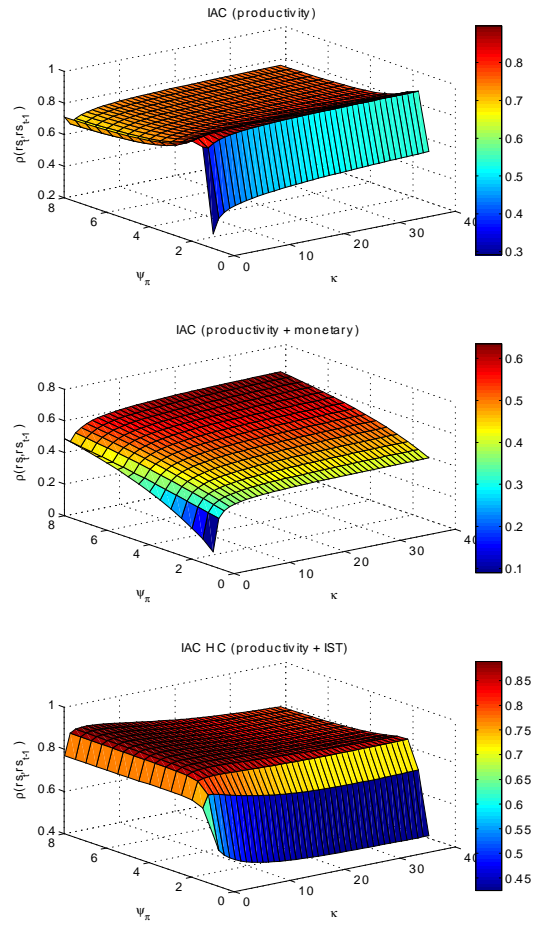
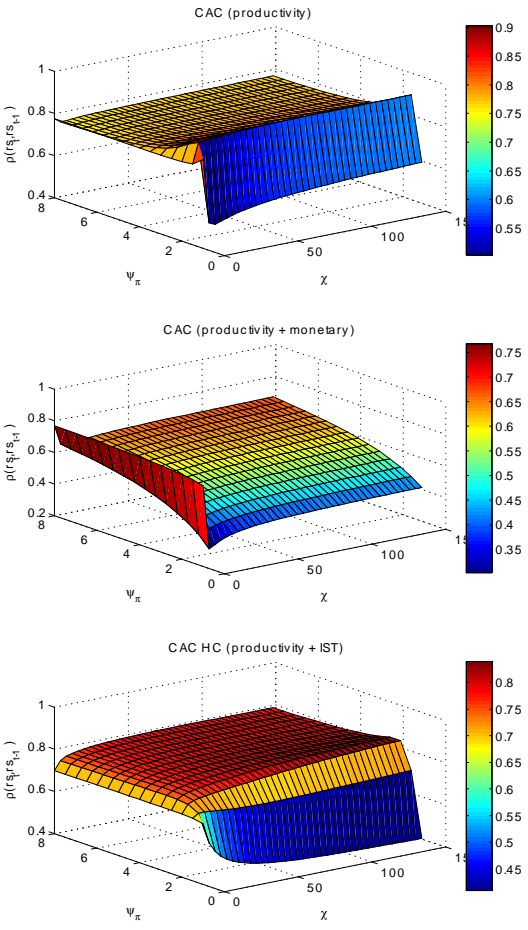

These graphs report the persistence of the RER whenever I allow the monetary policy response to inflation deviations and the adjustment cost parameter to vary within a range that includes the baseline parameterization. The weight on inflation deviations in the Taylor rule is always kept above one. All other structural parameters remain invariant. The statistics are computed after each series is H-P filtered (smoothing parameter $=1600$ ). The baseline parameterization is unmarked and aims at matching the investment volatility ratio, while $\mathrm{HC}$ indicates the alternative case where I approximate the consumption volatility ratio. CAC denotes the capital adjustment cost case, and IAC denotes the investment adjustment cost case. Within parenthesis, 'productivity' refers to the experiment where business cycles are entirely driven by productivity shocks, 'productivity + monetary' indicates the experiment where monetary and productivity shocks jointly determine the cycle, while 'productivity + IST' indicates the experiment where productivity and investment-specific technology (IST) shocks jointly determine the cycle. I use Matlab 7.4.0 and Dynare v3.065 for the stochastic simulation. 
Figure 11. RER Volatility in Relation to the Adjustment Costs and Policy Response to Output Deviations
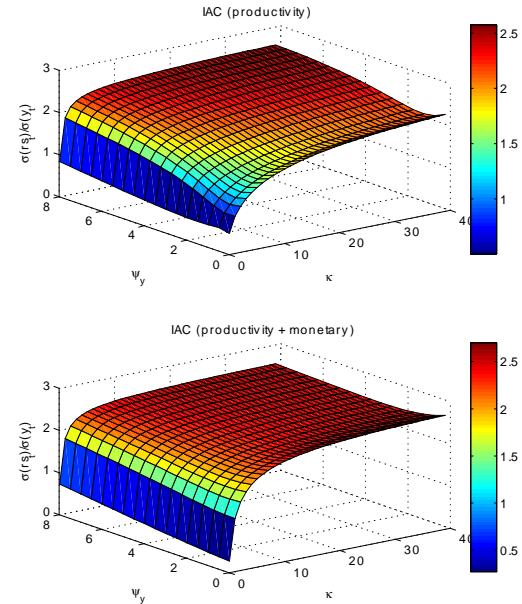

IAC HC (productivity + IST)

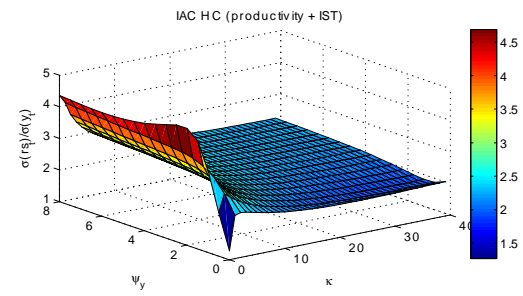

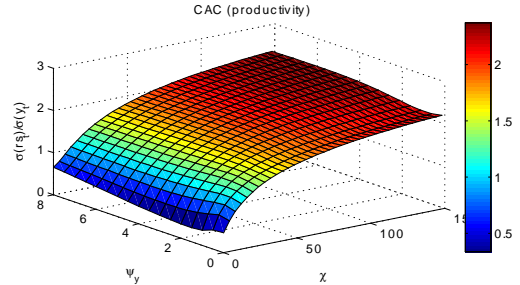
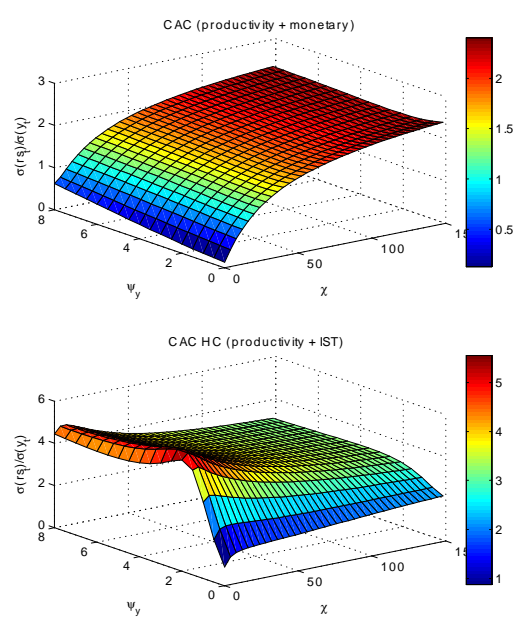

These graphs report the volatility of the RER whenever I allow the monetary policy response to output deviations and the adjustment cost parameter to vary within a range that includes the baseline parameterization. All other structural parameters remain invariant. The statistics are computed after each series is H-P filtered (smoothing parameter $=1600)$. The baseline parameterization is unmarked and aims at matching the investment volatility ratio, while $\mathrm{HC}$ indicates the alternative case where I approximate the consumption volatility ratio. CAC denotes the capital adjustment cost case, and IAC denotes the investment adjustment cost case. Within parenthesis, 'productivity' refers to the experiment where business cycles are entirely driven by productivity shocks, 'productivity + monetary' indicates the experiment where monetary and productivity shocks jointly determine the cycle, while 'productivity + IST' indicates the experiment where productivity and investment-specific technology (IST) shocks jointly determine the cycle. I use Matlab 7.4.0 and Dynare v3.065 for the stochastic simulation. 


\section{Figure 12. RER Persistence in Relation to the Adjustment Costs and Policy Response to Output Deviations}
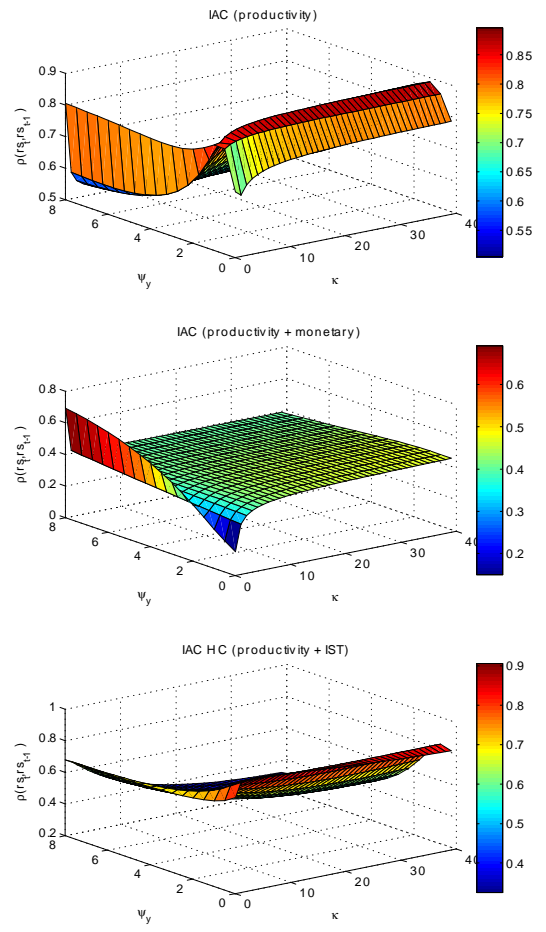
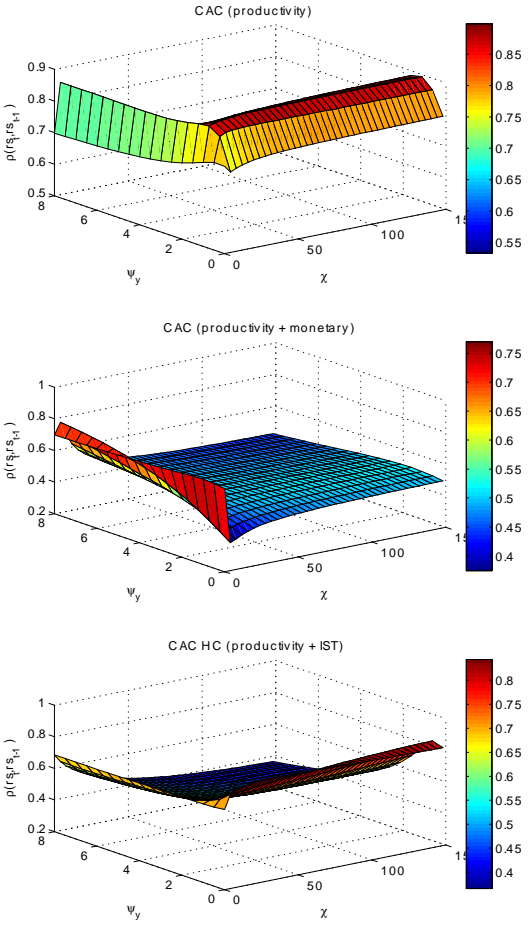

These graphs report the persistence of the RER whenever I allow the monetary policy response to output deviations and the adjustment cost parameter to vary within a range that includes the baseline parameterization. All other structural parameters remain invariant. The statistics are computed after each series is H-P filtered (smoothing parameter $=1600)$. The baseline parameterization is unmarked and aims at matching the investment volatility ratio, while $\mathrm{HC}$ indicates the alternative case where I approximate the consumption volatility ratio. CAC denotes the capital adjustment cost case, and IAC denotes the investment adjustment cost case. Within parenthesis, 'productivity' refers to the experiment where business cycles are entirely driven by productivity shocks, 'productivity + monetary' indicates the experiment where monetary and productivity shocks jointly determine the cycle, while 'productivity + IST' indicates the experiment where productivity and investment-specific technology (IST) shocks jointly determine the cycle. I use Matlab 7.4.0 and Dynare v3.065 for the stochastic simulation. 


\section{C.3 Figures under Local-Currency Pricing and Incomplete International Asset Markets}

Figure 13. RER Volatility in Relation to the Adjustment Costs and Monetary Policy Inertia
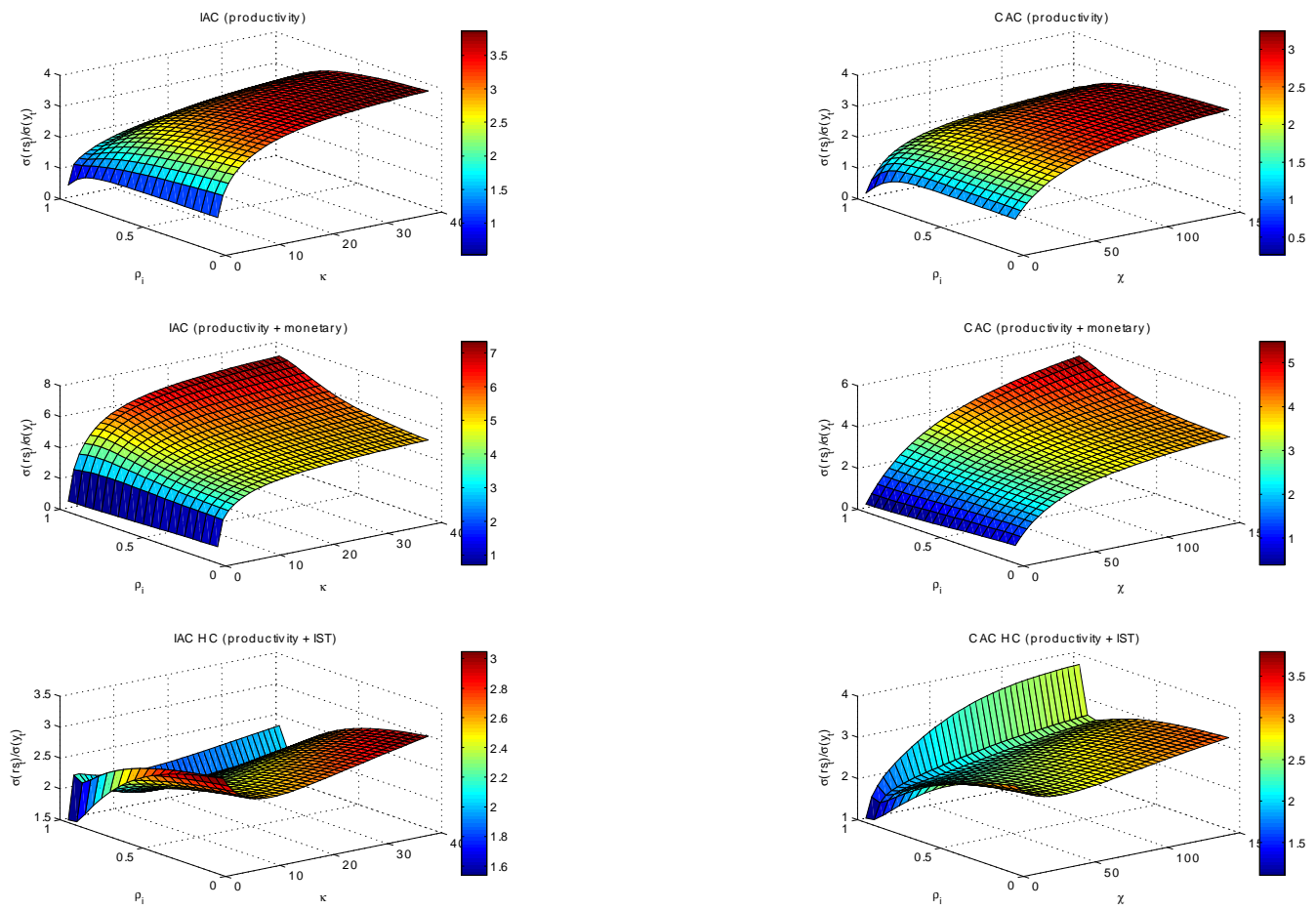

These graphs report the volatility of the RER whenever I allow the monetary policy inertia and the adjustment cost parameter to vary within a range that includes the baseline parameterization. All other structural parameters remain invariant. The statistics are computed after each series is H-P filtered (smoothing parameter $=1600$ ). CAC denotes the capital adjustment cost case, and IAC denotes the investment adjustment cost case. The baseline parameterization is unmarked and aims at matching the investment volatility ratio, while HC indicates the alternative case where I approximate the consumption volatility ratio. Within parenthesis, 'productivity' refers to the experiment where business cycles are entirely driven by productivity shocks, 'productivity + monetary' indicates the experiment where monetary and productivity shocks jointly determine the cycle, while 'productivity + IST' indicates the experiment where investment-specific technology (IST) and productivity shocks jointly determine the cycle. I use Matlab 7.4.0 and Dynare v3.065 for the stochastic simulation. 


\section{Figure 14. RER Persistence in Relation to the Adjustment Costs and Monetary Policy Inertia}
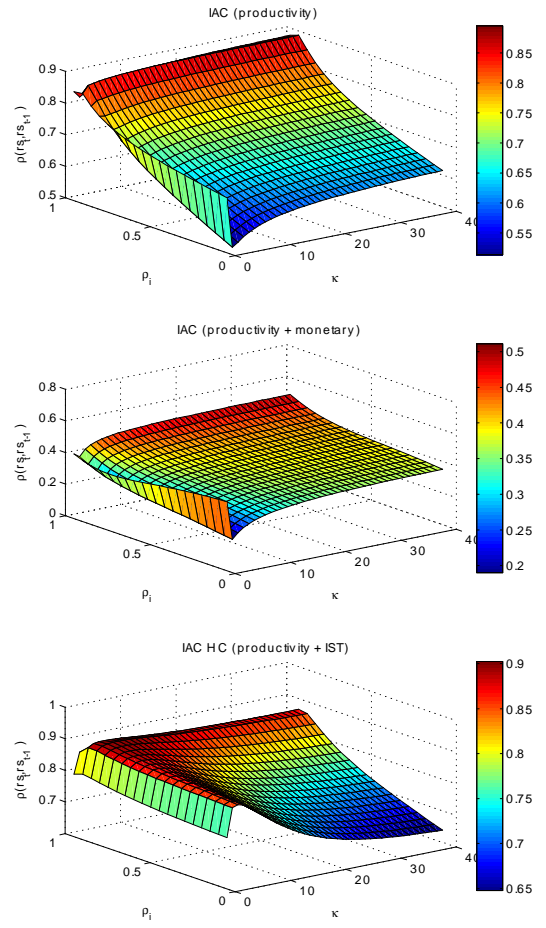
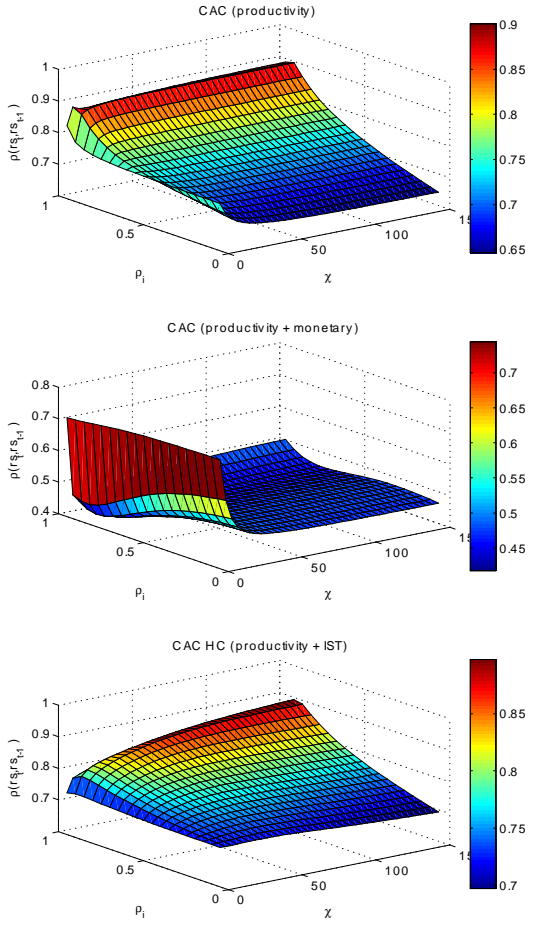

These graphs report the persistence of the RER whenever I allow the monetary policy inertia and the adjustment cost parameter to vary within a range that includes the baseline parameterization. All other structural parameters remain invariant. The statistics are computed after each series is H-P filtered (smoothing parameter=1600). The baseline parameterization is unmarked and aims at matching the investment volatility ratio, while HC indicates the alternative case where I approximate the consumption volatility ratio. CAC denotes the capital adjustment cost case, and IAC denotes the investment adjustment cost case. Within parenthesis, 'productivity' refers to the experiment where business cycles are entirely driven by productivity shocks, 'productivity + monetary' indicates the experiment where monetary and productivity shocks jointly determine the cycle, while 'productivity + IST' indicates the experiment where productivity and investment-specific technology (IST) shocks jointly determine the cycle. I use Matlab 7.4.0 and Dynare v3.065 for the stochastic simulation. 
Figure 15. RER Volatility in Relation to the Adjustment Costs and Policy Response to Inflation Deviations
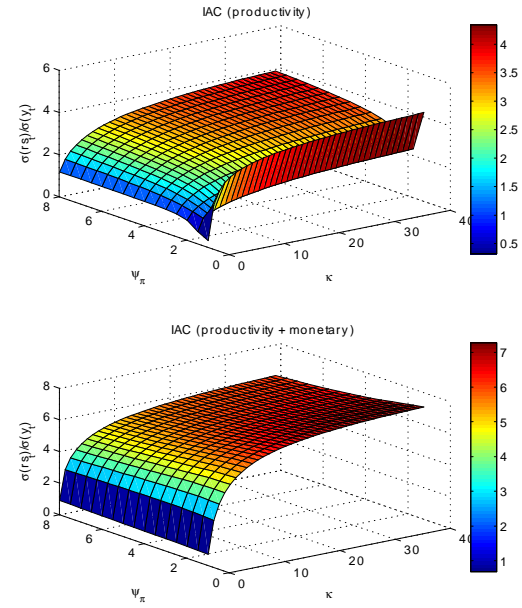

IAC HC (productivity + IST)

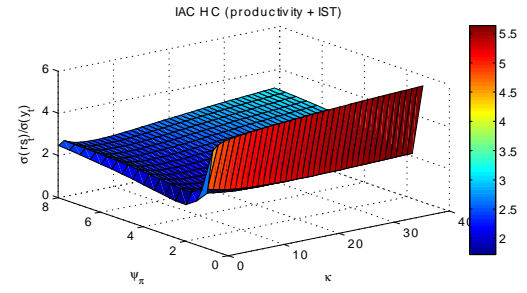

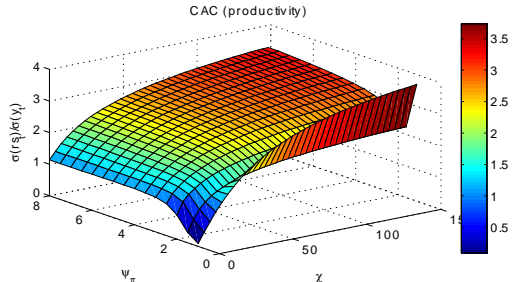
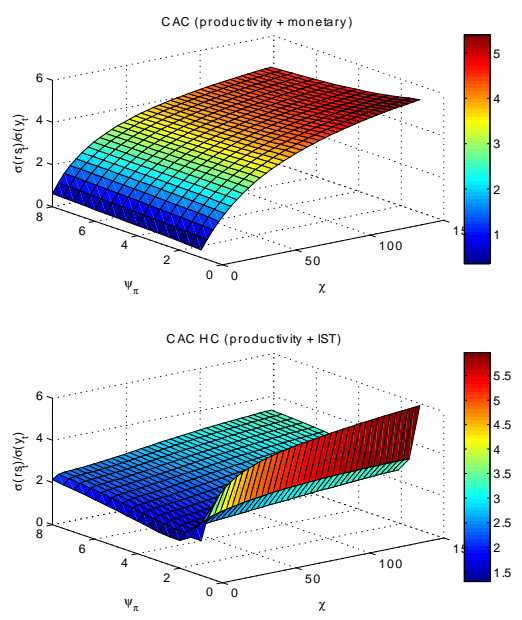

These graphs report the volatility of the RER whenever I allow the monetary policy response to inflation deviations and the adjustment cost parameter to vary within a range that includes the baseline parameterization. The weight on inflation deviations in the Taylor rule is always kept above one. All other structural parameters remain invariant. The statistics are computed after each series is H-P filtered (smoothing parameter $=1600$ ). The baseline parameterization is unmarked and aims at matching the investment volatility ratio, while $\mathrm{HC}$ indicates the alternative case where I approximate the consumption volatility ratio. CAC denotes the capital adjustment cost case, and IAC denotes the investment adjustment cost case. Within parenthesis, 'productivity' refers to the experiment where business cycles are entirely driven by productivity shocks, 'productivity + monetary' indicates the experiment where monetary and productivity shocks jointly determine the cycle, while 'productivity + IST' indicates the experiment where productivity and investment-specific technology (IST) shocks jointly determine the cycle. I use Matlab 7.4.0 and Dynare v3.065 for the stochastic simulation. 


\section{Figure 16. RER Persistence in Relation to the Adjustment Costs and Policy Response to Inflation Deviations}
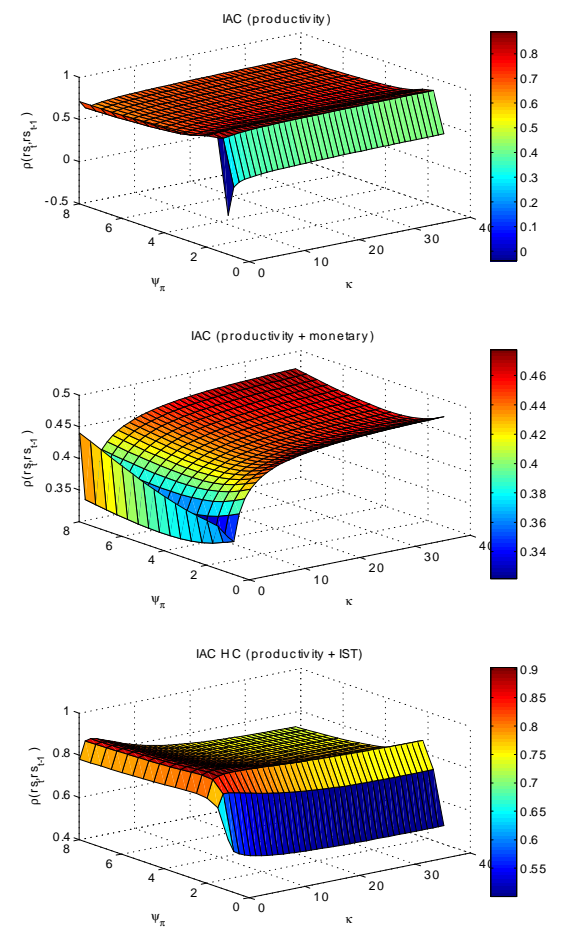
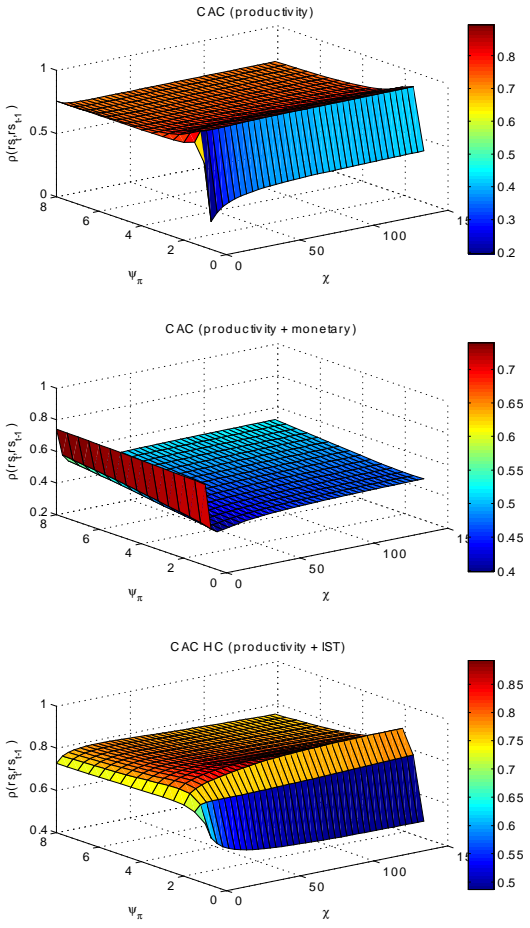

These graphs report the persistence of the RER whenever I allow the monetary policy response to inflation deviations and the adjustment cost parameter to vary within a range that includes the baseline parameterization. The weight on inflation deviations in the Taylor rule is always kept above one. All other structural parameters remain invariant. The statistics are computed after each series is H-P filtered (smoothing parameter $=1600$ ). The baseline parameterization is unmarked and aims at matching the investment volatility ratio, while $\mathrm{HC}$ indicates the alternative case where I approximate the consumption volatility ratio. CAC denotes the capital adjustment cost case, and IAC denotes the investment adjustment cost case. Within parenthesis, 'productivity' refers to the experiment where business cycles are entirely driven by productivity shocks, 'productivity + monetary' indicates the experiment where monetary and productivity shocks jointly determine the cycle, while 'productivity + IST' indicates the experiment where productivity and investment-specific technology (IST) shocks jointly determine the cycle. I use Matlab 7.4.0 and Dynare v3.065 for the stochastic simulation. 
Figure 17. RER Volatility in Relation to the Adjustment Costs and Policy Response to Output Deviations
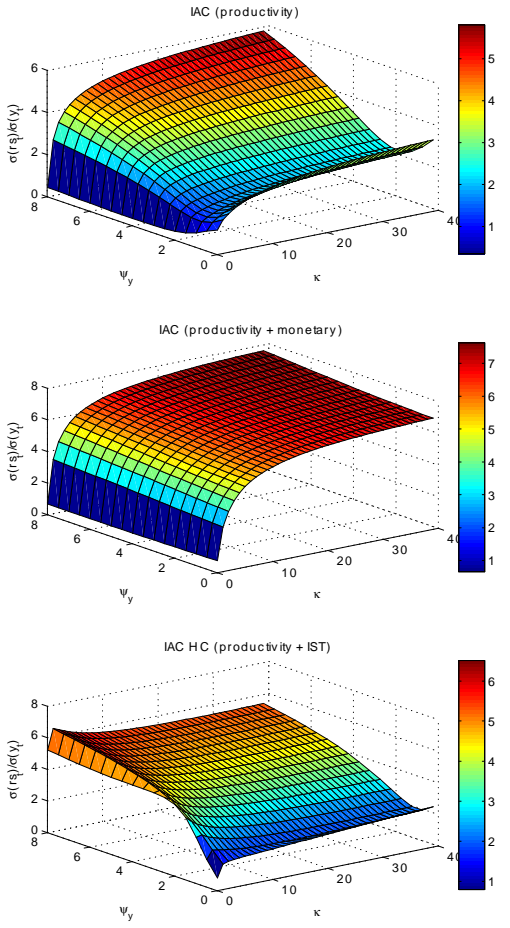
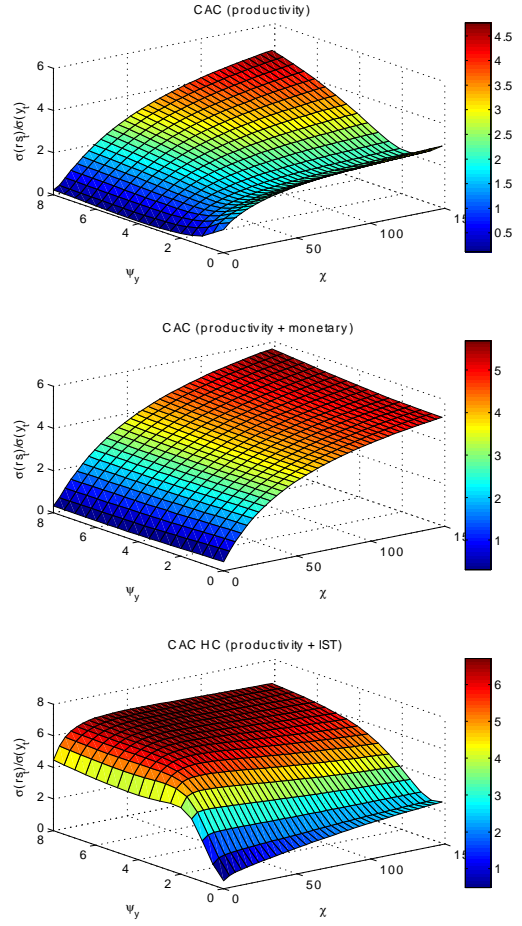

These graphs report the volatility of the RER whenever I allow the monetary policy response to output deviations and the adjustment cost parameter to vary within a range that includes the baseline parameterization. All other structural parameters remain invariant. The statistics are computed after each series is H-P filtered (smoothing parameter $=1600)$. The baseline parameterization is unmarked and aims at matching the investment volatility ratio, while $\mathrm{HC}$ indicates the alternative case where I approximate the consumption volatility ratio. CAC denotes the capital adjustment cost case, and IAC denotes the investment adjustment cost case. Within parenthesis, 'productivity' refers to the experiment where business cycles are entirely driven by productivity shocks, 'productivity + monetary' indicates the experiment where monetary and productivity shocks jointly determine the cycle, while 'productivity + IST' indicates the experiment where productivity and investment-specific technology (IST) shocks jointly determine the cycle. I use Matlab 7.4.0 and Dynare v3.065 for the stochastic simulation. 


\section{Figure 18. RER Persistence in Relation to the Adjustment Costs and Policy Response to Output Deviations}
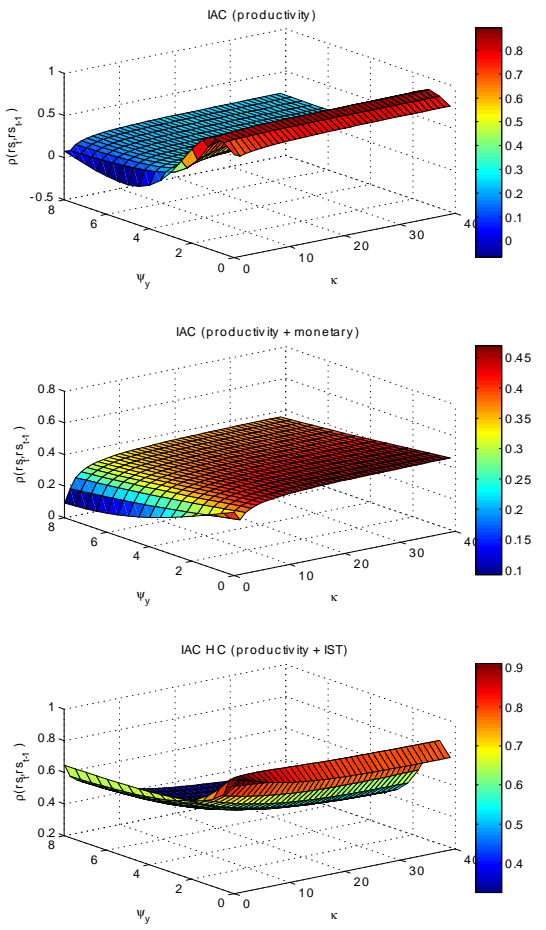
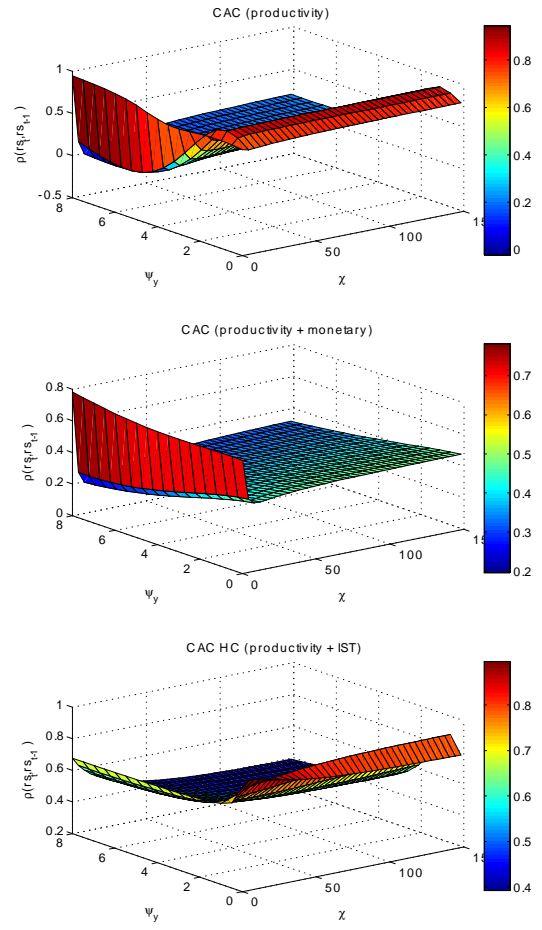

These graphs report the persistence of the RER whenever I allow the monetary policy response to output deviations and the adjustment cost parameter to vary within a range that includes the baseline parameterization. All other structural parameters remain invariant. The statistics are computed after each series is H-P filtered (smoothing parameter $=1600)$. The baseline parameterization is unmarked and aims at matching the investment volatility ratio, while $\mathrm{HC}$ indicates the alternative case where I approximate the consumption volatility ratio. CAC denotes the capital adjustment cost case, and IAC denotes the investment adjustment cost case. Within parenthesis, 'productivity' refers to the experiment where business cycles are entirely driven by productivity shocks, 'productivity + monetary' indicates the experiment where monetary and productivity shocks jointly determine the cycle, while 'productivity + IST' indicates the experiment where productivity and investment-specific technology (IST) shocks jointly determine the cycle. I use Matlab 7.4.0 and Dynare v3.065 for the stochastic simulation. 